$6-1-2020$

\title{
Behavioral Sentinel Surveillance Survey in Nigeria
}

Emily White Johansson

Paul Hutchinson

Elizabeth Omoluabi

Akanni Akinyemi

Andrea Smith

See next page for additional authors

Follow this and additional works at: https://knowledgecommons.popcouncil.org/departments_sbsr-pgy

Part of the Health Communication Commons, and the Public Health Commons

How does access to this work benefit you? Let us know!

\section{Recommended Citation}

White Johansson, Emily, Paul Hutchinson, Elizabeth Omoluabi, Akanni Akinyemi, Andrea Smith, J. Clint Welty, Udochisom Anaba, Dominique Meekers, Alejandra Leyton, Dele Abegunde, and Paul C. Hewett. 2020. "Behavioral Sentinel Surveillance Survey in Nigeria," Breakthrough RESEARCH Baseline Technical Report. Washington, DC: Population Council. 


\section{Authors}

Emily White Johansson, Paul Hutchinson, Elizabeth Omoluabi, Akanni Akinyemi, Andrea Smith, J. Clint Welty, Udochisom Anaba, Dominique Meekers, Alejandra Leyton, Dele Abegunde, and Paul C. Hewett 
BASELINE TECHNICAL REPORT

\section{Behavioral Sentinel Surveillance Survey in Nigeria}

JUNE 2020

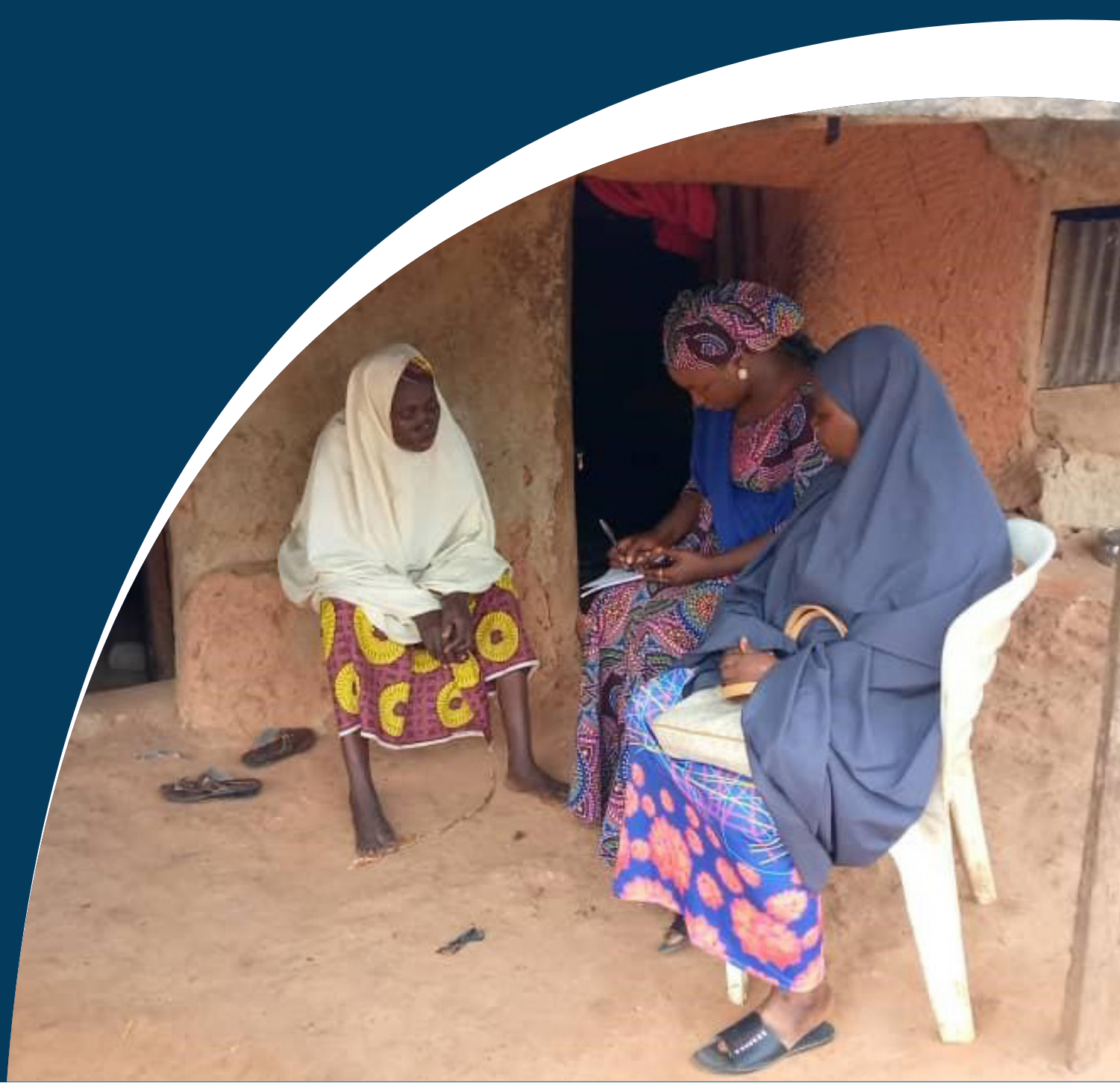




\section{Acknowledgments}

This research report was prepared by Emily White Johansson, Paul Hutchinson, Andrea Smith, J Clint Welty, Udochisom Anaba, Alejandra Leyton, and Dominique Meekers of Tulane University; Elizabeth Omoluabi and Akanni Akinyemi of the Centre for Research, Evaluation Resources and Development (CRERD); and Dele Abegunde and Paul Hewett of Population Council. We would like to offer our special thanks to the leadership and teams of supervisors and interviewers at CRERD for their diligent work and attention to detail. We would also like to express our sincere gratitude to lan Tweedie, Mathew Okoh, Stella Babalola, and countless others at Breakthrough ACTION for their input on the evaluation design and invaluable contributions towards the completion of this study. Thanks to Laurie Chamberlain and Alison Collier for helpful edits on the report and to Mariko Hewer, Rachel Yavinsky, and Sherry Hutchinson for assistance with preparing the report. Our gratitude also goes to Foyeke Oyedokun-Adebagbo, Rubama Ahmed, Amobi Onovo, Minal Amin, Hope Hempstone, and Angie Brasington at the United States Agency for International Development for their support and insightful inputs throughout the study.

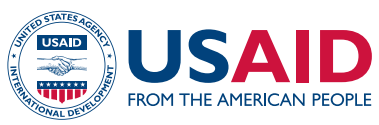

Breakthrough RESEARCH is made possible by the generous support of the American people through the United States Agency for International Development (USAID) under the terms of cooperative agreement no. AID-OAA-A-17-00018. The contents of this document are the sole responsibility of the Breakthrough RESEARCH and Population Council and do not necessarily reflect the views of USAID or the United States Government.

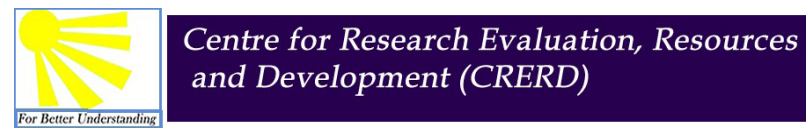

Centre for Research, Evaluation Resources and Development (CRERD) is a non-profit, non governmental organization concerned with improving the understanding and delivery of population and health services and environmental development in Nigeria. The organization is an outgrowth of the Operations Research Unit and Network, Obafemi Awolowo University, Ile-Ife, sponsored by The Population Council of New York, U.S.A. for an initial period of four years. CRERD has a network of multidisciplinary researchers all over Nigeria.

\section{Từ Tulane 2 University}

As stewards of the first school of public health in the United States, the Tulane University School of Public Health and Tropical Medicine cultivates independent thinkers, innovative leaders, fierce advocates, and accomplished scholars.

From the neighborhoods of New Orleans to communities worldwide, we conduct research and collaborate with our partners to ensure that all of humanity has an equitable opportunity to be healthy and pursue optimal well-being.

We train the problem solvers. Find us on the Front Lines.

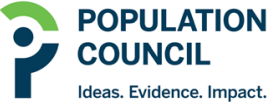

The Population Council confronts critical health and development issuesfrom stopping the spread of HIV to improving reproductive health and ensuring that young people lead full and productive lives. Through biomedical, social science and public health research in about 50 countries, the Council works with our partners to deliver solutions that lead to more effective policies, programs, and technologies to improve lives worldwide. Established in 1952 and headquartered in New York, the Council is a nongovernmental, nonprofit organization with an international board of trustees.

\section{Breakthrough RESEARCH FOR SOCIAL \& BEHAVIOR CHANGE}

Breakthrough RESEARCH catalyzes social and behavior change (SBC) by conducting state-of-the-art research and evaluation and promoting evidence-based solutions to improve health and development programs around the world. Breakthrough RESEARCH is a consortium led by the Population Council in partnership with Avenir Health, ideas42, Institute for Reproductive Health at Georgetown University, Population Reference Bureau, and Tulane University.

(C)2020 The Population Council. All rights reserved.

Cover photo by CRERD.

\section{Suggested Citation}

Johansson, E.W., P.L. Hutchinson, E. Omoluabi, A. Akinyemi, A. Smith, J.C. Welty, U. Anaba, D. Meekers, A. Leyton, D. Abegunde, and P. Hewett. 2020. "Behavioral sentinel surveillance survey in Nigeria: baseline technical report," Breakthrough RESEARCH Baseline Technical Report. Washington DC: Population Council.

\section{Contact}

Breakthrough RESEARCH/Nigeria

House 1, Plot 839 Idris Ibrahim Crescent | Jabi, Abuja - Nigeria

4301 Connecticut Avenue NW, Suite 280 | Washington, DC 20008

+1202 2379400 | BreakthroughResearch@popcouncil.org

breakthroughactionandresearch.org 


\section{Behavioral Sentinel Surveillance Survey in Nigeria: baseline technical report}

Emily White Johansson ${ }^{1,2}$ Paul Hutchinson ${ }^{1,2}$

Akanni Akinyemi ${ }^{3}$

Udochisom Anaba ${ }^{1,2}$

Dele Abegunde 2,4
Andrea Smith ${ }^{1}$

Dominique Meekers ${ }^{1}$

Paul Hewett ${ }^{2,4}$
Elizabeth $0 \mathrm{moluabi}^{3}$

J Clint Welty ${ }^{1}$

Alejandra Leyton ${ }^{1}$

\section{'Tulane University}

2Breakthrough RESEARCH/Nigeria

${ }^{3}$ Centre for Research, Evaluation Resources and Development (CRERD)

${ }^{4}$ Population Council 


\section{List of Acronyms}

$\begin{array}{ll}\text { ACT } & \text { Artemisinin-based combination therapies } \\ \text { ANC } & \text { Antenatal care } \\ \text { ARI } & \text { Acute respiratory infections } \\ \text { BCG } & \text { Bacille Calmette-Guerin } \\ \text { BSS } & \text { Behavioral Sentinel Surveillance } \\ \text { CI } & \text { Confidence intervals } \\ \text { CRERD } & \text { Center for Research, Evaluation Resources, and Development } \\ \text { CV } & \text { Community volunteer } \\ \text { DTP3 } & \text { Diphtheria-tetanus-pertussis } \\ \text { HC3 } & \text { Health Communication Capacity Collaborative } \\ \text { IPTp } & \text { Intermittent preventive treatment for malaria during pregnancy } \\ \text { IRB } & \text { Institutional Review Board } \\ \text { JHU CCP } & \text { Johns Hopkins Center for Communication Programs } \\ \text { LGA } & \text { Local government area } \\ \text { LLIN } & \text { Long-lasting insecticidal net } \\ \text { MMR } & \text { Maternal mortality ratio } \\ \text { MNCH+N } & \text { Maternal, newborn, and child health plus nutrition } \\ \text { NDHS } & \text { Nigeria Demographic and Health Survey } \\ \text { NHREC } & \text { National Health Research Ethics Committee } \\ \text { NURHI } & \text { Nigerian Urban Reproductive Health Initiative } \\ \text { ORS } & \text { Oral rehydration solutions } \\ \text { OVC } & \text { Orphaned and vulnerable children } \\ \text { PHC } & \text { Primary health care } \\ \text { PMI } & \text { President's Malaria Initiative } \\ \text { SBC } & \text { Social and behavior change } \\ \text { TFR } & \text { Total fertility rate } \\ \text { U5MR } & \text { Under-5 mortality rate } \\ \text { USAID } & \text { United States Agency for International Development } \\ & \end{array}$




\section{Table of Contents}

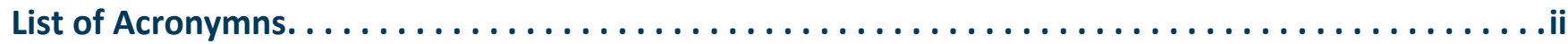

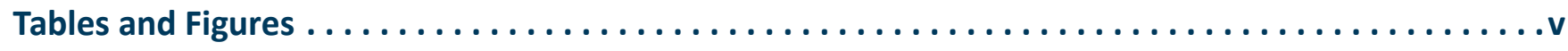

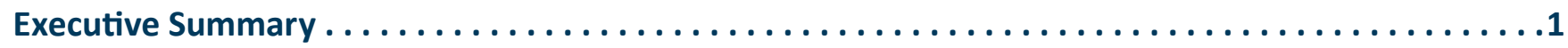

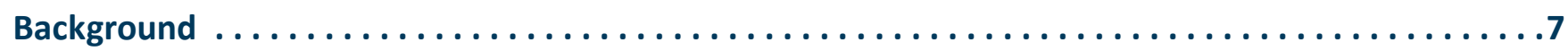

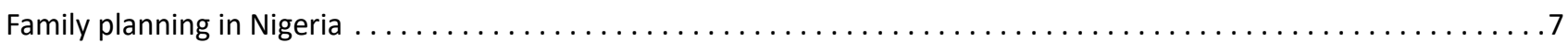

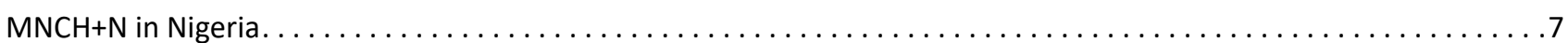

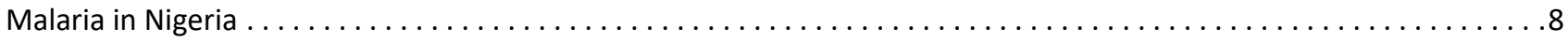

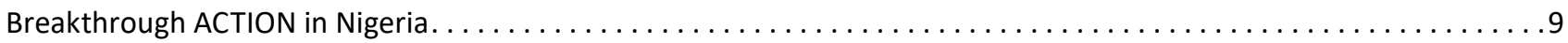

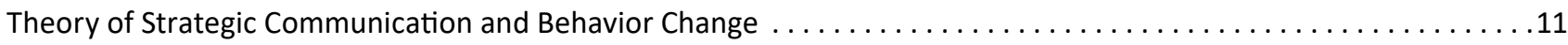

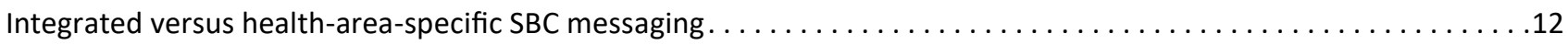

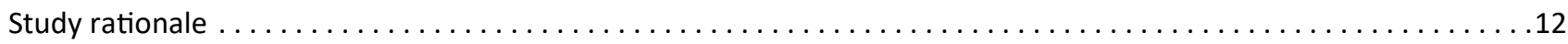

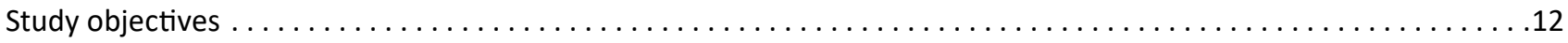

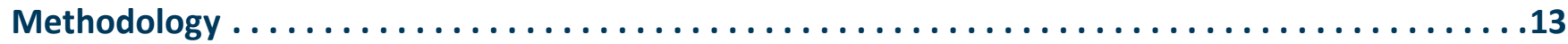

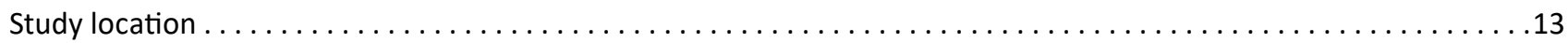

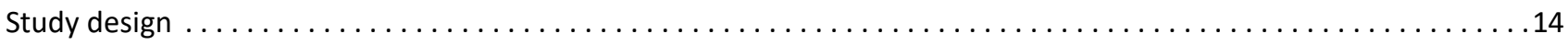

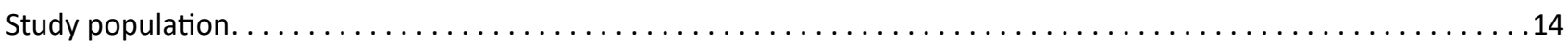

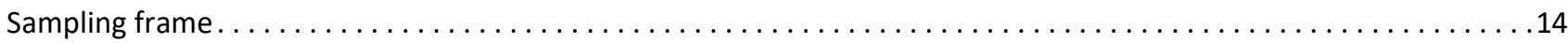

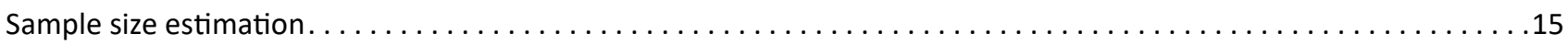

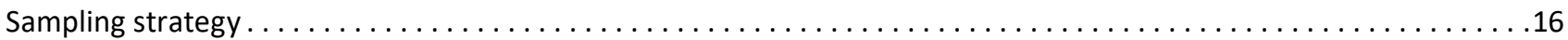

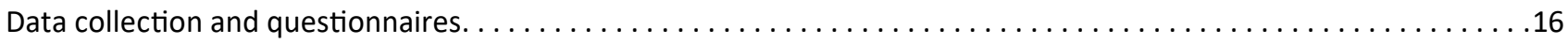

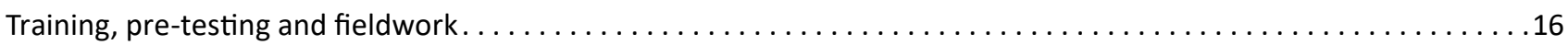

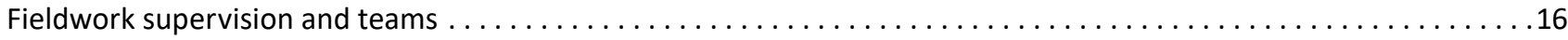

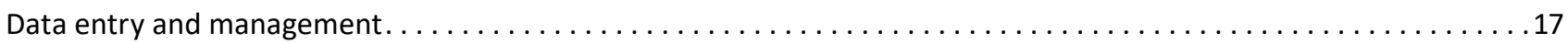

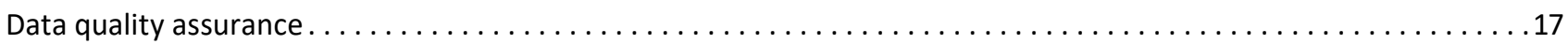

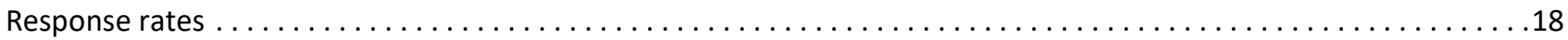

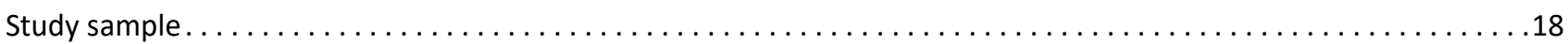

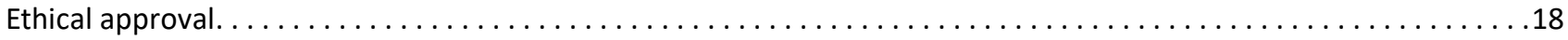

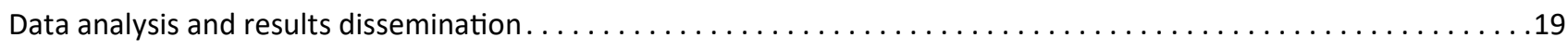

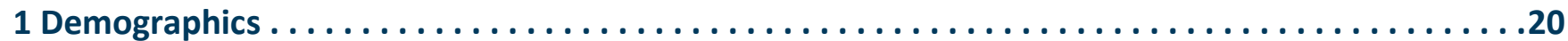

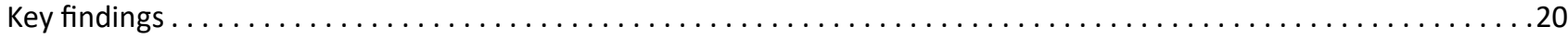

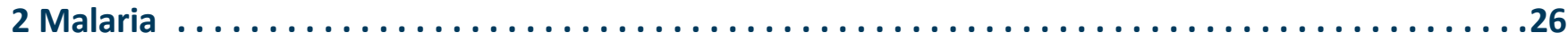

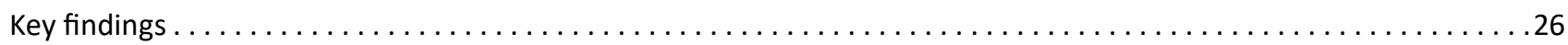

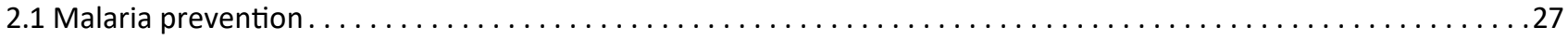

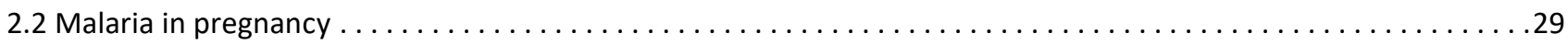

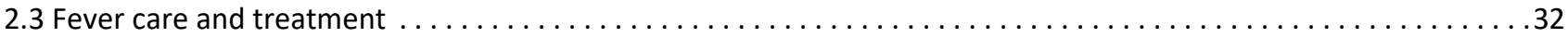

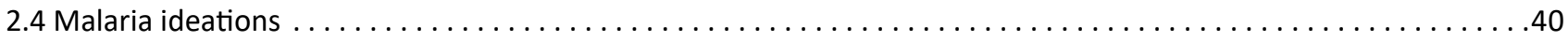




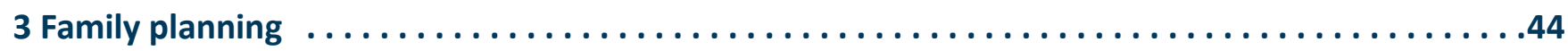

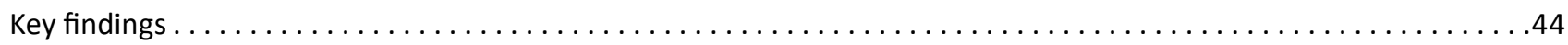

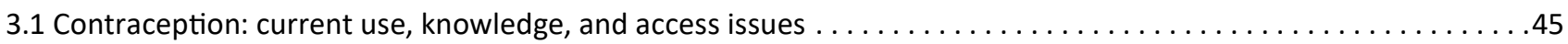

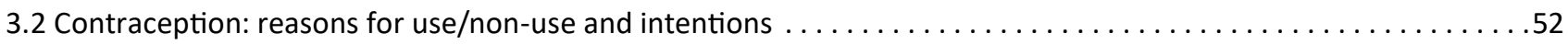

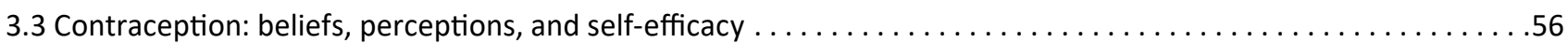

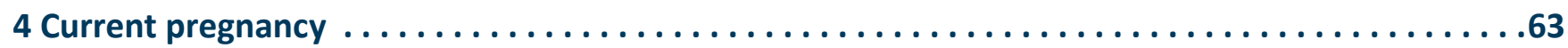

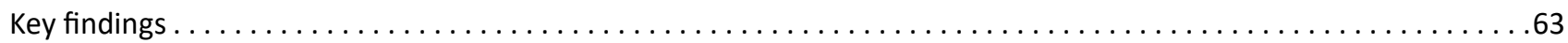

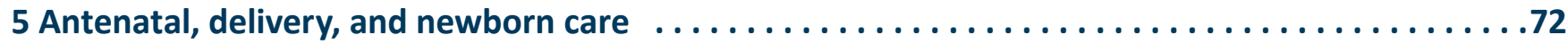

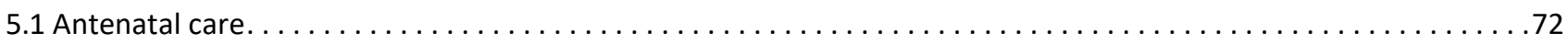

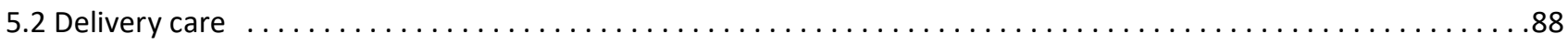

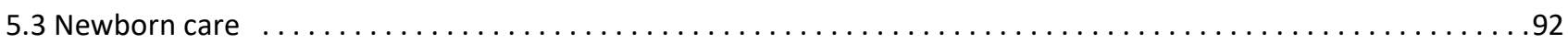

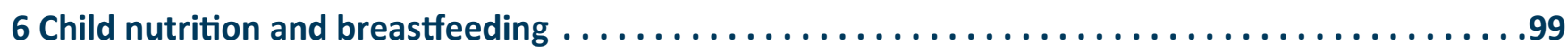

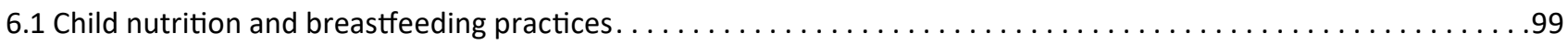

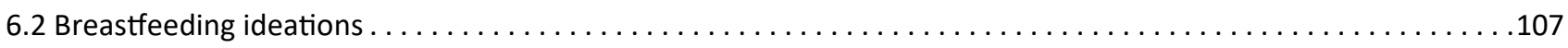

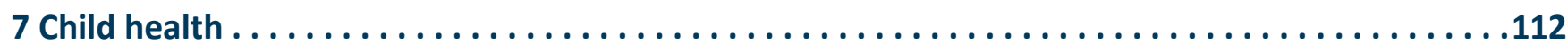

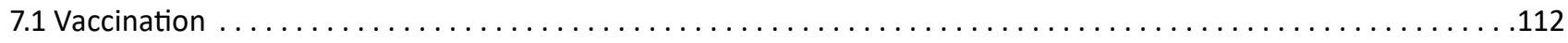

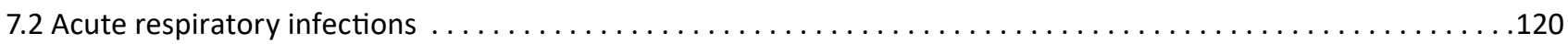

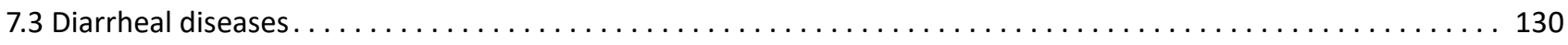

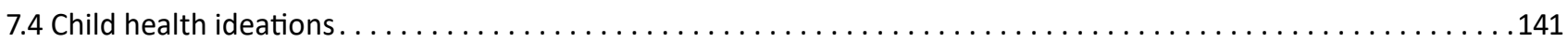

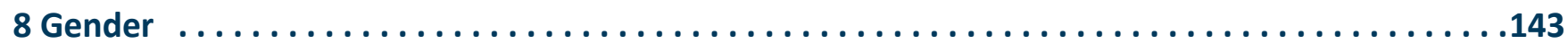

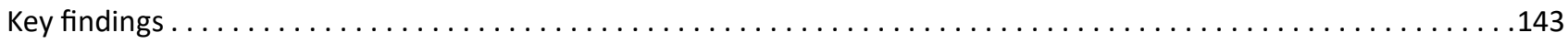

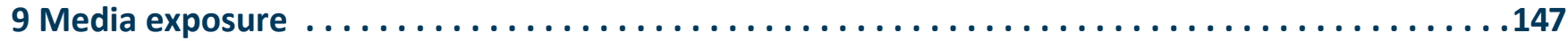

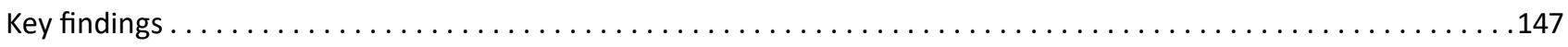

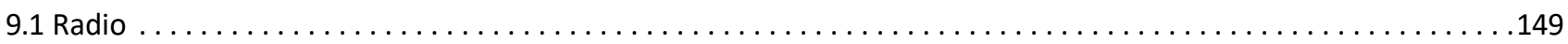

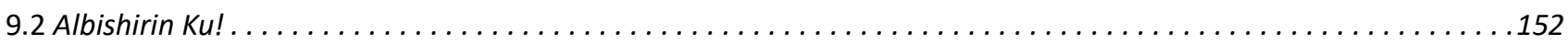

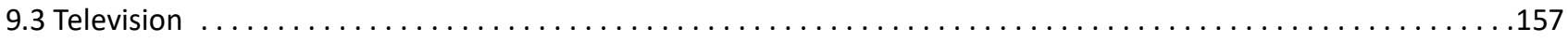

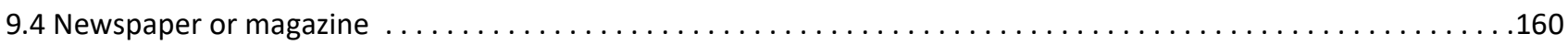

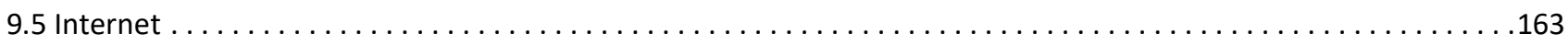

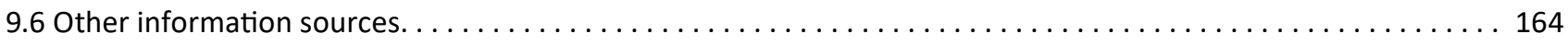

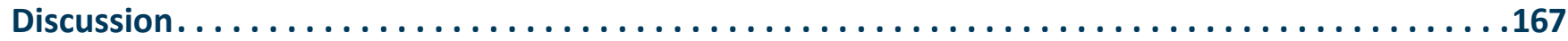

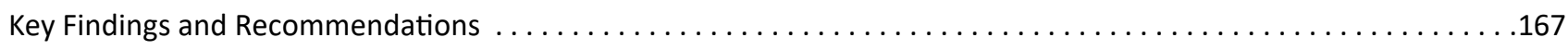

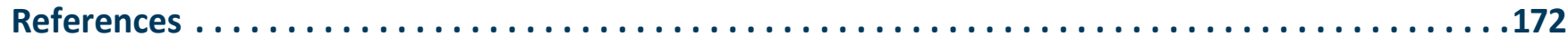




\section{Tables and Figures}

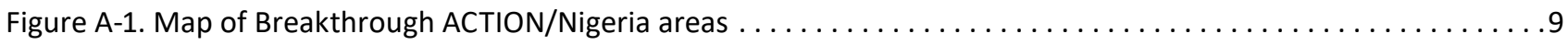

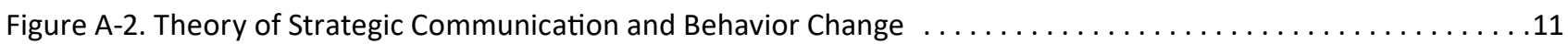

Figure A-3. Distribution of BSS sampling wards in Kebbi, Sokoto, and Zamfara States . . . . . . . . . . . . . . 15

Table A-1. Key objectives of life stage approach for integrated SBC programming areas . . . . . . . . . . . . .

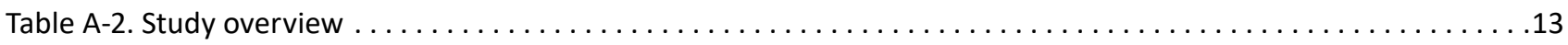

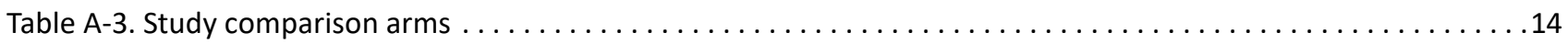

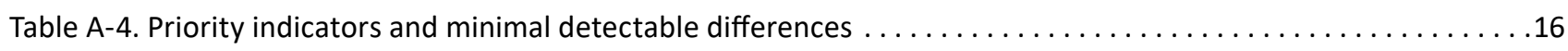

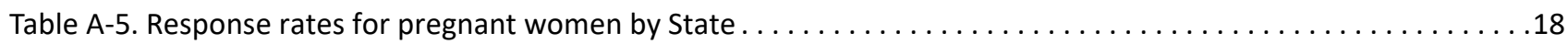

Table A-6. Response rates for women with a child under 2 years by State $\ldots \ldots \ldots \ldots \ldots$

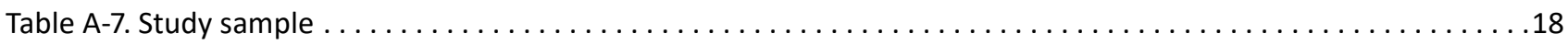

Table A-8. Notes on comparing NDHS and BSS baseline results. . . . . . . . . . . . . . . . . .

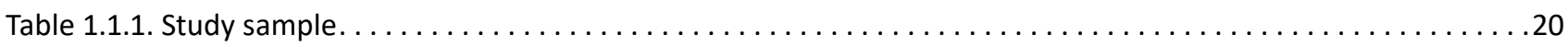

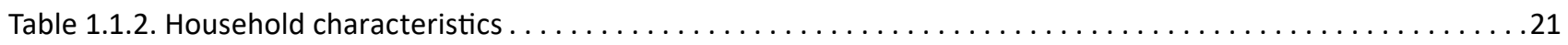

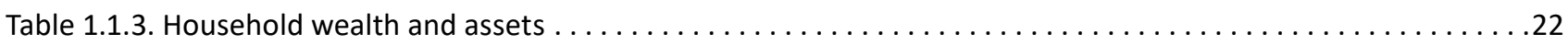

Table 1.1.4. Household distances to education, health, and economic centers . . . . . . . . . . . . . . .

Table 1.1.5. Respondent characteristics . . . . . . . . . . . . . . . . . . . . . . . . . . . . . 24

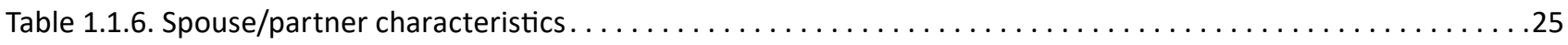

Table 2.1.A. LLIN availability in household (at least one) by sociodemographic characteristics . . . . . . . . . . . . 27

Table 2.1.B. LLIN availability in household (at least one for every two household members) by sociodemographic

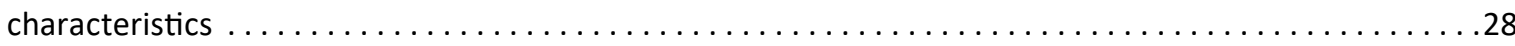

Table 2.1.C. LLIN use among pregnant women living in a household with at least one LLIN for every two household

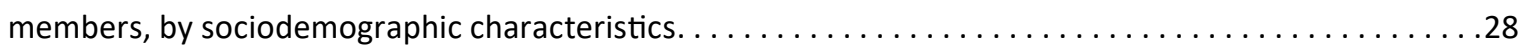

Table 2.1.D. LLIN use among under-twos living in a household with at least one LLIN for every two household members, by sociodemographic characteristics. . . . . . . . . . . . . . . . . . . 29

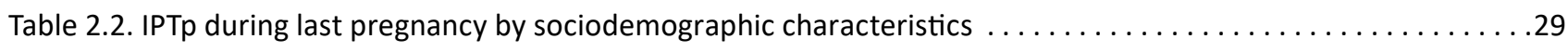

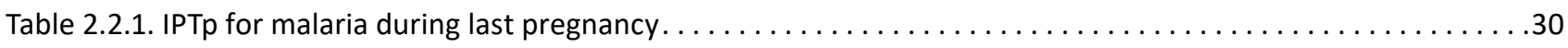

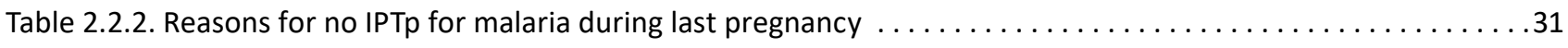

Table 2.2.3. What are some risks to the baby if a woman gets malaria during pregnancy? $\ldots \ldots \ldots \ldots \ldots \ldots \ldots$

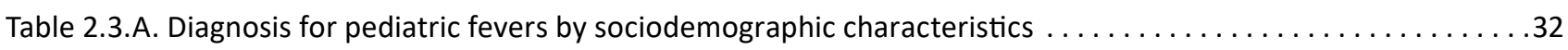

Table 2.3.B. ACT treatment for pediatric fevers by sociodemographic characteristics . . . . . . . . . . . . 33

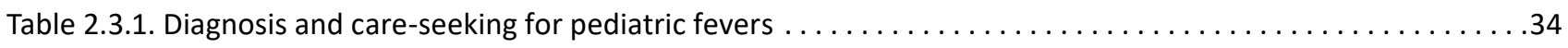

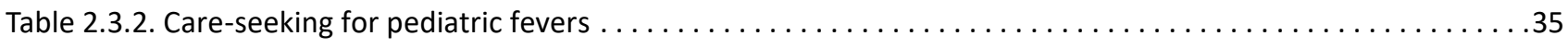

Table 2.3.3. Reasons for choosing source of care for pediatric fevers. . . . . . . . . . . . . . . . . . . .

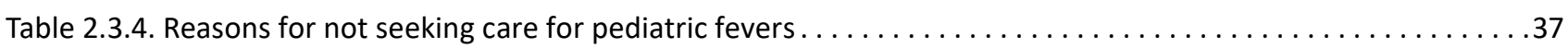

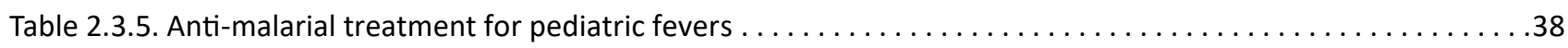

Table 2.3.6. Anti-malarial care-seeking timing and completion of ACT course for pediatric fevers ..............39

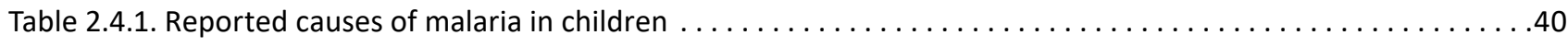

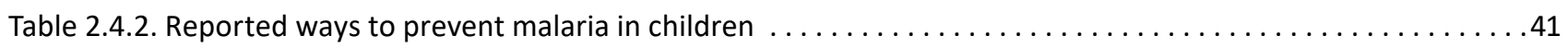

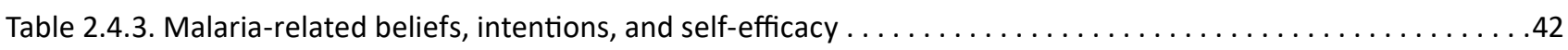

Table 3.1.A. Contraceptive prevalence rate by sociodemographic characteristics . . . . . . . . . . . . . 45

Table 3.1.B. Postpartum contraceptive prevalence rate by sociodemographic characteristics $\ldots \ldots \ldots \ldots \ldots \ldots \ldots$ 


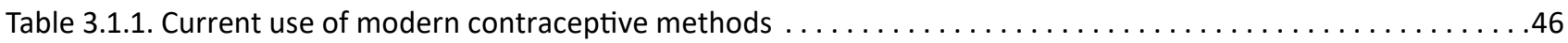

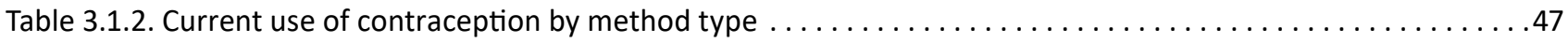

Table 3.1.3. Have you ever heard of the following contraception methods? . . . . . . . . . . . . . . . . 48

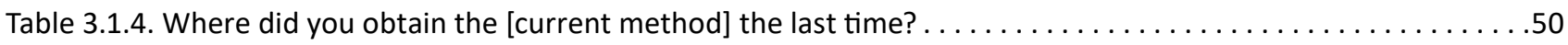

Table 3.1.5. Difficulties faced obtaining [current method] the last time? . . . . . . . . . . . . . . . . 51

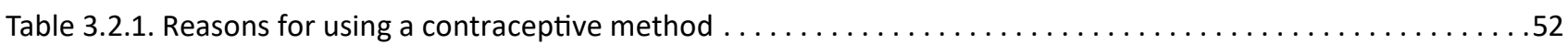

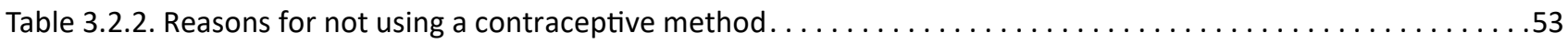

Table 3.2.3. Intention to use/continue to use contraception in the next six months. . . . . . . . . . . . . 55

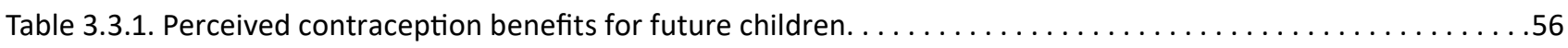

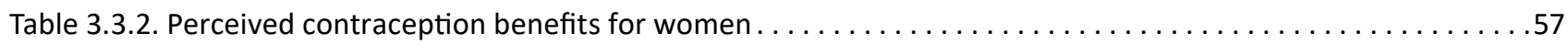

Table 3.3.3. Contraception beliefs, attitudes, and perceived social norms. . . . . . . . . . . . . . . . 58

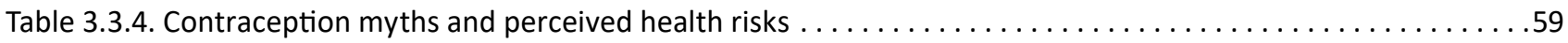

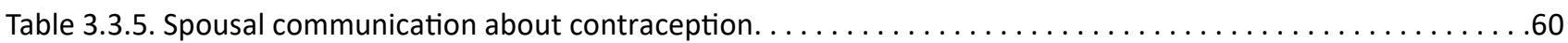

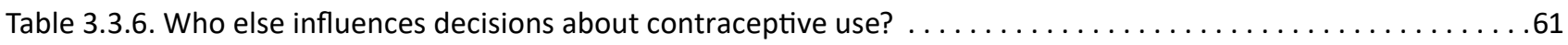

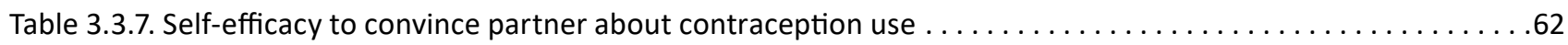

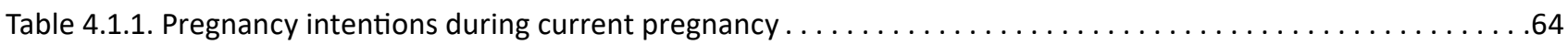

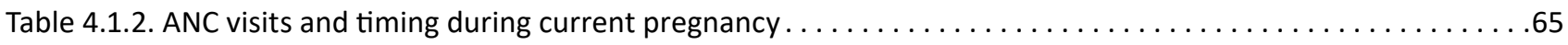

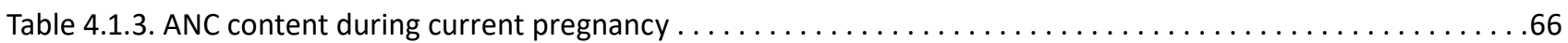

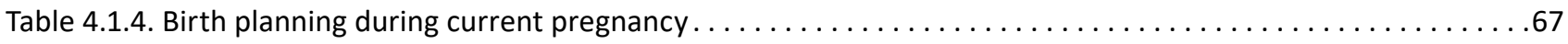

Table 4.1.5. Reasons for not attending ANC during current pregnancy $\ldots \ldots \ldots \ldots \ldots$

Table 4.1.6. Medicines or vaccinations received during current pregnancy . . . . . . . . . . . . . . . 69

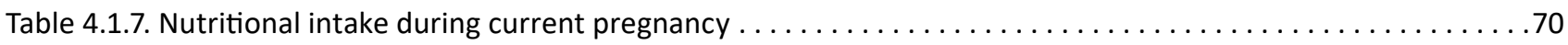

Table 5.1.A. ANC 1+ during last pregnancy by sociodemographic characteristics . . . . . . . . . . . . . 73

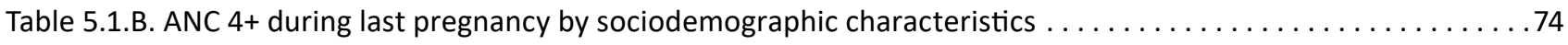

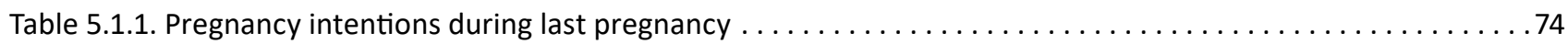

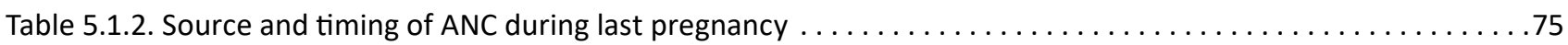

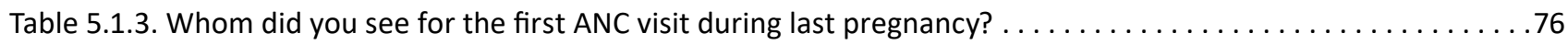

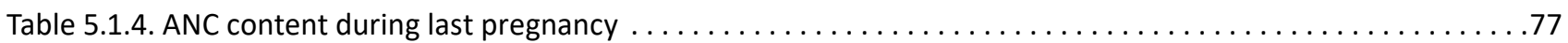

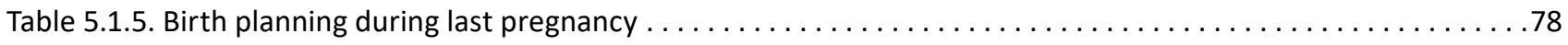

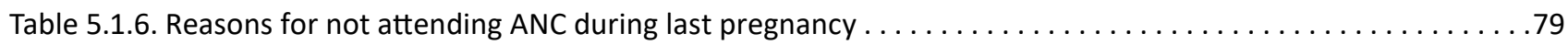

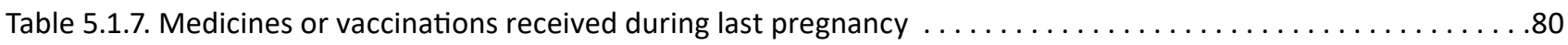

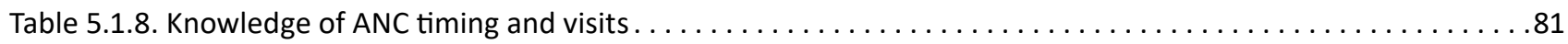

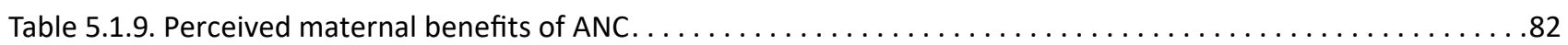

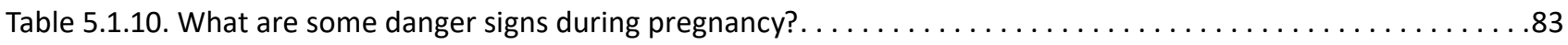

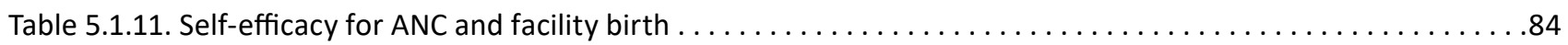

Table 5.1.12. Intentions for ANC and facility birth during next pregnancy $\ldots \ldots \ldots \ldots \ldots$

Table 5.1.13. Who else influences decisions about ANC and facility delivery? . . . . . . . . . . . . . . . 86

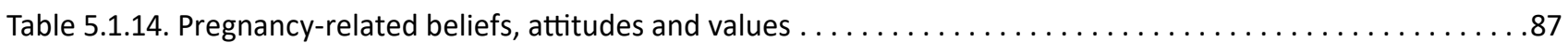

Table 5.2. Facility delivery during last pregnancy by sociodemographic characteristics . . . . . . . . . . . 88

Table 5.2.1. Who assisted in the delivery of your child during the last pregnancy? . . . . . . . . . . . . . 89

Table 5.2.2. Delivery location and respectful care during last pregnancy . . . . . . . . . . . . . . . . . 90

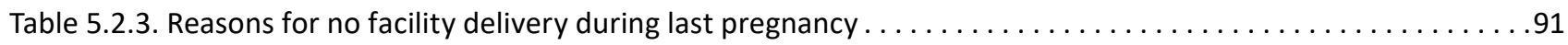

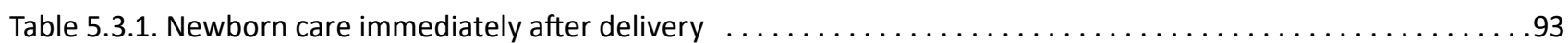

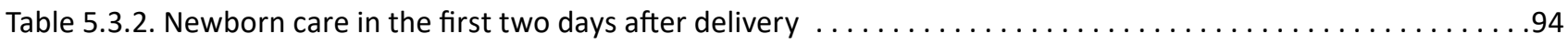

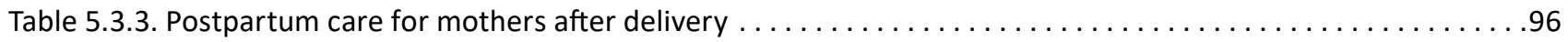

Table 5.3.4. What are some ways to protect a newborn immediately after birth? ................97 
Table 5.3.5. What are some ways to help a mother recover well after birth? $\ldots \ldots \ldots \ldots$

Table 6.1.A. Immediate breastfeeding after birth by sociodemographic characteristics . . . . . . . . . . 100

Table 6.1.B. Exclusive breastfeeding in first six months of life by sociodemographic characteristics .......... 100

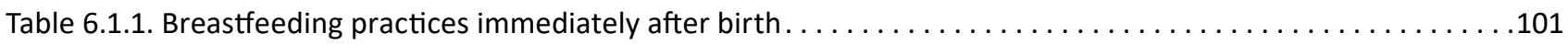

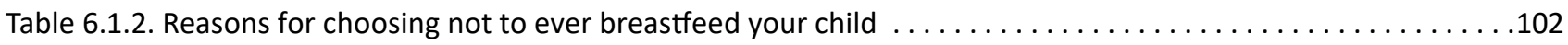

Table 6.1.3. Reasons for giving non-breastmilk liquids to a newborn in first three days after birth. . . . . . . . . 103

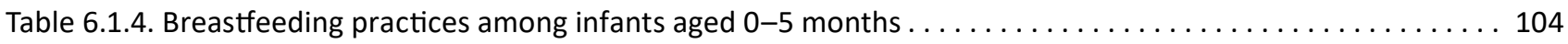

Table 6.1.5. Breastfeeding and nutrition among children aged $6-23$ months $\ldots \ldots \ldots \ldots$

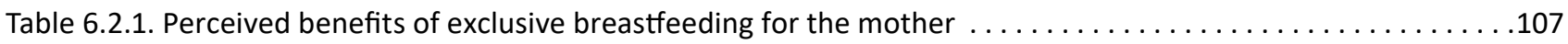

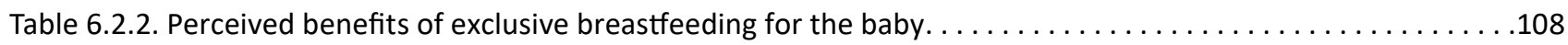

Table 6.2.3. Who else influences decisions about breastfeeding? . . . . . . . . . . . . . . . . . . 109

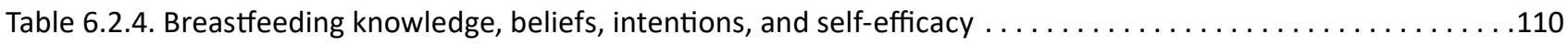

Table 6.2.5. What are some ways to recognize poor nutrition in a child? . . . . . . . . . . . . . . . . . . 111

Table 7.1. Fully vaccinated children $12-23$ months by sociodemographic characteristics. . . . . . . . . . 113

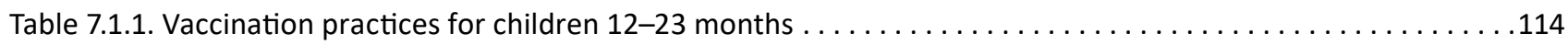

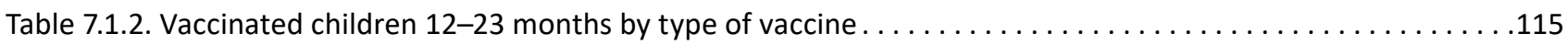

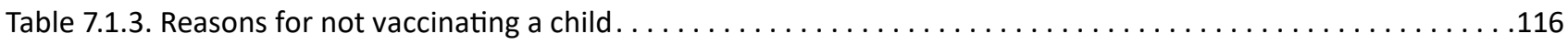

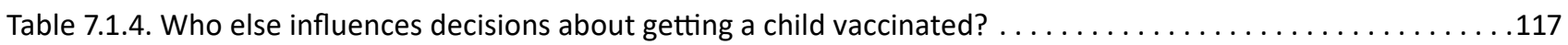

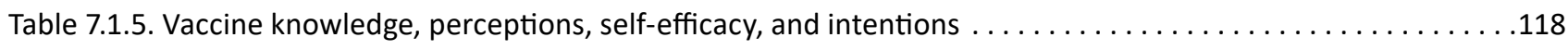

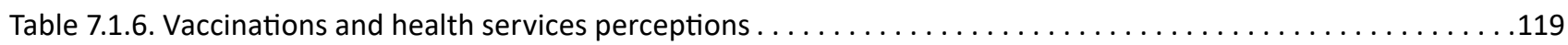

Table 7.2. ARI care-seeking from formal medical sources, by sociodemographic characteristics ............120

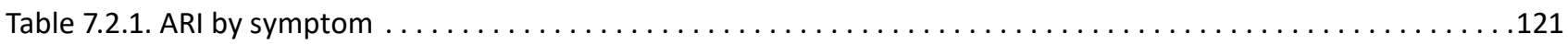

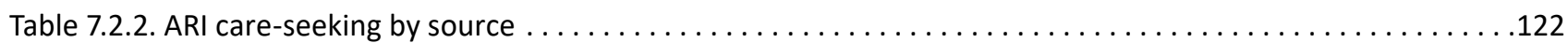

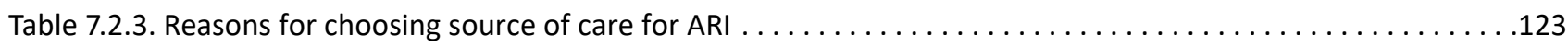

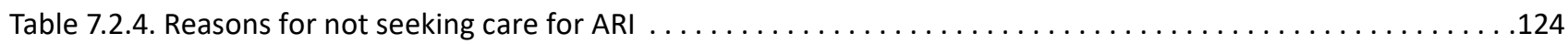

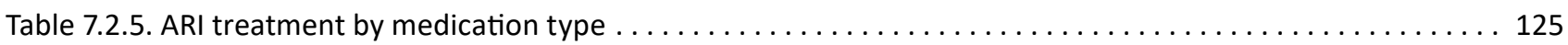

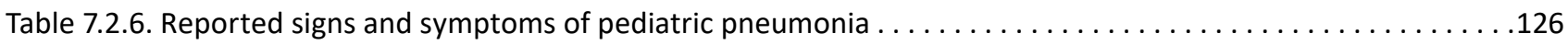

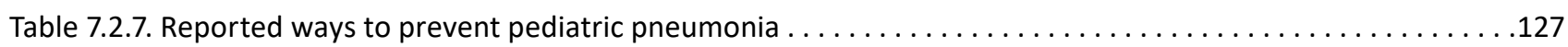

Table 7.2.8. Reported treatment perceived as effective against pediatric pneumonia . . . . . . . . . . . . 128

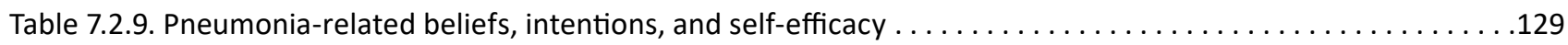

Table 7.3.A. ORS treatment for pediatric diarrhea by sociodemographic characteristics . . . . . . . . . . . . . . 131

Table 7.3.B. ORS and zinc treatment for pediatric diarrhea by sociodemographic characteristics. . . . . . . . . . 131

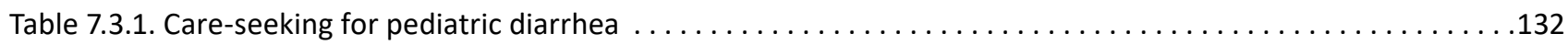

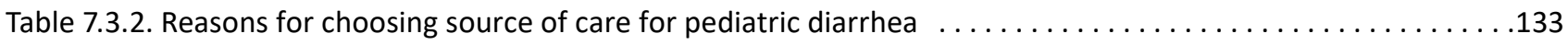

Table 7.3.3. Reasons for not seeking care for pediatric diarrhea . . . . . . . . . . . . . . . . . . 134

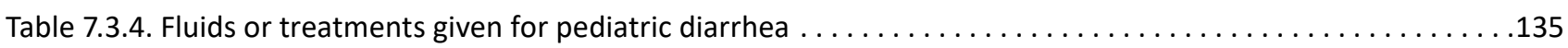

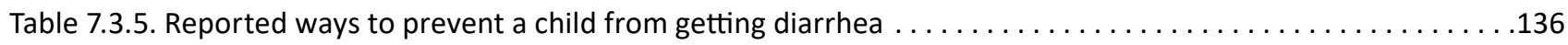

Table 7.3.6. Reported treatments perceived as effective against pediatric diarrhea . . . . . . . . . . . . . 137

Table 7.3.7. Reported ways ORS may help a child with diarrhea $\ldots \ldots \ldots \ldots \ldots \ldots$

Table 7.3.8. Reported ways zinc may help a child with diarrhea $\ldots \ldots \ldots \ldots \ldots \ldots \ldots$

Table 7.3.9. Diarrhea-related beliefs, intentions, and self-efficacy $\ldots \ldots \ldots \ldots \ldots \ldots$

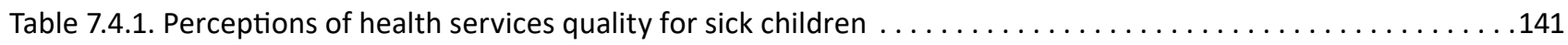

Table 7.4.2. Self-efficacy in convincing partner to seek care for a sick child . . . . . . . . . . . . . . . . . 141

Table 7.4.3. Who else influences decisions about seeking care for a sick child?. . . . . . . . . . . . . . . . 142

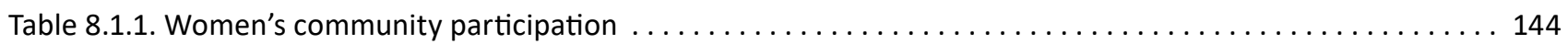

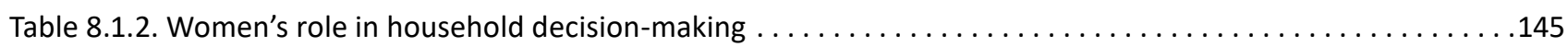


Table 8.1.3. Gender-related beliefs, attitudes, and perceptions

Table 9.1. Albishirin Ku! radio program exposure by sociodemographic

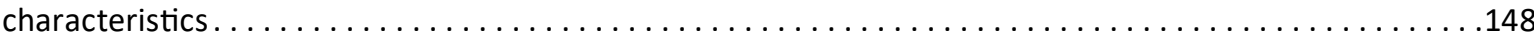

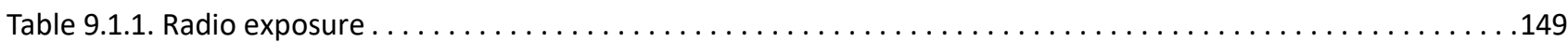

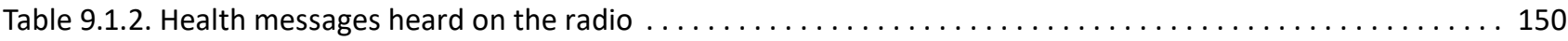

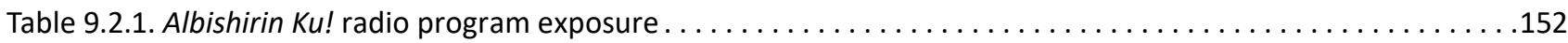

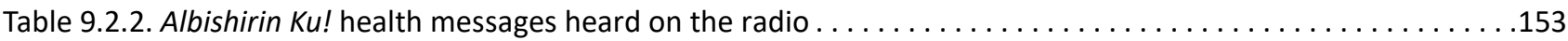

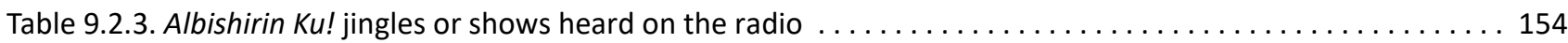

Table 9.2.4. Albishirin Ku! logo seen during household visit with community volunteer. . . . . . . . . . . . 155

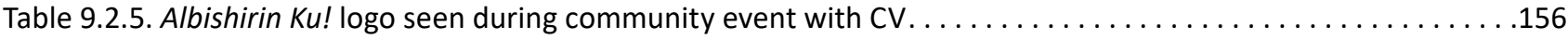

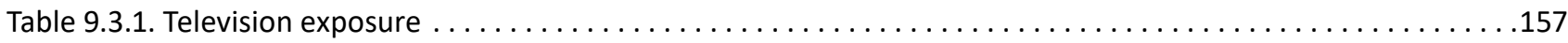

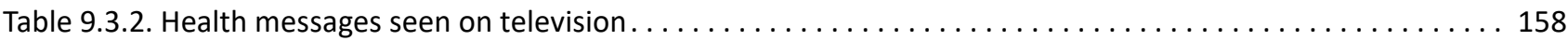

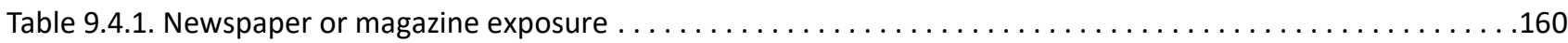

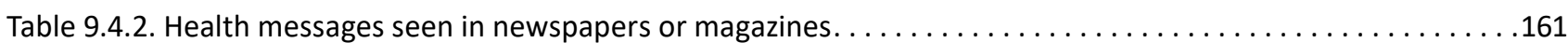

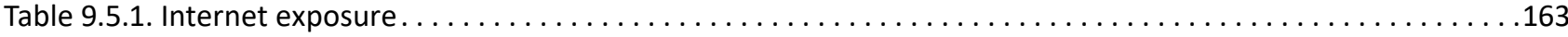

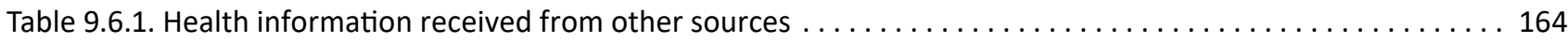

Table 9.6.2. Health information received from health system sources $\ldots \ldots \ldots \ldots \ldots \ldots$

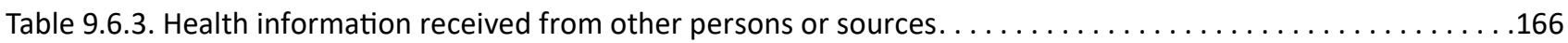




\section{Executive Summary}

This technical report presents results of the baseline Behavioral Sentinel Surveillance (BSS) survey undertaken by Breakthrough RESEARCH/Nigeria between September and October 2019. From 2019 to 2021, baseline, midline, and endline waves of the BSS will be used to assess the effectiveness of the Breakthrough ACTION/Nigeria integrated social and behavior change (SBC) activities for malaria; family planning; and maternal, newborn, and child health plus nutrition ( $\mathrm{MNCH}+\mathrm{N}$ ) in Kebbi and Sokoto states relative to Breakthrough ACTION/ Nigeria's malaria-only SBC activities in Zamfara State.

Breakthrough ACTION/Nigeria uses three primary SBC approaches that span across societal levels including: 1) advocacy outreach to opinion leaders and community influencers at state and local government area (LGA) level, 2) direct engagement of community members through community dialogues and group meetings that includes referrals for services, and 3) complementary SBC messaging through mass and mid-media. The official launch of integrated SBC activities in Kebbi and Sokoto occurred on 19 September 2019, although staged implementation rolled out across these states between early and late September, which coincided with BSS baseline survey fieldwork. The Albishirin Ku! radio program began airing in integrated states at the end of August 2019. Malaria-only SBC activities were initiated in Zamfara in October 2019.

This technical report presents detailed baseline results, including comparisons of results for the Breakthrough ACTION/Nigeria integrated (Kebbi/Sokoto) versus malaria-only program areas (Zamfara). The report also presents findings regarding the contextual factors and drivers that influence health behavior and service utilization outcomes, as these results have important and direct implications for Breakthrough ACTION/Nigeria SBC program adaption and scale-up.

\section{About the BSS}

The BSS is unique in its focus on the drivers of behavioral outcomes in the areas of family planning, malaria, and $\mathrm{MNCH}+\mathrm{N}$. Measures of these drivers are reflected in questions about the psychosocial influences-across cognitive, emotional, and social domains-that have been posited by various theories as intermediate determinants of behavioral outcomes. Indeed, as these theories suggest, a person's actions are strongly influenced by their beliefs, feelings, and sense of self-efficacy, and changing these perceptions may lead to future positive behavioral change.

The BSS focuses on women aged 15 to 49 years who are currently pregnant or with a child under 2 years living in areas targeted for integrated (Kebbi/Sokoto) and malaria-only (Zamfara) Breakthrough ACTION/Nigeria $\mathrm{SBC}$ programming. This subset of women is the primary target audience for Breakthrough ACTION/Nigeria's programming. A total of 3,026 pregnant women and 3,040 women with a child under 2 years from randomly selected locations in the Breakthrough ACTION/Nigeria program areas were included in the BSS baseline survey. Study populations are referred to as "respondents" throughout this report. Female interviewers used a structured questionnaire to collect information across a wide variety of health areas. The BSS asks respondents about current health behaviors and key behavioral determinants, including: (cognitive) knowledge, attitudes, beliefs, and perceived risk; (emotional) empathy, self-efficacy, and emotional response; and (social) subjective 
norms, social support, social influence, and interpersonal communication. By understanding these key behavioral determinants, the BSS provides new and important evidence that will directly inform programs in Nigeria to tailor messaging and promote activities that address the psychosocial influences that promote behavior change.

Such evidence is critical to inform Breakthrough ACTION/ Nigeria's SBC program, which works across multiple societal levels and through various communication channels to promote positive normative and behavioral change across family planning, malaria, and $\mathrm{MNCH}+\mathrm{N}$. The BSS results also complement findings from the recent Nigeria Demographic and Health Survey (NDHS) 2018. While the NDHS 2018 reports on a wide range of health and demographic outcomes across Nigeria, the BSS collects additional program-relevant evidence on behavioral determinants. Such evidence will inform programs about pregnant women's and mothers of young children's beliefs or perceptions that must be addressed to help improve outcomes by the next NDHS survey round.

Specifically, the BSS results presented in this reportand highlighted in this Executive Summary-answer the following unique and important program-relevant questions:

- Are respondents aware of the health behaviors that are being promoted, e.g., how to prevent or treat certain diseases?

- Are certain myths or beliefs held by respondents that could impede progress across health areas?

- How do respondents view health services in their communities and what are their main reasons for choosing certain treatment locations or for not seeking care at all? What types of difficulties, if any, have they had accessing services?

- How do health decisions get made in the household? Who mainly influences decisions and practices across health areas? How much autonomy or influence do respondents have in making family health decisions?

- What are key social norms around certain health behaviors? How prevalent are certain practices and how do respondents feel they would be perceived in their community if they took up the promoted practice?

- Do respondents feel confident in their ability to take up the promoted practice, or to negotiate with their husband/spouse about initiating the new practice?

\section{Key Findings and Recommendations}

\section{Albishirin Ku!}

Awareness of Breakthrough ACTION/Nigeria's Albishirin $\mathrm{Ku}$ ! radio programming is already apparent even at this baseline period. In Kebbi/Sokoto, 24 percent of respondents have heard the Albishirin Ku! radio slogan, as have 13 percent in Zamfara. Albishirin Ku! radio program awareness in Kebbi and Sokoto is significantly lower among respondents in the poorest households (12\%) than in the wealthiest households (42\%), which is likely due to lower radio ownership in the poorest households and thus fewer opportunities to listen to radio programming. This result suggests that Breakthrough ACTION/ Nigeria radio programming may not reach the poorest households that are shown to have demonstrably worse health outcomes described in this report (see below sections). Going forward, Breakthrough ACTION/Nigeria should ensure that ongoing SBC outreach efforts (e.g., household visits or community events) are targeting this population as a priority in order to raise overall rates.

\section{Family planning}

Modern contraceptive use: Non-pregnant respondents reported low modern contraceptive use, with higher use in Zamfara (15\%) than in Kebbi (9\%) and Sokoto (12\%). Modern contraceptive use was lower among respondents in the poorest households (6\% in Kebbi/Sokoto and $7 \%$ in Zamfara) than in wealthiest households (24\% in Kebbi/Sokoto and 34\% in Zamfara). Most respondents had heard of at least one modern method, with the most recognized methods being injectables, implants, and daily pills. Higher recall of methods was noted in Kebbi/Sokoto (30\% for injectables, 38\% for implants, and 33\% for daily pills) than in Zamfara (16\% for injectables, 23\% for implants, and $19 \%$ for daily pills). Consistent with existing evidence/literature, the most common methods used were injectables (48\%) and implants (25\%), with similar proportions across study areas. Finally, among non-users, 15 percent intended to start contraceptive use in the next six months, which presents an opportunity to fill this latent demand among current non-users who have a stated intention to act.

Reasons for non-use, attitudes, and social norms: The most common reasons non-pregnant respondents stated for not currently using contraception were fatalism ("It's up to God") (25\%), currently breastfeeding (23\%), partner opposition (21\%), and respondent opposition (18\%). In 
line with this result, approximately half (52\%) of respondents agreed that they do not personally approve of using contraception for birth spacing, underscoring a critical hurdle to Breakthrough ACTION/Nigeria SBC messaging for changing family planning practices. In addition, one in five respondents believed that people in their community would call them bad names or avoid their company if others knew they were using contraception for birth spacing, which further highlights the importance of addressing social norms in family planning. About half of the respondents agreed that religious leaders should speak publicly about using modern contraceptives, which underscores an important pillar of Breakthrough ACTION/ Nigeria programming.

Contraceptive myths: Common contraceptive myths also persist across study areas, with firmer belief in these myths in Kebbi/Sokoto than in Zamfara. About one in three respondents in Kebbi/Sokoto agreed that contraception could make a woman permanently infertile, harm a woman's womb, or lead to health problems, compared with about one-quarter of respondents in Zamfara. About one in five respondents in Kebbi/Sokoto believed that contraceptives cause cancer, result in deformed babies, increase promiscuity, or reduce sexual urges of women and men. SBC programming needs to actively work to dispel these specific myths going forward to help promote behavior change.

\section{Spousal communication and decision-making: There} were low rates of spousal communication about contraceptive use, although rates of spouse communication were higher in Zamfara (22\%) than in Kebbi/Sokoto (6\%). While approximately half of respondents across study areas reported that contraceptive use decisions were made jointly with their partner, a significant proportion-30 percent in Kebbi/Sokoto and 17 percent in Zamfara-noted that contraceptive decisions were mainly made by their partners. When respondents were asked who else influences a woman's decision about contraceptive use, the most commonly mentioned influencers were no one else (61\%) and partners/spouses (30\%). Self-efficacy for negotiating contraceptive use with a partner was low. Only about half of respondents across study areas were confident in their ability to convince their partner to use modern contraception for spacing births. These findings further underscore the need for SBC activities to engage couples in decision-making about birth spacing and to support women's agency in health decision-making to help raise contraceptive use rates.

\section{Malaria}

Malaria prevention: Overall, ownership of long-lasting insecticide nets (LLIN) was high. Approximately 75 percent and 69 percent of households surveyed in Zamfara and Kebbi/Sokoto, respectively, owned at least one LLIN. However, fewer households had the recommended ratio of at least one LLIN for every two household members, with slightly higher proportions in Kebbi/Sokoto (25\%) than in Zamfara (18\%). In households where there were sufficient nets (at least one LLIN for every two household members), 81 percent and 88 percent of pregnant respondents slept under an LLIN the previous night in Kebbi/Sokoto and Zamfara, with little difference across wealth quintiles or maternal education. Similarly, in households with sufficient nets, over 90 percent of children under 2 years slept under an LLIN the previous night in integrated and malaria-only areas, with limited differences across sociodemographics.

There was also extremely high overall awareness about the importance of LLINs in malaria prevention. Almost all respondents agreed that malaria was caused by mosquito bites (97\%), and that sleeping under mosquito nets was an effective malaria prevention method (93\%). In addition, nearly all respondents stated they were expected 
to be able to get all their children under 5 years sleeping under a mosquito net every night of the year (93\%). The results suggest that SBC programming to raise awareness about malaria prevention measures is not needed, but improving access, acceptability, and efficacy to use LLINs as directed may be a useful programmatic focus.

Malaria during pregnancy: Less than one in five respondents in study areas took intermittent preventive treatment as directed (three or more sulfadoxine/ pyrimethamine(SP)/Fansidar doses) during their last pregnancy, with lower rates among respondents in the poorest households (10\% and $12 \%$ ) compared with the wealthiest (33\% and 34\%). Lower percentages were also observed among the less educated (15\% and 14\%) compared to respondents who attended at least some primary school (36\% and 25\%). The most common reasons for not taking SP/Fansidar during the last pregnancy were opposition by the respondent (34\%) or spouse (33\%), and further research is needed to understand this opposition. Moreover, the "other" category was disproportionately high and largely included the following responses: did not attend antenatal care (ANC) or facility to obtain medicines, not sick or did not have pregnancy complications, and not necessary or customary to take medications. In addition, more frequent ANC visits is also associated with a higher likelihood of IPTp uptake. BSS baseline results show that women who attended ANC four or more times during the last pregnancy were about six times more likely to receive IPTp than those attending ANC only one time.

Taken together, these responses underscore the need for $\mathrm{SBC}$ messaging to reinforce the importance of intermittent preventive treatment for malaria during pregnancy (IPTp) as a key component of essential antenatal care services. In addition, while 91 percent of respondents agreed that malaria in pregnancy can affect the fetus, far fewer could state the specific risks to the baby. Only about half reported fetal death (45\%) or miscarriage $(44 \%)$ as specific risks from malaria in pregnancy, while far fewer reported premature birth (26\%) and low birth weight (17\%) as specific risks. Building knowledge of malaria risks and specific health consequences for the fetus may be a driver toward greater adoption of prevention behaviors.

Malaria treatment: While there were high rates of care-seeking for children under 2 years of age with fever, 33 percent and 54 percent of those who were taken to care in Kebbi/Sokoto and Zamfara, respectively, went to a pharmacy where poor-quality care persists. The most important reasons for choosing the treatment location for pediatric fever care were trust (36\%), nearby location (33\%), and effective treatment (28\%). These findings underscore the importance to women of convenient, trusted, and high-quality health services provision. Importantly, respondents also reported that few of their febrile children were tested for malaria (19\% and $24 \%$ ), and few of their febrile children were given artemisinin-based combination therapies (ACT) (22\% and 36\%) in Kebbi/Sokoto and Zamfara. The low prevalence of testing may be due in large part to care-seeking from pharmacies. Nearly all respondents reported intentions to take a child with a fever to care the same/next day (92\%) and to make sure the child takes the entire treatment course (92\%). This finding suggests that while respondents know the recommendation and have stated intentions to act, such knowledge and intentions may still not translate to behavioral action. However, while 74 percent agreed that a blood test is the only way to know whether a person has malaria, most respondents (61\%) agreed that they still worry the illness could be malaria even if the test result is negative. This implies some lack of confidence in negative malaria test results that reinforces Breakthrough ACTION/Nigeria's ongoing efforts to change malaria diagnosis perceptions and practices.

\section{$\mathrm{MNCH+N}$}

Antenatal care: There is low ANC attendance across Breakthrough ACTION program areas, with approximately one-third (32\% and $38 \%$ ) and one-quarter $(20 \%$ and $26 \%$ ) of respondents attending ANC one or more or four or more times during the last pregnancy in Kebbi/ Sokoto and Zamfara, respectively. The most common reasons for not attending ANC were lack of perceived need (42\%), spousal opposition (25\%), fatalism ("It's up to God") (20\%), and perceptions that ANC is not the norm (13\%). This finding suggests lack of information, poor risk perceptions, and lack of awareness about the benefits of ANC to a mother and her newborn. In addition, more than two-thirds (67\%) of respondents said their spouses influence their decision to go to ANC four or more times, making spousal support or opposition a critical facilitator or barrier to uptake. The data suggest that capturing husband ideations may be important to inform future spousal communications interventions. There are also common ANC myths that persist among respondents. Overall, 48 percent and 35 percent of respondents agreed that pregnant women need ANC only when sick, and 25 percent agreed that only first-time mothers 
need ANC. A significant percentage of respondents -40 percent and 30 percent in Kebbi/Sokoto and Zamfara, respectively-noted that it is better to use a traditional provider than a health facility for ANC. These perceptions point to areas for SBC messaging to improve awareness of the benefits of ANC even for healthy pregnancies that could lead to additional ANC uptake going forward. Finally, birth planning with a health worker during ANC does not appear to be standard practice, although one in three respondents reported having discussed birth plans. This suggests a strong starting point for making further inroads in this important area.

Facility delivery: Facility delivery was an uncommon practice in Kebbi/Sokoto (14\%) and Zamfara (16\%) among respondents during their last pregnancy. More than 80 percent of respondents delivered at their own or another home, and births are commonly assisted by family members (36\%) or traditional birth attendants (29\%). The most common reasons for not delivering at a facility were that they did not feel it was necessary $(75 \%$ and $61 \%$ ) followed by spousal opposition (15\% and 36\%) in Kebbi/Sokoto and Zamfara, respectively. Similar to ANC results, this finding suggests there is a critical lack of information, poor risk perceptions, and lack of awareness about the benefits of facility-based delivery to make this service feel necessary to both women and their spouses. When respondents were asked who influences the decision to have a facility delivery, partner/spouse $(57 \%)$ was the most common response, making spousal opposition another critical barrier to service utilization. About half (54\%) of respondents agreed that the health facility is the best place to deliver a baby, but fewer (44\%) felt confident that they could get to a health facility for delivery. About half (55\%) were confident that they could start a conversation with their husband about facility delivery, and a similar proportion (51\%) intended to deliver their next child at a health facility. These positive intentions around facility-based delivery represent an opportunity for SBC programs to support women with interventions to make it easier for them to act in accordance with their beliefs and intentions.

Breastfeeding: Nearly all respondents (96\%) reported ever breastfeeding their last-born child under 2 years. Yet initiating breastfeeding within one hour of birth was low across study areas, although the rate was higher in Zamfara (46\%) than in Kebbi/Sokoto (36\%). Exclusive breastfeeding for the first six months of life was low overall, with higher rates in Zamfara (46\%) than in Kebbi/ Sokoto (26\%). This low coverage is in large part due to high rates of giving non-breastmilk liquids to a child in the first three days after birth, with much higher practice rates in Kebbi/Sokoto (73\%) than in Zamfara (50\%). In relation to this practice, 23 percent of respondents also agreed that mother's breastmilk after birth (colostrum) is bad, suggesting an area for future SBC messaging. Taken together, these responses suggest ways SBC messaging may help promote exclusive breastfeeding in this area. In addition, while there was high awareness of breastfeeding and its benefits among respondents, only 55 percent of respondents felt confident to exclusively breastfeed their child for the first six months, with higher confidence in Zamfara (64\%) than Kebbi/Sokoto (42\%). Forty-one percent of respondents believed that most other women in their communities gave breastmilk exclusively to their infants, suggesting perceived social norms about breastfeeding. There was also higher intent to exclusively breastfeed their next child in Zamfara (59\%) and Kebbi/ Sokoto (46\%) compared to current exclusive breastfeeding rates, indicating an opportunity to support mothers with their breastfeeding intentions, such as increasing family or social supports.

Vaccination: There was very low vaccination coverage, with only 4 percent and 8 percent of children aged 12 to 23 months in Kebbi/Sokoto and Zamfara, respectively, who were fully vaccinated (bacilli Calmette-Guerin or BCG, measles, diphtheria-tetanus-pertussis or DTP3, and polio3) by the survey interview. The most important reason for not vaccinating the child was spousal opposition (33\%) followed by distance to the health facility (14\%) and fear of needles (11\%). Across study areas, 73 percent of respondents reported that their spouses influence their decision about child vaccinations, making spousal opposition an important barrier to vaccine uptake. The result suggests that capturing husband ideations may be important to inform future SBC interventions. While respondents generally gave positive reports about their own vaccine knowledge and attitudes, more specific questions elicited a less rosy picture. Specifically, only about half (52\%) of respondents in Kebbi/Sokoto believed that health facilities frequently had vaccines available, compared with 61 percent in Zamfara. Only 47 percent of respondents in Kebbi/Sokoto and 66 percent in Zamfara believed most women in their communities took children to facilities for routine vaccination. Approximately one in four respondents (26\%) agreed that vaccines contain dangerous chemicals that could harm a child's health. Few respondents knew when a child should first be vaccinated and the number of vaccinations children should receive by their first birthdays. Taken together, 
results suggest relatively low vaccine knowledge, common perceptions of frequent vaccine stockouts at health facilities, spousal opposition to vaccine uptake, and the persistence of vaccine myths across the study area.

Acute respiratory infections: While there were high rates of care-seeking for children under 2 years with cough, rapid breathing, or other chest-related problems, only 49 percent and 33 percent of those who sought care were taken to a formal medical source in Kebbi/ Sokoto and Zamfara. Even fewer children with these respiratory symptoms were given antibiotics ( $41 \%$ and $26 \%)$. The most common reasons for choosing the source of care across study areas were nearby location (40\%), provider trust (36\%), family/friend recommendation (30\%), and effective treatment (27\%), which underscores the importance to women of convenient, trusted, and high-quality health care providers in their communities. The most important reasons for not seeking care were expense/cost (33\%) and symptoms not perceived as severe or home care was provided (25\%). Pediatric pneumonia knowledge is low across study areas. Only 28 percent and 37 percent of respondents reported rapid or difficult breathing as a symptom of pediatric pneumonia; 45 percent and 31 percent could not report any way to help prevent a child from getting pneumonia; and 62 percent and 56 percent could not report any effective medicine to treat pneumonia in Kebbi/Sokoto and Zamfara, respectively. These results point to the need to shift care-seeking from pharmacies to formal medical sources and to improve overall pneumonia knowledge in study areas.

Diarrhea: Few children with diarrhea were given both oral rehydration solutions (ORS) and zinc, although this proportion was higher in Zamfara (27\%) than in Kebbi/ Sokoto (13\%). Among children with diarrhea, 36 percent and 56 percent were given ORS alone in Kebbi/Sokoto and Zamfara, respectively. While there were high rates of care-seeking for children under 2 years with diarrhea, 34 percent and 56 percent of these children were taken to a pharmacy instead of the formal medical sector. The most important reasons for choosing the source of care were provider trust (39\%), nearby location (29\%), and effective treatment (24\%), which underscores the importance to women of convenient, trusted, and high-quality health care provision in their communities. The most important reasons for not seeking care were expense/cost (32\%), fatalism ("It's up to God") (29\%), and symptoms not perceived as severe or care was provided at home (25\%). Approximately one in four respondents (24\%) had never heard of zinc across study areas. Across study areas, 63 percent of respondents reported that ORS replaces lost water in a child with diarrhea, while 44 percent incorrectly noted that it stops diarrhea. These results point to the need to shift care-seeking from pharmacies to formal medical sources in the study areas. 


\section{Background}

United States Agency for International Development (USAID)/Nigeria works to increase the utilization of quality primary health care services in targeted areas and population groups. Working with the Nigerian government, USAID/Nigeria aims to improve human resources for health, deliver high-impact services, and strengthen leadership, management, governance, and accountability in order to improve a wide set of health outcomes related to family planning, malaria, and maternal, newborn, and child health plus nutrition $(\mathrm{MNCH}+\mathrm{N})$ in Nigeria. ${ }^{1}$

\section{Family planning in Nigeria}

Modern contraceptive use in Nigeria has been historically low, without significant increases in the past two decades. ${ }^{2}$ In the three States of this study (Kebbi, Sokoto, and Zamfara), these indicators tend to be lower than national estimates. The 2018 Nigeria Demographic and Health Survey (NDHS) reported a persistently high total fertility rate (TFR) of 5.3 births per woman, which has been attributed to high rates of teenage pregnancy (19\%), early marriage, low modern contraceptive prevalence among married women (12\%), and high unmet need for family planning. ${ }^{3} \mathrm{~A}$ high fertility rate not only affects women and children's health and survival, but also their long-term education and employment prospects. Early childbearing is common among the poor; almost 60 percent of 20- to 24-year-olds living in the poorest households have had a child before the age of $18 .{ }^{4}$

Misconceptions and negative perceptions about family planning use, such as beliefs that contraceptives are dangerous to a woman's health or that they can harm a woman's womb, 5,6 are common. Opposition to use and wanting more children are also the predominant reasons women typically do not use modern contraceptives. ${ }^{4}$ Spousal communication about contraceptive use is also low: The Nigerian Urban Reproductive Health Initiative (NURHI) reported that less than 20 percent of married women discussed family planning and fertility options with their spouses within the past six months. ${ }^{6}$

There is ample opportunity to effect change through social and behavior change (SBC) programming since few women have heard family planning SBC messaging. Nationwide, less than one-quarter of women and less than one-third of men aged 15 to 19 years reported having heard any messages regarding family planning in the past few months, although 60 percent of young women in Lagos had heard such messages. ${ }^{3}$ In the six cities where NURHI conducted their endline survey, nearly two out of three women reported exposure to NURHI family planning television programs, and approximately three out of four had heard NURHI family planning radio programs. ${ }^{6}$

\section{$\mathrm{MNCH}+\mathrm{N}$ in Nigeria}

Nigeria has one of the highest child mortality rates in the world. ${ }^{7}$ NDHS 2018 reported that the under-5 mortality rate (U5MR) was estimated at 132 deaths per 1,000 live births in the five-year period before the survey, making Nigeria the largest contributor to child deaths in the African region. ${ }^{8}$ Moreover, the highest U5MR was found in the northern States, including Sokoto, Kebbi, and Zamfara. The leading causes of child

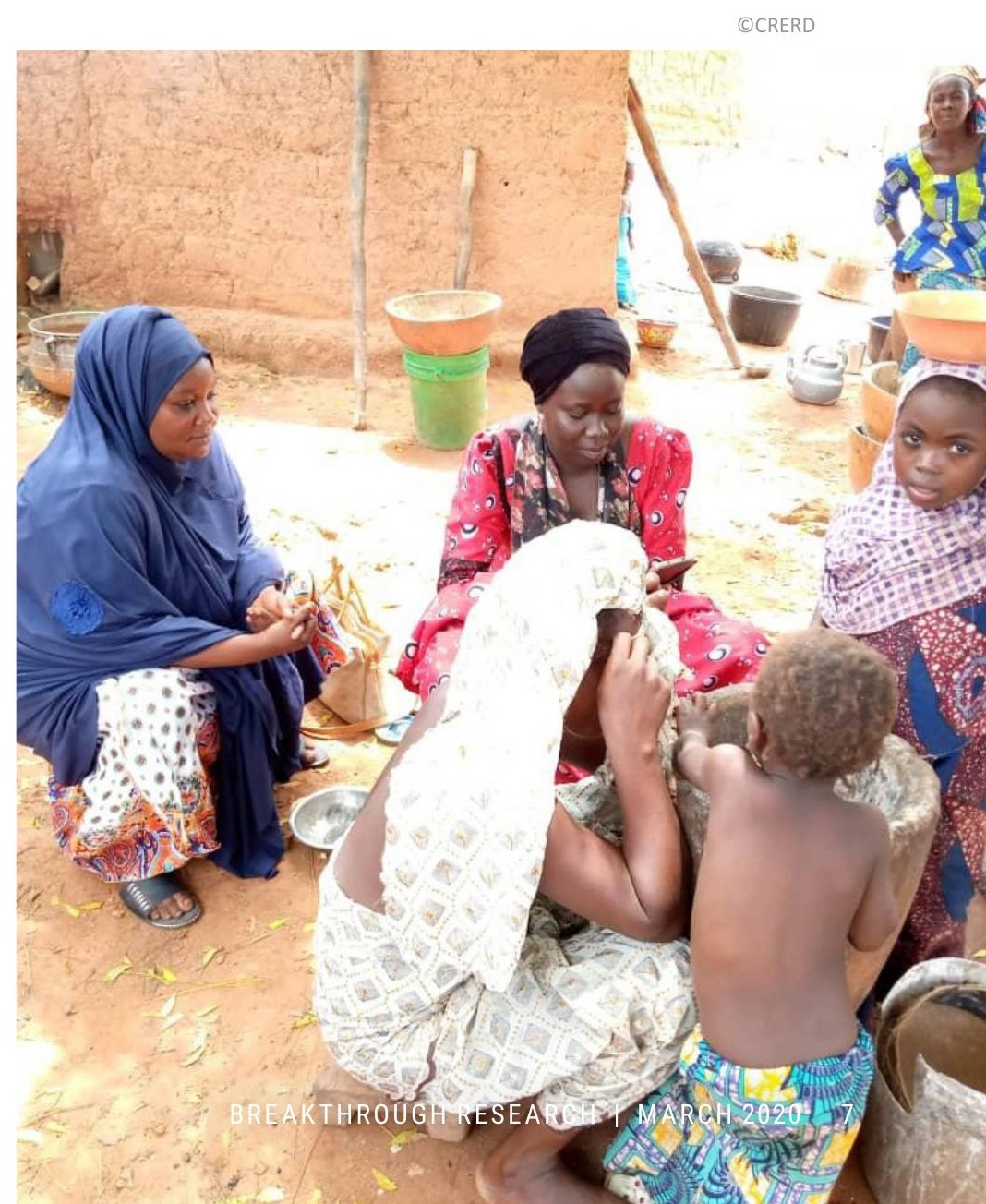


deaths include neonatal conditions, pneumonia, diarrhea, and malaria. Routine immunization rates remain low in Nigeria, with only approximately 50 percent of one-year-old children receiving measles and diphtheria-tetanus-pertussis (DPT3) vaccines in 2018, with far lower rates in the northwestern region.

Appropriate treatment for common causes of childhood morbidity and mortality is low. Only 23 percent of children under 5 years with diarrhea received the recommended treatment of ORS and zinc, while only 37 percent with symptoms of pneumonia were taken to a public sector provider for care. In terms of nutrition, one-third (37\%) of children under 5 years were stunted in 2018. In addition, only 29 percent of infants were exclusively breastfed for the first six months of their life in $2018 .^{3}$

According to NDHS 2018, the Nigerian maternal mortality ratio remains among the highest in the world, at 512 maternal deaths per 100,000 live births in the seven-year period before the 2018 survey. ${ }^{3}$ The main causes of maternal deaths include hemorrhage, hypertension, and indirect causes. In 2018, 57 percent of women attended antenatal care (ANC) at least four times during their last pregnancies, and only 39 percent of births took place in a facility, while 43 percent were attended by a skilled health professional.

\section{Malaria in Nigeria}

Based on NDHS 2018, four Nigerian States are considered high-transmission zones, including Jigawa, Kebbi, Zamfara, and Sokoto. The latter three states are the setting for the Behavioral Sentinel Surveillance (BSS) study. In the north, transmission is year-round with seasonal peaks within a four-month window from June to September. Plasmodium falciparum is the principal malaria species, and Anopheles (An.) gambiae is the predominant mosquito vector.

While there has been great progress over the past decade in malaria control activities, the availability of key prevention tools and engagement in malaria preventive behaviors remains sub-optimal. Only 61 percent of households owned at least one long-lasting insecticidal net (LLIN), although this represents significant improvements, given that only 8 percent reported the same in NDHS 2008 and 50 percent in NDHS 2013. Approximately 58 percent of pregnant women reported sleeping under an LLIN in 2018, and only 17 percent received intermittent preventive treatment during pregnancy (IPTp) to prevent malaria. ${ }^{9}$ Exposure to malaria messaging-generally through radio, community drama, printed materials, community and religious leaders, community support groups, and household visits - has led to high levels of knowledge of malaria interventions, but misconceptions about the causes of and ways to manage malaria still remain. ${ }^{10}$

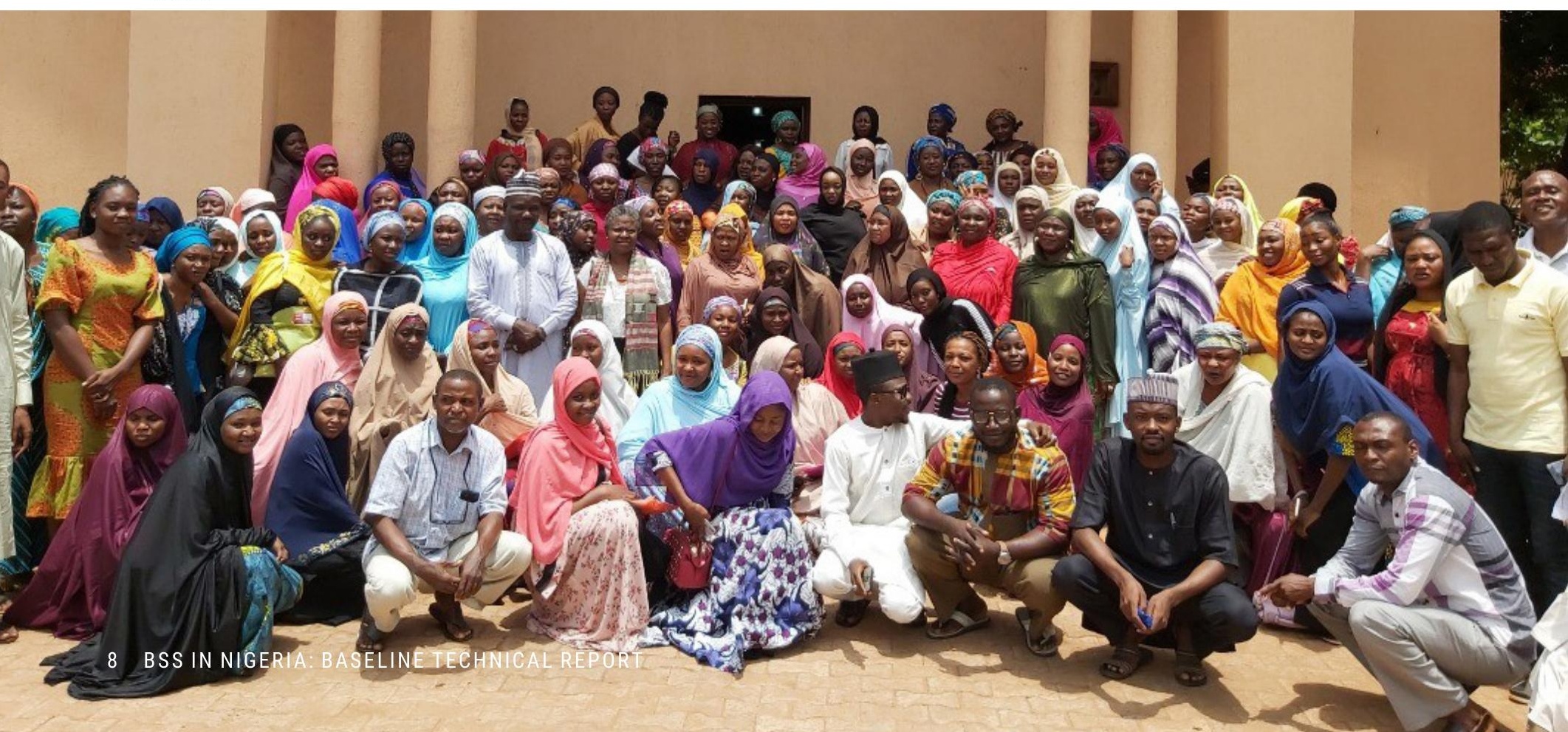




\section{Breakthrough ACTION in Nigeria}

Breakthrough ACTION/Nigeria is the follow-up project to the Johns Hopkins Center for Communications Programs (JHU CCP) Health Communication Capacity Collaborative (HC3) project (2014-2018), which operated in three northern states (Bauchi, Ebonyi, and Sokoto). HC3 used a selection of SBC approaches to promote positive birth spacing and use of family planning methods by women of reproductive age. In the states supported by the President's Malaria Initiative (PMI), HC3 also used SBC approaches to support the government of Nigeria's goal of attaining 80 percent of the population practicing appropriate malaria prevention and management by 2020.

Under HC3, an ideational theory of change for birth spacing was posited (see next section for a full description of this theory). ${ }^{11}$ Three primary SBC approaches were implemented across socio-ecological levels: 1) advocacy outreach to opinion leaders and community influencers at state and local government area (LGA) level, 2) direct engagement of community members through community dialogues and group meetings that included referrals for services, and 3) complementary SBC messaging through mass and mid-media. ${ }^{12}$ The reported impact of $\mathrm{HC} 3$ efforts was positive normative change supporting birth spacing, acceptability of contraception to achieve optimal birth spacing, and sizable increases in the utilization of family planning methods reported by health facilities in program areas. For malaria, the combination of advocacy visits, community dialogues, and training of community health workers allowed $\mathrm{HC} 3$ to reach more than 8,000 opinion leaders in Zamfara State alone. Furthermore, in an analysis in four of the five states in which $\mathrm{HC} 3$ malaria SBC activities were undertaken, exposure to malaria SBC messaging was associated with an approximately two-fold increase in the percent of respondents who slept under an LLIN.

Breakthrough ACTION/Nigeria, led by JHU CCP, leverages and expands the HC3 activities in Nigeria (Figure A-1). In particular, advocacy outreach expanded from state to LGA levels, and integrated $\mathrm{MNCH}+\mathrm{N}$ and malaria messaging using a life-stage approach is embedded with family planning SBC messaging and expanded to additional wards and LGAs in three targeted states (Bauchi, Sokoto, and Kebbi). The official launch of these expanded, integrated SBC activities in the targeted states occurred on 19 September 2019, in Abuja, although staged implementation rolled out between early and late September in LGAs/wards across the three states.

Table A-1 describes the key objectives of each life stage messaging within the Breakthrough ACTION/Nigeria approach used in those three states. This approach was based on extensive formative research conducted by Breakthrough ACTION/Nigeria to develop messaging materials for communities within these states. Based on this formative research, Breakthrough ACTION/Nigeria $\mathrm{MNCH}+\mathrm{N}$ messaging has focused on gateway and/or multiplier effect behaviors, such as ANC and routine immunization. In addition, Breakthrough ACTION/Nigeria continues to support SBC messaging that promotes the use of malaria control interventions, including IPTp, LLINs, and seasonal malaria chemoprevention. Breakthrough ACTION/Nigeria also continues to implement SBC programming in support of USAID PMI malaria programming, service delivery partners, and commodities distribution.

\section{FIGURE A-1. MAP OF BREAKTHROUGH ACTION/NIGERIA AREAS}

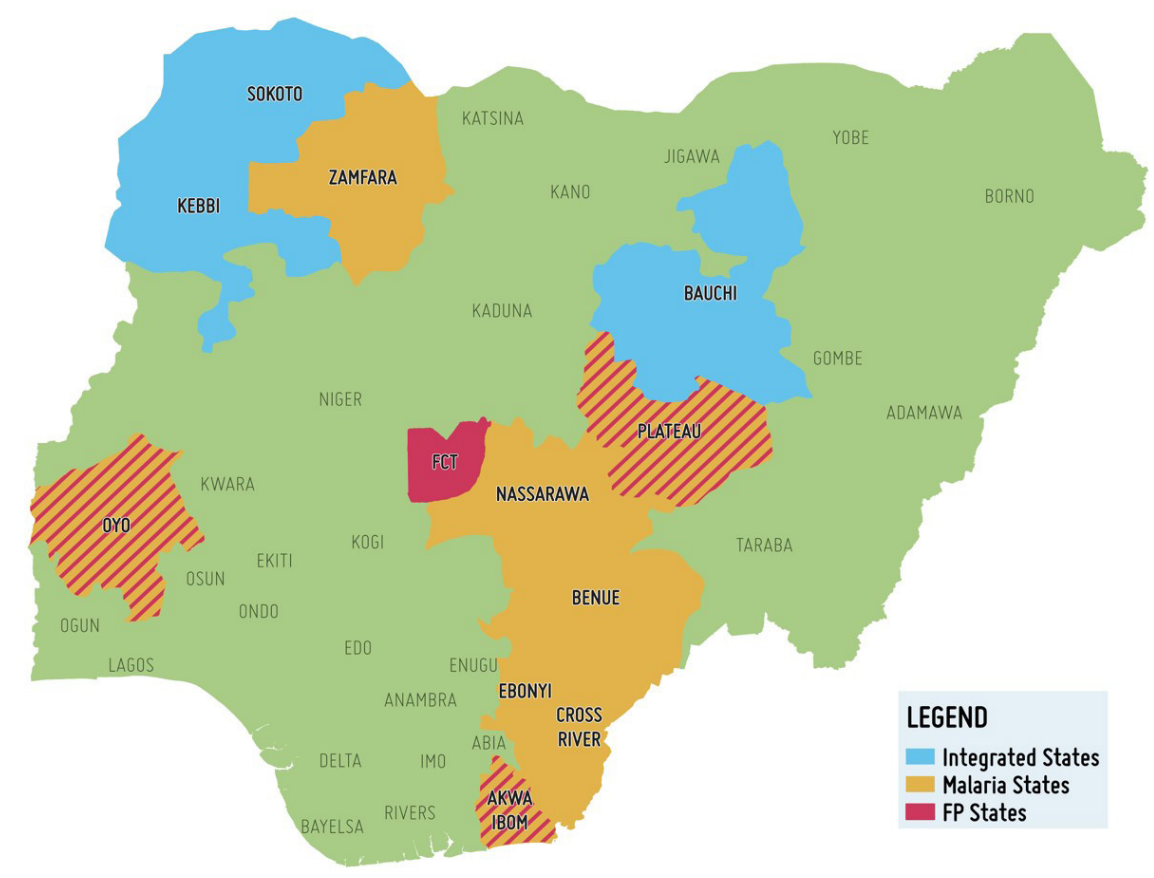




\section{TABLE A-1. KEY OBJECTIVES OF LIFE STAGE APPROACH FOR INTEGRATED SBC PROGRAMMING AREAS}

\begin{tabular}{|c|c|}
\hline LIFE & OBJECTIVE \\
\hline \multirow{7}{*}{$\begin{array}{l}\text { Life Stage } 1 \\
\text { SBC messag- } \\
\text { ing targeted } \\
\text { to women } \\
\text { planning for } \\
\text { pregnancy and } \\
\text { those in early } \\
\text { pregnancy }\end{array}$} & $\begin{array}{l}\text { Increase proportion of pregnant women who attend first ANC as soon as they become aware of pregnancy and at least } \\
\text { four times during pregnancy and up to eight times }\end{array}$ \\
\hline & Increase proportion of pregnant women taking three or more doses of IPTp as prescribed by health worker during ANC \\
\hline & Increase proportion of pregnant women who adhere to nutrition counseling to improve dietary intake during pregnancy \\
\hline & Increase proportion of pregnant women who sleep inside an insecticide-treated net \\
\hline & $\begin{array}{l}\text { Increase proportion of pregnant women/partners who develop a birth plan to increase intention to deliver at a health } \\
\text { facility }\end{array}$ \\
\hline & Increase proportion of pregnant women who deliver at a health facility and/or with a skilled birth attendant \\
\hline & $\begin{array}{l}\text { Increase proportion of pregnant women/partners who intend to use a modern contraceptive to space their children } \\
\text { after pregnancy }\end{array}$ \\
\hline \multirow{10}{*}{$\begin{array}{l}\text { Life Stage } 2 \\
\text { SBC messaging } \\
\text { targeted to } \\
\text { women in late } \\
\text { pregnancy and } \\
\text { those with a } \\
\text { newborn (first } 4 \\
\text { weeks of life) }\end{array}$} & $\begin{array}{l}\text { Increase proportion of pregnant women and their partners who develop a birth plan to increase intention to deliver at a } \\
\text { health facility }\end{array}$ \\
\hline & Increase proportion of new mothers placing their baby on their abdomen for warmth immediately after birth \\
\hline & Increase proportion of new mothers who start breastfeeding within 30 minutes of giving birth \\
\hline & Increase proportion of new mothers who use chlorhexidine to cleanse their baby's umbilical cord for seven days \\
\hline & Increase proportion of mothers who complete all immunizations scheduled at birth per Nigerian policy \\
\hline & Increase proportion of new mothers who use misoprostol to stop bleeding for post-partum hemorrhage \\
\hline & Increase proportion of new mothers who adhere to nutrition counseling to improve dietary intake post-partum \\
\hline & Increase proportion of new mothers and babies who sleep inside an insecticide-treated net \\
\hline & Increase proportion of new mothers who attend postnatal care check-ups \\
\hline & Increase proportion of pregnant women/partners using modern contraception to space births after pregnancy \\
\hline \multirow{8}{*}{$\begin{array}{l}\text { Life Stage } 3 \\
\text { SBC messaging } \\
\text { targeted to } \\
\text { women with } \\
\text { young children } \\
\text { under } 5 \text { years }\end{array}$} & Increase proportion of children between 12 and 23 months who are fully immunized per Nigerian policy \\
\hline & Increase proportion of children under 6 months who are exclusively breastfed \\
\hline & $\begin{array}{l}\text { Increase proportion of children six months to } 2 \text { years whose caregivers follow recommended infant and young child } \\
\text { feeding practices }\end{array}$ \\
\hline & Increase proportion of children under 5 years who sleep inside an insecticide-treated net \\
\hline & Increase proportion of children under 5 years with fever promptly taken for care and tested for malaria before treatment \\
\hline & Increase proportion of malaria-positive cases that take a full course of ACT \\
\hline & Increase proportion of children under 5 years with diarrhea promptly taken for care and given appropriate treatment \\
\hline & Increase proportion of children between 12 and 23 months who are fully immunized per Nigerian policy \\
\hline \multirow{4}{*}{$\begin{array}{l}\text { Life Stage } 3 \\
\text { SBC messaging } \\
\text { targeted to } \\
\text { male partners }\end{array}$} & Increase proportion of men and women who use modern contraceptive methods \\
\hline & Increase proportion of women who receive nutrition counseling to improve dietary intake \\
\hline & Increase proportion of women, men, youth, and children who sleep inside an insecticide-treated net \\
\hline & Increase proportion of women who intend to attend ANC early in their next pregnancy \\
\hline
\end{tabular}




\section{Theory of Strategic Communication and Behavior Change}

The BSS questionnaire was largely informed by Kincaid's Theory of Strategic Communication and Behavior Change (Figure A-2). ${ }^{12}$ In this model, ideations consist of three main domains-cognitive, emotional, and social. The two defining elements of attitude are beliefs and values, which indicate the perception of either the positive or negative consequence of a health behavior. Subjective norms involve an individual's thoughts around what other individuals within their communities are doing or what they think they are expected by others to do. Selfimage refers to an individual's belief about themselves in relation to a behavior, and self-efficacy measures how confident a person feels to perform a certain behavior. The social component involves interpersonal processes aimed at persuasion to adopt a behavior.

Furthermore, in this model, communication is considered an external factor impacting other variables in the model and shows how and why it impacts both intention and behavior. Communication has an indirect impact on behavior through its effect on skills and knowledge, ideations, and environmental support and constraints. Thus, the components in this model can be described as the "cognitive, emotional, and social outcomes of communication and determinants of intention and behavior." Because these components of ideation are regarded as intermediate outcome variables impacting intention and behavior, the Kincaid model represents a causal chain of the determinants of behavior where change is interdependent, simultaneous, and interactive.

The Breakthrough ACTION Integrated Health SBC Strategy (January 2019) was informed by this theory of change model, which was used to develop the SBC multi-level approach for influencing sociocultural and environmental determinants, as well as relationships and structural factors that affect $\mathrm{MNCH}+\mathrm{N}$, malaria, and family planning behaviors. Within these domains, Breakthrough ACTION's programming expicitly recognizes the centrality of community, household, and service delivery systems to effect desired change given the strong influence of social norms on behaviors and the critical role of health providers. Breakthrough ACTION also places a strong emphasis on the supportive environment as the foundational support to behavior change. Ideational factors are central to Breakthrough ACTION's

\section{FIGURE A-2. THEORY OF STRATEGIC COMMUNICATION AND BEHAVIOR CHANGE}

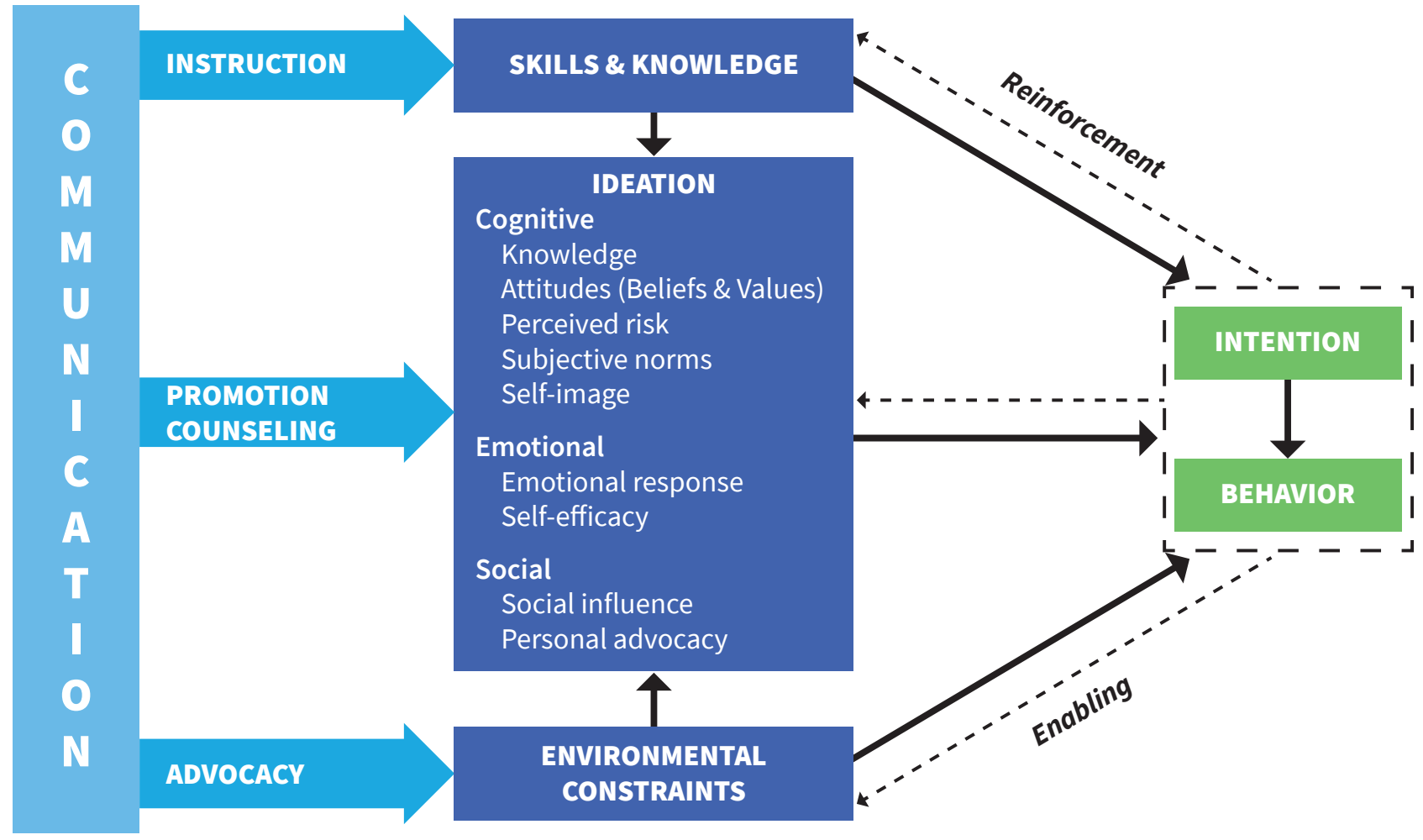

Kincaid DL, Delate R, Storey JD \& Figueroa ME. (2013). Closing the gaps in practice and in theory: evaluation of the Scrutinize HIV Campaign in South Africa. In Rice R \& Atkin C. (eds.) Public Communication Campaigns, 4th edition. Newbury Park, CA: Sage, pp. 305-319. 
SBC programming to help guide priorities and messaging, including knowledge, beliefs, values, risk perceptions, self-efficacy, social norms, perceived social support from key influencers, and spousal communication, among others. The more positive ideational factors held by a person about a given behavior, the more likely they are to engage in that behavior. Breakthrough ACTION has prioritized messages and activities to shift specific, actionable ideational factors for low prevalence behaviors, such as spousal communication about contraceptive use, as detailed in their strategy document.

\section{Integrated versus health-area- specific SBC messaging}

There has been recent and growing interest in and implementation of integrated, multi-component SBC programming. These programs often operate at multiple levels of the socio-ecosystem (individual, household, and community) and span multiple health components (e.g., family planning, $\mathrm{MNCH}+\mathrm{N}$, and malaria), or may even address cross-sectoral outcomes (e.g., agriculture, health, and education). This shift has been partly motivated by the desire to meet the complex and multi-faceted needs of target populations, to effectively address underlying common causes and determinants of closely related health outcomes, and to increase cost-efficiencies. Yet there have been few systematic evaluations of integrated SBC programs and limited robust evidence on the effectiveness of integrated SBC programs in improving target health and development outcomes. ${ }^{13}$

\section{Study rationale}

The partnership of Breakthrough ACTION/Nigeria and Breakthrough RESEARCH/Nigeria presents a unique opportunity to dovetail rigorous research design with state-of-the-art SBC programming to identify whether or not integrated SBC programming is more effective than vertical programming in promoting select healthy family behaviors. The study focused on pregnant women and women within the 1,000-day window of opportunity, and is closely tied to the primary Breakthrough ACTION/ Nigeria objective of improving key behaviors and ideations related to $\mathrm{MNCH}+\mathrm{N}$, malaria, and family planning among this target group in order to improve the lives and well-being of women and families.

\section{Study objectives}

This research exploited Breakthrough ACTION/Nigeria's variation in integrated programming in Nigeria to assess the effectiveness of integrated SBC approaches to improve healthy behaviors and ideations across the Breakthrough ACTION/Nigeria project life cycle. To date, there has been little research on best practices in SBC integration methods to promote a wide range of healthy family behaviors and outcomes. Key study objectives include:

- Objective 1: Assess the effectiveness of integrated SBC programming relative to malaria-only SBC programming focusing on pregnant women and women with a child under 2 years of age.

- Objective 2: Collect and report on a selection of ideational and priority behavioral indicators across $\mathrm{MNCH}+\mathrm{N}$, malaria, and family planning to inform Breakthrough ACTION/Nigeria implementation and scale-up. 


\section{Methodology}

Tulane University and Population Council, in collaboration with the Center for Research, Evaluation Resources, and Development (CRERD), conducted the BSS baseline survey in September 2019. Table A-2 provides an overview of the research activities, methods, and study instruments with a detailed discussion of these methods in the following sub-sections.

\section{Study location}

Three states were identified, in collaboration with USAID/ Nigeria and JHU CCP, for the study setting: Kebbi and Sokoto States targeted by Breakthrough ACTION/Nigeria for integrated SBC programming and Zamfara State targeted by Breakthrough ACTION/Nigeria for malaria-only SBC programming. These three states, located in northwestern Nigeria, are contiguous and share a border with
Niger Republic, while Kebbi also borders Benin to the west. The dominant religion and ethnicity in these states are Islam and Hausa, respectively. The total populations are 5.4 million (Sokoto), 3.5 million (Kebbi), and 4.1 million (Zamfara). The northwestern region has among the highest poverty rates and poorest health outcomes in Nigeria, including under-5 mortality rates of 119 (Sokoto), 174 (Kebbi), and 210 (Zamfara) per 1,000 live births. Additionally, the maternal mortality ratio (MMR) in Nigeria was estimated at 917 maternal deaths per 100,000 live births in 2017, and is likely much higher in the northwest region. ${ }^{14-16}$ Agriculture is the main source of income for people living in this region. ${ }^{13,17-21}$

\section{TABLE A-2. STUDY OVERVIEW}

\begin{tabular}{|c|c|}
\hline Study design & $\begin{array}{l}\text { A quasi-experimental and dose-response study. Women in the 1,000-day window in Breakthrough ACTION/Nigeria in- } \\
\text { tegrated areas (Sokoto, Kebbi) will be compared with women in Breakthrough ACTION/Nigeria vertical areas (Zamfara). } \\
\text { Furthermore, the study will include dose-response design to compare women in high-dose integrated messaging areas } \\
\text { (e.g., additional household visits) with women in standard-dose messaging areas. Community screening will be used } \\
\text { to identify pregnant women to form a cohort to be followed over the life of the Breakthrough ACTION/Nigeria project. } \\
\text { Women within the 1,000-day window will also be identified and interviewed cross-sectionally over the life of the Break- } \\
\text { through ACTION/Nigeria project. }\end{array}$ \\
\hline Study location & $\begin{array}{l}\text { Breakthrough ACTION/Nigeria integrated implementation LGAs/wards in Kebbi and Sokoto States; LGA/wards with } \\
\text { vertical malaria-only Breakthrough ACTION/Nigeria implementation in Zamfara State }\end{array}$ \\
\hline $\begin{array}{l}\text { Study } \\
\text { population }\end{array}$ & $\begin{array}{l}\text { Pregnant women and women with a child under } 2 \text { years old living within Breakthrough ACTION/Nigeria programming } \\
\text { wards in Kebbi, Sokoto, and Zamfara States. For the purpose of this report, the study population is referred to as } \\
\text { respondents. }\end{array}$ \\
\hline Study period & June 2019 to March 2022 \\
\hline Sample size & 6,034 respondents \\
\hline \multirow{7}{*}{$\begin{array}{l}\text { Annex } \\
\text { materials }\end{array}$} & Annex 1-a: Adult consent form \\
\hline & Annex 1-b: Guardian consent form \\
\hline & Annex 1-c: Adolescent consent form \\
\hline & Annex 1-d: Adult caregiver consent form for orphaned and vulnerable children (OVC) interview \\
\hline & Annex 2: Community screening instrument \\
\hline & Annex 3-a: Household questionnaire \\
\hline & Annex 3-b: Female questionnaire \\
\hline
\end{tabular}




\section{Study design}

The BSS survey targets pregnant women and women with a child under 2 years living in Breakthrough ACTION/ Nigeria programming areas targeted for integrated SBC messaging (Kebbi/Sokoto) and targeted for malaria-only SBC messaging (Zamfara). Since Breakthrough ACTION/ Nigeria covers only parts of these states, the data are representative of these populations within Breakthrough ACTION/Nigeria programming areas but not across the states at large. The BSS aims to measure changes in behaviors and ideations across family planning, malaria, and $\mathrm{MNCH}+\mathrm{N}$ among this study population at baseline, midline, and endline periods of the Breakthrough ACTION/Nigeria project cycle.

The BSS will include both quasi-experimental and dose-response study design components. For the quasi-experimental design, ideational factors, behaviors, and outcomes among women in the 1,000-day window of opportunity in the integrated programming states of Kebbi and Sokoto will be measured and compared across time with those indicators for similar women in Zamfara State, which has been targeted for malaria-only SBC messaging. For the dose-response design, and to measure the effect of more intensive integrated programming in terms of household visits, wards within a subsample of Breakthrough ACTION/Nigeria program areas will be purposefully chosen with Breakthrough ACTION/ Nigeria's assistance to receive additional programming (e.g., additional household visits). In previous studies, the effectiveness of SBC interventions has been shown to hinge upon the intensity of interventions. ${ }^{22}$ Table A-3 highlights the three study comparison arms in terms of household visit activities.

\section{Study population}

The study population includes currently pregnant women aged 15 to 49 years and women aged 15 to 49 years with a child under 2 years who are living in Breakthrough ACTION/Nigeria program areas targeted for integrated SBC messaging (Kebbi/Sokoto) and for malaria-only SBC messaging (Zamfara). The study was powered to assess Breakthrough ACTION/Nigeria SBC program impact for these two population groups, as described in the next section.

\section{Sampling frame}

The sampling frame for the study population was generated through community screening conducted in 108 clusters/wards across the three states (36 wards/clusters per state), which were selected using digital maps and grid sampling methodology (Figure A-3). Within each sampled cluster/ward, the community screening tool was used to identify all pregnant women and a random selection of women with a child under 2 years for inclusion in the BSS baseline survey using the following criteria:

1. The household must include at least one currently pregnant woman or at least one woman with a child under age 2 based on the community screening instrument.

\section{TABLE A-3. STUDY COMPARISON ARMS}

\begin{tabular}{|c|c|}
\hline \multirow{3}{*}{$\begin{array}{l}\text { Standard-dose } \\
\text { integrated SBC } \\
\text { program }\end{array}$} & 36 randomly selected wards in Kebbi/Sokoto: \\
\hline & - Women currently in one life stage receive one household visit (with one life stage dialogue) \\
\hline & - Women in two life stages receive two household visits (with two life stage dialogues) \\
\hline \multirow{3}{*}{$\begin{array}{l}\text { High-dose } \\
\text { integrated SBC } \\
\text { program }\end{array}$} & 36 randomly selected wards in Kebbi/Sokoto: \\
\hline & $\begin{array}{l}\text { Women currently in one life stage receive two household visits (with one life stage dialogue and a } \\
\text { second visit as a follow-up within seven days to reinforce key messages from the previous life stage } \\
\text { dialogue and to follow-up on referrals) }\end{array}$ \\
\hline & $\begin{array}{l}\text { Women in two life stages receive three household visits (with two different life stage dialogues given } \\
\text { within seven days of each other, and a third visit as a follow-up within } 10 \text { to } 14 \text { days to reinforce key } \\
\text { messages from the previous two life stage dialogues and for referral follow-up) }\end{array}$ \\
\hline Malaria-only & All wards in Zamfara: \\
\hline & - Women receive one household visit with standard malaria-only dialogue \\
\hline
\end{tabular}


FIGURE A-3. DISTRIBUTION OF BSS SAMPLING WARDS IN KEBBI, SOKOTO, AND ZAMFARA STATES

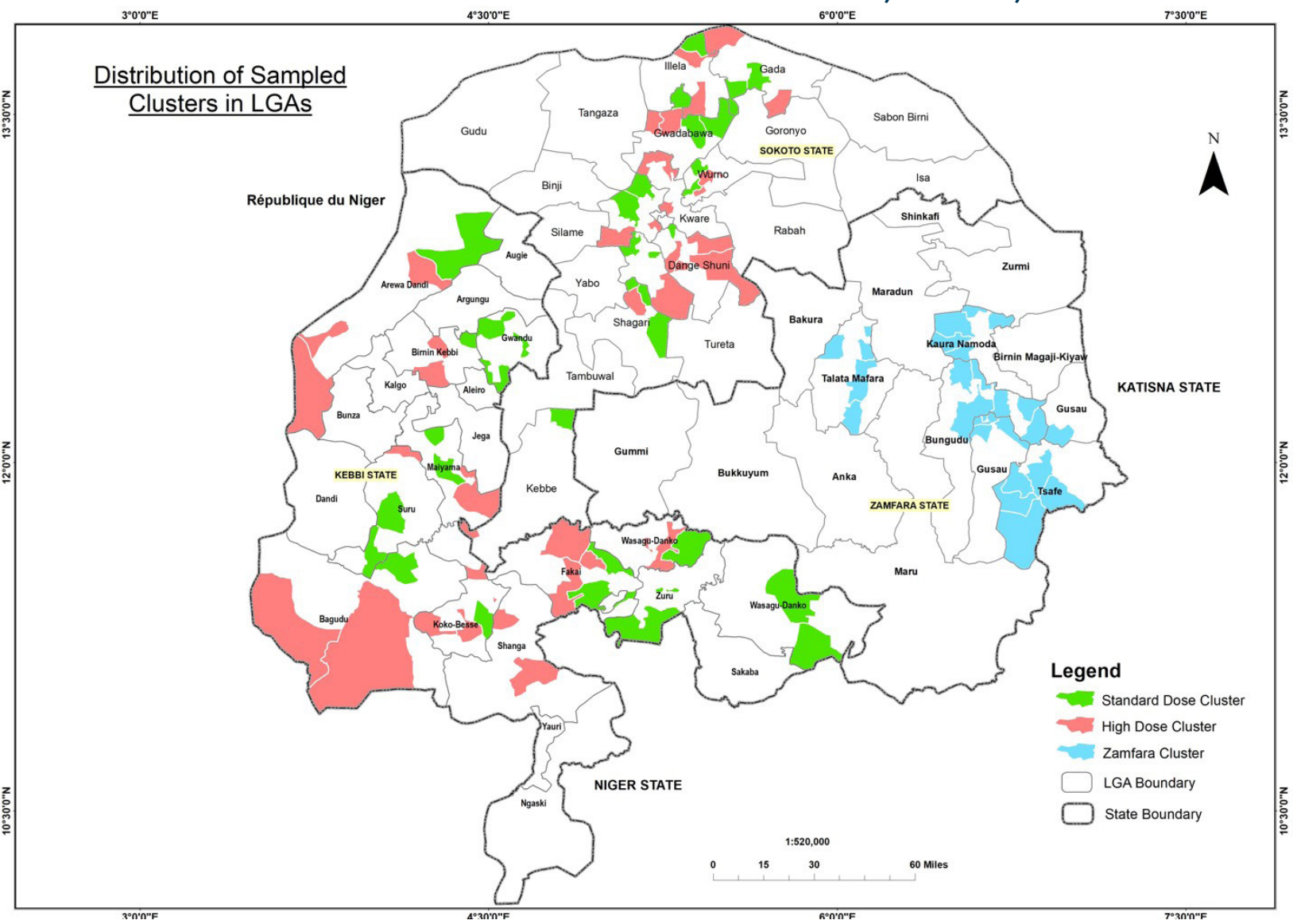

2. The woman must be between the ages of 15 and 49 years.

3. The woman must be willing and able to provide informed consent to participate.

Importantly, since only a quarter of women in the northern states attend ANC in the formal health sector, a community screening approach was necessary to prevent the omission of pregnant women who do not use formal sector care. Recruiting only women who present for ANC at health facilities would not reach all women targeted by Breakthrough ACTION/Nigeria programming and would introduce biases for understanding program effectiveness.

\section{Sample size estimation}

The sample size was estimated based on the quasi-experimental study design that contains three comparison arms: (1) high-dose intervention in integrated SBC areas, (2) standard-dose intervention in integrated SBC areas, and (3) malaria-only SBC programming. The sample was chosen to ensure the detection of expected changes in priority behavioral outcomes across integrated and malaria-only SBC areas in the quasi-experimental design, to allow for assessment of the impact of variations in Breakthrough ACTION/Nigeria programming, and to account for potential attrition from the BSS cohort. To reduce attrition, pregnant women selected for the BSS cohort will be tracked across time by collecting detailed contact information, including name, address, GPS coordinates of dwelling, and phone numbers.

To determine the required sample size for the baseline survey, a Stata 15.0 sample size routine for cluster sampling was used.23 The parameters specified for the sample size estimation included a power criterion of 0.80 , alpha coefficient of 0.05 , and intra-cluster correlations that varied by key outcomes as shown below. Table A-4 presents the minimal detectable differences for priority outcomes. Based on this estimation, a sample size of approximately 3,000 pregnant women and 3,000 women with a child under 2 years were targeted for inclusion in the baseline survey, with similar sample sizes expected in future BSS rounds. 
TABLE A-4. PRIORITY INDICATORS AND MINIMAL DETECTABLE DIFFERENCES

\begin{tabular}{|lrrr|}
\hline INDICATOR & PREVALENCE & ICC & $\begin{array}{r}\text { MINIMAL } \\
\text { DETECTABLE } \\
\text { DIFFERENCE }\end{array}$ \\
\hline ANC four or more & $17.5 \%$ & 0.29473 & $12-16 \mathrm{pp}(\mathrm{a})$ \\
Facility delivery & $6.0 \%$ & 0.15223 & $5-9 \mathrm{pp}(\mathrm{b})$ \\
$\begin{array}{l}\text { Pregnant women } \\
\text { slept under LLINs }\end{array}$ & $17.7 \%$ & 0.30883 & $12-17 \mathrm{pp}(\mathrm{c})$ \\
$\begin{array}{l}\text { Measles vacci- } \\
\text { nation (12-23 } \\
\text { months) }\end{array}$ & $5.2 \%$ & 0.1234 & $4-8 \mathrm{pp}(\mathrm{d})$ \\
$\begin{array}{l}\text { DPT3 vaccination } \\
\text { (12-23 months) }\end{array}$ & $3.9 \%$ & 0.07575 & $3-6 \mathrm{pp} \mathrm{(e)}$ \\
$\begin{array}{l}\text { Modern contra- } \\
\text { ceptive use }\end{array}$ & $<1.0 \%$ & 0.03894 & $\mathrm{pp} \mathrm{(f)}$ \\
\hline
\end{tabular}

Notes: (a) clustersampsi, binomial detectable difference p1(0.1749) k(36) $\mathrm{m}(25)$ rho(0.28064); (b) clustersampsi, binomial detectable difference $\mathrm{pl}(0.0599) \mathrm{k}(36) \mathrm{m}(25) \mathrm{rho}(0.15223)$; (c) clustersampsi, binomial detectable difference $\mathrm{p} 1(0.1768) \mathrm{k}(36) \mathrm{m}(25)$ rho(0.30883); (d) clustersampsi, binomial detectable difference p1(0.052) k(36) m(25) rho(0.12304); (e) clustersampsi, binomial detectable difference p1(.0389) k(36) m(25) rho $(0.07575)$

\section{Sampling strategy}

We used the following sampling strategy to obtain the estimated sample size. We selected all pregnant women in a cluster based on a community screening tool. If the average cluster size is 150 households, this would yield approximately 25 pregnant women per cluster. With 36 clusters per group, this yields 900 pregnant women per group. The total sample size would be 2,700 pregnant women, or 3,000 to account for 10 percent attrition. We further estimated the need to select 25 women per cluster with a child under 2 years. Again, with 36 clusters per group, this yields 900 women with a child under 2 per group, or 2,700. As before, it was planned to sample an additional 300 women with a child under 2 years to allow for 10 percent attrition.

\section{Data collection and questionnaires}

All eligible women were asked to respond to an interviewer-directed BSS questionnaire. Development of the questionnaire was done through a collaborative process involving relevant stakeholders, including: Breakthrough ACTION/Nigeria, JHU CCP, USAID Headquarters and
Nigeria Mission, and PMI. The household questionnaire collected information on usual resident household members, bed net ownership and use, and household assets and characteristics. The female questionnaire asked all respondents about their demographics, reproductive history, contraceptive use, media exposure, gender norms, and ideations related to family planning, malaria, and $\mathrm{MNCH}+\mathrm{N}$. All pregnant women were specifically asked about current pregnancy behaviors, including ANC, malaria prevention in pregnancy, and nutritional practices. All women with a child under 2 years were specifically asked about behaviors for their last-born child within the past two years, including antenatal and delivery care, newborn care, breastfeeding, routine vaccination, malaria prevention and treatment, child and maternal nutrition, and childhood illness care-seeking and treatment, including for pneumonia symptoms.

\section{Training, pre-testing and fieldwork}

Fieldwork was conducted simultaneously across the three states over a four-week period from 4 September 2019 to 7 October 2019. All 168 fieldworkers (144 female interviewers and 24 supervisors) were trained together in Sokoto during a one-week period from 29 August 2019 to 2 September 2019. A training manual was produced for each fieldworker to facilitate training sessions on the study objectives and survey instruments, ethical considerations, and fieldwork procedures, with participation in a questionnaire pilot exercise. The questionnaire was translated into Hausa and pre-tested to confirm translations and skip patterns, and to assess question appropriateness and sequencing.

\section{Fieldwork supervision and teams}

CRERD supervised and managed the BSS baseline survey implementation, as described in these sections. The fieldwork team in each state consisted of a state coordinator who oversaw all the activities in the state and reported to the CRERD management team, as well as 168 fieldworkers (144 female interviewers and 24 supervisors). There were data quality assurance personnel in each state who monitored the data for each upload and who flagged any issues of concern immediately for discussion and resolution. There were five supervisors in each state, with a minimum of five and maximum of eight data collectors to one supervisor in line with the sample distribution. The management team traveled around 
the states to monitor the fieldwork as it was being conducted.

\section{Data entry and management}

Survey questions were administered face-to-face by trained interviewers who entered responses into a secured handheld device (tablet). The handheld device was password-protected and the password was provided only to designated staff. Data from the handheld devices were removed from the device when uploaded to the central database. The completed data files on the handheld devices were encrypted after being completed. They were transmitted to the centralized storage in their encrypted format. Skips and data-entry checks were in place during the interview to prevent illogical data values. Survey data were uniquely identified through a numeric ID and were stored in central databases on password-protected computers that were transmitted regularly to the study investigators. All data were kept confidential and stored in a safe and secure place at Tulane and CRERD project offices.

\section{Data quality assurance}

CRERD selected the field teams based on proven integrity in data collection during a previous performance. More than 90 percent of the field team participated in previous CRERD survey activities or with their close collaborators. The following quality assurance checks were also conducted during data collection:

- Use of spatial data to ensure that the interviewer went to the assigned location for the interview. This was done based on geolocated household positioning and Google Earth maps, which show key visual features to determine whether the location of the interview was correct.

- The use of time stamps in the data (at the beginning and end of each interview) provided two important pieces of information to evaluate data quality: interview start time and duration. A typical interview should occur between 7:30am and 8pm. Any interview outside these times was flagged as suspicious. If the interview was too long or too short (and if this happened in consecutive or frequent interviews), the supervisor or interviewer was asked to explain.
- Interviewer performance dashboard allowed the CRERD management team to monitor interviewer errors, time taken for interviews, and the number of interviews completed. All of the information consolidated on a dashboard was used to monitor the progress of each interviewer. The dashboard helped the survey management team to check on field workers' productivity. It was also a tool for identifying top-performing individuals and teams for recognition.

- Field inspection allowed CRERD management and field supervisory teams to monitor all aspects of fieldwork activities. These teams inspected interviews to ensure the respondents had enough privacy, consent was obtained, and questions were clearly asked. They also ensured that interviewers behaved in a culturally sensitive way during fieldwork activities.

Uploaded data files were also reviewed for completeness, clarity, and consistency of reporting:

- Completeness: All data files were checked to ensure that all planned interviews were conducted. This was done by matching the number of interviews documented in the field report with what was found in the uploaded files. If there were any discrepancies, this was flagged by the data auditors, and field supervisors were asked to explain the difference and resolve the problem. We checked that the same interview was not uploaded twice, and, if duplicates occurred, the interviewer was asked to identify the correct version for upload. We also checked that all variables were properly captured, and we performed a skip analysis to ensure no incorrect skip patterns occurred within the data.

- Clarity: We ensured that the data variables followed the questionnaire sequence. All value and variable labels were clearly worded in a concise manner in the final dataset for ease of analysis.

- Consistency: To ensure that the data files made logical sense, the following checks were conducted:

- Review of outliers: Using central tendency, all continuous variables were evaluated for outliers. Numbers that seemed too low or too high were communicated to the teams. These were most often typographical errors (e.g., double digits instead of a single digit). 
- Population pyramid: We looked at the population pyramids across all three states to determine if the age and sex distributions we obtained from the household roster were realistic.

- Average household size: Since we knew the average household size of each state, we were able to flag fieldworkers who returned household rosters with too few household members. In these cases, field supervisors were asked to verify data collected and to correct any issues if found.

- Skip pattern analysis: We checked whether the skips were enforced before and after data cleaning.

\section{Response rates}

Tables A-5 and A-6 present the response rates across states for pregnant women and women with a child under 2 years, respectively.

\section{Study sample}

Table A-7 presents the final study sample in the BSS baseline survey by Breakthrough ACTION/Nigeria program areas with either integrated (Kebbi/Sokoto) or malaria-only (Zamfara) SBC programming.

\section{Ethical approval}

Ethical approval for this study was obtained from the National Health Research Ethics Committee (NHREC) in Nigeria (NHREC/01/01/2007-02/09/2019) and the Tulane University Institutional Review Board (IRB) in Louisiana, USA (2019-1047). Written informed consent to participate in the survey was obtained from all willing participants for the household and female questionnaires. Each participant signed or marked her thumbprint on the consent form to signify willingness to participate..

\section{TABLE A-7. STUDY SAMPLE}

\begin{tabular}{|c|c|c|c|}
\hline \multirow[b]{2}{*}{ SAMPLE } & \multicolumn{3}{|c|}{$\begin{array}{l}\text { BREAKTHROUGH ACTION } \\
\text { PROGRAM AREA }\end{array}$} \\
\hline & $\begin{array}{l}\text { KEBBI/ } \\
\text { SOKOTO }\end{array}$ & ZAMFARA & TOTAL \\
\hline N (\%) households & $\begin{array}{r}4,022 \\
(66.7 \%)\end{array}$ & $\begin{array}{r}2,012 \\
(33.3 \%)\end{array}$ & $\begin{array}{r}6,034 \\
(100.0 \%)\end{array}$ \\
\hline $\begin{array}{l}\mathrm{N}(\%) \text { currently } \\
\text { pregnant women aged } \\
15-49 \text { years }\end{array}$ & $\begin{array}{r}2,023 \\
(66.9 \%)\end{array}$ & $\begin{array}{r}1,003 \\
(33.2 \%)\end{array}$ & $\begin{array}{r}3,026 \\
(100.0 \%)\end{array}$ \\
\hline $\begin{array}{l}\mathrm{N}(\%) \text { women } 15 \text { to } 49 \\
\text { years old with a child } \\
\text { under } 2 \text { years, or ob- } \\
\text { servations of last-born } \\
\text { children in the past } 2 \\
\text { years }\end{array}$ & $\begin{array}{r}1,971 \\
(64.8 \%)\end{array}$ & $\begin{array}{r}1,069 \\
(35.2 \%)\end{array}$ & $\begin{array}{r}3,040 \\
(100.0 \%)\end{array}$ \\
\hline
\end{tabular}

TABLE A-5. RESPONSE RATES FOR PREGNANT WOMEN BY STATE

\begin{tabular}{|c|c|c|c|c|c|c|c|}
\hline \multirow[t]{2}{*}{ STATE } & \multirow{2}{*}{$\begin{array}{l}\text { CLUSTERS } \\
\text { SELECTED }\end{array}$} & \multirow{2}{*}{$\begin{array}{c}\text { TOTAL } \\
\text { HOUSEHOLDS } \\
\text { SCREENED }\end{array}$} & \multicolumn{5}{|c|}{ PREGNANT WOMEN } \\
\hline & & & $\begin{array}{c}\text { ELIGIBLE } \\
\text { HOUSEHOLDS }\end{array}$ & $\begin{array}{l}\text { ELIGIBLE } \\
\text { WOMEN }\end{array}$ & SELECTED & INTERVIEWED & $\begin{array}{l}\text { RESPONSE } \\
\text { RATE }\end{array}$ \\
\hline Kebbi & 35 & 6,347 & 1,279 & 1,336 & 980 & 980 & $100 \%$ \\
\hline Sokoto & 37 & 6,987 & 1,443 & 1,527 & 1,036 & 1,035 & $99 \%$ \\
\hline Zamfara & 36 & 6,931 & 1,722 & 1,833 & 1,008 & 1,007 & $99 \%$ \\
\hline All & 108 & 20,265 & 4,444 & 4,696 & 3,024 & 3,022 & $99 \%$ \\
\hline
\end{tabular}

TABLE A-6. RESPONSE RATES FOR WOMEN WITH A CHILD UNDER 2 YEARS BY STATE

\begin{tabular}{|c|c|c|c|c|c|c|c|}
\hline \multirow[t]{2}{*}{ STATE } & \multirow{2}{*}{$\begin{array}{l}\text { CLUSTERS } \\
\text { SELECTED }\end{array}$} & \multirow{2}{*}{$\begin{array}{l}\text { TOTAL } \\
\text { HOUSEHOLDS } \\
\text { SCREENED }\end{array}$} & \multicolumn{5}{|c|}{ WOMEN WITH A CHILD UNDER 2 YEARS } \\
\hline & & & $\begin{array}{c}\text { ELIGIBLE } \\
\text { HOUSEHOLDS }\end{array}$ & $\begin{array}{l}\text { ELIGIBLE } \\
\text { WOMEN }\end{array}$ & SELECTED & INTERVIEWED & $\begin{array}{l}\text { RESPONSE } \\
\text { RATE }\end{array}$ \\
\hline Kebbi & 35 & 6,347 & 2,510 & 3,288 & 980 & 980 & $100 \%$ \\
\hline Sokoto & 37 & 6,987 & 3,249 & 4,018 & 1,036 & 1,032 & $99 \%$ \\
\hline Zamfara & 36 & 6,931 & 2,989 & 4,112 & 1,008 & 1,008 & $100 \%$ \\
\hline All & 108 & 20,265 & 8,748 & 11,418 & 3,024 & 3,020 & $99 \%$ \\
\hline
\end{tabular}




\section{Data analysis and results dissemination}

The primary statistical comparison in the quasi-experimental study is between respondents who have been exposed to integrated SBC programming and those who have been exposed to malaria-only SBC programming. Final results, as presented in this baseline report, evaluate whether the characteristics of the samples across the study arms are statistically equivalent. All point estimates were tabulated using weights to account for unequal probabilities of selection due to over- and under-sampling. Standard error estimation accounted for data clustering in the complex survey design. All analyses were conducted in Stata 16 (STATA Corp, College Station, TX). implementing partners, governmental decision-makers, and other stakeholders. Finally, results will feed into regional presentations where applicable, and we anticipate that the rigor of this study design will evoke substantial interest in these findings from the global SBC community.

\section{Comparison between NDHS and BSS results}

Table A-8 summarizes some issues to keep in mind when comparing results from the NDHS 2018 and the BSS baseline survey. For most BSS and NDHS indicators, confidence intervals around estimates overlap, indicating no real difference in results.

Information generated from the study will be shared with research communities through well-established relationships with Obafemi Awolowo University, Ile-Ife (where our research partner CRERD is housed), and other academic institutions in Nigeria and internationally. We will also present the study findings to key stakeholders including Breakthrough ACTION/Nigeria and other USAID

TABLE A-8. NOTES ON COMPARING NDHS AND BSS BASELINE RESULTS

\begin{tabular}{|c|c|c|c|}
\hline & BSS BASELINE & NDHS 2018 & COMMENTS \\
\hline Survey timing & September 2019 & August-December 2018 & $\begin{array}{l}\text { Differences in some results may be due to program- } \\
\text { matic activities at the time of survey collection that } \\
\text { lead to rapid increases in coverage estimates that } \\
\text { then could wane in subsequent months or years, e.g., } \\
\text { LLIN distribution campaigns }\end{array}$ \\
\hline Representation & $\begin{array}{l}\text { Representative of wards tar- } \\
\text { geted by Breakthrough } \\
\text { ACTION for integrated SBC } \\
\text { (Kebbi/Sokoto) and } \\
\text { malaria-only SBC (Zamfara) } \\
\text { programming }\end{array}$ & $\begin{array}{l}\text { Representative at state level } \\
\text { (Kebbi, Sokoto, Zamfara) }\end{array}$ & $\begin{array}{l}\text { Differences in some results may be due to differences } \\
\text { in the representativeness of BSS and NDHS estimates. } \\
\text { Breakthrough ACTION/Nigeria works only in a subset } \\
\text { of state LGAs. }\end{array}$ \\
\hline $\begin{array}{l}\text { Sample } \\
\text { population }\end{array}$ & $\begin{array}{l}\text { Women } 15 \text { to } 49 \text { years who are } \\
\text { currently pregnant or have a } \\
\text { child under } 2 \text { years }\end{array}$ & Women 15 to 49 years & $\begin{array}{l}\text { Differences in some results may be due to different } \\
\text { target populations from which the samples were } \\
\text { drawn. Women } 15 \text { to } 49 \text { years old who are currently } \\
\text { pregnant or have a child under } 2 \text { years may be differ- } \\
\text { ent in systematic ways than the overall women } 15 \text { - to } \\
\text { 49-year-old population that is sampled for NDHS. }\end{array}$ \\
\hline $\begin{array}{l}\text { Confidence } \\
\text { intervals }(\mathrm{CI})\end{array}$ & \multicolumn{3}{|c|}{$\begin{array}{l}\text { All point estimates have associated confidence intervals within which the "true" population value lies (if samples are } \\
\text { repeatedly drawn from a target population, the true population value will lie within the } 95 \% \text { Cl range in } 95 \text { of } 100 \text { times } \\
\text { of repeated sampling). For most BSS and NDHS indicators, Cls overlap, indicating there is no real difference between } \\
\text { our estimates. }\end{array}$} \\
\hline $\begin{array}{l}\text { Indicator } \\
\text { definitions }\end{array}$ & \multicolumn{3}{|c|}{$\begin{array}{l}\text { Most indicators in BSS and NDHS are measured using the same methods and definitions. For some indicators, there } \\
\text { are differences in indicator definitions based on how data were collected that could lead to differences in results (e.g., } \\
\text { exclusive breastfeeding) }\end{array}$} \\
\hline
\end{tabular}




\section{Demographics}

\section{Key findings}

Study sample: The BSS included 6,034 households, with 4,022 (67\%) in Kebbi/Sokoto and 2,012 (33\%) in Zamfara. There were 3,026 currently pregnant women 15 to 49 years old, including 2,023 (67\%) in Kebbi/Sokoto and 1,003 (33\%) in Zamfara. There were 3,040 women 15 to 49 years old with a child under 2 years, or observations of last-born children in the past two years, including 1,971 (65\%) in Kebbi/Sokoto and 1,069 (35\%) in Zamfara.

Household characteristics: Among sampled households, a statistically significant higher percentage had an improved drinking water source in Zamfara (75\%) than in Kebbi/Sokoto (34\%). Zamfara also had a statistically significant higher percentage of households with improved sanitation facilities (48\%) compared with Kebbi/Sokoto (27\%). There were important differences in household wealth and assets across Kebbi/Sokoto and Zamfara. In Kebbi/Sokoto, 29 percent of households were in the poorest wealth quintile, compared with 10 percent in Zamfara. Similarly, 17 percent of households were in the wealthiest quintile in Kebbi/Sokoto, compared with 28 percent in Zamfara.

Radio ownership was statistically significant higher in Zamfara (41\%) than in Kebbi/Sokoto (28\%), which has important implications for Breakthrough ACTION/Nigeria radio programming going forward as a means to disseminate health messages to populations. As discussed in Chapter 9 (Media Exposure), radio listening was the most common form of media exposure among respondents compared to television, newspapers, internet, or other media forms. Mobile phone ownership was similarly higher in Zamfara (57\%) and Kebbi/Sokoto (48\%), which again may have implications for Breakthrough ACTION/Nigeria programming of health messages. About three-quarters of households across study areas were reported to lie within 30 minutes' travel time of a school, health center, road, or market. Less than half of households (46\%) were reportedly within 30 minutes' travel time of a general hospital, which has important implications for health services utilization promoted by Breakthrough ACTION/Nigeria SBC activities.

Respondent characteristics: There were no real differences in respondents' ages across Kebbi/Sokoto and Zamfara, with more than half of respondents (55\%) aged 20 to 29 years across study areas. Nearly all respondents (98\%) across study areas were married, and most respondents (73\%) never attended school or received Islamic education (11\%), with a slightly higher proportion with no education in Kebbi/Sokoto (78\%) than in Zamfara (69\%). As a result, there was also high illiteracy among respondents across all areas (79\%). Nearly all respondents were Muslim (>99\%), and Hausa was the main ethnic group $(88 \%)$ across study areas. In terms of spouse characteristics, more than half (55\%) of spouses never attended school, with higher proportions in Kebbi/Sokoto (63\%) than in Zamfara (51\%). Spouses tended to be older, with nearly half (45\%) reported to be 40 years or older across study areas. Across study areas, 66 percent of spouses had no other wife, while 24 percent had two other wives.

\section{TABLE 1.1.1. STUDY SAMPLE}

Percentage of households, pregnant women and women 15 to 49 years with a child under 2 years in the BSS baseline study sample, September-October 2019

\begin{tabular}{|c|c|c|c|c|}
\hline \multirow[t]{2}{*}{ SAMPLE } & \multicolumn{4}{|c|}{ BREAKTHROUGH ACTION/NIGERIA PROGRAM AREA } \\
\hline & KEBBI & SOKOTO & ZAMFARA & TOTAL \\
\hline N (\%) households & $1,960(32.5 \%)$ & $2,062(34.2 \%)$ & $2,012(33.3 \%)$ & $6,034(100.0 \%)$ \\
\hline $\mathrm{N}(\%)$ currently pregnant women 15 to 49 years & $984(32.5 \%)$ & $1,040(34.3 \%)$ & $1,004(33.2 \%)$ & $3,028(100.0 \%)$ \\
\hline $\begin{array}{l}\mathrm{N}(\%) \text { women } 15 \text { to } 49 \text { years with a child under } 2 \text { years (or observa- } \\
\text { tions of last-born children in the past } 2 \text { years) }\end{array}$ & $893(29.4 \%)$ & $1,078(35.4 \%)$ & $1,069(35.2 \%)$ & $3,040(100.0 \%)$ \\
\hline
\end{tabular}


TABLE 1.1.2. HOUSEHOLD CHARACTERISTICS

Percentage of households by characteristics, September-October 2019

\begin{tabular}{|c|c|c|c|c|c|c|}
\hline & KEBBI & SOKOTO & $\begin{array}{l}\text { MALARIA-ONLY } \\
\text { (ZAMFARA) }\end{array}$ & $\begin{array}{c}\text { INTEGRATED } \\
\text { (KEBBI/SOKOTO) }\end{array}$ & & $\begin{array}{c}\text { CHI }^{2} \\
\text { VALUE }\end{array}$ \\
\hline & $\%$ & $\%$ & $\%$ & $\%$ & $\mathbf{N}$ & \\
\hline \multicolumn{7}{|l|}{$\begin{array}{l}\text { Main source of } \\
\text { drinking water }\end{array}$} \\
\hline Improved source & 44.9 & 23.6 & 74.6 & 33.5 & 3,035 & 1122.209 \\
\hline Unimproved source & 55.1 & 76.4 & 25.4 & 66.5 & 2,997 & 17.663 \\
\hline Total & 100.0 & 100.0 & 100.0 & 100.0 & 6,032 & *** \\
\hline $\mathrm{N}$ & 1,960 & 2,061 & 2,011 & 4,021 & & \\
\hline \multicolumn{7}{|l|}{$\begin{array}{l}\text { Main sanitation } \\
\text { facility }\end{array}$} \\
\hline Improved & 26.1 & 27.0 & 48.0 & 26.6 & 2,340 & 280.414 \\
\hline Unimproved & 73.9 & 73.0 & 52.0 & 73.4 & 3,689 & 5.444 \\
\hline Total & 100.0 & 100.0 & 100.0 & 100.0 & 6,029 & ** \\
\hline $\mathrm{N}$ & 1,959 & 2,061 & 2,009 & 4,020 & & \\
\hline \multicolumn{7}{|l|}{$\begin{array}{l}\text { Main flooring } \\
\text { material }\end{array}$} \\
\hline Earth/sand & 67.7 & 67.0 & 55.0 & 67.3 & 3,490 & 170.903 \\
\hline Ceramic tiles & 2.6 & 3.3 & 6.0 & 3.0 & 297 & 2.190 \\
\hline Cement & 29.6 & 28.3 & 38.4 & 28.9 & 2,188 & \\
\hline $\begin{array}{l}\text { Parquet/Polished } \\
\text { wood }\end{array}$ & 0.1 & 0.1 & 0.5 & 0.1 & 12 & \\
\hline Other & 0.0 & 1.5 & 0.1 & 0.8 & 47 & \\
\hline Total & 100.0 & 100.0 & 100.0 & 100.0 & 6,034 & \\
\hline $\mathrm{N}$ & 1,960 & 2,062 & 2,012 & 4,022 & & \\
\hline \multicolumn{7}{|l|}{ Main roofing material } \\
\hline Thatched/Palm leaf & 16.4 & 23.0 & 11.1 & 20.0 & 978 & 134.733 \\
\hline Metal/Iron sheets & 83.5 & 74.8 & 87.5 & 78.8 & 4,986 & 1.733 \\
\hline $\begin{array}{l}\text { Calamine/Cement } \\
\text { fiber }\end{array}$ & 0.1 & 1.3 & 0.8 & 0.8 & 42 & \\
\hline Other & 0.0 & 0.9 & 0.6 & 0.5 & 28 & \\
\hline Total & 100.0 & 100.0 & 100.0 & 100.0 & 6,034 & \\
\hline N & 1,960 & 2,062 & 2,012 & 4,022 & & \\
\hline
\end{tabular}

Estimates based on low Ns (approx. $<30$ obs) have large relative standard errors and should be interpreted with caution. ${ }^{* * *}$ indicates $p<0.001,{ }^{* *}$ indicates $p<0.05$ and ${ }^{*}$ indicates $p<.0 .01$ for the chi ${ }^{2}$ statistic comparing integrated versus malaria-only study areas.

Improved drinking water sources include piped supplies and non-piped supplies (boreholes/tube wells, protected wells and springs, rainwater, packaged water, and delivered water); unimproved drinking water sources include unprotected wells and springs.

Improved sanitation facilities include network sanitation and on-site sanitation (flush or pour flush toilets or latrines connected to septic tanks or pits, ventilated improved pit latrines, pit latrines with slabs, composting toilets including twin pit latrines, and container-based systems; unimproved sanitation facilities include on-site sanitation (pit latrines without slabs, hanging latrines, bucket latrines). 
TABLE 1.1.3. HOUSEHOLD WEALTH AND ASSETS

Percentage of households by wealth index quintile and asset ownership, September-October 2019

\begin{tabular}{|c|c|c|c|c|c|c|}
\hline & $\begin{array}{l}\text { KEBBI } \\
\%\end{array}$ & $\begin{array}{l}\text { SOKOTO } \\
\%\end{array}$ & $\begin{array}{c}\text { MALARIA-ONLY } \\
\text { (ZAMFARA) } \\
\%\end{array}$ & $\begin{array}{c}\text { INTEGRATED } \\
\text { (KEBBI/SOKOTO) } \\
\%\end{array}$ & $\mathbf{N}$ & $\begin{array}{c}\mathrm{CHI}^{2} \\
\text { VALUE }\end{array}$ \\
\hline \multicolumn{7}{|c|}{ Household wealth index } \\
\hline Lowest & 29.4 & 34.2 & 11.8 & 32.0 & 1,367 & 470.004 \\
\hline Second & 21.8 & 20.4 & 19.3 & 21.1 & 1,167 & 5.319 \\
\hline Middle & 18.7 & 20.0 & 20.4 & 19.4 & 1,178 & *** \\
\hline Fourth & 13.9 & 12.2 & 24.8 & 13.0 & 1,050 & \\
\hline Highest & 16.2 & 13.2 & 23.7 & 14.6 & 1,272 & \\
\hline Total & 100.0 & 100.0 & 100.0 & 100.0 & 6,034 & \\
\hline $\mathrm{N}$ & 1,960 & 2,062 & 2,012 & 4,022 & & \\
\hline \multicolumn{7}{|c|}{ Household owns } \\
\hline \multicolumn{7}{|c|}{$\begin{array}{l}\text { Livestock, other farm animals, } \\
\text { or poultry }\end{array}$} \\
\hline No & 29.9 & 28.2 & 34.9 & 29.0 & 1,962 & 23.425 \\
\hline Yes & 70.1 & 71.8 & 65.1 & 71.0 & 4,072 & 0.857 \\
\hline Total & 100.0 & 100.0 & 100.0 & 100.0 & 6,034 & \\
\hline $\mathrm{N}$ & 1,960 & 2,062 & 2,012 & 4,022 & & \\
\hline \multicolumn{7}{|c|}{ Any agricultural land } \\
\hline No & 15.2 & 11.5 & 25.6 & 13.2 & 1,236 & 144.819 \\
\hline Yes & 84.5 & 87.8 & 74.1 & 86.3 & 4,773 & 3.561 \\
\hline Don't know & 0.3 & 0.7 & 0.3 & 0.5 & 25 & * \\
\hline Total & 100.0 & 100.0 & 100.0 & 100.0 & 6,034 & \\
\hline $\mathrm{N}$ & 1,960 & 2,062 & 2,012 & 4,022 & & \\
\hline \multicolumn{7}{|l|}{ Radio } \\
\hline No & 76.5 & 71.0 & 59.3 & 73.5 & 4,090 & 137.896 \\
\hline Yes & 23.6 & 29.0 & 40.7 & 26.5 & 1,944 & 5.937 \\
\hline Total & 100.0 & 100.0 & 100.0 & 100.0 & 6,034 & \\
\hline N & 1,960 & 2,062 & 2,012 & 4,022 & & \\
\hline \multicolumn{7}{|l|}{ Television } \\
\hline No & 87.2 & 89.0 & 85.3 & 88.1 & 5,071 & 11.928 \\
\hline Yes & 12.8 & 11.0 & 14.7 & 11.9 & 963 & 0.390 \\
\hline Total & 100.0 & 100.0 & 100.0 & 100.0 & 6,034 & \\
\hline $\mathrm{N}$ & 1,960 & 2,062 & 2,012 & 4,022 & & \\
\hline \multicolumn{7}{|l|}{ Mobile phone } \\
\hline No & 51.3 & 56.0 & 45.0 & 53.8 & 2,965 & 50.904 \\
\hline Yes & 48.8 & 44.0 & 55.0 & 46.2 & 3,069 & 1.538 \\
\hline Total & 100.0 & 100.0 & 100.0 & 100.0 & 6,034 & \\
\hline $\mathrm{N}$ & 1,960 & 2,062 & 2,012 & 4,022 & & \\
\hline \multicolumn{7}{|l|}{ Computer } \\
\hline No & 99.0 & 97.6 & 97.9 & 98.3 & 5,902 & 7.580 \\
\hline Yes & 1.0 & 2.4 & 2.1 & 1.7 & 132 & 1.325 \\
\hline Total & 100.0 & 100.0 & 100.0 & 100.0 & 6,034 & \\
\hline $\mathrm{N}$ & 1,960 & 2,062 & 2,012 & 4,022 & & \\
\hline \multicolumn{7}{|c|}{ Household owns bicycle } \\
\hline No & 88.6 & 86.9 & 81.8 & 87.7 & 5,170 & 38.948 \\
\hline Yes & 11.4 & 13.1 & 18.2 & 12.4 & 864 & 1.844 \\
\hline Total & 100.0 & 100.0 & 100.0 & 100.0 & 6,034 & \\
\hline N & 1,960 & 2,062 & 2,012 & 4,022 & & \\
\hline \multicolumn{7}{|c|}{ Motorcycle or motor scooter } \\
\hline No & 67.7 & 72.0 & 65.8 & 70.0 & 4,129 & 17.002 \\
\hline Yes & 32.3 & 28.0 & 34.2 & 30.0 & 1,905 & 0.965 \\
\hline Total & 100.0 & 100.0 & 100.0 & 100.0 & 6,034 & \\
\hline N & 1,960 & 2,062 & 2,012 & 4,022 & & \\
\hline \multicolumn{7}{|l|}{ Car or truck } \\
\hline No & 95.2 & 94.3 & 92.9 & 94.7 & 5,625 & 8.680 \\
\hline Yes & 4.8 & 5.7 & 7.1 & 5.3 & 409 & 0.726 \\
\hline Total & 100.0 & 100.0 & 100.0 & 100.0 & 6,034 & \\
\hline $\mathrm{N}$ & 1,960 & 2,062 & 2,012 & 4,022 & & \\
\hline
\end{tabular}

Estimates based on low Ns (approx. $<30$ obs) have large relative standard errors and should be interpreted with caution. ${ }^{* * *}$ indicates $p<0.001,{ }^{* *}$ indicates $p<0.05$ and ${ }^{*}$ indicates $p<.0 .01$ for the chi ${ }^{2}$ statistic comparing integrated versus malaria-only study areas. 
TABLE 1.1.4. HOUSEHOLD DISTANCES TO EDUCATION, HEALTH, AND ECONOMIC CENTERS

Percentage of households by reported distances to education, health, and economic centers, September-October 2019

\begin{tabular}{|c|c|c|c|c|c|c|}
\hline & $\begin{array}{c}\text { KEBBI } \\
\% \\
(\mathrm{~N}=1,960)\end{array}$ & $\begin{array}{c}\text { SOKOTO } \\
\% \\
(\mathrm{~N}=2,062)\end{array}$ & $\begin{array}{c}\text { MALARIA-ONLY } \\
\text { (ZAMFARA) } \\
\% \\
(\mathrm{~N}=\mathbf{2 , 0 1 2})\end{array}$ & $\begin{array}{c}\text { INTEGRATED } \\
\text { (KEBBI/SOKOTO) } \\
\% \\
(\mathrm{~N}=4,022)\end{array}$ & $\begin{array}{c}N \\
(N=6,034)\end{array}$ & $\begin{array}{c}\mathrm{CHI}^{2} \\
\text { VALUE }\end{array}$ \\
\hline \multicolumn{7}{|l|}{ School } \\
\hline 0-30 minutes & 75.2 & 58.3 & 75.6 & 66.1 & 4,245 & 392.410 \\
\hline $31-60$ minutes & 15.0 & 15.2 & 8.1 & 15.1 & 770 & 2.110 \\
\hline$>60$ minutes & 2.2 & 6.4 & 0.0 & 4.5 & 165 & \\
\hline Don't know & 7.6 & 20.0 & 16.3 & 14.3 & 854 & \\
\hline Total & 100.0 & 100.0 & 100.0 & 100.0 & 6,034 & \\
\hline$N$ & 1,960 & 2,062 & 2,012 & 4,022 & & \\
\hline \multicolumn{7}{|l|}{ Health center } \\
\hline 0-30 minutes & 73.4 & 74.3 & 86.2 & 73.9 & 4,672 & 250.750 \\
\hline $31-60$ minutes & 16.0 & 12.7 & 7.1 & 14.2 & 775 & 1.686 \\
\hline$>60$ minutes & 4.9 & 1.1 & 0.9 & 2.9 & 130 & \\
\hline Don't know & 5.7 & 11.9 & 5.8 & 9.0 & 457 & \\
\hline Total & 100.0 & 100.0 & 100.0 & 100.0 & 6,034 & \\
\hline $\mathrm{N}$ & 1,960 & 2,062 & 2,012 & 4,022 & & \\
\hline \multicolumn{7}{|l|}{ General hospital } \\
\hline 0-30 minutes & 40.1 & 42.3 & 46.4 & 41.3 & 2,776 & 218.741 \\
\hline $31-60$ minutes & 35.2 & 29.1 & 37.6 & 31.9 & 2,030 & 1.095 \\
\hline$>60$ minutes & 16.5 & 11.7 & 5.1 & 13.9 & 553 & \\
\hline Don't know & 8.3 & 16.9 & 11.0 & 12.9 & 675 & \\
\hline Total & 100.0 & 100.0 & 100.0 & 100.0 & 6,034 & \\
\hline $\mathrm{N}$ & 1,960 & 2,062 & 2,012 & 4,022 & & \\
\hline \multicolumn{7}{|l|}{ Bank } \\
\hline 0-30 minutes & 32.2 & 15.6 & 31.6 & 23.3 & 1,788 & 656.162 \\
\hline $31-60$ minutes & 24.7 & 17.6 & 35.8 & 20.9 & 1,529 & 3.263 \\
\hline$>60$ minutes & 15.9 & 14.0 & 2.9 & 14.9 & 598 & ** \\
\hline Don't know & 27.2 & 52.8 & 29.7 & 41.0 & 2,119 & \\
\hline Total & 100.0 & 100.0 & 100.0 & 100.0 & 6,034 & \\
\hline N & 1,960 & 2,062 & 2,012 & 4,022 & & \\
\hline \multicolumn{7}{|l|}{ Tarred road } \\
\hline 0-30 minutes & 73.3 & 65.7 & 71.0 & 69.2 & 4,526 & 49.014 \\
\hline $31-60$ minutes & 19.2 & 19.9 & 16.9 & 19.6 & 930 & 0.184 \\
\hline$>60$ minutes & 1.5 & 6.3 & 5.3 & 4.1 & 182 & \\
\hline Don't know & 6.0 & 8.1 & 6.8 & 7.1 & 396 & \\
\hline Total & 100.0 & 100.0 & 100.0 & 100.0 & 6,034 & \\
\hline $\mathrm{N}$ & 1,960 & 2,062 & 2,012 & 4,022 & & \\
\hline \multicolumn{7}{|l|}{ Local market } \\
\hline 0-30 minutes & 83.4 & 79.2 & 79.4 & 81.1 & 4,891 & 119.425 \\
\hline $31-60$ minutes & 5.9 & 9.1 & 10.5 & 7.6 & 478 & 0.596 \\
\hline$>60$ minutes & 4.7 & 2.6 & 0.6 & 3.5 & 153 & \\
\hline Don't know & 6.0 & 9.2 & 9.5 & 7.7 & 512 & \\
\hline Total & 100.0 & 100.0 & 100.0 & 100.0 & 6,034 & \\
\hline $\mathrm{N}$ & 1,960 & 2,062 & 2,012 & 4,022 & & \\
\hline \multicolumn{7}{|l|}{ State capital } \\
\hline 0-30 minutes & 10.7 & 12.1 & 26.5 & 11.4 & 1,146 & 448.449 \\
\hline $31-60$ minutes & 10.5 & 24.7 & 25.0 & 18.1 & 1,320 & 2.001 \\
\hline$>60$ minutes & 63.5 & 42.3 & 37.0 & 52.1 & 2,628 & \\
\hline Don't know & 15.4 & 21.0 & 11.6 & 18.4 & 940 & \\
\hline Total & 100.0 & 100.0 & 100.0 & 100.0 & 6,034 & \\
\hline $\mathrm{N}$ & 1,960 & 2,062 & 2,012 & 4,022 & & \\
\hline
\end{tabular}

Estimates based on low Ns (approx. $<30$ obs) have large relative standard errors and should be interpreted with caution. ${ }^{* \star *}$ indicates $p<0.001,{ }^{* *}$ indicates $p<0.05$ and * indicates $p<.0 .01$ for the chi statistic comparing integrated versus malaria-only study areas. Note: While respondents must decide for themselves how to interpret health center and general hospital in this question, we believe health center would mainly be interpreted by respondents as PHC while general hospital would be interpreted as nearest referral or general hospital. 
TABLE 1.1.5. RESPONDENT CHARACTERISTICS

Percentage of women aged 15 to 49 years currently pregnant or with a child under 2 years by sociodemographic characteristics, September-October 2019

\begin{tabular}{|c|c|c|c|c|c|c|}
\hline & KEBBI & SOKото & $\begin{array}{l}\text { MALARIA-ONLY } \\
\text { (ZAMFARA) }\end{array}$ & $\begin{array}{c}\text { INTEGRATED } \\
\text { (KEBBI/SOKOTO) }\end{array}$ & $\mathbf{N}$ & $\begin{array}{c}\mathrm{CHI}^{2} \\
\text { VALUE }\end{array}$ \\
\hline & $\%$ & $\%$ & $\%$ & $\%$ & & \\
\hline \multicolumn{7}{|l|}{ Education (highest level attended) } \\
\hline None & 75.4 & 80.2 & 69.4 & 78.0 & 4,411 & 81.408 \\
\hline Primary & 4.8 & 4.8 & 5.2 & 4.8 & 319 & 1.133 \\
\hline Secondary or higher & 9.4 & 4.6 & 13.8 & 6.8 & 692 & \\
\hline Islamic & 10.5 & 10.3 & 11.6 & 10.4 & 611 & \\
\hline Total & 100.0 & 100.0 & 100.0 & 100.0 & 6,033 & \\
\hline $\mathrm{N}$ & 1,960 & 2,062 & 2,011 & 4,022 & & \\
\hline \multicolumn{7}{|l|}{ Age (in years) } \\
\hline $15-24$ years & 41.0 & 46.8 & 41.8 & 44.1 & 2,715 & 3.688 \\
\hline $25-34$ years & 45.0 & 41.7 & 44.4 & 43.2 & 2,575 & 0.436 \\
\hline $35-49$ years & 14.0 & 11.6 & 13.8 & 12.7 & 743 & \\
\hline Total & 100.0 & 100.0 & 100.0 & 100.0 & 6,033 & \\
\hline N & 1,960 & 2,062 & 2,011 & 4,022 & & \\
\hline \multicolumn{7}{|l|}{ Ethnicity } \\
\hline Hausa & 72.9 & 91.1 & 92.2 & 82.7 & 5,067 & 188.541 \\
\hline Fulani & 10.3 & 5.7 & 5.6 & 7.8 & 466 & 4.061 \\
\hline Badakare/Dakarkari & 4.2 & 0.0 & 0.0 & 1.9 & 101 & ** \\
\hline Other & 12.6 & 3.2 & 2.2 & 7.5 & 399 & \\
\hline Total & 100.0 & 100.0 & 100.0 & 100.0 & 6,033 & \\
\hline $\mathrm{N}$ & 1,960 & 2,062 & 2,011 & 4,022 & & \\
\hline \multicolumn{7}{|l|}{ Literacy } \\
\hline Cannot read at all & 84.9 & 75.2 & 78.8 & 79.7 & 4,668 & 138.322 \\
\hline Able to read only parts of sentence & 6.4 & 4.7 & 9.9 & 5.5 & 489 & 3.169 \\
\hline Able to read whole sentence & 6.1 & 4.6 & 7.7 & 5.3 & 473 & * \\
\hline Blind/visually impaired & 0.1 & 0.0 & 0.2 & 0.1 & 8 & \\
\hline Unable to read the language of the card & 2.4 & 15.5 & 3.4 & 9.4 & 395 & \\
\hline Total & 100.0 & 100.0 & 100.0 & 100.0 & 6,033 & \\
\hline N & 1,960 & 2,062 & 2,011 & 4,022 & & \\
\hline \multicolumn{7}{|l|}{ Religion } \\
\hline Islam & 99.4 & 99.8 & 99.8 & 99.6 & 6,013 & 3.069 \\
\hline Christianity & 0.6 & 0.2 & 0.2 & 0.4 & 19 & 1.184 \\
\hline Traditional religion & 0.0 & 0.0 & 0.0 & 0.0 & 1 & \\
\hline Total & 100.0 & 100.0 & 100.0 & 100.0 & 6,033 & \\
\hline $\mathrm{N}$ & 1,960 & 2,062 & 2,011 & 4,022 & & \\
\hline \multicolumn{7}{|l|}{ Occupation } \\
\hline No work outside home & 42.4 & 52.2 & 42.8 & 47.6 & 2,726 & 26.298 \\
\hline Work outside home & 54.4 & 44.1 & 51.3 & 48.9 & 2,966 & 0.401 \\
\hline Student & 0.4 & 0.9 & 0.9 & 0.6 & 51 & \\
\hline Other & 2.9 & 2.8 & 5.0 & 2.8 & 218 & \\
\hline Total & 100.0 & 100.0 & 100.0 & 100.0 & 5,961 & \\
\hline N & 1,942 & 2,042 & 1,977 & 3,984 & & \\
\hline \multicolumn{7}{|l|}{ Marital status } \\
\hline Married or living together to one person & 96.6 & 95.2 & 91.8 & 95.8 & 5,765 & 55.561 \\
\hline Married to more than one person & 2.7 & 4.0 & 5.8 & 3.4 & 196 & 1.875 \\
\hline Divorced/separated & 0.6 & 0.5 & 0.5 & 0.5 & 32 & \\
\hline Widowed & 0.2 & 0.3 & 1.9 & 0.2 & 37 & \\
\hline Never married and never lived together & 0.1 & 0.0 & 0.0 & 0.0 & 3 & \\
\hline Total & 100.0 & 100.0 & 100.0 & 100.0 & 6,033 & \\
\hline $\mathrm{N}$ & 1,960 & 2,062 & 2,011 & & & \\
\hline
\end{tabular}

Estimates based on low Ns (approx. $<30$ obs) have large relative standard errors and should be interpreted with caution. ${ }^{* * *}$ indicates $p<0.001,{ }^{* *}$ indicates $p<0.05$ and * indicates $p<.0 .01$ for the chi statistic comparing integrated versus malaria-only study areas. 
TABLE 1.1.6. SPOUSE/PARTNER CHARACTERISTICS

Percentage of women aged 15 to 49 years currently pregnant or with a child under 2 years by partner/spouse sociodemographic characteristics, September-October 2019

\begin{tabular}{|c|c|c|c|c|c|c|}
\hline & KEBBI & SOKOTO & $\begin{array}{l}\text { MALARIA-ONLY } \\
\text { (ZAMFARA) }\end{array}$ & $\begin{array}{c}\text { INTEGRATED } \\
\text { (KEBBI/SOKOTO) }\end{array}$ & $\mathbf{N}$ & $\begin{array}{c}\mathrm{CHI}^{2} \\
\text { VALUE }\end{array}$ \\
\hline \multicolumn{7}{|l|}{ Age } \\
\hline$<20$ years & 0.5 & 0.6 & 0.9 & 0.5 & 42 & 4.023 \\
\hline 20-24 years & 3.0 & 2.3 & 2.1 & 2.7 & 163 & *** \\
\hline 25-29 years & 9.8 & 12.2 & 9.3 & 11.1 & 715 & \\
\hline 30-34 years & 17.7 & 20.3 & 18.8 & 19.1 & 1,182 & \\
\hline $35-39$ years & 17.6 & 17.0 & 14.6 & 17.3 & 990 & \\
\hline 40-44 years & 16.0 & 19.4 & 20.0 & 17.8 & 1,075 & \\
\hline $45+$ years & 20.5 & 20.7 & 30.1 & 20.6 & 1,348 & \\
\hline No spousal information & 0.8 & 0.8 & 2.5 & 0.8 & 73 & \\
\hline Don't know & 14.1 & 6.6 & 1.8 & 10.1 & 446 & 366.304 \\
\hline Total & 100.0 & 100.0 & 100.0 & 100.0 & 6,034 & \\
\hline $\mathrm{N}$ & 1,960 & 2,062 & 2,012 & 4,022 & & \\
\hline \multicolumn{7}{|c|}{ Education, highest school level attended } \\
\hline No education & 60.2 & 64.9 & 51.3 & 62.7 & 3,390 & 144.893 \\
\hline Primary & 5.9 & 6.0 & 5.8 & 5.9 & 360 & 1.154 \\
\hline Secondary or higher & 21.5 & 16.4 & 30.3 & 18.7 & 1,571 & \\
\hline Islamic education & 11.7 & 12.0 & 10.2 & 11.8 & 640 & \\
\hline No spousal information & 0.8 & 0.8 & 2.5 & 0.8 & 73 & \\
\hline Total & 100.0 & 100.0 & 100.0 & 100.0 & 6,034 & \\
\hline N & 1,960 & 2,062 & 2,012 & 4,022 & & \\
\hline \multicolumn{7}{|l|}{ Occupation } \\
\hline No work outside home & 4.6 & 4.0 & 4.4 & 4.2 & 265 & 61.192 \\
\hline Works outside home & 93.6 & 91.1 & 88.2 & 92.2 & 5,457 & 1.330 \\
\hline Student & 0.2 & 0.7 & 0.5 & 0.5 & 35 & \\
\hline No spousal information & 0.8 & 0.8 & 2.5 & 0.8 & 73 & \\
\hline Other & 0.9 & 3.4 & 4.5 & 2.3 & 204 & \\
\hline Total & 100.0 & 100.0 & 100.0 & 100.0 & 6,034 & \\
\hline $\mathrm{N}$ & 1,960 & 2,062 & 2,012 & 4,022 & & \\
\hline \multicolumn{7}{|l|}{ Total wives } \\
\hline No other wife & 68.8 & 75.1 & 61.6 & 72.2 & 4,324 & 171.543 \\
\hline Yes, 1 other wife & 1.5 & 1.6 & 2.1 & 1.6 & 92 & 3.306 \\
\hline Yes, 2 other wives & 22.8 & 19.6 & 26.5 & 21.1 & 1,247 & ** \\
\hline Yes, 3 other wives & 3.5 & 2.1 & 6.1 & 2.8 & 200 & \\
\hline Yes, 4 other wives & 0.3 & 0.1 & 0.9 & 0.2 & 26 & \\
\hline No spousal information & 0.8 & 0.8 & 2.5 & 0.8 & 73 & \\
\hline Don't know & 2.3 & 0.7 & 0.3 & 1.4 & 69 & \\
\hline Total & 100.0 & 100.0 & 100.0 & 100.0 & 6,031 & \\
\hline $\mathrm{N}$ & 1,960 & 2,062 & 2,009 & 4,022 & & \\
\hline
\end{tabular}

Estimates based on low Ns (approx. $<30$ obs) have large relative standard errors and should be interpreted with caution. ${ }^{* * *}$ indicates $p<0.001,{ }^{* *}$ indicates $p<0.05$ and ${ }^{*}$ indicates $p<.0 .01$ for the chi ${ }^{2}$ statistic comparing integrated versus malaria-only study areas. 


\section{Malaria}

\section{Key findings}

Malaria prevention: Overall, ownership of long-lasting insecticide nets (LLIN) was high. Approximately 75 percent and 69 percent of households in Zamfara and Kebbi/ Sokoto, respectively, own at least one LLIN. However, fewer households had the recommended ratio of at least one LLIN for every two household members, with slightly higher proportions in Kebbi/Sokoto (25\%) than in Zamfara (18\%). In households where there were sufficient nets (at least one LLIN for every two household members), 81 percent and 88 percent of pregnant respondents slept under an LLIN the previous night in Kebbi/Sokoto and Zamfara, with little difference across wealth quintiles or maternal education. Similarly, in households with sufficient nets, over 90 percent of children under 2 years slept under an LLIN the previous night in integrated and malaria-only areas, with limited differences across sociodemographic characteristics.

There was also extremely high overall awareness about the importance of LLINs in malaria prevention. Almost all respondents agreed that malaria was caused by mosquito bites (97\%), and that sleeping under mosquito nets was an effective malaria prevention method (93\%). In addition, nearly all respondents stated they were likely to get all their children under 5 sleeping under a mosquito net every night of the year (93\%). The results suggest that SBC programming to raise awareness about malaria prevention measures is not needed, but improving access, acceptability, and efficacy to use LLINs as directed may be a useful programmatic focus.

Malaria during pregnancy: Less than one in five respondents in study areas who attended any ANC visit received intermittent preventive treatment (three or more doses of SP/Fansidar) to protect against malaria during their last pregnancy, with significantly lower rates among respondents in the poorest households (10\% and $12 \%)$ and among less-educated respondents (15\% and 14\%) in Kebbi/Sokoto and in Zamfara compared with their wealthiest counterparts (33\% and 34\%) and those with at least primary education (36\% and 25\%). Doses of sulfadoxine/pyrimethamine (SP/Fansidar) were administered almost exclusively during ANC visits (74\% in Kebbi)
Sokoto and $84 \%$ in Zamfara). In addition, more frequent ANC visits is also associated with a higher likelihood of IPTp uptake. BSS baseline results show that women who attended ANC four or more times during the last pregnancy were about six times more likely to receive IPTp than those attending ANC only one time.

The most common reasons for not taking SP/Fansidar during pregnancy were opposition by respondent (27\%) or spouse (22\%). There were also many "other" responses that largely consisted of the following responses: did not attend ANC or facility to collect medicines, did not get sick during pregnancy or have pregnancy complications, not customary or not necessary to take medications. Among respondents attending any ANC visit, few (21\% in Kebbi/Sokoto and $36 \%$ in Zamfara) were given a mosquito net during ANC. While 91 percent reported that malaria in pregnancy can affect the fetus, few respondents were able to report specific risks to the baby, with only about half reporting fetal death (45\%) or miscarriage (44\%), and even fewer reporting premature birth (26\%) and low birth weight (17\%). This suggests areas for future SBC programming to raise awareness about the specific and serious risks of malaria in pregnancy to the unborn child.

Pediatric fever prevalence and care-seeking: Among last-born children in the past two years, one in five were reported to have had a fever in the previous two weeks. Among these children, 68 percent and 74 percent were taken for advice or treatment from any source in Kebbi/ Sokoto and Zamfara, and most respondents who sought advice from any source for the febrile child went the same or next day. However, 32 percent and 54 percent of febrile children who were taken to any care were brought to a pharmacy in Kebbi/Sokoto and Zamfara instead of formal medical care.

Reasons for source of care or not seeking care for pediatric fevers: The most important reasons for choosing the source of care were trust in provider (36\%), nearby location (33\%), and effective treatment (28\%) across study areas. This result underscores the importance to respondents in this community of convenient, trusted, and high-quality health care provision for effective treatment of their sick children. The most important reasons for not seeking care included fatalism ("It's up to God") (33\%), 
expense or cost (31\%), and symptoms were not perceived as severe or home care was provided (21\%).

Diagnosis and treatment of pediatric fevers: Among febrile children under 2 years, few were tested for malaria (19\% and 24\%) and few were given artemisinin-based combination therapies (ACT) treatment (22\% and 36\%) in Kebbi/Sokoto and Zamfara, respectively. Similar to other priority behavioral outcomes, there was lower testing and ACT treatment among febrile children living in the poorest households and with less-educated mothers as compared with their wealthier and better-educated counterparts. There was strong intent to take a child with a fever to care the same/next day (92\%) and to make sure the child takes the entire treatment course (92\%). However, while 74 percent agreed that a blood test is the only way to know if a person has malaria, most respondents (61\%) said they still worry the illness could be malaria even if the test result is negative, which implies a lack of confidence in negative malaria test results.

\subsection{Malaria prevention}

\section{TABLE 2.1.A. LLIN AVAILABILITY IN HOUSEHOLD (AT LEAST ONE) BY SOCIODEMOGRAPHIC CHARACTERISTICS}

Percentage of households with at least one LLIN, September-October 2019

\begin{tabular}{|c|c|c|c|c|c|c|c|c|}
\hline & \multicolumn{2}{|c|}{ KEBBI } & \multicolumn{2}{|c|}{ SOKOTO } & \multicolumn{2}{|c|}{$\begin{array}{l}\text { MALARIA-ONLY } \\
\text { (ZAMFARA) }\end{array}$} & \multicolumn{2}{|c|}{$\begin{array}{c}\text { INTEGRATED } \\
\text { (KEBBI/SOKOTO) }\end{array}$} \\
\hline & $\%$ & $\mathbf{N}$ & $\%$ & $\mathbf{N}$ & $\%$ & $\mathbf{N}$ & $\%$ & $\mathbf{N}$ \\
\hline Total & 68.0 & 1,960 & 68.9 & 2,062 & 74.8 & 2,012 & 68.5 & 4,022 \\
\hline \multicolumn{9}{|l|}{ Household wealth } \\
\hline Lowest & 67.1 & 541 & 67.3 & 618 & 62.9 & 208 & 67.2 & 1,159 \\
\hline Second & 60.8 & 419 & 70.1 & 407 & 74.7 & 341 & 65.7 & 826 \\
\hline Middle & 68.8 & 363 & 71.5 & 408 & 75.5 & 407 & 70.4 & 771 \\
\hline Fourth & 72.9 & 276 & 76.6 & 286 & 74.1 & 488 & 74.8 & 562 \\
\hline Highest & 74.3 & 361 & 60.2 & 343 & 81.1 & 568 & 68.5 & 704 \\
\hline \multicolumn{9}{|l|}{ Maternal age (in years) } \\
\hline 15-24 years & 63.9 & 866 & 70.4 & 1015 & 78.5 & 834 & 67.6 & 1,881 \\
\hline $25-34$ years & 70.3 & 850 & 67.9 & 837 & 74.2 & 888 & 69.0 & 1,687 \\
\hline $35-49$ years & 73.3 & 244 & 66.5 & 210 & 65.6 & 289 & 69.9 & 454 \\
\hline \multicolumn{9}{|c|}{$\begin{array}{l}\text { Maternal education (high- } \\
\text { est level attended) }\end{array}$} \\
\hline None & 65.9 & 1,487 & 69.3 & 1624 & 71.8 & 1,300 & 67.8 & 3,111 \\
\hline Primary & 76.0 & 99 & 74.6 & 101 & 82.6 & 119 & 75.2 & 200 \\
\hline Secondary or higher & 82.3 & 210 & 46.9 & 141 & 80.7 & 341 & 69.4 & 351 \\
\hline Islamic & 66.5 & 164 & 73.9 & 196 & 81.5 & 251 & 70.5 & 360 \\
\hline
\end{tabular}

Estimates based on low Ns (approx. $<30$ obs) have large relative standard errors and should be interpreted with caution. ${ }^{* * *}$ indicates $p<0.001,{ }^{* *}$ indicates $p<0.05$ and ${ }^{*}$ indicates $p<.0 .01$ for the chi $^{2}$ statistic comparing integrated versus malaria-only study areas. 


\section{TABLE 2.1.B. LLIN AVAILABILITY IN HOUSEHOLD (AT LEAST ONE FOR EVERY TWO HOUSEHOLD MEMBERS) BY SOCIODEMOGRAPHIC CHARACTERISTICS}

Percentage of households with at least one LLIN for every two household members, September-October 2019

\begin{tabular}{|c|c|c|c|c|c|c|c|c|}
\hline & \multicolumn{2}{|c|}{ KEBBI } & \multicolumn{2}{|c|}{ SOKOTO } & \multicolumn{2}{|c|}{$\begin{array}{l}\text { INTEGRATED } \\
\text { (KEBBI/SOKOTO) }\end{array}$} & \multicolumn{2}{|c|}{$\begin{array}{l}\text { MALARIA-ONLY } \\
\text { (ZAMFARA) }\end{array}$} \\
\hline & $\%$ & $\mathbf{N}$ & $\%$ & $\mathbf{N}$ & $\%$ & $\mathbf{N}$ & $\%$ & $\mathbf{N}$ \\
\hline Total & 26.4 & 1,960 & 23.6 & 2,062 & 24.9 & 4,022 & 17.7 & 2,012 \\
\hline \multicolumn{9}{|l|}{ Household wealth } \\
\hline Lowest & 25.7 & 541 & 29.1 & 618 & 27.7 & 1,159 & 8.8 & 208 \\
\hline Second & 19.9 & 419 & 21.8 & 407 & 20.9 & 826 & 18.8 & 341 \\
\hline Middle & 24.0 & 363 & 19.5 & 408 & 21.4 & 771 & 15.0 & 407 \\
\hline Fourth & 29.7 & 276 & 26.0 & 286 & 27.8 & 562 & 20.3 & 488 \\
\hline Highest & 36.3 & 361 & 17.3 & 343 & 27.1 & 704 & 21.2 & 568 \\
\hline \multicolumn{9}{|l|}{ Maternal age (in years) } \\
\hline 15-24 years & 31.5 & 866 & 29.4 & 1015 & 30.3 & 1,881 & 25.6 & 834 \\
\hline $25-34$ years & 23.6 & 850 & 19.5 & 837 & 21.5 & 1,687 & 14.0 & 888 \\
\hline $35-49$ years & 19.8 & 244 & 5.2 & 210 & 17.2 & 454 & 5.2 & 289 \\
\hline \multicolumn{9}{|c|}{$\begin{array}{l}\text { Maternal education (highest level } \\
\text { attended) }\end{array}$} \\
\hline None & 24.1 & 1,487 & 22.1 & 1,624 & 23.0 & 3,111 & 16.5 & 1,300 \\
\hline Primary & 33.1 & 99 & 23.4 & 101 & 27.8 & 200 & 19.2 & 119 \\
\hline Secondary or higher & 42.0 & 210 & 20.3 & 141 & 34.0 & 351 & 23.5 & 341 \\
\hline Islamic & 26.2 & 164 & 37.8 & 196 & 32.6 & 360 & 16.9 & 251 \\
\hline
\end{tabular}

Estimates based on low Ns (approx. $<30$ obs) have large relative standard errors and should be interpreted with caution. ${ }^{* * *}$ indicates $p<0.001,{ }^{* *}$ indicates $p<0.05$ and $^{*}$ indicates $p<.0 .01$ for the chi ${ }^{2}$ statistic comparing integrated versus malaria-only study areas.

\section{TABLE 2.1.C. LLIN USE AMONG PREGNANT WOMEN LIVING IN A HOUSEHOLD WITH AT LEAST ONE LLIN FOR EVERY TWO HOUSEHOLD MEMBERS, BY SOCIODEMOGRAPHIC CHARACTERISTICS}

Percentage of pregnant women living in households with at least one LLIN for every two people who slept under an LLIN the previous night, September-October 2019

\begin{tabular}{|c|c|c|c|c|c|c|c|c|}
\hline & \multicolumn{2}{|c|}{ KEBBI } & \multicolumn{2}{|c|}{ SOKOTO } & \multicolumn{2}{|c|}{$\begin{array}{l}\text { MALARIA-ONLY } \\
\text { (ZAMFARA) }\end{array}$} & \multicolumn{2}{|c|}{$\begin{array}{l}\text { INTEGRATED } \\
\text { (KEBBI/SOKOTO) }\end{array}$} \\
\hline & $\%$ & $\mathbf{N}$ & $\%$ & $\mathbf{N}$ & $\%$ & $\mathbf{N}$ & $\%$ & $\mathbf{N}$ \\
\hline Total & 86.7 & 284 & 76.7 & 323 & 88.4 & 259 & 81.2 & 607 \\
\hline \multicolumn{9}{|l|}{ Household wealth } \\
\hline Lowest & 92.6 & 78 & 78.7 & 90 & 100.0 & 10 & 84.3 & 168 \\
\hline Second & 92.2 & 49 & 76.0 & 63 & 85.0 & 33 & 83.1 & 112 \\
\hline Middle & 83.0 & 49 & 79.2 & 61 & 82.0 & 53 & 80.8 & 110 \\
\hline Fourth & 78.6 & 41 & 74.0 & 60 & 90.7 & 73 & 75.8 & 101 \\
\hline Highest & 84.3 & 67 & 72.5 & 49 & 90.2 & 90 & 79.7 & 116 \\
\hline \multicolumn{9}{|l|}{ Maternal age (in years) } \\
\hline 15-24 years & 88.8 & 157 & 78.5 & 218 & 87.4 & 177 & 82.6 & 375 \\
\hline $25-34$ years & 84.7 & 101 & 76.4 & 86 & 91.3 & 76 & 80.8 & 187 \\
\hline $35-49$ years & 82.7 & 26 & 53.5 & 19 & 86.0 & 6 & 71.0 & 45 \\
\hline \multicolumn{9}{|c|}{$\begin{array}{l}\text { Maternal education (highest level } \\
\text { attended) }\end{array}$} \\
\hline None & 89.5 & 199 & 77 & 255 & 88.6 & 150 & 82.4 & 454 \\
\hline Primary & 81.8 & 18 & 70.1 & 13 & 91.0 & 22 & 76.4 & 31 \\
\hline Secondary or higher & 77.9 & 55 & 63.3 & 25 & 94.0 & 59 & 73.4 & 80 \\
\hline Islamic & 80.3 & 12 & 82.9 & 30 & 78.0 & 28 & 82.2 & 42 \\
\hline
\end{tabular}




\section{TABLE 2.1.D. LLIN USE AMONG UNDER-TWOS LIVING IN A HOUSEHOLD WITH AT LEAST ONE LLIN FOR EVERY TWO HOUSEHOLD MEMBERS, BY SOCIODEMOGRAPHIC CHARACTERISTICS}

Percentage of last-born children under 2 years living in households with at least one LLIN for every two people who slept under an LLIN the previous night, September-October 2019

\begin{tabular}{|c|c|c|c|c|c|c|c|c|}
\hline & \multicolumn{2}{|c|}{ KEBBI } & \multicolumn{2}{|c|}{ SOKOTO } & \multicolumn{2}{|c|}{$\begin{array}{l}\text { MALARIA-ONLY } \\
\text { (ZAMFARA) }\end{array}$} & \multicolumn{2}{|c|}{$\begin{array}{c}\text { INTEGRATED } \\
\text { (KEBBI/SOKOTO) }\end{array}$} \\
\hline & $\%$ & $\mathbf{N}$ & $\%$ & $\mathbf{N}$ & $\%$ & $\mathbf{N}$ & $\%$ & $\mathbf{N}$ \\
\hline Total & 97.6 & 158 & 89.0 & 113 & 92.8 & 124 & 94.2 & 271 \\
\hline \multicolumn{9}{|l|}{ Household wealth } \\
\hline Lowest & 98.6 & 99 & 95.4 & 62 & 100.0 & 2 & 97.5 & 161 \\
\hline Second & 100.0 & 11 & 86.0 & 24 & 100.0 & 26 & 89.7 & 35 \\
\hline Middle & 92.7 & 9 & 100.0 & 15 & 93.0 & 27 & 98.2 & 25 \\
\hline Fourth & 90.6 & 4 & 50.0 & 12 & 100.0 & 32 & 64.0 & 17 \\
\hline Highest & 96.5 & 31 & 0 & 0 & 80.5 & 37 & 96.5 & 33 \\
\hline \multicolumn{9}{|l|}{ Maternal age (in years) } \\
\hline $15-24$ years & 96.1 & 92 & 88.5 & 66 & 99.1 & 61 & 93.0 & 158 \\
\hline $25-34$ years & 99.3 & 61 & 85.0 & 35 & 88.6 & 63 & 94.5 & 96 \\
\hline $35-49$ years & 100.0 & 5 & 100.0 & 12 & 0.0 & 0 & 100.0 & 17 \\
\hline \multicolumn{9}{|c|}{ Maternal education (highest level attended) } \\
\hline None & 98.6 & 141 & 87.4 & 98 & 98.2 & 82 & 94.2 & 239 \\
\hline Primary & 94.2 & 7 & 0.0 & 0 & 100.0 & 4 & 94.2 & 7 \\
\hline Secondary or higher & 81.3 & 10 & 0.0 & 0 & 79.6 & 38 & 81.3 & 10 \\
\hline Islamic & 0.0 & 0 & 100.0 & 15 & 0.0 & 0 & 100.0 & 15 \\
\hline
\end{tabular}

Estimates based on low Ns (approx. $<30$ obs) have large relative standard errors and should be interpreted with caution. ${ }^{* * *}$ indicates $p<0.001,{ }^{* *}$ indicates $\mathrm{p}<0.05$ and * indicates $\mathrm{p}<.0 .01$ for the $\mathrm{chi}^{2}$ statistic comparing integrated versus malaria-only study areas.

\subsection{Malaria in pregnancy}

\section{TABLE 2.2. IPTP DURING LAST PREGNANCY BY SOCIODEMOGRAPHIC CHARACTERISTICS}

Percentage of women 15 to 49 years with a child under 2 years who received IPTp (three or more doses SP/Fansidar) during pregnancy for their last-born child by sociodemographic characteristics, September-October 2019

\begin{tabular}{|c|c|c|c|c|c|c|c|c|}
\hline & \multicolumn{2}{|c|}{ KEBBI } & \multicolumn{2}{|c|}{ SOKOTO } & \multicolumn{2}{|c|}{$\begin{array}{c}\text { MALARIA-ONLY } \\
\text { (ZAMFARA) }\end{array}$} & \multicolumn{2}{|c|}{$\begin{array}{c}\text { INTEGRATED } \\
\text { (KEBBI/SOKOTO) }\end{array}$} \\
\hline & $\%$ & $\mathbf{N}$ & $\%$ & $\mathbf{N}$ & $\%$ & $\mathrm{~N}$ & $\%$ & $\mathbf{N}$ \\
\hline Total & 23.7 & 892 & 14.9 & 1,078 & 19.1 & 1,069 & 18.6 & 1,971 \\
\hline \multicolumn{9}{|l|}{ Household wealth } \\
\hline Lowest & 8.4 & 264 & 11.6 & 341 & 11.8 & 111 & 10.3 & 606 \\
\hline Second & 29.0 & 192 & 10.2 & 229 & 11.6 & 183 & 18.4 & 421 \\
\hline Middle & 27.6 & 157 & 11.7 & 220 & 14.5 & 223 & 18.1 & 377 \\
\hline Fourth & 34.8 & 113 & 23.5 & 135 & 17.5 & 248 & 28.8 & 249 \\
\hline Highest & 33.3 & 166 & 32.0 & 153 & 33.8 & 304 & 32.6 & 318 \\
\hline \multicolumn{9}{|l|}{ Maternal age (in years) } \\
\hline $15-24$ years & 18.2 & 367 & 14.7 & 485 & 15.9 & 423 & 16.0 & 852 \\
\hline 25-34 years & 23.1 & 412 & 14.8 & 478 & 20.7 & 486 & 18.6 & 890 \\
\hline $35-49$ years & 40.7 & 113 & 16.0 & 115 & 23.1 & 160 & 27.5 & 229 \\
\hline \multicolumn{9}{|c|}{ Maternal education (highest level attended) } \\
\hline None & 20.1 & 675 & 11.3 & 855 & 13.9 & 698 & 14.9 & 1,530 \\
\hline Primary & 33.8 & 47 & 37.1 & 57 & 25.0 & 60 & 35.6 & 104 \\
\hline Secondary or higher & 28.5 & 95 & 53.1 & 60 & 43.4 & 180 & 37.3 & 155 \\
\hline Islamic & 38.8 & 75 & 18.2 & 106 & 20.2 & 131 & 27.3 & 182 \\
\hline
\end{tabular}

Estimates based on low Ns (approx. $<30$ obs) have large relative standard errors and should be interpreted with caution. ${ }^{* * *}$ indicates $p<0.001,{ }^{* \star}$ indicates $\mathrm{p}<0.05$ and * indicates $\mathrm{p}<.0 .01$ for the chi² statistic comparing integrated versus malaria-only study areas. 
TABLE 2.2.1. IPTP FOR MALARIA DURING LAST PREGNANCY

Percentage of women 15 to 49 years who received IPTp (three or more doses SP/Fansidar) for malaria during pregnancy for their last-born child in past two years, September-October 2019

\begin{tabular}{|c|c|c|c|c|c|c|}
\hline & KEBBI & SOKOTO & $\begin{array}{l}\text { MALARIA-ONLY } \\
\text { (ZAMFARA) }\end{array}$ & $\begin{array}{l}\text { INTEGRATED } \\
\text { (KEBBI/SOKOTO) }\end{array}$ & $\mathbf{N}$ & $\begin{array}{c}\mathrm{CHI}^{2} \\
\text { VALUE }\end{array}$ \\
\hline & $\%$ & $\%$ & $\%$ & $\%$ & & \\
\hline \multicolumn{7}{|c|}{$\begin{array}{l}\text { During your pregnancy with child, did } \\
\text { you take SP/Fansidar to keep you from } \\
\text { getting malaria }\end{array}$} \\
\hline No & 45.8 & 59.4 & 45.2 & 53.5 & 1,462 & 72.309 \\
\hline Yes & 48.6 & 38.1 & 54.3 & 42.6 & 1,498 & 4.011 \\
\hline Don't know & 5.6 & 2.5 & 0.6 & 3.8 & 79 & * \\
\hline Total & 100.0 & 100.0 & 100.0 & 100.0 & 3,039 & \\
\hline N & 892 & 1,078 & 1,069 & 1,970 & & \\
\hline \multicolumn{7}{|c|}{$\begin{array}{l}\text { How many months pregnant were you } \\
\text { at your first dose of SP/Fansidar? }\end{array}$} \\
\hline 0-3 months & 33.4 & 15.9 & 20.3 & 24.5 & 334 & 5.772 \\
\hline 4-6 months & 50.3 & 68.6 & 64.3 & 59.6 & 916 & 0.437 \\
\hline $7+$ months & 13.3 & 12.4 & 13.5 & 12.8 & 205 & \\
\hline Don't know & 3.0 & 3.1 & 1.9 & 3.1 & 43 & \\
\hline Total & 100.0 & 100.0 & 100.0 & 100.0 & 1,498 & \\
\hline N & 435 & 460 & 603 & 895 & & \\
\hline \multicolumn{7}{|c|}{$\begin{array}{l}\text { How many times did you take SP/ } \\
\text { Fansidar during your last pregnancy? }\end{array}$} \\
\hline None & 0.6 & 0.0 & 0.0 & 0.3 & 3 & 23.866 \\
\hline 1 & 22.3 & 30.6 & 25.4 & 26.5 & 389 & 1.745 \\
\hline 2 & 20.2 & 28.1 & 35.4 & 24.2 & 429 & \\
\hline 3 or more & 48.7 & 39.1 & 35.2 & 43.8 & 598 & \\
\hline Don't know & 8.2 & 2.2 & 4.1 & 5.2 & 79 & \\
\hline Total & 100.0 & 100.0 & 100.0 & 100.0 & 1,498 & \\
\hline $\mathrm{N}$ & 435 & 460 & 603 & 895 & & \\
\hline \multicolumn{7}{|c|}{ Where did you get SP/Fansidar? } \\
\hline \multicolumn{7}{|c|}{ Antenatal care visit } \\
\hline No & 23.0 & 28.6 & 15.6 & 25.8 & 315 & 22.864 \\
\hline Yes & 77.0 & 71.4 & 84.4 & 74.2 & 1,183 & 3.112 \\
\hline Total & 100.0 & 100.0 & 100.0 & 100.0 & 1,498 & \\
\hline $\mathrm{N}$ & 435 & 460 & 603 & 895 & & \\
\hline \multicolumn{7}{|c|}{ Another visit to a health facility } \\
\hline No & 92.6 & 94.5 & 97.4 & 93.6 & 1,431 & 12.847 \\
\hline Yes & 7.4 & 5.5 & 2.7 & 6.5 & 67 & 4.466 \\
\hline Total & 100.0 & 100.0 & 100.0 & 100.0 & 1,498 & \\
\hline N & 435 & 460 & 603 & 895 & & \\
\hline \multicolumn{7}{|l|}{ Pharmacy } \\
\hline No & 91.5 & 81.0 & 90.6 & 86.1 & 1,329 & 6.838 \\
\hline Yes & 8.5 & 19.0 & 9.4 & 13.9 & 169 & 1.093 \\
\hline Total & 100.0 & 100.0 & 100.0 & 100.0 & 1,498 & \\
\hline N & 435 & 460 & 603 & 895 & & \\
\hline \multicolumn{7}{|l|}{ Market/store } \\
\hline No & 87.3 & 93.4 & 96.9 & 90.4 & 1,394 & 28.443 \\
\hline Yes & 12.7 & 6.6 & 3.1 & 9.6 & 104 & 4.590 \\
\hline Total & 100.0 & 100.0 & 100.0 & 100.0 & 1,498 & \\
\hline$N$ & 435 & 460 & 603 & 895 & & \\
\hline \multicolumn{7}{|c|}{ Itinerant drug seller } \\
\hline No & 99.1 & 99.0 & 98.6 & 99.1 & 1,486 & 0.628 \\
\hline Yes & 0.9 & 1.0 & 1.4 & 1.0 & 12 & 0.186 \\
\hline Total & 100.0 & 100.0 & 100.0 & 100.0 & 1,498 & \\
\hline $\mathrm{N}$ & 435 & 460 & 603 & 895 & & \\
\hline
\end{tabular}

Estimates based on low Ns (approx. $<30$ obs) have large relative standard errors and should be interpreted with caution. ${ }^{\star \star *}$ indicates $p<0.001,{ }^{\star \star}$ indicates $p<0.05$ and ${ }^{*}$ indicates $p<.0 .01$ for the chi² statistic comparing integrated versus malaria-only study areas. 
TABLE 2.2.2. REASONS FOR NO IPTP FOR MALARIA DURING LAST PREGNANCY

Percentage of women 15 to 49 years who did not receive IPTp for their last-born child in past two years by reason, September-October 2019

\begin{tabular}{|c|c|c|c|c|c|c|}
\hline & KEBBI & SOKOTO & $\begin{array}{l}\text { MALARIA-ONLY } \\
\text { (ZAMFARA) }\end{array}$ & $\begin{array}{c}\text { INTEGRATED } \\
\text { (KEBBI/SOKOTO) }\end{array}$ & $\mathbf{N}$ & $\begin{array}{c}\mathrm{CHI}^{2} \\
\text { VALUE }\end{array}$ \\
\hline & $\%$ & $\%$ & $\%$ & $\%$ & & \\
\hline \multicolumn{7}{|c|}{ None available at facility } \\
\hline No & 88.4 & 98.1 & 89.1 & 94.5 & 1,353 & 13.762 \\
\hline Yes & 11.6 & 1.9 & 10.9 & 5.5 & 109 & 2.418 \\
\hline Total & 100.0 & 100.0 & 100.0 & 100.0 & 1,462 & \\
\hline $\mathrm{N}$ & 410 & 593 & 459 & 1,003 & & \\
\hline \multicolumn{7}{|c|}{ Provider did not offer medicine } \\
\hline No & 88.2 & 89.4 & 93.7 & 89.0 & 1,343 & 10.471 \\
\hline Yes & 11.8 & 10.6 & 6.3 & 11.0 & 119 & 2.946 \\
\hline Total & 100.0 & 100.0 & 100.0 & 100.0 & 1,462 & \\
\hline $\mathrm{N}$ & 410 & 593 & 459 & 1,003 & & \\
\hline \multicolumn{7}{|c|}{ Afraid of side effects } \\
\hline No & 92.8 & 96.6 & 96.8 & 95.2 & 1,395 & 2.598 \\
\hline Yes & 7.2 & 3.4 & 3.2 & 4.8 & 67 & 0.237 \\
\hline Total & 100.0 & 100.0 & 100.0 & 100.0 & 1,462 & \\
\hline N & 410 & 593 & 459 & 1,003 & & \\
\hline \multicolumn{7}{|c|}{ Too costly } \\
\hline No & 90.5 & 95.9 & 95.3 & 93.9 & 1,376 & 1.312 \\
\hline Yes & 9.5 & 4.1 & 4.7 & 6.1 & 86 & 0.218 \\
\hline Total & 100.0 & 100.0 & 100.0 & 100.0 & 1,462 & \\
\hline $\mathrm{N}$ & 410 & 593 & 459 & 1,003 & & \\
\hline \multicolumn{7}{|c|}{ Respondent opposes } \\
\hline No & 83.1 & 71.4 & 70.6 & 75.7 & 1,051 & 4.730 \\
\hline Yes & 16.9 & 28.6 & 29.4 & 24.3 & 411 & 0.444 \\
\hline Total & 100.0 & 100.0 & 100.0 & 100.0 & 1,462 & \\
\hline $\mathrm{N}$ & 410 & 593 & 459 & 1,003 & & \\
\hline \multicolumn{7}{|c|}{ Husband/partner opposes } \\
\hline No & 79.9 & 88.0 & 72.1 & 85.1 & 1,163 & 34.713 \\
\hline Yes & 20.1 & 12.0 & 27.9 & 15.0 & 299 & 3.397 \\
\hline Total & 100.0 & 100.0 & 100.0 & 100.0 & 1,462 & \\
\hline $\mathrm{N}$ & 410 & 593 & 459 & 1,003 & & \\
\hline \multicolumn{7}{|c|}{ Religious/community leaders oppose } \\
\hline No & 99.3 & 98.8 & 99.7 & 99.0 & 1,446 & 2.745 \\
\hline Yes & 0.7 & 1.2 & 0.4 & 1.1 & 16 & 1.219 \\
\hline Total & 100.0 & 100.0 & 100.0 & 100.0 & 1,462 & \\
\hline $\mathrm{N}$ & 410 & 593 & 459 & 1,003 & & \\
\hline \multicolumn{7}{|l|}{ Other } \\
\hline No & 79.8 & 69.0 & 76.2 & 73.0 & 1,130 & 1.975 \\
\hline Yes & 20.2 & 31.0 & 23.8 & 27.0 & 332 & 0.201 \\
\hline Total & 100.0 & 100.0 & 100.0 & 100.0 & 1,462 & \\
\hline $\mathrm{N}$ & 410 & 593 & 459 & 1,003 & & \\
\hline \multicolumn{7}{|c|}{ Don't know } \\
\hline No & 85.3 & 81.1 & 86.7 & 82.6 & 1,219 & 4.609 \\
\hline Yes & 14.8 & 18.9 & 13.3 & 17.4 & 243 & 0.491 \\
\hline Total & 100.0 & 100.0 & 100.0 & 100.0 & 1,462 & \\
\hline $\mathrm{N}$ & 410 & 593 & 459 & 1,003 & & \\
\hline
\end{tabular}

Estimates based on low Ns (approx. $<30$ obs) have large relative standard errors and should be interpreted with caution. ${ }^{* \star *}$ indicates $p<0.001,{ }^{* *}$ indicates $p<0.05$ and ${ }^{*}$ indicates $p<.0 .01$ for the chi ${ }^{2}$ statistic comparing integrated versus malaria-only study areas. 


\section{TABLE 2.2.3. WHAT ARE SOME RISKS TO THE BABY IF A WOMAN GETS MALARIA DURING}

PREGNANCY?

Percentage of women aged 15 to 49 years currently pregnant or with a child under 2 years who report risks (unprompted) to the baby if a woman gets malaria during pregnancy by type of risk, September-October 2019

\begin{tabular}{|c|c|c|c|c|c|c|}
\hline & KEBBI & SOKOTO & $\begin{array}{l}\text { MALARIA-ONLY } \\
\text { (ZAMFARA) }\end{array}$ & $\begin{array}{c}\text { INTEGRATED } \\
\text { (KEBBI/SOKOTO) }\end{array}$ & $\mathbf{N}$ & $\begin{array}{c}\mathrm{CHI}^{2} \\
\text { VALUE }\end{array}$ \\
\hline & $\%$ & $\%$ & $\%$ & $\%$ & & \\
\hline \multicolumn{7}{|c|}{ Fetal death } \\
\hline No & 62.2 & 69.3 & 46.9 & 66.0 & 3,629 & 213.871 \\
\hline Yes & 37.8 & 30.7 & 53.1 & 34.0 & 2,405 & 8.224 \\
\hline Total & 100.0 & 100.0 & 100.0 & 100.0 & 6,034 & \\
\hline N & 1,960 & 2,062 & 2,012 & 4,022 & & \\
\hline \multicolumn{7}{|c|}{ Premature birth } \\
\hline No & 74.2 & 81.8 & 70.7 & 78.3 & 4,547 & 42.927 \\
\hline Yes & 25.8 & 18.3 & 29.3 & 21.7 & 1,487 & 1.637 \\
\hline Total & 100.0 & 100.0 & 100.0 & 100.0 & 6,034 & \\
\hline N & 1,960 & 2,062 & 2,012 & 4,022 & & \\
\hline \multicolumn{7}{|c|}{ Low birth weight } \\
\hline No & 86.5 & 88.9 & 79.1 & 87.8 & 5,114 & 76.631 \\
\hline Yes & 13.5 & 11.1 & 20.9 & 12.2 & 920 & 2.936 \\
\hline Total & 100.0 & 100.0 & 100.0 & 100.0 & 6,034 & \\
\hline $\mathrm{N}$ & 1,960 & 2,062 & 2,012 & 4,022 & & \\
\hline \multicolumn{7}{|c|}{ Miscarriage } \\
\hline No & 65.6 & 65.4 & 49.4 & 65.5 & 3,595 & 152.659 \\
\hline Yes & 34.4 & 34.6 & 50.6 & 34.5 & 2,439 & 6.114 \\
\hline Total & 100.0 & 100.0 & 100.0 & 100.0 & 6,034 & \\
\hline $\mathrm{N}$ & 1,960 & 2,062 & 2,012 & 4,022 & & \\
\hline
\end{tabular}

Estimates based on low Ns (approx. $<30$ obs) have large relative standard errors and should be interpreted with caution. ${ }^{* * *}$ indicates $p<0.001,{ }^{* *}$ indicates $p<0.05$ and ${ }^{*}$ indicates $p<.0 .01$ for the chi ${ }^{2}$ statistic comparing integrated versus malaria-only study areas.

\subsection{Fever care and treatment}

\section{TABLE 2.3.A. DIAGNOSIS FOR PEDIATRIC FEVERS BY SOCIODEMOGRAPHIC CHARACTERISTICS}

Percentage of last-born children under 2 years with fever in the past two weeks who received a finger or heel stick for testing by sociodemographic characteristics, September-October 2019

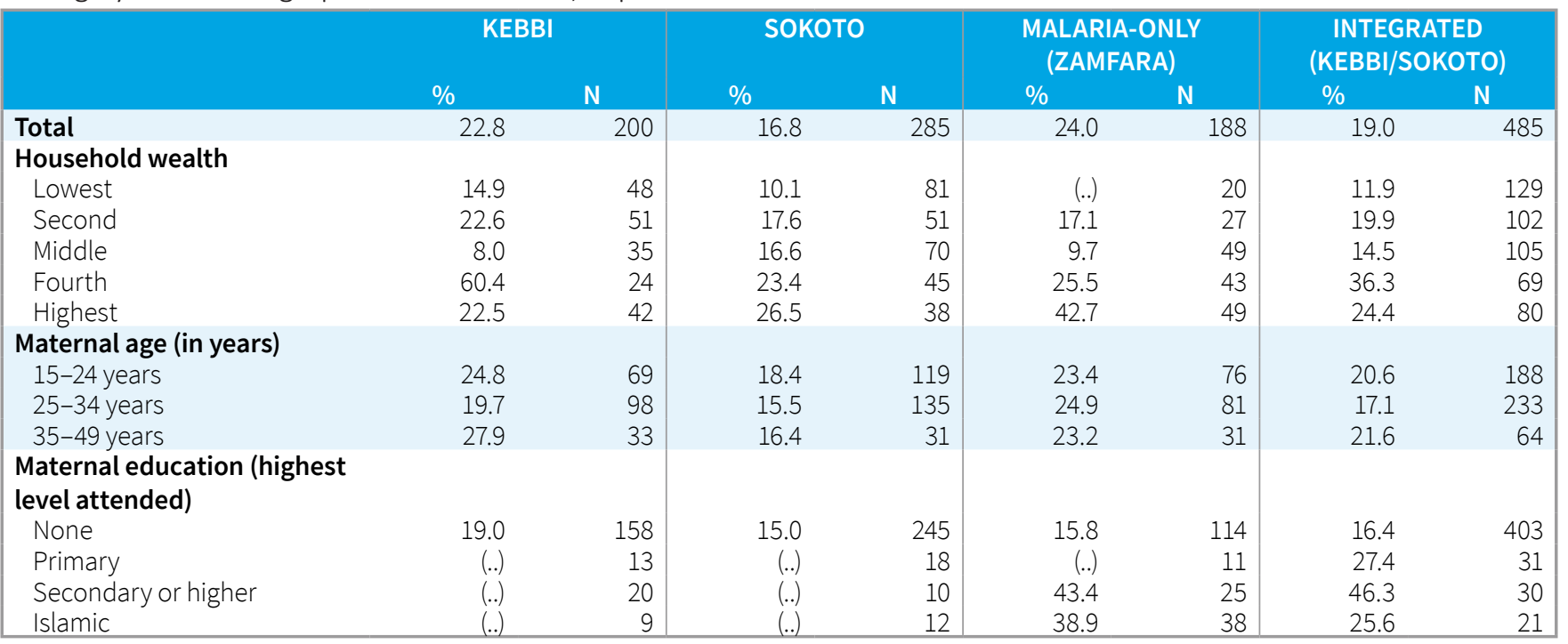

(..) Value not shown due to low number of observations. Estimates based on low Ns (approx.<30 obs) have large relative standard errors and should be interpreted with caution. ${ }^{* \star *}$ indicates $p<0.001,{ }^{* *}$ indicates $p<0.05$ and ${ }^{*}$ indicates $p<.0 .01$ for the chi ${ }^{2}$ statistic comparing integrated versus malaria-only study areas. 
TABLE 2.3.B. ACT TREATMENT FOR PEDIATRIC FEVERS BY SOCIODEMOGRAPHIC CHARACTERISTICS

Percentage of last-born children under 2 years with fever in the past two weeks who received ACT treatment by sociodemographic characteristics, September-October 2019

\begin{tabular}{|c|c|c|c|c|c|c|c|c|}
\hline & \multicolumn{2}{|c|}{ KEBBI } & \multicolumn{2}{|c|}{ sокото } & \multicolumn{2}{|c|}{$\begin{array}{l}\text { MALARIA-ONLY } \\
\text { (ZAMFARA) }\end{array}$} & \multicolumn{2}{|c|}{$\begin{array}{c}\text { INTEGRATED } \\
\text { (KEBBI/SOKOTO) }\end{array}$} \\
\hline & $\%$ & $\mathbf{N}$ & $\%$ & $\mathbf{N}$ & $\%$ & $\mathbf{N}$ & $\%$ & $\mathbf{N}$ \\
\hline Total & 28.8 & 200 & 17.9 & 285 & 36.1 & 188 & 22.0 & 485 \\
\hline \multicolumn{9}{|l|}{ Household wealth } \\
\hline Lowest & 21.6 & 48 & 12.2 & 81 & (..) & 20 & 15.7 & 129 \\
\hline Second & 31.3 & 51 & 21.1 & 51 & 29.6 & 27 & 25.7 & 102 \\
\hline Middle & 22.7 & 35 & 17.1 & 70 & 34.9 & 49 & 18.5 & 105 \\
\hline Fourth & 38.5 & 24 & 19.6 & 45 & 28.8 & 43 & 26.2 & 69 \\
\hline Highest & 36.0 & 42 & 29.6 & 38 & 53.9 & 49 & 32.9 & 80 \\
\hline \multicolumn{9}{|l|}{ Maternal age (in years) } \\
\hline 15-24 years & 27.6 & 69 & (..) & 19 & 23.4 & 76 & 23.7 & 188 \\
\hline $25-34$ years & 25.3 & 98 & 18.8 & 135 & 45.1 & 81 & 21.3 & 233 \\
\hline $35-49$ years & 41.0 & 33 & 3.3 & 31 & 47.3 & 31 & 20.1 & 64 \\
\hline \multicolumn{9}{|c|}{$\begin{array}{l}\text { Maternal education (highest } \\
\text { level attended) }\end{array}$} \\
\hline None & 31.0 & 158 & 16.7 & 245 & 31.2 & 114 & 21.7 & 403 \\
\hline Primary & 27.7 & 13 & (..) & 18 & (..) & 11 & 34.1 & 31 \\
\hline Secondary or higher & (..) & 20 & (..) & 10 & 46.8 & 25 & 22.5 & 30 \\
\hline Islamic & (..) & 9 & (..) & 12 & 51.8 & 38 & 11.2 & 21 \\
\hline
\end{tabular}

(..) Value not shown due to low number of observations. Estimates based on low Ns (approx. <30 obs) have large relative standard errors and should be interpreted with caution. ${ }^{* * *}$ indicates $p<0.001,{ }^{* *}$ indicates $p<0.05$ and ${ }^{*}$ indicates $p<.0 .01$ for the chi ${ }^{2}$ statistic comparing integrated versus malaria-only study areas. 
TABLE 2.3.1. DIAGNOSIS AND CARE-SEEKING FOR PEDIATRIC FEVERS

Percentage of last-born children under 2 years with fever in the past two weeks who were tested for malaria and sought any advice or treatment, September-October 2019

\begin{tabular}{|c|c|c|c|c|c|c|}
\hline & KEBBI & SOKOTO & $\begin{array}{l}\text { MALARIA-ONLY } \\
\text { (ZAMFARA) }\end{array}$ & $\begin{array}{l}\text { INTEGRATED } \\
\text { (KEBBI/SOKOTO) }\end{array}$ & $\mathbf{N}$ & $\begin{array}{c}\mathrm{CHI}^{2} \\
\text { VALUE }\end{array}$ \\
\hline & $\%$ & $\%$ & $\%$ & $\%$ & & \\
\hline \multicolumn{7}{|c|}{$\begin{array}{l}\text { Has the child had an illness with a fever } \\
\text { at any time in the last } 2 \text { weeks? }\end{array}$} \\
\hline No & 78.0 & 71.7 & 83.3 & 74.4 & 2,344 & 36.039 \\
\hline Yes & 21.8 & 27.5 & 16.4 & 25.0 & 673 & 4.021 \\
\hline Don't know & 0.2 & 0.9 & 0.3 & 0.6 & 22 & * \\
\hline Total & 100.0 & 100.0 & 100.0 & 100.0 & 3,039 & \\
\hline $\mathrm{N}$ & 892 & 1,078 & 1,069 & 1,970 & & \\
\hline \multicolumn{7}{|c|}{$\begin{array}{l}\text { At any time during the illness did child } \\
\text { have blood taken for testing? }\end{array}$} \\
\hline No & 76.7 & 82.6 & 76.0 & 80.4 & 533 & 4.210 \\
\hline Yes & 22.8 & 16.8 & 24.0 & 19.0 & 135 & 1.199 \\
\hline Don't know & 0.5 & 0.6 & 0.0 & 0.6 & 5 & . \\
\hline Total & 100.0 & 100.0 & 100.0 & 100.0 & 673 & \\
\hline N & 200 & 285 & 188 & 485 & & \\
\hline \multicolumn{7}{|c|}{$\begin{array}{l}\text { Did you seek advice or treatment from } \\
\text { any source for child's fever? }\end{array}$} \\
\hline No & 16.2 & 41.5 & 25.6 & 32.0 & 187 & 3.450 \\
\hline Yes & 83.8 & 58.5 & 74.5 & 68.0 & 486 & 1.089 \\
\hline Total & 100.0 & 100.0 & 100.0 & 100.0 & 673 & \\
\hline $\mathrm{N}$ & 200 & 285 & 188 & 485 & & \\
\hline \multicolumn{7}{|c|}{$\begin{array}{l}\text { How many days after illness started was } \\
\text { treatment sought? }\end{array}$} \\
\hline Same/next day & 61.7 & 64.5 & 70.9 & 63.2 & 314 & 3.849 \\
\hline 2 days later & 24.7 & 25.9 & 21.7 & 25.3 & 114 & 0.670 \\
\hline $3+$ days later & 13.6 & 9.6 & 7.4 & 11.5 & 58 & \\
\hline Total & 100.0 & 100.0 & 100.0 & 100.0 & 486 & \\
\hline $\mathrm{N}$ & 167 & 174 & 145 & 341 & & \\
\hline \multicolumn{7}{|c|}{$\begin{array}{l}\text { Among children who were taken to any } \\
\text { care, did child have blood taken for } \\
\text { testing? }\end{array}$} \\
\hline No & 72.2 & 70.8 & 68.4 & 71.5 & 314 & 3.849 \\
\hline Yes & 27.2 & 28.7 & 31.6 & 28.0 & 114 & 0.670 \\
\hline Don't know & 0.5 & 0.5 & 0.0 & 0.5 & 58 & \\
\hline Total & 100.0 & 100.0 & 100.0 & 100.0 & 486 & \\
\hline$N$ & 167 & 174 & 145 & 341 & & \\
\hline
\end{tabular}

Estimates based on low Ns (approx. $<30$ obs) have large relative standard errors and should be interpreted with caution. ${ }^{* * *}$ indicates $p<0.001,{ }^{* *}$ indicates $p<0.05$ and * indicates $p<.0 .01$ for the chi ${ }^{2}$ statistic comparing integrated versus malaria-only study areas. 
TABLE 2.3.2. CARE-SEEKING FOR PEDIATRIC FEVERS

Percentage of last-born children under 2 years with fever in the past two weeks who sought any advice or treatment by source of care, September-October 2019

\begin{tabular}{|c|c|c|c|c|c|c|}
\hline & KEBBI & SOKOTO & $\begin{array}{l}\text { MALARIA-ONLY } \\
\text { (ZAMFARA) }\end{array}$ & $\begin{array}{c}\text { INTEGRATED } \\
\text { (KEBBI/SOKOTO) }\end{array}$ & $\mathbf{N}$ & $\begin{array}{c}\mathrm{CHI}^{2} \\
\text { VALUE }\end{array}$ \\
\hline & $\%$ & $\%$ & $\%$ & $\%$ & & \\
\hline \multicolumn{7}{|c|}{ Government hospital } \\
\hline No & 62.7 & 89.8 & 79.4 & 77.3 & 374 & 0.325 \\
\hline Yes & 37.3 & 10.2 & 20.6 & 22.7 & 112 & 0.054 \\
\hline Total & 100.0 & 100.0 & 100.0 & 100.0 & 486 & \\
\hline N & 167 & 174 & 145 & 341 & & \\
\hline \multicolumn{7}{|c|}{ Governmental PHC } \\
\hline No & 78.9 & 67.6 & 79.0 & 72.8 & 367 & 2.547 \\
\hline Yes & 21.1 & 32.4 & 21.0 & 27.2 & 119 & 0.597 \\
\hline Total & 100.0 & 100.0 & 100.0 & 100.0 & 486 & \\
\hline $\mathrm{N}$ & 167 & 174 & 145 & 341 & & \\
\hline \multicolumn{7}{|c|}{ Dispensary/health post } \\
\hline No & 97.9 & 91.0 & 95.9 & 94.2 & 456 & 0.763 \\
\hline Yes & 2.1 & 9.0 & 4.1 & 5.8 & 30 & 0.124 \\
\hline Total & 100.0 & 100.0 & 100.0 & 100.0 & 486 & \\
\hline N & 167 & 174 & 145 & 341 & & \\
\hline \multicolumn{7}{|c|}{ Community health outreach post } \\
\hline No & 92.7 & 99.6 & 100.0 & 96.4 & 473 & 9.270 \\
\hline Yes & 7.3 & 0.4 & 0.0 & 3.6 & 13 & 3.423 \\
\hline Total & 100.0 & 100.0 & 100.0 & 100.0 & 486 & \\
\hline $\mathrm{N}$ & 167 & 174 & 145 & 341 & & \\
\hline \multicolumn{7}{|c|}{ Private hospital/clinic } \\
\hline No & 98.2 & 93.1 & 98.8 & 95.5 & 470 & 4.927 \\
\hline Yes & 1.8 & 6.9 & 1.2 & 4.5 & 16 & 1.788 \\
\hline Total & 100.0 & 100.0 & 100.0 & 100.0 & 486 & \\
\hline $\mathrm{N}$ & 167 & 174 & 145 & 341 & & \\
\hline \multicolumn{7}{|c|}{ Pharmacy/chemist } \\
\hline No & 70.9 & 66.0 & 45.7 & 68.3 & 298 & 25.316 \\
\hline Yes & 29.1 & 34.0 & 54.4 & 31.7 & 188 & 7.456 \\
\hline Total & 100.0 & 100.0 & 100.0 & 100.0 & 486 & ** \\
\hline $\mathrm{N}$ & 167 & 174 & 145 & 341 & & \\
\hline
\end{tabular}

Estimates based on low Ns (approx. $<30$ obs) have large relative standard errors and should be interpreted with caution. ${ }^{* * *}$ indicates $p<0.001,{ }^{* \star}$ indicates $p<0.05$ and * indicates $p<.0 .01$ for the chi ${ }^{2}$ statistic comparing integrated versus malaria-only study areas. 
TABLE 2.3.3. REASONS FOR CHOOSING SOURCE OF CARE FOR PEDIATRIC FEVERS

Percentage of last-born children under 2 years with fever in the past two weeks taken to any source of care by reason for choosing treatment location, September-October 2019

\begin{tabular}{|c|c|c|c|c|c|c|}
\hline & KEBBI & SOKOTO & $\begin{array}{l}\text { MALARIA-ONLY } \\
\text { (ZAMFARA) }\end{array}$ & $\begin{array}{l}\text { INTEGRATED } \\
\text { (KEBBI/SOKOTO) }\end{array}$ & $\mathbf{N}$ & $\begin{array}{c}\mathrm{CHI}^{2} \\
\text { VALUE }\end{array}$ \\
\hline & $\%$ & $\%$ & $\%$ & $\%$ & & \\
\hline \multicolumn{7}{|c|}{ Free or inexpensive } \\
\hline No & 91.6 & 82.2 & 92.5 & 86.5 & 436 & 33.859 \\
\hline Yes & 8.4 & 17.8 & 7.5 & 13.5 & 47 & 0.794 \\
\hline Total & 100.0 & 100.0 & 100.0 & 100.0 & 483 & \\
\hline $\mathrm{N}$ & 166 & 172 & 145 & 338 & & \\
\hline \multicolumn{7}{|c|}{ Nearby/transport available } \\
\hline No & 61.9 & 62.1 & 71.1 & 62.0 & 325 & 32.807 \\
\hline Yes & 38.1 & 37.9 & 28.9 & 38.0 & 158 & 0.576 \\
\hline Total & 100.0 & 100.0 & 100.0 & 100.0 & 483 & \\
\hline $\mathrm{N}$ & 166 & 172 & 145 & 338 & & \\
\hline \multicolumn{7}{|c|}{ Medicines often in stock } \\
\hline No & 93.2 & 86.9 & 94.3 & 89.8 & 447 & 25.198 \\
\hline Yes & 6.9 & 13.1 & 5.7 & 10.2 & 36 & 1.421 \\
\hline Total & 100.0 & 100.0 & 100.0 & 100.0 & 483 & \\
\hline $\mathrm{N}$ & 166 & 172 & 145 & 338 & & \\
\hline \multicolumn{7}{|c|}{ Respectful care } \\
\hline No & 94.8 & 88.5 & 83.4 & 91.4 & 431 & 49.790 \\
\hline Yes & 5.3 & 11.5 & 16.6 & 8.6 & 52 & 2.770 \\
\hline Total & 100.0 & 100.0 & 100.0 & 100.0 & 483 & \\
\hline$N$ & 166 & 172 & 145 & 338 & & \\
\hline \multicolumn{7}{|c|}{ Trust to care for my child } \\
\hline No & 63.3 & 73.8 & 59.6 & 68.9 & 307 & 32.930 \\
\hline Yes & 36.7 & 26.2 & 40.4 & 31.1 & 176 & 1.081 \\
\hline Total & 100.0 & 100.0 & 100.0 & 100.0 & 483 & \\
\hline $\mathrm{N}$ & 166 & 172 & 145 & 338 & & \\
\hline \multicolumn{7}{|c|}{ Effective treatment } \\
\hline No & 80.1 & 75.2 & 68.1 & 77.5 & 358 & 38.892 \\
\hline Yes & 19.9 & 24.8 & 31.9 & 22.5 & 125 & 1.562 \\
\hline Total & 100.0 & 100.0 & 100.0 & 100.0 & 483 & \\
\hline N & 166 & 172 & 145 & 338 & & \\
\hline \multicolumn{7}{|c|}{ Short wait time } \\
\hline No & 92.0 & 91.3 & 86.0 & 91.6 & 428 & 27.473 \\
\hline Yes & 8.0 & 8.7 & 14.0 & 8.4 & 55 & 0.906 \\
\hline Total & 100.0 & 100.0 & 100.0 & 100.0 & 483 & \\
\hline$N$ & 166 & 172 & 145 & 338 & & \\
\hline \multicolumn{7}{|l|}{ Privacy } \\
\hline No & 99.7 & 98.9 & 98.5 & 99.2 & 477 & 4.712 \\
\hline Yes & 0.3 & 1.1 & 1.6 & 0.8 & 6 & 0.440 \\
\hline Total & 100.0 & 100.0 & 100.0 & 100.0 & 483 & \\
\hline $\mathrm{N}$ & 166 & 172 & 145 & 338 & & \\
\hline \multicolumn{7}{|c|}{ Family/friends } \\
\hline No & 90.0 & 88.3 & 80.9 & 89.1 & 414 & 46.131 \\
\hline Yes & 10.0 & 11.7 & 19.1 & 10.9 & 69 & 1.687 \\
\hline Total & 100.0 & 100.0 & 100.0 & 100.0 & 483 & \\
\hline$N$ & 166 & 172 & 145 & 338 & & \\
\hline \multicolumn{7}{|c|}{$\begin{array}{l}\text { Recommended by religious or } \\
\text { community leader }\end{array}$} \\
\hline No & 84.9 & 100.0 & 100.0 & 93.0 & 453 & 133.310 \\
\hline Yes & 15.1 & 0.0 & 0.0 & 7.0 & 30 & 1.507 \\
\hline Total & 100.0 & 100.0 & 100.0 & 100.0 & 483 & \\
\hline $\mathrm{N}$ & 166 & 172 & 145 & 338 & & \\
\hline
\end{tabular}

Estimates based on low Ns (approx. $<30$ obs) have large relative standard errors and should be interpreted with caution. ${ }^{* * *}$ indicates $p<0.001,{ }^{* *}$ indicates $p<0.05$ and * indicates $p<.0 .01$ for the chi ${ }^{2}$ statistic comparing integrated versus malaria-only study areas. 
TABLE 2.3.4. REASONS FOR NOT SEEKING CARE FOR PEDIATRIC FEVERS

Percentage of last-born children under 2 years with fever in the past two weeks not taken to any source of care by reasons for no care-seeking, September-October 2019

\begin{tabular}{|c|c|c|c|c|c|c|}
\hline & $\begin{array}{c}\text { KEBBI } \\
\%\end{array}$ & $\begin{array}{c}\text { SOKOTO } \\
\%\end{array}$ & $\begin{array}{c}\text { MALARIA-ONLY } \\
\text { (ZAMFARA) } \\
\%\end{array}$ & $\begin{array}{c}\text { INTEGRATED } \\
\text { (KEBBI/SOKOTO) } \\
\%\end{array}$ & $\mathbf{N}$ & $\begin{array}{c}\mathrm{CHI}^{2} \\
\text { VALUE }\end{array}$ \\
\hline \multicolumn{7}{|c|}{$\begin{array}{l}\text { Too expensive/could not find } \\
\text { money }\end{array}$} \\
\hline No & 63.7 & 73.0 & 66.0 & 71.2 & 130 & 0.574 \\
\hline Yes & 36.3 & 27.0 & 34.0 & 28.8 & 57 & 0.154 \\
\hline Total & 100.0 & 100.0 & 100.0 & 100.0 & 187 & \\
\hline N & 33 & 111 & 43 & 144 & & \\
\hline \multicolumn{7}{|c|}{ Facility closed/no one there } \\
\hline No & 94.4 & 100.0 & 100.0 & 98.9 & 185 & 0.884 \\
\hline Yes & 5.6 & 0.0 & 0.0 & 1.1 & 2 & 1.350 \\
\hline Total & 100.0 & 100.0 & 100.0 & 100.0 & 187 & . \\
\hline N & 33 & 111 & 43 & 144 & & \\
\hline \multicolumn{7}{|c|}{$\begin{array}{l}\text { Distance/transportation not avail- } \\
\text { able }\end{array}$} \\
\hline No & 100.0 & 94.8 & 97.9 & 95.8 & 181 & 0.652 \\
\hline Yes & 0.0 & 5.2 & 2.1 & 4.2 & 6 & 0.377 \\
\hline Total & 100.0 & 100.0 & 100.0 & 100.0 & 187 & . \\
\hline $\mathrm{N}$ & 33 & 111 & 43 & 144 & & \\
\hline \multicolumn{7}{|c|}{ Medicine often not available } \\
\hline No & 93.5 & 99.0 & 100.0 & 98.0 & 184 & 1.696 \\
\hline Yes & 6.5 & 1.0 & 0.0 & 2.0 & 3 & 1.827 \\
\hline Total & 100.0 & 100.0 & 100.0 & 100.0 & 187 & . \\
\hline $\mathrm{N}$ & 33 & 111 & 43 & 144 & & \\
\hline \multicolumn{7}{|c|}{ Poor quality service } \\
\hline No & 100.0 & 99.2 & 98.0 & 99.3 & 185 & 0.636 \\
\hline Yes & 0.0 & 0.8 & 2.0 & 0.7 & 2 & 0.682 \\
\hline Total & 100.0 & 100.0 & 100.0 & 100.0 & 187 & \\
\hline $\mathrm{N}$ & 33 & 111 & 43 & 144 & & \\
\hline \multicolumn{7}{|c|}{$\begin{array}{l}\text { Symptoms not severe/provided } \\
\text { home care }\end{array}$} \\
\hline No & 74.2 & 76.5 & 83.3 & 76.1 & 146 & 1.473 \\
\hline Yes & 25.8 & 23.5 & 16.7 & 23.9 & 41 & 0.505 \\
\hline Total & 100.0 & 100.0 & 100.0 & 100.0 & 187 & . \\
\hline N & 33 & 111 & 43 & 144 & & \\
\hline \multicolumn{7}{|c|}{ Husband/partner oppose } \\
\hline No & 93.9 & 93.7 & 85.4 & 93.7 & 170 & 3.572 \\
\hline Yes & 6.1 & 6.3 & 14.6 & 6.3 & 17 & 2.004 \\
\hline Total & 100.0 & 100.0 & 100.0 & 100.0 & 187 & \\
\hline N & 33 & 111 & 43 & 144 & & \\
\hline \multicolumn{7}{|c|}{ Unable to leave work/home } \\
\hline No & 100.0 & 99.2 & 100.0 & 99.3 & 186 & 0.555 \\
\hline Yes & 0.0 & 0.8 & 0.0 & 0.7 & 1 & 0.885 \\
\hline Total & 100.0 & 100.0 & 100.0 & 100.0 & 187 & . \\
\hline $\mathrm{N}$ & 33 & 111 & 43 & 144 & & \\
\hline \multicolumn{7}{|c|}{ Up to God } \\
\hline No & 84.7 & 66.1 & 63.3 & 69.6 & 126 & 0.842 \\
\hline Yes & 15.3 & 33.9 & 36.7 & 30.4 & 61 & 0.183 \\
\hline Total & 100.0 & 100.0 & 100.0 & 100.0 & 187 & \\
\hline $\mathrm{N}$ & 33 & 111 & 43 & 144 & & \\
\hline
\end{tabular}

Estimates based on low Ns (approx. $<30$ obs) have large relative standard errors and should be interpreted with caution. ${ }^{* \star *}$ indicates $p<0.001,{ }^{* *}$ indicates $p<0.05$ and ${ }^{*}$ indicates $p<.0 .01$ for the chi $^{2}$ statistic comparing integrated versus malaria-only study areas. 
TABLE 2.3.5. ANTI-MALARIAL TREATMENT FOR PEDIATRIC FEVERS

Percentage of last-born children under 2 years with fever in the past two weeks given anti-malarials by type, September-October 2019

\begin{tabular}{|c|c|c|c|c|c|c|}
\hline & KEBBI & SOKOTO & $\begin{array}{l}\text { MALARIA-ONLY } \\
\text { (ZAMFARA) }\end{array}$ & $\begin{array}{c}\text { INTEGRATED } \\
\text { (KEBBI/SOKOTO) }\end{array}$ & $\mathbf{N}$ & $\begin{array}{c}\mathrm{CHI}^{2} \\
\text { VALUE }\end{array}$ \\
\hline & $\%$ & $\%$ & & & & \\
\hline \multicolumn{7}{|c|}{$\begin{array}{l}\text { At any time during the illness, did child } \\
\text { take any medicines for the illness? }\end{array}$} \\
\hline No & 6.4 & 25.9 & 18.6 & 18.6 & 121 & 0.000 \\
\hline Yes & 93.6 & 74.1 & 81.4 & 81.4 & 552 & 0.000 \\
\hline Total & 100.0 & 100.0 & 100.0 & 100.0 & 673 & \\
\hline N & 200 & 285 & 188 & 485 & & \\
\hline \multicolumn{7}{|c|}{ What medicines did the child take? } \\
\hline \multicolumn{7}{|c|}{ Any ACT } \\
\hline No & 71.2 & 82.1 & 63.9 & 78.0 & 499 & 16.288 \\
\hline Yes & 28.8 & 17.9 & 36.1 & 22.0 & 174 & 3.951 \\
\hline Total & 100.0 & 100.0 & 100.0 & 100.0 & 673 & * \\
\hline $\mathrm{N}$ & 200 & 285 & 188 & 485 & & \\
\hline \multicolumn{7}{|l|}{ ACT } \\
\hline No & 74.8 & 84.7 & 72.7 & 81.0 & 532 & 6.459 \\
\hline Yes & 25.2 & 15.3 & 27.3 & 19.0 & 141 & 1.649 \\
\hline Total & 100.0 & 100.0 & 100.0 & 100.0 & 673 & \\
\hline $\mathrm{N}$ & 200 & 285 & 188 & 485 & & \\
\hline \multicolumn{7}{|c|}{ SP/Fansidar } \\
\hline No & 83.5 & 96.4 & 88.3 & 91.6 & 604 & 2.005 \\
\hline Yes & 16.5 & 3.6 & 11.7 & 8.4 & 69 & 0.254 \\
\hline Total & 100.0 & 100.0 & 100.0 & 100.0 & 673 & \\
\hline $\mathrm{N}$ & 200 & 285 & 188 & 485 & & \\
\hline \multicolumn{7}{|c|}{ Chloroquine } \\
\hline No & 79.1 & 91.8 & 93.1 & 87.1 & 589 & 6.900 \\
\hline Yes & 20.9 & 8.2 & 6.9 & 12.9 & 84 & 1.300 \\
\hline Total & 100.0 & 100.0 & 100.0 & 100.0 & 673 & \\
\hline$N$ & 200 & 285 & 188 & 485 & & \\
\hline \multicolumn{7}{|c|}{ Amodiaquine } \\
\hline No & 95.3 & 97.1 & 99.2 & 96.4 & 649 & 6.013 \\
\hline Yes & 4.7 & 2.9 & 0.8 & 3.6 & 24 & 3.752 \\
\hline Total & 100.0 & 100.0 & 100.0 & 100.0 & 673 & \\
\hline N & 200 & 285 & 188 & 485 & & \\
\hline \multicolumn{7}{|c|}{ Quinine pills } \\
\hline No & 98.4 & 100.0 & 96.8 & 99.4 & 664 & 6.147 \\
\hline Yes & 1.6 & 0.0 & 3.2 & 0.6 & 9 & 6.362 \\
\hline Total & 100.0 & 100.0 & 100.0 & 100.0 & 673 & \\
\hline $\mathrm{N}$ & 200 & 285 & 188 & 485 & & \\
\hline \multicolumn{7}{|c|}{ Quinine injection } \\
\hline No & 93.7 & 98.2 & 98.3 & 96.5 & 647 & 2.039 \\
\hline Yes & 6.3 & 1.8 & 1.7 & 3.5 & 26 & 1.357 \\
\hline Total & 100.0 & 100.0 & 100.0 & 100.0 & 673 & \\
\hline N & 200 & 285 & 188 & 485 & & \\
\hline \multicolumn{7}{|c|}{ Artesunate rectal } \\
\hline No & 99.3 & 98.4 & 93.9 & 98.7 & 655 & 11.158 \\
\hline Yes & 0.7 & 1.6 & 6.1 & 1.3 & 18 & 5.438 \\
\hline Total & 100.0 & 100.0 & 100.0 & 100.0 & 673 & \\
\hline $\mathrm{N}$ & 200 & 285 & 188 & 485 & & \\
\hline \multicolumn{7}{|c|}{ Artesunate injection } \\
\hline No & 96.4 & 97.4 & 95.4 & 97.0 & 646 & 1.219 \\
\hline Yes & 3.6 & 2.6 & 4.6 & 3.0 & 27 & 0.935 \\
\hline Total & 100.0 & 100.0 & 100.0 & 100.0 & 673 & \\
\hline N & 200 & 285 & 188 & 485 & & \\
\hline \multicolumn{7}{|c|}{ Other anti-malarial } \\
\hline No & 99.5 & 99.4 & 99.5 & 99.4 & 669 & 0.021 \\
\hline Yes & 0.6 & 0.6 & 0.5 & 0.6 & 4 & 0.017 \\
\hline Total & 100.0 & 100.0 & 100.0 & 100.0 & 673 & \\
\hline $\mathrm{N}$ & 200 & 285 & 188 & 485 & & \\
\hline
\end{tabular}

Estimates based on low Ns (approx. $<30$ obs) have large relative standard errors and should be interpreted with caution. ${ }^{* * *}$ indicates $p<0.001,{ }^{* *}$ indicates $p<0.05$ and ${ }^{*}$ indicates $p<.0 .01$ for the chi ${ }^{2}$ statistic comparing integrated versus malaria-only study areas. 
TABLE 2.3.6. ANTI-MALARIAL CARE-SEEKING TIMING AND COMPLETION OF ACT COURSE FOR PEDIATRIC FEVERS

Percentage of last-born children under 2 years with fever in the past two weeks who sought treatment and went same/ next day, September-October 2019; Percentage of last-born children under 2 years with fever in the past two weeks who received ACT and completed the dose, September-October 2019

\begin{tabular}{|c|c|c|c|c|c|c|}
\hline & KEBBI & SOKOTO & $\begin{array}{l}\text { MALARIA-ONLY } \\
\text { (ZAMFARA) }\end{array}$ & $\begin{array}{l}\text { INTEGRATED } \\
\text { (KEBBI/SOKOTO) }\end{array}$ & $\mathrm{N}$ & $\begin{array}{c}\mathrm{CHI}^{2} \\
\text { VALUE }\end{array}$ \\
\hline & $\%$ & $\%$ & $\%$ & $\%$ & & \\
\hline \multicolumn{7}{|c|}{$\begin{array}{l}\text { How many days after illness started } \\
\text { was treatment sought? }\end{array}$} \\
\hline Same/next day & 69.6 & 72.0 & 74.7 & 70.8 & 103 & 1.964 \\
\hline 2 days later & 21.1 & 18.6 & 13.2 & 19.9 & 22 & 0.548 \\
\hline $3+$ days later & 7.6 & 9.5 & 8.7 & 8.5 & 13 & \\
\hline Don't know & 1.7 & 0.0 & 3.4 & 0.9 & 3 & \\
\hline Total & 100.0 & 100.0 & 100.0 & 100.0 & 141 & \\
\hline $\mathrm{N}$ & 42 & 46 & 53 & 88 & & \\
\hline \multicolumn{7}{|c|}{$\begin{array}{l}\text { Did child finish the complete ACT } \\
\text { dose? }\end{array}$} \\
\hline No & 37.8 & 18.2 & 19.3 & 27.9 & 32 & 2.318 \\
\hline Yes & 62.2 & 81.8 & 79.0 & 72.1 & 108 & 0.665 \\
\hline Don't know & 0.0 & 0.0 & 1.7 & 0.0 & 1 & \\
\hline Total & 100.0 & 100.0 & 100.0 & 100.0 & 141 & \\
\hline $\mathrm{N}$ & 42 & 46 & 53 & & & \\
\hline
\end{tabular}

Estimates based on low Ns (approx. $<30$ obs) have large relative standard errors and should be interpreted with caution. ${ }^{* * *}$ indicates $p<0.001,{ }^{* *}$ indicates $p<0.05$ and ${ }^{*}$ indicates $p<.0 .01$ for the chi ${ }^{2}$ statistic comparing integrated versus malaria-only study areas. 


\subsection{Malaria ideations}

\section{TABLE 2.4.1. REPORTED CAUSES OF MALARIA IN CHILDREN}

Percentage of women aged 15 to 49 years currently pregnant or with a child under 2 years reporting the causes of malaria in children, September-October 2019

\begin{tabular}{|c|c|c|c|c|c|c|}
\hline & KEBBI & Sокото & $\begin{array}{l}\text { MALARIA-ONLY } \\
\text { (ZAMFARA) }\end{array}$ & $\begin{array}{c}\text { INTEGRATED } \\
\text { (KEBBI/SOKOTO) }\end{array}$ & $\mathbf{N}$ & $\begin{array}{c}\text { CHI }^{2} \\
\text { VALUE }\end{array}$ \\
\hline & $\%$ & $\%$ & $\%$ & $\%$ & & \\
\hline \multicolumn{7}{|c|}{ Mosquito bites } \\
\hline No & 8.7 & 5.9 & 0.6 & 7.2 & 324 & 200.709 \\
\hline Yes & 91.3 & 94.1 & 99.4 & 92.8 & 5,710 & 52.808 \\
\hline Total & 100.0 & 100.0 & 100.0 & 100.0 & 6,034 & *** \\
\hline N & 1,960 & 2,062 & 2,012 & 4,022 & & \\
\hline \multicolumn{7}{|c|}{ Eating dirty food } \\
\hline No & 84.9 & 95.6 & 94.9 & 90.6 & 5,549 & 42.821 \\
\hline Yes & 15.2 & 4.4 & 5.1 & 9.4 & 485 & 1.268 \\
\hline Total & 100.0 & 100.0 & 100.0 & 100.0 & 6,034 & \\
\hline N & 1,960 & 2,062 & 2,012 & 4,022 & & \\
\hline \multicolumn{7}{|c|}{ Drinking dirty water } \\
\hline No & 80.7 & 96.1 & 89.4 & 89.0 & 5,397 & 0.289 \\
\hline Yes & 19.3 & 4.0 & 10.6 & 11.1 & 637 & 0.006 \\
\hline Total & 100.0 & 100.0 & 100.0 & 100.0 & 6,034 & \\
\hline N & 1,960 & 2,062 & 2,012 & 4,022 & & \\
\hline \multicolumn{7}{|c|}{ Dirty surroundings } \\
\hline No & 72.5 & 93.7 & 78.6 & 83.9 & 4,876 & 26.187 \\
\hline Yes & 27.5 & 6.4 & 21.4 & 16.1 & 1,158 & 0.824 \\
\hline Total & 100.0 & 100.0 & 100.0 & 100.0 & 6,034 & \\
\hline N & 1,960 & 2,062 & 2,012 & 4,022 & & \\
\hline \multicolumn{7}{|c|}{ Getting soaked with rain } \\
\hline No & 93.3 & 95.8 & 97.4 & 94.7 & 5,744 & 30.055 \\
\hline Yes & 6.7 & 4.2 & 2.6 & 5.3 & 290 & 1.869 \\
\hline Total & 100.0 & 100.0 & 100.0 & 100.0 & 6,034 & \\
\hline N & 1,960 & 2,062 & 2,012 & 4,022 & & \\
\hline \multicolumn{7}{|c|}{ Cold or changing weather } \\
\hline No & 92.7 & 93.9 & 92.8 & 93.3 & 5,610 & 0.574 \\
\hline Yes & 7.3 & 6.2 & 7.2 & 6.7 & 424 & 0.021 \\
\hline Total & 100.0 & 100.0 & 100.0 & 100.0 & 6,034 & \\
\hline N & 1,960 & 2,062 & 2,012 & 4,022 & & \\
\hline \multicolumn{7}{|c|}{ Don't know } \\
\hline No & 97.0 & 95.7 & 99.7 & 96.3 & 5,880 & 103.990 \\
\hline Yes & 3.0 & 4.3 & 0.3 & 3.7 & 154 & 21.329 \\
\hline Total & 100.0 & 100.0 & 100.0 & 100.0 & 6,034 & *** \\
\hline N & 1,960 & 2,062 & 2,012 & 4,022 & & \\
\hline
\end{tabular}

Estimates based on low Ns (approx. $<30$ obs) have large relative standard errors and should be interpreted with caution. ${ }^{* * *}$ indicates $p<0.001,{ }^{* *}$ indicates $p<0.05$ and * indicates $p<.0 .01$ for the chi ${ }^{2}$ statistic comparing integrated versus malaria-only study areas. 
TABLE 2.4.2. REPORTED WAYS TO PREVENT MALARIA IN CHILDREN

Percentage of women aged 15 to 49 years currently pregnant or with a child under 2 years reporting ways to prevent malaria in children, September-October 2019

\begin{tabular}{|c|c|c|c|c|c|c|}
\hline & $\begin{array}{c}\text { KEBBI } \\
\%\end{array}$ & $\begin{array}{c}\text { SOKOTO } \\
\%\end{array}$ & $\begin{array}{c}\text { MALARIA-ONLY } \\
\text { (ZAMFARA) } \\
\%\end{array}$ & $\begin{array}{c}\text { INTEGRATED } \\
\text { (KEBBI/SOKOTO) } \\
\%\end{array}$ & $\mathbf{N}$ & $\begin{array}{c}\mathrm{CHI}^{2} \\
\text { VALUE }\end{array}$ \\
\hline \multicolumn{7}{|c|}{ Sleep under a mosquito net } \\
\hline No & 8.9 & 9.7 & 5.0 & 9.3 & 522 & 43.044 \\
\hline Yes & 91.1 & 90.3 & 95.0 & 90.7 & 5,512 & 2.902 \\
\hline Total & 100.0 & 100.0 & 100.0 & 100.0 & 6,034 & \\
\hline $\mathrm{N}$ & 1,960 & 2,062 & 2,012 & 4,022 & & \\
\hline \multicolumn{7}{|c|}{ Avoid mosquito bites } \\
\hline No & 45.6 & 49.8 & 39.3 & 47.9 & 2,733 & 44.042 \\
\hline Yes & 54.4 & 50.2 & 60.8 & 52.1 & 3,301 & 1.311 \\
\hline Total & 100.0 & 100.0 & 100.0 & 100.0 & 6,034 & \\
\hline N & 1,960 & 2,062 & 2,012 & 4,022 & & \\
\hline \multicolumn{7}{|c|}{ Take preventative medication } \\
\hline No & 84.8 & 86.7 & 74.3 & 85.8 & 4,921 & 117.098 \\
\hline Yes & 15.3 & 13.3 & 25.8 & 14.2 & 1,113 & 4.030 \\
\hline Total & 100.0 & 100.0 & 100.0 & 100.0 & 6,034 & * \\
\hline $\mathrm{N}$ & 1,960 & 2,062 & 2,012 & 4,022 & & \\
\hline \multicolumn{7}{|c|}{ Spray house with insecticide } \\
\hline No & 77.4 & 86.7 & 78.8 & 82.4 & 4,854 & 11.896 \\
\hline Yes & 22.6 & 13.3 & 21.2 & 17.6 & 1,180 & 0.347 \\
\hline Total & 100.0 & 100.0 & 100.0 & 100.0 & 6,034 & \\
\hline N & 1,960 & 2,062 & 2,012 & 4,022 & & \\
\hline \multicolumn{7}{|c|}{ Cut grass around house } \\
\hline No & 74.2 & 90.7 & 81.9 & 83.1 & 4,946 & 1.551 \\
\hline Yes & 25.8 & 9.3 & 18.1 & 16.9 & 1,088 & 0.045 \\
\hline Total & 100.0 & 100.0 & 100.0 & 100.0 & 6,034 & \\
\hline $\mathrm{N}$ & 1,960 & 2,062 & 2,012 & 4,022 & & \\
\hline \multicolumn{7}{|c|}{ Fill puddles/stagnant water } \\
\hline No & 88.9 & 97.0 & 94.1 & 93.3 & 5,621 & 1.634 \\
\hline Yes & 11.1 & 3.0 & 5.9 & 6.8 & 413 & 0.060 \\
\hline Total & 100.0 & 100.0 & 100.0 & 100.0 & 6,034 & \\
\hline N & 1,960 & 2,062 & 2,012 & 4,022 & & \\
\hline \multicolumn{7}{|c|}{ Keep house surroundings clean } \\
\hline No & 77.7 & 90.7 & 78.8 & 84.7 & 4,929 & 33.155 \\
\hline Yes & 22.3 & 9.3 & 21.2 & 15.3 & 1,105 & 1.095 \\
\hline Total & 100.0 & 100.0 & 100.0 & 100.0 & 6,034 & \\
\hline $\mathrm{N}$ & 1,960 & 2,062 & 2,012 & 4,022 & & \\
\hline \multicolumn{7}{|c|}{ Burn leaves } \\
\hline No & 93.8 & 96.8 & 92.4 & 95.4 & 5,684 & 21.842 \\
\hline Yes & 6.2 & 3.2 & 7.6 & 4.6 & 350 & 0.666 \\
\hline Total & 100.0 & 100.0 & 100.0 & 100.0 & 6,034 & \\
\hline$N$ & 1,960 & 2,062 & 2,012 & 4,022 & & \\
\hline \multicolumn{7}{|c|}{ Don't know } \\
\hline No & 96.5 & 96.1 & 99.7 & 96.3 & 5,878 & 102.223 \\
\hline Yes & 3.5 & 3.9 & 0.3 & 3.7 & 156 & 20.041 \\
\hline Total & 100.0 & 100.0 & 100.0 & 100.0 & 6,034 & $* \star \star$ \\
\hline $\mathrm{N}$ & 1,960 & 2,062 & 2,012 & 4,022 & & \\
\hline
\end{tabular}

Estimates based on low Ns (approx. $<30$ obs) have large relative standard errors and should be interpreted with caution. ${ }^{* * *}$ indicates $p<0.001,{ }^{* *}$ indicates $p<0.05$ and ${ }^{*}$ indicates $p<.0 .01$ for the chi ${ }^{2}$ statistic comparing integrated versus malaria-only study areas. 
TABLE 2.4.3. MALARIA-RELATED BELIEFS, INTENTIONS, AND SELF-EFFICACY

Percentage of women aged 15 to 49 years currently pregnant or with a child under 2 years reporting malaria beliefs, intentions or self-efficacy, September-October 2019

\begin{tabular}{|c|c|c|c|c|c|c|}
\hline & $\begin{array}{c}\text { KEBBI } \\
\%\end{array}$ & $\begin{array}{c}\text { SOKOTO } \\
\%\end{array}$ & $\begin{array}{c}\text { MALARIA-ONLY } \\
\text { (ZAMFARA) } \\
\%\end{array}$ & $\begin{array}{c}\text { INTEGRATED } \\
\text { (KEBBI/SOKOTO) } \\
\%\end{array}$ & $\mathbf{N}$ & $\begin{array}{c}\mathrm{CHI}^{2} \\
\text { VALUE }\end{array}$ \\
\hline \multicolumn{7}{|c|}{$\begin{array}{l}\text { When a child has fever it's almost } \\
\text { always caused by malaria }\end{array}$} \\
\hline Agree & 86.6 & 85.3 & 85.5 & 85.9 & 5,180 & 60.563 \\
\hline Disagree & 6.5 & 10.3 & 12.3 & 8.6 & 572 & 1.192 \\
\hline Don't know & 6.9 & 4.3 & 2.3 & 5.5 & 281 & \\
\hline Total & 100.0 & 100.0 & 100.0 & 100.0 & 6,033 & \\
\hline$N$ & 1,960 & 2,062 & 2,011 & 4,022 & & \\
\hline \multicolumn{7}{|c|}{$\begin{array}{l}\text { Blood test is only way to know if a } \\
\text { person has malaria }\end{array}$} \\
\hline Agree & 74.8 & 60.6 & 79.4 & 67.2 & 4,364 & 115.751 \\
\hline Disagree & 15.5 & 23.5 & 11.8 & 19.8 & 1,000 & 2.026 \\
\hline Don't know & 9.7 & 16.0 & 8.8 & 13.1 & 669 & \\
\hline Total & 100.0 & 100.0 & 100.0 & 100.0 & 6,033 & \\
\hline $\mathrm{N}$ & 1,960 & 2,062 & 2,011 & 4,022 & & \\
\hline \multicolumn{7}{|c|}{$\begin{array}{l}\text { Even if blood test is negative, I still } \\
\text { worry about malaria }\end{array}$} \\
\hline Agree & 66.2 & 53.8 & 61.8 & 59.5 & 3,665 & 47.656 \\
\hline Disagree & 24.3 & 27.5 & 29.4 & 26.0 & 1,611 & 0.808 \\
\hline Don't know & 9.5 & 18.8 & 8.9 & 14.5 & 757 & \\
\hline Total & 100.0 & 100.0 & 100.0 & 100.0 & 6,033 & \\
\hline$N$ & 1,960 & 2,062 & 2,011 & 4,022 & & \\
\hline \multicolumn{7}{|c|}{$\begin{array}{l}\text { Children under- } 5 \text { should sleep under a } \\
\text { net every night of the year }\end{array}$} \\
\hline Agree & 91.1 & 85.3 & 97.3 & 88.0 & 5,478 & 206.111 \\
\hline Disagree & 5.7 & 10.3 & 1.9 & 8.2 & 383 & 6.801 \\
\hline Don't know & 3.3 & 4.4 & 0.9 & 3.9 & 172 & ** \\
\hline Total & 100.0 & 100.0 & 100.0 & 100.0 & 6,033 & \\
\hline $\mathrm{N}$ & 1,960 & 2,062 & 2,011 & 4,022 & & \\
\hline \multicolumn{7}{|c|}{$\begin{array}{l}\text { Pregnant women should sleep under a } \\
\text { net every night of the year }\end{array}$} \\
\hline Agree & 90.9 & 86.8 & 97.4 & 88.7 & 5,495 & 195.660 \\
\hline Disagree & 5.6 & 9.0 & 2.0 & 7.5 & 380 & 7.864 \\
\hline Don't know & 3.5 & 4.2 & 0.6 & 3.9 & 158 & 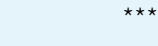 \\
\hline Total & 100.0 & 100.0 & 100.0 & 100.0 & 6,033 & \\
\hline $\mathrm{N}$ & 1,960 & 2,062 & 2,011 & 4,022 & & \\
\hline \multicolumn{7}{|c|}{$\begin{array}{l}\text { When a pregnant woman gets malaria, } \\
\text { it can affect the fetus }\end{array}$} \\
\hline Agree & 88.0 & 84.7 & 94.0 & 86.2 & 5,328 & 117.767 \\
\hline Disagree & 4.1 & 5.1 & 2.9 & 4.6 & 263 & 3.222 \\
\hline Don't know & 7.9 & 10.2 & 3.1 & 9.1 & 442 & \\
\hline Total & 100.0 & 100.0 & 100.0 & 100.0 & 6,033 & \\
\hline $\mathrm{N}$ & 1,960 & 2,062 & 2,011 & 4,022 & & \\
\hline \multicolumn{7}{|c|}{$\begin{array}{l}\text { Medicine given to pregnant women to } \\
\text { prevent malaria works well }\end{array}$} \\
\hline Agree & 88.0 & 83.8 & 91.3 & 85.8 & 5,277 & 66.662 \\
\hline Disagree & 3.9 & 3.4 & 3.7 & 3.7 & 228 & 1.770 \\
\hline Don't know & 8.1 & 12.7 & 5.0 & 10.6 & 528 & \\
\hline Total & 100.0 & 100.0 & 100.0 & 100.0 & 6,033 & \\
\hline $\mathrm{N}$ & 1,960 & 2,062 & 2,011 & 4,022 & & \\
\hline
\end{tabular}




\begin{tabular}{|c|c|c|c|c|c|c|}
\hline \multicolumn{7}{|c|}{$\begin{array}{l}\text { Likelihood to seek treatment for } \\
\text { febrile child the same/next day }\end{array}$} \\
\hline Likely & 89.3 & 84.5 & 96.0 & 86.7 & 5,449 & 192.218 \\
\hline Unlikely & 5.5 & 10.9 & 3.3 & 8.4 & 372 & 4.114 \\
\hline Don't know & 5.2 & 4.6 & 0.7 & 4.9 & 212 & * \\
\hline Total & 100.0 & 100.0 & 100.0 & 100.0 & 6,033 & \\
\hline $\mathrm{N}$ & 1,960 & 2,062 & 2,011 & 4,022 & & \\
\hline \multicolumn{7}{|c|}{$\begin{array}{l}\text { Likelihood to get all children under- } 5 \\
\text { sleeping under net every night }\end{array}$} \\
\hline Likely & 89.7 & 84.2 & 96.6 & 86.7 & 5,397 & 208.749 \\
\hline Unlikely & 7.3 & 11.3 & 2.8 & 9.4 & 475 & 6.295 \\
\hline Don't know & 3.0 & 4.6 & 0.7 & 3.8 & 161 & ** \\
\hline Total & 100.0 & 100.0 & 100.0 & 100.0 & 6,033 & \\
\hline$N$ & 1,960 & 2,062 & 2,011 & 4,022 & & \\
\hline \multicolumn{7}{|c|}{$\begin{array}{l}\text { Likelihood to get child to take entire } \\
\text { course of malaria medicine }\end{array}$} \\
\hline Likely & 86.9 & 87.5 & 95.1 & 87.2 & 5,422 & 139.727 \\
\hline Unlikely & 9.2 & 7.1 & 4.0 & 8.1 & 420 & 3.381 \\
\hline Don't know & 3.9 & 5.4 & 0.9 & 4.7 & 191 & \\
\hline Total & 100.0 & 100.0 & 100.0 & 100.0 & 6,033 & \\
\hline $\mathrm{N}$ & 1,960 & 2,062 & 2,011 & 4,022 & & \\
\hline
\end{tabular}

Estimates based on low Ns (approx. $<30$ obs) have large relative standard errors and should be interpreted with caution. ${ }^{* *}$ indicates $p<0.05$ and * indicates $p<.0 .01$ for the chi ${ }^{2}$ statistic 


\section{Family planning}

\section{Key findings}

Contraceptive use: There was overall low modern contraceptive use among non-pregnant respondents across study areas, with slightly higher use in Zamfara $(17 \%)$ than in Kebbi/Sokoto (11\%). Modern contraceptive use was lower among respondents in the poorest households (6\% and $7 \%$ ) than in the wealthiest households (24\% and 34\%), as well as among respondents with no schooling ( $8 \%$ and $10 \%$ ) compared with respondents with at least primary school education (18\% and 29\%) in Kebbi/Sokoto and Zamfara, respectively. There was also low modern contraceptive use among postpartum respondents (birth in the past 12 months) at 10 percent (Kebbi/Sokoto) and 14 percent (Zamfara), with similar patterns by sociodemographic characteristics across study areas.

Contraceptive methods and access issues: Most respondents had heard of at least one modern contraceptive method, with the most recognized methods being injectables, implants, and daily pills. There was significantly lower awareness in Kebbi/Sokoto (71\% for injectables, $62 \%$ for implants, $67 \%$ for daily pills) than in Zamfara (85\% for injectables, $77 \%$ for implants, $81 \%$ for daily pills) of these three methods. The most common methods used were injectables (48\%) and implants (25\%) among non-pregnant respondents currently using contraception. Most contraceptive users reported obtaining their current method the last time at a government hospital or government primary health care (PHC), and few respondents $(4 \%)$ reported difficulties in obtaining the method the last time. The most common reasons for not using contraception among current non-users were fatalism ("It's up to God") (25\%), current breastfeeding (23\%), partner opposition (21\%), or respondent opposition (18\%). Among non-users, only 15 percent reported intentions to start contraceptive use in the next six months.

Contraceptive beliefs and attitudes: All respondents were asked about their personal beliefs, attitudes, and perceptions of contraception. Across study areas, approximately half (52\%) of respondents agreed that they do not personally approve of using contraception for birth spacing, underscoring a critical hurdle to Breakthrough ACTION/Nigeria SBC messaging for changing family planning practices. Approximately one in five respondents believed that people in their community would call them bad names or avoid their company if others knew they were using contraception for birth spacing, which further highlights the importance of social norms related to family planning practices. About half of the respondents agreed that religious leaders should speak publicly about using modern contraceptives, which is a main component of Breakthrough ACTION/Nigeria SBC activities. Self-efficacy for negotiating contraceptive use with a partner was low. Only half of the respondents reported confidence in their ability to convince their partner to use modern contraception for birth spacing across study areas. Nevertheless, respondents were asked about perceived benefits of contraception use for birth spacing for both themselves and their children. The most commonly reported benefit for themselves was rest after giving birth (61\% and $71 \%$ ), while for children it was better growth (55\% and 63\%) in Kebbi/Sokoto and Zamfara, respectively.

Contraceptive myths: A large proportion of respondents agreed with various contraceptive myths in Kebbi/Sokoto and Zamfara. About one-third of respondents in Kebbi/ Sokoto agreed that contraception could make a woman permanently infertile, harm a woman's womb, and lead to health problems, compared with about one-quarter of respondents agreeing with those myths in Zamfara. About one in five respondents in Kebbi/Sokoto agreed that contraceptives cause cancer, can result in deformed babies, and can increase promiscuity or reduce sexual urges among women and men. In Zamfara, belief in these myths was lower at around one in 10 respondents, though 16 percent of respondents believed that contraception could cause cancer or deformed babies.

Spousal communication and decision-making: There were overall low rates of spousal communication about contraceptive use, although it was much higher in Zamfara (22\%) than in Kebbi/Sokoto (6\%). Unsurprisingly, respondents also reported that contraceptive use decisions were mainly made by their partners, with higher reports in Kebbi/Sokoto (30\%) than in Zamfara (17\%). When respondents were asked who else influences a woman's decision about contraception use, the most commonly mentioned influencers were no one else (61\%) and partners/spouses (30\%) across study areas. 


\subsection{Contraception: current use, knowledge, and access issues}

\section{TABLE 3.1.A. CONTRACEPTIVE PREVALENCE RATE BY SOCIODEMOGRAPHIC CHARACTERISTICS}

Percentage of non-pregnant women aged 15 to 49 years with a child under 2 years who are using any modern contraceptive method by sociodemographic characteristics, September-October 2019

\begin{tabular}{|c|c|c|c|c|c|c|c|c|}
\hline & \multicolumn{2}{|c|}{ KEBBI } & \multicolumn{2}{|c|}{ SOKOTO } & \multicolumn{2}{|c|}{$\begin{array}{l}\text { MALARIA-ONLY } \\
\text { (ZAMFARA) }\end{array}$} & \multicolumn{2}{|c|}{$\begin{array}{l}\text { INTEGRATED } \\
\text { (KEBBI/SOKOTO) }\end{array}$} \\
\hline & $\%$ & N & $\%$ & $\mathrm{~N}$ & $\%$ & $\mathrm{~N}$ & $\%$ & $\mathbf{N}$ \\
\hline Total & 9.1 & 974 & 11.9 & 1,021 & 15.2 & 1,007 & 10.6 & 1,999 \\
\hline \multicolumn{9}{|l|}{ Household wealth } \\
\hline Lowest & 2.4 & 284 & 9.2 & 324 & 7.6 & 107 & 6.3 & 609 \\
\hline Second & 8.3 & 213 & 7.8 & 208 & 4.8 & 176 & 8.0 & 422 \\
\hline Middle & 6.1 & 170 & 9.3 & 208 & 4.9 & 205 & 7.9 & 379 \\
\hline Fourth & 19.8 & 131 & 13.1 & 126 & 17.8 & 233 & 16.5 & 257 \\
\hline Highest & 18.0 & 176 & 30.5 & 155 & 33.8 & 286 & 24.0 & 332 \\
\hline \multicolumn{9}{|l|}{ Maternal age (in years) } \\
\hline $15-24$ years & 7.0 & 392 & 14.1 & 453 & 13.6 & 388 & 11.0 & 847 \\
\hline $25-34$ years & 8.8 & 454 & 10.3 & 450 & 17.3 & 461 & 9.6 & 906 \\
\hline $35-49$ years & 15.4 & 128 & 9.9 & 118 & 13.0 & 158 & 12.7 & 246 \\
\hline \multicolumn{9}{|c|}{$\begin{array}{l}\text { Maternal education (highest } \\
\text { level attended) }\end{array}$} \\
\hline None & 6.2 & 739 & 9.5 & 85 & 9.5 & 667 & 8.1 & 1546 \\
\hline Primary & 12.0 & 47 & 23.0 & 56 & 29.1 & 50 & 18.2 & 103 \\
\hline Secondary or higher & 3.0 & 102 & 39.8 & 58 & 42.5 & 170 & 33.1 & 161 \\
\hline Islamic & 9.7 & 86 & 14.7 & 102 & 12.2 & 120 & 12.3 & 189 \\
\hline
\end{tabular}

Estimates based on low Ns (approx. $<30$ obs) have large relative standard errors and should be interpreted with caution. ${ }^{* \star *}$ indicates $p<0.001,{ }^{* *}$ indicates $p<0.05$ and * indicates $p<.0 .01$ for the chi ${ }^{2}$ statistic comparing integrated versus malaria-only study areas.

\section{TABLE 3.1.B. POSTPARTUM CONTRACEPTIVE PREVALENCE RATE BY SOCIODEMOGRAPHIC CHARACTERISTICS}

Percentage of postpartum non-pregnant women aged 15 to 49 years (with a child under 1 year) who are using any modern contraceptive method by sociodemographic characteristics, September-October 2019

\begin{tabular}{|c|c|c|c|c|c|c|c|c|}
\hline & \multicolumn{2}{|c|}{ KEBBI } & \multicolumn{2}{|c|}{ SOKOTO } & \multicolumn{2}{|c|}{$\begin{array}{l}\text { MALARIA-ONLY } \\
\text { (ZAMFARA) }\end{array}$} & \multicolumn{2}{|c|}{$\begin{array}{c}\text { INTEGRATED } \\
\text { (KEBBI/SOKOTO) }\end{array}$} \\
\hline & $\%$ & $\mathbf{N}$ & $\%$ & $\mathbf{N}$ & $\%$ & $N$ & $\%$ & $\mathbf{N}$ \\
\hline Total & 9.2 & 473 & 9.7 & 546 & 13.6 & 528 & 9.5 & 1,021 \\
\hline \multicolumn{9}{|l|}{ Household wealth } \\
\hline Lowest & 3.3 & 136 & 5.9 & 162 & 4.8 & 47 & 4.9 & 299 \\
\hline Second & 7.5 & 95 & 8.0 & 126 & 2.5 & 81 & 7.7 & 222 \\
\hline Middle & 5.0 & 4 & 6.9 & 110 & 4.3 & 124 & 6.1 & 194 \\
\hline Fourth & 22.9 & 65 & 11.5 & 70 & 1.7 & 106 & 17.0 & 135 \\
\hline Highest & 15.0 & 93 & 27.2 & 78 & 34.1 & 170 & 20.8 & 171 \\
\hline \multicolumn{9}{|l|}{ Maternal age (in years) } \\
\hline $15-24$ years & 4.7 & 186 & 11.9 & 251 & 10.8 & 216 & 9.2 & 438 \\
\hline 25-34 years & 8.8 & 231 & 8.0 & 230 & 17.1 & 231 & 8.4 & 462 \\
\hline $35-49$ years & 23.4 & 56 & 7.3 & 65 & 11.3 & 81 & 13.9 & 121 \\
\hline \multicolumn{9}{|c|}{$\begin{array}{l}\text { Maternal education (highest } \\
\text { level attended) }\end{array}$} \\
\hline None & 6.7 & 361 & 8.0 & 465 & 6.8 & 367 & 7.5 & 827 \\
\hline Primary & 21.5 & 25 & 28.0 & 24 & 30.1 & 30 & 24.9 & 49 \\
\hline Secondary or higher & 30.7 & 47 & 33.3 & 28 & 44.1 & 94 & 31.6 & 75 \\
\hline Islamic & 4.6 & 40 & 7.9 & 29 & 12.1 & 37 & 6.0 & 70 \\
\hline
\end{tabular}

Estimates based on low Ns (approx. $<30$ obs) have large relative standard errors and should be interpreted with caution. ${ }^{* \star *}$ indicates $p<0.001,{ }^{* *}$ indicates $p<0.05$ and ${ }^{*}$ indicates $p<.0 .01$ for the chi ${ }^{2}$ statistic comparing integrated versus malaria-only study areas. 
TABLE 3.1.1. CURRENT USE OF MODERN CONTRACEPTIVE METHODS

Percentage of non-pregnant women aged 15 to 49 years with a child under 2 years who are using any modern contraceptive method, September-October 2019; Percentage of non-pregnant women aged 15 to 49 years who are postpartum (birth in the past 12 months) who are using any modern contraceptive method, September-October 2019

\begin{tabular}{|c|c|c|c|c|c|c|}
\hline & KEBBI & SOKOTO & $\begin{array}{c}\text { INTEGRATED } \\
\text { (KEBBI/SOKOTO) }\end{array}$ & $\begin{array}{l}\text { MALARIA-ONLY } \\
\text { (ZAMFARA) }\end{array}$ & $\mathbf{N}$ & $\begin{array}{c}\mathrm{CHI}^{2} \\
\text { VALUE }\end{array}$ \\
\hline & $\%$ & $\%$ & $\%$ & $\%$ & & \\
\hline \multicolumn{7}{|c|}{ Non-pregnant women } \\
\hline No & 90.9 & 88.1 & 89.4 & 84.8 & 2,607 & 13.372 \\
\hline Yes & 9.1 & 11.9 & 10.6 & 15.2 & 393 & 1.703 \\
\hline Total & 100.0 & 100.0 & 100.0 & 100.0 & 3,000 & \\
\hline N & 974 & 1,021 & 1,995 & 1,005 & & \\
\hline \multicolumn{7}{|c|}{ Postpartum non-pregnant women } \\
\hline No & 90.8 & 90.3 & 90.5 & 86.4 & 1,359 & 23.415 \\
\hline Yes & 9.2 & 9.7 & 9.5 & 13.6 & 186 & 1.252 \\
\hline Total & 100.0 & 100.0 & 100.0 & 100.0 & 1,545 & \\
\hline $\mathrm{N}$ & 473 & 546 & 1,019 & 526 & & \\
\hline
\end{tabular}

Estimates based on low Ns (approx. $<30$ obs) have large relative standard errors and should be interpreted with caution. ${ }^{* * *}$ indicates $p<0.001,{ }^{* *}$ indicates $p<0.05$ and ${ }^{*}$ indicates $p<.0 .01$ for the chi ${ }^{2}$ statistic comparing integrated versus malaria-only study areas. 
TABLE 3.1.2. CURRENT USE OF CONTRACEPTION BY METHOD TYPE

Percentage of non-pregnant women aged 15 to 49 years with a child under 2 years who are currently using contraception by method type, September-October 2019

\begin{tabular}{|c|c|c|c|c|c|c|}
\hline & KEBBI & SOKOTO & $\begin{array}{l}\text { MALARIA-ONLY } \\
\text { (ZAMFARA) }\end{array}$ & $\begin{array}{l}\text { INTEGRATED } \\
\text { (KEBBI/SOKOTO) }\end{array}$ & $\mathbf{N}$ & $\begin{array}{c}\mathrm{CHI}^{2} \\
\text { VALUE }\end{array}$ \\
\hline & $\%$ & $\%$ & $\%$ & $\%$ & & \\
\hline \multicolumn{7}{|c|}{$\begin{array}{l}\text { Are you or your partner currently using } \\
\text { any method of contraception? }\end{array}$} \\
\hline No & 90.1 & 87.1 & 82.9 & 88.5 & 2,571 & 18.060 \\
\hline Yes & 9.9 & 12.9 & 17.1 & 11.5 & 429 & 2.410 \\
\hline Total & 100.0 & 100.0 & 100.0 & 100.0 & 3,000 & \\
\hline N & 974 & 1,021 & 1,005 & 1,995 & & \\
\hline \multicolumn{7}{|c|}{ Which method are you using? } \\
\hline \multicolumn{7}{|l|}{ IUD } \\
\hline No & 98.5 & 99.6 & 92.4 & 99.2 & 412 & 8.383 \\
\hline Yes & 1.5 & 0.4 & 7.6 & 0.8 & 17 & 9.120 \\
\hline Total & 100.0 & 100.0 & 100.0 & 100.0 & 429 & ** \\
\hline N & 90 & 145 & 194 & 235 & & \\
\hline \multicolumn{7}{|c|}{ Injectables } \\
\hline No & 44.6 & 53.6 & 52.4 & 50.1 & 224 & 0.194 \\
\hline Yes & 55.4 & 46.4 & 47.7 & 49.9 & 205 & 0.083 \\
\hline Total & 100.0 & 100.0 & 100.0 & 100.0 & 429 & \\
\hline $\mathrm{N}$ & 90 & 145 & 194 & 235 & & \\
\hline \multicolumn{7}{|c|}{ Implants } \\
\hline No & 75.0 & 67.1 & 77.2 & 70.3 & 311 & 2.423 \\
\hline Yes & 25.0 & 32.9 & 22.8 & 29.8 & 118 & 1.105 \\
\hline Total & 100.0 & 100.0 & 100.0 & 100.0 & 429 & \\
\hline N & 90 & 145 & 194 & 235 & & \\
\hline \multicolumn{7}{|c|}{ Daily pill } \\
\hline No & 89.0 & 84.1 & 81.3 & 86.1 & 356 & 1.508 \\
\hline Yes & 11.0 & 15.9 & 18.7 & 13.9 & 73 & 1.109 \\
\hline Total & 100.0 & 100.0 & 100.0 & 100.0 & 429 & \\
\hline$N$ & 90 & 145 & 194 & 235 & & \\
\hline \multicolumn{7}{|c|}{ Male condom } \\
\hline No & 97.6 & 100.0 & 97.7 & 99.1 & 421 & 0.918 \\
\hline Yes & 2.4 & 0.0 & 2.3 & 0.9 & 8 & 1.009 \\
\hline Total & 100.0 & 100.0 & 100.0 & 100.0 & 429 & \\
\hline $\mathrm{N}$ & 90 & 145 & 194 & 235 & & \\
\hline \multicolumn{7}{|c|}{ Withdrawal } \\
\hline No & 96.8 & 98.4 & 95.3 & 97.8 & 412 & 1.475 \\
\hline Yes & 3.2 & 1.6 & 4.7 & 2.2 & 17 & 0.884 \\
\hline Total & 100.0 & 100.0 & 100.0 & 100.0 & 429 & \\
\hline $\mathrm{N}$ & 90 & 145 & 194 & 235 & & \\
\hline
\end{tabular}

Estimates based on low Ns (approx. $<30$ obs) have large relative standard errors and should be interpreted with caution. ${ }^{* * *}$ indicates $p<0.001,{ }^{* *}$ indicates $p<0.05$ and ${ }^{*}$ indicates $p<.0 .01$ for the chi $^{2}$ statistic comparing integrated versus malaria-only study areas. 
TABLE 3.1.3. HAVE YOU EVER HEARD OF THE FOLLOWING CONTRACEPTION METHODS?

Percentage of women aged 15 to 49 years currently pregnant or with a child under 2 years who have ever heard of contraceptive methods by type, September-October 2019

\begin{tabular}{|c|c|c|c|c|c|c|}
\hline & $\begin{array}{c}\text { KEBBI } \\
\%\end{array}$ & $\begin{array}{c}\text { SOKOTO } \\
\%\end{array}$ & $\begin{array}{c}\text { MALARIA-ONLY } \\
\text { (ZAMFARA) } \\
\%\end{array}$ & $\begin{array}{c}\text { INTEGRATED } \\
\text { (KEBBI/SOKOTO) } \\
\%\end{array}$ & $\mathbf{N}$ & $\begin{array}{c}\mathrm{CHI}^{2} \\
\text { VALUE }\end{array}$ \\
\hline \multicolumn{7}{|l|}{ Male condom } \\
\hline Mentioned spontaneously & 8.2 & 8.9 & 14.6 & 8.6 & 702 & 52.844 \\
\hline Recognised upon listening & 25.5 & 17.3 & 21.7 & 21.1 & 1,341 & 1.033 \\
\hline Neither mentioned nor recognised & 66.3 & 73.8 & 63.7 & 70.3 & 3,990 & \\
\hline Total & 100.0 & 100.0 & 100.0 & 100.0 & 6,033 & \\
\hline N & 1,960 & 2,062 & 2,011 & 4,022 & & \\
\hline \multicolumn{7}{|l|}{ Female sterilization/ tubal ligation } \\
\hline Mentioned spontaneously & 13.3 & 4.5 & 8.0 & 8.5 & 497 & 8.398 \\
\hline Recognised upon listening & 16.8 & 15.8 & 19.1 & 16.2 & 1,091 & 0.126 \\
\hline Neither mentioned nor recognised & 69.9 & 79.8 & 72.9 & 75.2 & 4,445 & \\
\hline Total & 100.0 & 100.0 & 100.0 & 100.0 & 6,033 & \\
\hline N & 1,960 & 2,062 & 2,011 & 4,022 & & \\
\hline \multicolumn{7}{|l|}{ Male sterilization/vasectomy } \\
\hline Mentioned spontaneously & 7.6 & 3.9 & 6.2 & 5.6 & 371 & 23.283 \\
\hline Recognised upon listening & 10.6 & 3.7 & 10.3 & 6.9 & 517 & 0.409 \\
\hline Neither mentioned nor recognised & 81.8 & 92.4 & 83.4 & 87.5 & 5,145 & \\
\hline Total & 100.0 & 100.0 & 100.0 & 100.0 & 6,033 & \\
\hline N & 1,960 & 2,062 & 2,011 & 4,022 & & \\
\hline \multicolumn{7}{|l|}{ IUD } \\
\hline Mentioned spontaneously & 10.6 & 9.4 & 18.7 & 10.0 & 825 & 87.544 \\
\hline Recognised upon listening & 15.5 & 15.9 & 14.7 & 15.7 & 970 & 1.781 \\
\hline Neither mentioned nor recognised & 73.9 & 74.7 & 66.6 & 74.3 & 4,238 & \\
\hline Total & 100.0 & 100.0 & 100.0 & 100.0 & 6,033 & \\
\hline N & 1,960 & 2,062 & 2,011 & 4,022 & & \\
\hline \multicolumn{7}{|l|}{ Injectables or injections } \\
\hline Mentioned spontaneously & 35.3 & 49.3 & 63.5 & 42.9 & 2,983 & 269.317 \\
\hline Recognised upon listening & 33.0 & 23.3 & 20.6 & 27.8 & 1,515 & 4.009 \\
\hline Neither mentioned nor recognised & 31.7 & 27.4 & 15.9 & 29.4 & 1,535 & * \\
\hline Total & 100.0 & 100.0 & 100.0 & 100.0 & 6,033 & \\
\hline $\mathrm{N}$ & 1,960 & 2,062 & 2,011 & 4,022 & & \\
\hline \multicolumn{7}{|l|}{ Implants/Implanon/Jadelle } \\
\hline Mentioned spontaneously & 24.8 & 36.0 & 58.4 & 30.8 & 2,400 & 443.199 \\
\hline Recognised upon listening & 37.3 & 26.2 & 18.5 & 31.3 & 1,586 & 7.901 \\
\hline Neither mentioned nor recognised & 37.9 & 37.8 & 23.2 & 37.9 & 2,047 & *** \\
\hline Total & 100.0 & 100.0 & 100.0 & 100.0 & 6,033 & \\
\hline $\mathrm{N}$ & 1,960 & 2,062 & 2,011 & 4,022 & & \\
\hline \multicolumn{7}{|l|}{ Daily pill } \\
\hline Mentioned spontaneously & 35.5 & 38.4 & 56.0 & 37.1 & 2,633 & 236.584 \\
\hline Recognised upon listening & 32.4 & 27.0 & 24.6 & 29.5 & 1,620 & 3.830 \\
\hline Neither mentioned nor recognised & 32.1 & 34.6 & 19.4 & 33.5 & 1,780 & * \\
\hline Total & 100.0 & 100.0 & 100.0 & 100.0 & 6,033 & \\
\hline N & 1,960 & 2,062 & 2,011 & 4,022 & & \\
\hline \multicolumn{7}{|l|}{ Female condom } \\
\hline Mentioned spontaneously & 3.6 & 4.1 & 4.9 & 3.9 & 289 & 4.193 \\
\hline Recognised upon listening & 11.4 & 8.9 & 10.4 & 10.1 & 663 & 0.110 \\
\hline Neither mentioned nor recognised & 85.1 & 87.0 & 84.7 & 86.1 & 5,081 & \\
\hline Total & 100.0 & 100.0 & 100.0 & 100.0 & 6,033 & \\
\hline N & 1,960 & 2,062 & 2,011 & 4,022 & & \\
\hline
\end{tabular}




\begin{tabular}{|c|c|c|c|c|c|c|}
\hline \multicolumn{7}{|l|}{$\begin{array}{l}\text { Emergency contraception/morning } \\
\text { after pill/postinor } 2\end{array}$} \\
\hline Mentioned spontaneously & 3.3 & 4.9 & 7.6 & 4.2 & 363 & 44.362 \\
\hline Recognised upon listening & 13.9 & 8.4 & 7.8 & 10.9 & 609 & 1.038 \\
\hline Neither mentioned nor recognised & 82.9 & 86.7 & 84.6 & 84.9 & 5,061 & \\
\hline Total & 100.0 & 100.0 & 100.0 & 100.0 & 6,033 & \\
\hline N & 1,960 & 2,062 & 2,011 & 4,022 & & \\
\hline \multicolumn{7}{|l|}{ Standard days/cycle beads } \\
\hline Mentioned spontaneously & 5.0 & 3.3 & 3.3 & 4.1 & 261 & 6.174 \\
\hline Recognised upon listening & 16.5 & 5.8 & 12.4 & 10.7 & 700 & 0.203 \\
\hline Neither mentioned nor recognised & 78.5 & 90.9 & 84.3 & 85.2 & 5,072 & \\
\hline Total & 100.0 & 100.0 & 100.0 & 100.0 & 6,033 & \\
\hline N & 1,960 & 2,062 & 2,011 & 4,022 & & \\
\hline \multicolumn{7}{|l|}{ Lactational amenorrhea (LAM) } \\
\hline Mentioned spontaneously & 11.6 & 4.9 & 9.9 & 8.0 & 536 & 7.940 \\
\hline Recognised upon listening & 29.1 & 19.9 & 22.3 & 24.2 & 1,372 & 0.117 \\
\hline Neither mentioned nor recognised & 59.3 & 75.2 & 67.9 & 67.9 & 4,125 & . \\
\hline Total & 100.0 & 100.0 & 100.0 & 100.0 & 6,033 & \\
\hline N & 1,960 & 2,062 & 2,011 & 4,022 & & \\
\hline \multicolumn{7}{|l|}{ Rhythm method } \\
\hline Mentioned spontaneously & 5.9 & 1.6 & 1.5 & 3.6 & 195 & 29.511 \\
\hline Recognised upon listening & 16.6 & 4.5 & 10.7 & 10.1 & 641 & 1.055 \\
\hline Neither mentioned nor recognised & 77.6 & 93.9 & 87.8 & 86.3 & 5,197 & . \\
\hline Total & 100.0 & 100.0 & 100.0 & 100.0 & 6,033 & \\
\hline N & 1,960 & 2,062 & 2,011 & 4,022 & & \\
\hline \multicolumn{7}{|l|}{ Withdrawal } \\
\hline Mentioned spontaneously & 8.8 & 7.9 & 10.0 & 8.3 & 543 & 18.484 \\
\hline Recognised upon listening & 29.5 & 17.8 & 18.9 & 23.2 & 1,262 & 0.285 \\
\hline Neither mentioned nor recognised & 61.8 & 74.4 & 71.1 & 68.6 & 4,228 & \\
\hline Total & 100.0 & 100.0 & 100.0 & 100.0 & 6,033 & \\
\hline $\mathrm{N}$ & 1,960 & 2,062 & 2,011 & 4,022 & & \\
\hline \multicolumn{7}{|l|}{ Spermicide } \\
\hline Mentioned spontaneously & 3.4 & 1.2 & 0.6 & 2.2 & 126 & 31.253 \\
\hline Recognised upon listening & 6.4 & 2.7 & 4.2 & 4.4 & 293 & 1.384 \\
\hline Neither mentioned nor recognised & 90.1 & 96.2 & 95.2 & 93.4 & 5,614 & \\
\hline Total & 100.0 & 100.0 & 100.0 & 100.0 & 6,033 & \\
\hline N & 1,960 & 2,062 & 2,011 & 4,022 & & \\
\hline \multicolumn{7}{|l|}{ Diaphragm } \\
\hline Mentioned spontaneously & 3.8 & 1.3 & 0.4 & 2.5 & 139 & 49.552 \\
\hline Recognised upon listening & 5.9 & 2.4 & 4.5 & 4.0 & 273 & 2.616 \\
\hline Neither mentioned nor recognised & 90.4 & 96.3 & 95.1 & 93.5 & 5,621 & \\
\hline Total & 100.0 & 100.0 & 100.0 & 100.0 & 6,033 & \\
\hline N & 1,960 & 2,062 & 2,011 & 4,022 & & \\
\hline \multicolumn{7}{|l|}{ Sayana Press } \\
\hline Mentioned spontaneously & 5.6 & 1.9 & 3.0 & 3.6 & 247 & 30.061 \\
\hline Recognised upon listening & 8.3 & 5.4 & 3.7 & 6.8 & 376 & 0.463 \\
\hline Neither mentioned nor recognised & 86.1 & 92.7 & 93.2 & 89.6 & 5,410 & . \\
\hline Total & 100.0 & 100.0 & 100.0 & 100.0 & 6,033 & \\
\hline $\mathrm{N}$ & 1,960 & 2,062 & 2,011 & 4,022 & & \\
\hline
\end{tabular}

Estimates based on low Ns (approx. $<30$ obs) have large relative standard errors and should be interpreted with caution. ${ }^{* \star *}$ indicates $p<0.001,{ }^{* *}$ indicates $p<0.05$ and ${ }^{*}$ indicates $p<.0 .01$ for the chi $^{2}$ statistic comparing integrated versus malaria-only study areas. 
TABLE 3.1.4. WHERE DID YOU OBTAIN THE [CURRENT METHOD] THE LAST TIME?

Percentage of non-pregnant women aged 15 to 49 years with a child under 2 years using any contraception method by location obtained the last time, September-October 2019

\begin{tabular}{|c|c|c|c|c|c|c|}
\hline & KEBBI & SOKOTO & $\begin{array}{l}\text { MALARIA-ONLY } \\
\text { (ZAMFARA) }\end{array}$ & $\begin{array}{c}\text { INTEGRATED } \\
\text { (KEBBI/SOKOTO) }\end{array}$ & $\mathbf{N}$ & $\begin{array}{c}\mathrm{CHI}^{2} \\
\text { VALUE }\end{array}$ \\
\hline & $\%$ & $\%$ & $\%$ & $\%$ & & \\
\hline Other private sector & 4.7 & 0.0 & 1.2 & 1.9 & 4 & \\
\hline Other public sector & 0.0 & 0.4 & 0.0 & 0.2 & 1 & \\
\hline Government hospital & 33.8 & 33.6 & 47.8 & 33.7 & 194 & \\
\hline Governmental PHC & 43.6 & 28.8 & 20.6 & 34.6 & 102 & \\
\hline Dispensary/health post & 2.3 & 12.3 & 2.0 & 8.3 & 24 & \\
\hline Community health outreach post & 1.2 & 0.8 & 1.3 & 0.9 & 4 & \\
\hline Nursing/maternity home & 2.9 & 0.4 & 1.9 & 1.4 & 9 & \\
\hline Private hospital/clinic & 3.5 & 2.3 & 1.4 & 2.8 & 10 & \\
\hline Pharmacy/chemist & 7.0 & 10.4 & 9.0 & 9.1 & 38 & \\
\hline Don't know & 0.0 & 0.0 & 1.3 & 0.0 & 3 & 27.880 \\
\hline Other & 1.0 & 11.3 & 13.6 & 7.2 & 40 & 1.329 \\
\hline Total & 100.0 & 100.0 & 100.0 & 100.0 & 429 & \\
\hline $\mathrm{N}$ & 90 & 145 & 194 & 235 & & \\
\hline
\end{tabular}

Estimates based on low Ns (approx. $<30$ obs) have large relative standard errors and should be interpreted with caution. ${ }^{* *}$ indicates $p<0.05$ and ${ }^{*}$ indicates $p<.0 .01$ for the chi ${ }^{2}$ statistic 


\section{TABLE 3.1.5. DIFFICULTIES FACED OBTAINING [CURRENT METHOD] THE LAST TIME?}

Percentage of non-pregnant women aged 15 to 49 years with a child under 2 years using any contraception method by difficulties faced in obtaining method last time, September-October 2019

\begin{tabular}{|c|c|c|c|c|c|c|}
\hline & $\begin{array}{c}\text { KEBBI } \\
\%\end{array}$ & $\begin{array}{l}\text { SOKOTO } \\
\%\end{array}$ & $\begin{array}{c}\text { MALARIA-ONLY } \\
\text { (ZAMFARA) } \\
\%\end{array}$ & $\begin{array}{c}\text { INTEGRATED } \\
\text { (KEBBI/SOKOTO) } \\
\%\end{array}$ & $\mathbf{N}$ & $\begin{array}{c}\mathrm{CHI}^{2} \\
\text { VALUE }\end{array}$ \\
\hline \multicolumn{7}{|c|}{$\begin{array}{l}\text { Have you or your partner had any difficul- } \\
\text { ties in obtaining CURRENT METHOD? }\end{array}$} \\
\hline No & 94.1 & 86.2 & 98.7 & 89.3 & 399 & 20.106 \\
\hline Yes & 5.9 & 13.8 & 1.3 & 10.7 & 30 & 10.516 \\
\hline Total & 100.0 & 100.0 & 100.0 & 100.0 & 429 & ** \\
\hline $\mathrm{N}$ & 90 & 145 & 194 & 235 & & \\
\hline \multicolumn{7}{|c|}{$\begin{array}{l}\text { Difficulties faced in obtaining CURRENT } \\
\text { METHOD? }\end{array}$} \\
\hline \multicolumn{7}{|c|}{$\begin{array}{l}\text { Fear of partner knowing; he opposes } \\
\text { contraception }\end{array}$} \\
\hline No & 80.2 & 74.7 & 56.9 & 76.0 & 23 & 0.875 \\
\hline Yes & 19.8 & 25.3 & 43.1 & 24.1 & 7 & 0.181 \\
\hline Total & 100.0 & 100.0 & 100.0 & 100.0 & 30 & \\
\hline $\mathrm{N}$ & 7 & 21 & 2 & 28 & & \\
\hline \multicolumn{7}{|c|}{ Fear of parents/other relatives knowing } \\
\hline No & 54.6 & 90.6 & 100.0 & 82.7 & 25 & 1.221 \\
\hline Yes & 45.4 & 9.4 & 0.0 & 17.3 & 5 & 1.188 \\
\hline Total & 100.0 & 100.0 & 100.0 & 100.0 & 30 & \\
\hline $\mathrm{N}$ & 7 & 21 & 2 & 28 & & \\
\hline \multicolumn{7}{|c|}{ High cost of transportation to facility } \\
\hline No & 80.2 & 83.6 & 100.0 & 82.9 & 25 & 1.209 \\
\hline Yes & 19.8 & 16.4 & 0.0 & 17.2 & 5 & 1.706 \\
\hline Total & 100.0 & 100.0 & 100.0 & 100.0 & 30 & \\
\hline $\mathrm{N}$ & 7 & 21 & 2 & 28 & & \\
\hline \multicolumn{7}{|c|}{ Long waiting times at facility } \\
\hline No & 65.0 & 86.1 & 100.0 & 81.5 & 24 & 1.324 \\
\hline Yes & 35.0 & 13.9 & 0.0 & 18.5 & 6 & 1.377 \\
\hline Total & 100.0 & 100.0 & 100.0 & 100.0 & 30 & \\
\hline $\mathrm{N}$ & 7 & 21 & 2 & 28 & & \\
\hline \multicolumn{7}{|c|}{ Periodic stock-outs at facility } \\
\hline No & 100.0 & 70.5 & 100.0 & 77.0 & 24 & 1.717 \\
\hline Yes & 0.0 & 29.5 & 0.0 & 23.0 & 6 & 0.906 \\
\hline Total & 100.0 & 100.0 & 100.0 & 100.0 & 30 & \\
\hline N & 7 & 21 & 2 & 28 & & \\
\hline
\end{tabular}

Estimates based on low Ns (approx. $<30$ obs) have large relative standard errors and should be interpreted with caution. ${ }^{* \star *}$ indicates $p<0.001,{ }^{* *}$ indicates $p<0.05$ and ${ }^{*}$ indicates $p<.0 .01$ for the chi ${ }^{2}$ statistic comparing integrated versus malaria-only study areas. 


\subsection{Contraception: reasons for use/non-use and intentions}

\section{TABLE 3.2.1. REASONS FOR USING A CONTRACEPTIVE METHOD}

Percentage of non-pregnant women aged 15 to 49 years with a child under 2 years using any contraception method by reasons for method use, September-October 2019

\begin{tabular}{|c|c|c|c|c|c|c|c|}
\hline & KEBBI & SOKото & $\begin{array}{l}\text { MALARIA-ONLY } \\
\text { (ZAMFARA) }\end{array}$ & $\begin{array}{l}\text { INTEGRATED } \\
\text { (KEBBI/SOKOTO) }\end{array}$ & TOTAL & $\mathbf{N}$ & $\begin{array}{c}\mathrm{CHI}^{2} \\
\text { VALUE }\end{array}$ \\
\hline & $\%$ & $\%$ & $\%$ & $\%$ & $\%$ & & \\
\hline \multicolumn{8}{|c|}{$\begin{array}{l}\text { Prefer to wait before having } \\
\text { more children }\end{array}$} \\
\hline No & 23.7 & 20.4 & 23.4 & 21.7 & 22.8 & 100 & 0.146 \\
\hline Yes & 76.3 & 79.6 & 76.7 & 78.3 & 77.2 & 329 & 0.035 \\
\hline Total & 100.0 & 100.0 & 100.0 & 100.0 & 100.0 & 429 & \\
\hline $\mathrm{N}$ & 90 & 145 & 194 & 235 & 429 & & \\
\hline \multicolumn{8}{|c|}{$\begin{array}{l}\text { Does not want any more } \\
\text { children }\end{array}$} \\
\hline No & 85.8 & 94.6 & 89.2 & 91.1 & 89.8 & 386 & 0.374 \\
\hline Yes & 14.2 & 5.4 & 10.8 & 8.9 & 10.2 & 43 & 0.213 \\
\hline Total & 100.0 & 100.0 & 100.0 & 100.0 & 100.0 & 429 & \\
\hline $\mathrm{N}$ & 90 & 145 & 194 & 235 & 429 & & \\
\hline \multicolumn{8}{|c|}{$\begin{array}{l}\text { My partner wants to use } \\
\text { contraception }\end{array}$} \\
\hline No & 72.8 & 60.5 & 68.7 & 65.4 & 67.6 & 291 & 0.452 \\
\hline Yes & 27.2 & 39.5 & 31.4 & 34.6 & 32.4 & 138 & 0.105 \\
\hline Total & 100.0 & 100.0 & 100.0 & 100.0 & 100.0 & 429 & \\
\hline$N$ & 90 & 145 & 194 & 235 & 429 & & \\
\hline \multicolumn{8}{|c|}{$\begin{array}{l}\text { Health provider says I should } \\
\text { use contraception }\end{array}$} \\
\hline No & 93.4 & 94.6 & 92.9 & 94.1 & 93.3 & 402 & 0.233 \\
\hline Yes & 6.6 & 5.4 & 7.2 & 5.9 & 6.7 & 27 & 0.177 \\
\hline Total & 100.0 & 100.0 & 100.0 & 100.0 & 100.0 & 429 & \\
\hline $\mathrm{N}$ & 90 & 145 & 194 & 235 & 429 & & \\
\hline \multicolumn{8}{|c|}{ Protect against STIs } \\
\hline No & 97.8 & 100.0 & 98.9 & 99.1 & 99.0 & 424 & 0.040 \\
\hline Yes & 2.3 & 0.0 & 1.1 & 0.9 & 1.0 & 5 & 0.043 \\
\hline Total & 100.0 & 100.0 & 100.0 & 100.0 & 100.0 & 429 & \\
\hline $\mathrm{N}$ & 90 & 145 & 194 & 235 & 429 & & \\
\hline
\end{tabular}

Estimates based on low Ns (approx. $<30$ obs) have large relative standard errors and should be interpreted with caution. ${ }^{\star \star *}$ indicates $p<0.001,{ }^{\star *}$ indicates $p<0.05$ and * indicates $p<.0 .01$ for the chi ${ }^{2}$ statistic comparing integrated versus malaria-only study areas. 
TABLE 3.2.2. REASONS FOR NOT USING A CONTRACEPTIVE METHOD

Percentage of non-pregnant women aged 15 to 49 years with a child under 2 years not using any contraception method by reasons for method non-use, September-October 2019

\begin{tabular}{|c|c|c|c|c|c|c|}
\hline & $\begin{array}{c}\text { KEBBI } \\
\%\end{array}$ & $\begin{array}{c}\text { SOKOTO } \\
\%\end{array}$ & $\begin{array}{c}\text { MALARIA-ONLY } \\
\text { (ZAMFARA) } \\
\%\end{array}$ & $\begin{array}{c}\text { INTEGRATED } \\
\text { (KEBBI/SOKOTO) } \\
\%\end{array}$ & $\mathbf{N}$ & $\begin{array}{c}\mathrm{CHI}^{2} \\
\text { VALUE }\end{array}$ \\
\hline \multicolumn{7}{|c|}{ Knows no method } \\
\hline No & 96.1 & 98.9 & 99.3 & 97.6 & 2,522 & 13.186 \\
\hline Yes & 3.9 & 1.2 & 0.7 & 2.4 & 49 & 3.058 \\
\hline Total & 100.0 & 100.0 & 100.0 & 100.0 & 2,571 & \\
\hline N & 884 & 876 & 811 & 1,760 & & \\
\hline \multicolumn{7}{|c|}{ Doesn't know where to get method } \\
\hline No & 98.8 & 99.2 & 99.2 & 99.0 & 2,551 & 0.171 \\
\hline Yes & 1.2 & 0.8 & 0.8 & 1.0 & 20 & 0.081 \\
\hline Total & 100.0 & 100.0 & 100.0 & 100.0 & 2,571 & \\
\hline N & 884 & 876 & 811 & 1,760 & & \\
\hline \multicolumn{7}{|c|}{ Health concerns/Fear of side effects } \\
\hline No & 97.1 & 96.8 & 97.2 & 96.9 & 2,501 & 0.215 \\
\hline Yes & 2.9 & 3.2 & 2.8 & 3.1 & 70 & 0.052 \\
\hline Total & 100.0 & 100.0 & 100.0 & 100.0 & 2,571 & \\
\hline $\mathrm{N}$ & 884 & 876 & 811 & 1,760 & & \\
\hline \multicolumn{7}{|c|}{ Distance to health facility too far } \\
\hline No & 99.1 & 100.0 & 99.7 & 99.6 & 2,561 & 0.131 \\
\hline Yes & 0.9 & 0.0 & 0.4 & 0.4 & 10 & 0.052 \\
\hline Total & 100.0 & 100.0 & 100.0 & 100.0 & 2,571 & \\
\hline N & 884 & 876 & 811 & 1,760 & & \\
\hline \multicolumn{7}{|c|}{$\begin{array}{l}\text { Difficult to get transport to health } \\
\text { facility }\end{array}$} \\
\hline No & 99.3 & 99.5 & 99.7 & 99.4 & 2,558 & 1.054 \\
\hline Yes & 0.7 & 0.5 & 0.3 & 0.6 & 13 & 0.491 \\
\hline Total & 100.0 & 100.0 & 100.0 & 100.0 & 2,571 & \\
\hline $\mathrm{N}$ & 884 & 876 & 811 & 1,760 & & \\
\hline \multicolumn{7}{|c|}{ Costs too much } \\
\hline No & 99.5 & 99.3 & 99.6 & 99.4 & 2,555 & 0.350 \\
\hline Yes & 0.5 & 0.7 & 0.4 & 0.6 & 16 & 0.295 \\
\hline Total & 100.0 & 100.0 & 100.0 & 100.0 & 2,571 & \\
\hline $\mathrm{N}$ & 884 & 876 & 811 & 1,760 & & \\
\hline \multicolumn{7}{|c|}{ Inconvenient to use } \\
\hline No & 97.6 & 96.8 & 97.7 & 97.2 & 2,498 & 0.748 \\
\hline Yes & 2.4 & 3.2 & 2.3 & 2.8 & 73 & 0.108 \\
\hline Total & 100.0 & 100.0 & 100.0 & 100.0 & 2,571 & \\
\hline N & 884 & 876 & 811 & 1,760 & & \\
\hline \multicolumn{7}{|c|}{ Preferred method not available } \\
\hline No & 99.8 & 100.0 & 99.9 & 99.9 & 2,567 & 0.002 \\
\hline Yes & 0.2 & 0.0 & 0.1 & 0.1 & 4 & 0.002 \\
\hline Total & 100.0 & 100.0 & 100.0 & 100.0 & 2,571 & \\
\hline N & 884 & 876 & 811 & 1,760 & & \\
\hline \multicolumn{7}{|c|}{ Fear of infertility } \\
\hline No & 86.5 & 90.0 & 94.7 & 88.4 & 2,323 & 33.650 \\
\hline Yes & 13.5 & 10.0 & 5.4 & 11.6 & 248 & 3.795 \\
\hline Total & 100.0 & 100.0 & 100.0 & 100.0 & 2,571 & \\
\hline N & 884 & 876 & 811 & 1,760 & & \\
\hline
\end{tabular}




\begin{tabular}{|c|c|c|c|c|c|c|}
\hline \multicolumn{7}{|c|}{ Infrequent sex/not having sex } \\
\hline No & 98.2 & 98.8 & 97.5 & 98.5 & 2,523 & 3.140 \\
\hline Yes & 1.8 & 1.2 & 2.5 & 1.5 & 48 & 0.992 \\
\hline Total & 100.0 & 100.0 & 100.0 & 100.0 & 2,571 & \\
\hline N & 884 & 876 & 811 & 1,760 & & \\
\hline \multicolumn{7}{|c|}{ Can't get pregnant } \\
\hline No & 94.6 & 98.9 & 93.7 & 96.9 & 2,458 & 14.134 \\
\hline Yes & 5.4 & 1.1 & 6.3 & 3.1 & 113 & 1.723 \\
\hline Total & 100.0 & 100.0 & 100.0 & 100.0 & 2,571 & . \\
\hline N & 884 & 876 & 811 & 1,760 & & \\
\hline \multicolumn{7}{|c|}{ Breastfeeding } \\
\hline No & 83.8 & 88.6 & 69.8 & 86.3 & 2,069 & 96.863 \\
\hline Yes & 16.2 & 11.4 & 30.2 & 13.7 & 502 & 8.956 \\
\hline Total & 100.0 & 100.0 & 100.0 & 100.0 & 2,571 & ** \\
\hline $\mathrm{N}$ & 884 & 876 & 811 & 1,760 & & \\
\hline \multicolumn{7}{|c|}{ Away from husband/partner } \\
\hline No & 98.9 & 97.2 & 97.3 & 98.0 & 2,512 & 1.470 \\
\hline Yes & 1.1 & 2.8 & 2.7 & 2.0 & 59 & 0.810 \\
\hline Total & 100.0 & 100.0 & 100.0 & 100.0 & 2,571 & \\
\hline N & 884 & 876 & 811 & 1,760 & & \\
\hline \multicolumn{7}{|c|}{ Trying to get pregnant } \\
\hline No & 98.3 & 96.6 & 98.7 & 97.4 & 2,511 & 5.610 \\
\hline Yes & 1.7 & 3.4 & 1.3 & 2.6 & 60 & 1.952 \\
\hline Total & 100.0 & 100.0 & 100.0 & 100.0 & 2,571 & . \\
\hline N & 884 & 876 & 811 & 1,760 & & \\
\hline \multicolumn{7}{|c|}{ Wants more children } \\
\hline No & 83.2 & 85.2 & 88.3 & 84.3 & 2,193 & 8.840 \\
\hline Yes & 16.8 & 14.8 & 11.7 & 15.7 & 378 & 0.651 \\
\hline Total & 100.0 & 100.0 & 100.0 & 100.0 & 2,571 & \\
\hline N & 884 & 876 & 811 & 1,760 & & \\
\hline \multicolumn{7}{|c|}{ Respondent opposes } \\
\hline No & 87.7 & 84.0 & 79.4 & 85.7 & 2,163 & 16.840 \\
\hline Yes & 12.3 & 16.1 & 20.6 & 14.3 & 408 & 1.729 \\
\hline Total & 100.0 & 100.0 & 100.0 & 100.0 & 2,571 & . \\
\hline $\mathrm{N}$ & 884 & 876 & 811 & 1,760 & & \\
\hline \multicolumn{7}{|c|}{ Husband/partner opposes } \\
\hline No & 84.7 & 82.2 & 75.5 & 83.4 & 2,087 & 23.633 \\
\hline Yes & 15.3 & 17.8 & 24.5 & 16.6 & 484 & 2.826 \\
\hline Total & 100.0 & 100.0 & 100.0 & 100.0 & 2,571 & . \\
\hline N & 884 & 876 & 811 & 1,760 & & \\
\hline \multicolumn{7}{|c|}{ Others oppose } \\
\hline No & 99.4 & 99.5 & 99.5 & 99.4 & 2,555 & 0.152 \\
\hline Yes & 0.6 & 0.5 & 0.5 & 0.6 & 16 & 0.123 \\
\hline Total & 100.0 & 100.0 & 100.0 & 100.0 & 2,571 & \\
\hline $\mathrm{N}$ & 884 & 876 & 811 & 1,760 & & \\
\hline \multicolumn{7}{|c|}{ Interferes with body's natural process } \\
\hline No & 96.4 & 94.4 & 93.1 & 95.4 & 2,452 & 5.690 \\
\hline Yes & 3.6 & 5.6 & 6.9 & 4.6 & 119 & 0.657 \\
\hline Total & 100.0 & 100.0 & 100.0 & 100.0 & 2,571 & . \\
\hline $\mathrm{N}$ & 884 & 876 & 811 & 1,760 & & \\
\hline \multicolumn{7}{|c|}{ Up to God } \\
\hline No & 73.2 & 65.8 & 79.9 & 69.3 & 1,889 & 37.974 \\
\hline Yes & 26.8 & 34.2 & 20.1 & 30.7 & 682 & 3.037 \\
\hline Total & 100.0 & 100.0 & 100.0 & 100.0 & 2,571 & \\
\hline $\mathrm{N}$ & 884 & 876 & 811 & 1,760 & & \\
\hline
\end{tabular}

Estimates based on low Ns (approx. $<30$ obs) have large relative standard errors and should be interpreted with caution. ${ }^{* * *}$ indicates $p<0.001,{ }^{* *}$ indicates $p<0.05$ and * indicates $p<.0 .01$ for the chi ${ }^{2}$ statistic comparing integrated versus malaria-only study areas. 
TABLE 3.2.3. INTENTION TO USE/CONTINUE TO USE CONTRACEPTION IN THE NEXT SIX MONTHS

Percentage of women aged 15 to 49 years currently pregnant or with a child under 2 years intending to start or continue to use contraception in next six months by method, September-October 2019

\begin{tabular}{|c|c|c|c|c|c|c|}
\hline & $\begin{array}{c}\text { KEBBI } \\
\%\end{array}$ & $\begin{array}{c}\text { SOKOTO } \\
\%\end{array}$ & $\begin{array}{c}\text { MALARIA-ONLY } \\
\text { (ZAMFARA) } \\
\%\end{array}$ & $\begin{array}{c}\text { INTEGRATED } \\
\text { (KEBBI/SOKOTO) } \\
\%\end{array}$ & $\mathbf{N}$ & $\begin{array}{c}\mathrm{CHI}^{2} \\
\text { VALUE }\end{array}$ \\
\hline \multicolumn{7}{|c|}{$\begin{array}{l}\text { In the next } 6 \text { months, do you plan to start } \\
\text { using a method of contraception? }\end{array}$} \\
\hline No & 79.0 & 85.7 & 78.3 & 82.5 & 2,073 & 34.195 \\
\hline Yes & 13.8 & 7.8 & 17.7 & 10.6 & 333 & 2.739 \\
\hline Don't know & 7.3 & 6.6 & 3.9 & 6.9 & 165 & \\
\hline Total & 100.0 & 100.0 & 100.0 & 100.0 & 2,571 & \\
\hline $\mathrm{N}$ & 884 & 876 & 811 & 1,760 & & \\
\hline \multicolumn{7}{|c|}{$\begin{array}{l}\text { In the next } 6 \text { months, do you plan to con- } \\
\text { tinue using a method of contraception? }\end{array}$} \\
\hline No & 14.1 & 6.4 & 27.7 & 9.5 & 76 & 18.871 \\
\hline Yes & 85.2 & 91.9 & 71.8 & 89.2 & 350 & 5.518 \\
\hline Don't know & 0.8 & 1.7 & 0.5 & 1.3 & 3 & ** \\
\hline Total & 100.0 & 100.0 & 100.0 & 100.0 & 429 & \\
\hline N & 90 & 145 & 194 & 235 & & \\
\hline \multicolumn{7}{|c|}{ Method you intend to use? } \\
\hline \multicolumn{7}{|l|}{ IUD } \\
\hline No & 98.4 & 99.8 & 97.4 & 99.1 & 670 & 2.230 \\
\hline Yes & 1.6 & 0.3 & 2.6 & 0.9 & 13 & 2.393 \\
\hline Total & 100.0 & 100.0 & 100.0 & 100.0 & 683 & \\
\hline N & 185 & 209 & 289 & 394 & & \\
\hline \multicolumn{7}{|l|}{ Injectables } \\
\hline No & 44.1 & 48.4 & 52.2 & 46.3 & 346 & 2.113 \\
\hline Yes & 55.9 & 51.6 & 47.8 & 53.7 & 337 & 0.705 \\
\hline Total & 100.0 & 100.0 & 100.0 & 100.0 & 683 & \\
\hline $\mathrm{N}$ & 185 & 209 & 289 & 394 & & \\
\hline \multicolumn{7}{|l|}{ Implants } \\
\hline No & 75.2 & 70.4 & 71.4 & 72.7 & 484 & 0.131 \\
\hline Yes & 24.8 & 29.6 & 28.6 & 27.3 & 199 & 0.039 \\
\hline Total & 100.0 & 100.0 & 100.0 & 100.0 & 683 & \\
\hline $\mathrm{N}$ & 185 & 209 & 289 & 394 & & \\
\hline \multicolumn{7}{|l|}{ Daily pill } \\
\hline No & 90.8 & 82.6 & 81.3 & 86.6 & 576 & 2.997 \\
\hline Yes & 9.3 & 17.4 & 18.7 & 13.4 & 107 & 1.653 \\
\hline Total & 100.0 & 100.0 & 100.0 & 100.0 & 683 & \\
\hline N & 185 & 209 & 289 & 394 & & \\
\hline \multicolumn{7}{|l|}{ Male condom } \\
\hline No & 99.0 & 100.0 & 99.5 & 99.5 & 678 & 0.015 \\
\hline Yes & 1.0 & 0.0 & 0.6 & 0.5 & 5 & 0.021 \\
\hline Total & 100.0 & 100.0 & 100.0 & 100.0 & 683 & \\
\hline $\mathrm{N}$ & 185 & 209 & 289 & 394 & & \\
\hline \multicolumn{7}{|c|}{ Lactational amenorrhea method } \\
\hline No & 100.0 & 99.5 & 99.0 & 99.7 & 679 & 1.308 \\
\hline Yes & 0.0 & 0.5 & 1.1 & 0.3 & 4 & 2.269 \\
\hline Total & 100.0 & 100.0 & 100.0 & 100.0 & 683 & \\
\hline N & 185 & 209 & 289 & 394 & & \\
\hline \multicolumn{7}{|l|}{ Withdrawal } \\
\hline No & 99.8 & 99.4 & 97.6 & 99.6 & 673 & 3.565 \\
\hline Yes & 0.2 & 0.6 & 2.4 & 0.4 & 10 & 5.609 \\
\hline Total & 100.0 & 100.0 & 100.0 & 100.0 & 683 & * \\
\hline $\mathrm{N}$ & 185 & 209 & 289 & 394 & & \\
\hline
\end{tabular}

Estimates based on low Ns (approx. $<30$ obs) have large relative standard errors and should be interpreted with caution. ${ }^{* \star *}$ indicates $p<0.001,{ }^{* \star}$ indicates $p<0.05$ and * indicates $p<.0 .01$ for the chi ${ }^{2}$ statistic comparing integrated versus malaria-only study areas. 


\subsection{Contraception: beliefs, perceptions, and self-efficacy}

\section{TABLE 3.3.1. PERCEIVED CONTRACEPTION BENEFITS FOR FUTURE CHILDREN}

Percentage of women 15 to 49 years currently pregnant or with a child under 2 years reporting contraceptive benefits for children by benefit, September-October 2019

\begin{tabular}{|c|c|c|c|c|c|c|}
\hline & KEBBI & SOKOTO & $\begin{array}{c}\text { MALARIA-ONLY } \\
\text { (ZAMFARA) }\end{array}$ & $\begin{array}{c}\text { INTEGRATED } \\
\text { (KEBBI/SOKOTO) }\end{array}$ & $\mathbf{N}$ & $\begin{array}{c}\mathrm{CHI}^{2} \\
\text { VALUE }\end{array}$ \\
\hline & $\%$ & $\%$ & $\%$ & $\%$ & & \\
\hline \multicolumn{7}{|c|}{ No benefits } \\
\hline No & 89.8 & 81.5 & 89.7 & 85.4 & 5,236 & 25.914 \\
\hline Yes & 10.2 & 18.5 & 10.3 & 14.6 & 798 & 1.433 \\
\hline Total & 100.0 & 100.0 & 100.0 & 100.0 & 6,034 & \\
\hline $\mathrm{N}$ & 1,960 & 2,062 & 2,012 & 4,022 & & \\
\hline \multicolumn{7}{|c|}{ Better growth } \\
\hline No & 43.0 & 47.7 & 37.0 & 45.5 & 2,550 & 43.701 \\
\hline Yes & 57.1 & 52.3 & 63.0 & 54.5 & 3,484 & 1.673 \\
\hline Total & 100.0 & 100.0 & 100.0 & 100.0 & 6,034 & \\
\hline N & 1,960 & 2,062 & 2,012 & 4,022 & & \\
\hline \multicolumn{7}{|c|}{ Better nutritional status } \\
\hline No & 81.6 & 80.4 & 62.2 & 81.0 & 4,505 & 243.768 \\
\hline Yes & 18.4 & 19.6 & 37.8 & 19.0 & 1,529 & 10.710 \\
\hline Total & 100.0 & 100.0 & 100.0 & 100.0 & 6,034 & *** \\
\hline $\mathrm{N}$ & 1,960 & 2,062 & 2,012 & 4,022 & & \\
\hline \multicolumn{7}{|c|}{ Better overall health } \\
\hline No & 75.6 & 83.6 & 58.1 & 80.0 & 4,355 & 314.285 \\
\hline Yes & 24.4 & 16.4 & 42.0 & 20.1 & 1,679 & 13.681 \\
\hline Total & 100.0 & 100.0 & 100.0 & 100.0 & 6,034 & *** \\
\hline N & 1,960 & 2,062 & 2,012 & 4,022 & & \\
\hline \multicolumn{7}{|c|}{ Better survival chance } \\
\hline No & 93.2 & 90.3 & 79.3 & 91.6 & 5,259 & 167.649 \\
\hline Yes & 6.8 & 9.8 & 20.7 & 8.4 & 775 & 9.225 \\
\hline Total & 100.0 & 100.0 & 100.0 & 100.0 & 6,034 & ** \\
\hline $\mathrm{N}$ & 1,960 & 2,062 & 2,012 & 4,022 & & \\
\hline \multicolumn{7}{|c|}{ More attention by mother } \\
\hline No & 79.8 & 78.5 & 68.0 & 79.1 & 4,526 & 91.448 \\
\hline Yes & 20.2 & 21.5 & 32.1 & 21.0 & 1,508 & 3.912 \\
\hline Total & 100.0 & 100.0 & 100.0 & 100.0 & 6,034 & \\
\hline $\mathrm{N}$ & 1,960 & 2,062 & 2,012 & 4,022 & & \\
\hline \multicolumn{7}{|c|}{ Better education } \\
\hline No & 96.3 & 96.7 & 91.0 & 96.5 & 5,634 & 72.819 \\
\hline Yes & 3.7 & 3.3 & 9.1 & 3.5 & 400 & 6.505 \\
\hline Total & 100.0 & 100.0 & 100.0 & 100.0 & 6,034 & * \\
\hline $\mathrm{N}$ & 1,960 & 2,062 & 2,012 & 4,022 & & \\
\hline \multicolumn{7}{|c|}{ More opportunities } \\
\hline No & 97.6 & 98.0 & 90.0 & 97.8 & 5,758 & 139.643 \\
\hline Yes & 2.4 & 2.0 & 10.0 & 2.2 & 276 & 9.951 \\
\hline Total & 100.0 & 100.0 & 100.0 & 100.0 & 6,034 & ** \\
\hline $\mathrm{N}$ & 1,960 & 2,062 & 2,012 & 4,022 & & \\
\hline
\end{tabular}

Estimates based on low Ns (approx. $<30$ obs) have large relative standard errors and should be interpreted with caution. ${ }^{* \star *}$ indicates $p<0.001,{ }^{\star *}$ indicates $p<0.05$ and ${ }^{*}$ indicates $p<.0 .01$ for the chi ${ }^{2}$ statistic comparing integrated versus malaria-only study areas. 
TABLE 3.3.2. PERCEIVED CONTRACEPTION BENEFITS FOR WOMEN

Percentage of women aged 15 to 49 years currently pregnant or with a child under 2 years who report any contraceptive benefits for themselves by type of benefit, September-October 2019

\begin{tabular}{|c|c|c|c|c|c|c|}
\hline & KEBBI & SOKOTO & $\begin{array}{l}\text { MALARIA-ONLY } \\
\text { (ZAMFARA) }\end{array}$ & $\begin{array}{c}\text { INTEGRATED } \\
\text { (KEBBI/SOKOTO) }\end{array}$ & $\mathbf{N}$ & $\begin{array}{c}\mathrm{CHI}^{2} \\
\text { VALUE }\end{array}$ \\
\hline & $\%$ & $\%$ & $\%$ & $\%$ & & \\
\hline \multicolumn{7}{|c|}{ No benefits } \\
\hline No & 89.7 & 84.5 & 89.6 & 86.9 & 5,269 & 10.803 \\
\hline Yes & 10.3 & 15.5 & 10.4 & 13.1 & 765 & 0.576 \\
\hline Total & 100.0 & 100.0 & 100.0 & 100.0 & 6,034 & \\
\hline N & 1,960 & 2,062 & 2,012 & 4,022 & & \\
\hline \multicolumn{7}{|c|}{ Woman gets rest after birth } \\
\hline No & 39.3 & 39.0 & 29.5 & 39.1 & 2,227 & 61.025 \\
\hline Yes & 60.7 & 61.0 & 70.5 & 60.9 & 3,807 & 2.201 \\
\hline Total & 100.0 & 100.0 & 100.0 & 100.0 & 6,034 & \\
\hline N & 1,960 & 2,062 & 2,012 & 4,022 & & \\
\hline \multicolumn{7}{|c|}{ Better health and nutrition } \\
\hline No & 71.0 & 77.1 & 56.7 & 74.3 & 4,052 & 194.827 \\
\hline Yes & 29.0 & 22.9 & 43.3 & 25.8 & 1,982 & 8.955 \\
\hline Total & 100.0 & 100.0 & 100.0 & 100.0 & 6,034 & ** \\
\hline N & 1,960 & 2,062 & 2,012 & 4,022 & & \\
\hline \multicolumn{7}{|c|}{ Beauty and youthfulness } \\
\hline No & 86.1 & 84.1 & 67.5 & 85.0 & 4,729 & 236.897 \\
\hline Yes & 13.9 & 15.9 & 32.6 & 15.0 & 1,305 & 10.885 \\
\hline Total & 100.0 & 100.0 & 100.0 & 100.0 & 6,034 & *** \\
\hline $\mathrm{N}$ & 1,960 & 2,062 & 2,012 & 4,022 & & \\
\hline \multicolumn{7}{|c|}{ Fewer pregnancy complications } \\
\hline No & 91.4 & 85.9 & 80.9 & 88.4 & 5,193 & 61.231 \\
\hline Yes & 8.6 & 14.2 & 19.1 & 11.6 & 841 & 2.207 \\
\hline Total & 100.0 & 100.0 & 100.0 & 100.0 & 6,034 & \\
\hline $\mathrm{N}$ & 1,960 & 2,062 & 2,012 & 4,022 & & \\
\hline \multicolumn{7}{|c|}{ Reduce unwanted pregnancies } \\
\hline No & 90.8 & 89.3 & 80.7 & 90.0 & 5,240 & 96.667 \\
\hline Yes & 9.2 & 10.7 & 19.3 & 10.0 & 794 & 2.888 \\
\hline Total & 100.0 & 100.0 & 100.0 & 100.0 & 6,034 & \\
\hline N & 1,960 & 2,062 & 2,012 & 4,022 & & \\
\hline \multicolumn{7}{|c|}{ Fewer children to educate } \\
\hline No & 98.5 & 98.3 & 94.6 & 98.4 & 5,818 & 57.258 \\
\hline Yes & 1.5 & 1.7 & 5.4 & 1.6 & 216 & 7.019 \\
\hline Total & 100.0 & 100.0 & 100.0 & 100.0 & 6,034 & ** \\
\hline N & 1,960 & 2,062 & 2,012 & 4,022 & & \\
\hline \multicolumn{7}{|c|}{ Family has more money } \\
\hline No & 98.9 & 99.0 & 96.9 & 99.0 & 5,909 & 28.352 \\
\hline Yes & 1.1 & 1.0 & 3.1 & 1.1 & 125 & 4.047 \\
\hline Total & 100.0 & 100.0 & 100.0 & 100.0 & 6,034 & * \\
\hline $\mathrm{N}$ & 1,960 & 2,062 & 2,012 & 4,022 & & \\
\hline
\end{tabular}

Estimates based on low Ns (approx. $<30$ obs) have large relative standard errors and should be interpreted with caution. ${ }^{* * *}$ indicates $p<0.001,{ }^{* *}$ indicates $p<0.05$ and ${ }^{*}$ indicates $p<.0 .01$ for the chi ${ }^{2}$ statistic comparing integrated versus malaria-only study areas. 
TABLE 3.3.3. CONTRACEPTION BELIEFS, ATTITUDES, AND PERCEIVED SOCIAL NORMS

Percentage of women aged 15 to 49 years currently pregnant or with a child under 2 years reporting contraception beliefs, attitudes, or perceived social norms, September-October 2019

\begin{tabular}{|c|c|c|c|c|c|c|}
\hline & KEBBI & sокото & $\begin{array}{l}\text { MALARIA-ONLY } \\
\text { (ZAMFARA) }\end{array}$ & $\begin{array}{l}\text { INTEGRATED } \\
\text { (KEBBI/SOKOTO) }\end{array}$ & $\mathbf{N}$ & $\begin{array}{c}\mathrm{CHI}^{2} \\
\text { VALUE }\end{array}$ \\
\hline & $\%$ & $\%$ & $\%$ & $\%$ & & \\
\hline \multicolumn{7}{|c|}{ Do you personally approve of using contraception for SPACING BIRTHS? } \\
\hline No & 50.3 & 58.7 & 50.1 & 54.8 & 3,126 & 59.974 \\
\hline Yes & 43.3 & 35.1 & 46.8 & 38.9 & 2,576 & 1.745 \\
\hline Don't know & 6.4 & 6.2 & 3.1 & 6.3 & 331 & \\
\hline Total & 100.0 & 100.0 & 100.0 & 100.0 & 6,033 & \\
\hline N & 1,960 & 2,062 & 2,011 & 4,022 & & \\
\hline \multicolumn{7}{|c|}{ Do you think people would call you bad names or avoid your company if they knew you were using contraception to space births? } \\
\hline No & 65.1 & 43.5 & 68.8 & 53.5 & 3,556 & 198.886 \\
\hline Yes & 14.9 & 34.2 & 21.5 & 25.2 & 1,441 & 4.209 \\
\hline Don't know & 20.0 & 22.4 & 9.7 & 21.3 & 1,036 & * \\
\hline Total & 100.0 & 100.0 & 100.0 & 100.0 & 6,033 & \\
\hline$N$ & 1,960 & 2,062 & 2,011 & 4,022 & & \\
\hline \multicolumn{7}{|c|}{ Couples who use modern contraception have a better quality of life } \\
\hline Agree & 62.8 & 55.9 & 59.7 & 59.1 & 3,638 & 107.833 \\
\hline Disagree & 24.2 & 27.3 & 33.0 & 25.9 & 1,646 & 2.518 \\
\hline Don't know & 13.0 & 16.8 & 7.3 & 15.0 & 749 & \\
\hline Total & 100.0 & 100.0 & 100.0 & 100.0 & 6,033 & \\
\hline N & 1,960 & 2,062 & 2,011 & 4,022 & & \\
\hline \multicolumn{7}{|c|}{ Side effects are normal and usually go away in a few months } \\
\hline Agree & 50.1 & 42.7 & 52.3 & 46.2 & 2,990 & 58.092 \\
\hline Disagree & 25.0 & 30.6 & 29.9 & 28.0 & 1,583 & 1.484 \\
\hline Don't know & 24.9 & 26.7 & 17.8 & 25.9 & 1,460 & \\
\hline Total & 100.0 & 100.0 & 100.0 & 100.0 & 6,033 & \\
\hline N & 1,960 & 2,062 & 2,011 & 4,022 & & \\
\hline \multicolumn{7}{|c|}{ Religious leaders should speak publicly about modern contraception } \\
\hline Agree & 51.5 & 49.5 & 50.0 & 50.5 & 3,079 & 106.955 \\
\hline Disagree & 32.6 & 37.5 & 43.2 & 35.3 & 2,187 & 2.344 \\
\hline Don't know & 15.9 & 13.0 & 6.8 & 14.3 & 767 & \\
\hline Total & 100.0 & 100.0 & 100.0 & 100.0 & 6,033 & \\
\hline N & 1,960 & 2,062 & 2,011 & 4,022 & & \\
\hline \multicolumn{7}{|c|}{ Most couples in my community use modern contraception for birth spacing } \\
\hline Agree & 42.8 & 34.2 & 51.7 & 38.2 & 2,752 & 138.535 \\
\hline Disagree & 37.6 & 38.1 & 34.1 & 37.9 & 2,033 & 2.398 \\
\hline Don't know & 19.6 & 27.7 & 14.2 & 23.9 & 1,248 & \\
\hline Total & 100.0 & 100.0 & 100.0 & 100.0 & 6,033 & \\
\hline N & 1,960 & 2,062 & 2,011 & 4,022 & & \\
\hline \multicolumn{7}{|c|}{ It is important that husbands and wives discuss contraception } \\
\hline Agree & 69.4 & 62.5 & 72.4 & 65.7 & 4,165 & 82.298 \\
\hline Disagree & 19.4 & 29.7 & 23.8 & 24.9 & 1,388 & 1.943 \\
\hline Don't know & 11.2 & 7.8 & 3.9 & 9.3 & 480 & \\
\hline Total & 100.0 & 100.0 & 100.0 & 100.0 & 6,033 & \\
\hline N & 1,960 & 2,062 & 2,011 & 4,022 & & \\
\hline \multicolumn{7}{|c|}{ A woman's body is not ready for childbirth until she is 18} \\
\hline Agree & 27.4 & 22.6 & 26.7 & 24.8 & 1,546 & 125.008 \\
\hline Disagree & 59.9 & 69.9 & 70.3 & 65.3 & 4,026 & 2.506 \\
\hline Don't know & 12.8 & 7.5 & 3.1 & 10.0 & 461 & \\
\hline Total & 100.0 & 100.0 & 100.0 & 100.0 & 6,033 & \\
\hline N & 1,960 & 2,062 & 2,011 & 4,022 & & \\
\hline \multicolumn{7}{|c|}{$\begin{array}{l}\text { Women over } 35 \text { have a higher risk of complications and } \\
\text { should consider limiting births }\end{array}$} \\
\hline Agree & 36.6 & 32.4 & 42.8 & 34.3 & 2,265 & 173.162 \\
\hline Disagree & 48.3 & 53.8 & 52.2 & 51.3 & 3,092 & 3.470 \\
\hline Don't know & 15.2 & 13.8 & 5.0 & 14.4 & 676 & * \\
\hline Total & 100.0 & 100.0 & 100.0 & 100.0 & 6,033 & \\
\hline N & 1,960 & 2,062 & 2,011 & 4,022 & & \\
\hline
\end{tabular}

Estimates based on low Ns (approx. $<30$ obs) have large relative standard errors and should be interpreted with caution. ${ }^{* \star *}$ indicates $p<0.001,{ }^{* *}$ indicates $p<0.05$ and ${ }^{*}$ indicates $p<.0 .01$ for the chi ${ }^{2}$ statistic comparing integrated versus malaria-only study areas. 
TABLE 3.3.4. CONTRACEPTION MYTHS AND PERCEIVED HEALTH RISKS

Percentage of women aged 15 to 49 years currently pregnant or with a child under 2 years reporting contraception myths and perceived health risks, September-October 2019

\begin{tabular}{|c|c|c|c|c|c|}
\hline & \multicolumn{3}{|c|}{ INTERVENTION AREA } & \multirow[b]{2}{*}{$\mathbf{N}$} & \multirow[b]{2}{*}{$\begin{array}{c}\mathrm{CHI}^{2} \\
\text { VALUE }\end{array}$} \\
\hline & $\begin{array}{c}\text { INTEGRATED } \\
\text { (KEBBI/SOKOTO) } \\
\%\end{array}$ & $\begin{array}{c}\text { MALARIA-ONLY } \\
\text { (ZAMFARA) } \\
\%\end{array}$ & $\begin{array}{c}\text { TOTAL } \\
\quad \%\end{array}$ & & \\
\hline \multicolumn{6}{|c|}{$\begin{array}{l}\text { Use of some contraceptives can make a woman } \\
\text { permanently infertile }\end{array}$} \\
\hline Agree & 31.4 & 25.1 & 27.6 & 1,728 & 101.959 \\
\hline Disagree & 40.9 & 54.1 & 48.7 & 2,684 & 2.063 \\
\hline Don't know & 27.7 & 20.8 & 23.6 & 1,621 & \\
\hline Total & 100.0 & 100.0 & 100.0 & 6,033 & \\
\hline $\mathrm{N}$ & 4,022 & 2,011 & 6,033 & & \\
\hline \multicolumn{6}{|c|}{ Contraceptives can harm a woman's womb } \\
\hline Agree & 32.8 & 23.8 & 27.5 & 1,738 & 135.019 \\
\hline Disagree & 39.2 & 54.4 & 48.2 & 2,645 & 2.738 \\
\hline Don't know & 28.0 & 21.9 & 24.3 & 1,650 & \\
\hline Total & 100.0 & 100.0 & 100.0 & 6,033 & \\
\hline $\mathrm{N}$ & 4,022 & 2,011 & 6,033 & & \\
\hline \multicolumn{6}{|c|}{ Contraceptives can cause cancer } \\
\hline Agree & 21.3 & 16.0 & 18.2 & 1,173 & 157.593 \\
\hline Disagree & 36.5 & 52.9 & 46.2 & 2,513 & 3.183 \\
\hline Don't know & 42.2 & 31.1 & 35.6 & 2,347 & * \\
\hline Total & 100.0 & 100.0 & 100.0 & 6,033 & \\
\hline $\mathrm{N}$ & 4,022 & 2,011 & 6,033 & & \\
\hline \multicolumn{6}{|c|}{ Contraceptives can give you deformed babies } \\
\hline Agree & 20.6 & 15.5 & 17.6 & 1,128 & 103.150 \\
\hline Disagree & 44.8 & 58.1 & 52.7 & 2,978 & 2.324 \\
\hline Don't know & 34.6 & 26.4 & 29.7 & 1,927 & \\
\hline Total & 100.0 & 100.0 & 100.0 & 6,033 & \\
\hline$N$ & 4,022 & 2,011 & 6,033 & & \\
\hline \multicolumn{6}{|c|}{$\begin{array}{l}\text { Women who use contraception end up with } \\
\text { health problems }\end{array}$} \\
\hline Agree & 35.0 & 30.5 & 32.3 & 1,974 & 123.174 \\
\hline Disagree & 36.8 & 50.5 & 45.0 & 2,490 & 2.640 \\
\hline Don't know & 28.2 & 19.0 & 22.7 & 1,569 & \\
\hline Total & 100.0 & 100.0 & 100.0 & 6,033 & \\
\hline $\mathrm{N}$ & 4,022 & 2,011 & 6,033 & & \\
\hline \multicolumn{6}{|c|}{ Contraceptives can reduce a man's sexual urge } \\
\hline Agree & 17.8 & 11.1 & 13.8 & 954 & 162.542 \\
\hline Disagree & 38.2 & 54.5 & 47.9 & 2,613 & 3.377 \\
\hline Don't know & 44.0 & 34.4 & 38.3 & 2,466 & * \\
\hline Total & 100.0 & 100.0 & 100.0 & 6,033 & \\
\hline $\mathrm{N}$ & 4,022 & 2,011 & 6,033 & & \\
\hline \multicolumn{6}{|c|}{$\begin{array}{l}\text { Contraceptives can reduce a woman's sexual } \\
\text { urge }\end{array}$} \\
\hline Agree & 17.6 & 12.8 & 14.8 & 991 & 147.498 \\
\hline Disagree & 38.4 & 54.3 & 47.8 & 2,612 & 3.151 \\
\hline Don't know & 44.1 & 32.9 & 37.4 & 2,430 & \\
\hline Total & 100.0 & 100.0 & 100.0 & 6,033 & \\
\hline$N$ & 4,022 & 2,011 & 6,033 & & \\
\hline \multicolumn{6}{|c|}{$\begin{array}{l}\text { Women who use contraceptives may become } \\
\text { promiscuous }\end{array}$} \\
\hline Agree & 19.6 & 11.1 & 14.6 & 1,064 & 166.608 \\
\hline Disagree & 40.8 & 56.6 & 50.1 & 2,753 & 3.281 \\
\hline Don't know & 39.6 & 32.4 & 35.3 & 2,216 & * \\
\hline Total & 100.0 & 100.0 & 100.0 & 6,033 & \\
\hline $\mathrm{N}$ & 4,022 & 2,011 & 6,033 & & \\
\hline
\end{tabular}

Estimates based on low Ns (approx. $<30$ obs) have large relative standard errors and should be interpreted with caution. ${ }^{* * *}$ indicates $p<0.001,{ }^{* *}$ indicates $p<0.05$ and ${ }^{*}$ indicates $p<.0 .01$ for the chi ${ }^{2}$ statistic comparing integrated versus malaria-only study areas. 
TABLE 3.3.5. SPOUSAL COMMUNICATION ABOUT CONTRACEPTION

Percentage of women aged 15 to 49 years currently pregnant or with a child under 2 years reporting on spousal communication about contraception, September-October 2019

\begin{tabular}{|c|c|c|c|c|c|c|}
\hline & KEBBI & SOKOTO & $\begin{array}{l}\text { MALARIA-ONLY } \\
\text { (ZAMFARA) }\end{array}$ & $\begin{array}{l}\text { INTEGRATED } \\
\text { (KEBBI/SOKOTO) }\end{array}$ & $\mathbf{N}$ & $\begin{array}{c}\mathrm{CHI}^{2} \\
\text { VALUE }\end{array}$ \\
\hline & $\%$ & $\%$ & $\%$ & $\%$ & & \\
\hline \multicolumn{7}{|c|}{$\begin{array}{l}\text { Ever talked to your husband/partner } \\
\text { about the number of children to have? }\end{array}$} \\
\hline No & 94.5 & 92.1 & 91.0 & 93.2 & 5,565 & 36.612 \\
\hline Yes & 4.8 & 6.6 & 8.8 & 5.7 & 423 & 3.104 \\
\hline Don't know & 0.7 & 1.4 & 0.2 & 1.1 & 45 & \\
\hline Total & 100.0 & 100.0 & 100.0 & 100.0 & 6,033 & \\
\hline N & 1,960 & 2,062 & 2,011 & 4,022 & & \\
\hline \multicolumn{7}{|c|}{$\begin{array}{l}\text { Ever talked with husband/partner } \\
\text { about using modern contraception? }\end{array}$} \\
\hline No & 85.2 & 83.4 & 73.9 & 84.2 & 4,898 & 96.933 \\
\hline Yes & 14.2 & 15.3 & 25.3 & 14.8 & 1,079 & 4.811 \\
\hline Don't know & 0.7 & 1.3 & 0.8 & 1.0 & 56 & * \\
\hline Total & 100.0 & 100.0 & 100.0 & 100.0 & 6,033 & \\
\hline $\mathrm{N}$ & 1,960 & 2,062 & 2,011 & 4,022 & & \\
\hline \multicolumn{7}{|l|}{ Discussion with partner included } \\
\hline \multicolumn{7}{|l|}{ Which method to use } \\
\hline No & 54.9 & 44.8 & 40.1 & 49.3 & 498 & 7.614 \\
\hline Yes & 45.1 & 55.2 & 59.9 & 50.7 & 581 & 0.817 \\
\hline Total & 100.0 & 100.0 & 100.0 & 100.0 & 1,079 & \\
\hline $\mathrm{N}$ & 245 & 321 & 513 & 566 & & \\
\hline \multicolumn{7}{|l|}{ Using a method to space births } \\
\hline No & 29.2 & 31.2 & 27.1 & 30.3 & 308 & 1.133 \\
\hline Yes & 70.8 & 68.8 & 72.9 & 69.7 & 771 & 0.170 \\
\hline Total & 100.0 & 100.0 & 100.0 & 100.0 & 1,079 & \\
\hline $\mathrm{N}$ & 245 & 321 & 513 & 566 & & \\
\hline \multicolumn{7}{|l|}{$\begin{array}{l}\text { Using a method to not have more } \\
\text { births }\end{array}$} \\
\hline No & 94.3 & 94.6 & 78.5 & 94.5 & 954 & 39.866 \\
\hline Yes & 5.7 & 5.4 & 21.5 & 5.5 & 125 & 5.225 \\
\hline Total & 100.0 & 100.0 & 100.0 & 100.0 & 1,079 & * \\
\hline $\mathrm{N}$ & 245 & 321 & 513 & 566 & & \\
\hline \multicolumn{7}{|l|}{ Side effects of methods } \\
\hline No & 89.4 & 88.9 & 77.9 & 89.1 & 926 & 18.151 \\
\hline Yes & 10.6 & 11.1 & 22.1 & 10.9 & 153 & 1.389 \\
\hline Total & 100.0 & 100.0 & 100.0 & 100.0 & 1,079 & \\
\hline $\mathrm{N}$ & 245 & 321 & 513 & 566 & & \\
\hline \multicolumn{7}{|c|}{$\begin{array}{l}\text { Who decides if you use a contraceptive } \\
\text { method? }\end{array}$} \\
\hline Mainly your decision & 22.9 & 17.2 & 26.8 & 19.9 & 1,458 & 137.967 \\
\hline Mainly your partner's decision & 32.9 & 26.9 & 17.2 & 29.7 & 1,494 & 2.548 \\
\hline Both decide together & 44.2 & 55.9 & 56.0 & 50.5 & 3,081 & \\
\hline Total & 100.0 & 100.0 & 100.0 & 100.0 & 6,033 & \\
\hline $\mathrm{N}$ & 1,960 & 2,062 & 2,011 & 4,022 & & \\
\hline
\end{tabular}

Estimates based on low Ns (approx. $<30$ obs) have large relative standard errors and should be interpreted with caution. ${ }^{* \star *}$ indicates $p<0.001,{ }^{* *}$ indicates $p<0.05$ and ${ }^{*}$ indicates $p<.0 .01$ for the chi ${ }^{2}$ statistic comparing integrated versus malaria-only study areas. 
TABLE 3.3.6. WHO ELSE INFLUENCES DECISIONS ABOUT CONTRACEPTIVE USE?

Percentage of women aged 15 to 49 years currently pregnant or with a child under 2 years reporting on who else influences a woman's decision about contraceptive use, September-October 2019

\begin{tabular}{|c|c|c|c|c|c|}
\hline & $\begin{array}{c}\text { INTEGRATED } \\
\text { (KEBBI/SOKOTO) } \\
\%\end{array}$ & $\begin{array}{c}\text { ITERVENTION AREA } \\
\text { MALARIA-ONLY } \\
\text { (ZAMFARA) } \\
\%\end{array}$ & $\begin{array}{c}\text { TOTAL } \\
\% \\
\%\end{array}$ & $\mathbf{N}$ & $\begin{array}{c}\mathrm{CHI}^{2} \\
\text { VALUE }\end{array}$ \\
\hline \multicolumn{6}{|c|}{ No one else } \\
\hline $\begin{array}{l}\text { No } \\
\text { Yes } \\
\text { Total } \\
\text { N }\end{array}$ & $\begin{array}{r}38.7 \\
61.3 \\
100.0 \\
4,022\end{array}$ & $\begin{array}{r}39.0 \\
61.0 \\
100.0 \\
2,012\end{array}$ & $\begin{array}{r}38.9 \\
61.1 \\
100.0 \\
6,034\end{array}$ & $\begin{array}{l}2,294 \\
3,740 \\
6,034\end{array}$ & $\begin{array}{l}0.044 \\
0.002\end{array}$ \\
\hline \multicolumn{6}{|c|}{ Partner } \\
\hline $\begin{array}{l}\text { No } \\
\text { Yes } \\
\text { Total } \\
\text { N }\end{array}$ & $\begin{array}{r}69.9 \\
30.1 \\
100.0 \\
4,022\end{array}$ & $\begin{array}{r}69.9 \\
30.1 \\
100.0 \\
2,012\end{array}$ & $\begin{array}{r}69.9 \\
30.1 \\
100.0 \\
6,034\end{array}$ & $\begin{array}{l}4,236 \\
1,798 \\
6,034\end{array}$ & $\begin{array}{r}0.001 \\
0.000\end{array}$. \\
\hline \multicolumn{6}{|c|}{ Mother-in-law } \\
\hline $\begin{array}{l}\text { No } \\
\text { Yes } \\
\text { Total } \\
\text { N }\end{array}$ & $\begin{array}{r}94.0 \\
6.0 \\
100.0 \\
4,022\end{array}$ & $\begin{array}{r}98.4 \\
1.6 \\
100.0 \\
2,012\end{array}$ & $\begin{array}{r}96.6 \\
3.4 \\
100.0 \\
6,034\end{array}$ & $\begin{array}{r}5,780 \\
254 \\
6,034\end{array}$ & $\begin{array}{r}83.068 \\
14.751 \\
\star \star \star\end{array}$ \\
\hline \multicolumn{6}{|l|}{ Mother } \\
\hline $\begin{array}{l}\text { No } \\
\text { Yes } \\
\text { Total } \\
\text { N }\end{array}$ & $\begin{array}{r}95.4 \\
4.6 \\
100.0 \\
4,022\end{array}$ & $\begin{array}{r}95.9 \\
4.1 \\
100.0 \\
2,012\end{array}$ & $\begin{array}{r}95.7 \\
4.3 \\
100.0 \\
6,034\end{array}$ & $\begin{array}{r}5,786 \\
248 \\
6,034\end{array}$ & $\begin{array}{r}0.784 \\
0.064 \\
.\end{array}$ \\
\hline $\begin{array}{l}\text { Other o } \\
\text { No } \\
\text { Yes } \\
\text { Total } \\
\text { N }\end{array}$ & $\begin{array}{r}99.5 \\
0.5 \\
100.0 \\
4,022\end{array}$ & $\begin{array}{r}98.7 \\
1.3 \\
100.0 \\
2,012\end{array}$ & $\begin{array}{r}99.0 \\
1.0 \\
100.0 \\
6,034\end{array}$ & $\begin{array}{r}5,986 \\
48 \\
6,034\end{array}$ & $\begin{array}{l}9.401 \\
2.905\end{array}$ \\
\hline $\begin{array}{l}\text { Other } \mathbf{p} \\
\text { No } \\
\text { Yes } \\
\text { Total } \\
\text { N }\end{array}$ & $\begin{array}{r}99.8 \\
0.2 \\
100.0 \\
4,022\end{array}$ & $\begin{array}{r}99.3 \\
0.7 \\
100.0 \\
2,012\end{array}$ & $\begin{array}{r}99.5 \\
0.5 \\
100.0 \\
6,034\end{array}$ & $\begin{array}{r}6,020 \\
14 \\
6,034\end{array}$ & $\begin{array}{r}7.725 \\
3.674 \\
.\end{array}$ \\
\hline $\begin{array}{l}\text { Friends } \\
\text { No } \\
\text { Yes } \\
\text { Total } \\
\text { N }\end{array}$ & $\begin{array}{r}98.4 \\
1.7 \\
100.0 \\
4,022\end{array}$ & $\begin{array}{r}97.4 \\
2.7 \\
100.0 \\
2,012\end{array}$ & $\begin{array}{r}97.8 \\
2.2 \\
100.0 \\
6,034\end{array}$ & $\begin{array}{r}5,914 \\
120 \\
6,034\end{array}$ & $\begin{array}{l}6.675 \\
1.071\end{array}$ \\
\hline $\begin{array}{l}\text { Health } \\
\text { No } \\
\text { Yes } \\
\text { Total } \\
\text { N }\end{array}$ & $\begin{array}{r}96.7 \\
3.3 \\
100.0 \\
4,022\end{array}$ & $\begin{array}{r}96.1 \\
3.9 \\
100.0 \\
2,012\end{array}$ & $\begin{array}{r}96.4 \\
3.6 \\
100.0 \\
6,034\end{array}$ & $\begin{array}{r}5,829 \\
205 \\
6,034\end{array}$ & $\begin{array}{r}1.281 \\
0.116 \\
.\end{array}$ \\
\hline $\begin{array}{l}\text { Comm } \\
\text { No } \\
\text { Yes } \\
\text { Total } \\
\text { N }\end{array}$ & $\begin{array}{r}99.6 \\
0.4 \\
100.0 \\
4,022\end{array}$ & $\begin{array}{r}99.9 \\
0.1 \\
100.0 \\
2,012\end{array}$ & $\begin{array}{r}99.8 \\
0.2 \\
100.0 \\
6,034\end{array}$ & $\begin{array}{r}6,019 \\
15 \\
6,034\end{array}$ & $\begin{array}{r}4.513 \\
1.995 \\
.\end{array}$ \\
\hline $\begin{array}{l}\text { Other } \\
\text { No } \\
\text { Yes } \\
\text { Total } \\
\text { N }\end{array}$ & $\begin{array}{r}99.8 \\
0.2 \\
100.0 \\
4,022\end{array}$ & $\begin{array}{r}99.1 \\
0.9 \\
100.0 \\
2,012 \\
\end{array}$ & $\begin{array}{r}99.4 \\
0.6 \\
100.0 \\
6,034\end{array}$ & $\begin{array}{r}6,007 \\
27 \\
6,034\end{array}$ & $\begin{array}{r}12.058 \\
5.329 \\
\text { * }\end{array}$ \\
\hline
\end{tabular}

Estimates based on low Ns (approx. $<30$ obs) have large relative standard errors and should be interpreted with caution. ${ }^{* * *}$ indicates $p<0.001,{ }^{* *}$ indicates $p<0.05$ and ${ }^{*}$ indicates $p<.0 .01$ for the chi ${ }^{2}$ statistic comparing integrated versus malaria-only study areas. 
TABLE 3.3.7. SELF-EFFICACY TO CONVINCE PARTNER ABOUT CONTRACEPTION USE

Percentage of women aged 15 to 49 years currently pregnant or with a child under 2 years reporting on self-efficacy to convince partner about contraceptive use, September-October 2019

\begin{tabular}{|c|c|c|c|c|c|c|}
\hline & KEBBI & SOKOTO & $\begin{array}{l}\text { MALARIA-ONLY } \\
\text { (ZAMFARA) }\end{array}$ & $\begin{array}{c}\text { INTEGRATED } \\
\text { (KEBBI/SOKOTO) }\end{array}$ & $\mathbf{N}$ & $\begin{array}{c}\mathrm{CHI}^{2} \\
\text { VALUE }\end{array}$ \\
\hline & $\%$ & $\%$ & $\%$ & $\%$ & & \\
\hline \multicolumn{7}{|c|}{$\begin{array}{l}\text { Confident in ability to convince } \\
\text { partner to use modern } \\
\text { contraception to SP }\end{array}$} \\
\hline Confident & 53.8 & 41.2 & 52.2 & 47.0 & 3,022 & 99.746 \\
\hline Uncertain & 37.9 & 52.1 & 45.6 & 45.5 & 2,641 & 2.695 \\
\hline Don't know & 8.3 & 6.8 & 2.2 & 7.5 & 370 & \\
\hline Total & 100.0 & 100.0 & 100.0 & 100.0 & 6,033 & \\
\hline$N$ & 1,960 & 2,062 & 2,011 & 4,022 & & \\
\hline \multicolumn{7}{|c|}{$\begin{array}{l}\text { Confidence in ability to use } \\
\text { modern contraception even if } \\
\text { partner opposes }\end{array}$} \\
\hline Confident & 35.1 & 29.6 & 40.9 & 32.1 & 2,134 & 104.217 \\
\hline Uncertain & 55.8 & 65.4 & 56.7 & 60.9 & 3,549 & 2.831 \\
\hline Don't know & 9.2 & 5.1 & 2.4 & 7.0 & 350 & . \\
\hline Total & 100.0 & 100.0 & 100.0 & 100.0 & 6,033 & \\
\hline $\mathrm{N}$ & 1,960 & 2,062 & 2,011 & 4,022 & & \\
\hline
\end{tabular}

Estimates based on low Ns (approx. $<30$ obs) have large relative standard errors and should be interpreted with caution. ${ }^{* * *}$ indicates $p<0.001,{ }^{* *}$ indicates $p<0.05$ and * indicates $p<.0 .01$ for the chi ${ }^{2}$ statistic comparing integrated versus malaria-only study areas. 


\section{Current pregnancy}

\section{Key findings}

Among respondents who were currently pregnant, 87 percent reported that they wanted to get pregnant at the time they did for this pregnancy, and 84 percent planned to have another child in the future. Among those currently in late pregnancy (7-9 months pregnant), only half (53\%) reported attending any ANC; 66 percent of respondents went for their first visit during the second trimester. In Kebbi/Sokoto, the most common sources for the first ANC visit were government PHC (49\%) or government hospital (37\%), whereas in Zamfara respondents in late pregnancy who attended ANC were 1.5 times more likely to go to a government hospital than a government PHC. Among respondents currently in late pregnancy (7-9 months) who did not attend antenatal care, the most common reasons for non-attendance included: lack of perceived need (47\%), spousal opposition (37\%), and fatalism ("It's up to God") (20\%).

Most respondents currently in late pregnancy (7-9 months) and who attended ANC reported that the following checks were done: blood pressure measured (90\%), urine sample taken (72\%), blood sample taken (76\%), HIV testing (50\%), weight measured (79\%), pregnancy danger signs discussed (67\%), and baby's due date discussed (50\%). About half of these respondents were given iron tablets or folic acid supplements, and only 39 percent and 17 percent, respectively, reported receiving a tetanus injection or being given deworming medication. Only 16 percent of respondents currently in late pregnancy and who attended ANC reported receiving a mosquito net during ANC. In addition, few of these respondents discussed a birth plan with a health worker, with significantly lower birth planning practiced in Kebbi/ Sokoto (25\%) than in Zamfara (45\%). This suggests that developing a birth plan is not a standard practice in these areas, and that birth planning could be a potential topic for more focused SBC programming.

All currently pregnant respondents were asked about their dietary intake in the previous 24 hours. Seven out of 10 respondents (71\%) reported eating at least three main meals during the previous day or night. Nearly all pregnant respondents (92\%) reported eating breads, rice, tuwo (stodgy, tuber- or grain-based meal), or other grains. Other commonly reported foods eaten in the previous 24-hour period included: beans, peas, or lentils (59\%); oil, fats, and butters (59\%); dark green leafy vegetables (58\%); meat, poultry, or fish (48\%); milk, cheese, yogurt, or other dairy (43\%); and sugary foods (36\%). Few pregnant respondents across study areas reportedly ate eggs (21\%) or mangoes or papaya (14\%). 
TABLE 4.1.1. PREGNANCY INTENTIONS DURING CURRENT PREGNANCY

Percentage of currently pregnant women aged 15 to 49 years who report pregnancy intentions for this current pregnancy, September-October 2019

\begin{tabular}{|c|c|c|c|c|c|c|}
\hline & KEBBI & SOKOTO & $\begin{array}{l}\text { MALARIA-ONLY } \\
\text { (ZAMFARA) }\end{array}$ & $\begin{array}{c}\text { INTEGRATED } \\
\text { (KEBBI/SOKOTO) }\end{array}$ & $\mathbf{N}$ & $\begin{array}{c}\mathrm{CHI}^{2} \\
\text { VALUE }\end{array}$ \\
\hline & $\%$ & $\%$ & $\%$ & $\%$ & & \\
\hline \multicolumn{7}{|c|}{$\begin{array}{l}\text { How many months pregnant are } \\
\text { you? }\end{array}$} \\
\hline 0-3 months & 22.9 & 22.4 & 26.5 & 22.6 & 685 & 44.990 \\
\hline 4-6 months & 40.1 & 35.7 & 37.4 & 37.7 & 1,114 & 3.140 \\
\hline 7+ months & 32.2 & 31.2 & 33.3 & 31.7 & 1,031 & * \\
\hline Don't know & 4.8 & 10.8 & 2.8 & 8.0 & 198 & \\
\hline Total & 100.0 & 100.0 & 100.0 & 100.0 & 3,028 & \\
\hline $\mathrm{N}$ & 984 & 1,040 & 1,004 & 2,024 & & \\
\hline \multicolumn{7}{|c|}{$\begin{array}{l}\text { When you got pregnant, did you } \\
\text { want to get pregnant at that time? }\end{array}$} \\
\hline No & 14.7 & 8.5 & 13.3 & 11.4 & 375 & 2.327 \\
\hline Yes & 85.3 & 91.5 & 86.7 & 88.6 & 2,653 & 0.331 \\
\hline Total & 100.0 & 100.0 & 100.0 & 100.0 & 3,028 & \\
\hline$N$ & 984 & 1,040 & 1,004 & 2,024 & & \\
\hline \multicolumn{7}{|c|}{$\begin{array}{l}\text { Want to have a baby later or no } \\
\text { more children? (among mothers) }\end{array}$} \\
\hline No more/None & 37.0 & 19.1 & 19.3 & 29.5 & 84 & 4.422 \\
\hline Later & 63.0 & 80.9 & 80.7 & 70.5 & 235 & 1.325 \\
\hline Total & 100.0 & 100.0 & 100.0 & 100.0 & 319 & \\
\hline $\mathrm{N}$ & 117 & 85 & 117 & 202 & & \\
\hline \multicolumn{7}{|c|}{$\begin{array}{l}\text { Want to have a baby later or no } \\
\text { more children? (among never } \\
\text { mothers) }\end{array}$} \\
\hline No more/None & 16.8 & 17.0 & 19.6 & 16.9 & 12 & 0.051 \\
\hline Later & 83.2 & 83.0 & 80.4 & 83.1 & 44 & 0.039 \\
\hline Total & 100.0 & 100.0 & 100.0 & 100.0 & 56 & \\
\hline$N$ & 22 & 10 & 24 & 32 & & \\
\hline \multicolumn{7}{|c|}{$\begin{array}{l}\text { How much longer did you want to } \\
\text { wait (in years)? }\end{array}$} \\
\hline Don't know & 20.2 & 11.6 & 1.8 & 16.3 & 30 & 23.365 \\
\hline $0-2$ & 35.9 & 19.4 & 41.2 & 28.5 & 90 & 3.620 \\
\hline 3 & 30.6 & 39.1 & 30.9 & 34.4 & 92 & * \\
\hline $4-8$ & 13.4 & 29.9 & 26.2 & 20.8 & 67 & \\
\hline Total & 100.0 & 100.0 & 100.0 & 100.0 & 279 & \\
\hline$N$ & 92 & 76 & 111 & 168 & & \\
\hline \multicolumn{7}{|c|}{$\begin{array}{l}\text { Do you plan to have another child } \\
\text { in the future? }\end{array}$} \\
\hline No & 10.3 & 6.7 & 17.7 & 8.4 & 343 & 109.335 \\
\hline Yes & 83.3 & 90.0 & 81.9 & 86.9 & 2,578 & 7.791 \\
\hline Don't know & 6.4 & 3.4 & 0.5 & 4.8 & 107 & ** \\
\hline Total & 100.0 & 100.0 & 100.0 & 100.0 & 3,028 & \\
\hline $\mathrm{N}$ & 984 & 1,040 & 1,004 & 2,024 & & \\
\hline
\end{tabular}

Estimates based on low Ns (approx. $<30$ obs) have large relative standard errors and should be interpreted with caution. ${ }^{* * *}$ indicates $p<0.001,{ }^{* \star}$ indicates $p<0.05$ and ${ }^{*}$ indicates $p<.0 .01$ for the $c h{ }^{2}$ statistic comparing integrated versus malaria-only study areas. 
TABLE 4.1.2. ANC VISITS AND TIMING DURING CURRENT PREGNANCY

Percentage of women aged 15 to 49 currently in late pregnancy (7-9 months) reporting ANC visits and timing for current pregnancy, September-October 2019

\begin{tabular}{|c|c|c|c|c|c|c|}
\hline & $\begin{array}{c}\text { KEBBI } \\
\%\end{array}$ & $\begin{array}{c}\text { SOKOTO } \\
\%\end{array}$ & $\begin{array}{c}\text { MALARIA-ONLY } \\
\text { (ZAMFARA) } \\
\%\end{array}$ & $\begin{array}{c}\text { INTEGRATED } \\
\text { (KEBBI/SOKOTO) } \\
\%\end{array}$ & $\mathbf{N}$ & $\begin{array}{c}\mathrm{CHI}^{2} \\
\text { VALUE }\end{array}$ \\
\hline \multicolumn{7}{|l|}{$\begin{array}{l}\text { At anytime during this pregnancy, } \\
\text { have you seen anyone for ANC? }\end{array}$} \\
\hline No & 47.8 & 48.7 & 47.0 & 48.3 & 451 & 0.903 \\
\hline Yes & 52.2 & 51.3 & 53.0 & 51.7 & 580 & 0.018 \\
\hline Total & 100.0 & 100.0 & 100.0 & 100.0 & 1,031 & \\
\hline N & 334 & 340 & 357 & 674 & & \\
\hline \multicolumn{7}{|c|}{$\begin{array}{l}\text { When did you first receive ANC for this } \\
\text { pregnancy? }\end{array}$} \\
\hline $0-3$ months & 18.6 & 21.5 & 15.8 & 20.1 & 104 & 75.416 \\
\hline 4-6 months & 64.7 & 63.6 & 67.6 & 64.2 & 380 & 1.519 \\
\hline $7+$ months & 13.8 & 14.6 & 16.6 & 14.2 & 90 & \\
\hline Don't know & 2.9 & 0.4 & 0.0 & 1.6 & 6 & \\
\hline Total & 100.0 & 100.0 & 100.0 & 100.0 & 580 & \\
\hline $\mathrm{N}$ & 181 & 190 & 209 & 371 & & \\
\hline \multicolumn{7}{|c|}{$\begin{array}{l}\text { Where did you receive ANC for this first } \\
\text { visit during the pregnancy? }\end{array}$} \\
\hline Don't know & 1.3 & 0.0 & 0.0 & 0.6 & 2 & \\
\hline Other & 0.0 & 1.0 & 0.0 & 0.5 & 2 & \\
\hline Other private sector & 0.0 & 0.0 & 0.0 & 0.0 & 0 & \\
\hline Government hospital & 38.9 & 35.8 & 55.0 & 37.3 & 267 & \\
\hline Governmental PHC & 49.3 & 48.7 & 37.0 & 49.0 & 249 & \\
\hline Dispensary/health post & 2.9 & 9.8 & 1.6 & 6.5 & 26 & \\
\hline Community health outreach post & 3.0 & 0.8 & 3.9 & 1.8 & 15 & \\
\hline Nursing/maternity home & 0.9 & 0.0 & 0.8 & 0.4 & 3 & \\
\hline Private hospital/clinic & 3.2 & 3.3 & 0.0 & 3.3 & 11 & \\
\hline Pharmacy/chemist & 0.5 & 0.7 & 1.7 & 0.6 & 5 & \\
\hline Total & 100.0 & 100.0 & 100.0 & 100.0 & 580 & \\
\hline N & 181 & 190 & 209 & 371 & & \\
\hline \multicolumn{7}{|l|}{$\begin{array}{l}\text { Whom did you see when you first re- } \\
\text { ceived ANC during this pregnancy? }\end{array}$} \\
\hline Don't know/don't remember & 0.0 & 0.0 & 0.0 & 0.0 & 0 & \\
\hline Other & 0.7 & 0.5 & 0.0 & 0.6 & 2 & \\
\hline Other health personnel & 0.5 & 0.0 & 0.0 & 0.2 & 1 & \\
\hline Doctor & 11.4 & 20.1 & 8.4 & 16.0 & 77 & \\
\hline Nurse/midwife & 60.2 & 51.6 & 66.8 & 55.7 & 352 & \\
\hline Community health worker & 25.6 & 27.3 & 24.9 & 26.5 & 144 & \\
\hline Chemist & 1.0 & 0.0 & 0.0 & 0.5 & 2 & \\
\hline Traditional healer/birth attendant & 0.0 & 0.5 & 0.0 & 0.3 & 1 & \\
\hline Family & 0.7 & 0.0 & 0.0 & 0.3 & 1 & \\
\hline Friends/neighbors & 0.0 & 0.0 & 0.0 & 0.0 & 0 & \\
\hline Total & 100.0 & 100.0 & 100.0 & 100.0 & 580 & \\
\hline $\mathrm{N}$ & 181 & 190 & 209 & 371 & & \\
\hline
\end{tabular}

Estimates based on low Ns (approx. $<30$ obs) have large relative standard errors and should be interpreted with caution. ${ }^{* \star *}$ indicates $p<0.001,{ }^{* \star}$ indicates $p<0.05$ and ${ }^{*}$ indicates $p<.0 .01$ for the chi ${ }^{2}$ statistic comparing integrated versus malaria-only study areas. 
TABLE 4.1.3. ANC CONTENT DURING CURRENT PREGNANCY

Percentage of women aged 15 to 49 currently in late pregnancy (7-9 months) reporting ANC content for current pregnancy, September-October 2019

\begin{tabular}{|c|c|c|c|c|c|c|}
\hline & $\begin{array}{c}\text { KEBBI } \\
\%\end{array}$ & $\begin{array}{c}\text { SOKOTO } \\
\%\end{array}$ & $\begin{array}{c}\text { MALARIA-ONLY } \\
\text { (ZAMFARA) } \\
\%\end{array}$ & $\begin{array}{c}\text { INTEGRATED } \\
\text { (KEBBI/SOKOTO) } \\
\%\end{array}$ & $\mathbf{N}$ & $\begin{array}{c}\mathrm{CHI}^{2} \\
\text { VALUE }\end{array}$ \\
\hline \multicolumn{7}{|c|}{$\begin{array}{l}\text { How many checkups have you had for this preg- } \\
\text { nancy? }\end{array}$} \\
\hline None & 2.0 & 0.0 & 0.0 & 0.9 & 2 & 267.055 \\
\hline 1 & 18.2 & 19.7 & 17.1 & 19.0 & 98 & 3.363 \\
\hline 2 & 16.4 & 14.9 & 26.6 & 15.6 & 114 & ** \\
\hline 3 & 15.3 & 16.5 & 21.8 & 15.9 & 111 & \\
\hline $4+$ & 45.1 & 46.7 & 34.5 & 45.9 & 245 & \\
\hline Don't know & 3.2 & 2.3 & 0.0 & 2.7 & 10 & \\
\hline Total & 100.0 & 100.0 & 100.0 & 100.0 & 580 & \\
\hline $\mathrm{N}$ & 181 & 190 & 209 & 371 & & \\
\hline \multicolumn{7}{|c|}{ Was your blood pressure measured? } \\
\hline No & 21.1 & 13.7 & 5.3 & 17.2 & 71 & 220.168 \\
\hline Yes & 78.9 & 85.9 & 94.7 & 82.6 & 508 & 8.029 \\
\hline Don't know & 0.0 & 0.4 & 0.0 & 0.2 & 1 & ** \\
\hline Total & 100.0 & 100.0 & 100.0 & 100.0 & 580 & \\
\hline $\mathrm{N}$ & 181 & 190 & 209 & 371 & & \\
\hline \multicolumn{7}{|c|}{ Did you give a urine sample? } \\
\hline No & 40.7 & 38.6 & 21.6 & 39.6 & 173 & 210.334 \\
\hline Yes & 59.3 & 61.4 & 78.4 & 60.4 & 407 & 5.113 \\
\hline Total & 100.0 & 100.0 & 100.0 & 100.0 & 580 & * \\
\hline N & 181 & 190 & 209 & 371 & & \\
\hline \multicolumn{7}{|c|}{ Did you give a blood sample? } \\
\hline No & 30.7 & 28.2 & 20.5 & 29.4 & 142 & \\
\hline Yes & 69.3 & 71.8 & 79.5 & 70.6 & 438 & \\
\hline Don't know & 0.0 & 0.0 & 0.0 & 0.0 & 0 & \\
\hline Total & 100.0 & 100.0 & 100.0 & 100.0 & 580 & \\
\hline $\mathrm{N}$ & 181 & 190 & 209 & 371 & & \\
\hline \multicolumn{7}{|c|}{ Were you tested for HIV? } \\
\hline No & 43.6 & 37.7 & 29.3 & 40.5 & 202 & 501.476 \\
\hline Yes & 34.7 & 30.5 & 61.0 & 32.5 & 260 & 6.291 \\
\hline Don't know & 21.8 & 31.8 & 9.7 & 27.0 & 118 & ** \\
\hline Total & 100.0 & 100.0 & 100.0 & 100.0 & 580 & \\
\hline $\mathrm{N}$ & 181 & 190 & 209 & 371 & & \\
\hline \multicolumn{7}{|c|}{ Was your weight measured? } \\
\hline No & 42.3 & 18.2 & 14.7 & 29.7 & 125 & 218.840 \\
\hline Yes & 56.4 & 81.3 & 85.4 & 69.4 & 451 & 4.299 \\
\hline Don't know & 1.3 & 0.4 & 0.0 & 0.9 & 4 & * \\
\hline Total & 100.0 & 100.0 & 100.0 & 100.0 & 580 & \\
\hline N & 181 & 190 & 209 & 371 & & \\
\hline \multicolumn{7}{|c|}{$\begin{array}{l}\text { Did you discuss with a health provider when the } \\
\text { baby is due? }\end{array}$} \\
\hline No & 59.6 & 58.8 & 43.7 & 59.2 & 293 & 176.048 \\
\hline Yes & 40.4 & 39.0 & 56.3 & 39.7 & 282 & 3.154 \\
\hline Don't know & 0.0 & 2.3 & 0.0 & 1.2 & 5 & \\
\hline Total & 100.0 & 100.0 & 100.0 & 100.0 & 580 & \\
\hline N & 181 & 190 & 209 & 371 & & \\
\hline \multicolumn{7}{|c|}{$\begin{array}{l}\text { Told by health worker about danger signs in } \\
\text { pregnancy? }\end{array}$} \\
\hline No & 44.0 & 36.1 & 29.4 & 39.8 & 206 & \\
\hline Yes & 56.1 & 63.9 & 70.7 & 60.2 & 374 & \\
\hline Don't know & 0.0 & 0.0 & 0.0 & 0.0 & 0 & \\
\hline Total & 100.0 & 100.0 & 100.0 & 100.0 & 580 & \\
\hline $\mathrm{N}$ & 181 & 190 & 209 & 371 & & \\
\hline \multicolumn{7}{|c|}{ Ever been given a mosquito net as part of ANC? } \\
\hline No & 95.4 & 82.4 & 81.9 & 88.6 & 497 & 45.192 \\
\hline Yes & 4.6 & 17.6 & 18.1 & 11.4 & 83 & 2.336 \\
\hline Total & 100.0 & 100.0 & 100.0 & 100.0 & 580 & \\
\hline N & 181 & 190 & 209 & 371 & & \\
\hline
\end{tabular}

Estimates based on low Ns (approx. $<30$ obs) have large relative standard errors and should be interpreted with caution. ${ }^{* \star *}$ indicates $p<0.001,{ }^{* *}$ indicates $p<0.05$ and ${ }^{*}$ indicates $p<.0 .01$ for the chi $^{2}$ statistic comparing integrated versus malaria-only study areas. 


\section{TABLE 4.1.4. BIRTH PLANNING DURING CURRENT PREGNANCY}

Percentage of women aged 15 to 49 currently in late pregnancy (7-9 months) reporting on childbirth planning with health worker during current pregnancy, September-October 2019

\begin{tabular}{|c|c|c|c|c|c|c|}
\hline & $\begin{array}{c}\text { KEBBI } \\
\%\end{array}$ & $\begin{array}{c}\text { SOKOTO } \\
\%\end{array}$ & $\begin{array}{c}\text { MALARIA-ONLY } \\
\text { (ZAMFARA) } \\
\%\end{array}$ & $\begin{array}{c}\text { INTEGRATED } \\
\text { (KEBBI/SOKOTO) } \\
\%\end{array}$ & $\mathbf{N}$ & $\begin{array}{c}\mathrm{CHI}^{2} \\
\text { VALUE }\end{array}$ \\
\hline \multicolumn{7}{|c|}{$\begin{array}{l}\text { Discussed with a health worker a plan } \\
\text { for your delivery? }\end{array}$} \\
\hline No & 77.9 & 71.6 & 54.6 & 74.6 & 383 & 231.967 \\
\hline Yes & 21.7 & 28.4 & 45.4 & 25.2 & 196 & 4.155 \\
\hline Don't know & 0.5 & 0.0 & 0.0 & 0.2 & 1 & * \\
\hline Total & 100.0 & 100.0 & 100.0 & 100.0 & 580 & \\
\hline $\mathrm{N}$ & 181 & 190 & 209 & 371 & & \\
\hline \multicolumn{7}{|c|}{ Where you plan to deliver the baby? } \\
\hline No & 26.1 & 14.8 & 3.6 & 19.5 & 22 & 351.219 \\
\hline Yes & 73.9 & 85.2 & 96.4 & 80.5 & 174 & 13.204 \\
\hline Total & 100.0 & 100.0 & 100.0 & 100.0 & 196 & 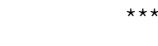 \\
\hline$N$ & 38 & 56 & 102 & 94 & & \\
\hline \multicolumn{7}{|c|}{$\begin{array}{l}\text { If you wanted to have a relative/friend/ } \\
\text { neighbor with you during labor }\end{array}$} \\
\hline No & 26.4 & 42.7 & 33.2 & 36.0 & 67 & 46.143 \\
\hline Yes & 71.0 & 57.3 & 66.8 & 62.9 & 128 & 0.444 \\
\hline Don't know & 2.6 & 0.0 & 0.0 & 1.1 & 1 & \\
\hline Total & 100.0 & 100.0 & 100.0 & 100.0 & 196 & \\
\hline$N$ & 38 & 56 & 102 & 94 & & \\
\hline \multicolumn{7}{|c|}{$\begin{array}{l}\text { Transportation to where the baby } \\
\text { would be born? }\end{array}$} \\
\hline No & 53.7 & 42.3 & 33.4 & 47.0 & 79 & 160.490 \\
\hline Yes & 41.7 & 57.7 & 66.6 & 51.1 & 115 & 1.690 \\
\hline Don't know & 4.6 & 0.0 & 0.0 & 1.9 & 2 & \\
\hline Total & 100.0 & 100.0 & 100.0 & 100.0 & 196 & \\
\hline$N$ & 38 & 56 & 102 & 94 & & \\
\hline \multicolumn{7}{|c|}{$\begin{array}{l}\text { What funds or finances you would use } \\
\text { for delivery? }\end{array}$} \\
\hline No & 49.4 & 31.7 & 24.9 & 39.0 & 60 & 177.141 \\
\hline Yes & 45.9 & 68.3 & 75.1 & 59.1 & 134 & 2.284 \\
\hline Don't know & 4.6 & 0.0 & 0.0 & 1.9 & 2 & \\
\hline Total & 100.0 & 100.0 & 100.0 & 100.0 & 196 & \\
\hline$N$ & 38 & 56 & 102 & 94 & & \\
\hline \multicolumn{7}{|c|}{$\begin{array}{l}\text { Finding a skilled birth attendant to } \\
\text { deliver your child }\end{array}$} \\
\hline No & 32.6 & 22.8 & 20.6 & 26.8 & 47 & 99.559 \\
\hline Yes & 62.8 & 77.3 & 79.4 & 71.3 & 147 & 1.613 \\
\hline Don't know & 4.6 & 0.0 & 0.0 & 1.9 & 2 & \\
\hline Total & 100.0 & 100.0 & 100.0 & 100.0 & 196 & \\
\hline $\mathrm{N}$ & 38 & 56 & 102 & 94 & & \\
\hline
\end{tabular}

Estimates based on low Ns (approx. $<30$ obs) have large relative standard errors and should be interpreted with caution. ${ }^{* \star *}$ indicates $p<0.001,{ }^{* *}$ indicates $p<0.05$ and ${ }^{*}$ indicates $p<.0 .01$ for the chi ${ }^{2}$ statistic comparing integrated versus malaria-only study areas. 
TABLE 4.1.5. REASONS FOR NOT ATTENDING ANC DURING CURRENT PREGNANCY

Percentage of women aged 15 to 49 currently in late pregnancy (7-9 months) reporting reasons for not attending any antenatal care during current pregnancy, September-October 2019

\begin{tabular}{|c|c|c|c|c|c|c|}
\hline & $\begin{array}{c}\text { KEBBI } \\
\%\end{array}$ & $\begin{array}{c}\text { SOKOTO } \\
\%\end{array}$ & $\begin{array}{c}\text { MALARIA-ONLY } \\
\text { (ZAMFARA) } \\
\%\end{array}$ & $\begin{array}{c}\text { INTEGRATED } \\
\text { (KEBBI/SOKOTO) } \\
\%\end{array}$ & $\mathbf{N}$ & $\begin{array}{c}\mathrm{CHI}^{2} \\
\text { VALUE }\end{array}$ \\
\hline \multicolumn{7}{|c|}{$\begin{array}{l}\text { Costs too much/Difficulty getting } \\
\text { money }\end{array}$} \\
\hline No & 85.0 & 85.2 & 90.9 & 85.1 & 397 & 43.674 \\
\hline Yes & 15.0 & 14.8 & 9.1 & 14.9 & 54 & 0.836 \\
\hline Total & 100.0 & 100.0 & 100.0 & 100.0 & 451 & \\
\hline $\mathrm{N}$ & 153 & 150 & 148 & 303 & & \\
\hline \multicolumn{7}{|c|}{ Don't know where to go } \\
\hline No & 96.7 & 97.4 & 96.7 & 97.1 & 436 & 0.738 \\
\hline Yes & 3.3 & 2.6 & 3.3 & 2.9 & 15 & 0.027 \\
\hline Total & 100.0 & 100.0 & 100.0 & 100.0 & 451 & \\
\hline N & 153 & 150 & 148 & 303 & & \\
\hline \multicolumn{7}{|c|}{ Health facility not open } \\
\hline No & 97.3 & 95.9 & 98.1 & 96.5 & 437 & 12.601 \\
\hline Yes & 2.7 & 4.1 & 1.9 & 3.5 & 14 & 0.303 \\
\hline Total & 100.0 & 100.0 & 100.0 & 100.0 & 451 & \\
\hline $\mathrm{N}$ & 153 & 150 & 148 & 303 & & \\
\hline \multicolumn{7}{|c|}{ Distance to health facility too far } \\
\hline No & 95.6 & 98.8 & 97.3 & 97.3 & 437 & 0.003 \\
\hline Yes & 4.4 & 1.2 & 2.7 & 2.7 & 14 & 0.000 \\
\hline Total & 100.0 & 100.0 & 100.0 & 100.0 & 451 & \\
\hline $\mathrm{N}$ & 153 & 150 & 148 & 303 & & \\
\hline \multicolumn{7}{|c|}{ Poor quality of service } \\
\hline No & 98.5 & 99.6 & 99.6 & 99.1 & 446 & 6.378 \\
\hline Yes & 1.5 & 0.4 & 0.4 & 0.9 & 5 & 0.609 \\
\hline Total & 100.0 & 100.0 & 100.0 & 100.0 & 451 & \\
\hline $\mathrm{N}$ & 153 & 150 & 148 & 303 & & \\
\hline \multicolumn{7}{|c|}{ No female provider at facility } \\
\hline No & 100.0 & 96.0 & 75.0 & 97.9 & 423 & 498.139 \\
\hline Yes & 0.0 & 4.0 & 25.0 & 2.1 & 28 & 5.916 \\
\hline Total & 100.0 & 100.0 & 100.0 & 100.0 & 451 & * \\
\hline N & 153 & 150 & 148 & 303 & & \\
\hline \multicolumn{7}{|c|}{ Husband/partner oppose } \\
\hline No & 66.8 & 72.7 & 58.7 & 70.0 & 303 & 70.411 \\
\hline Yes & 33.2 & 27.3 & 41.3 & 30.0 & 148 & 0.537 \\
\hline Total & 100.0 & 100.0 & 100.0 & 100.0 & 451 & \\
\hline N & 153 & 150 & 148 & 303 & & \\
\hline \multicolumn{7}{|c|}{ Religious leader opposes } \\
\hline No & 95.0 & 99.4 & 98.7 & 97.3 & 440 & 13.595 \\
\hline Yes & 5.1 & 0.6 & 1.3 & 2.7 & 11 & 0.459 \\
\hline Total & 100.0 & 100.0 & 100.0 & 100.0 & 451 & \\
\hline $\mathrm{N}$ & 153 & 150 & 148 & 303 & & \\
\hline \multicolumn{7}{|c|}{ Not necessary to go } \\
\hline No & 65.1 & 52.0 & 49.5 & 58.2 & 274 & 39.169 \\
\hline Yes & 34.9 & 48.0 & 50.5 & 41.8 & 177 & 0.337 \\
\hline Total & 100.0 & 100.0 & 100.0 & 100.0 & 451 & \\
\hline $\mathrm{N}$ & 153 & 150 & 148 & 303 & & \\
\hline \multicolumn{7}{|c|}{ Not customary } \\
\hline No & 93.5 & 68.5 & 91.6 & 80.3 & 392 & 148.691 \\
\hline Yes & 6.5 & 31.5 & 8.4 & 19.7 & 59 & 1.632 \\
\hline Total & 100.0 & 100.0 & 100.0 & 100.0 & 451 & \\
\hline N & 153 & 150 & 148 & 303 & & \\
\hline \multicolumn{7}{|c|}{ Up to God } \\
\hline No & 82.3 & 68.8 & 82.4 & 75.1 & 356 & 42.339 \\
\hline Yes & 17.8 & 31.2 & 17.6 & 24.9 & 95 & 0.940 \\
\hline Total & 100.0 & 100.0 & 100.0 & 100.0 & 451 & \\
\hline N & 153 & 150 & 148 & 303 & & \\
\hline
\end{tabular}

Estimates based on low Ns (approx. <30 obs) have large relative standard errors and should be interpreted with caution. ${ }^{* * *}$ indicates $p<0.001,{ }^{* *}$ indicates $p<0.05$ and ${ }^{*}$ indicates $p<.0 .01$ for the chi ${ }^{2}$ statistic comparing integrated versus malaria-only study areas. 
TABLE 4.1.6. MEDICINES OR VACCINATIONS RECEIVED DURING CURRENT PREGNANCY

Percentage of women 15 to 49 years currently in late pregnancy (7-9 months) reporting medicines or vaccinations received during ANC for current pregnancy, September-October 2019

\begin{tabular}{|c|c|c|c|c|c|c|}
\hline & $\begin{array}{c}\text { KEBBI } \\
\%\end{array}$ & $\begin{array}{c}\text { SOKOTO } \\
\%\end{array}$ & $\begin{array}{c}\text { MALARIA-ONLY } \\
\text { (ZAMFARA) } \\
\%\end{array}$ & $\begin{array}{c}\text { INTEGRATED } \\
\text { (KEBBI/SOKOTO) } \\
\%\end{array}$ & $\mathbf{N}$ & $\begin{array}{c}\mathrm{CHI}^{2} \\
\text { VALUE }\end{array}$ \\
\hline \multicolumn{7}{|c|}{$\begin{array}{l}\text { Were you given an injection in the arm } \\
\text { to prevent tetanus? }\end{array}$} \\
\hline No & 63.9 & 63.3 & 58.9 & 63.6 & 597 & 47.500 \\
\hline Yes & 34.4 & 36.7 & 41.2 & 35.6 & 427 & 1.057 \\
\hline Don't know & 1.8 & 0.0 & 0.0 & 0.8 & 7 & \\
\hline Total & 100.0 & 100.0 & 100.0 & 100.0 & 1,031 & \\
\hline $\mathrm{N}$ & 334 & 340 & 357 & 674 & & \\
\hline \multicolumn{7}{|c|}{$\begin{array}{l}\text { Before this pregnancy, how many } \\
\text { times did you get a tetanus injection? }\end{array}$} \\
\hline None & 44.3 & 62.2 & 63.4 & 53.7 & 552 & 287.726 \\
\hline 1 & 5.9 & 5.3 & 3.1 & 5.6 & 54 & 2.710 \\
\hline 2 & 5.8 & 5.9 & 11.2 & 5.9 & 84 & \\
\hline 3 or more & 4.2 & 7.4 & 9.0 & 5.9 & 80 & \\
\hline Don't know & 39.7 & 19.3 & 13.3 & 29.0 & 258 & \\
\hline Total & 100.0 & 100.0 & 100.0 & 100.0 & 1,028 & \\
\hline $\mathrm{N}$ & 333 & 339 & 356 & 672 & & \\
\hline \multicolumn{7}{|c|}{$\begin{array}{l}\text { During this pregnancy, have you taken } \\
\text { any iron tablet or iron syrup? }\end{array}$} \\
\hline No & 54.2 & 56.4 & 50.3 & 55.4 & 513 & 35.616 \\
\hline Yes & 45.6 & 42.8 & 49.7 & 44.1 & 513 & 0.651 \\
\hline Don't know & 0.2 & 0.8 & 0.0 & 0.5 & 5 & \\
\hline Total & 100.0 & 100.0 & 100.0 & 100.0 & 1,031 & \\
\hline$N$ & 334 & 340 & 357 & 674 & & \\
\hline \multicolumn{7}{|c|}{$\begin{array}{l}\text { During this pregnancy, have you taken } \\
\text { any folic acid supplements? }\end{array}$} \\
\hline No & 56.5 & 56.0 & 50.7 & 56.2 & 521 & 81.318 \\
\hline Yes & 40.0 & 43.0 & 49.1 & 41.6 & 490 & 1.441 \\
\hline Don't know & 3.5 & 1.0 & 0.2 & 2.2 & 20 & \\
\hline Total & 100.0 & 100.0 & 100.0 & 100.0 & 1,031 & \\
\hline$N$ & 334 & 340 & 357 & 674 & & \\
\hline \multicolumn{7}{|c|}{$\begin{array}{l}\text { During this pregnancy, have you taken } \\
\text { any drugs for intestinal worms? }\end{array}$} \\
\hline No & 65.3 & 75.6 & 81.3 & 70.7 & 742 & 90.720 \\
\hline Yes & 25.8 & 18.3 & 14.1 & 21.8 & 215 & 2.258 \\
\hline Don't know & 8.9 & 6.1 & 4.6 & 7.4 & 74 & \\
\hline Total & 100.0 & 100.0 & 100.0 & 100.0 & 1,031 & \\
\hline $\mathrm{N}$ & 334 & 340 & 357 & 674 & & \\
\hline
\end{tabular}

Estimates based on low Ns (approx. $<30$ obs) have large relative standard errors and should be interpreted with caution. ${ }^{* * *}$ indicates $p<0.001,{ }^{* *}$ indicates $p<0.05$ and ${ }^{*}$ indicates $p<.0 .01$ for the chi ${ }^{2}$ statistic comparing integrated versus malaria-only study areas. 
TABLE 4.1.7. NUTRITIONAL INTAKE DURING CURRENT PREGNANCY

Percentage of women aged 15 to 49 who are currently pregnant reporting foods eaten during the previous 24 hours, September-October 2019

\begin{tabular}{|c|c|c|c|c|c|c|}
\hline & $\begin{array}{c}\text { KEBBI } \\
\%\end{array}$ & $\begin{array}{l}\text { SOKOTO } \\
\%\end{array}$ & $\begin{array}{l}\text { MALARIA-ONLY } \\
\text { (ZAMFARA) } \\
\%\end{array}$ & $\begin{array}{c}\text { INTEGRATED } \\
\text { (KEBBI/SOKOTO) } \\
\%\end{array}$ & $\mathbf{N}$ & $\begin{array}{c}\mathrm{CHI}^{2} \\
\text { VALUE }\end{array}$ \\
\hline \multicolumn{7}{|c|}{$\begin{array}{l}\text { How many meals did you have at any } \\
\text { time yesterday? }\end{array}$} \\
\hline None & 0.1 & 0.2 & 0.1 & 0.1 & 4 & 113.635 \\
\hline 1 & 1.7 & 7.3 & 1.8 & 4.7 & 106 & 5.742 \\
\hline 2 & 17.2 & 25.2 & 9.5 & 21.5 & 523 & *** \\
\hline 3 or more & 81.0 & 66.5 & 88.3 & 73.3 & 2,380 & \\
\hline Don't know & 0.1 & 0.8 & 0.4 & 0.5 & 15 & \\
\hline Total & 100.0 & 100.0 & 100.0 & 100.0 & 3,028 & \\
\hline $\mathrm{N}$ & 984 & 1,040 & 1,004 & 2,024 & & \\
\hline \multicolumn{7}{|c|}{$\begin{array}{l}\text { Did you eat any of the following at } \\
\text { any time yesterday (during the day or } \\
\text { night)? }\end{array}$} \\
\hline \multicolumn{7}{|c|}{ Milk, cheese, yoghurt, other dairy } \\
\hline No & 65.6 & 53.9 & 55.5 & 59.4 & 1,792 & 5.515 \\
\hline Yes & 34.4 & 45.9 & 44.2 & 40.5 & 1,230 & 0.280 \\
\hline Don't know & 0.0 & 0.2 & 0.3 & 0.1 & 6 & \\
\hline Total & 100.0 & 100.0 & 100.0 & 100.0 & 3,028 & \\
\hline $\mathrm{N}$ & 984 & 1,040 & 1,004 & 2,024 & & \\
\hline \multicolumn{7}{|c|}{ Bread, rice, tuwo or other grains } \\
\hline No & 8.3 & 3.5 & 7.6 & 5.8 & 173 & 5.756 \\
\hline Yes & 91.67 & 96.4 & 92.2 & 94.2 & 2,851 & 0.240 \\
\hline Don't know & 0.0 & 0.1 & 0.2 & 0.0 & 4 & \\
\hline Total & 100.0 & 100.0 & 100.0 & 100.0 & 3,028 & \\
\hline N & 984 & 1,040 & 1,004 & 2,024 & & \\
\hline \multicolumn{7}{|c|}{$\begin{array}{l}\text { White potato, cassava, yam, eba, or } \\
\text { other foods from roots or tubers }\end{array}$} \\
\hline No & 58.1 & 72.4 & 56.9 & 65.8 & 1,890 & 23.621 \\
\hline Yes & 41.5 & 27.3 & 42.6 & 33.9 & 1,127 & 1.562 \\
\hline Don't know & 0.3 & 0.3 & 0.5 & 0.3 & 11 & \\
\hline Total & 100.0 & 100.0 & 100.0 & 100.0 & 3,028 & \\
\hline $\mathrm{N}$ & 984 & 1,040 & 1,004 & 2,024 & & \\
\hline \multicolumn{7}{|c|}{$\begin{array}{l}\text { Pumpkin, squash, or other that is yel- } \\
\text { low or orange inside }\end{array}$} \\
\hline No & 82.8 & 86.4 & 79.3 & 84.8 & 2,532 & 15.024 \\
\hline Yes & 16.5 & 13.3 & 19.7 & 14.8 & 477 & 0.719 \\
\hline Don't know & 0.6 & 0.3 & 1.0 & 0.5 & 19 & \\
\hline Total & 100.0 & 100.0 & 100.0 & 100.0 & 3,028 & \\
\hline N & 984 & 1,040 & 1,004 & 2,024 & & \\
\hline \multicolumn{7}{|c|}{ Any dark and leafy vegetables } \\
\hline No & 37.5 & 51.4 & 40.1 & 44.9 & 1,330 & 7.046 \\
\hline Yes & 62.2 & 48.3 & 59.6 & 54.7 & 1,686 & 0.501 \\
\hline Don't know & 0.4 & 0.3 & 0.4 & 0.3 & 12 & \\
\hline Total & 100.0 & 100.0 & 100.0 & 100.0 & 3,028 & \\
\hline $\mathrm{N}$ & 984 & 1,040 & 1,004 & 2,024 & & \\
\hline \multicolumn{7}{|c|}{ Ripe mangoes, pawpaw } \\
\hline No & 87.7 & 91.9 & 82.6 & 90.0 & 2,665 & 31.884 \\
\hline Yes & 12.2 & 7.8 & 16.7 & 9.8 & 352 & 1.806 \\
\hline Don't know & 0.1 & 0.3 & 0.7 & 0.2 & 11 & \\
\hline Total & 100.0 & 100.0 & 100.0 & 100.0 & 3,028 & \\
\hline $\mathrm{N}$ & 984 & 1,040 & 1,004 & 2,024 & & \\
\hline
\end{tabular}




\begin{tabular}{|c|c|c|c|c|c|c|}
\hline \multicolumn{7}{|c|}{$\begin{array}{l}\text { Other kinds of fruits/vegetables (other } \\
\text { local vitamin A rich fruits) }\end{array}$} \\
\hline No & 57.6 & 79.9 & 67.2 & 69.5 & 2,062 & 1.950 \\
\hline Yes & 42.0 & 19.8 & 32.4 & 30.1 & 952 & 0.104 \\
\hline Don't know & 0.4 & 0.3 & 0.5 & 0.4 & 14 & \\
\hline Total & 100.0 & 100.0 & 100.0 & 100.0 & 3,028 & \\
\hline$N$ & 984 & 1,040 & 1,004 & 2,024 & & \\
\hline \multicolumn{7}{|l|}{ Eggs } \\
\hline No & 80.7 & 86.1 & 75.4 & 83.6 & 2,429 & 29.028 \\
\hline Yes & 19.2 & 13.6 & 24.4 & 16.2 & 592 & 1.890 \\
\hline Don't know & 0.1 & 0.3 & 0.2 & 0.2 & 7 & \\
\hline Total & 100.0 & 100.0 & 100.0 & 100.0 & 3,028 & \\
\hline$N$ & 984 & 1,040 & 1,004 & 2,024 & & \\
\hline \multicolumn{7}{|c|}{$\begin{array}{l}\text { Any kinds of meat, poultry, fish, or } \\
\text { shellfish (or organ meats) }\end{array}$} \\
\hline No & 53.9 & 56.5 & 49.1 & 55.3 & 1,586 & 11.563 \\
\hline Yes & 45.9 & 43.2 & 50.5 & 44.5 & 1,432 & 0.716 \\
\hline Don't know & 0.1 & 0.3 & 0.4 & 0.2 & 10 & \\
\hline Total & 100.0 & 100.0 & 100.0 & 100.0 & 3,028 & \\
\hline $\mathrm{N}$ & 984 & 1,040 & 1,004 & 2,024 & & \\
\hline \multicolumn{7}{|c|}{$\begin{array}{l}\text { Any foods made from beans, peas, } \\
\text { lentils or nuts }\end{array}$} \\
\hline No & 44.8 & 58.3 & 32.5 & 52.0 & 1,363 & 117.060 \\
\hline Yes & 53.7 & 41.4 & 67.0 & 47.2 & 1,642 & 5.578 \\
\hline Don't know & 1.4 & 0.3 & 0.5 & 0.8 & 23 & ** \\
\hline Total & 100.0 & 100.0 & 100.0 & 100.0 & 3,028 & \\
\hline$N$ & 984 & 1,040 & 1,004 & 2,024 & & \\
\hline \multicolumn{7}{|c|}{$\begin{array}{l}\text { Any oil, fats, butter or foods made with } \\
\text { these }\end{array}$} \\
\hline No & 41.4 & 53.5 & 35.7 & 47.8 & 1,317 & 47.337 \\
\hline Yes & 56.8 & 46.2 & 63.7 & 51.1 & 1,681 & 1.590 \\
\hline Don't know & 1.9 & 0.3 & 0.6 & 1.0 & 30 & \\
\hline Total & 100.0 & 100.0 & 100.0 & 100.0 & 3,028 & \\
\hline$N$ & 984 & 1,040 & 1,004 & 2,024 & & \\
\hline \multicolumn{7}{|c|}{$\begin{array}{l}\text { Sugary foods such as dates, sweets, } \\
\text { biscuits }\end{array}$} \\
\hline No & 70.1 & 67.9 & 58.7 & 68.9 & 1,945 & 34.988 \\
\hline Yes & 28.2 & 31.7 & 40.6 & 30.0 & 1,051 & 1.536 \\
\hline Don't know & 1.8 & 0.4 & 0.7 & 1.1 & 32 & \\
\hline Total & 100.0 & 100.0 & 100.0 & 100.0 & 3,028 & \\
\hline$N$ & 984 & 1,040 & 1,004 & 2,024 & & \\
\hline
\end{tabular}

Estimates based on low Ns (approx. $<30$ obs) have large relative standard errors and should be interpreted with caution. ${ }^{* * *}$ indicates $p<0.001,{ }^{* *}$ indicates $p<0.05$ and ${ }^{*}$ indicates $p<.0 .01$ for the chi ${ }^{2}$ statistic comparing integrated versus malaria-only study areas. 


\section{Antenatal, delivery, and newborn care}

\subsection{Antenatal care}

\section{Key findings}

ANC attendance: Among respondents with a completed pregnancy in the past two years, only 32 percent in Kebbi/Sokoto and 38 percent in Zamfara made at least one ANC visit with a skilled health provider. This percentage was significantly higher among respondents in the wealthiest households (58\% and 75\%) than in the poorest households (18\% and 12\%) in Kebbi/Sokoto and Zamfara, respectively. Similarly, respondents who attended primary or secondary/higher education in both areas were more likely to attend ANC at least one time than their less educated counterparts. As a result, only 20 percent of respondents in Kebbi/Sokoto and 26 percent in Zamfara made the recommended four or more ANC visits during their last pregnancy, with similar patterns by sociodemographic characteristics.

ANC awareness and non-use reasons: Among respondents who chose not to make any ANC visits during the last pregnancy, the most common reasons were lack of perceived need (42\%), spousal opposition (25\%), fatalism ("It's up to God") (20\%), and the perception that ANC is not the norm (13\%). Few respondents were aware of the suggested number and timing of ANC visits during pregnancy. There was higher awareness about attending ANC four or more times in Zamfara (49\%) than in Kebbi/ Sokoto (34\%). Only one quarter of respondents reported that a woman should go for her first ANC visit as soon as she thinks she is pregnant or in the first trimester.

ANC decision-making, self-efficacy, and intentions: All respondents were asked about who influences a woman's decision to go to four or more ANC visits. The most common responses were spouse (67\%) and no one else (26\%). Nearly all respondents (92\%) agreed that it is important to discuss their pregnancies with their husbands yet only 73 percent of respondents had the confidence to start a conversation with their husband about attending ANC. Only about two-thirds of respondents (66\%) intended to make at least four ANC visits during their next pregnancies with fewer (48\%) intending to go to ANC eight or more times.
ANC myths: All respondents were asked about the benefits of ANC to a woman. The most common responses were to monitor the mother's health (66\%) and the baby's growth (65\%). Nevertheless, there were some common myths about ANC, with a greater proportion of respondents agreeing with these myths in Kebbi/Sokoto than in Zamfara. Overall, 48 percent and 35 percent of respondents in Kebbi/Sokoto and Zamfara, respectively, agreed that pregnant women need ANC only when sick, while 40 percent and 30 percent of respondents in these areas agreed that it is better to use a traditional provider than a health facility for ANC. One-quarter of respondents (25\%) believed that only first-time mothers need ANC. These responses point to areas for SBC activities to improve ANC attendance by dispelling myths and raising awareness of ANC benefits among women in these areas.

ANC content: Beyond malaria prevention during pregnancy, few respondents received other essential pregnancy-related care during their last pregnancy. For example, roughly half of respondents (47\%) in Zamfara and one-third (35\%) in Kebbi/Sokoto received a tetanus injection during their most recent pregnancy, even though the majority (66\% in Kebbi/Sokoto and 61\% in Zamfara) had not received any tetanus injection prior to the most recent pregnancy. Only about half of respondents reported having taken iron tablets/iron syrup (42\% in Kebbi/Sokoto and 53\% in Zamfara). Similarly, only 41 percent of respondents in Kebbi/Sokoto and 54 percent of respondents in Zamfara took any folic acid supplements during their most recent pregnancy.

Birth planning: Developing a birth plan with the assistance of a health worker does not appear to be standard practice in study areas, and baseline results point to the need for more intensified SBC messaging to improve this practice going forward. Only one in three respondents reported discussing birth plans with a health worker, with the majority of these respondents reporting that discussions focused on delivery location (92\%), delivery financing (74\%), finding a skilled birth attendant (72\%), choosing personnel to have present during the delivery (67\%), and transportation (60\%). 


\section{TABLE 5.1.A. ANC 1+ DURING LAST PREGNANCY BY SOCIODEMOGRAPHIC CHARACTERISTICS}

Percentage of women 15 to 49 years with a child under 2 years who attended ANC one or more times where at least one visit was with a skilled provider for their last-born child by sociodemographic characteristics, September-October 2019

\begin{tabular}{|c|c|c|c|c|c|c|c|c|}
\hline & \multicolumn{2}{|c|}{ KEBBI } & \multicolumn{2}{|c|}{ SOKOTO } & \multicolumn{2}{|c|}{$\begin{array}{l}\text { MALARIA-ONLY } \\
\text { (ZAMFARA) }\end{array}$} & \multicolumn{2}{|c|}{$\begin{array}{l}\text { INTEGRATED } \\
\text { (KEBBI/SOKOTO) }\end{array}$} \\
\hline & $\%$ & $\mathbf{N}$ & $\%$ & $\mathbf{N}$ & $\%$ & $\mathbf{N}$ & $\%$ & $\mathrm{~N}$ \\
\hline Total & 41.9 & 892 & 24.4 & 1,078 & 38.2 & 1,069 & 32.0 & 1,971 \\
\hline \multicolumn{9}{|l|}{ Household wealth } \\
\hline Lowest & 26.2 & 264 & 11.7 & 341 & 11.7 & 111 & 17.5 & 606 \\
\hline Second & 38.9 & 192 & 19.2 & 229 & 18.0 & 183 & 27.8 & 421 \\
\hline Middle & 44.4 & 157 & 31.7 & 220 & 24.6 & 223 & 36.8 & 377 \\
\hline Fourth & 57.7 & 113 & 32.9 & 135 & 41.1 & 248 & 44.6 & 249 \\
\hline Highest & 61.8 & 166 & 54.4 & 153 & 75.3 & 304 & 58.4 & 318 \\
\hline \multicolumn{9}{|l|}{ Maternal age (in years) } \\
\hline 15-24 years & 36.2 & 367 & 24.8 & 485 & 32.8 & 423 & 29.3 & 852 \\
\hline $25-34$ years & 43.7 & 412 & 23.7 & 478 & 41.6 & 486 & 32.8 & 890 \\
\hline $35-49$ years & 52.0 & 113 & 25.5 & 115 & 43.7 & 160 & 37.8 & 229 \\
\hline \multicolumn{9}{|c|}{$\begin{array}{l}\text { Maternal education (highest } \\
\text { level attended) }\end{array}$} \\
\hline None & 33.5 & 675 & 22.4 & 855 & 28.9 & 698 & 27.0 & 1,530 \\
\hline Primary & 64.7 & 47 & 48.9 & 57 & 74.1 & 60 & 55.8 & 104 \\
\hline Secondary or higher & 83.5 & 95 & 62.2 & 60 & 85.3 & 180 & 75.9 & 155 \\
\hline Islamic & 54.7 & 75 & 15.6 & 106 & 23 & 131 & 32.9 & 182 \\
\hline
\end{tabular}

Estimates based on low Ns (approx. $<30$ obs) have large relative standard errors and should be interpreted with caution. ${ }^{* \star *}$ indicates $p<0.001,{ }^{* *}$ indicates $p<0.05$ and ${ }^{*}$ indicates $p<.0 .01$ for the chi $^{2}$ statistic comparing integrated versus malaria-only study areas. 


\section{TABLE 5.1.B. ANC 4+ DURING LAST PREGNANCY BY SOCIODEMOGRAPHIC CHARACTERISTICS}

Percentage of women 15 to 49 years with a child under 2 years who attended ANC four or more times where at least one visit was with a skilled provider for their last-born child by sociodemographic characteristics, September-October 2019

\begin{tabular}{|c|c|c|c|c|c|c|c|c|}
\hline & \multicolumn{2}{|c|}{ KEBBI } & \multicolumn{2}{|c|}{ SOKOTO } & \multicolumn{2}{|c|}{$\begin{array}{l}\text { MALARIA-ONLY } \\
\text { (ZAMFARA) }\end{array}$} & \multicolumn{2}{|c|}{$\begin{array}{c}\text { INTEGRATED } \\
\text { (KEBBI/SOKOTO) }\end{array}$} \\
\hline & $\%$ & $\mathbf{N}$ & $\%$ & $\mathbf{N}$ & $\%$ & $\mathrm{~N}$ & $\%$ & $\mathbf{N}$ \\
\hline Total & 23.5 & 892 & 16.9 & 1,078 & 26.1 & 1,069 & 19.7 & 1,971 \\
\hline \multicolumn{9}{|l|}{ Household wealth } \\
\hline Lowest & 12.1 & 264 & 6.2 & 341 & 5.9 & 111 & 8.5 & 606 \\
\hline Second & 23.5 & 192 & 10.5 & 229 & 12.7 & 183 & 16.2 & 421 \\
\hline Middle & 19.7 & 157 & 23.3 & 220 & 13.9 & 223 & 21.9 & 377 \\
\hline Fourth & 37.8 & 113 & 25.7 & 135 & 25.7 & 248 & 31.3 & 249 \\
\hline Highest & 39.1 & 166 & 43.7 & 153 & 56.8 & 304 & 41.4 & 318 \\
\hline \multicolumn{9}{|l|}{ Maternal age (in years) } \\
\hline 15-24 years & 19.5 & 367 & 18.2 & 485 & 19.2 & 423 & 18.7 & 852 \\
\hline $25-34$ years & 24.0 & 412 & 15.9 & 478 & 29.3 & 486 & 19.6 & 890 \\
\hline $35-49$ years & 33.1 & 113 & 15.5 & 115 & 35.9 & 160 & 23.7 & 229 \\
\hline \multicolumn{9}{|c|}{$\begin{array}{l}\text { Maternal education (highest } \\
\text { level attended) }\end{array}$} \\
\hline None & 17.6 & 675 & 11.1 & 855 & 17.6 & 698 & 15.9 & 1,530 \\
\hline Primary & 43.6 & 47 & 23.8 & 57 & 53.6 & 60 & 42.0 & 104 \\
\hline Secondary or higher & 53.2 & 95 & 41.7 & 60 & 67.7 & 180 & 53.2 & 155 \\
\hline Islamic & 30.5 & 75 & 17.2 & 106 & 15.8 & 131 & 19.0 & 182 \\
\hline
\end{tabular}

Estimates based on low Ns (approx. $<30$ obs) have large relative standard errors and should be interpreted with caution. ${ }^{\star * *}$ indicates $p<0.001,{ }^{* *}$ indicates $p<0.05$ and * indicates $p<.0 .01$ for the chi $^{2}$ statistic comparing integrated versus malaria-only study areas.

\section{TABLE 5.1.1. PREGNANCY INTENTIONS DURING LAST PREGNANCY}

Percentage of women 15 to 49 years with a child under 2 years reporting pregnancy intentions for their last-born child in the past two years, September-October 2019

\begin{tabular}{|c|c|c|c|c|c|c|}
\hline & KEBBI & SOKOTO & $\begin{array}{l}\text { MALARIA-ONLY } \\
\text { (ZAMFARA) }\end{array}$ & $\begin{array}{l}\text { INTEGRATED } \\
\text { (KEBBI/SOKOTO) }\end{array}$ & $\mathbf{N}$ & $\begin{array}{c}\mathrm{CH}^{2} \\
\text { VALUE }\end{array}$ \\
\hline & $\%$ & $\%$ & $\%$ & $\%$ & & \\
\hline \multicolumn{7}{|c|}{$\begin{array}{l}\text { When you got pregnant, did you want } \\
\text { to pregnant at that time? }\end{array}$} \\
\hline No & 8.8 & 7.8 & 14.1 & 8.2 & 316 & 23.870 \\
\hline Yes & 91.2 & 92.2 & 85.9 & 91.8 & 2,723 & 2.660 \\
\hline Total & 100.0 & 100.0 & 100.0 & 100.0 & 3,039 & \\
\hline $\mathrm{N}$ & 892 & 1,078 & 1,069 & 1,970 & & \\
\hline \multicolumn{7}{|c|}{$\begin{array}{l}\text { Did you want to have a baby later on or } \\
\text { did you not want any more children? }\end{array}$} \\
\hline No more/None & 24.4 & 22.7 & 23.7 & 23.5 & 80 & 0.001 \\
\hline Later & 75.6 & 77.3 & 76.3 & 76.5 & 236 & 0.000 \\
\hline Total & 100.0 & 100.0 & 100.0 & 100.0 & 316 & \\
\hline N & 86 & 87 & 143 & 173 & & \\
\hline \multicolumn{7}{|c|}{$\begin{array}{l}\text { How much longer did you want to } \\
\text { wait? }\end{array}$} \\
\hline $0-6$ months & 97.3 & 87.2 & 95.6 & 91.7 & 219 & 8.289 \\
\hline 7-12 months & 0.7 & 1.4 & 3.6 & 1.1 & 6 & 3.889 \\
\hline Don't know & 2.0 & 11.4 & 0.9 & 7.2 & 11 & * \\
\hline Total & 100.0 & 100.0 & 100.0 & 100.0 & 236 & \\
\hline $\mathrm{N}$ & 64 & 66 & 106 & 130 & & \\
\hline
\end{tabular}

Estimates based on low Ns (approx. $<30$ obs) have large relative standard errors and should be interpreted with caution. ${ }^{* * *}$ indicates $p<0.001,{ }^{* *}$ indicates $p<0.05$ and * indicates $p<.0 .01$ for the chi ${ }^{2}$ statistic comparing integrated versus malaria-only study areas. 
TABLE 5.1.2. SOURCE AND TIMING OF ANC DURING LAST PREGNANCY

Percentage of women 15 to 49 years with a child under 2 years reporting source and timing of ANC for their last-born child in the past two years, September-October 2019

\begin{tabular}{|c|c|c|c|c|c|c|}
\hline & KEBBI & SOKOTO & $\begin{array}{l}\text { MALARIA-ONLY } \\
\text { (ZAMFARA) }\end{array}$ & $\begin{array}{c}\text { INTEGRATED } \\
\text { (KEBBI/SOKOTO) }\end{array}$ & $\mathbf{N}$ & $\begin{array}{c}\mathrm{CHI}^{2} \\
\text { VALUE }\end{array}$ \\
\hline & $\%$ & $\%$ & $\%$ & $\%$ & & \\
\hline \multicolumn{7}{|l|}{$\begin{array}{l}\text { During your pregnancy, did you see } \\
\text { anyone for antenatal care? }\end{array}$} \\
\hline No & 47.0 & 61.8 & 47.4 & 55.4 & 1,523 & 29.969 \\
\hline Yes & 52.7 & 37.5 & 52.6 & 44.1 & 1,502 & 1.894 \\
\hline Don't know & 0.3 & 0.7 & 0.0 & 0.6 & 14 & \\
\hline Total & 100.0 & 100.0 & 100.0 & 100.0 & 3,039 & \\
\hline $\mathrm{N}$ & 892 & 1,078 & 1,069 & 1,970 & & \\
\hline \multicolumn{7}{|l|}{$\begin{array}{l}\text { When did you first receive antenatal } \\
\text { care for last pregnancy? }\end{array}$} \\
\hline 0-3 months & 30.7 & 25.5 & 20.5 & 28.1 & 356 & 32.632 \\
\hline 4-6 months & 53.7 & 60.6 & 63.2 & 57.0 & 912 & 3.300 \\
\hline $7+$ months & 9.0 & 11.0 & 14.9 & 10.0 & 188 & * \\
\hline Don't know & 6.6 & 3.0 & 1.4 & 4.9 & 46 & \\
\hline Total & 100.0 & 100.0 & 100.0 & 100.0 & 1,502 & \\
\hline $\mathrm{N}$ & 452 & 457 & 593 & 909 & & \\
\hline \multicolumn{7}{|c|}{$\begin{array}{l}\text { Where did you receive ANC for this first } \\
\text { visit during your pregnancy }\end{array}$} \\
\hline Her home & 4.2 & 2.5 & 0.8 & 3.4 & 37 & \\
\hline Other home & 0.6 & 0.0 & 0.1 & 0.3 & 5 & \\
\hline Government hospital & 31.8 & 30.8 & 46.6 & 31.4 & 616 & \\
\hline Governmental PHC & 55.2 & 48.2 & 41.0 & 51.8 & 668 & \\
\hline Dispensary/health post & 2.0 & 12.6 & 3.2 & 7.1 & 85 & \\
\hline Community health outreach post & 2.7 & 0.9 & 4.1 & 1.8 & 31 & \\
\hline Nursing/maternity home & 0.2 & 0.6 & 0.0 & 0.4 & 3 & \\
\hline Private hospital/clinic & 1.4 & 3.2 & 0.8 & 2.3 & 29 & \\
\hline Pharmacy & 0.5 & 0.4 & 2.6 & 0.4 & 15 & \\
\hline Don't know/don't remember & 1.0 & 0.0 & 0.0 & 0.5 & 3 & 84.832 \\
\hline Other & 0.3 & 0.0 & 0.5 & 0.2 & 3 & 2.001 \\
\hline Other public source & 0.2 & 0.0 & 0.0 & 0.1 & 1 & \\
\hline Other private source & 0.0 & 0.9 & 0.4 & 0.4 & 6 & \\
\hline Total & 100.0 & 100.0 & 100.0 & 100.0 & 1,502 & \\
\hline $\mathrm{N}$ & 452 & 457 & 593 & 909 & & \\
\hline
\end{tabular}

Estimates based on low Ns (approx. $<30$ obs) have large relative standard errors and should be interpreted with caution. ${ }^{* \star *}$ indicates $p<0.001,{ }^{* *}$ indicates $p<0.05$ and ${ }^{*}$ indicates $p<0.01$ for the chi ${ }^{2}$ statistic comparing integrated versus malaria-only study areas. 
TABLE 5.1.3. WHOM DID YOU SEE FOR THE FIRST ANC VISIT DURING LAST PREGNANCY?

Percentage of women 15 to 49 years with a child under 2 years reporting whom they saw for their first ANC visit for their last-born child in the past two years, September-October 2019

\begin{tabular}{|c|c|c|c|c|c|c|}
\hline & KEBBI & SOKото & $\begin{array}{l}\text { MALARIA-ONLY } \\
\text { (ZAMFARA) }\end{array}$ & $\begin{array}{c}\text { INTEGRATED } \\
\text { (KEBBI/SOKOTO) }\end{array}$ & $\mathbf{N}$ & $\begin{array}{c}\mathrm{CHI}^{2} \\
\text { VALUE }\end{array}$ \\
\hline & $\%$ & $\%$ & $\%$ & $\%$ & & \\
\hline \multicolumn{7}{|l|}{ Doctor } \\
\hline No & 83.9 & 80.7 & 89.4 & 82.4 & 1,255 & 15.179 \\
\hline Yes & 16.2 & 19.3 & 10.6 & 17.7 & 247 & 1.733 \\
\hline Total & 100.0 & 100.0 & 100.0 & 100.0 & 1,502 & \\
\hline N & 452 & 457 & 593 & 909 & & \\
\hline \multicolumn{7}{|c|}{ Nurse/midwife } \\
\hline No & 35.2 & 48.5 & 32.1 & 41.6 & 565 & 13.480 \\
\hline Yes & 64.8 & 51.5 & 67.9 & 58.4 & 937 & 1.217 \\
\hline Total & 100.0 & 100.0 & 100.0 & 100.0 & 1,502 & \\
\hline $\mathrm{N}$ & 452 & 457 & 593 & 909 & & \\
\hline \multicolumn{7}{|c|}{ Community health worker } \\
\hline No & 80.8 & 62.8 & 69.0 & 72.0 & 1,085 & 1.496 \\
\hline Yes & 19.2 & 37.3 & 31.0 & 28.0 & 417 & 0.119 \\
\hline Total & 100.0 & 100.0 & 100.0 & 100.0 & 1,502 & \\
\hline $\mathrm{N}$ & 452 & 457 & 593 & 909 & & \\
\hline \multicolumn{7}{|c|}{ Chemist } \\
\hline No & 99.3 & 100.0 & 98.6 & 99.6 & 1,492 & 3.320 \\
\hline Yes & 0.8 & 0.0 & 1.4 & 0.4 & 10 & 1.645 \\
\hline Total & 100.0 & 100.0 & 100.0 & 100.0 & 1,502 & \\
\hline N & 452 & 457 & 593 & 909 & & \\
\hline \multicolumn{7}{|c|}{ Traditional healer/birth attendant } \\
\hline No & 99.7 & 100.0 & 99.8 & 99.9 & 1,499 & 0.051 \\
\hline Yes & 0.3 & 0.0 & 0.2 & 0.2 & 3 & 0.055 \\
\hline Total & 100.0 & 100.0 & 100.0 & 100.0 & 1,502 & \\
\hline$N$ & 452 & 457 & 593 & 909 & & \\
\hline \multicolumn{7}{|l|}{ Family } \\
\hline No & 98.8 & 99.9 & 99.7 & 99.3 & 1,494 & 1.166 \\
\hline Yes & 1.2 & 0.1 & 0.3 & 0.7 & 8 & 1.035 \\
\hline Total & 100.0 & 100.0 & 100.0 & 100.0 & 1,502 & \\
\hline $\mathrm{N}$ & 452 & 457 & 593 & 909 & & \\
\hline \multicolumn{7}{|c|}{ Friends/neighbors } \\
\hline No & 99.8 & 100.0 & 100.0 & 99.9 & 1,501 & 0.933 \\
\hline Yes & 0.2 & 0.0 & 0.0 & 0.1 & 1 & 1.788 \\
\hline Total & 100.0 & 100.0 & 100.0 & 100.0 & 1,502 & \\
\hline $\mathrm{N}$ & 452 & 457 & 593 & 909 & & \\
\hline
\end{tabular}

Estimates based on low Ns (approx. $<30$ obs) have large relative standard errors and should be interpreted with caution. ${ }^{* \star *}$ indicates $p<0.001,{ }^{* *}$ indicates $p<0.05$ and ${ }^{*}$ indicates $p<.0 .01$ for the chi ${ }^{2}$ statistic comparing integrated versus malaria-only study areas. 


\section{TABLE 5.1.4. ANC CONTENT DURING LAST PREGNANCY}

Percentage of women 15 to 49 years with a child under 2 years reporting on content of ANC visits for their last-born child in the past two years, September-October 2019

\begin{tabular}{|c|c|c|c|c|c|c|}
\hline & $\begin{array}{l}\text { KEBBI } \\
\%\end{array}$ & $\begin{array}{l}\text { SOKOTO } \\
\%\end{array}$ & $\begin{array}{l}\text { MALARIA-ONLY } \\
\text { (ZAMFARA) } \\
\%\end{array}$ & $\begin{array}{c}\text { INTEGRATED } \\
\text { (KEBBI/SOKOTO) } \\
\%\end{array}$ & N & $\begin{array}{c}\mathrm{CHI}^{2} \\
\text { VALUE }\end{array}$ \\
\hline \multicolumn{7}{|c|}{ How many check-ups did you have for your last pregnancy? } \\
\hline Don't know & 11.5 & 5.1 & 1.7 & 8.4 & 96 & 42.962 \\
\hline 0 & 0.3 & 0.0 & 0.8 & 0.1 & & 3.173 \\
\hline $1-3$ & 34.4 & 29.5 & 36.0 & 32.0 & 481 & \\
\hline $4-6$ & 43.3 & 43.8 & 46.7 & 43.5 & 692 & \\
\hline $7-60$ & 10.7 & 21.6 & 14.8 & 16.0 & 227 & \\
\hline Total & 100.0 & 100.0 & 100.0 & 100.0 & 1,502 & \\
\hline N & 452 & 457 & 593 & 909 & & \\
\hline \multicolumn{7}{|c|}{ Was your blood pressure measured? } \\
\hline No & 21.1 & 11.4 & 9.7 & 16.4 & 181 & 15.139 \\
\hline Yes & 78.1 & 88.6 & 90.1 & 83.2 & 1,317 & 1.178 \\
\hline Don't know & 0.8 & 0.0 & 0.2 & 0.4 & & \\
\hline Total & 100.0 & 100.0 & 100.0 & 100.0 & 1,502 & \\
\hline N & 452 & 457 & 593 & 909 & & \\
\hline \multicolumn{7}{|c|}{ Did you give a urine sample? } \\
\hline No & 33.3 & 32.8 & 20.4 & 33.1 & 380 & 30.888 \\
\hline Yes & 66.2 & 67.2 & 79.6 & 66.7 & 1,119 & 2.810 \\
\hline Don't know & 0.5 & 0.0 & 0.1 & 0.3 & & \\
\hline Total & 100.0 & 100.0 & 100.0 & 100.0 & 1,502 & \\
\hline N & 452 & 457 & 593 & 909 & & \\
\hline \multicolumn{7}{|c|}{ Did you give a blood sample? } \\
\hline No & 27.9 & 22.3 & 17.8 & 25.2 & 310 & 11.604 \\
\hline Yes & 71.8 & 77.6 & 82.0 & 74.6 & 1,186 & 1.085 \\
\hline Don't know & 0.3 & 0.1 & 0.3 & 0.2 & & \\
\hline Total & 100.0 & 100.0 & 100.0 & 100.0 & 1,502 & \\
\hline $\mathrm{N}$ & 452 & 457 & 593 & 909 & & \\
\hline \multicolumn{7}{|c|}{ Were you tested for HIV? } \\
\hline No & 36.8 & 37.6 & 24.4 & 37.2 & 465 & 67.647 \\
\hline Yes & 42.6 & 38.7 & 62.7 & 40.7 & 761 & 3.856 \\
\hline Don't know & 20.6 & 23.7 & 12.9 & 22.1 & 276 & \\
\hline Total & 100.0 & 100.0 & 100.0 & 100.0 & 1,502 & \\
\hline N & 452 & 457 & 593 & 909 & & \\
\hline \multicolumn{7}{|c|}{ Was your weight measured? } \\
\hline No & 40.6 & 16.1 & 9.8 & 28.7 & 298 & 94.643 \\
\hline Yes & 58.8 & 82.9 & 90.1 & 70.5 & 1,191 & 7.987 \\
\hline Don't know & 0.6 & 1.0 & 0.1 & 0.8 & $\begin{aligned} 1,+1 \\
13\end{aligned}$ & $* *$ \\
\hline Total & 100.0 & 100.0 & 100.0 & 100.0 & 1,502 & \\
\hline N & 452 & 457 & 593 & 909 & & \\
\hline \multicolumn{7}{|c|}{ Did a health provider ask if you had vaginal bleeding? } \\
\hline No & 50.9 & 52.4 & 44.4 & 51.7 & 708 & 7.840 \\
\hline Yes & 48.2 & 45.9 & 54.6 & 47.1 & 772 & 0.624 \\
\hline Don't know & 0.9 & 1.6 & 0.9 & 1.2 & 22 & \\
\hline Total & 100.0 & 100.0 & 100.0 & 100.0 & 1,502 & \\
\hline \multirow{2}{*}{\multicolumn{7}{|c|}{ Were you told to pay attention to baby movements? }} \\
\hline & & & & & & \\
\hline No & 34.2 & 26.0 & 27.2 & 30.2 & 416 & 6.529 \\
\hline Yes & 64.9 & 72.7 & 72.5 & 68.7 & 1,073 & 0.562 \\
\hline Don't know & 0.9 & 1.3 & 0.3 & 1.1 & 13 & \\
\hline Total & 100.0 & 100.0 & 100.0 & 100.0 & 1,502 & \\
\hline N & 452 & 457 & 593 & 909 & & \\
\hline \multicolumn{7}{|c|}{ Did you discuss baby due date? } \\
\hline No & 54.1 & 57.4 & 46.1 & 55.7 & 753 & 24.670 \\
\hline Yes & 44.7 & 39.7 & 53.5 & 42.3 & 726 & 1.903 \\
\hline Don't know & 1.2 & 2.9 & 0.4 & 2.0 & 23 & \\
\hline Total & 100.0 & 100.0 & 100.0 & 100.0 & 1,502 & \\
\hline N & 452 & 457 & 593 & 909 & & \\
\hline \multicolumn{7}{|c|}{ Told about danger signs in pregnancy? } \\
\hline No & 43.7 & 35.4 & 36.9 & 39.7 & 566 & 1.893 \\
\hline Yes & 54.9 & 63.7 & 62.3 & 59.2 & 919 & 0.146 \\
\hline Don't know & 1.4 & 0.9 & 0.8 & 1.2 & 17 & \\
\hline Total & 100.0 & 100.0 & 100.0 & 100.0 & 1,502 & \\
\hline $\mathrm{N}$ & 452 & 457 & 593 & 909 & & \\
\hline \multicolumn{7}{|c|}{$\begin{array}{l}\text { As part of your ANC during your pregnancy were you ever } \\
\text { given a mosquito net? }\end{array}$} \\
\hline No & 74.0 & 82.9 & 63.8 & 78.3 & 1,079 & 34.177 \\
\hline Yes & 25.6 & 17.1 & 36.0 & 21.5 & 420 & 6.990 \\
\hline Don't know & 0.4 & 0.0 & 0.2 & 0.2 & 3 & $\star \star$ \\
\hline Total & 100.0 & 100.0 & 100.0 & 100.0 & 1,502 & \\
\hline N & 452 & 457 & 593 & 909 & & \\
\hline
\end{tabular}

Estimates based on low Ns (approx. $<30$ obs) have large relative standard errors and should be interpreted with caution. ${ }^{* \star *}$ indicates $p<0.001,{ }^{* *}$ indicates $p<0.05$ and ${ }^{*}$ indicates $p<.0 .01$ for the chi ${ }^{2}$ statistic comparing integrated versus malaria-only study areas. 
TABLE 5.1.5. BIRTH PLANNING DURING LAST PREGNANCY

Percentage of women 15 to 49 years with a child under 2 years who discussed a birth plan with a health worker for their last-born child in past two years by content, September-October 2019

\begin{tabular}{|c|c|c|c|c|c|c|}
\hline & KEBBI & SOKOTO & $\begin{array}{l}\text { MALARIA-ONLY } \\
\text { (ZAMFARA) }\end{array}$ & $\begin{array}{l}\text { INTEGRATED } \\
\text { (KEBBI/SOKOTO) }\end{array}$ & $\mathbf{N}$ & $\begin{array}{c}\mathrm{CHI}^{2} \\
\text { VALUE }\end{array}$ \\
\hline & $\%$ & $\%$ & $\%$ & $\%$ & & \\
\hline \multicolumn{7}{|c|}{$\begin{array}{l}\text { Discuss with a health worker a plan for } \\
\text { your delivery during your last pregnancy? }\end{array}$} \\
\hline No & 74.4 & 69.7 & 64.9 & 72.1 & 1,019 & 8.167 \\
\hline Yes & 25.2 & 30.3 & 34.7 & 27.7 & 478 & 0.651 \\
\hline Don't know & 0.4 & 0.0 & 0.4 & 0.2 & 5 & \\
\hline Total & 100.0 & 100.0 & 100.0 & 100.0 & 1,502 & \\
\hline N & 452 & 457 & 593 & 909 & & \\
\hline \multicolumn{7}{|c|}{ Where you plan to deliver the baby? } \\
\hline No & 18.6 & 10.5 & 4.5 & 14.3 & 45 & 14.040 \\
\hline Yes & 81.0 & 89.3 & 95.3 & 85.4 & 430 & 5.085 \\
\hline Don't know & 0.4 & 0.2 & 0.1 & 0.3 & 3 & * \\
\hline Total & 100.0 & 100.0 & 100.0 & 100.0 & 478 & \\
\hline $\mathrm{N}$ & 116 & 147 & 215 & 263 & & \\
\hline \multicolumn{7}{|c|}{$\begin{array}{l}\text { Wanted to have relative/friend/neighbor } \\
\text { with you during labor? }\end{array}$} \\
\hline No & 29.5 & 42.8 & 30.9 & 36.5 & 164 & 1.862 \\
\hline Yes & 70.1 & 57.0 & 68.2 & 63.2 & 309 & 0.254 \\
\hline Don't know & 0.4 & 0.2 & 0.9 & 0.3 & 5 & \\
\hline Total & 100.0 & 100.0 & 100.0 & 100.0 & 478 & \\
\hline $\mathrm{N}$ & 116 & 147 & 215 & 263 & & \\
\hline \multicolumn{7}{|c|}{$\begin{array}{l}\text { Transport to where the baby would be } \\
\text { born? }\end{array}$} \\
\hline No & 47.0 & 50.7 & 34.5 & 49.0 & 189 & 9.735 \\
\hline Yes & 52.6 & 49.3 & 64.2 & 50.8 & 283 & 1.743 \\
\hline Don't know & 0.4 & 0.0 & 1.3 & 0.2 & 6 & \\
\hline Total & 100.0 & 100.0 & 100.0 & 100.0 & 478 & \\
\hline $\mathrm{N}$ & 116 & 147 & 215 & 263 & & \\
\hline \multicolumn{7}{|c|}{ What funds you would use for delivery? } \\
\hline No & 49.4 & 34.3 & 18.3 & 41.4 & 146 & 28.711 \\
\hline Yes & 49.2 & 65.5 & 80.4 & 57.8 & 324 & 5.518 \\
\hline Don't know & 1.5 & 0.2 & 1.4 & 0.8 & 8 & ** \\
\hline Total & 100.0 & 100.0 & 100.0 & 100.0 & 478 & \\
\hline N & 116 & 147 & 215 & 263 & & \\
\hline \multicolumn{7}{|c|}{$\begin{array}{l}\text { Finding a skilled birth attendant for } \\
\text { delivery? }\end{array}$} \\
\hline No & 35.8 & 27.3 & 24.5 & 31.3 & 134 & 3.278 \\
\hline Yes & 63.8 & 72.1 & 73.8 & 68.2 & 336 & 0.558 \\
\hline Don't know & 0.4 & 0.7 & 1.7 & 0.5 & 8 & \\
\hline Total & 100.0 & 100.0 & 100.0 & 100.0 & 478 & \\
\hline $\mathrm{N}$ & 116 & 147 & 215 & 263 & & \\
\hline
\end{tabular}

Estimates based on low Ns (approx. $<30$ obs) have large relative standard errors and should be interpreted with caution. ${ }^{* \star *}$ indicates $p<0.001,{ }^{* *}$ indicates $p<0.05$ and ${ }^{*}$ indicates $p<.0 .01$ for the chi ${ }^{2}$ statistic comparing integrated versus malaria-only study areas. 
TABLE 5.1.6. REASONS FOR NOT ATTENDING ANC DURING LAST PREGNANCY

Percentage of women 15 to 49 years who did not attend ANC for their last-born child in past two years by reason, September-October 2019

\begin{tabular}{|c|c|c|c|c|c|c|}
\hline & $\begin{array}{c}\text { KEBBI } \\
\%\end{array}$ & $\begin{array}{c}\text { SOKOTO } \\
\%\end{array}$ & $\begin{array}{c}\text { MALARIA-ONLY } \\
\text { (ZAMFARA) } \\
\%\end{array}$ & $\begin{array}{c}\text { INTEGRATED } \\
\text { (KEBBI/SOKOTO) } \\
\%\end{array}$ & $\mathbf{N}$ & $\begin{array}{c}\mathrm{CHI}^{2} \\
\text { VALUE }\end{array}$ \\
\hline \multicolumn{7}{|c|}{$\begin{array}{l}\text { Costs too much/Difficulty getting } \\
\text { money }\end{array}$} \\
\hline No & 91.4 & 93.9 & 93.6 & 93.0 & 1,416 & 0.219 \\
\hline Yes & 8.6 & 6.2 & 6.4 & 7.0 & 107 & 0.040 \\
\hline Total & 100.0 & 100.0 & 100.0 & 100.0 & 1,523 & \\
\hline $\mathrm{N}$ & 435 & 612 & 476 & 1,047 & & \\
\hline \multicolumn{7}{|c|}{ Don't know where to go } \\
\hline No & 97.3 & 97.0 & 93.9 & 97.1 & 1,467 & 8.440 \\
\hline Yes & 2.7 & 3.1 & 6.1 & 2.9 & 56 & 1.064 \\
\hline Total & 100.0 & 100.0 & 100.0 & 100.0 & 1,523 & \\
\hline $\mathrm{N}$ & 435 & 612 & 476 & 1,047 & & \\
\hline \multicolumn{7}{|c|}{ Health facility not open } \\
\hline No & 96.5 & 98.5 & 96.0 & 97.8 & 1,487 & 3.874 \\
\hline Yes & 3.5 & 1.5 & 4.0 & 2.2 & 36 & 0.369 \\
\hline Total & 100.0 & 100.0 & 100.0 & 100.0 & 1,523 & \\
\hline $\mathrm{N}$ & 435 & 612 & 476 & 1,047 & & \\
\hline \multicolumn{7}{|c|}{ Distance to health facility too far } \\
\hline No & 95.6 & 96.8 & 88.7 & 96.4 & 1,434 & 29.570 \\
\hline Yes & 4.4 & 3.2 & 11.3 & 3.7 & 89 & 4.038 \\
\hline Total & 100.0 & 100.0 & 100.0 & 100.0 & 1,523 & * \\
\hline $\mathrm{N}$ & 435 & 612 & 476 & 1,047 & & \\
\hline \multicolumn{7}{|c|}{ Poor quality of service } \\
\hline No & 98.6 & 99.2 & 98.2 & 99.0 & 1,501 & 1.639 \\
\hline Yes & 1.4 & 0.8 & 1.8 & 1.0 & 22 & 0.575 \\
\hline Total & 100.0 & 100.0 & 100.0 & 100.0 & 1,523 & \\
\hline $\mathrm{N}$ & 435 & 612 & 476 & 1,047 & & \\
\hline \multicolumn{7}{|c|}{ No female provider at facility } \\
\hline No & 98.9 & 95.1 & 94.1 & 96.5 & 1,446 & 4.642 \\
\hline Yes & 1.1 & 4.9 & 5.9 & 3.5 & & 0.212 \\
\hline Total & 100.0 & 100.0 & 100.0 & 100.0 & 1,523 & \\
\hline N & 435 & 612 & 476 & 1,047 & & \\
\hline \multicolumn{7}{|c|}{ Husband/partner oppose } \\
\hline No & 76.2 & 82.5 & 70.5 & 80.2 & 1,151 & 18.770 \\
\hline Yes & 23.8 & 17.5 & 29.6 & 19.8 & 372 & 1.797 \\
\hline Total & 100.0 & 100.0 & 100.0 & 100.0 & 1,523 & \\
\hline $\mathrm{N}$ & 435 & 612 & 476 & 1,047 & & \\
\hline \multicolumn{7}{|c|}{ Not wanting to go alone } \\
\hline No & 98.1 & 98.3 & 98.7 & 98.2 & 1,501 & 0.625 \\
\hline Yes & 1.9 & 1.7 & 1.3 & 1.8 & 22 & 0.185 \\
\hline Total & 100.0 & 100.0 & 100.0 & 100.0 & 1,523 & \\
\hline N & 435 & 612 & 476 & 1,047 & & \\
\hline \multicolumn{7}{|c|}{ Not necessary to go } \\
\hline No & 47.1 & 52.7 & 64.3 & 50.7 & 847 & 28.662 \\
\hline Yes & 52.9 & 47.3 & 35.7 & 49.3 & 676 & 2.495 \\
\hline Total & 100.0 & 100.0 & 100.0 & 100.0 & 1,523 & \\
\hline N & 435 & 612 & 476 & 1,047 & & \\
\hline \multicolumn{7}{|c|}{ Not customary } \\
\hline No & 93.0 & 79.1 & 89.7 & 84.2 & 1,332 & 10.193 \\
\hline Yes & 7.0 & 20.9 & 10.3 & 15.8 & 191 & 0.784 \\
\hline Total & 100.0 & 100.0 & 100.0 & 100.0 & 1,523 & \\
\hline N & 435 & 612 & 476 & 1,047 & & \\
\hline \multicolumn{7}{|c|}{ Up to God } \\
\hline No & 90.2 & 70.2 & 81.3 & 77.6 & 1,228 & 3.247 \\
\hline Yes & 9.8 & 29.8 & 18.7 & 22.5 & 295 & 0.505 \\
\hline Total & 100.0 & 100.0 & 100.0 & 100.0 & 1,523 & \\
\hline $\mathrm{N}$ & 435 & 612 & 476 & 1,047 & & \\
\hline
\end{tabular}

Estimates based on low Ns (approx. $<30$ obs) have large relative standard errors and should be interpreted with caution. ${ }^{* * *}$ indicates $p<0.001,{ }^{* *}$ indicates $p<0.05$ and $^{*}$ indicates $p<.0 .01$ for the chi ${ }^{2}$ statistic comparing integrated versus malaria-only study areas. 
TABLE 5.1.7. MEDICINES OR VACCINATIONS RECEIVED DURING LAST PREGNANCY

Percentage of women 15 to 49 years who received certain medicines or vaccinations during pregnancy for their lastborn child in past two years, September-October 2019

\begin{tabular}{|c|c|c|c|c|c|c|}
\hline & KEBBI & SOKOTO & $\begin{array}{l}\text { MALARIA-ONLY } \\
\text { (ZAMFARA) }\end{array}$ & $\begin{array}{l}\text { INTEGRATED } \\
\text { (KEBBI/SOKOTO) }\end{array}$ & $\mathrm{N}$ & $\begin{array}{c}\mathrm{CHI}^{2} \\
\text { VALUE }\end{array}$ \\
\hline & $\%$ & $\%$ & $\%$ & $\%$ & & \\
\hline \multicolumn{7}{|c|}{$\begin{array}{l}\text { Given an injection in the arm to prevent the } \\
\text { baby from getting tetanus }\end{array}$} \\
\hline No & 60.6 & 66.7 & 52.4 & 64.0 & 1,775 & 54.182 \\
\hline Yes & 38.3 & 32.1 & 47.3 & 34.8 & 1,233 & 4.344 \\
\hline Don't know & 1.2 & 1.2 & 0.3 & 1.2 & 31 & * \\
\hline Total & 100.0 & 100.0 & 100.0 & 100.0 & 3,039 & \\
\hline$N$ & 892 & 1,078 & 1,069 & 1,970 & & \\
\hline \multicolumn{7}{|c|}{$\begin{array}{l}\text { During your last pregnancy, how many } \\
\text { times were you given a tetanus injection? }\end{array}$} \\
\hline None & 42.3 & 59.4 & 43.8 & 52.0 & 1,457 & 87.691 \\
\hline 1 & 13.2 & 11.2 & 11.6 & 12.0 & 385 & 2.081 \\
\hline 2 & 14.7 & 14.5 & 21.0 & 14.6 & 527 & . \\
\hline 3 or more & 9.6 & 6.4 & 15.8 & 7.8 & 327 & \\
\hline Don't know & 20.2 & 8.6 & 7.8 & 13.6 & 343 & \\
\hline Total & 100.0 & 100.0 & 100.0 & 100.0 & 3,039 & \\
\hline $\mathrm{N}$ & 892 & 1,078 & 1,069 & 1,970 & & \\
\hline \multicolumn{7}{|c|}{$\begin{array}{l}\text { Before your last pregnancy, how many } \\
\text { times did you get a tetanus injection? }\end{array}$} \\
\hline & 0.0 & 0.1 & 0.0 & 0.0 & 1 & 93.569 \\
\hline None & 59.3 & 70.9 & 60.6 & 65.9 & 1,905 & 2.617 \\
\hline 1 & 5.4 & 5.2 & 5.8 & 5.3 & 175 & \\
\hline 2 & 4.0 & 6.3 & 10.4 & 5.3 & 222 & \\
\hline 3 or more & 3.0 & 2.9 & 9.6 & 3.0 & 169 & \\
\hline Don't know & 28.3 & 14.7 & 13.7 & 20.5 & 567 & \\
\hline Total & 100.0 & 100.0 & 100.0 & 100.0 & 3,039 & \\
\hline $\mathrm{N}$ & 892 & 1,078 & 1,069 & 1,970 & & \\
\hline \multicolumn{7}{|c|}{$\begin{array}{l}\text { During your last pregnancy with child, did } \\
\text { you take any iron tables or iron syrup? }\end{array}$} \\
\hline No & 48.0 & 61.4 & 47.2 & 55.6 & 1,542 & 62.296 \\
\hline Yes & 49.4 & 36.9 & 52.7 & 42.3 & 1,449 & 5.025 \\
\hline Don't know & 2.7 & 1.8 & 0.1 & 2.2 & 48 & * \\
\hline Total & 100.0 & 100.0 & 100.0 & 100.0 & 3,039 & \\
\hline$N$ & 892 & 1,078 & 1,069 & 1,970 & & \\
\hline \multicolumn{7}{|c|}{$\begin{array}{l}\text { During your last pregnancy with child, did } \\
\text { you take any folic acid supplements? }\end{array}$} \\
\hline No & 49.5 & 59.2 & 46.3 & 55.0 & 1,522 & 89.884 \\
\hline Yes & 45.3 & 38.3 & 53.5 & 41.3 & 1,438 & 6.945 \\
\hline Don't know & 5.2 & 2.5 & 0.2 & 3.6 & 79 & ** \\
\hline Total & 100.0 & 100.0 & 100.0 & 100.0 & 3,039 & \\
\hline $\mathrm{N}$ & 892 & 1,078 & 1,069 & 1,970 & & \\
\hline \multicolumn{7}{|c|}{$\begin{array}{l}\text { During your last pregnancy, did you take } \\
\text { any drugs for intestinal worms? }\end{array}$} \\
\hline No & 59.2 & 74.9 & 77.4 & 68.1 & 2,110 & 35.497 \\
\hline Yes & 28.9 & 18.2 & 17.3 & 22.8 & 685 & 2.163 \\
\hline Don't know & 11.9 & 7.0 & 5.3 & 9.1 & 244 & \\
\hline Total & 100.0 & 100.0 & 100.0 & 100.0 & 3,039 & \\
\hline $\mathrm{N}$ & 892 & 1,078 & 1,069 & 1,970 & & \\
\hline
\end{tabular}

Estimates based on low Ns (approx. $<30$ obs) have large relative standard errors and should be interpreted with caution. ${ }^{* \star *}$ indicates $p<0.001,{ }^{* *}$ indicates $p<0.05$ and ${ }^{*}$ indicates $p<.0 .01$ for the chi ${ }^{2}$ statistic comparing integrated versus malaria-only study areas. 
TABLE 5.1.8. KNOWLEDGE OF ANC TIMING AND VISITS

Percentage of women aged 15 to 49 years currently pregnant or with a child under 2 years who report the number and timing of ANC visits during pregnancy, September-October 2019

\begin{tabular}{|c|c|c|c|c|c|c|}
\hline & KEBBI & SOKото & $\begin{array}{l}\text { MALARIA-ONLY } \\
\text { (ZAMFARA) }\end{array}$ & $\begin{array}{l}\text { INTEGRATED } \\
\text { (KEBBI/SOKOTO) }\end{array}$ & $\mathbf{N}$ & $\begin{array}{c}\mathrm{CHI}^{2} \\
\text { VALUE }\end{array}$ \\
\hline & $\%$ & $\%$ & $\%$ & $\%$ & & \\
\hline \multicolumn{7}{|l|}{$\begin{array}{l}\text { Number of times women should get } \\
\text { a checkup during pregnancy }\end{array}$} \\
\hline None & 4.4 & 1.8 & 5.5 & 3.0 & 228 & 260.703 \\
\hline $1-3$ & 5.4 & 5.4 & 8.8 & 5.4 & 385 & 3.713 \\
\hline 4 or more & 29.3 & 37.5 & 48.7 & 33.7 & 2,469 & * \\
\hline Don't know & 60.8 & 55.3 & 36.9 & 57.9 & 2,951 & \\
\hline Total & 100.0 & 100.0 & 100.0 & 100.0 & 6,033 & \\
\hline $\mathrm{N}$ & 1,960 & 2,062 & 2,011 & 4,022 & & \\
\hline \multicolumn{7}{|l|}{$\begin{array}{l}\text { Opinion on timing of 1st ANC visit for } \\
\text { pregnant woman }\end{array}$} \\
\hline As soon as she thinks she is pregnant & 22.2 & 15.6 & 11.5 & 18.7 & 963 & 260.078 \\
\hline In the 1st trimester & 7.5 & 10.0 & 14.7 & 8.9 & 689 & 2.355 \\
\hline At the beginning of the 2 nd trimester & 12.5 & 17.7 & 27.4 & 15.3 & 1,236 & * \\
\hline At the beginning of the 3rd trimester & 12.4 & 7.9 & 5.9 & 10.0 & 565 & \\
\hline Any time during pregnancy & 19.6 & 12.4 & 17.0 & 15.7 & 944 & \\
\hline Other & 1.9 & 1.1 & 1.9 & 1.5 & 95 & \\
\hline Don't know & 24.0 & 35.3 & 21.8 & 30.0 & 1,541 & \\
\hline Total & 100.0 & 100.0 & 100.0 & 100.0 & 6,033 & \\
\hline $\mathrm{N}$ & 1,960 & 2,062 & 2,011 & 4,022 & & \\
\hline
\end{tabular}

Estimates based on low Ns (approx. $<30$ obs) have large relative standard errors and should be interpreted with caution. ${ }^{* * *}$ indicates $p<0.001,{ }^{* *}$ indicates $p<0.05$ and ${ }^{*}$ indicates $p<.0 .01$ for the chi $^{2}$ statistic comparing integrated versus malaria-only study areas. 
TABLE 5.1.9. PERCEIVED MATERNAL BENEFITS OF ANC

Percentage of women aged 15 to 49 years currently pregnant or with a child under 2 years who report ANC benefits for themselves by type of benefit, September-October 2019

\begin{tabular}{|c|c|c|c|c|c|c|}
\hline & KEBBI & SOKOTO & $\begin{array}{l}\text { MALARIA-ONLY } \\
\text { (ZAMFARA) }\end{array}$ & $\begin{array}{l}\text { INTEGRATED } \\
\text { (KEBBI/SOKOTO) }\end{array}$ & $\mathbf{N}$ & $\begin{array}{c}\mathrm{CHI}^{2} \\
\text { VALUE }\end{array}$ \\
\hline & $\%$ & $\%$ & $\%$ & $\%$ & & \\
\hline \multicolumn{7}{|c|}{ No benefits to mother } \\
\hline No & 93.9 & 93.2 & 95.8 & 93.6 & 5,710 & 15.240 \\
\hline Yes & 6.1 & 6.8 & 4.2 & 6.4 & 324 & 0.385 \\
\hline Total & 100.0 & 100.0 & 100.0 & 100.0 & 6,034 & \\
\hline N & 1,960 & 2,062 & 2,012 & 4,022 & & \\
\hline \multicolumn{7}{|c|}{ Monitor baby's growth by provider } \\
\hline No & 41.3 & 41.1 & 31.4 & 41.2 & 2,211 & 61.550 \\
\hline Yes & 58.7 & 58.9 & 68.6 & 58.8 & 3,823 & 2.063 \\
\hline Total & 100.0 & 100.0 & 100.0 & 100.0 & 6,034 & \\
\hline N & 1,960 & 2,062 & 2,012 & 4,022 & & \\
\hline \multicolumn{7}{|c|}{ Monitor mother's health by provider } \\
\hline No & 43.2 & 41.6 & 28.1 & 42.3 & 2,270 & 130.938 \\
\hline Yes & 56.8 & 58.4 & 71.9 & 57.7 & 3,764 & 4.644 \\
\hline Total & 100.0 & 100.0 & 100.0 & 100.0 & 6,034 & * \\
\hline N & 1,960 & 2,062 & 2,012 & 4,022 & & \\
\hline \multicolumn{7}{|c|}{ Receive mosquito net } \\
\hline No & 78.4 & 90.4 & 73.7 & 84.9 & 4,923 & 106.175 \\
\hline Yes & 21.6 & 9.6 & 26.3 & 15.1 & 1,111 & 3.916 \\
\hline Total & 100.0 & 100.0 & 100.0 & 100.0 & 6,034 & * \\
\hline $\mathrm{N}$ & 1,960 & 2,062 & 2,012 & 4,022 & & \\
\hline \multicolumn{7}{|c|}{$\begin{array}{l}\text { Receive medicine to prevent malaria } \\
\text { during pregnancy }\end{array}$} \\
\hline No & 75.4 & 84.6 & 71.5 & 80.3 & 4,653 & 60.786 \\
\hline Yes & 24.6 & 15.5 & 28.5 & 19.7 & 1,381 & 2.238 \\
\hline Total & 100.0 & 100.0 & 100.0 & 100.0 & 6,034 & \\
\hline$N$ & 1,960 & 2,062 & 2,012 & 4,022 & & \\
\hline \multicolumn{7}{|c|}{$\begin{array}{l}\text { Reduce risk of pregnancy complica- } \\
\text { tions }\end{array}$} \\
\hline No & 85.7 & 87.2 & 82.0 & 86.5 & 5,118 & 22.212 \\
\hline Yes & 14.3 & 12.8 & 18.1 & 13.5 & 916 & 0.914 \\
\hline Total & 100.0 & 100.0 & 100.0 & 100.0 & 6,034 & \\
\hline$N$ & 1,960 & 2,062 & 2,012 & 4,022 & & \\
\hline \multicolumn{7}{|c|}{ Reduce risk of delivery complications } \\
\hline No & 89.8 & 91.6 & 83.5 & 90.8 & 5,315 & 66.454 \\
\hline Yes & 10.2 & 8.4 & 16.6 & 9.2 & 719 & 3.386 \\
\hline Total & 100.0 & 100.0 & 100.0 & 100.0 & 6,034 & \\
\hline N & 1,960 & 2,062 & 2,012 & 4,022 & & \\
\hline
\end{tabular}

Estimates based on low Ns (approx. $<30$ obs) have large relative standard errors and should be interpreted with caution. ${ }^{* \star *}$ indicates $p<0.001,{ }^{* *}$ indicates $p<0.05$ and * indicates $p<.0 .01$ for the chi ${ }^{2}$ statistic comparing integrated versus malaria-only study areas. 
TABLE 5.1.10. WHAT ARE SOME DANGER SIGNS DURING PREGNANCY?

Percentage of women 15 to 49 years currently pregnant or with a child under 2 years who report any danger signs during pregnancy by type, September-October 2019

\begin{tabular}{|c|c|c|c|c|c|c|}
\hline & KEBBI & SOKOTO & $\begin{array}{l}\text { MALARIA-ONLY } \\
\text { (ZAMFARA) }\end{array}$ & $\begin{array}{c}\text { INTEGRATED } \\
\text { (KEBBI/SOKOTO) }\end{array}$ & $\mathbf{N}$ & $\begin{array}{c}\text { CHI }^{2} \\
\text { VALUE }\end{array}$ \\
\hline & $\%$ & $\%$ & $\%$ & $\%$ & & \\
\hline \multicolumn{7}{|c|}{$\begin{array}{l}\text { No knowledge of pregnancy danger } \\
\text { signs }\end{array}$} \\
\hline No & 87.5 & 89.9 & 91.9 & 88.8 & 5,425 & 16.868 \\
\hline Yes & 12.5 & 10.1 & 8.1 & 11.2 & 609 & 0.788 \\
\hline Total & 100.0 & 100.0 & 100.0 & 100.0 & 6,034 & \\
\hline $\mathrm{N}$ & 1,960 & 2,062 & 2,012 & 4,022 & & \\
\hline \multicolumn{7}{|c|}{ Severe weakness } \\
\hline No & 63.2 & 67.5 & 49.9 & 65.5 & 3,659 & 144.598 \\
\hline Yes & 36.8 & 32.5 & 50.1 & 34.5 & 2,375 & 5.559 \\
\hline Total & 100.0 & 100.0 & 100.0 & 100.0 & 6,034 & * \\
\hline $\mathrm{N}$ & 1,960 & 2,062 & 2,012 & 4,022 & & \\
\hline \multicolumn{7}{|c|}{ Convulsions or fits } \\
\hline No & 78.7 & 84.4 & 71.4 & 81.8 & 4,788 & 85.798 \\
\hline Yes & 21.3 & 15.6 & 28.7 & 18.2 & 1,246 & 2.999 \\
\hline Total & 100.0 & 100.0 & 100.0 & 100.0 & 6,034 & \\
\hline $\mathrm{N}$ & 1,960 & 2,062 & 2,012 & 4,022 & & \\
\hline \multicolumn{7}{|c|}{ Severe headache } \\
\hline No & 68.2 & 73.8 & 58.8 & 71.2 & 4,069 & 97.236 \\
\hline Yes & 31.9 & 26.2 & 41.2 & 28.8 & 1,965 & 4.326 \\
\hline Total & 100.0 & 100.0 & 100.0 & 100.0 & 6,034 & * \\
\hline N & 1,960 & 2,062 & 2,012 & 4,022 & & \\
\hline \multicolumn{7}{|c|}{ Blurred vision } \\
\hline No & 82.4 & 87.2 & 84.7 & 85.0 & 5,143 & 0.106 \\
\hline Yes & 17.6 & 12.8 & 15.3 & 15.0 & 891 & 0.004 \\
\hline Total & 100.0 & 100.0 & 100.0 & 100.0 & 6,034 & \\
\hline$N$ & 1,960 & 2,062 & 2,012 & 4,022 & & \\
\hline \multicolumn{7}{|l|}{ Fever } \\
\hline No & 62.6 & 56.1 & 56.1 & 59.1 & 3,484 & 5.388 \\
\hline Yes & 37.4 & 43.9 & 43.9 & 40.9 & 2,550 & 0.217 \\
\hline Total & 100.0 & 100.0 & 100.0 & 100.0 & 6,034 & \\
\hline N & 1,960 & 2,062 & 2,012 & 4,022 & & \\
\hline \multicolumn{7}{|c|}{ Severe stomach pain } \\
\hline No & 64.4 & 67.4 & 67.9 & 66.0 & 4,007 & 2.416 \\
\hline Yes & 35.6 & 32.7 & 32.1 & 34.0 & 2,027 & 0.134 \\
\hline Total & 100.0 & 100.0 & 100.0 & 100.0 & 6,034 & \\
\hline $\mathrm{N}$ & 1,960 & 2,062 & 2,012 & 4,022 & & \\
\hline \multicolumn{7}{|c|}{ Fast or difficult breathing } \\
\hline No & 85.7 & 85.9 & 87.7 & 85.8 & 5,260 & 4.709 \\
\hline Yes & 14.3 & 14.1 & 12.3 & 14.2 & 774 & 0.245 \\
\hline Total & 100.0 & 100.0 & 100.0 & 100.0 & 6,034 & \\
\hline $\mathrm{N}$ & 1,960 & 2,062 & 2,012 & 4,022 & & \\
\hline \multicolumn{7}{|c|}{ Swollen feet } \\
\hline No & 83.2 & 79.5 & 74.4 & 81.2 & 4,747 & 38.639 \\
\hline Yes & 16.8 & 20.5 & 25.6 & 18.8 & 1,287 & 1.846 \\
\hline Total & 100.0 & 100.0 & 100.0 & 100.0 & 6,034 & \\
\hline $\mathrm{N}$ & 1,960 & 2,062 & 2,012 & 4,022 & & \\
\hline \multicolumn{7}{|c|}{ Swollen hands } \\
\hline No & 86.7 & 88.2 & 86.6 & 87.6 & 5,310 & 1.216 \\
\hline Yes & 13.3 & 11.8 & 13.4 & 12.5 & 724 & 0.054 \\
\hline Total & 100.0 & 100.0 & 100.0 & 100.0 & 6,034 & \\
\hline $\mathrm{N}$ & 1,960 & 2,062 & 2,012 & 4,022 & & \\
\hline
\end{tabular}

Estimates based on low Ns (approx. $<30$ obs) have large relative standard errors and should be interpreted with caution. ${ }^{* * *}$ indicates $p<0.001,{ }^{* *}$ indicates $p<0.05$ and * indicates $p<.0 .01$ for the chi ${ }^{2}$ statistic comparing integrated versus malaria-only study areas. 
TABLE 5.1.11. SELF-EFFICACY FOR ANC AND FACILITY BIRTH

Percentage of women aged 15 to 49 years currently pregnant or with a child under 2 years who report confidence to start a conversation or get to a facility for ANC/delivery, September-October 2019

\begin{tabular}{|c|c|c|c|c|c|c|}
\hline & KEBBI & SOKOTO & $\begin{array}{l}\text { MALARIA-ONLY } \\
\text { (ZAMFARA) }\end{array}$ & $\begin{array}{l}\text { INTEGRATED } \\
\text { (KEBBI/SOKOTO) }\end{array}$ & $\mathbf{N}$ & $\begin{array}{c}\mathrm{CHI}^{2} \\
\text { VALUE }\end{array}$ \\
\hline & $\%$ & $\%$ & $\%$ & $\%$ & & \\
\hline \multicolumn{7}{|c|}{$\begin{array}{l}\text { Confidence to start a conversation } \\
\text { with husband about attending ANC }\end{array}$} \\
\hline Confident & 73.7 & 67.1 & 74.9 & 70.2 & 4,414 & 44.298 \\
\hline Uncertain & 23.3 & 29.9 & 24.2 & 26.8 & 1,484 & 1.228 \\
\hline Don't know & 3.0 & 3.0 & 0.9 & 3.0 & 135 & \\
\hline Total & 100.0 & 100.0 & 100.0 & 100.0 & 6,033 & \\
\hline$N$ & 1,960 & 2,062 & 2,011 & 4,022 & & \\
\hline \multicolumn{7}{|c|}{$\begin{array}{l}\text { Confidence that you could get to a } \\
\text { health facility for ANC }\end{array}$} \\
\hline Confident & 67.4 & 63.0 & 71.3 & 65.0 & 4,161 & 41.605 \\
\hline Uncertain & 28.6 & 33.6 & 27.1 & 31.3 & 1,707 & 0.984 \\
\hline Don't know & 4.0 & 3.4 & 1.7 & 3.7 & 165 & \\
\hline Total & 100.0 & 100.0 & 100.0 & 100.0 & 6,033 & \\
\hline$N$ & 1,960 & 2,062 & 2,011 & 4,022 & & \\
\hline \multicolumn{7}{|c|}{$\begin{array}{l}\text { Confidence to start a conversation } \\
\text { with husband about facility delivery }\end{array}$} \\
\hline Confident & 56.8 & 47.5 & 49.7 & 51.8 & 3,213 & 63.754 \\
\hline Uncertain & 38.2 & 49.5 & 49.3 & 44.3 & 2,646 & 1.578 \\
\hline Don't know & 5.0 & 3.1 & 1.0 & 3.9 & 174 & \\
\hline Total & 100.0 & 100.0 & 100.0 & 100.0 & 6,033 & \\
\hline$N$ & 1,960 & 2,062 & 2,011 & 4,022 & & \\
\hline \multicolumn{7}{|c|}{$\begin{array}{l}\text { Confidence that you could get to a } \\
\text { health facility for delivery }\end{array}$} \\
\hline Confident & 48.6 & 43.3 & 43.5 & 45.8 & 2,855 & 76.230 \\
\hline Uncertain & 46.7 & 53.1 & 55.6 & 50.1 & 3,000 & 1.957 \\
\hline Don't know & 4.7 & 3.6 & 0.9 & 4.1 & 178 & \\
\hline Total & 100.0 & 100.0 & 100.0 & 100.0 & 6,033 & \\
\hline $\mathrm{N}$ & 1,960 & 2,062 & 2,011 & 4,022 & & \\
\hline
\end{tabular}

Estimates based on low Ns (approx. $<30$ obs) have large relative standard errors and should be interpreted with caution. ${ }^{* * *}$ indicates $p<0.001,{ }^{* *}$ indicates $p<0.05$ and ${ }^{*}$ indicates $p<.0 .01$ for the chi $^{2}$ statistic comparing integrated versus malaria-only study areas. 
TABLE 5.1.12. INTENTIONS FOR ANC AND FACILITY BIRTH DURING NEXT PREGNANCY

Percentage of women aged 15 to 49 years currently pregnant or with a child under 2 years who report intent to attend ANC and have a facility birth during next pregnancy, September-October 2019

\begin{tabular}{|c|c|c|c|c|c|c|}
\hline & KEBBI & SOKOTO & $\begin{array}{l}\text { MALARIA-ONLY } \\
\text { (ZAMFARA) }\end{array}$ & $\begin{array}{l}\text { INTEGRATED } \\
\text { (KEBBI/SOKOTO) }\end{array}$ & $\mathbf{N}$ & $\begin{array}{c}\mathrm{CHI}^{2} \\
\text { VALUE }\end{array}$ \\
\hline & $\%$ & $\%$ & $\%$ & $\%$ & & \\
\hline \multicolumn{7}{|c|}{$\begin{array}{l}\text { Make at least } 1 \text { ANC visit during next } \\
\text { pregnancy }\end{array}$} \\
\hline Likely & 65.4 & 58.7 & 65.7 & 61.8 & 3,907 & 95.242 \\
\hline Unlikely & 28.5 & 34.6 & 32.6 & 31.8 & 1,827 & 2.492 \\
\hline Don't know & 6.1 & 6.8 & 1.7 & 6.5 & 299 & \\
\hline Total & 100.0 & 100.0 & 100.0 & 100.0 & 6,033 & \\
\hline N & 1,960 & 2,062 & 2,011 & 4,022 & & \\
\hline \multicolumn{7}{|c|}{$\begin{array}{l}\text { Make at least } 4 \text { ANC visits during next } \\
\text { pregnancy }\end{array}$} \\
\hline Likely & 65.2 & 58.6 & 68.8 & 61.7 & 3,983 & 127.378 \\
\hline Unlikely & 27.8 & 34.0 & 29.6 & 31.1 & 1,714 & 3.422 \\
\hline Don't know & 7.0 & 7.4 & 1.7 & 7.2 & 336 & . \\
\hline Total & 100.0 & 100.0 & 100.0 & 100.0 & 6,033 & \\
\hline N & 1,960 & 2,062 & 2,011 & 4,022 & & \\
\hline \multicolumn{7}{|c|}{$\begin{array}{l}\text { Make at least } 8 \text { ANC visits during next } \\
\text { pregnancy }\end{array}$} \\
\hline Likely & 52.7 & 46.2 & 46.4 & 49.2 & 2,938 & 103.863 \\
\hline Unlikely & 37.3 & 44.6 & 49.8 & 41.2 & 2,612 & 2.614 \\
\hline Don't know & 10.0 & 9.2 & 3.8 & 9.6 & 483 & \\
\hline Total & 100.0 & 100.0 & 100.0 & 100.0 & 6,033 & \\
\hline $\mathrm{N}$ & 1,960 & 2,062 & 2,011 & 4,022 & & \\
\hline \multicolumn{7}{|c|}{$\begin{array}{l}\text { Take SP/Fansidar to prevent malaria } \\
\text { during next pregnancy }\end{array}$} \\
\hline Likely & 76.3 & 66.9 & 81.4 & 71.3 & 4,581 & 185.937 \\
\hline Unlikely & 15.6 & 22.6 & 16.6 & 19.3 & 1,020 & 4.749 \\
\hline Don't know & 8.1 & 10.5 & 2.0 & 9.4 & 432 & * \\
\hline Total & 100.0 & 100.0 & 100.0 & 100.0 & 6,033 & \\
\hline N & 1,960 & 2,062 & 2,011 & 4,022 & & \\
\hline \multicolumn{7}{|c|}{$\begin{array}{l}\text { Have facility delivery during next } \\
\text { pregnancy }\end{array}$} \\
\hline Likely & 56.3 & 45.2 & 51.7 & 50.3 & 3,209 & 98.262 \\
\hline Unlikely & 37.2 & 48.6 & 46.7 & 43.3 & 2,537 & 2.500 \\
\hline Don't know & 6.6 & 6.1 & 1.6 & 6.3 & 287 & \\
\hline Total & 100.0 & 100.0 & 100.0 & 100.0 & 6,033 & \\
\hline $\mathrm{N}$ & 1,960 & 2,062 & 2,011 & 4,022 & & \\
\hline
\end{tabular}

Estimates based on low Ns (approx. $<30$ obs) have large relative standard errors and should be interpreted with caution. ${ }^{* \star *}$ indicates $p<0.001,{ }^{* *}$ indicates $p<0.05$ and * indicates $p<.0 .01$ for the chi ${ }^{2}$ statistic comparing integrated versus malaria-only study areas. 
TABLE 5.1.13. WHO ELSE INFLUENCES DECISIONS ABOUT ANC AND FACILITY DELIVERY?

Percentage of women aged 15 to 49 years currently pregnant or with a child under 2 years who report influences on their decision to go for ANC or facility delivery, September-October 2019

\begin{tabular}{|c|c|c|c|c|c|c|}
\hline & KEBBI & SOKOTO & $\begin{array}{l}\text { MALARIA-ONLY } \\
\text { (ZAMFARA) }\end{array}$ & $\begin{array}{c}\text { INTEGRATED } \\
\text { (KEBBI/SOKOTO) }\end{array}$ & $\mathbf{N}$ & $\begin{array}{c}\mathrm{CHI}^{2} \\
\text { VALUE }\end{array}$ \\
\hline & $\%$ & $\%$ & $\%$ & $\%$ & & \\
\hline \multicolumn{7}{|l|}{ To attend 4+ ANC visits } \\
\hline No one else & 27.3 & 27.3 & 25.5 & 27.3 & 1,582 & 117.315 \\
\hline Partner & 67.0 & 63.6 & 67.4 & 65.2 & 3,988 & 1.818 \\
\hline Mother-in-law & 2.8 & 3.6 & 0.9 & 3.3 & 153 & \\
\hline Mother & 1.6 & 1.0 & 1.2 & 1.3 & 89 & \\
\hline Other own family & 0.2 & 0.3 & 0.3 & 0.2 & 18 & \\
\hline Other partner's family & 0.1 & 0.1 & 0.3 & 0.1 & 10 & \\
\hline Friends & 0.2 & 0.1 & 0.4 & 0.1 & 14 & \\
\hline Health provider & 0.7 & 3.7 & 1.5 & 2.3 & 117 & \\
\hline Community/religious leader & 0.0 & 0.2 & 0.0 & 0.1 & 4 & \\
\hline Other & 0.1 & 0.1 & 2.5 & 0.1 & 58 & \\
\hline Total & 100.0 & 100.0 & 100.0 & 100.0 & 6,033 & \\
\hline $\mathrm{N}$ & 1,960 & 2,062 & 2,011 & 4,022 & & \\
\hline \multicolumn{7}{|l|}{ For facility delivery } \\
\hline No one else & 30.4 & 31.1 & 36.8 & 30.7 & 1,969 & 173.590 \\
\hline Partner & 60.3 & 57.2 & 56.5 & 58.6 & 3,508 & 2.752 \\
\hline Mother-in-law & 4.1 & 4.3 & 0.8 & 4.2 & 173 & * \\
\hline Mother & 2.4 & 1.4 & 1.3 & 1.9 & 110 & \\
\hline Other own family & 0.1 & 0.3 & 0.6 & 0.2 & 18 & \\
\hline Other partner's family & 0.1 & 0.1 & 0.2 & 0.1 & 9 & \\
\hline Friends & 0.2 & 0.2 & 0.1 & 0.2 & 13 & \\
\hline Health provider & 2.5 & 5.4 & 2.0 & 4.0 & 188 & \\
\hline Community/religious leader & 0.0 & 0.1 & 0.0 & 0.1 & 3 & \\
\hline Other & 0.0 & 0.1 & 1.8 & 0.1 & 42 & \\
\hline Total & 100.0 & 100.0 & 100.0 & 100.0 & 6,033 & \\
\hline $\mathrm{N}$ & 1,960 & 2,062 & 2,011 & 4,022 & & \\
\hline
\end{tabular}

Estimates based on low Ns (approx. $<30$ obs) have large relative standard errors and should be interpreted with caution. ${ }^{\star \star \star}$ indicates $p<0.001,{ }^{\star \star}$ indicates $p<0.05$ and $^{*}$ indicates $p<.0 .01$ for the chi ${ }^{2}$ statistic comparing integrated versus malaria-only study areas. 
TABLE 5.1.14. PREGNANCY-RELATED BELIEFS, ATTITUDES AND VALUES

Percentage of women aged 15 to 49 years currently pregnant or with a child under 2 years who agree with certain beliefs, attitudes, or values about ANC or facility delivery, September-October 2019

\begin{tabular}{|c|c|c|c|c|c|c|}
\hline & KEBBI & SOKOTO & $\begin{array}{l}\text { MALARIA-ONLY } \\
\text { (ZAMFARA) }\end{array}$ & $\begin{array}{l}\text { INTEGRATED } \\
\text { (KEBBI/SOKOTO) }\end{array}$ & $\mathbf{N}$ & $\begin{array}{c}\mathrm{CHI}^{2} \\
\text { VALUE }\end{array}$ \\
\hline & $\%$ & $\%$ & $\%$ & $\%$ & & \\
\hline \multicolumn{7}{|c|}{$\begin{array}{l}\text { It is important for a woman to discuss } \\
\text { her pregnancy with her husband }\end{array}$} \\
\hline Agree & 89.7 & 87.8 & 94.8 & 88.7 & 5,480 & 88.462 \\
\hline Disagree & 6.9 & 8.3 & 4.3 & 7.7 & 404 & 2.293 \\
\hline Don't know & 3.4 & 3.9 & 0.9 & 3.7 & 149 & \\
\hline Total & 100.0 & 100.0 & 100.0 & 100.0 & 6,033 & \\
\hline $\mathrm{N}$ & 1,960 & 2,062 & 2,011 & 4,022 & & \\
\hline \multicolumn{7}{|c|}{$\begin{array}{l}\text { Pregnant women need ANC only when } \\
\text { sick }\end{array}$} \\
\hline Agree & 45.2 & 50.8 & 34.8 & 48.2 & 2,535 & 129.623 \\
\hline Disagree & 49.4 & 40.0 & 59.2 & 44.3 & 3,088 & 2.423 \\
\hline Don't know & 5.4 & 9.3 & 6.1 & 7.5 & 410 & \\
\hline Total & 100.0 & 100.0 & 100.0 & 100.0 & 6,033 & \\
\hline$N$ & 1,960 & 2,062 & 2,011 & 4,022 & & \\
\hline \multicolumn{7}{|c|}{$\begin{array}{l}\text { Only first-time pregnant women need } \\
\text { ANC }\end{array}$} \\
\hline Agree & 33.5 & 26.2 & 21.2 & 29.6 & 1,638 & 55.400 \\
\hline Disagree & 58.6 & 62.4 & 67.0 & 60.6 & 3,766 & 0.976 \\
\hline Don't know & 7.9 & 11.5 & 11.7 & 9.8 & 629 & \\
\hline Total & 100.0 & 100.0 & 100.0 & 100.0 & 6,033 & \\
\hline $\mathrm{N}$ & 1,960 & 2,062 & 2,011 & 4,022 & & \\
\hline \multicolumn{7}{|c|}{$\begin{array}{l}\text { Better to use traditional provider than } \\
\text { health facility for ANC }\end{array}$} \\
\hline Agree & 41.3 & 38.2 & 29.9 & 39.7 & 2,116 & 83.144 \\
\hline Disagree & 51.0 & 54.6 & 64.6 & 52.9 & 3,515 & 2.009 \\
\hline Don't know & 7.7 & 7.2 & 5.5 & 7.4 & 402 & \\
\hline Total & 100.0 & 100.0 & 100.0 & 100.0 & 6,033 & \\
\hline$N$ & 1,960 & 2,062 & 2,011 & 4,022 & & \\
\hline \multicolumn{7}{|c|}{$\begin{array}{l}\text { The health facility is the best place to } \\
\text { deliver a baby }\end{array}$} \\
\hline Agree & 58.6 & 47.7 & 56.3 & 52.7 & 3,401 & 46.154 \\
\hline Disagree & 36.6 & 47.7 & 42.0 & 42.6 & 2,404 & 1.090 \\
\hline Don't know & 4.8 & 4.5 & 1.7 & 4.7 & 228 & \\
\hline Total & 100.0 & 100.0 & 100.0 & 100.0 & 6,033 & \\
\hline$N$ & 1,960 & 2,062 & 2,011 & 4,022 & & \\
\hline \multicolumn{7}{|c|}{$\begin{array}{l}\text { Pregnant women attending } 4+\text { ANC } \\
\text { visits have safer pregnancies }\end{array}$} \\
\hline Agree & 67.8 & 58.3 & 63.2 & 62.7 & 3,924 & 24.781 \\
\hline Disagree & 20.9 & 27.7 & 27.7 & 24.5 & 1,431 & 0.413 \\
\hline Don't know & 11.3 & 14.1 & 9.1 & 12.8 & 678 & \\
\hline Total & 100.0 & 100.0 & 100.0 & 100.0 & 6,033 & \\
\hline $\mathrm{N}$ & 1,960 & 2,062 & 2,011 & 4,022 & & \\
\hline
\end{tabular}

Estimates based on low Ns (approx. $<30$ obs) have large relative standard errors and should be interpreted with caution. ${ }^{* * *}$ indicates $p<0.001,{ }^{* *}$ indicates $p<0.05$ and * indicates $p<.0 .01$ for the chi ${ }^{2}$ statistic comparing integrated versus malaria-only study areas. 


\subsection{Delivery care}

\section{Key findings}

Across study areas, more than $80 \%$ of women 15 to 49 years with a completed pregnancy in the past two years delivered at home, either at their own home $(74 \%)$ or at another home (9\%). Births were most commonly assisted by family members (36\%) or traditional birth attendants (29\%). Only $14 \%$ and $16 \%$ of women delivered in a health facility in Kebbi/Sokoto and Zamfara, respectively. Facility deliveries were more common among women living in the wealthiest households (36\% and $43 \%$ ) than in the poorest households (5\% and 4\%) in Kebbi/Sokoto and Zamfara. Women who attended primary education (31\% and $19 \%$ ) had higher facility delivery rates than those with no school attendance (10\% and $9 \%$ ) in Kebbi/Sokoto and Zamfara, respectively. Among women who did not have a facility delivery, the most common reasons for not doing so were lack of perceived need (75\% and 61\%) and spousal opposition (15\% and 36\%) in Kebbi/Sokoto and Zamfara.

All respondents were asked about decision-making for facility deliveries and other related beliefs or attitudes. The most commonly reported influencers of a woman's decision to have a facility delivery were spouses (57\%) and no one else (34\%). Despite reasons for non-facility delivery, including lack of perceived need, $55 \%$ of all respondents nevertheless agreed that the health facility is the best place to deliver a baby. Moreover, only $44 \%$ felt confident that they could get to a health facility for delivery. Only 51\% were confident that they could start a conversation with their husband about facility delivery, and a similar proportion (51\%) intended to deliver their next child at a health facility. There was no real difference in responses to these questions across Kebbi/Sokoto and Zamfara.

\section{TABLE 5.2. FACILITY DELIVERY DURING LAST PREGNANCY BY SOCIODEMOGRAPHIC CHARACTERISTICS}

Percentage of women 15 to 49 years with a child under 2 years with a facility delivery for their last-born child by sociodemographic characteristics, September-October 2019

\begin{tabular}{|c|c|c|c|c|c|c|c|c|}
\hline & \multicolumn{2}{|c|}{ KEBBI } & \multicolumn{2}{|c|}{ SOKOTO } & \multicolumn{2}{|c|}{$\begin{array}{l}\text { MALARIA-ONLY } \\
\text { (ZAMFARA) }\end{array}$} & \multicolumn{2}{|c|}{$\begin{array}{l}\text { INTEGRATED (KEBBI/ } \\
\text { SOKOTO) }\end{array}$} \\
\hline & $\%$ & $\mathbf{N}$ & $\%$ & $\mathbf{N}$ & $\%$ & $\mathbf{N}$ & $\%$ & $\mathbf{N}$ \\
\hline Total & 14.8 & 892 & 13.8 & 1,078 & 16.3 & 1,069 & 14.2 & 1,971 \\
\hline \multicolumn{9}{|l|}{ Household wealth } \\
\hline Lowest & 5.7 & 264 & 5.3 & 341 & 3.5 & 111 & 5.4 & 606 \\
\hline Second & 11.0 & 192 & 11.8 & 229 & 5.5 & 183 & 11.5 & 421 \\
\hline Middle & 13.1 & 157 & 14.8 & 220 & 9.1 & 223 & 14.1 & 377 \\
\hline Fourth & 28.1 & 113 & 15.6 & 135 & 10.8 & 248 & 21.4 & 249 \\
\hline Highest & 29.5 & 166 & 42.1 & 153 & 42.5 & 304 & 35.6 & 318 \\
\hline \multicolumn{9}{|l|}{ Maternal age (in years) } \\
\hline $15-24$ years & 15.8 & 367 & 16.6 & 485 & 13.1 & 423 & 16.3 & 852 \\
\hline $25-34$ years & 13.8 & 412 & 11.7 & 478 & 17.7 & 486 & 12.7 & 890 \\
\hline $35-49$ years & 15.5 & 113 & 10.8 & 115 & 21.7 & 160 & 13.0 & 229 \\
\hline \multicolumn{9}{|c|}{$\begin{array}{l}\text { Maternal education (highest } \\
\text { level attended) }\end{array}$} \\
\hline None & 8.8 & 675 & 10.3 & 855 & 9.0 & 698 & 9.7 & 1,530 \\
\hline Primary & 36.9 & 47 & 26.9 & 57 & 18.9 & 60 & 31.2 & 104 \\
\hline Secondary or higher & 40.5 & 95 & 68.1 & 60 & 54.4 & 180 & 50.4 & 155 \\
\hline Islamic & 24.8 & 75 & 15.5 & 106 & 15.4 & 131 & 19.6 & 182 \\
\hline
\end{tabular}

Estimates based on low Ns (approx. $<30$ obs) have large relative standard errors and should be interpreted with caution. ${ }^{* \star *}$ indicates $p<0.001,{ }^{* *}$ indicates $p<0.05$ and ${ }^{*}$ indicates $p<.0 .01$ for the chi ${ }^{2}$ statistic comparing integrated versus malaria-only study areas. 
TABLE 5.2.1. WHO ASSISTED IN THE DELIVERY OF YOUR CHILD DURING THE LAST PREGNANCY?

Percentage of women 15 to 49 years with a child under 2 years reporting who assisted in the delivery of their last-born child in past two years by reason, September-October 2019

\begin{tabular}{|c|c|c|c|c|c|c|}
\hline & $\begin{array}{c}\text { KEBBI } \\
\%\end{array}$ & $\begin{array}{c}\text { SOKOTO } \\
\%\end{array}$ & $\begin{array}{c}\text { MALARIA-ONLY } \\
\text { (ZAMFARA) } \\
\%\end{array}$ & $\begin{array}{c}\text { INTEGRATED } \\
\text { (KEBBI/SOKOTO) } \\
\%\end{array}$ & $\mathbf{N}$ & $\begin{array}{c}\mathrm{CHI}^{2} \\
\text { VALUE }\end{array}$ \\
\hline \multicolumn{7}{|l|}{ Doctor } \\
\hline No & 96.1 & 95.9 & 97.0 & 96.0 & 2,912 & 2.355 \\
\hline Yes & 3.9 & 4.1 & 3.0 & 4.0 & 127 & 0.467 \\
\hline Total & 100.0 & 100.0 & 100.0 & 100.0 & 3,039 & \\
\hline $\mathrm{N}$ & 892 & 1,078 & 1,069 & 1,970 & & \\
\hline \multicolumn{7}{|c|}{ Nurse/midwife } \\
\hline No & 77.9 & 89.8 & 83.1 & 84.7 & 2,507 & 1.384 \\
\hline Yes & 22.2 & 10.2 & 17.0 & 15.3 & 532 & 0.145 \\
\hline Total & 100.0 & 100.0 & 100.0 & 100.0 & 3,039 & \\
\hline$N$ & 892 & 1,078 & 1,069 & 1,970 & & \\
\hline \multicolumn{7}{|c|}{ Community health worker } \\
\hline No & 94.9 & 94.8 & 92.5 & 94.8 & 2,851 & 6.675 \\
\hline Yes & 5.1 & 5.2 & 7.5 & 5.2 & 188 & 1.582 \\
\hline Total & 100.0 & 100.0 & 100.0 & 100.0 & 3,039 & \\
\hline N & 892 & 1,078 & 1,069 & 1,970 & & \\
\hline \multicolumn{7}{|c|}{ Chemist } \\
\hline No & 100.0 & 100.0 & 100.0 & 100.0 & 3,038 & 0.280 \\
\hline Yes & 0.0 & 0.0 & 0.0 & 0.0 & 1 & 1.555 \\
\hline Total & 100.0 & 100.0 & 100.0 & 100.0 & 3,039 & \\
\hline $\mathrm{N}$ & 892 & 1,078 & 1,069 & 1,970 & & \\
\hline \multicolumn{7}{|c|}{ Traditional birth attendant } \\
\hline No & 84.5 & 63.5 & 70.1 & 72.5 & 2,203 & 2.062 \\
\hline Yes & 15.5 & 36.5 & 29.9 & 27.5 & 836 & 0.133 \\
\hline Total & 100.0 & 100.0 & 100.0 & 100.0 & 3,039 & \\
\hline N & 892 & 1,078 & 1,069 & 1,970 & & \\
\hline \multicolumn{7}{|c|}{ Religious leader } \\
\hline No & 99.7 & 99.9 & 99.9 & 99.8 & 3,034 & 0.786 \\
\hline Yes & 0.3 & 0.1 & 0.1 & 0.2 & 5 & 0.848 \\
\hline Total & 100.0 & 100.0 & 100.0 & 100.0 & 3,039 & \\
\hline $\mathrm{N}$ & 892 & 1,078 & 1,069 & 1,970 & & \\
\hline \multicolumn{7}{|l|}{ Family } \\
\hline No & 58.0 & 67.5 & 64.6 & 63.4 & 1,918 & 0.415 \\
\hline Yes & 42.0 & 32.5 & 35.5 & 36.6 & 1,121 & 0.033 \\
\hline Total & 100.0 & 100.0 & 100.0 & 100.0 & 3,039 & \\
\hline N & 892 & 1,078 & 1,069 & 1,970 & & \\
\hline \multicolumn{7}{|c|}{ Friends/neighbors } \\
\hline No & 82.1 & 92.0 & 84.6 & 87.7 & 2,627 & 5.874 \\
\hline Yes & 17.9 & 8.0 & 15.4 & 12.3 & 412 & 0.627 \\
\hline Total & 100.0 & 100.0 & 100.0 & 100.0 & 3,039 & \\
\hline $\mathrm{N}$ & 892 & 1,078 & 1,069 & 1,970 & & \\
\hline \multicolumn{7}{|c|}{ Itinerant drug seller } \\
\hline No & 99.9 & 100.0 & 99.9 & 100.0 & 3,037 & 0.452 \\
\hline Yes & 0.1 & 0.0 & 0.1 & 0.0 & 2 & 0.571 \\
\hline Total & 100.0 & 100.0 & 100.0 & 100.0 & 3,039 & \\
\hline $\mathrm{N}$ & 892 & 1,078 & 1,069 & 1,970 & & \\
\hline
\end{tabular}

Estimates based on low Ns (approx. $<30$ obs) have large relative standard errors and should be interpreted with caution. ${ }^{\star \star *}$ indicates $p<0.001,{ }^{\star \star}$ indicates $p<0.05$ and * indicates $p<.0 .01$ for the chi ${ }^{2}$ statistic comparing integrated versus malaria-only study areas. 
TABLE 5.2.2. DELIVERY LOCATION AND RESPECTFUL CARE DURING LAST PREGNANCY

Percentage of women 15 to 49 years with a child under 2 years reporting where they gave birth during the delivery of their last-born child in past two years and respectful care among facility births, September-October 2019

\begin{tabular}{|c|c|c|c|c|c|c|}
\hline & KEBBI & SOKOTO & $\begin{array}{l}\text { MALARIA-ONLY } \\
\text { (ZAMFARA) }\end{array}$ & $\begin{array}{l}\text { INTEGRATED } \\
\text { (KEBBI/SOKOTO) }\end{array}$ & $\mathrm{N}$ & $\begin{array}{c}\mathrm{CHI}^{2} \\
\text { VALUE }\end{array}$ \\
\hline & $\%$ & $\%$ & $\%$ & $\%$ & & \\
\hline \multicolumn{7}{|l|}{ Where did you give birth to child? } \\
\hline Her home & 77.1 & 75.6 & 72.2 & 76.3 & 2,208 & 74.368 \\
\hline Other home & 7.1 & 6.3 & 10.2 & 6.6 & 236 & 1.812 \\
\hline Government hospital & 7.4 & 7.6 & 11.4 & 7.5 & 326 & \\
\hline Governmental PHC & 7.0 & 4.8 & 3.1 & 5.8 & 144 & \\
\hline Dispensary/health post & 0.1 & 0.5 & 0.0 & 0.3 & 8 & \\
\hline Community health outreach post & 0.1 & 0.0 & 0.8 & 0.0 & 10 & \\
\hline Nursing/maternity home & 0.0 & 0.0 & 0.6 & 0.0 & 11 & \\
\hline Private hospital/clinic & 0.2 & 0.9 & 0.5 & 0.6 & 21 & \\
\hline Pharmacy & 0.0 & 0.0 & 0.0 & 0.0 & 1 & \\
\hline Other & 0.8 & 4.1 & 0.9 & 2.7 & 62 & \\
\hline Don't know & 0.2 & 0.2 & 0.4 & 0.2 & 12 & \\
\hline Total & 100.0 & 100.0 & 100.0 & 100.0 & 3,039 & \\
\hline $\mathrm{N}$ & 892 & 1,078 & 1,069 & 1,970 & & \\
\hline \multicolumn{7}{|l|}{$\begin{array}{l}\text { When giving birth (in a facility), } \\
\text { did you feel ignored or neglected by } \\
\text { health staff? }\end{array}$} \\
\hline No & 83.8 & 85.2 & 85.3 & 84.6 & 405 & 5.692 \\
\hline Yes & 15.1 & 12.4 & 14.8 & 13.6 & 76 & 1.490 \\
\hline Don't know & 1.1 & 2.4 & 0.0 & 1.8 & 7 & \\
\hline Total & 100.0 & 100.0 & 100.0 & 100.0 & 488 & \\
\hline $\mathrm{N}$ & 138 & 156 & 194 & 294 & & \\
\hline \multicolumn{7}{|l|}{$\begin{array}{l}\text { When giving birth (in a facility), } \\
\text { did you have privacy? }\end{array}$} \\
\hline No & 25.5 & 3.2 & 13.1 & 13.5 & 70 & 28.982 \\
\hline Yes, separate room & 44.5 & 47.2 & 25.7 & 45.9 & 182 & 2.535 \\
\hline Yes, curtains & 28.8 & 48.5 & 59.8 & 39.5 & 228 & \\
\hline Other & 0.0 & 0.0 & 1.3 & 0.0 & 3 & \\
\hline Don't know & 1.2 & 1.1 & 0.0 & 1.2 & 5 & \\
\hline Total & 100.0 & 100.0 & 100.0 & 100.0 & 488 & \\
\hline $\mathrm{N}$ & 138 & 156 & 194 & 294 & & \\
\hline
\end{tabular}

Estimates based on low Ns (approx. $<30$ obs) have large relative standard errors and should be interpreted with caution. ${ }^{* \star *}$ indicates $p<0.001$, ${ }^{* *}$ indicates $p<0.05$ and ${ }^{*}$ indicates $p<.0 .01$ for the chi statistic comparing integrated versus malaria-only study areas. Note: The respectful care questions were only asked for respondents that had a facility delivery, or more specifically, who gave birth during their last pregnancy at a government hospital, government PHC, dispensary/health post, or community health outreach post. 
TABLE 5.2.3. REASONS FOR NO FACILITY DELIVERY DURING LAST PREGNANCY

Percentage of women 15 to 49 years with a child under 2 years who did not deliver their last-born child in the past two years in a health facility by reasons for non-facility delivery, September-October 2019

\begin{tabular}{|c|c|c|c|c|c|c|}
\hline & KEBBI & SOKOTO & $\begin{array}{l}\text { MALARIA-ONLY } \\
\text { (ZAMFARA) }\end{array}$ & $\begin{array}{c}\text { INTEGRATED } \\
\text { (KEBBI/SOKOTO) }\end{array}$ & $\mathbf{N}$ & $\begin{array}{c}\mathrm{CHI}^{2} \\
\text { VALUE }\end{array}$ \\
\hline & $\%$ & $\%$ & $\%$ & $\%$ & & \\
\hline \multicolumn{7}{|c|}{ Costs too much } \\
\hline No & 92.7 & 95.5 & 95.5 & 94.3 & 2,386 & 1.700 \\
\hline Yes & 7.3 & 4.5 & 4.5 & 5.7 & 132 & 0.228 \\
\hline Total & 100.0 & 100.0 & 100.0 & 100.0 & 2,518 & \\
\hline N & 750 & 908 & 860 & 1,658 & & \\
\hline \multicolumn{7}{|c|}{ Facility not open } \\
\hline No & 97.8 & 98.7 & 95.3 & 98.3 & 2,455 & 16.722 \\
\hline Yes & 2.2 & 1.3 & 4.7 & 1.7 & 63 & 1.934 \\
\hline Total & 100.0 & 100.0 & 100.0 & 100.0 & 2,518 & \\
\hline $\mathrm{N}$ & 750 & 908 & 860 & 1,658 & & \\
\hline \multicolumn{7}{|c|}{$\begin{array}{l}\text { Health facility too far away/ } \\
\text { no transportation }\end{array}$} \\
\hline No & 97.1 & 97.5 & 92.2 & 97.3 & 2,408 & 28.694 \\
\hline Yes & 3.0 & 2.6 & 7.8 & 2.7 & 110 & 4.151 \\
\hline Total & 100.0 & 100.0 & 100.0 & 100.0 & 2,518 & * \\
\hline $\mathrm{N}$ & 750 & 908 & 860 & 1,658 & & \\
\hline \multicolumn{7}{|c|}{ No female provider at facility } \\
\hline No & 99.4 & 98.7 & 95.6 & 99.0 & 2,461 & 24.011 \\
\hline Yes & 0.6 & 1.3 & 4.4 & 1.0 & 57 & 4.418 \\
\hline Total & 100.0 & 100.0 & 100.0 & 100.0 & 2,518 & * \\
\hline $\mathrm{N}$ & 750 & 908 & 860 & 1,658 & & \\
\hline \multicolumn{7}{|c|}{ Poor quality of service } \\
\hline No & 99.3 & 99.4 & 99.6 & 99.4 & 2,501 & 0.483 \\
\hline Yes & 0.7 & 0.6 & 0.4 & 0.6 & 17 & 0.418 \\
\hline Total & 100.0 & 100.0 & 100.0 & 100.0 & 2,518 & \\
\hline $\mathrm{N}$ & 750 & 908 & 860 & 1,658 & & \\
\hline \multicolumn{7}{|c|}{ Husband/family does not allow } \\
\hline No & 84.1 & 85.9 & 64.3 & 85.1 & 1,942 & 131.939 \\
\hline Yes & 15.9 & 14.1 & 35.7 & 14.9 & 576 & 11.712 \\
\hline Total & 100.0 & 100.0 & 100.0 & 100.0 & 2,518 & 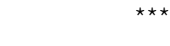 \\
\hline $\mathrm{N}$ & 750 & 908 & 860 & 1,658 & & \\
\hline \multicolumn{7}{|c|}{ Not necessary to go } \\
\hline No & 24.3 & 25.7 & 38.6 & 25.1 & 761 & 49.618 \\
\hline Yes & 75.7 & 74.3 & 61.4 & 74.9 & 1,757 & 3.736 \\
\hline Total & 100.0 & 100.0 & 100.0 & 100.0 & 2,518 & \\
\hline $\mathrm{N}$ & 750 & 908 & 860 & 1,658 & & \\
\hline
\end{tabular}

Estimates based on low Ns (approx. $<30$ obs) have large relative standard errors and should be interpreted with caution. ${ }^{* * *}$ indicates $p<0.001,{ }^{* *}$ indicates $p<0.05$ and ${ }^{*}$ indicates $p<.0 .01$ for the chi ${ }^{2}$ statistic comparing integrated versus malaria-only study areas. 


\subsection{Newborn care}

\section{Key findings}

Newborn care practices: Among respondents with a percent and 22 percent reported placing the newborn on a bare chest directly after birth in Kebbi/Sokoto and Zamfara, respectively. Less than one in five newborns had chlorhexidine applied to their stump across study areas. In the two days following birth, less than one in five newborns were provided with all other essential measures, including examining the cord, measuring temperature, and checking on the child for feeding practices, breathing problems, weakness, convulsions, swollen/red umbilical cord, skin boils, and pus in the eyes.

All respondents were asked about ways to protect a newborn after delivery. While nearly two-thirds of respondents across study areas (65\%) reported immediate breastfeeding, awareness of other measures was far lower: getting the child immunized (23\%), skin-to-skin contact (19\%), and using chlorhexidine for the child's stump (7\%), with generally higher awareness in Zamfara than in Kebbi/Sokoto for each of these measures. Taken together, these results point to low awareness and provision of newborn care in study areas, which could be an area for SBC activities to help raise rates of essential newborn care.
Postpartum care and counseling: Among respondents with a completed pregnancy in the past two years, only 13 percent and 20 percent were given misoprostol after their most recent birth in Kebbi/Sokoto and Zamfara, respectively. Less than one-third received a postpartum check following birth. Of those who did, nearly all received a check within the first 12 hours post-birth, either by a nurse/midwife (33\%), community health worker $(27 \%)$, or doctor (22\%). Few respondents (10\% and $12 \%$ ) in Kebbi/Sokoto and Zamfara were counseled on postpartum family planning in the first few days after delivery. In addition, few respondents were counseled on newborn danger signs (16\% and 15\%) and breastfeeding practices (17\% and 16\%) within the first 2 days after birth in Kebbi/Sokoto and Zamfara.

All respondents were asked about ways to help a mother recover from childbirth. While most respondents reported rest (62\%) and good nutrition (58\%), far fewer reported the need to take misoprostol immediately after birth (14\%), thereby indicating low awareness of this practice. There was much lower awareness of misoprostol in Kebbi/Sokoto (9\%) than in Zamfara (18\%). Taken together, these results point to low awareness and provision of postpartum care and counseling in study areas to help women recover from childbirth. 
TABLE 5.3.1. NEWBORN CARE IMMEDIATELY AFTER DELIVERY

Percentage of women 15 to 49 years with a child under 2 years reporting the following procedures done immediately after delivery for her last-born child in the past two years, September-October 2019

\begin{tabular}{|c|c|c|c|c|c|c|}
\hline & KEBBI & SOKOTO & $\begin{array}{l}\text { MALARIA-ONLY } \\
\text { (ZAMFARA) }\end{array}$ & $\begin{array}{l}\text { INTEGRATED } \\
\text { (KEBBI/SOKOTO) }\end{array}$ & $\mathbf{N}$ & $\begin{array}{c}\mathrm{CHI}^{2} \\
\text { VALUE }\end{array}$ \\
\hline & $\%$ & $\%$ & $\%$ & $\%$ & & \\
\hline \multicolumn{7}{|c|}{$\begin{array}{l}\text { Was child wiped dry within a few min- } \\
\text { utes after birth? }\end{array}$} \\
\hline No & 41.1 & 48.5 & 45.4 & 45.3 & 1,330 & 2.204 \\
\hline Yes & 53.9 & 47.5 & 51.2 & 50.2 & 1,564 & 0.087 \\
\hline Don't know & 5.0 & 4.1 & 3.4 & 4.5 & 145 & \\
\hline Total & 100.0 & 100.0 & 100.0 & 100.0 & 3,039 & \\
\hline $\mathrm{N}$ & 892 & 1,078 & 1,069 & 1,970 & & \\
\hline \multicolumn{7}{|c|}{$\begin{array}{l}\text { Was child bathed immediately after } \\
\text { birth? }\end{array}$} \\
\hline No & 30.9 & 28.5 & 31.9 & 29.5 & 1,000 & 5.035 \\
\hline Yes & 65.9 & 68.2 & 65.9 & 67.2 & 1,941 & 0.204 \\
\hline Don't know & 3.2 & 3.4 & 2.2 & 3.3 & 98 & \\
\hline Total & 100.0 & 100.0 & 100.0 & 100.0 & 3,039 & \\
\hline$N$ & 892 & 1,078 & 1,069 & 1,970 & & \\
\hline \multicolumn{7}{|c|}{$\begin{array}{l}\text { How long after birth was child bathed } \\
\text { for the first time (in hours)? }\end{array}$} \\
\hline 1 & 58.5 & 50.6 & 48.8 & 53.9 & 1,015 & 113.686 \\
\hline 2 & 10.1 & 14.8 & 13.5 & 12.8 & 260 & 3.139 \\
\hline $3-5$ & 4.8 & 10.8 & 21.5 & 8.3 & 243 & * \\
\hline $6-11$ & 2.1 & 7.2 & 6.2 & 5.0 & 107 & \\
\hline $12-23$ & 2.8 & 7.2 & 5.1 & 5.3 & 106 & \\
\hline $24+$ & 9.1 & 5.9 & 3.9 & 7.3 & 113 & \\
\hline Don't know & 12.6 & 3.6 & 1.2 & 7.4 & 97 & \\
\hline Total & 100.0 & 100.0 & 100.0 & 100.0 & 1,941 & \\
\hline N & 539 & 725 & 677 & 1,264 & & \\
\hline \multicolumn{7}{|c|}{$\begin{array}{l}\text { Child placed directly on bare skin of } \\
\text { chest }\end{array}$} \\
\hline No & 66.4 & 64.7 & 76.5 & 65.4 & 2,056 & 45.522 \\
\hline Yes & 31.5 & 32.9 & 21.5 & 32.3 & 905 & 2.397 \\
\hline Don't know & 2.1 & 2.4 & 2.0 & 2.3 & 78 & \\
\hline Total & 100.0 & 100.0 & 100.0 & 100.0 & 3,039 & \\
\hline $\mathrm{N}$ & 892 & 1,078 & 1,069 & 1,970 & & \\
\hline \multicolumn{7}{|c|}{ New blade to cut child's cord } \\
\hline No & 13.2 & 7.0 & 7.7 & 9.7 & 290 & 4.057 \\
\hline Yes & 85.6 & 90.6 & 90.6 & 88.4 & 2,687 & 0.204 \\
\hline Don't know & 1.2 & 2.5 & 1.7 & 1.9 & 62 & \\
\hline Total & 100.0 & 100.0 & 100.0 & 100.0 & 3,039 & \\
\hline $\mathrm{N}$ & 892 & 1,078 & 1,069 & 1,970 & & \\
\hline \multicolumn{7}{|c|}{ Chlorhexidine applied to child's stump } \\
\hline No & 75.2 & 79.0 & 77.7 & 77.4 & 2,268 & 5.964 \\
\hline Yes & 17.5 & 15.9 & 18.1 & 16.6 & 583 & 0.327 \\
\hline Don't know & 7.3 & 5.2 & 4.3 & 6.1 & 188 & \\
\hline Total & 100.0 & 100.0 & 100.0 & 100.0 & 3,039 & \\
\hline $\mathrm{N}$ & 892 & 1,078 & 1,069 & 1,970 & & \\
\hline \multicolumn{7}{|c|}{ Given misoprostol (miso miso) } \\
\hline No & 78.7 & 83.2 & 75.0 & 81.3 & 2,348 & 26.393 \\
\hline Yes & 13.7 & 12.6 & 20.3 & 13.1 & 509 & 1.562 \\
\hline Don't know & 7.6 & 4.1 & 4.7 & 5.6 & 182 & \\
\hline Total & 100.0 & 100.0 & 100.0 & 100.0 & 3,039 & \\
\hline N & 892 & 1,078 & 1,069 & 1,970 & & \\
\hline
\end{tabular}

Estimates based on low Ns (approx. $<30$ obs) have large relative standard errors and should be interpreted with caution. ${ }^{* * *}$ indicates $p<0.001,{ }^{* *}$ indicates $p<0.05$ and ${ }^{*}$ indicates $p<.0 .01$ for the chi ${ }^{2}$ statistic comparing integrated versus malaria-only study areas. 
TABLE 5.3.2. NEWBORN CARE IN THE FIRST TWO DAYS AFTER DELIVERY

Percentage of women 15 to 49 years with a child under 2 years reporting the following procedures done within two days after delivery for her last-born child in the past two years, September-October 2019

\begin{tabular}{|c|c|c|c|c|c|c|}
\hline & $\begin{array}{c}\text { KEBBI } \\
\%\end{array}$ & $\begin{array}{c}\text { SOKOTO } \\
\%\end{array}$ & $\begin{array}{c}\text { MALARIA-ONLY } \\
\text { (ZAMFARA) } \\
\%\end{array}$ & $\begin{array}{c}\text { INTEGRATED } \\
\text { (KEBBI/SOKOTO) } \\
\%\end{array}$ & $\mathbf{N}$ & $\begin{array}{c}\mathrm{CHI}^{2} \\
\text { VALUE }\end{array}$ \\
\hline \multicolumn{7}{|c|}{ Examine the cord } \\
\hline No & 80.6 & 71.2 & 75.2 & 75.2 & 2,242 & 10.093 \\
\hline Yes & 16.1 & 25.2 & 23.0 & 21.3 & 712 & 0.615 \\
\hline Don't know & 3.3 & 3.6 & 1.7 & 3.5 & 85 & \\
\hline Total & 100.0 & 100.0 & 100.0 & 100.0 & 3,039 & \\
\hline $\mathrm{N}$ & 892 & 1,078 & 1,069 & 1,970 & & \\
\hline \multicolumn{7}{|c|}{ Measure child temperature } \\
\hline No & 85.4 & 80.5 & 80.0 & 82.6 & 2,424 & 9.591 \\
\hline Yes & 10.7 & 16.6 & 17.7 & 14.1 & 528 & 0.747 \\
\hline Don't know & 3.9 & 2.9 & 2.3 & 3.3 & 87 & \\
\hline Total & 100.0 & 100.0 & 100.0 & 100.0 & 3,039 & \\
\hline N & 892 & 1,078 & 1,069 & 1,970 & & \\
\hline \multicolumn{7}{|c|}{ Check child feeding problems } \\
\hline No & 83.5 & 79.6 & 82.4 & 81.3 & 2,422 & 7.653 \\
\hline Yes & 12.1 & 17.3 & 15.5 & 15.0 & 520 & 0.596 \\
\hline Don't know & 4.4 & 3.1 & 2.0 & 3.7 & 97 & \\
\hline Total & 100.0 & 100.0 & 100.0 & 100.0 & 3,039 & \\
\hline N & 892 & 1,078 & 1,069 & 1,970 & & \\
\hline \multicolumn{7}{|c|}{ Check child for jaundice } \\
\hline No & 83.9 & 80.7 & 80.2 & 82.1 & 2,414 & 30.729 \\
\hline Yes & 10.2 & 15.0 & 17.7 & 12.9 & 496 & 2.688 \\
\hline Don't know & 6.0 & 4.3 & 2.1 & 5.0 & 129 & \\
\hline Total & 100.0 & 100.0 & 100.0 & 100.0 & 3,039 & \\
\hline $\mathrm{N}$ & 892 & 1,078 & 1,069 & 1,970 & & \\
\hline \multicolumn{7}{|c|}{ Check child breathing problems } \\
\hline No & 82.2 & 80.7 & 81.0 & 81.3 & 2,407 & 6.158 \\
\hline Yes & 13.2 & 15.8 & 16.5 & 14.7 & 523 & 0.477 \\
\hline Don't know & 4.6 & 3.6 & 2.6 & 4.0 & 109 & \\
\hline Total & 100.0 & 100.0 & 100.0 & 100.0 & 3,039 & \\
\hline $\mathrm{N}$ & 892 & 1,078 & 1,069 & 1,970 & & \\
\hline \multicolumn{7}{|c|}{ Check child for convulsions } \\
\hline No & 84.2 & 82.6 & 83.2 & 83.3 & 2,483 & 10.034 \\
\hline Yes & 9.7 & 12.6 & 13.5 & 11.4 & 411 & 0.778 \\
\hline Don't know & 6.1 & 4.8 & 3.3 & 5.4 & 145 & \\
\hline Total & 100.0 & 100.0 & 100.0 & 100.0 & 3,039 & \\
\hline $\mathrm{N}$ & 892 & 1,078 & 1,069 & 1,970 & & \\
\hline \multicolumn{7}{|c|}{ Check child for weakness/lethargy } \\
\hline No & 83.5 & 81.7 & 83.5 & 82.5 & 2,465 & 17.773 \\
\hline Yes & 10.4 & 12.3 & 13.5 & 11.5 & 414 & 1.417 \\
\hline Don't know & 6.1 & 6.0 & 3.1 & 6.0 & 160 & \\
\hline Total & 100.0 & 100.0 & 100.0 & 100.0 & 3,039 & \\
\hline N & 892 & 1,078 & 1,069 & 1,970 & & \\
\hline \multicolumn{7}{|c|}{$\begin{array}{l}\text { Check child for swollen/red umbilicus } \\
\text { with pus }\end{array}$} \\
\hline No & 83.9 & 81.5 & 81.6 & 82.5 & 2,438 & 10.786 \\
\hline Yes & 11.1 & 13.2 & 15.1 & 12.3 & 456 & 0.849 \\
\hline Don't know & 5.1 & 5.3 & 3.3 & 5.2 & 145 & \\
\hline Total & 100.0 & 100.0 & 100.0 & 100.0 & 3,039 & \\
\hline $\mathrm{N}$ & 892 & 1,078 & 1,069 & 1,970 & & \\
\hline \multicolumn{7}{|c|}{ Check child for skin boils } \\
\hline No & 83.3 & 82.4 & 82.8 & 82.8 & 2,467 & 4.835 \\
\hline
\end{tabular}




\begin{tabular}{|c|c|c|c|c|c|c|}
\hline Yes & 12.0 & 13.0 & 13.9 & 12.5 & 442 & 0.391 \\
\hline Don't know & 4.8 & 4.6 & 3.3 & 4.7 & 130 & \\
\hline Total & 100.0 & 100.0 & 100.0 & 100.0 & 3,039 & \\
\hline $\mathrm{N}$ & 892 & 1,078 & 1,069 & 1,970 & & \\
\hline \multicolumn{7}{|c|}{ Check child for pus in eyes } \\
\hline No & 83.8 & 80.8 & 82.4 & 82.1 & 2,446 & 3.607 \\
\hline Yes & 11.6 & 14.4 & 14.2 & 13.2 & 461 & 0.276 \\
\hline Don't know & 4.6 & 4.8 & 3.4 & 4.7 & 132 & \\
\hline Total & 100.0 & 100.0 & 100.0 & 100.0 & 3,039 & \\
\hline N & 892 & 1,078 & 1,069 & 1,970 & & \\
\hline \multicolumn{7}{|c|}{ Counsel on newborn danger signs } \\
\hline No & 85.3 & 76.7 & 83.5 & 80.4 & 2,447 & 9.732 \\
\hline Yes & 10.6 & 19.9 & 14.5 & 15.9 & 490 & 0.502 \\
\hline Don't know & 4.1 & 3.4 & 2.0 & 3.7 & 102 & \\
\hline Total & 100.0 & 100.0 & 100.0 & 100.0 & 3,039 & \\
\hline N & 892 & 1,078 & 1,069 & 1,970 & & \\
\hline \multicolumn{7}{|c|}{ Counsel on breastfeeding } \\
\hline No & 84.5 & 76.0 & 82.2 & 79.6 & 2,411 & 5.565 \\
\hline Yes & 12.0 & 21.4 & 15.9 & 17.4 & 546 & 0.291 \\
\hline Don't know & 3.6 & 2.6 & 1.9 & 3.0 & 82 & \\
\hline Total & 100.0 & 100.0 & 100.0 & 100.0 & 3,039 & \\
\hline $\mathrm{N}$ & 892 & 1,078 & 1,069 & 1,970 & & \\
\hline \multicolumn{7}{|c|}{ Counsel on nutrition for new mothers } \\
\hline No & 84.5 & 76.2 & 83.2 & 79.8 & 2,436 & 8.750 \\
\hline Yes & 11.5 & 21.3 & 15.0 & 17.1 & 518 & 0.432 \\
\hline Don't know & 4.1 & 2.5 & 1.8 & 3.2 & 85 & \\
\hline Total & 100.0 & 100.0 & 100.0 & 100.0 & 3,039 & \\
\hline $\mathrm{N}$ & 892 & 1,078 & 1,069 & 1,970 & & \\
\hline
\end{tabular}

Estimates based on low Ns (approx. $<30$ obs) have large relative standard errors and should be interpreted with caution. ${ }^{* * *}$ indicates $p<0.001,{ }^{* *}$ indicates $p<0.05$ and ${ }^{*}$ indicates $p<.0 .01$ for the chi ${ }^{2}$ statistic comparing integrated versus malaria-only study areas. 
TABLE 5.3.3. POSTPARTUM CARE FOR MOTHERS AFTER DELIVERY

Percentage of women 15 to 49 years with a child under 2 years reporting the following procedures done within two days after delivery for her last-born child in the past two years, September-October 2019

\begin{tabular}{|c|c|c|c|c|c|c|}
\hline & KEBBI & SOKOTO & $\begin{array}{l}\text { MALARIA-ONLY } \\
\text { (ZAMFARA) }\end{array}$ & $\begin{array}{l}\text { INTEGRATED } \\
\text { (KEBBI/SOKOTO) }\end{array}$ & $\mathbf{N}$ & $\begin{array}{c}\mathrm{CHI}^{2} \\
\text { VALUE }\end{array}$ \\
\hline & $\%$ & $\%$ & $\%$ & $\%$ & & \\
\hline \multicolumn{7}{|c|}{$\begin{array}{l}\text { Did anyone check on your health after } \\
\text { you gave birth to child? }\end{array}$} \\
\hline No & 80.6 & 76.7 & 66.0 & 78.4 & 2,201 & 57.431 \\
\hline Yes & 18.2 & 22.2 & 33.1 & 20.5 & 804 & 3.973 \\
\hline Don't know & 1.2 & 1.1 & 0.9 & 1.1 & 34 & * \\
\hline Total & 100.0 & 100.0 & 100.0 & 100.0 & 3,039 & \\
\hline N & 892 & 1,078 & 1,069 & 1,970 & & \\
\hline \multicolumn{7}{|l|}{$\begin{array}{l}\text { How long after delivery did the first } \\
\text { check take place? }\end{array}$} \\
\hline $0-11$ hours & 82.5 & 65.7 & 63.8 & 72.2 & 541 & 5.538 \\
\hline 12-23 hours & 2.1 & 0.7 & 1.0 & 1.2 & 9 & 1.193 \\
\hline $24+$ hours & 15.4 & 33.7 & 35.2 & 26.5 & 239 & \\
\hline Total & 100.0 & 100.0 & 100.0 & 100.0 & 789 & \\
\hline $\mathrm{N}$ & 168 & 244 & 377 & 412 & & \\
\hline \multicolumn{7}{|l|}{$\begin{array}{l}\text { Who checked on your health at that } \\
\text { time? }\end{array}$} \\
\hline Other health personnel & 0.0 & 0.0 & 0.4 & 0.0 & 1 & 95.866 \\
\hline Doctor & 19.4 & 22.1 & 22.6 & 21.1 & 186 & 3.837 \\
\hline Nurse/midwife & 52.5 & 23.0 & 31.8 & 34.4 & 277 & *** \\
\hline Community health worker & 20.1 & 18.9 & 30.0 & 19.4 & 198 & \\
\hline Chemist & 0.8 & 0.1 & 8.0 & 0.4 & 29 & \\
\hline Traditional healer/birth attendant & 4.5 & 8.8 & 4.9 & 7.2 & 48 & \\
\hline Religious leader & 0.0 & 0.4 & 0.0 & 0.2 & 1 & \\
\hline Family & 2.8 & 22.8 & 1.1 & 15.1 & 50 & \\
\hline Friends/neighbors & 0.0 & 3.5 & 0.5 & 2.1 & 10 & \\
\hline Other & 0.0 & 0.0 & 0.6 & 0.0 & 2 & \\
\hline Don't know & 0.0 & 0.4 & 0.2 & 0.2 & 2 & \\
\hline Total & 100.0 & 100.0 & 100.0 & 100.0 & 804 & \\
\hline $\mathrm{N}$ & 171 & 250 & 383 & 421 & & \\
\hline \multicolumn{7}{|c|}{$\begin{array}{l}\text { After childbirth, did anyone talk to you } \\
\text { about using modern contraception? }\end{array}$} \\
\hline No & 90.8 & 87.9 & 87.4 & 89.2 & 2,659 & 14.162 \\
\hline Yes & 7.9 & 11.2 & 12.4 & 9.8 & 351 & 1.486 \\
\hline Don't know & 1.4 & 0.9 & 0.2 & 1.1 & 29 & \\
\hline Total & 100.0 & 100.0 & 100.0 & 100.0 & 3,039 & \\
\hline $\mathrm{N}$ & 892 & 1,078 & 1,069 & 1,970 & & \\
\hline \multicolumn{7}{|c|}{$\begin{array}{l}\text { After giving birth, did anyone counsel } \\
\text { you on nutrition for new mothers? }\end{array}$} \\
\hline No & 84.5 & 76.2 & 83.2 & 79.8 & 2,436 & 8.750 \\
\hline Yes & 11.5 & 21.3 & 15.0 & 17.1 & 518 & 0.432 \\
\hline Don't know & 4.1 & 2.5 & 1.8 & 3.2 & 85 & \\
\hline Total & 100.0 & 100.0 & 100.0 & 100.0 & 3,039 & \\
\hline $\mathrm{N}$ & 892 & 1,078 & 1,069 & 1,970 & & \\
\hline
\end{tabular}

Estimates based on low Ns (approx. $<30$ obs) have large relative standard errors and should be interpreted with caution. ${ }^{* * *}$ indicates $p<0.001,{ }^{* *}$ indicates $p<0.05$ and ${ }^{*}$ indicates $p<.0 .01$ for the chi ${ }^{2}$ statistic comparing integrated versus malaria-only study areas. 
TABLE 5.3.4. WHAT ARE SOME WAYS TO PROTECT A NEWBORN IMMEDIATELY AFTER BIRTH?

Percentage of women aged 15 to 49 years currently pregnant or with a child under 2 years who report ways to protect a newborn immediately after birth by method, September-October 2019

\begin{tabular}{|c|c|c|c|c|c|c|}
\hline & KEBBI & SOKOTO & $\begin{array}{l}\text { MALARIA-ONLY } \\
\text { (ZAMFARA) }\end{array}$ & $\begin{array}{l}\text { INTEGRATED } \\
\text { (KEBBI/SOKOTO) }\end{array}$ & $\mathbf{N}$ & $\begin{array}{c}\mathrm{CHI}^{2} \\
\text { VALUE }\end{array}$ \\
\hline & $\%$ & $\%$ & $\%$ & $\%$ & & \\
\hline \multicolumn{7}{|c|}{ Skin to skin contact } \\
\hline No & 81.7 & 79.0 & 81.1 & 80.2 & 4,888 & 0.627 \\
\hline Yes & 18.3 & 21.1 & 18.9 & 19.8 & 1,146 & 0.020 \\
\hline Total & 100.0 & 100.0 & 100.0 & 100.0 & 6,034 & \\
\hline $\mathrm{N}$ & 1,960 & 2,062 & 2,012 & 4,022 & & \\
\hline \multicolumn{7}{|c|}{ Immediate breastfeeding } \\
\hline No & 31.0 & 46.3 & 31.8 & 39.2 & 2,299 & 35.481 \\
\hline Yes & 69.1 & 53.7 & 68.2 & 60.8 & 3,735 & 1.132 \\
\hline Total & 100.0 & 100.0 & 100.0 & 100.0 & 6,034 & \\
\hline $\mathrm{N}$ & 1,960 & 2,062 & 2,012 & 4,022 & & \\
\hline \multicolumn{7}{|c|}{ Give fluids excluding breastmilk } \\
\hline No & 83.2 & 79.8 & 90.0 & 81.4 & 5,086 & 92.970 \\
\hline Yes & 16.8 & 20.3 & 10.0 & 18.6 & 948 & 2.464 \\
\hline Total & 100.0 & 100.0 & 100.0 & 100.0 & 6,034 & \\
\hline N & 1,960 & 2,062 & 2,012 & 4,022 & & \\
\hline \multicolumn{7}{|c|}{ Get child immunized } \\
\hline No & 83.4 & 81.2 & 73.2 & 82.3 & 4,667 & 67.682 \\
\hline Yes & 16.6 & 18.8 & 26.9 & 17.8 & 1,367 & 2.175 \\
\hline Total & 100.0 & 100.0 & 100.0 & 100.0 & 6,034 & \\
\hline $\mathrm{N}$ & 1,960 & 2,062 & 2,012 & 4,022 & & \\
\hline \multicolumn{7}{|c|}{ Chlorhexidine for baby's stump } \\
\hline No & 95.6 & 94.5 & 91.3 & 95.0 & 5,661 & 30.284 \\
\hline Yes & 4.4 & 5.5 & 8.7 & 5.0 & 373 & 0.966 \\
\hline Total & 100.0 & 100.0 & 100.0 & 100.0 & 6,034 & \\
\hline N & 1,960 & 2,062 & 2,012 & 4,022 & & \\
\hline \multicolumn{7}{|c|}{ Traditional medicine for stump } \\
\hline No & 85.2 & 78.5 & 83.0 & 81.6 & 5,027 & 2.096 \\
\hline Yes & 14.8 & 21.5 & 17.0 & 18.4 & 1,007 & 0.050 \\
\hline Total & 100.0 & 100.0 & 100.0 & 100.0 & 6,034 & \\
\hline $\mathrm{N}$ & 1,960 & 2,062 & 2,012 & 4,022 & & \\
\hline
\end{tabular}

Estimates based on low Ns (approx. $<30$ obs) have large relative standard errors and should be interpreted with caution. ${ }^{* \star *}$ indicates $p<0.001,{ }^{* *}$ indicates $p<0.05$ and ${ }^{*}$ indicates $p<.0 .01$ for the chi $^{2}$ statistic comparing integrated versus malaria-only study areas. 
TABLE 5.3.5. WHAT ARE SOME WAYS TO HELP A MOTHER RECOVER WELL AFTER BIRTH?

Percentage of women aged 15 to 49 years currently pregnant or with a child under 2 years who report ways to help a mother recover well from childbirth by method, September-October 2019

\begin{tabular}{|c|c|c|c|c|c|c|}
\hline & KEBBI & SOKOTO & $\begin{array}{l}\text { MALARIA-ONLY } \\
\text { (ZAMFARA) }\end{array}$ & $\begin{array}{l}\text { INTEGRATED } \\
\text { (KEBBI/SOKOTO) }\end{array}$ & $\mathbf{N}$ & $\begin{array}{c}\mathrm{CHI}^{2} \\
\text { VALUE }\end{array}$ \\
\hline & $\%$ & $\%$ & $\%$ & $\%$ & & \\
\hline \multicolumn{7}{|c|}{$\begin{array}{l}\text { Take misoprostol immediately after } \\
\text { birth }\end{array}$} \\
\hline No & 94.0 & 89.3 & 81.6 & 91.5 & 5,297 & 114.640 \\
\hline Yes & 6.0 & 10.7 & 18.4 & 8.5 & 737 & 4.576 \\
\hline Total & 100.0 & 100.0 & 100.0 & 100.0 & 6,034 & * \\
\hline $\mathrm{N}$ & 1,960 & 2,062 & 2,012 & 4,022 & & \\
\hline \multicolumn{7}{|c|}{ Ensure good nutrition is taken } \\
\hline No & 41.4 & 52.8 & 37.6 & 47.5 & 2,661 & 58.892 \\
\hline Yes & 58.6 & 47.3 & 62.4 & 52.5 & 3,373 & 1.869 \\
\hline Total & 100.0 & 100.0 & 100.0 & 100.0 & 6,034 & \\
\hline $\mathrm{N}$ & 1,960 & 2,062 & 2,012 & 4,022 & & \\
\hline \multicolumn{7}{|c|}{ Lose weight gained during pregnancy } \\
\hline No & 94.5 & 93.9 & 86.9 & 94.2 & 5,567 & 84.542 \\
\hline Yes & 5.5 & 6.1 & 13.1 & 5.8 & 467 & 3.285 \\
\hline Total & 100.0 & 100.0 & 100.0 & 100.0 & 6,034 & \\
\hline N & 1,960 & 2,062 & 2,012 & 4,022 & & \\
\hline \multicolumn{7}{|c|}{ Resume regular activity immediately } \\
\hline No & 85.0 & 86.2 & 75.3 & 85.6 & 5,016 & 95.435 \\
\hline Yes & 15.0 & 13.8 & 24.7 & 14.4 & 1,018 & 2.792 \\
\hline Total & 100.0 & 100.0 & 100.0 & 100.0 & 6,034 & \\
\hline $\mathrm{N}$ & 1,960 & 2,062 & 2,012 & 4,022 & & \\
\hline \multicolumn{7}{|l|}{ Rest } \\
\hline No & 47.9 & 26.9 & 38.4 & 36.6 & 2,342 & 1.868 \\
\hline Yes & 52.1 & 73.1 & 61.7 & 63.4 & 3,692 & 0.060 \\
\hline Total & 100.0 & 100.0 & 100.0 & 100.0 & 6,034 & \\
\hline$N$ & 1,960 & 2,062 & 2,012 & 4,022 & & \\
\hline
\end{tabular}

Estimates based on low Ns (approx. $<30$ obs) have large relative standard errors and should be interpreted with caution. ${ }^{* \star *}$ indicates $p<0.001,{ }^{\star \star}$ indicates $p<0.05$ and ${ }^{*}$ indicates $p<.0 .01$ for the chi ${ }^{2}$ statistic comparing integrated versus malaria-only study areas. 


\section{Child nutrition and breastfeeding}

\subsection{Child nutrition and breastfeeding practices}

\section{Key findings}

Nearly all respondents (96\%) reported ever breastfeeding their last-born child under 2 years old. Among the few respondents who never breastfed, the most common reasons were respondent and partner opposition. Despite high rates of ever breastfeeding, initiation of breastfeeding within one hour of birth was low overall but higher in Zamfara (46\%) than in Kebbi/Sokoto (36\%). Immediate breastfeeding was higher among respondents in the wealthiest ( $40 \%$ and $57 \%$ ) than in the poorest (35\% and $47 \%$ ) households and among respondents with secondary/higher schooling (47\% and 58\%) than among respondents with no education (33\% and $43 \%$ ) in Kebbi/ Sokoto and Zamfara, respectively.

Exclusive breastfeeding was also low across study areas, with higher rates in Zamfara (46\%) than in Kebbi/Sokoto (26\%) and with similar patterns by sociodemographic characteristics. This low coverage is in large part due to high rates of giving non-breastmilk liquids to a child in the first three days after birth, with much higher practice rates in Kebbi/Sokoto (73\%) than in Zamfara (50\%). In addition, 73 percent of respondents agreed that the mother's breastmilk after birth is bad, which may be linked with giving non-breastmilk liquids to a newborn.
Taken together, this points to a potential area for SBC activities to help promote exclusive breastfeeding.

All respondents were asked who else influences decisions about breastfeeding, with the most common responses being spouse (60\%) and no one else (34\%). Respondents were also asked about the benefits of breastfeeding for women. The most commonly reported benefits were promoting infant bonding (31\%) and convenience (20\%). One in five respondents reported no benefits whatsoever. Nearly two-thirds (64\%) of respondents reported that breastfeeding should last 12 to 23 months, and a similar proportion agreed that exclusive breastfeeding was important. Most respondents (86\%) also agreed that breastmilk contains essential nutrients for the child's first six months of life.

Yet despite this reported high awareness of breastfeeding and its benefits, only about half (55\%) of respondents felt confident to exclusively breastfeed their child for the first six months, with higher confidence in Zamfara (64\%) than in Kebbi/Sokoto (42\%). In Kebbi/Sokoto and Zamfara, respectively, 30 percent and 49 percent of respondents believed that most women in their communities gave breastmilk alone to infants, suggesting weak social norms favoring exclusive breastfeeding in this area. There was also relatively low intent to exclusively breastfeed their next child for the first six months, with higher intent in Zamfara (59\%) than in Kebbi/Sokoto (46\%). 


\section{TABLE 6.1.A. IMMEDIATE BREASTFEEDING AFTER BIRTH BY SOCIODEMOGRAPHIC CHARACTERISTICS}

Percentage of last-born children in past two years who were breastfed within one hour of birth by sociodemographic characteristics, September-October 2019

\begin{tabular}{|c|c|c|c|c|c|c|c|c|}
\hline & \multicolumn{2}{|c|}{ KEBBI } & \multicolumn{2}{|c|}{ sокото } & \multicolumn{2}{|c|}{$\begin{array}{l}\text { MALARIA-ONLY } \\
\text { (ZAMFARA) }\end{array}$} & \multicolumn{2}{|c|}{$\begin{array}{l}\text { INTEGRATED } \\
\text { (KEBBI/SOKOTO) }\end{array}$} \\
\hline & $\%$ & $\mathbf{N}$ & $\%$ & $\mathrm{~N}$ & $\%$ & $\mathrm{~N}$ & $\%$ & $\mathrm{~N}$ \\
\hline Total & 41.6 & 892 & 31.6 & 1,078 & 46.1 & 1,069 & 35.9 & 1,971 \\
\hline \multicolumn{9}{|l|}{ Household wealth } \\
\hline Lowest & 32.0 & 264 & 36.9 & 341 & 46.9 & 111 & 35.0 & 606 \\
\hline Second & 40.1 & 192 & 30.1 & 229 & 36.7 & 183 & 34.5 & 421 \\
\hline Middle & 40.4 & 157 & 24.9 & 220 & 32.3 & 223 & 31.1 & 377 \\
\hline Fourth & 63.7 & 113 & 28.2 & 135 & 55.0 & 248 & 44.9 & 249 \\
\hline Highest & 46.1 & 166 & 32.7 & 153 & 56.5 & 304 & 39.6 & 318 \\
\hline \multicolumn{9}{|l|}{ Maternal age (in years) } \\
\hline $15-24$ years & 46.8 & 367 & 27.9 & 485 & 38.9 & 423 & 35.4 & 852 \\
\hline $25-34$ years & 37.2 & 412 & 35.5 & 478 & 51.9 & 486 & 36.2 & 890 \\
\hline $35-49$ years & 42.3 & 113 & 31.3 & 115 & 48.8 & 160 & 36.4 & 229 \\
\hline \multicolumn{9}{|c|}{$\begin{array}{l}\text { Maternal education (highest } \\
\text { level attended) }\end{array}$} \\
\hline None & 40.2 & 675 & 28.4 & 855 & 42.5 & 698 & 33.2 & 1530 \\
\hline Primary & 55.2 & 47 & 28.0 & 57 & 56.0 & 60 & 39.8 & 104 \\
\hline Secondary or higher & 47.8 & 95 & 46.4 & 60 & 58.4 & 180 & 47.3 & 155 \\
\hline Islamic & 40.2 & 75 & 52.1 & 106 & 50.5 & 131 & 46.9 & 182 \\
\hline
\end{tabular}

Estimates based on low Ns (approx. $<30 \mathrm{obs}$ ) have large relative standard errors and should be interpreted with caution. ${ }^{* \star *}$ indicates $p<0.001,{ }^{* \star}$ indicates $p<0.05$ and * indicates $p<.0 .01$ for the chi $^{2}$ statistic comparing integrated versus malaria-only study areas.

\section{TABLE 6.1.B. EXCLUSIVE BREASTFEEDING IN FIRST SIX MONTHS OF LIFE BY SOCIODEMOGRAPHIC CHARACTERISTICS}

Percentage of last-born children aged 0-5 months who were exclusively breastfed (defined in BSS as current breastfeeding without solid or semi-solid foods and not given non-breastmilk liquids within first 3 days of life) by sociodemographic characteristics, September-October 2019

\begin{tabular}{|c|c|c|c|c|c|c|c|c|}
\hline & \multicolumn{2}{|c|}{ KEBBI } & \multicolumn{2}{|c|}{ SOKOTO } & \multicolumn{2}{|c|}{$\begin{array}{l}\text { MALARIA-ONLY } \\
\text { (ZAMFARA) }\end{array}$} & \multicolumn{2}{|c|}{$\begin{array}{c}\text { INTEGRATED } \\
\text { (KEBBI/SOKOTO) }\end{array}$} \\
\hline & $\%$ & $\mathbf{N}$ & $\%$ & $\mathbf{N}$ & $\%$ & $\mathrm{~N}$ & $\%$ & $\mathbf{N}$ \\
\hline Total & 20.3 & 212 & 29.3 & 276 & 45.9 & 233 & 25.6 & 488 \\
\hline \multicolumn{9}{|l|}{ Household wealth } \\
\hline Lowest & 14.6 & 58 & 27.4 & 96 & 9.2 & 21 & 23.2 & 154 \\
\hline Second & 20.9 & 44 & 35.3 & 61 & 43.6 & 38 & 29.1 & 105 \\
\hline Middle & 27.9 & 34 & 30.9 & 45 & 41.1 & 51 & 29.7 & 79 \\
\hline Fourth & 17.6 & 28 & 22.6 & 35 & 53.0 & 45 & 20.2 & 63 \\
\hline Highest & 23.3 & 48 & 28.0 & 39 & 60.9 & 78 & 25.6 & 87 \\
\hline \multicolumn{9}{|l|}{ Maternal age (in years) } \\
\hline $15-24$ years & 19.6 & 89 & 27.6 & 115 & 37.6 & 89 & 24.4 & 204 \\
\hline $25-34$ years & 17.5 & 100 & 30.9 & 132 & 52.3 & 109 & 25.3 & 232 \\
\hline $35-49$ years & 33.4 & 23 & 28.8 & 29 & 50.9 & 35 & 30.8 & 52 \\
\hline \multicolumn{9}{|c|}{$\begin{array}{l}\text { Maternal education (highest } \\
\text { level attended) }\end{array}$} \\
\hline None & 17.1 & 156 & 26.4 & 236 & 44.4 & 159 & 22.9 & 392 \\
\hline Primary & (..) & 11 & $(.)$. & 14 & (..) & 9 & 25.1 & 25 \\
\hline Secondary or higher & 43.4 & 24 & (..) & 14 & 62.8 & 42 & 44.4 & 38 \\
\hline Islamic & 21.2 & 21 & (..) & 12 & 24.2 & 23 & 36.4 & 33 \\
\hline
\end{tabular}

Estimates based on low Ns (approx. $<30$ obs) have large relative standard errors and should be interpreted with caution. ${ }^{* \star *}$ indicates $p<0.001,{ }^{* *}$ indicates $p<0.05$ and * indicates $p<.0 .01$ for the chi ${ }^{2}$ statistic comparing integrated versus malaria-only study areas. 
TABLE 6.1.1. BREASTFEEDING PRACTICES IMMEDIATELY AFTER BIRTH

Percentage of last-born children in past two years who ever breastfed, initiated breastfeeding within one hour of birth or received non-breastmilk liquids in first three days, September-October 2019

\begin{tabular}{|c|c|c|c|c|c|c|}
\hline & KEBBI & SOKOTO & $\begin{array}{l}\text { MALARIA-ONLY } \\
\text { (ZAMFARA) }\end{array}$ & $\begin{array}{l}\text { INTEGRATED } \\
\text { (KEBBI/SOKOTO) }\end{array}$ & $\mathbf{N}$ & $\begin{array}{c}\mathrm{CHI}^{2} \\
\text { VALUE }\end{array}$ \\
\hline & $\%$ & $\%$ & $\%$ & $\%$ & & \\
\hline \multicolumn{7}{|c|}{$\begin{array}{l}\text { Did you ever breastfeed your } \\
\text { last-born child? }\end{array}$} \\
\hline No & 1.9 & 2.4 & 4.9 & 2.2 & 126 & 14.336 \\
\hline Yes & 98.1 & 97.6 & 95.1 & 97.8 & 2,913 & 2.471 \\
\hline Total & 100.0 & 100.0 & 100.0 & 100.0 & 3,039 & \\
\hline$N$ & 892 & 1,078 & 1,069 & 1,970 & & \\
\hline \multicolumn{7}{|c|}{$\begin{array}{l}\text { Did the child start breastfeeding } \\
\text { within } 1 \text { hour of birth? }\end{array}$} \\
\hline No & 57.6 & 67.4 & 53.6 & 63.2 & 1,812 & 34.620 \\
\hline Yes & 41.6 & 31.6 & 46.1 & 35.9 & 1,201 & 2.158 \\
\hline Don't know & 0.8 & 1.0 & 0.3 & 0.9 & 26 & \\
\hline Total & 100.0 & 100.0 & 100.0 & 100.0 & 3,039 & \\
\hline N & 892 & 1,078 & 1,069 & 1,970 & & \\
\hline \multicolumn{7}{|c|}{$\begin{array}{l}\text { In the first } 3 \text { days, was the child } \\
\text { given something to drink apart } \\
\text { from breastmilk? }\end{array}$} \\
\hline No & 21.6 & 29.1 & 49.9 & 25.9 & 979 & 168.895 \\
\hline Yes & 76.9 & 70.3 & 49.7 & 73.2 & 1,903 & 12.643 \\
\hline Don't know & 1.5 & 0.5 & 0.5 & 1.0 & 31 & 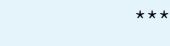 \\
\hline Total & 100.0 & 100.0 & 100.0 & 100.0 & 2,913 & \\
\hline $\mathrm{N}$ & 864 & 1,045 & 1,004 & 1,909 & & \\
\hline
\end{tabular}

Estimates based on low Ns (approx. $<30$ obs) have large relative standard errors and should be interpreted with caution. ${ }^{* \star *}$ indicates $p<0.001,{ }^{* \star}$ indicates $p<0.05$ and * indicates $p<.0 .01$ for the chi ${ }^{2}$ statistic comparing integrated versus malaria-only study areas. 
TABLE 6.1.2. REASONS FOR CHOOSING NOT TO EVER BREASTFEED YOUR CHILD

Percentage of women 15 to 49 years with a child under 2 years who did not ever breastfeed their last-born child by reasons for choosing not to breastfeed, September-October 2019

\begin{tabular}{|c|c|c|c|c|c|c|}
\hline & KEBBI & SOKOTO & $\begin{array}{l}\text { MALARIA-ONLY } \\
\text { (ZAMFARA) }\end{array}$ & $\begin{array}{c}\text { INTEGRATED } \\
\text { (KEBBI/SOKOTO) }\end{array}$ & $\mathbf{N}$ & $\begin{array}{c}\mathrm{CHI}^{2} \\
\text { VALUE }\end{array}$ \\
\hline & $\%$ & $\%$ & $\%$ & $\%$ & & \\
\hline \multicolumn{7}{|c|}{ Respondent opposed } \\
\hline No & 95.4 & 65.6 & 46.0 & 76.8 & 82 & 8.447 \\
\hline Yes & 4.6 & 34.4 & 54.1 & 23.3 & 44 & 3.435 \\
\hline Total & 100.0 & 100.0 & 100.0 & 100.0 & 126 & \\
\hline $\mathrm{N}$ & 28 & 33 & 65 & 61 & & \\
\hline \multicolumn{7}{|c|}{ Partner opposed } \\
\hline No & 63.2 & 74.9 & 70.4 & 70.5 & 89 & 0.000 \\
\hline Yes & 36.8 & 25.1 & 29.6 & 29.5 & 37 & 0.000 \\
\hline Total & 100.0 & 100.0 & 100.0 & 100.0 & 126 & \\
\hline N & 28 & 33 & 65 & 61 & & \\
\hline \multicolumn{7}{|c|}{ Inadequate milk } \\
\hline No & 92.4 & 90.7 & 96.5 & 91.3 & 116 & 1.337 \\
\hline Yes & 7.6 & 9.3 & 3.5 & 8.7 & 10 & 1.082 \\
\hline Total & 100.0 & 100.0 & 100.0 & 100.0 & 126 & \\
\hline N & 28 & 33 & 65 & 61 & & \\
\hline \multicolumn{7}{|c|}{ Not necessary } \\
\hline No & 77.2 & 86.0 & 92.4 & 82.7 & 114 & 2.363 \\
\hline Yes & 22.8 & 14.0 & 7.6 & 17.3 & 12 & 1.079 \\
\hline Total & 100.0 & 100.0 & 100.0 & 100.0 & 126 & \\
\hline N & 28 & 33 & 65 & 61 & & \\
\hline \multicolumn{7}{|c|}{ Up to God } \\
\hline No & 77.5 & 80.3 & 90.5 & 79.3 & 110 & 2.649 \\
\hline Yes & 22.5 & 19.7 & 9.5 & 20.7 & 16 & 0.684 \\
\hline Total & 100.0 & 100.0 & 100.0 & 100.0 & 126 & \\
\hline N & 28 & 33 & 65 & 61 & & \\
\hline \multicolumn{7}{|c|}{ Introduced other fluids } \\
\hline No & 89.9 & 97.8 & 95.3 & 94.9 & 119 & 0.009 \\
\hline Yes & 10.1 & 2.2 & 4.7 & 5.1 & 7 & 0.009 \\
\hline Total & 100.0 & 100.0 & 100.0 & 100.0 & 126 & \\
\hline N & 28 & 33 & 65 & 61 & & \\
\hline
\end{tabular}

Estimates based on low Ns (approx. $<30$ obs) have large relative standard errors and should be interpreted with caution. ${ }^{\star * *}$ indicates $p<0.001,{ }^{\star \star}$ indicates $p<0.05$ and * indicates $p<.0 .01$ for the chi ${ }^{2}$ statistic comparing integrated versus malaria-only study areas. 
TABLE 6.1.3. REASONS FOR GIVING NON-BREASTMILK LIQUIDS TO A NEWBORN IN FIRST THREE DAYS AFTER BIRTH

Percentage of women 15 to 49 years with a child under 2 years who gave non-breastmilk liquids in first three days to last-born child by reasons for not exclusively breastfeeding, September-October 2019

\begin{tabular}{|c|c|c|c|c|c|c|}
\hline & KEBBI & SOKOTO & $\begin{array}{l}\text { MALARIA-ONLY } \\
\text { (ZAMFARA) }\end{array}$ & $\begin{array}{l}\text { INTEGRATED } \\
\text { (KEBBI/SOKOTO) }\end{array}$ & $\mathbf{N}$ & $\begin{array}{l}\mathrm{CHI}^{2} \\
\text { VALUE }\end{array}$ \\
\hline & $\%$ & $\%$ & $\%$ & $\%$ & & \\
\hline \multicolumn{7}{|c|}{ Respondent opposed } \\
\hline No & 62.7 & 63.7 & 56.8 & 63.2 & 1,177 & 8.042 \\
\hline Yes & 37.3 & 36.3 & 43.2 & 36.8 & 726 & 0.484 \\
\hline Total & 100.0 & 100.0 & 100.0 & 100.0 & 1,903 & \\
\hline N & 657 & 738 & 508 & 1,395 & & \\
\hline \multicolumn{7}{|c|}{ Partner opposed } \\
\hline No & 70.3 & 74.0 & 60.8 & 72.3 & 1,303 & 28.517 \\
\hline Yes & 29.7 & 26.0 & 39.2 & 27.7 & 600 & 2.324 \\
\hline Total & 100.0 & 100.0 & 100.0 & 100.0 & 1,903 & \\
\hline N & 657 & 738 & 508 & 1,395 & & \\
\hline \multicolumn{7}{|c|}{$\begin{array}{l}\text { Religious/community leader } \\
\text { opposed }\end{array}$} \\
\hline No & 97.9 & 92.9 & 97.3 & 95.2 & 1,817 & 5.561 \\
\hline Yes & 2.1 & 7.1 & 2.8 & 4.8 & 86 & 0.830 \\
\hline Total & 100.0 & 100.0 & 100.0 & 100.0 & 1,903 & \\
\hline $\mathrm{N}$ & 657 & 738 & 508 & 1,395 & & \\
\hline \multicolumn{7}{|c|}{ Inadequate milk for newborn } \\
\hline No & 85.5 & 86.5 & 82.3 & 86.1 & 1,608 & 5.129 \\
\hline Yes & 14.5 & 13.5 & 17.7 & 13.9 & 295 & 0.354 \\
\hline Total & 100.0 & 100.0 & 100.0 & 100.0 & 1,903 & \\
\hline $\mathrm{N}$ & 657 & 738 & 508 & 1,395 & & \\
\hline \multicolumn{7}{|c|}{ Painful breastfeeding } \\
\hline No & 99.1 & 99.0 & 99.0 & 99.0 & 1,884 & 0.000 \\
\hline Yes & 0.9 & 1.1 & 1.0 & 1.0 & 19 & 0.000 \\
\hline Total & 100.0 & 100.0 & 100.0 & 100.0 & 1,903 & \\
\hline $\mathrm{N}$ & 657 & 738 & 508 & 1,395 & & \\
\hline \multicolumn{7}{|c|}{ Not necessary } \\
\hline No & 75.1 & 82.9 & 78.2 & 79.4 & 1,509 & 0.372 \\
\hline Yes & 24.9 & 17.1 & 21.8 & 20.6 & 394 & 0.038 \\
\hline Total & 100.0 & 100.0 & 100.0 & 100.0 & 1,903 & \\
\hline N & 657 & 738 & 508 & 1,395 & & \\
\hline \multicolumn{7}{|c|}{ Healthworker attitude } \\
\hline No & 100.0 & 99.9 & 99.6 & 100.0 & 1,899 & 2.919 \\
\hline Yes & 0.0 & 0.1 & 0.4 & 0.0 & 4 & 5.676 \\
\hline Total & 100.0 & 100.0 & 100.0 & 100.0 & 1,903 & . \\
\hline N & 657 & 738 & 508 & 1,395 & & \\
\hline \multicolumn{7}{|c|}{ Up to God } \\
\hline No & 87.6 & 88.6 & 87.2 & 88.1 & 1,668 & 0.381 \\
\hline Yes & 12.4 & 11.4 & 12.8 & 11.9 & 235 & 0.040 \\
\hline Total & 100.0 & 100.0 & 100.0 & 100.0 & 1,903 & \\
\hline N & 657 & 738 & 508 & 1,395 & & \\
\hline \multicolumn{7}{|c|}{ Introduced food $<6$ months } \\
\hline No & 98.0 & 96.3 & 91.7 & 97.1 & 1,825 & 26.028 \\
\hline Yes & 2.0 & 3.7 & 8.3 & 2.9 & 78 & 3.578 \\
\hline Total & 100.0 & 100.0 & 100.0 & 100.0 & 1,903 & \\
\hline N & 657 & 738 & 508 & 1,395 & & \\
\hline
\end{tabular}

Estimates based on low Ns (approx. $<30$ obs) have large relative standard errors and should be interpreted with caution. ${ }^{* * *}$ indicates $p<0.001,{ }^{* *}$ indicates $p<0.05$ and * indicates $p<.0 .01$ for the chi ${ }^{2}$ statistic comparing integrated versus malaria-only study areas. 
TABLE 6.1.4. BREASTFEEDING PRACTICES AMONG INFANTS AGED 0-5 MONTHS

Percentage of last-born children in past two years aged 0-5 months who were ever breastfed, are still breastfeeding, and are exclusively breastfed, ${ }^{* *}$ September-October 2019

\begin{tabular}{|c|c|c|c|c|c|c|}
\hline & $\begin{array}{c}\text { KEBBI } \\
\%\end{array}$ & $\begin{array}{l}\text { SOKOTO } \\
\%\end{array}$ & $\begin{array}{c}\text { MALARIA-ONLY } \\
\text { (ZAMFARA) } \\
\%\end{array}$ & $\begin{array}{c}\text { INTEGRATED } \\
\text { (KEBBI/SOKOTO) } \\
\% \\
\end{array}$ & $\mathbf{N}$ & $\begin{array}{c}\mathrm{CHI}^{2} \\
\text { VALUE }\end{array}$ \\
\hline \multicolumn{7}{|c|}{$\begin{array}{l}\text { Did you ever breastfeed your } \\
\text { last-born child? }\end{array}$} \\
\hline No & 1.3 & 0.8 & 4.3 & 1.0 & 21 & 53.748 \\
\hline Yes & 98.7 & 99.2 & 95.7 & 99.0 & 700 & 4.223 \\
\hline Total & 100.0 & 100.0 & 100.0 & 100.0 & 721 & * \\
\hline $\mathrm{N}$ & 212 & 276 & 233 & 488 & & \\
\hline \multicolumn{7}{|c|}{$\begin{array}{l}\text { In the first } 3 \text { days, was the child } \\
\text { given something to drink apart } \\
\text { from breastmilk? }\end{array}$} \\
\hline No & 25.7 & 37.4 & 52.2 & 32.6 & 264 & 232.426 \\
\hline Yes & 73.9 & 62.0 & 47.7 & 66.9 & 431 & 4.751 \\
\hline Don't know & 0.4 & 0.6 & 0.2 & 0.5 & 5 & * \\
\hline Total & 100.0 & 100.0 & 100.0 & 100.0 & 700 & \\
\hline$N$ & 206 & 273 & 221 & 479 & & \\
\hline \multicolumn{7}{|c|}{$\begin{array}{l}\text { Are you still breastfeeding the } \\
\text { child? }\end{array}$} \\
\hline No & 3.9 & 5.2 & 2.6 & 4.7 & 56 & 19.285 \\
\hline Yes & 96.1 & 94.8 & 97.4 & 95.3 & 644 & 1.332 \\
\hline Total & 100.0 & 100.0 & 100.0 & 100.0 & 700 & \\
\hline$N$ & 206 & 273 & 221 & 479 & & \\
\hline \multicolumn{7}{|c|}{$\begin{array}{l}\text { Are you giving the child soft or } \\
\text { semi-solid food? }\end{array}$} \\
\hline No & 85.4 & 81.7 & 82.3 & 83.2 & 581 & 0.788 \\
\hline Yes & 14.7 & 18.3 & 17.7 & 16.8 & 140 & 0.035 \\
\hline Total & 100.0 & 100.0 & 100.0 & 100.0 & 721 & \\
\hline$N$ & 212 & 276 & 233 & 488 & & \\
\hline \multicolumn{7}{|c|}{$\begin{array}{l}\text { Was the child exclusively breastfed } \\
(0-5 \text { months)? }\end{array}$} \\
\hline No & 79.3 & 70.2 & 54.1 & 74.0 & 512 & 271.828 \\
\hline Yes & 20.3 & 29.3 & 45.9 & 25.6 & 206 & 7.402 \\
\hline Don't know & 0.4 & 0.5 & 0.0 & 0.5 & 3 & ** \\
\hline Total & 100.0 & 100.0 & 100.0 & 100.0 & 721 & \\
\hline $\mathrm{N}$ & 212 & 276 & 233 & 488 & & \\
\hline
\end{tabular}

Estimates based on low Ns (approx. $<30$ obs) have large relative standard errors and should be interpreted with caution. ${ }^{* \star *}$ indicates $p<0.001,{ }^{* *}$ indicates $p<0.05$ and * indicates $p<.0 .01$ for the chi ${ }^{2}$ statistic comparing integrated versus malaria-only study areas. Note that exclusive breastfeeding in BSS is defined as infants 0-5 months who are currently breastfeeding and who received no solid or semi-solid foods in the past 24 hours and no non-breastmilk liquids in first three days after birth. 
TABLE 6.1.5. BREASTFEEDING AND NUTRITION AMONG CHILDREN AGED 6-23 MONTHS

Percentage of last-born children in past two years aged 6-23 months who are still breastfeeding, or given solid or semisolid foods by type of food, September-October 2019

\begin{tabular}{|c|c|c|c|c|c|c|}
\hline & KEBBI & sOKOTO & $\begin{array}{l}\text { MALARIA-ONLY } \\
\text { (ZAMFARA) }\end{array}$ & $\begin{array}{l}\text { INTEGRATED } \\
\text { (KEBBI/SOKOTO) }\end{array}$ & $\mathbf{N}$ & $\begin{array}{c}\mathrm{CHI}^{2} \\
\text { VALUE }\end{array}$ \\
\hline & $\%$ & $\%$ & $\%$ & $\%$ & & \\
\hline \multicolumn{7}{|c|}{ Are you still breastfeeding the child? } \\
\hline No & 12.6 & 10.2 & 12.6 & 11.3 & 357 & 2.347 \\
\hline Yes & 87.5 & 89.8 & 87.4 & 88.8 & 1,817 & 0.239 \\
\hline Total & 100.0 & 100.0 & 100.0 & 100.0 & 2,174 & \\
\hline$N$ & 649 & 757 & 768 & 1,406 & & \\
\hline \multicolumn{7}{|c|}{$\begin{array}{l}\text { Are you giving the child soft or } \\
\text { semi-solid food? }\end{array}$} \\
\hline No & 16.4 & 12.5 & 13.0 & 14.2 & 332 & 1.631 \\
\hline Yes & 83.7 & 87.5 & 87.0 & 85.8 & 1,945 & 0.220 \\
\hline Total & 100.0 & 100.0 & 100.0 & 100.0 & 2,277 & \\
\hline $\mathrm{N}$ & 671 & 787 & 819 & 1,458 & & \\
\hline \multicolumn{7}{|c|}{$\begin{array}{l}\text { How many times did the child eat } \\
\text { solid/semi-solid food yesterday? }\end{array}$} \\
\hline No times & 0.5 & 1.2 & 0.4 & 0.9 & 12 & 20.039 \\
\hline 1 time & 4.5 & 8.8 & 8.3 & 7.0 & 133 & 0.338 \\
\hline 2 times & 23.8 & 29.0 & 28.9 & 26.8 & 500 & \\
\hline $3+$ times & 66.6 & 53.2 & 57.8 & 58.9 & 1,164 & \\
\hline Don't know & 4.7 & 7.9 & 4.6 & 6.5 & 136 & \\
\hline Total & 100.0 & 100.0 & 100.0 & 100.0 & 1,945 & \\
\hline N & 551 & 681 & 713 & 1,232 & & \\
\hline \multicolumn{7}{|c|}{ Did child eat any of these yesterday? } \\
\hline \multicolumn{7}{|c|}{ Milk, cheese, yoghurt, other dairy } \\
\hline No & 51.8 & 46.8 & 42.9 & 48.9 & 893 & 21.744 \\
\hline Yes & 48.2 & 52.8 & 57.0 & 50.9 & 1,046 & 0.698 \\
\hline Don't know & 0.0 & 0.4 & 0.1 & 0.2 & 6 & \\
\hline Total & 100.0 & 100.0 & 100.0 & 100.0 & 1,945 & \\
\hline $\mathrm{N}$ & 551 & 681 & 713 & 1,232 & & \\
\hline \multicolumn{7}{|c|}{ Bread, rice, tuwo, other grains } \\
\hline No & 15.5 & 11.9 & 16.0 & 13.4 & 257 & 9.745 \\
\hline Yes & 84.5 & 88.1 & 83.8 & 86.5 & 1,684 & 0.647 \\
\hline Don't know & 0.0 & 0.1 & 0.2 & 0.1 & 4 & \\
\hline Total & 100.0 & 100.0 & 100.0 & 100.0 & 1,945 & \\
\hline N & 551 & 681 & 713 & 1,232 & & \\
\hline \multicolumn{7}{|c|}{ Potato, other roots or tubers } \\
\hline No & 78.9 & 83.2 & 72.3 & 81.4 & 1,485 & 62.234 \\
\hline Yes & 20.7 & 16.2 & 27.2 & 18.1 & 447 & 2.269 \\
\hline Don't know & 0.4 & 0.6 & 0.5 & 0.5 & 13 & \\
\hline Total & 100.0 & 100.0 & 100.0 & 100.0 & 1,945 & \\
\hline N & 551 & 681 & 713 & 1,232 & & \\
\hline \multicolumn{7}{|c|}{ Pumpkin squash or similar food } \\
\hline No & 89.0 & 91.9 & 86.5 & 90.7 & 1,716 & 22.580 \\
\hline Yes & 11.0 & 7.6 & 13.1 & 9.0 & 221 & 0.881 \\
\hline Don't know & 0.0 & 0.5 & 0.4 & 0.3 & 8 & \\
\hline Total & 100.0 & 100.0 & 100.0 & 100.0 & 1,945 & \\
\hline $\mathrm{N}$ & 551 & 681 & 713 & 1,232 & & \\
\hline \multicolumn{7}{|c|}{ Dark or leafy vegetables } \\
\hline No & 56.9 & 73.1 & 74.3 & 66.2 & 1,344 & 43.575 \\
\hline Yes & 43.1 & 26.0 & 25.3 & 33.2 & 591 & 1.933 \\
\hline Don't know & 0.0 & 1.0 & 0.4 & 0.6 & 10 & \\
\hline Total & 100.0 & 100.0 & 100.0 & 100.0 & 1,945 & \\
\hline $\mathrm{N}$ & 551 & 681 & 713 & 1,232 & & \\
\hline
\end{tabular}




\begin{tabular}{|c|c|c|c|c|c|c|}
\hline \multicolumn{7}{|c|}{ Mango, pawpaw or vitamin A fruits } \\
\hline No & 90.6 & 93.8 & 88.2 & 92.4 & 1,738 & 26.325 \\
\hline Yes & 9.3 & 5.5 & 11.1 & 7.1 & 192 & 1.132 \\
\hline Don't know & 0.1 & 0.7 & 0.7 & 0.5 & 15 & \\
\hline Total & 100.0 & 100.0 & 100.0 & 100.0 & 1,945 & \\
\hline $\mathrm{N}$ & 551 & 681 & 713 & 1,232 & & \\
\hline \multicolumn{7}{|c|}{ Any other fruits or vegetables } \\
\hline No & 72.0 & 81.8 & 73.3 & 77.6 & 1,452 & 13.764 \\
\hline Yes & 27.9 & 17.6 & 26.0 & 22.0 & 479 & 0.506 \\
\hline Don't know & 0.1 & 0.7 & 0.7 & 0.4 & 14 & \\
\hline Total & 100.0 & 100.0 & 100.0 & 100.0 & 1,945 & \\
\hline N & 551 & 681 & 713 & 1,232 & & \\
\hline \multicolumn{7}{|l|}{ Eggs } \\
\hline No & 79.5 & 79.8 & 71.4 & 79.7 & 1,466 & 60.324 \\
\hline Yes & 20.2 & 19.0 & 28.3 & 19.5 & 465 & 2.296 \\
\hline Don't know & 0.3 & 1.3 & 0.4 & 0.9 & 14 & \\
\hline Total & 100.0 & 100.0 & 100.0 & 100.0 & 1,945 & \\
\hline N & 551 & 681 & 713 & 1,232 & & \\
\hline \multicolumn{7}{|c|}{ Meat, poultry, fish } \\
\hline No & 61.5 & 70.7 & 56.8 & 66.8 & 1,181 & 64.787 \\
\hline Yes & 38.2 & 28.4 & 42.9 & 32.5 & 752 & 2.189 \\
\hline Don't know & 0.3 & 1.0 & 0.3 & 0.7 & 12 & \\
\hline Total & 100.0 & 100.0 & 100.0 & 100.0 & 1,945 & \\
\hline N & 551 & 681 & 713 & 1,232 & & \\
\hline \multicolumn{7}{|c|}{ Bean food, peas, lentils or nuts } \\
\hline No & 52.6 & 69.4 & 42.3 & 62.3 & 1,016 & 220.035 \\
\hline Yes & 47.2 & 29.9 & 57.4 & 37.2 & 918 & 7.469 \\
\hline Don't know & 0.2 & 0.7 & 0.4 & 0.5 & 11 & ** \\
\hline Total & 100.0 & 100.0 & 100.0 & 100.0 & 1,945 & \\
\hline N & 551 & 681 & 713 & 1,232 & & \\
\hline \multicolumn{7}{|c|}{$\begin{array}{l}\text { Any oil, fats, butter, or foods made } \\
\text { with these }\end{array}$} \\
\hline No & 48.9 & 65.6 & 49.5 & 58.5 & 1,029 & 51.290 \\
\hline Yes & 50.9 & 33.6 & 50.3 & 40.9 & 905 & 1.230 \\
\hline Don't know & 0.2 & 0.8 & 0.2 & 0.6 & 11 & . \\
\hline Total & 100.0 & 100.0 & 100.0 & 100.0 & 1,945 & \\
\hline N & 551 & 681 & 713 & 1,232 & & \\
\hline \multicolumn{7}{|c|}{ Dates, sweets, biscuits } \\
\hline No & 57.2 & 52.8 & 42.9 & 54.7 & 931 & 76.651 \\
\hline Yes & 42.6 & 46.2 & 56.5 & 44.6 & 995 & 1.960 \\
\hline Don't know & 0.2 & 1.0 & 0.6 & 0.7 & 19 & \\
\hline Total & 100.0 & 100.0 & 100.0 & 100.0 & 1,945 & \\
\hline $\mathrm{N}$ & 551 & 681 & 713 & 1,232 & & \\
\hline
\end{tabular}

Estimates based on low Ns (approx. $<30$ obs) have large relative standard errors and should be interpreted with caution. ${ }^{* \star *}$ indicates $p<0.001,{ }^{* *}$ indicates $p<0.05$ and ${ }^{*}$ indicates $p<.0 .01$ for the chi $^{2}$ statistic comparing integrated versus malaria-only study areas. 


\subsection{Breastfeeding ideations}

\section{TABLE 6.2.1. PERCEIVED BENEFITS OF EXCLUSIVE BREASTFEEDING FOR THE MOTHER}

Percentage of women aged 15 to 49 years currently pregnant or with a child under 2 years who report breastfeeding benefits for the mother by perceived benefit, September-October 2019

\begin{tabular}{|c|c|c|c|c|c|c|}
\hline & $\begin{array}{c}\text { KEBBI } \\
\%\end{array}$ & $\begin{array}{c}\text { SOKOTO } \\
\%\end{array}$ & $\begin{array}{c}\text { MALARIA-ONLY } \\
\text { (ZAMFARA) } \\
\%\end{array}$ & $\begin{array}{c}\text { INTEGRATED } \\
\text { (KEBBI/SOKOTO) } \\
\%\end{array}$ & $\mathbf{N}$ & $\begin{array}{c}\mathrm{CHI}^{2} \\
\text { VALUE }\end{array}$ \\
\hline \multicolumn{7}{|l|}{ None } \\
\hline No & 69.3 & 77.9 & 79.2 & 73.9 & 4,560 & 22.578 \\
\hline Yes & 30.7 & 22.1 & 20.8 & 26.1 & 1,474 & 0.760 \\
\hline Total & 100.0 & 100.0 & 100.0 & 100.0 & 6,034 & \\
\hline $\mathrm{N}$ & 1,960 & 2,062 & 2,012 & 4,022 & & \\
\hline \multicolumn{7}{|c|}{ Free/No cost } \\
\hline No & 88.8 & 88.2 & 83.9 & 88.5 & 5,224 & 24.655 \\
\hline Yes & 11.2 & 11.8 & 16.1 & 11.5 & 810 & 0.908 \\
\hline Total & 100.0 & 100.0 & 100.0 & 100.0 & 6,034 & \\
\hline N & 1,960 & 2,062 & 2,012 & 4,022 & & \\
\hline \multicolumn{7}{|c|}{ Convenient } \\
\hline No & 89.5 & 84.7 & 74.7 & 86.9 & 5,000 & 134.187 \\
\hline Yes & 10.5 & 15.3 & 25.3 & 13.1 & 1,034 & 4.386 \\
\hline Total & 100.0 & 100.0 & 100.0 & 100.0 & 6,034 & * \\
\hline $\mathrm{N}$ & 1,960 & 2,062 & 2,012 & 4,022 & & \\
\hline \multicolumn{7}{|c|}{ Promotes bonding with infant } \\
\hline No & 89.4 & 71.7 & 61.5 & 79.9 & 4,431 & 230.443 \\
\hline Yes & 10.6 & 28.3 & 38.5 & 20.1 & 1,603 & 8.242 \\
\hline Total & 100.0 & 100.0 & 100.0 & 100.0 & 6,034 & ** \\
\hline N & 1,960 & 2,062 & 2,012 & 4,022 & & \\
\hline \multicolumn{7}{|c|}{ Promotes weight loss } \\
\hline No & 95.2 & 94.6 & 82.3 & 94.9 & 5,479 & 209.408 \\
\hline Yes & 4.8 & 5.4 & 17.7 & 5.1 & 555 & 10.031 \\
\hline Total & 100.0 & 100.0 & 100.0 & 100.0 & 6,034 & ** \\
\hline $\mathrm{N}$ & 1,960 & 2,062 & 2,012 & 4,022 & & \\
\hline \multicolumn{7}{|c|}{ Reduces blood post-delivery } \\
\hline No & 96.6 & 94.4 & 83.4 & 95.5 & 5,535 & 204.831 \\
\hline Yes & 3.4 & 5.6 & 16.6 & 4.6 & 499 & 8.728 \\
\hline Total & 100.0 & 100.0 & 100.0 & 100.0 & 6,034 & ** \\
\hline $\mathrm{N}$ & 1,960 & 2,062 & 2,012 & 4,022 & & \\
\hline \multicolumn{7}{|c|}{ Relieves pain after birth } \\
\hline No & 96.1 & 93.6 & 87.3 & 94.8 & 5,573 & 91.711 \\
\hline Yes & 3.9 & 6.4 & 12.7 & 5.2 & 461 & 3.511 \\
\hline Total & 100.0 & 100.0 & 100.0 & 100.0 & 6,034 & \\
\hline N & 1,960 & 2,062 & 2,012 & 4,022 & & \\
\hline \multicolumn{7}{|c|}{ Promotes maternal health } \\
\hline No & 94.8 & 91.6 & 86.8 & 93.1 & 5,515 & 61.027 \\
\hline Yes & 5.2 & 8.4 & 13.2 & 6.9 & 519 & 2.282 \\
\hline Total & 100.0 & 100.0 & 100.0 & 100.0 & 6,034 & \\
\hline N & 1,960 & 2,062 & 2,012 & 4,022 & & \\
\hline \multicolumn{7}{|c|}{ Improves emotional well-being } \\
\hline No & 93.9 & 93.5 & 87.7 & 93.7 & 5,526 & 57.895 \\
\hline Yes & 6.1 & 6.5 & 12.3 & 6.3 & 508 & 2.807 \\
\hline Total & 100.0 & 100.0 & 100.0 & 100.0 & 6,034 & \\
\hline $\mathrm{N}$ & 1,960 & 2,062 & 2,012 & 4,022 & & \\
\hline
\end{tabular}

Estimates based on low Ns (approx. $<30$ obs) have large relative standard errors and should be interpreted with caution. ${ }^{* \star *}$ indicates $p<0.001,{ }^{* *}$ indicates $p<0.05$ and ${ }^{*}$ indicates $p<.0 .01$ for the chi ${ }^{2}$ statistic comparing integrated versus malaria-only study areas. 
TABLE 6.2.2. PERCEIVED BENEFITS OF EXCLUSIVE BREASTFEEDING FOR THE BABY

Percentage of women aged 15-49 years with a child under 2 years who report breastfeeding benefits for the baby by perceived benefit, September-October 2019

\begin{tabular}{|c|c|c|c|c|c|c|}
\hline & KEBBI & SOKOTO & $\begin{array}{l}\text { MALARIA-ONLY } \\
\text { (ZAMFARA) }\end{array}$ & $\begin{array}{c}\text { INTEGRATED } \\
\text { (KEBBI/SOKOTO) }\end{array}$ & $\mathbf{N}$ & $\begin{array}{c}\mathrm{CHI}^{2} \\
\text { VALUE }\end{array}$ \\
\hline & $\%$ & $\%$ & $\%$ & $\%$ & & \\
\hline \multicolumn{7}{|c|}{ Good nutrition } \\
\hline No & 92.5 & 90.0 & 92.2 & 91.2 & 2,860 & 1.031 \\
\hline Yes & 7.6 & 10.0 & 7.8 & 8.8 & 267 & 0.124 \\
\hline Total & 100.0 & 100.0 & 100.0 & 100.0 & 3,127 & \\
\hline N & 1,240 & 1,062 & 825 & 2,302 & & \\
\hline \multicolumn{7}{|c|}{ Prevents illness } \\
\hline No & 98.4 & 93.8 & 93.4 & 96.1 & 2,966 & 12.047 \\
\hline Yes & 1.6 & 6.2 & 6.6 & 3.9 & 161 & 0.960 \\
\hline Total & 100.0 & 100.0 & 100.0 & 100.0 & 3,127 & \\
\hline N & 1,240 & 1,062 & 825 & 2,302 & & \\
\hline \multicolumn{7}{|c|}{ Promotes long-term health } \\
\hline No & 99.3 & 98.2 & 94.8 & 98.8 & 3,036 & 39.490 \\
\hline Yes & 0.7 & 1.9 & 5.2 & 1.3 & 91 & 6.465 \\
\hline Total & 100.0 & 100.0 & 100.0 & 100.0 & 3,127 & * \\
\hline $\mathrm{N}$ & 1,240 & 1,062 & 825 & 2,302 & & \\
\hline \multicolumn{7}{|c|}{ Promotes bonding with mother } \\
\hline No & 99.3 & 98.2 & 98.7 & 98.8 & 3,089 & 0.073 \\
\hline Yes & 0.7 & 1.9 & 1.4 & 1.2 & 38 & 0.017 \\
\hline Total & 100.0 & 100.0 & 100.0 & 100.0 & 3,127 & \\
\hline N & 1,240 & 1,062 & 825 & 2,302 & & \\
\hline \multicolumn{7}{|c|}{ Leads to weight gain } \\
\hline No & 99.8 & 98.5 & 97.8 & 99.1 & 3,084 & 9.555 \\
\hline Yes & 0.3 & 1.5 & 2.3 & 0.9 & 43 & 1.735 \\
\hline Total & 100.0 & 100.0 & 100.0 & 100.0 & 3,127 & \\
\hline $\mathrm{N}$ & 1,240 & 1,062 & 825 & 2,302 & & \\
\hline \multicolumn{7}{|c|}{ Leads to higher IQ } \\
\hline No & 99.6 & 99.3 & 95.2 & 99.5 & 3,072 & 56.275 \\
\hline Yes & 0.4 & 0.7 & 4.8 & 0.5 & 55 & 11.855 \\
\hline Total & 100.0 & 100.0 & 100.0 & 100.0 & 3,127 & *** \\
\hline $\mathrm{N}$ & 1,240 & 1,062 & 825 & 2,302 & & \\
\hline
\end{tabular}

Estimates based on low Ns (approx. $<30$ obs) have large relative standard errors and should be interpreted with caution. ${ }^{* \star *}$ indicates $p<0.001,{ }^{\star \star}$ indicates $p<0.05$ and * indicates $p<.0 .01$ for the chi ${ }^{2}$ statistic comparing integrated versus malaria-only study areas. 
TABLE 6.2.3. WHO ELSE INFLUENCES DECISIONS ABOUT BREASTFEEDING?

Percentage of women aged 15 to 49 years who are currently pregnant or with a child under 2 years who report influencers on a woman's decisions about breastfeeding, September-October 2019

\begin{tabular}{|c|c|c|c|c|c|c|}
\hline & KEBBI & SOKOTO & $\begin{array}{l}\text { MALARIA-ONLY } \\
\text { (ZAMFARA) }\end{array}$ & $\begin{array}{c}\text { INTEGRATED } \\
\text { (KEBBI/SOKOTO) }\end{array}$ & $\mathbf{N}$ & $\begin{array}{c}\mathrm{CHI}^{2} \\
\text { VALUE }\end{array}$ \\
\hline & $\%$ & $\%$ & $\%$ & $\%$ & & \\
\hline \multicolumn{7}{|c|}{ No one else } \\
\hline No & 71.6 & 62.2 & 65.0 & 66.5 & 3,951 & 1.439 \\
\hline Yes & 28.4 & 37.8 & 35.0 & 33.5 & 2,083 & 0.040 \\
\hline Total & 100.0 & 100.0 & 100.0 & 100.0 & 6,034 & \\
\hline$N$ & 1,960 & 2,062 & 2,012 & 4,022 & & \\
\hline \multicolumn{7}{|c|}{ Partner } \\
\hline No & 32.8 & 46.4 & 39.5 & 40.1 & 2,455 & 0.233 \\
\hline Yes & 67.2 & 53.6 & 60.5 & 59.9 & 3,579 & 0.006 \\
\hline Total & 100.0 & 100.0 & 100.0 & 100.0 & 6,034 & \\
\hline N & 1,960 & 2,062 & 2,012 & 4,022 & & \\
\hline \multicolumn{7}{|c|}{ Mother in-law } \\
\hline No & 91.1 & 86.4 & 95.7 & 88.6 & 5,536 & 110.887 \\
\hline Yes & 8.9 & 13.6 & 4.3 & 11.5 & 498 & 10.482 \\
\hline Total & 100.0 & 100.0 & 100.0 & 100.0 & 6,034 & ** \\
\hline$N$ & 1,960 & 2,062 & 2,012 & 4,022 & & \\
\hline \multicolumn{7}{|l|}{ Mother } \\
\hline No & 92.7 & 91.4 & 93.9 & 92.0 & 5,575 & 7.719 \\
\hline Yes & 7.3 & 8.6 & 6.1 & 8.0 & 459 & 0.549 \\
\hline Total & 100.0 & 100.0 & 100.0 & 100.0 & 6,034 & \\
\hline $\mathrm{N}$ & 1,960 & 2,062 & 2,012 & 4,022 & & \\
\hline \multicolumn{7}{|c|}{ Own other family members } \\
\hline No & 99.5 & 98.7 & 99.3 & 99.1 & 5,984 & 0.812 \\
\hline Yes & 0.5 & 1.3 & 0.7 & 0.9 & 50 & 0.262 \\
\hline Total & 100.0 & 100.0 & 100.0 & 100.0 & 6,034 & \\
\hline N & 1,960 & 2,062 & 2,012 & 4,022 & & \\
\hline \multicolumn{7}{|c|}{ Partner's other family members } \\
\hline No & 99.8 & 99.3 & 99.3 & 99.5 & 6,005 & 0.969 \\
\hline Yes & 0.3 & 0.7 & 0.7 & 0.5 & 29 & 0.304 \\
\hline Total & 100.0 & 100.0 & 100.0 & 100.0 & 6,034 & \\
\hline $\mathrm{N}$ & 1,960 & 2,062 & 2,012 & 4,022 & & \\
\hline \multicolumn{7}{|c|}{ Friends } \\
\hline No & 99.4 & 99.1 & 97.1 & 99.2 & 5,935 & 32.939 \\
\hline Yes & 0.6 & 0.9 & 2.9 & 0.8 & 99 & 6.445 \\
\hline Total & 100.0 & 100.0 & 100.0 & 100.0 & 6,034 & * \\
\hline $\mathrm{N}$ & 1,960 & 2,062 & 2,012 & 4,022 & & \\
\hline \multicolumn{7}{|c|}{ Health provider } \\
\hline No & 97.8 & 96.6 & 93.6 & 97.2 & 5,792 & 40.263 \\
\hline Yes & 2.2 & 3.4 & 6.4 & 2.8 & 242 & 2.266 \\
\hline Total & 100.0 & 100.0 & 100.0 & 100.0 & 6,034 & \\
\hline $\mathrm{N}$ & 1,960 & 2,062 & 2,012 & 4,022 & & \\
\hline \multicolumn{7}{|c|}{ Religious/community leader } \\
\hline No & 99.2 & 99.8 & 99.7 & 99.5 & 6,010 & 1.441 \\
\hline Yes & 0.8 & 0.2 & 0.3 & 0.5 & 24 & 0.459 \\
\hline Total & 100.0 & 100.0 & 100.0 & 100.0 & 6,034 & \\
\hline $\mathrm{N}$ & 1,960 & 2,062 & 2,012 & 4,022 & & \\
\hline
\end{tabular}

Estimates based on low Ns (approx. $<30$ obs) have large relative standard errors and should be interpreted with caution. ${ }^{* * *}$ indicates $p<0.001,{ }^{* *}$ indicates $p<0.05$ and ${ }^{*}$ indicates $p<.0 .01$ for the chi ${ }^{2}$ statistic comparing integrated versus malaria-only study areas. 
TABLE 6.2.4. BREASTFEEDING KNOWLEDGE, BELIEFS, INTENTIONS, AND SELF-EFFICACY

Percentage of women aged 15 to 49 years who are currently pregnant or with a child under 2 years with breastfeeding knowledge, beliefs, intentions, and self-efficacy, September-October 2019

\begin{tabular}{|c|c|c|c|c|c|c|}
\hline & $\begin{array}{c}\text { KEBBI } \\
\%\end{array}$ & $\begin{array}{c}\text { SOKOTO } \\
\%\end{array}$ & $\begin{array}{c}\text { MALARIA-ONLY } \\
\text { (ZAMFARA) } \\
\%\end{array}$ & $\begin{array}{c}\text { INTEGRATED } \\
\text { (KEBBI/SOKOTO) } \\
\%\end{array}$ & $\mathbf{N}$ & $\begin{array}{c}\text { CHI }^{2} \\
\text { VALUE }\end{array}$ \\
\hline \multicolumn{7}{|c|}{ In your opinion, how long should a woman breastfeed her child? } \\
\hline 6 months or less & 2.7 & 1.1 & 2.3 & 1.9 & 133 & 129.116 \\
\hline $6-11$ months & 3.1 & 7.2 & 8.1 & 5.3 & 327 & 1.027 \\
\hline 12-23 months & 64.2 & 68.3 & 62.5 & 66.4 & 3,957 & \\
\hline 24 months or more & & & & 246 & 1353 & \\
\hline Other & $\begin{array}{r}21.9 \\
1.2\end{array}$ & $\begin{array}{r}21.1 \\
1.0\end{array}$ & $\begin{array}{r}19.8 \\
6.1\end{array}$ & $\begin{array}{r}24.6 \\
1.1\end{array}$ & $\begin{array}{r}1,353 \\
178\end{array}$ & \\
\hline Don't know & 0.9 & 0.7 & 1.2 & 0.8 & 85 & \\
\hline Total & 100.0 & 100.0 & 100.0 & 100.0 & 6,033 & \\
\hline N & 1,960 & 2,062 & 2,011 & 4,022 & & \\
\hline \multicolumn{7}{|c|}{ Breastmilk contains essential nutrients for first 6 months of life } \\
\hline Agree & 77.1 & 85.0 & 88.7 & 81.3 & 5,005 & 95.845 \\
\hline Disagree & 15.8 & 9.9 & 9.5 & 12.6 & 691 & 2.510 \\
\hline Don't know & 7.1 & 5.2 & 1.8 & 6.1 & 337 & \\
\hline Total & 100.0 & 100.0 & 100.0 & 100.0 & 6,033 & \\
\hline N & 1,960 & 2,062 & 2,011 & 4,022 & & \\
\hline \multicolumn{7}{|c|}{ Breastmilk immediately after birth is bad } \\
\hline Agree & 29.9 & 28.2 & 18.8 & 29.0 & 1,489 & 190.173 \\
\hline Disagree & 61.3 & 65.5 & 78.7 & 63.6 & 4,145 & 5.282 \\
\hline Don't know & 8.8 & 6.3 & 2.5 & 7.5 & 399 & ** \\
\hline Total & 100.0 & 100.0 & 100.0 & 100.0 & 6,033 & \\
\hline N & 1,960 & 2,062 & 2,011 & 4,022 & & \\
\hline \multicolumn{7}{|c|}{ Likelihood you would exclusively breastfeed next child for first 6 months } \\
\hline Likely & 45.4 & 45.8 & 59.3 & 45.6 & 2,987 & 147.534 \\
\hline Unlikely & 47.8 & 49.9 & 39.1 & 49.0 & 2,759 & 2.871 \\
\hline Don't know & 6.8 & 4.3 & 1.7 & 5.4 & 287 & \\
\hline Total & 100.0 & 100.0 & 100.0 & 100.0 & 6,033 & \\
\hline $\mathrm{N}$ & 1,960 & 2,062 & 2,011 & 4,022 & & \\
\hline \multicolumn{7}{|c|}{ Most women in my community only give infants breastmilk } \\
\hline Agree & 31.5 & 28.8 & 48.8 & 30.0 & 2,270 & 211.334 \\
\hline Disagree & 58.2 & 60.0 & 43.6 & 59.2 & 3,127 & 3.611 \\
\hline Don't know & 10.3 & 11.3 & 7.6 & 10.8 & 636 & * \\
\hline Total & 100.0 & 100.0 & 100.0 & 100.0 & 6,033 & \\
\hline N & 1,960 & 2,062 & 2,011 & 4,022 & & \\
\hline \multicolumn{7}{|c|}{ Important for mothers to only give child breastmilk for first 6 months } \\
\hline Agree & 48.6 & 53.0 & 70.6 & 51.0 & 3,462 & 246.712 \\
\hline Disagree & 41.8 & 39.9 & 25.6 & 40.8 & 2,150 & 4.297 \\
\hline Don't know & 9.6 & 7.1 & 3.8 & 8.3 & 421 & * \\
\hline Total & 100.0 & 100.0 & 100.0 & 100.0 & 6,033 & \\
\hline N & 1,960 & 2,062 & 2,011 & 4,022 & & \\
\hline \multicolumn{7}{|c|}{ Confidence to exclusively breastfeed your child for first 6 months } \\
\hline Confident & 40.4 & 43.5 & 64.2 & 42.1 & 2,921 & 334.042 \\
\hline Uncertain & 50.7 & 53.4 & 34.7 & 52.2 & 2,829 & 6.956 \\
\hline Don't know & 8.9 & 3.0 & 1.2 & 5.7 & 283 & ** \\
\hline Total & 100.0 & 100.0 & 100.0 & 100.0 & 6,033 & \\
\hline N & 1,960 & 2,062 & 2,011 & 4,022 & & \\
\hline \multicolumn{7}{|c|}{ Confidence to start conversation with husband about breastfeeding child } \\
\hline Confident & 59.3 & 61.5 & 75.1 & 60.5 & 3,928 & 184.155 \\
\hline Uncertain & 34.8 & 35.4 & 24.0 & 35.1 & 1,903 & 4.678 \\
\hline Don't know & 5.9 & 3.1 & 0.9 & 4.4 & 202 & * \\
\hline Total & 100.0 & 100.0 & 100.0 & 100.0 & 6,033 & \\
\hline $\mathrm{N}$ & 1,960 & 2,062 & 2,011 & 4,022 & & \\
\hline
\end{tabular}

Estimates based on low Ns (approx. $<30$ obs) have large relative standard errors and should be interpreted with caution. ${ }^{* * *}$ indicates $p<0.001,{ }^{* *}$ indicates $p<0.05$ and ${ }^{*}$ indicates $p<.0 .01$ for the chi $^{2}$ statistic comparing integrated versus malaria-only study areas. 
TABLE 6.2.5. WHAT ARE SOME WAYS TO RECOGNIZE POOR NUTRITION IN A CHILD?

Percentage of women aged 15 to 49 years who are pregnant or with a child under 2 years who report ways of recognizing poor nutrition in a child by symptom, September-October 2019

\begin{tabular}{|c|c|c|c|c|c|c|}
\hline & KEBBI & SOKOTO & $\begin{array}{l}\text { MALARIA-ONLY } \\
\text { (ZAMFARA) }\end{array}$ & $\begin{array}{c}\text { INTEGRATED } \\
\text { (KEBBI/SOKOTO) }\end{array}$ & $\mathbf{N}$ & $\begin{array}{c}\mathrm{CHI}^{2} \\
\text { VALUE }\end{array}$ \\
\hline & $\%$ & $\%$ & $\%$ & $\%$ & & \\
\hline \multicolumn{7}{|c|}{ Lack of energy } \\
\hline No & 56.7 & 62.7 & 52.3 & 59.9 & 3,392 & 34.015 \\
\hline Yes & 43.3 & 37.3 & 47.7 & 40.1 & 2,642 & 1.448 \\
\hline Total & 100.0 & 100.0 & 100.0 & 100.0 & 6,034 & \\
\hline $\mathrm{N}$ & 1,960 & 2,062 & 2,012 & 4,022 & & \\
\hline \multicolumn{7}{|c|}{ Trouble paying attention } \\
\hline No & 74.2 & 81.7 & 74.4 & 78.2 & 4,677 & 11.927 \\
\hline Yes & 25.8 & 18.3 & 25.6 & 21.8 & 1,357 & 0.362 \\
\hline Total & 100.0 & 100.0 & 100.0 & 100.0 & 6,034 & \\
\hline N & 1,960 & 2,062 & 2,012 & 4,022 & & \\
\hline \multicolumn{7}{|c|}{ Trouble learning } \\
\hline No & 83.3 & 89.8 & 82.4 & 86.8 & 5,142 & 21.107 \\
\hline Yes & 16.7 & 10.2 & 17.6 & 13.2 & 892 & 0.790 \\
\hline Total & 100.0 & 100.0 & 100.0 & 100.0 & 6,034 & \\
\hline N & 1,960 & 2,062 & 2,012 & 4,022 & & \\
\hline \multicolumn{7}{|c|}{ Irritability } \\
\hline No & 77.2 & 91.1 & 85.7 & 84.7 & 5,106 & 1.205 \\
\hline Yes & 22.8 & 8.9 & 14.3 & 15.3 & 928 & 0.040 \\
\hline Total & 100.0 & 100.0 & 100.0 & 100.0 & 6,034 & \\
\hline $\mathrm{N}$ & 1,960 & 2,062 & 2,012 & 4,022 & & \\
\hline \multicolumn{7}{|c|}{ Becomes ill easily } \\
\hline No & 68.0 & 77.5 & 73.8 & 73.1 & 4,476 & 0.399 \\
\hline Yes & 32.0 & 22.5 & 26.2 & 26.9 & 1,558 & 0.011 \\
\hline Total & 100.0 & 100.0 & 100.0 & 100.0 & 6,034 & \\
\hline$N$ & 1,960 & 2,062 & 2,012 & 4,022 & & \\
\hline \multicolumn{7}{|c|}{ Weakness } \\
\hline No & 71.0 & 75.9 & 63.9 & 73.6 & 4,298 & 63.379 \\
\hline Yes & 29.0 & 24.1 & 36.1 & 26.4 & 1,736 & 2.418 \\
\hline Total & 100.0 & 100.0 & 100.0 & 100.0 & 6,034 & \\
\hline $\mathrm{N}$ & 1,960 & 2,062 & 2,012 & 4,022 & & \\
\hline \multicolumn{7}{|c|}{ Dry/scaly skin } \\
\hline No & 88.6 & 80.2 & 76.8 & 84.1 & 4,926 & 48.345 \\
\hline Yes & 11.4 & 19.8 & 23.2 & 15.9 & 1,108 & 2.021 \\
\hline Total & 100.0 & 100.0 & 100.0 & 100.0 & 6,034 & \\
\hline $\mathrm{N}$ & 1,960 & 2,062 & 2,012 & 4,022 & & \\
\hline \multicolumn{7}{|c|}{ Longer time to healing for wounds } \\
\hline No & 94.6 & 94.7 & 92.4 & 94.6 & 5,706 & 11.847 \\
\hline Yes & 5.4 & 5.3 & 7.6 & 5.4 & 328 & 0.824 \\
\hline Total & 100.0 & 100.0 & 100.0 & 100.0 & 6,034 & \\
\hline $\mathrm{N}$ & 1,960 & 2,062 & 2,012 & 4,022 & & \\
\hline \multicolumn{7}{|c|}{ Stunted growth } \\
\hline No & 78.5 & 66.4 & 64.1 & 72.0 & 4,272 & 41.217 \\
\hline Yes & 21.5 & 33.7 & 35.9 & 28.0 & 1,762 & 1.660 \\
\hline Total & 100.0 & 100.0 & 100.0 & 100.0 & 6,034 & \\
\hline N & 1,960 & 2,062 & 2,012 & 4,022 & & \\
\hline \multicolumn{7}{|c|}{ Thin arms and legs } \\
\hline No & 81.6 & 71.8 & 67.2 & 76.3 & 4,501 & 58.853 \\
\hline Yes & 18.4 & 28.2 & 32.8 & 23.7 & 1,533 & 1.829 \\
\hline Total & 100.0 & 100.0 & 100.0 & 100.0 & 6,034 & \\
\hline $\mathrm{N}$ & 1,960 & 2,062 & 2,012 & 4,022 & & \\
\hline \multicolumn{7}{|c|}{ Visible ribs } \\
\hline No & 87.0 & 72.7 & 67.4 & 79.3 & 4,616 & 102.197 \\
\hline Yes & 13.1 & 27.3 & 32.6 & 20.7 & 1,418 & 3.534 \\
\hline Total & 100.0 & 100.0 & 100.0 & 100.0 & 6,034 & \\
\hline $\mathrm{N}$ & 1,960 & 2,062 & 2,012 & 4,022 & & \\
\hline
\end{tabular}

Estimates based on low Ns (approx. $<30$ obs) have large relative standard errors and should be interpreted with caution. ${ }^{* * *}$ indicates $p<0.001,{ }^{* *}$ indicates $p<0.05$ and * indicates $p<.0 .01$ for the chi ${ }^{2}$ statistic comparing integrated versus malaria-only study areas. 


\section{Child health}

\subsection{Vaccination}

\section{Key findings}

From the baseline results, it is clear that promoting norms and awareness of the value of routine vaccinations should be a priority area for SBC efforts. There was low vaccination coverage in study areas, with generally higher vaccination rates in Zamfara than in Kebbi/Sokoto. Based on BSS baseline results, only 4 percent and 8 percent of children 12-23 months in Kebbi/Sokoto and Zamfara received all basic vaccinations (BCG, measles, DPT3, polio3) before the survey interview. Children 12 to 23 months in the poorest households (2\% and $2 \%$ ) and with least educated mothers (4\% and $5 \%$ ) had lower rates of full vaccination than those in the wealthiest households (9\% and $18 \%$ ) and with mothers who attended secondary/higher school (17\% and 19\%) in Kebbi/Sokoto and Zamfara, respectively. The most important reasons for not vaccinating a child across study areas were spousal opposition (33\%), distance to health facilities (14\%), and fear of needles (11\%). The person most commonly reported to influence a woman's decision to vaccinate her child was her spouse (73\%), making their opposition an important barrier to vaccine uptake.

All respondents were asked about their views of health services for child vaccinations. Most respondents (76\%) agreed that vaccination services in their communities were free of charge, and 75 percent agreed that people in their communities trust health providers with vaccination services. Yet in Kebbi/Sokoto, only about half (52\%) of respondents believed that health facilities frequently had vaccines available, as compared with 61 percent in Zamfara. Only 47 percent of respondents in Kebbi/Sokoto and 66 percent in Zamfara believed that most women in their communities take children to facilities for routine vaccinations. Approximately one-quarter (26\%) believed that vaccines contain dangerous chemicals that could harm a child.

Nevertheless, most respondents (72\%) agreed that childhood vaccinations were effective in preventing illnesses, and 70 percent agreed that the illnesses vaccines prevent are severe. Seventy-two percent also agreed that they knew when and where to get a child vaccinated. Yet results from more specific vaccine questions elicit a less rosy picture: Only 17 percent and 30 percent of respondents in Kebbi/Sokoto and Zamfara, respectively, reported that a child should be given his/ her first vaccination at birth. Two-thirds of respondents could not recall the number of times a child should be vaccinated before his or her first birthday. This suggests some disconnect between respondents' positive reports of their own vaccine knowledge and the more realistic picture derived from specific questions. Indeed, BSS baseline results suggest that vaccine-specific knowledge is relatively low, that there are common perceptions of frequent vaccine stock-outs at health facilities, and that vaccine myths persist in this area. 


\section{TABLE 7.1. FULLY VACCINATED CHILDREN 12-23 MONTHS BY SOCIODEMOGRAPHIC CHARACTERISTICS}

Percentage of last-born children aged 12-23 months who received all basic vaccinations (BCG, measles1, polio3, DPT3) at any time before the survey by sociodemographic characteristics, September-October 2019

\begin{tabular}{|c|c|c|c|c|c|c|c|c|}
\hline & \multicolumn{2}{|c|}{ KEBBI } & \multicolumn{2}{|c|}{ SOKOTO } & \multicolumn{2}{|c|}{$\begin{array}{l}\text { MALARIA-ONLY } \\
\text { (ZAMFARA) }\end{array}$} & \multicolumn{2}{|c|}{$\begin{array}{c}\text { INTEGRATED } \\
\text { (KEBBI/SOKOTO) }\end{array}$} \\
\hline & $\%$ & $\mathbf{N}$ & $\%$ & $\mathbf{N}$ & $\%$ & $\mathbf{N}$ & $\%$ & $\mathbf{N}$ \\
\hline Total & 3.6 & 482 & 4.5 & 548 & 7.7 & 578 & 4.1 & 1,030 \\
\hline \multicolumn{9}{|l|}{ Household wealth } \\
\hline Lowest & 1.5 & 149 & 1.9 & 172 & 2.0 & 65 & 1.7 & 321 \\
\hline Second & 2.7 & 104 & 1.2 & 106 & 1.7 & 105 & 1.9 & 210 \\
\hline Middle & 3.0 & 90 & 6.2 & 121 & 3.1 & 120 & 4.8 & 211 \\
\hline Fourth & 11.5 & 58 & 6.8 & 67 & 9.0 & 144 & 9.1 & 126 \\
\hline Highest & 4.2 & 81 & 13.3 & 82 & 18.1 & 144 & 8.7 & 162 \\
\hline \multicolumn{9}{|l|}{ Maternal age (in years) } \\
\hline $15-24$ years & 2.4 & 201 & 5.4 & 240 & 5.0 & 227 & 4.1 & 440 \\
\hline 25-34 years & 4.4 & 213 & 4.0 & 253 & 7.2 & 269 & 4.2 & 466 \\
\hline 35-49 years & 4.5 & 68 & 2.4 & 55 & 16.5 & 82 & 3.6 & 124 \\
\hline \multicolumn{9}{|c|}{$\begin{array}{l}\text { Maternal education } \\
\text { (highest level attended) }\end{array}$} \\
\hline None & 3.7 & 365 & 3.6 & 401 & 5.3 & 365 & 3.6 & 766 \\
\hline Primary & (..) & 21 & 4.5 & 32 & 33.7 & 32 & 2.6 & 53 \\
\hline Secondary or higher & 9.3 & 58 & 3.1 & 35 & 19.3 & 88 & 17.0 & 93 \\
\hline Islamic & $(.)$. & 38 & 1.1 & 80 & 0.0 & 93 & 0.7 & 118 \\
\hline
\end{tabular}

(..) Value not shown due to low number of observations. Estimates based on low Ns (approx. $<30$ obs) have large relative standard errors and should be interpreted with caution. ${ }^{* * *}$ indicates $p<0.001,{ }^{* *}$ indicates $p<0.05$ and ${ }^{*}$ indicates $p<.0 .01$ for the chi ${ }^{2}$ statistic comparing integrated versus malaria-only study areas. 
TABLE 7.1.1. VACCINATION PRACTICES FOR CHILDREN 12-23 MONTHS

Percentage of last-born children aged 12-23 months whoever received vaccinations, and among those, total times taken for vaccination and vaccine card receipt, September-October 2019

\begin{tabular}{|c|c|c|c|c|c|c|}
\hline & KEBBI & SOKOTO & $\begin{array}{l}\text { MALARIA-ONLY } \\
\text { (ZAMFARA) }\end{array}$ & $\begin{array}{l}\text { INTEGRATED } \\
\text { (KEBBI/SOKOTO) }\end{array}$ & $\mathbf{N}$ & $\begin{array}{c}\mathrm{CHI}^{2} \\
\text { VALUE }\end{array}$ \\
\hline & $\%$ & $\%$ & $\%$ & $\%$ & & \\
\hline \multicolumn{7}{|l|}{$\begin{array}{l}\text { Has the child ever received any } \\
\text { immunizations? }\end{array}$} \\
\hline No & 48.6 & 59.2 & 60.1 & 54.4 & 896 & 82.007 \\
\hline Yes & 48.9 & 38.3 & 39.7 & 43.1 & 680 & 2.126 \\
\hline Don't know & 2.5 & 2.6 & 0.2 & 2.6 & 32 & \\
\hline Total & 100.0 & 100.0 & 100.0 & 100.0 & 1,608 & \\
\hline $\mathrm{N}$ & 482 & 548 & 578 & 1,030 & & \\
\hline \multicolumn{7}{|l|}{$\begin{array}{l}\text { Total times child taken for routine } \\
\text { immunization since birth }\end{array}$} \\
\hline No times & 5.9 & 4.5 & 3.0 & 5.2 & 27 & 300.385 \\
\hline $1-3$ times & 40.2 & 47.2 & 46.1 & 43.6 & 300 & 3.269 \\
\hline 4-6 times & 35.4 & 30.8 & 39.3 & 33.2 & 256 & * \\
\hline $7+$ times & 9.4 & 5.8 & 11.0 & 7.7 & 55 & \\
\hline Don't know & 9.1 & 11.6 & 0.6 & 10.3 & 42 & \\
\hline Total & 100.0 & 100.0 & 100.0 & 100.0 & 680 & \\
\hline $\mathrm{N}$ & 240 & 221 & 219 & 461 & & \\
\hline \multicolumn{7}{|l|}{$\begin{array}{l}\text { Do you have a card where child } \\
\text { vaccinations are written down? }\end{array}$} \\
\hline Yes, only has a card & 56.0 & 39.3 & 58.8 & 47.9 & 348 & 70.600 \\
\hline Yes, has only another document & 2.8 & 0.0 & 2.1 & 1.5 & 8 & 1.119 \\
\hline Yes, has card and other document & 1.3 & 4.7 & 1.7 & 3.0 & 13 & \\
\hline $\begin{array}{l}\text { No, has no card and no other } \\
\text { document }\end{array}$ & 39.8 & 56.0 & 37.4 & 47.7 & 311 & \\
\hline Total & 100.0 & 100.0 & 100.0 & 100.0 & 680 & \\
\hline $\mathrm{N}$ & 240 & 221 & 219 & 461 & & \\
\hline
\end{tabular}

Estimates based on low Ns (approx. $<30$ obs) have large relative standard errors and should be interpreted with caution. ${ }^{* * *}$ indicates $p<0.001,{ }^{* *}$ indicates $p<0.05$ and ${ }^{*}$ indicates $p<.0 .01$ for the chi ${ }^{2}$ statistic comparing integrated versus malaria-only study areas. 
TABLE 7.1.2. VACCINATED CHILDREN 12-23 MONTHS BY TYPE OF VACCINE

Percentage of last-born children aged 12-23 months who received specific vaccines at any time before the survey, September-October 2019

\begin{tabular}{|c|c|c|c|c|c|c|}
\hline & KEBBI & SOKOTO & $\begin{array}{l}\text { MALARIA-ONLY } \\
\text { (ZAMFARA) }\end{array}$ & $\begin{array}{c}\text { INTEGRATED } \\
\text { (KEBBI/SOKOTO) }\end{array}$ & $\mathbf{N}$ & $\begin{array}{c}\mathrm{CHI}^{2} \\
\text { VALUE }\end{array}$ \\
\hline & $\%$ & $\%$ & $\%$ & $\%$ & & \\
\hline \multicolumn{7}{|l|}{ BCG coverage } \\
\hline No & 57.4 & 73.0 & 69.7 & 65.9 & 1,051 & 74.678 \\
\hline Yes & 32.8 & 21.9 & 27.8 & 26.8 & 448 & 1.796 \\
\hline Don't know & 9.9 & 5.1 & 2.6 & 7.3 & 109 & \\
\hline Total & 100.0 & 100.0 & 100.0 & 100.0 & 1,608 & \\
\hline $\mathrm{N}$ & 482 & 548 & 578 & 1,030 & & \\
\hline \multicolumn{7}{|c|}{ DPT1 coverage } \\
\hline No & 65.3 & 75.6 & 71.1 & 70.9 & 1,125 & 61.258 \\
\hline Yes & 19.4 & 18.1 & 23.4 & 18.7 & 343 & 1.115 \\
\hline Don't know & 15.3 & 6.3 & 5.5 & 10.4 & 140 & \\
\hline Total & 100.0 & 100.0 & 100.0 & 100.0 & 1,608 & \\
\hline $\mathrm{N}$ & 482 & 548 & 578 & 1,030 & & \\
\hline \multicolumn{7}{|c|}{ DPT2 coverage } \\
\hline No & 73.9 & 79.3 & 78.6 & 76.8 & 1,233 & 56.504 \\
\hline Yes & 10.8 & 14.4 & 15.9 & 12.8 & 235 & 1.216 \\
\hline Don't know & 15.3 & 6.3 & 5.5 & 10.4 & 140 & \\
\hline Total & 100.0 & 100.0 & 100.0 & 100.0 & 1,608 & \\
\hline $\mathrm{N}$ & 482 & 548 & 578 & 1,030 & & \\
\hline \multicolumn{7}{|c|}{ DPT3 coverage } \\
\hline No & 79.1 & 84.1 & 83.8 & 81.8 & 1,325 & 59.564 \\
\hline Yes & 5.7 & 9.6 & 10.7 & 7.8 & 143 & 1.300 \\
\hline Don't know & 15.3 & 6.3 & 5.5 & 10.4 & 140 & \\
\hline Total & 100.0 & 100.0 & 100.0 & 100.0 & 1,608 & \\
\hline $\mathrm{N}$ & 482 & 548 & 578 & 1,030 & & \\
\hline \multicolumn{7}{|c|}{ Polio1 coverage } \\
\hline No & 50.5 & 61.1 & 63.6 & 56.3 & 930 & 177.505 \\
\hline Yes & 37.7 & 31.1 & 34.3 & 34.1 & 560 & 4.168 \\
\hline Don't know & 11.9 & 7.8 & 2.0 & 9.6 & 116 & * \\
\hline Total & 100.0 & 100.0 & 100.0 & 100.0 & 1,606 & \\
\hline $\mathrm{N}$ & 482 & 546 & 578 & 1,028 & & \\
\hline \multicolumn{7}{|c|}{ Polio2 coverage } \\
\hline No & 52.2 & 64.4 & 65.3 & 58.9 & 974 & 174.445 \\
\hline Yes & 35.9 & 27.8 & 32.7 & 31.5 & 518 & 4.167 \\
\hline Don't know & 11.9 & 7.8 & 2.0 & 9.6 & 116 & * \\
\hline Total & 100.0 & 100.0 & 100.0 & 100.0 & 1,608 & \\
\hline N & 482 & 548 & 578 & 1,030 & & \\
\hline \multicolumn{7}{|c|}{ Polio3 coverage } \\
\hline No & 59.2 & 71.3 & 69.8 & 65.9 & 1,079 & 174.762 \\
\hline Yes & 28.9 & 20.9 & 28.1 & 24.5 & 413 & 4.123 \\
\hline Don't know & 11.9 & 7.8 & 2.0 & 9.6 & 116 & * \\
\hline Total & 100.0 & 100.0 & 100.0 & 100.0 & 1,608 & \\
\hline $\mathrm{N}$ & 482 & 548 & 578 & 1,030 & & \\
\hline \multicolumn{7}{|c|}{ Measles1 coverage } \\
\hline No & 67.6 & 78.6 & 76.4 & 73.7 & 1,189 & 80.083 \\
\hline Yes & 15.9 & 16.5 & 19.2 & 16.2 & 288 & 1.792 \\
\hline Don't know & 16.5 & 4.9 & 4.4 & 10.1 & 131 & \\
\hline Total & 100.0 & 100.0 & 100.0 & 100.0 & 1,608 & \\
\hline N & 482 & 548 & 578 & 1,030 & & \\
\hline \multicolumn{7}{|c|}{$\begin{array}{l}\text { All basic vaccinations (BCG, Mea- } \\
\text { sles1, DPT3, Polio3) }\end{array}$} \\
\hline No & 96.4 & 95.6 & 92.4 & 95.9 & 1,521 & 31.269 \\
\hline Yes & 3.7 & 4.5 & 7.7 & 4.1 & 87 & 2.128 \\
\hline Total & 100.0 & 100.0 & 100.0 & 100.0 & 1,608 & \\
\hline $\mathrm{N}$ & 482 & 548 & 578 & 1,030 & & \\
\hline
\end{tabular}

Estimates based on low Ns (approx. $<30$ obs) have large relative standard errors and should be interpreted with caution. ${ }^{* \star *}$ indicates $p<0.001,{ }^{* *}$ indicates $p<0.05$ and * indicates $p<.0 .01$ for the chi ${ }^{2}$ statistic comparing integrated versus malaria-only study areas. 
TABLE 7.1.3. REASONS FOR NOT VACCINATING A CHILD

Percentage of pregnant women and women with a child under 2 years who reported specific reasons why a mother in her community may not get her child vaccinated, September-October 2019

\begin{tabular}{|c|c|c|c|c|c|c|}
\hline & KEBBI & SOKOTO & $\begin{array}{l}\text { MALARIA-ONLY } \\
\text { (ZAMFARA) }\end{array}$ & $\begin{array}{c}\text { INTEGRATED } \\
\text { (KEBBI/SOKOTO) }\end{array}$ & $\mathbf{N}$ & $\begin{array}{c}\mathrm{CHI}^{2} \\
\text { VALUE }\end{array}$ \\
\hline & $\%$ & $\%$ & $\%$ & $\%$ & & \\
\hline Too exp & & & & & & \\
\hline No & 92.8 & 91.7 & 96.1 & 92.1 & 1,734 & 13.424 \\
\hline Yes & 7.2 & 8.3 & 3.9 & 7.9 & 113 & 1.571 \\
\hline Total & 100.0 & 100.0 & 100.0 & 100.0 & 1,847 & \\
\hline $\mathrm{N}$ & 496 & 688 & 663 & 1,184 & & \\
\hline Facility & & & & & & \\
\hline No & 89.0 & 96.9 & 88.8 & 93.8 & 1,697 & 13.029 \\
\hline Yes & 11.0 & 3.1 & 11.2 & 6.2 & 150 & 1.051 \\
\hline Total & 100.0 & 100.0 & 100.0 & 100.0 & 1,847 & \\
\hline$N$ & 496 & 688 & 663 & 1,184 & & \\
\hline Distanc & & & & & & \\
\hline No & 90.5 & 90.4 & 83.0 & 90.5 & 1,631 & 20.203 \\
\hline Yes & 9.5 & 9.6 & 17.0 & 9.6 & 216 & 1.864 \\
\hline Total & 100.0 & 100.0 & 100.0 & 100.0 & 1,847 & \\
\hline $\mathrm{N}$ & 496 & 688 & 663 & 1,184 & & \\
\hline No fem & & & & & & \\
\hline No & 98.5 & 96.9 & 95.2 & 97.5 & 1,773 & 6.394 \\
\hline Yes & 1.5 & 3.1 & 4.8 & 2.5 & 74 & 0.519 \\
\hline Total & 100.0 & 100.0 & 100.0 & 100.0 & 1,847 & \\
\hline $\mathrm{N}$ & 496 & 688 & 663 & 1,184 & & \\
\hline Disresp & & & & & & \\
\hline No & 98.6 & 97.9 & 99.3 & 98.2 & 1,817 & 5.058 \\
\hline Yes & 1.4 & 2.1 & 0.7 & 1.8 & 30 & 2.684 \\
\hline Total & 100.0 & 100.0 & 100.0 & 100.0 & 1,847 & \\
\hline $\mathrm{N}$ & 496 & 688 & 663 & 1,184 & & \\
\hline Poor qu & & & & & & \\
\hline No & 95.9 & 96.9 & 96.4 & 96.5 & 1,776 & 0.035 \\
\hline Yes & 4.1 & 3.1 & 3.7 & 3.5 & 71 & 0.006 \\
\hline Total & 100.0 & 100.0 & 100.0 & 100.0 & 1,847 & \\
\hline $\mathrm{N}$ & 496 & 688 & 663 & 1,184 & & \\
\hline Vaccine & & & & & & \\
\hline No & 96.1 & 96.8 & 99.6 & 96.5 & 1,798 & 25.885 \\
\hline Yes & 3.9 & 3.2 & 0.4 & 3.5 & 49 & 15.040 \\
\hline Total & 100.0 & 100.0 & 100.0 & 100.0 & 1,847 & 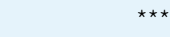 \\
\hline $\mathrm{N}$ & 496 & 688 & 663 & 1,184 & & \\
\hline Vaccine & & & & & & \\
\hline No & 80.5 & 83.7 & 95.8 & 82.4 & 1,602 & 92.640 \\
\hline Yes & 19.5 & 16.3 & 4.2 & 17.6 & 245 & 10.598 \\
\hline Total & 100.0 & 100.0 & 100.0 & 100.0 & 1,847 & ** \\
\hline $\mathrm{N}$ & 496 & 688 & 663 & 1,184 & & \\
\hline Fear ne & & & & & & \\
\hline No & 93.1 & 91.0 & 86.4 & 91.8 & 1,652 & 12.784 \\
\hline Yes & 6.9 & 9.0 & 13.6 & 8.2 & 195 & 1.349 \\
\hline Total & 100.0 & 100.0 & 100.0 & 100.0 & 1,847 & \\
\hline $\mathrm{N}$ & 496 & 688 & 663 & 1,184 & & \\
\hline Husban & & & & & & \\
\hline No & 70.8 & 65.4 & 66.8 & 67.5 & 1,210 & 0.105 \\
\hline Yes & 29.2 & 34.6 & 33.2 & 32.5 & 637 & 0.010 \\
\hline Total & 100.0 & 100.0 & 100.0 & 100.0 & 1,847 & \\
\hline $\mathrm{N}$ & 496 & 688 & 663 & 1,184 & & \\
\hline Religiou & & & & & & \\
\hline No & 98.8 & 98.9 & 99.4 & 98.8 & 1,823 & 1.767 \\
\hline Yes & 1.2 & 1.1 & 0.6 & 1.2 & 24 & 0.661 \\
\hline Total & 100.0 & 100.0 & 100.0 & 100.0 & 1,847 & \\
\hline $\mathrm{N}$ & 496 & 688 & 663 & 1,184 & & \\
\hline Don't k & & & & & & \\
\hline No & 95.1 & 96.5 & 97.9 & 95.9 & 1,788 & 6.110 \\
\hline Yes & 4.9 & 3.5 & 2.1 & 4.1 & 59 & 1.363 \\
\hline Total & 100.0 & 100.0 & 100.0 & 100.0 & 1,847 & \\
\hline $\mathrm{N}$ & 496 & 688 & 663 & 1,184 & & \\
\hline
\end{tabular}

Estimates based on low Ns (approx. $<30$ obs) have large relative standard errors and should be interpreted with caution. ${ }^{* * *}$ indicates $p<0.001,{ }^{* *}$ indicates $p<0.05$ and ${ }^{*}$ indicates $p<.0 .01$ for the chi ${ }^{2}$ statistic comparing integrated versus malaria-only study areas. 
TABLE 7.1.4. WHO ELSE INFLUENCES DECISIONS ABOUT GETTING A CHILD VACCINATED?

Percentage of pregnant women and women with a child under 2 years reporting who influences decisions to vaccinate a child, September-October 2019

\begin{tabular}{|c|c|c|c|c|c|c|}
\hline & KEBBI & SOKOTO & $\begin{array}{l}\text { MALARIA-ONLY } \\
\text { (ZAMFARA) }\end{array}$ & $\begin{array}{c}\text { INTEGRATED } \\
\text { (KEBBI/SOKOTO) }\end{array}$ & $\mathbf{N}$ & $\begin{array}{c}\mathrm{CHI}^{2} \\
\text { VALUE }\end{array}$ \\
\hline & $\%$ & $\%$ & $\%$ & $\%$ & & \\
\hline \multicolumn{7}{|c|}{ No one else } \\
\hline No & 76.0 & 79.4 & 78.1 & 77.9 & 2,364 & 0.025 \\
\hline Yes & 24.0 & 20.6 & 21.9 & 22.1 & 675 & 0.002 \\
\hline Total & 100.0 & 100.0 & 100.0 & 100.0 & 3,039 & \\
\hline N & 892 & 1,078 & 1,069 & 1,970 & & \\
\hline \multicolumn{7}{|c|}{ Husband/partner } \\
\hline No & 27.0 & 26.5 & 27.2 & 26.7 & 819 & 0.086 \\
\hline Yes & 73.0 & 73.5 & 72.8 & 73.3 & 2,220 & 0.005 \\
\hline Total & 100.0 & 100.0 & 100.0 & 100.0 & 3,039 & \\
\hline N & 892 & 1,078 & 1,069 & 1,970 & & \\
\hline \multicolumn{7}{|c|}{ Mother-in-law } \\
\hline No & 92.5 & 83.7 & 95.5 & 87.5 & 2,771 & 65.394 \\
\hline Yes & 7.5 & 16.3 & 4.5 & 12.5 & 268 & 11.386 \\
\hline Total & 100.0 & 100.0 & 100.0 & 100.0 & 3,039 & *** \\
\hline N & 892 & 1,078 & 1,069 & 1,970 & & \\
\hline \multicolumn{7}{|c|}{ Mother } \\
\hline No & 94.2 & 94.7 & 93.8 & 94.5 & 2,853 & 0.587 \\
\hline Yes & 5.8 & 5.3 & 6.2 & 5.5 & 186 & 0.090 \\
\hline Total & 100.0 & 100.0 & 100.0 & 100.0 & 3,039 & \\
\hline N & 892 & 1,078 & 1,069 & 1,970 & & \\
\hline \multicolumn{7}{|c|}{ Own other family members } \\
\hline No & 99.7 & 99.3 & 99.1 & 99.5 & 3,019 & 1.859 \\
\hline Yes & 0.3 & 0.7 & 1.0 & 0.5 & 20 & 1.164 \\
\hline Total & 100.0 & 100.0 & 100.0 & 100.0 & 3,039 & \\
\hline N & 892 & 1,078 & 1,069 & 1,970 & & \\
\hline \multicolumn{7}{|c|}{ Partner's other family members } \\
\hline No & 99.9 & 99.3 & 99.2 & 99.6 & 3,026 & 1.831 \\
\hline Yes & 0.1 & 0.7 & 0.9 & 0.4 & 13 & 0.897 \\
\hline Total & 100.0 & 100.0 & 100.0 & 100.0 & 3,039 & \\
\hline N & 892 & 1,078 & 1,069 & 1,970 & & \\
\hline \multicolumn{7}{|c|}{ Friends } \\
\hline No & 99.3 & 98.9 & 96.1 & 99.1 & 2,975 & 25.194 \\
\hline Yes & 0.7 & 1.1 & 3.9 & 0.9 & 64 & 7.136 \\
\hline Total & 100.0 & 100.0 & 100.0 & 100.0 & 3,039 & ** \\
\hline $\mathrm{N}$ & 892 & 1,078 & 1,069 & 1,970 & & \\
\hline \multicolumn{7}{|c|}{ Health provider } \\
\hline No & 97.3 & 95.8 & 93.9 & 96.4 & 2,910 & 9.636 \\
\hline Yes & 2.7 & 4.2 & 6.1 & 3.6 & 129 & 1.287 \\
\hline Total & 100.0 & 100.0 & 100.0 & 100.0 & 3,039 & \\
\hline N & 892 & 1,078 & 1,069 & 1,970 & & \\
\hline \multicolumn{7}{|c|}{ Community/religious leader } \\
\hline No & 99.3 & 98.9 & 98.5 & 99.0 & 3,008 & 1.546 \\
\hline Yes & 0.7 & 1.2 & 1.5 & 1.0 & 31 & 0.626 \\
\hline Total & 100.0 & 100.0 & 100.0 & 100.0 & 3,039 & \\
\hline $\mathrm{N}$ & 892 & 1,078 & 1,069 & 1,970 & & \\
\hline
\end{tabular}

Estimates based on low Ns (approx. $<30$ obs) have large relative standard errors and should be interpreted with caution. ${ }^{* * *}$ indicates $p<0.001,{ }^{* *}$ indicates $p<0.05$ and ${ }^{*}$ indicates $p<.0 .01$ for the chi $^{2}$ statistic comparing integrated versus malaria-only study areas. 
TABLE 7.1.5. VACCINE KNOWLEDGE, PERCEPTIONS, SELF-EFFICACY, AND INTENTIONS

Percentage of pregnant women and women with a child under 2 years reporting specific knowledge, perceptions, self-efficacy, and intentions for child vaccinations, September-October 2019

\begin{tabular}{|c|c|c|c|c|c|c|}
\hline & KEBBI & SOKото & $\begin{array}{l}\text { MALARIA-ONLY } \\
\text { (ZAMFARA) }\end{array}$ & $\begin{array}{c}\text { INTEGRATED } \\
\text { (KEBBI/SOKOTO) }\end{array}$ & $\mathbf{N}$ & $\begin{array}{c}\mathrm{CH}^{2} \\
\text { VALUE }\end{array}$ \\
\hline & $\%$ & $\%$ & $\%$ & $\%$ & & \\
\hline \multicolumn{7}{|c|}{ Before 12 months, how many times should a child be vaccinated? } \\
\hline No times & 5.4 & 3.6 & 6.0 & 4.4 & 158 & 76.635 \\
\hline $1-5$ times & 15.0 & 16.8 & 18.3 & 16.0 & 566 & 2.066 \\
\hline $6-10$ times & 2.0 & 7.7 & 14.0 & 5.2 & 281 & \\
\hline $11+$ times & 1.7 & 0.1 & 0.7 & 0.8 & 22 & \\
\hline Don't know & 75.9 & 71.9 & 61.0 & 73.6 & 2,012 & \\
\hline Total & 100.0 & 100.0 & 100.0 & 100.0 & 3,039 & \\
\hline $\mathrm{N}$ & 892 & 1,078 & 1,069 & 1,970 & & \\
\hline \multicolumn{7}{|c|}{ What age should child go for first routine vaccination? } \\
\hline At birth & 24.6 & 11.8 & 29.6 & 17.3 & 681 & 87.751 \\
\hline Before naming ceremony & 12.4 & 12.0 & 9.7 & 12.2 & 394 & 1.717 \\
\hline After naming ceremony but before coming out & 9.7 & 15.3 & 10.7 & 12.9 & 395 & \\
\hline At coming out $/ 6$ weeks & 6.2 & 2.5 & 1.9 & 4.1 & 102 & \\
\hline 3 months & 4.7 & 3.7 & 1.5 & 4.1 & 96 & \\
\hline After 1 year & 0.8 & 0.9 & 0.6 & 0.8 & 22 & \\
\hline Other & 0.9 & 1.2 & 1.4 & 1.1 & 43 & \\
\hline Don't know & 40.8 & 52.8 & 44.6 & 47.6 & 1,306 & \\
\hline Total & 100.0 & 100.0 & 100.0 & 100.0 & 3,039 & \\
\hline N & 892 & 1,078 & 1,069 & 1,970 & & \\
\hline \multicolumn{7}{|c|}{ In your opinion, how effective are childhood vaccines? } \\
\hline Effective & 73.6 & 67.5 & 72.6 & 70.2 & 2,204 & 15.116 \\
\hline Not effective & 4.8 & 7.4 & 8.8 & 6.3 & 204 & 0.640 \\
\hline Don't know & 21.6 & 25.1 & 18.7 & 23.6 & 631 & \\
\hline Total & 100.0 & 100.0 & 100.0 & 100.0 & 3,039 & \\
\hline $\mathrm{N}$ & 892 & 1,078 & 1,069 & 1,970 & & \\
\hline \multicolumn{7}{|l|}{ Many of the illnesses vaccines prevent are severe } \\
\hline Agree & 70.3 & 68.9 & 71.1 & 69.5 & 2,145 & 8.207 \\
\hline Disagree & 16.8 & 12.7 & 16.3 & 14.5 & 458 & 0.344 \\
\hline Don't know & 12.9 & 18.5 & 12.6 & 16.1 & 436 & \\
\hline Total & 100.0 & 100.0 & 100.0 & 100.0 & 3,039 & \\
\hline $\mathrm{N}$ & 892 & 1,078 & 1,069 & 1,970 & & \\
\hline \multicolumn{7}{|l|}{ I know where and when to get a child vaccinated } \\
\hline Agree & 64.2 & 63.6 & 75.6 & 63.9 & 2,138 & 50.324 \\
\hline Disagree & 25.4 & 21.7 & 14.6 & 23.3 & 579 & 1.660 \\
\hline Don't know & 10.4 & 14.7 & 9.8 & 12.9 & 322 & \\
\hline Total & 100.0 & 100.0 & 100.0 & 100.0 & 3,039 & \\
\hline $\mathrm{N}$ & 892 & 1,078 & 1,069 & 1,970 & & \\
\hline \multicolumn{7}{|c|}{ Easy for mothers in my community to take child for routine immunizations } \\
\hline Agree & 53.1 & 45.2 & 66.3 & 48.6 & 1,773 & 120.177 \\
\hline Disagree & 40.3 & 44.6 & 24.0 & 42.8 & 1,008 & 3.359 \\
\hline Don't know & 6.6 & 10.2 & 9.7 & 8.6 & 258 & \\
\hline Total & 100.0 & 100.0 & 100.0 & 100.0 & 3,039 & \\
\hline $\mathrm{N}$ & 892 & 1,078 & 1,069 & 1,970 & & \\
\hline \multicolumn{7}{|c|}{ Easy for mothers in my community to track child routine immunizations } \\
\hline Agree & 46.6 & 38.3 & 62.1 & 41.9 & 1,583 & 172.165 \\
\hline Disagree & 46.4 & 50.9 & 25.8 & 49.0 & 1,149 & 4.879 \\
\hline Don't know & 7.0 & 10.8 & 12.1 & 9.1 & 307 & ** \\
\hline Total & 100.0 & 100.0 & 100.0 & 100.0 & 3,039 & \\
\hline $\mathrm{N}$ & 892 & 1,078 & 1,069 & 1,970 & & \\
\hline \multicolumn{7}{|c|}{ Vaccines have chemicals that can be dangerous to a child's health } \\
\hline Agree & 34.4 & 26.0 & 23.1 & 29.6 & 835 & 16.814 \\
\hline Disagree & 42.3 & 44.0 & 48.6 & 43.2 & 1,437 & 0.592 \\
\hline Don't know & 23.3 & 30.1 & 28.3 & 27.2 & 767 & \\
\hline Total & 100.0 & 100.0 & 100.0 & 100.0 & 3,039 & \\
\hline $\mathrm{N}$ & 892 & 1,078 & 1,069 & 1,970 & & \\
\hline \multicolumn{7}{|c|}{ Likelihood to make sure next child receives all vaccines } \\
\hline Likely & 78.4 & 68.5 & 78.4 & 72.8 & 2,277 & 14.627 \\
\hline Unlikely & 14.1 & 23.4 & 16.3 & 19.4 & 560 & 0.572 \\
\hline Don't know & 7.6 & 8.1 & 5.3 & 7.9 & 202 & \\
\hline Total & 100.0 & 100.0 & 100.0 & 100.0 & 3,039 & \\
\hline$N$ & 892 & 1,078 & 1,069 & 1,970 & & \\
\hline
\end{tabular}

Estimates based on low Ns (approx. $<30$ obs) have large relative standard errors and should be interpreted with caution. ${ }^{\star \star *}$ indicates $p<0.001,{ }^{\star *}$ indicates $p<0.05$ and ${ }^{*}$ indicates $p<.0 .01$ for the chi ${ }^{2}$ statistic comparing integrated versus malaria-only study areas. 
TABLE 7.1.6. VACCINATIONS AND HEALTH SERVICES PERCEPTIONS

Percentage of pregnant women and women with a child under 2 years who reported specific perceptions about health services for child vaccinations, September-October 2019

\begin{tabular}{|c|c|c|c|c|c|c|}
\hline & $\begin{array}{c}\text { KEBBI } \\
\%\end{array}$ & $\begin{array}{l}\text { SOKOTO } \\
\%\end{array}$ & $\begin{array}{l}\text { MALARIA-ONLY } \\
\text { (ZAMFARA) } \\
\%\end{array}$ & $\begin{array}{c}\text { INTEGRATED } \\
\text { (KEBBI/SOKOTO) } \\
\%\end{array}$ & $\mathbf{N}$ & $\begin{array}{c}\mathrm{CHI}^{2} \\
\text { VALUE }\end{array}$ \\
\hline \multicolumn{7}{|c|}{$\begin{array}{l}\text { Immunization services in my } \\
\text { community are free }\end{array}$} \\
\hline Agree & 75.5 & 68.4 & 78.7 & 71.5 & 2,288 & 33.447 \\
\hline Disagree & 13.7 & 15.9 & 8.4 & 15.0 & 376 & 1.089 \\
\hline Don't know & 10.8 & 15.7 & 12.9 & 13.6 & 375 & \\
\hline Total & 100.0 & 100.0 & 100.0 & 100.0 & 3,039 & \\
\hline N & 892 & 1,078 & 1,069 & 1,970 & & \\
\hline \multicolumn{7}{|c|}{$\begin{array}{l}\text { Most people in my community trust } \\
\text { immunization services }\end{array}$} \\
\hline Agree & 75.5 & 60.8 & 80.1 & 67.1 & 2,203 & 69.938 \\
\hline Disagree & 15.4 & 25.1 & 11.2 & 20.9 & 536 & 2.274 \\
\hline Don't know & 9.1 & 14.2 & 8.7 & 12.0 & 300 & \\
\hline Total & 100.0 & 100.0 & 100.0 & 100.0 & 3,039 & \\
\hline $\mathrm{N}$ & 892 & 1,078 & 1,069 & 1,970 & & \\
\hline \multicolumn{7}{|c|}{$\begin{array}{l}\text { Health facilities in my community } \\
\text { frequently have vaccines available }\end{array}$} \\
\hline Agree & 57.8 & 47.8 & 60.6 & 52.1 & 1,736 & 21.536 \\
\hline Disagree & 30.3 & 33.0 & 26.8 & 31.9 & 880 & 0.684 \\
\hline Don't know & 11.8 & 19.2 & 12.7 & 16.0 & 423 & \\
\hline Total & 100.0 & 100.0 & 100.0 & 100.0 & 3,039 & \\
\hline $\mathrm{N}$ & 892 & 1,078 & 1,069 & 1,970 & & \\
\hline \multicolumn{7}{|c|}{$\begin{array}{l}\text { Most parents take children to a health } \\
\text { facility for routine immunizations }\end{array}$} \\
\hline Agree & 48.2 & 45.9 & 65.9 & 46.9 & 1,724 & 155.535 \\
\hline Disagree & 43.6 & 44.7 & 22.8 & 44.2 & 1,010 & 5.040 \\
\hline Don't know & 8.2 & 9.5 & 11.3 & 8.9 & 305 & ** \\
\hline Total & 100.0 & 100.0 & 100.0 & 100.0 & 3,039 & \\
\hline $\mathrm{N}$ & 892 & 1,078 & 1,069 & 1,970 & & \\
\hline
\end{tabular}

Estimates based on low Ns (approx. $<30$ obs) have large relative standard errors and should be interpreted with caution. ${ }^{* * *}$ indicates $p<0.001,{ }^{* *}$ indicates $p<0.05$ and * indicates $p<.0 .01$ for the chi ${ }^{2}$ statistic comparing integrated versus malaria-only study areas. 


\subsection{Acute respiratory infections}

\section{Key findings}

Care-seeking and treatment: Among last-born children in the past two years, 7 percent were reported to have had a cough, rapid breaths, and a chest-related problem in the past two weeks, or acute respiratory infections (ARI). Among these children, 81 percent and 77 percent were taken for any care in Kebbi/Sokoto and Zamfara, respectively. However, only 49 percent and 33 percent of children with these respiratory symptoms who were taken for any care were brought to a formal medical source in Kebbi/Sokoto and Zamfara, indicating that many children may not have received adequate health care. Few children with these symptoms were given antibiotics across study areas, although Zamfara (41\%) had higher antibiotic treatment rates than Kebbi/Sokoto (26\%).

\section{Reasons for source of care or not seeking any care:}

The most important reasons for choosing the source of care were nearby location (40\%), trust in provider (36\%), recommendation by family member or friend (30\%), and effective treatment (27\%) across study areas. These results point to the importance to respondents of convenient, trusted, and high-quality health services in their communities as a complement to SBC activities. The most important reasons for not seeking care included expense or cost (33\%) and perceptions that symptoms were not severe or that home care was provided (25\%).

Pneumonia ideations: Pediatric pneumonia knowledge is low across study areas. Only 28 percent and 37 percent of respondents currently pregnant or with a child under 2 years reported rapid or difficult breathing as a sign or symptom of pediatric pneumonia; 45 percent and 31 percent of respondents were unable to report any way to help prevent a child from getting pneumonia; and 62 percent and 56 percent were unable to report any effective medicine to treat pneumonia in Kebbi/Sokoto and Zamfara, respectively. Across study areas, most respondents (72\%) agreed that pneumonia is a serious illness that can result in hospitalization and even death. Most respondents (80\%) said that they were likely to have their child complete a full course of antibiotics, and most $(86 \%)$ also said that they intended to seek treatment the same or next day for pneumonia symptoms.

\section{TABLE 7.2. ARI CARE-SEEKING FROM FORMAL MEDICAL SOURCES, BY SOCIODEMOGRAPHIC CHARACTERISTICS}

Percentage of last-born children under 2 years with ARI in the past two weeks who were taken to a formal medical source by sociodemographic characteristics, September-October 2019

\begin{tabular}{|c|c|c|c|c|c|c|c|c|}
\hline & \multicolumn{2}{|c|}{ KEBBI } & \multicolumn{2}{|c|}{ SOKOTO } & \multicolumn{2}{|c|}{$\begin{array}{l}\text { MALARIA-ONLY } \\
\text { (ZAMFARA) }\end{array}$} & \multicolumn{2}{|c|}{$\begin{array}{c}\text { INTEGRATED } \\
\text { (KEBBI/SOKOTO) }\end{array}$} \\
\hline & $\%$ & $\mathbf{N}$ & $\%$ & $\mathbf{N}$ & $\%$ & $\mathbf{N}$ & $\%$ & $\mathbf{N}$ \\
\hline Total & 38.0 & 42 & 53.9 & 84 & 32.6 & 77 & 48.8 & 126 \\
\hline \multicolumn{9}{|l|}{ Household wealth } \\
\hline Lowest & (..) & 11 & 49.8 & 30 & (..) & 5 & 48.1 & 41 \\
\hline Second & (..) & 7 & (..) & 10 & (..) & 13 & (..) & 17 \\
\hline Middle & (..) & 8 & 61.3 & 24 & (..) & 14 & 56.1 & 32 \\
\hline Fourth & (..) & 10 & (..) & 10 & 34.7 & 25 & (..) & 20 \\
\hline Highest & (..) & 6 & (..) & 10 & (..) & 20 & (..) & 16 \\
\hline \multicolumn{9}{|l|}{ Maternal age (in years) } \\
\hline $15-24$ years & (..) & 9 & 49.4 & 30 & 29.4 & 33 & 42.1 & 39 \\
\hline 25-34 years & 42.4 & 31 & 55.8 & 45 & 27.1 & 33 & 50.5 & 76 \\
\hline $35-49$ years & (..) & 2 & (..) & 9 & (..) & 11 & (..) & 11 \\
\hline \multicolumn{9}{|c|}{$\begin{array}{l}\text { Maternal education } \\
\text { (highest level attended) }\end{array}$} \\
\hline None & 48.7 & 27 & 51.9 & 64 & 25.2 & 45 & 51.0 & 91 \\
\hline Primary & (..) & 3 & (..) & 5 & (..) & 10 & (..) & 8 \\
\hline Secondary or higher & (..) & 8 & (..) & 1 & (..) & 8 & (..) & 9 \\
\hline Islamic & (..) & 4 & (..) & 14 & (..) & 14 & (..) & 18 \\
\hline
\end{tabular}

(..) Value is not shown due to few observations. Estimates based on low Ns (approx. $<30$ obs) have large relative standard errors and should be interpreted with caution. ${ }^{* * *}$ indicates $p<0.001,{ }^{* *}$ indicates $p<0.05$ and ${ }^{*}$ indicates $p<.0 .01$ for the chi ${ }^{2}$ statistic comparing integrated versus malaria-only study areas. 
TABLE 7.2.1. ARI BY SYMPTOM

Percentage of last-born children under 2 years with ARI symptoms in the past two weeks, September-October 2019

\begin{tabular}{|c|c|c|c|c|c|c|}
\hline & $\begin{array}{c}\text { KEBBI } \\
\%\end{array}$ & $\begin{array}{c}\text { SOKOTO } \\
\%\end{array}$ & $\begin{array}{c}\text { MALARIA-ONLY } \\
\text { (ZAMFARA) } \\
\%\end{array}$ & $\begin{array}{c}\text { INTEGRATED } \\
\text { (KEBBI/SOKOTO) } \\
\%\end{array}$ & $\mathbf{N}$ & $\begin{array}{c}\mathrm{CHI}^{2} \\
\text { VALUE }\end{array}$ \\
\hline \multicolumn{7}{|c|}{$\begin{array}{l}\text { Has child had cough, rapid breaths, } \\
\text { chest issue in past } 2 \text { weeks? }\end{array}$} \\
\hline No & 94.1 & 90.8 & 92.9 & 92.2 & 2,802 & 6.033 \\
\hline Yes & 5.1 & 8.1 & 6.9 & 6.8 & 203 & 0.959 \\
\hline Don't know & 0.8 & 1.1 & 0.3 & 0.9 & 28 & \\
\hline Total & 100.0 & 100.0 & 100.0 & 100.0 & 3,033 & \\
\hline $\mathrm{N}$ & 887 & 1,077 & 1,069 & 1,964 & & \\
\hline \multicolumn{7}{|c|}{$\begin{array}{l}\text { Has child had an illness with a cough } \\
\text { at any time in the last } 2 \text { weeks? }\end{array}$} \\
\hline No & 84.0 & 75.7 & 77.1 & 79.3 & 2,373 & 8.434 \\
\hline Yes & 15.3 & 23.3 & 22.6 & 19.9 & 639 & 0.949 \\
\hline Don't know & 0.8 & 1.0 & 0.3 & 0.9 & 27 & \\
\hline Total & 100.0 & 100.0 & 100.0 & 100.0 & 3,039 & \\
\hline $\mathrm{N}$ & 892 & 1,078 & 1,069 & 1,970 & & \\
\hline \multicolumn{7}{|c|}{$\begin{array}{l}\text { Did child have short, rapid breaths or } \\
\text { difficulty breathing? }\end{array}$} \\
\hline No & 46.4 & 38.9 & 47.8 & 41.4 & 283 & 7.894 \\
\hline Yes & 49.2 & 61.1 & 52.2 & 57.2 & 351 & 1.289 \\
\hline Don't know & 4.4 & 0.0 & 0.0 & 1.5 & 5 & \\
\hline Total & 100.0 & 100.0 & 100.0 & 100.0 & 639 & \\
\hline $\mathrm{N}$ & 138 & 245 & 256 & 383 & & \\
\hline \multicolumn{7}{|c|}{$\begin{array}{l}\text { Was this a problem in the chest or a } \\
\text { blocked/runny nose? }\end{array}$} \\
\hline Chest only & 12.3 & 14.1 & 14.9 & 13.6 & 45 & 2.072 \\
\hline Nose only & 32.3 & 42.0 & 42.0 & 39.2 & 146 & 0.294 \\
\hline Both chest and nose & 55.4 & 42.8 & 43.1 & 46.4 & 158 & \\
\hline Other & 0.0 & 0.5 & 0.0 & 0.4 & 1 & \\
\hline Don't know & 0.0 & 0.5 & 0.0 & 0.4 & 1 & \\
\hline Total & 100.0 & 100.0 & 100.0 & 100.0 & 351 & \\
\hline $\mathrm{N}$ & 69 & 151 & 131 & 220 & & \\
\hline
\end{tabular}

Estimates based on low Ns (approx. $<30$ obs) have large relative standard errors and should be interpreted with caution. ${ }^{* * *}$ indicates $p<0.001,{ }^{* *}$ indicates $p<0.05$ and ${ }^{*}$ indicates $p<.0 .01$ for the chi ${ }^{2}$ statistic comparing integrated versus malaria-only study areas. 
TABLE 7.2.2. ARI CARE-SEEKING BY SOURCE

Percentage of last-born children under 2 years with ARI in the past two weeks who was taken to any care by source, September-October 2019

\begin{tabular}{|c|c|c|c|c|c|c|}
\hline & $\begin{array}{c}\text { KEBBI } \\
\%\end{array}$ & $\begin{array}{c}\text { SOKOTO } \\
\%\end{array}$ & $\begin{array}{c}\text { MALARIA-ONLY } \\
\text { (ZAMFARA) } \\
\%\end{array}$ & $\begin{array}{c}\text { INTEGRATED } \\
\text { (KEBBI/SOKOTO) } \\
\%\end{array}$ & $\mathbf{N}$ & $\begin{array}{c}\mathrm{CHI}^{2} \\
\text { VALUE }\end{array}$ \\
\hline \multicolumn{7}{|c|}{ Did you seek advice or treatment for the illness? } \\
\hline No & 12.2 & 22.8 & 22.9 & 19.4 & 41 & 10.684 \\
\hline Yes & 87.8 & 77.2 & 77.1 & 80.6 & 162 & 0.200 \\
\hline Total & 100.0 & 100.0 & 100.0 & 100.0 & 203 & \\
\hline N & 42 & 84 & 77 & 126 & & \\
\hline \multicolumn{7}{|c|}{ Source of care for respiratory symptoms } \\
\hline No care sought & 12.2 & 22.8 & 22.9 & 19.4 & 41 & 163.354 \\
\hline Informal private source & 49.8 & 23.3 & 44.5 & 31.9 & 76 & 1.545 \\
\hline Formal medical source & 38.0 & 53.9 & 32.6 & 48.8 & 86 & \\
\hline Total & 100.0 & 100.0 & 100.0 & 100.0 & 203 & \\
\hline N & 42 & 84 & 77 & 126 & & \\
\hline \multicolumn{7}{|c|}{ Where did you seek advice or treatment? } \\
\hline \multicolumn{7}{|l|}{ Government hospital } \\
\hline No & 82.8 & 93.4 & 81.9 & 89.7 & 139 & 68.305 \\
\hline Yes & 17.2 & 6.6 & 18.1 & 10.4 & 23 & 1.236 \\
\hline Total & 100.0 & 100.0 & 100.0 & 100.0 & 162 & \\
\hline N & 37 & 64 & 61 & 101 & & \\
\hline \multicolumn{7}{|l|}{ Governmental PHC } \\
\hline No & 89.6 & 63.6 & 79.6 & 72.7 & 122 & 39.094 \\
\hline Yes & 10.4 & 36.4 & 20.4 & 27.3 & 40 & 0.420 \\
\hline Total & 100.0 & 100.0 & 100.0 & 100.0 & 162 & \\
\hline N & 37 & 64 & 61 & 101 & & \\
\hline \multicolumn{7}{|l|}{ Dispensary/health post } \\
\hline No & 97.6 & 90.1 & 98.4 & 92.7 & 152 & 120.759 \\
\hline Yes & 2.5 & 9.9 & 1.7 & 7.3 & 10 & 2.240 \\
\hline Total & 100.0 & 100.0 & 100.0 & 100.0 & 162 & \\
\hline $\mathrm{N}$ & 37 & 64 & 61 & 101 & & \\
\hline \multicolumn{7}{|c|}{ Community health outreach post } \\
\hline No & 90.7 & 91.0 & 100.0 & 90.9 & 156 & 335.472 \\
\hline Yes & 9.3 & 9.0 & 0.0 & 9.1 & 6 & 3.137 \\
\hline Total & 100.0 & 100.0 & 100.0 & 100.0 & 162 & \\
\hline N & 37 & 64 & 61 & 101 & & \\
\hline \multicolumn{7}{|l|}{ Nursing/maternity home } \\
\hline No & 97.6 & 100.0 & 97.1 & 99.2 & 160 & 29.524 \\
\hline Yes & 2.4 & 0.0 & 2.9 & 0.8 & 2 & 0.831 \\
\hline Total & 100.0 & 100.0 & 100.0 & 100.0 & 162 & \\
\hline $\mathrm{N}$ & 37 & 64 & 61 & 101 & & \\
\hline \multicolumn{7}{|l|}{ Private hospital/clinic } \\
\hline No & 96.1 & 97.8 & 97.0 & 97.2 & 156 & 0.226 \\
\hline Yes & 3.9 & 2.2 & 3.0 & 2.8 & 6 & 0.004 \\
\hline Total & 100.0 & 100.0 & 100.0 & 100.0 & 162 & \\
\hline N & 37 & 64 & 61 & 101 & & \\
\hline \multicolumn{7}{|l|}{ Pharmacy/chemist } \\
\hline No & 44.6 & 73.9 & 50.0 & 63.6 & 94 & 109.370 \\
\hline Yes & 55.4 & 26.1 & 50.0 & 36.4 & 68 & 1.536 \\
\hline Total & 100.0 & 100.0 & 100.0 & 100.0 & 162 & \\
\hline $\mathrm{N}$ & 37 & 64 & 61 & 101 & & \\
\hline
\end{tabular}

Estimates based on low Ns (approx. $<30$ obs) have large relative standard errors and should be interpreted with caution. ${ }^{* * *}$ indicates $p<0.001,{ }^{* *}$ indicates $p<0.05$ and * indicates $p<.0 .01$ for the chi $^{2}$ statistic comparing integrated versus malaria-only study areas. Note that the respondent was not prompted about the type of facility attended. The interviewer is trained to code any secondary level government (or general) hospital mentioned as "government hospital." 
TABLE 7.2.3. REASONS FOR CHOOSING SOURCE OF CARE FOR ARI

Percentage of last-born children under 2 years with ARI in the past two weeks who were taken to care by reason for treatment location, September-October 2019

\begin{tabular}{|c|c|c|c|c|c|c|}
\hline & KEBBI & SOKOTO & $\begin{array}{l}\text { MALARIA-ONLY } \\
\text { (ZAMFARA) }\end{array}$ & $\begin{array}{c}\text { INTEGRATED } \\
\text { (KEBBI/SOKOTO) }\end{array}$ & $\mathbf{N}$ & $\begin{array}{c}\text { CHI }^{2} \\
\text { VALUE }\end{array}$ \\
\hline & $\%$ & $\%$ & $\%$ & $\%$ & & \\
\hline \multicolumn{7}{|c|}{ Free or inexpensive } \\
\hline No & 94.6 & 69.4 & 89.2 & 78.2 & 143 & 133.929 \\
\hline Yes & 5.4 & 30.6 & 10.8 & 21.8 & 19 & 1.300 \\
\hline Total & 100.0 & 100.0 & 100.0 & 100.0 & 162 & \\
\hline $\mathrm{N}$ & 37 & 64 & 61 & 101 & & \\
\hline \multicolumn{7}{|c|}{ Nearby/transport available } \\
\hline No & 55.5 & 47.6 & 66.7 & 50.4 & 102 & 161.706 \\
\hline Yes & 44.6 & 52.4 & 33.3 & 49.7 & 60 & 2.164 \\
\hline Total & 100.0 & 100.0 & 100.0 & 100.0 & 162 & \\
\hline $\mathrm{N}$ & 37 & 64 & 61 & 101 & & \\
\hline \multicolumn{7}{|c|}{ Medicines often in stock } \\
\hline No & 94.1 & 97.4 & 97.1 & 96.3 & 155 & 3.488 \\
\hline Yes & 5.9 & 2.6 & 2.9 & 3.8 & 7 & 0.074 \\
\hline Total & 100.0 & 100.0 & 100.0 & 100.0 & 162 & \\
\hline N & 37 & 64 & 61 & 101 & & \\
\hline \multicolumn{7}{|c|}{ Respectful care } \\
\hline No & 97.6 & 97.1 & 89.0 & 97.3 & 153 & 141.101 \\
\hline Yes & 2.4 & 2.9 & 11.0 & 2.7 & 9 & 3.483 \\
\hline Total & 100.0 & 100.0 & 100.0 & 100.0 & 162 & \\
\hline N & 37 & 64 & 61 & 101 & & \\
\hline \multicolumn{7}{|c|}{ Trust to care for child } \\
\hline No & 62.6 & 75.7 & 58.8 & 71.1 & 102 & 95.120 \\
\hline Yes & 37.4 & 24.3 & 41.3 & 28.9 & 60 & 1.216 \\
\hline Total & 100.0 & 100.0 & 100.0 & 100.0 & 162 & \\
\hline $\mathrm{N}$ & 37 & 64 & 61 & 101 & & \\
\hline \multicolumn{7}{|c|}{ Effective treatment } \\
\hline No & 76.8 & 83.1 & 68.0 & 80.9 & 119 & 121.602 \\
\hline Yes & 23.2 & 16.9 & 32.0 & 19.1 & 43 & 1.467 \\
\hline Total & 100.0 & 100.0 & 100.0 & 100.0 & 162 & \\
\hline $\mathrm{N}$ & 37 & 64 & 61 & 101 & & \\
\hline \multicolumn{7}{|c|}{ Short wait time } \\
\hline No & 68.6 & 90.5 & 87.7 & 82.8 & 135 & 28.788 \\
\hline Yes & 31.4 & 9.5 & 12.3 & 17.2 & 27 & 0.284 \\
\hline Total & 100.0 & 100.0 & 100.0 & 100.0 & 162 & \\
\hline $\mathrm{N}$ & 37 & 64 & 61 & 101 & & \\
\hline \multicolumn{7}{|l|}{ Privacy } \\
\hline No & 97.6 & 96.9 & 100.0 & 97.1 & 158 & 103.063 \\
\hline Yes & 2.4 & 3.1 & 0.0 & 2.9 & 4 & 3.501 \\
\hline Total & 100.0 & 100.0 & 100.0 & 100.0 & 162 & \\
\hline$N$ & 37 & 64 & 61 & 101 & & \\
\hline \multicolumn{7}{|c|}{ Family/friends recommend } \\
\hline No & 84.2 & 94.0 & 55.6 & 90.5 & 121 & 836.974 \\
\hline Yes & 15.8 & 6.1 & 44.4 & 9.5 & 41 & 10.966 \\
\hline Total & 100.0 & 100.0 & 100.0 & 100.0 & 162 & *** \\
\hline N & 37 & 64 & 61 & 101 & & \\
\hline \multicolumn{7}{|c|}{ Religious/community leaders } \\
\hline No & 98.7 & 100.0 & 100.0 & 99.5 & 161 & 16.387 \\
\hline Yes & 1.3 & 0.0 & 0.0 & 0.5 & 1 & 1.423 \\
\hline Total & 100.0 & 100.0 & 100.0 & 100.0 & 162 & \\
\hline $\mathrm{N}$ & 37 & 64 & 61 & 101 & & \\
\hline
\end{tabular}

Estimates based on low Ns (approx. $<30$ obs) have large relative standard errors and should be interpreted with caution. ${ }^{* \star *}$ indicates $p<0.001,{ }^{* \star}$ indicates $p<0.05$ and ${ }^{*}$ indicates $p<.0 .01$ for the chi ${ }^{2}$ statistic comparing integrated versus malaria-only study areas. 
TABLE 7.2.4. REASONS FOR NOT SEEKING CARE FOR ARI

Percentage of last-born children under 2 years with ARI in the past two weeks not taken to care by reason for no care-seeking, September-October 2019

\begin{tabular}{|c|c|c|c|c|c|c|c|}
\hline & KEBBI & sокото & $\begin{array}{l}\text { MALARIA-ONLY } \\
\text { (ZAMFARA) }\end{array}$ & $\begin{array}{l}\text { INTEGRATED } \\
\text { (KEBBI/SOKOTO) }\end{array}$ & TOTAL & $\mathbf{N}$ & $\begin{array}{c}\mathrm{CHI}^{2} \\
\text { VALUE }\end{array}$ \\
\hline & $\%$ & $\%$ & $\%$ & $\%$ & $\%$ & & \\
\hline \multicolumn{8}{|c|}{$\begin{array}{l}\text { Too expensive/could not } \\
\text { find money }\end{array}$} \\
\hline No & 56.9 & 50.5 & 76.1 & 51.8 & 67.5 & 27 & 361.503 \\
\hline Yes & 43.1 & 49.5 & 23.9 & 48.2 & 32.5 & 14 & 2.620 \\
\hline Total & 100.0 & 100.0 & 100.0 & 100.0 & 100.0 & 41 & \\
\hline $\mathrm{N}$ & 5 & 20 & 16 & 25 & 41 & & \\
\hline \multicolumn{8}{|c|}{ Facility closed/no one there } \\
\hline No & 100.0 & 97.4 & 87.7 & 97.9 & 91.4 & 39 & 178.178 \\
\hline Yes & 0.0 & 2.6 & 12.3 & 2.1 & 8.7 & 2 & 2.065 \\
\hline Total & 100.0 & 100.0 & 100.0 & 100.0 & 100.0 & 41 & \\
\hline N & 5 & 20 & 16 & 25 & 41 & & \\
\hline \multicolumn{8}{|c|}{$\begin{array}{l}\text { Distance/transportation not } \\
\text { available }\end{array}$} \\
\hline No & 86.1 & 100.0 & 100.0 & 97.2 & 99.0 & 40 & 107.571 \\
\hline Yes & 13.9 & 0.0 & 0.0 & 2.8 & 1.0 & 1 & 1.630 \\
\hline Total & 100.0 & 100.0 & 100.0 & 100.0 & 100.0 & 41 & \\
\hline $\mathrm{N}$ & 5 & 20 & 16 & 25 & 41 & & \\
\hline \multicolumn{8}{|c|}{ Medicine often not available } \\
\hline No & 80.3 & 100.0 & 100.0 & 96.0 & 98.6 & 40 & 153.740 \\
\hline Yes & 19.8 & 0.0 & 0.0 & 4.0 & 1.4 & 1 & 1.677 \\
\hline Total & 100.0 & 100.0 & 100.0 & 100.0 & 100.0 & 41 & \\
\hline $\mathrm{N}$ & 5 & 20 & 16 & 25 & 41 & & \\
\hline \multicolumn{8}{|c|}{ Treatment not effective } \\
\hline No & 100.0 & 93.8 & 94.5 & 95.1 & 94.7 & 39 & 0.847 \\
\hline Yes & 0.0 & 6.2 & 5.5 & 4.9 & 5.3 & 2 & 0.006 \\
\hline Total & 100.0 & 100.0 & 100.0 & 100.0 & 100.0 & 41 & \\
\hline $\mathrm{N}$ & 5 & 20 & 16 & 25 & 41 & & \\
\hline \multicolumn{8}{|c|}{$\begin{array}{l}\text { Symptoms not severe/pro- } \\
\text { vided home care }\end{array}$} \\
\hline No & 76.7 & 89.2 & 67.9 & 86.7 & 74.6 & 31 & 250.013 \\
\hline Yes & 23.3 & 10.8 & 32.1 & 13.3 & 25.4 & 10 & 1.139 \\
\hline Total & 100.0 & 100.0 & 100.0 & 100.0 & 100.0 & 41 & \\
\hline N & 5 & 20 & 16 & 25 & 41 & & \\
\hline \multicolumn{8}{|c|}{ Husband/partner oppose } \\
\hline No & 100.0 & 87.3 & 90.3 & 89.9 & 90.1 & 37 & 0.207 \\
\hline Yes & 0.0 & 12.7 & 9.7 & 10.1 & 9.9 & 4 & 0.001 \\
\hline Total & 100.0 & 100.0 & 100.0 & 100.0 & 100.0 & 41 & \\
\hline $\mathrm{N}$ & 5 & 20 & 16 & 25 & 41 & & \\
\hline \multicolumn{8}{|c|}{ Up to God } \\
\hline No & 94.0 & 69.2 & 91.5 & 74.2 & 85.4 & 31 & 322.832 \\
\hline Yes & 6.1 & 30.8 & 8.5 & 25.8 & 14.6 & 10 & 1.166 \\
\hline Total & 100.0 & 100.0 & 100.0 & 100.0 & 100.0 & 41 & \\
\hline$N$ & 5 & 20 & 16 & 25 & 41 & & \\
\hline
\end{tabular}

Estimates based on low Ns (approx. $<30$ obs) have large relative standard errors and should be interpreted with caution. ${ }^{* * *}$ indicates $p<0.001,{ }^{* *}$ indicates $p<0.05$ and * indicates $p<.0 .01$ for the chi $^{2}$ statistic comparing integrated versus malaria-only study areas. 
TABLE 7.2.5. ARI TREATMENT BY MEDICATION TYPE

Percentage of last-born children under 2 years with ARI in the past two weeks who were given antibiotics to treat the condition, September-October 2019

\begin{tabular}{|c|c|c|c|c|c|c|}
\hline & $\begin{array}{c}\text { KEBBI } \\
\%\end{array}$ & $\begin{array}{c}\text { SOKOTO } \\
\%\end{array}$ & $\begin{array}{c}\text { MALARIA-ONLY } \\
\text { (ZAMFARA) } \\
\%\end{array}$ & $\begin{array}{c}\text { INTEGRATED } \\
\text { (KEBBI/SOKOTO) } \\
\%\end{array}$ & $\mathbf{N}$ & $\begin{array}{c}\mathrm{CHI}^{2} \\
\text { VALUE }\end{array}$ \\
\hline \multicolumn{7}{|c|}{$\begin{array}{l}\text { At any time during the illness, } \\
\text { did the child take any medicines? }\end{array}$} \\
\hline No & 5.2 & 16.6 & 14.7 & 12.9 & 27 & 3.757 \\
\hline Yes & 94.8 & 83.4 & 85.3 & 87.1 & 176 & 0.062 \\
\hline Total & 100.0 & 100.0 & 100.0 & 100.0 & 203 & \\
\hline N & 42 & 84 & 77 & 126 & & \\
\hline \multicolumn{7}{|c|}{ What medicines did the child take? } \\
\hline \multicolumn{7}{|c|}{ Any antibiotic drug } \\
\hline No & 54.5 & 83.8 & 58.9 & 74.3 & 136 & 150.251 \\
\hline Yes & 45.5 & 16.3 & 41.1 & 25.7 & 67 & 1.884 \\
\hline Total & 100.0 & 100.0 & 100.0 & 100.0 & 203 & \\
\hline N & 42 & 84 & 77 & 126 & & \\
\hline \multicolumn{7}{|c|}{ Antibiotic drugs: pills/syrup } \\
\hline No & 52.0 & 81.6 & 54.1 & 71.2 & 111 & 176.736 \\
\hline Yes & 48.0 & 18.4 & 45.9 & 28.8 & 65 & 2.024 \\
\hline Total & 100.0 & 100.0 & 100.0 & 100.0 & 176 & \\
\hline $\mathrm{N}$ & 39 & 70 & 67 & 109 & & \\
\hline \multicolumn{7}{|c|}{ Antibiotic drugs: IV/Injection } \\
\hline No & 97.7 & 98.9 & 93.4 & 98.5 & 170 & 87.299 \\
\hline Yes & 2.3 & 1.1 & 6.7 & 1.5 & 6 & 3.688 \\
\hline Total & 100.0 & 100.0 & 100.0 & 100.0 & 176 & \\
\hline N & 39 & 70 & 67 & 109 & & \\
\hline
\end{tabular}

Estimates based on low Ns (approx. $<30$ obs) have large relative standard errors and should be interpreted with caution. ${ }^{* *}$ indicates $p<0.05$ and ${ }^{*}$ indicates $p<.0 .01$ for the chi ${ }^{2}$ statistic 
TABLE 7.2.6. REPORTED SIGNS AND SYMPTOMS OF PEDIATRIC PNEUMONIA

Percentage of women aged 15 to 49 years currently pregnant or with a child under 2 years reporting signs and symptoms of pediatric pneumonia, September-October 2019

\begin{tabular}{|c|c|c|c|c|c|c|}
\hline & $\begin{array}{c}\text { KEBBI } \\
\%\end{array}$ & $\begin{array}{c}\text { SOKOTO } \\
\%\end{array}$ & $\begin{array}{l}\text { MALARIA-ONLY } \\
\text { (ZAMFARA) } \\
\%\end{array}$ & $\begin{array}{c}\text { INTEGRATED } \\
\text { (KEBBI/SOKOTO) } \\
\%\end{array}$ & $\mathbf{N}$ & $\begin{array}{c}\mathrm{CHI}^{2} \\
\text { VALUE }\end{array}$ \\
\hline \multicolumn{7}{|l|}{ Fever } \\
\hline No & 70.4 & 73.6 & 67.2 & 72.1 & 4,316 & 16.420 \\
\hline Yes & 29.7 & 26.4 & 32.8 & 27.9 & 1,718 & 0.537 \\
\hline Total & 100.0 & 100.0 & 100.0 & 100.0 & 6,034 & \\
\hline N & 1,960 & 2,062 & 2,012 & 4,022 & & \\
\hline \multicolumn{7}{|l|}{ Chills } \\
\hline No & 84.1 & 87.3 & 90.2 & 85.8 & 5,262 & 27.544 \\
\hline Yes & 15.9 & 12.7 & 9.8 & 14.2 & 772 & 1.100 \\
\hline Total & 100.0 & 100.0 & 100.0 & 100.0 & 6,034 & \\
\hline $\mathrm{N}$ & 1,960 & 2,062 & 2,012 & 4,022 & & \\
\hline \multicolumn{7}{|c|}{ Headache } \\
\hline No & 80.6 & 85.8 & 79.9 & 83.4 & 4,938 & 11.614 \\
\hline Yes & 19.4 & 14.2 & 20.1 & 16.6 & 1,096 & 0.366 \\
\hline Total & 100.0 & 100.0 & 100.0 & 100.0 & 6,034 & \\
\hline N & 1,960 & 2,062 & 2,012 & 4,022 & & \\
\hline \multicolumn{7}{|c|}{ Body pain } \\
\hline No & 84.1 & 87.5 & 82.4 & 85.9 & 5,060 & 13.024 \\
\hline Yes & 15.9 & 12.5 & 17.6 & 14.1 & 974 & 0.689 \\
\hline Total & 100.0 & 100.0 & 100.0 & 100.0 & 6,034 & \\
\hline N & 1,960 & 2,062 & 2,012 & 4,022 & & \\
\hline \multicolumn{7}{|c|}{ Stiff neck } \\
\hline No & 93.8 & 97.2 & 94.2 & 95.7 & 5,725 & 6.572 \\
\hline Yes & 6.2 & 2.8 & 5.9 & 4.4 & 309 & 0.516 \\
\hline Total & 100.0 & 100.0 & 100.0 & 100.0 & 6,034 & \\
\hline N & 1,960 & 2,062 & 2,012 & 4,022 & & \\
\hline \multicolumn{7}{|c|}{ Wheezing } \\
\hline No & 78.2 & 82.4 & 78.4 & 80.5 & 4,839 & 3.742 \\
\hline Yes & 21.8 & 17.6 & 21.6 & 19.5 & 1,195 & 0.169 \\
\hline Total & 100.0 & 100.0 & 100.0 & 100.0 & 6,034 & \\
\hline $\mathrm{N}$ & 1,960 & 2,062 & 2,012 & 4,022 & & \\
\hline \multicolumn{7}{|l|}{ Cough } \\
\hline No & 57.1 & 56.8 & 44.6 & 56.9 & 3,245 & 89.086 \\
\hline Yes & 42.9 & 43.2 & 55.4 & 43.1 & 2,789 & 3.684 \\
\hline Total & 100.0 & 100.0 & 100.0 & 100.0 & 6,034 & \\
\hline N & 1,960 & 2,062 & 2,012 & 4,022 & & \\
\hline \multicolumn{7}{|c|}{ Rapid or difficult breathing } \\
\hline No & 75.7 & 68.4 & 62.9 & 71.8 & 4,295 & 50.961 \\
\hline Yes & 24.3 & 31.6 & 37.1 & 28.2 & 1,739 & 1.650 \\
\hline Total & 100.0 & 100.0 & 100.0 & 100.0 & 6,034 & \\
\hline $\mathrm{N}$ & 1,960 & 2,062 & 2,012 & 4,022 & & \\
\hline \multicolumn{7}{|c|}{ Diarrhea } \\
\hline No & 94.5 & 96.7 & 94.1 & 95.7 & 5,728 & 7.194 \\
\hline Yes & 5.5 & 3.3 & 5.9 & 4.3 & 306 & 0.418 \\
\hline Total & 100.0 & 100.0 & 100.0 & 100.0 & 6,034 & \\
\hline N & 1,960 & 2,062 & 2,012 & 4,022 & & \\
\hline \multicolumn{7}{|c|}{ Vomiting } \\
\hline No & 91.4 & 92.3 & 90.3 & 91.9 & 5,511 & 4.583 \\
\hline Yes & 8.6 & 7.7 & 9.7 & 8.1 & 523 & 0.275 \\
\hline Total & 100.0 & 100.0 & 100.0 & 100.0 & 6,034 & \\
\hline N & 1,960 & 2,062 & 2,012 & 4,022 & & \\
\hline \multicolumn{7}{|c|}{ Poor appetite } \\
\hline No & 95.2 & 95.5 & 92.4 & 95.4 & 5,719 & 21.449 \\
\hline Yes & 4.8 & 4.6 & 7.6 & 4.7 & 315 & 0.856 \\
\hline Total & 100.0 & 100.0 & 100.0 & 100.0 & 6,034 & \\
\hline N & 1,960 & 2,062 & 2,012 & 4,022 & & \\
\hline \multicolumn{7}{|c|}{ Crying all the time } \\
\hline No & 96.8 & 93.7 & 88.3 & 95.2 & 5,622 & 84.121 \\
\hline Yes & 3.2 & 6.3 & 11.7 & 4.8 & 412 & 5.779 \\
\hline Total & 100.0 & 100.0 & 100.0 & 100.0 & 6,034 & * \\
\hline N & 1,960 & 2,062 & 2,012 & 4,022 & & \\
\hline \multicolumn{7}{|c|}{ Don't know } \\
\hline No & 70.3 & 62.9 & 72.5 & 66.3 & 4,045 & 26.537 \\
\hline Yes & 29.7 & 37.1 & 27.5 & 33.7 & 1,989 & 1.119 \\
\hline Total & 100.0 & 100.0 & 100.0 & 100.0 & 6,034 & \\
\hline N & 1,960 & 2,062 & 2,012 & 4,022 & & \\
\hline
\end{tabular}

Estimates based on low Ns (approx. $<30$ obs) have large relative standard errors and should be interpreted with caution. ${ }^{* * *}$ indicates $p<0.001,{ }^{* *}$ indicates $p<0.05$ and * indicates $p<.0 .01$ for the chi ${ }^{2}$ statistic comparing integrated versus malaria-only study areas. 
TABLE 7.2.7. REPORTED WAYS TO PREVENT PEDIATRIC PNEUMONIA

Percentage of women aged 15 to 49 years currently pregnant or with a child under 2 years reporting ways to prevent pediatric pneumonia, September-October 2019

\begin{tabular}{|c|c|c|c|c|c|c|}
\hline & KEBBI & SOKото & $\begin{array}{l}\text { MALARIA-ONLY } \\
\text { (ZAMFARA) }\end{array}$ & $\begin{array}{c}\text { INTEGRATED } \\
\text { (KEBBI/SOKOTO) }\end{array}$ & $\mathbf{N}$ & $\begin{array}{c}\mathrm{CHI}^{2} \\
\text { VALUE }\end{array}$ \\
\hline & $\%$ & $\%$ & $\%$ & $\%$ & & \\
\hline \multicolumn{7}{|c|}{ Ensure child vaccinated } \\
\hline No & 73.7 & 74.1 & 67.6 & 73.9 & 4,355 & 27.882 \\
\hline Yes & 26.3 & 25.9 & 32.4 & 26.1 & 1,679 & 0.714 \\
\hline Total & 100.0 & 100.0 & 100.0 & 100.0 & 6,034 & \\
\hline $\mathrm{N}$ & 1,960 & 2,062 & 2,012 & 4,022 & & \\
\hline \multicolumn{7}{|c|}{ Keep house/surroundings clean } \\
\hline No & 78.8 & 88.8 & 78.1 & 84.2 & 4,905 & 34.250 \\
\hline Yes & 21.2 & 11.2 & 21.9 & 15.8 & 1,129 & 1.442 \\
\hline Total & 100.0 & 100.0 & 100.0 & 100.0 & 6,034 & \\
\hline N & 1,960 & 2,062 & 2,012 & 4,022 & & \\
\hline \multicolumn{7}{|c|}{ Only breastmilk first 6 months } \\
\hline No & 92.4 & 94.1 & 85.4 & 93.3 & 5,467 & 90.616 \\
\hline Yes & 7.6 & 5.9 & 14.6 & 6.7 & 567 & 2.881 \\
\hline Total & 100.0 & 100.0 & 100.0 & 100.0 & 6,034 & \\
\hline N & 1,960 & 2,062 & 2,012 & 4,022 & & \\
\hline \multicolumn{7}{|c|}{ Safe storage of drinking water } \\
\hline No & 91.6 & 93.0 & 83.6 & 92.4 & 5,330 & 99.703 \\
\hline Yes & 8.4 & 7.0 & 16.4 & 7.6 & 704 & 4.946 \\
\hline Total & 100.0 & 100.0 & 100.0 & 100.0 & 6,034 & * \\
\hline $\mathrm{N}$ & 1,960 & 2,062 & 2,012 & 4,022 & & \\
\hline \multicolumn{7}{|c|}{ Don't give dirty water } \\
\hline No & 91.0 & 94.5 & 90.9 & 92.9 & 5,537 & 7.824 \\
\hline Yes & 9.0 & 5.5 & 9.2 & 7.1 & 497 & 0.571 \\
\hline Total & 100.0 & 100.0 & 100.0 & 100.0 & 6,034 & \\
\hline $\mathrm{N}$ & 1,960 & 2,062 & 2,012 & 4,022 & & \\
\hline \multicolumn{7}{|c|}{ Good nutrition } \\
\hline No & 87.7 & 92.2 & 87.8 & 90.1 & 5,370 & 7.510 \\
\hline Yes & 12.3 & 7.8 & 12.2 & 9.9 & 664 & 0.311 \\
\hline Total & 100.0 & 100.0 & 100.0 & 100.0 & 6,034 & \\
\hline $\mathrm{N}$ & 1,960 & 2,062 & 2,012 & 4,022 & & \\
\hline \multicolumn{7}{|c|}{ Handwashing } \\
\hline No & 93.5 & 95.7 & 94.1 & 94.7 & 5,671 & 0.844 \\
\hline Yes & 6.5 & 4.3 & 5.9 & 5.3 & 363 & 0.045 \\
\hline Total & 100.0 & 100.0 & 100.0 & 100.0 & 6,034 & \\
\hline $\mathrm{N}$ & 1,960 & 2,062 & 2,012 & 4,022 & & \\
\hline \multicolumn{7}{|l|}{ Other } \\
\hline No & 89.3 & 91.7 & 87.7 & 90.6 & 5,445 & 12.261 \\
\hline Yes & 10.7 & 8.3 & 12.3 & 9.4 & 589 & 0.568 \\
\hline Total & 100.0 & 100.0 & 100.0 & 100.0 & 6,034 & \\
\hline $\mathrm{N}$ & 1,960 & 2,062 & 2,012 & 4,022 & & \\
\hline \multicolumn{7}{|c|}{ Don't know } \\
\hline No & 62.4 & 47.9 & 68.7 & 54.6 & 3,581 & 122.903 \\
\hline Yes & 37.6 & 52.1 & 31.3 & 45.4 & 2,453 & 5.031 \\
\hline Total & 100.0 & 100.0 & 100.0 & 100.0 & 6,034 & * \\
\hline $\mathrm{N}$ & 1,960 & 2,062 & 2,012 & 4,022 & & \\
\hline
\end{tabular}

Estimates based on low Ns (approx. $<30$ obs) have large relative standard errors and should be interpreted with caution. ${ }^{* * *}$ indicates $p<0.001,{ }^{* *}$ indicates $p<0.05$ and ${ }^{*}$ indicates $p<.0 .01$ for the chi ${ }^{2}$ statistic comparing integrated versus malaria-only study areas. 
TABLE 7.2.8. REPORTED TREATMENT PERCEIVED AS EFFECTIVE AGAINST PEDIATRIC PNEUMONIA

Percentage of women aged 15 to 49 years currently pregnant or with a child under 2 years reporting which medicines are effective to treat pneumonia, September-October 2019

\begin{tabular}{|c|c|c|c|c|c|c|}
\hline & KEBBI & SOKOTO & $\begin{array}{l}\text { MALARIA-ONLY } \\
\text { (ZAMFARA) }\end{array}$ & $\begin{array}{c}\text { INTEGRATED } \\
\text { (KEBBI/SOKOTO) }\end{array}$ & $\mathbf{N}$ & $\begin{array}{c}\mathrm{CHI}^{2} \\
\text { VALUE }\end{array}$ \\
\hline & $\%$ & $\%$ & $\%$ & $\%$ & & \\
\hline \multicolumn{7}{|c|}{ Amoxicillin } \\
\hline No & 71.0 & 82.4 & 71.6 & 77.1 & 4,592 & 22.678 \\
\hline Yes & 29.0 & 17.7 & 28.4 & 22.9 & 1,442 & 0.833 \\
\hline Total & 100.0 & 100.0 & 100.0 & 100.0 & 6,034 & \\
\hline $\mathrm{N}$ & 1,960 & 2,062 & 2,012 & 4,022 & & \\
\hline \multicolumn{7}{|c|}{ Cotrimoxazole } \\
\hline No & 87.4 & 93.0 & 78.3 & 90.4 & 5,313 & 152.736 \\
\hline Yes & 12.7 & 7.0 & 21.7 & 9.6 & 721 & 5.800 \\
\hline Total & 100.0 & 100.0 & 100.0 & 100.0 & 6,034 & * \\
\hline$N$ & 1,960 & 2,062 & 2,012 & 4,022 & & \\
\hline \multicolumn{7}{|c|}{ Other antibiotics } \\
\hline No & 98.8 & 98.9 & 97.8 & 98.9 & 5,953 & 9.537 \\
\hline Yes & 1.2 & 1.1 & 2.2 & 1.1 & 81 & 1.567 \\
\hline Total & 100.0 & 100.0 & 100.0 & 100.0 & 6,034 & \\
\hline $\mathrm{N}$ & 1,960 & 2,062 & 2,012 & 4,022 & & \\
\hline \multicolumn{7}{|c|}{ Anti-malarials } \\
\hline No & 93.2 & 96.1 & 88.5 & 94.7 & 5,591 & 69.809 \\
\hline Yes & 6.8 & 3.9 & 11.5 & 5.3 & 443 & 2.592 \\
\hline Total & 100.0 & 100.0 & 100.0 & 100.0 & 6,034 & \\
\hline $\mathrm{N}$ & 1,960 & 2,062 & 2,012 & 4,022 & & \\
\hline \multicolumn{7}{|l|}{ ORS } \\
\hline No & 93.6 & 99.1 & 98.2 & 96.6 & 5,845 & 16.757 \\
\hline Yes & 6.4 & 0.9 & 1.8 & 3.4 & 189 & 1.644 \\
\hline Total & 100.0 & 100.0 & 100.0 & 100.0 & 6,034 & \\
\hline $\mathrm{N}$ & 1,960 & 2,062 & 2,012 & 4,022 & & \\
\hline \multicolumn{7}{|l|}{ Other } \\
\hline No & 96.9 & 87.6 & 92.7 & 91.9 & 5,612 & 1.233 \\
\hline Yes & 3.1 & 12.4 & 7.3 & 8.1 & 422 & 0.053 \\
\hline Total & 100.0 & 100.0 & 100.0 & 100.0 & 6,034 & \\
\hline N & 1,960 & 2,062 & 2,012 & 4,022 & & \\
\hline \multicolumn{7}{|c|}{ Don't know } \\
\hline No & 43.1 & 34.2 & 43.9 & 38.3 & 2,336 & 18.902 \\
\hline Yes & 56.9 & 65.8 & 56.1 & 61.7 & 3,698 & 0.685 \\
\hline Total & 100.0 & 100.0 & 100.0 & 100.0 & 6,034 & \\
\hline $\mathrm{N}$ & 1,960 & 2,062 & 2,012 & 4,022 & & \\
\hline
\end{tabular}

Estimates based on low Ns (approx. $<30$ obs) have large relative standard errors and should be interpreted with caution. ${ }^{* * *}$ indicates $p<0.001,{ }^{* *}$ indicates $p<0.05$ and ${ }^{*}$ indicates $p<.0 .01$ for the chi $^{2}$ statistic comparing integrated versus malaria-only study areas. 
TABLE 7.2.9. PNEUMONIA-RELATED BELIEFS, INTENTIONS, AND SELF-EFFICACY

Percentage of women aged 15 to 49 years currently pregnant or with a child under 2 years reporting certain pneumonia-related beliefs, intentions, and self-efficacy, September-October 2019

\begin{tabular}{|c|c|c|c|c|c|c|}
\hline & KEBBI & SOKOTO & $\begin{array}{l}\text { MALARIA-ONLY } \\
\text { (ZAMFARA) }\end{array}$ & $\begin{array}{l}\text { INTEGRATED } \\
\text { (KEBBI/SOKOTO) }\end{array}$ & $\mathbf{N}$ & $\begin{array}{c}\mathrm{CHI}^{2} \\
\text { VALUE }\end{array}$ \\
\hline & $\%$ & $\%$ & $\%$ & $\%$ & & \\
\hline \multicolumn{7}{|c|}{$\begin{array}{l}\text { Young children may get severely ill from } \\
\text { pneumonia, hospitalized, or die }\end{array}$} \\
\hline Agree & 75.0 & 70.6 & 72.4 & 72.6 & 4,305 & 12.669 \\
\hline Disagree & 4.3 & 7.6 & 8.3 & 6.1 & 403 & 0.224 \\
\hline Don't know & 20.7 & 21.8 & 19.3 & 21.3 & 1,307 & \\
\hline Total & 100.0 & 100.0 & 100.0 & 100.0 & 6,015 & \\
\hline N & 1,954 & 2,060 & 2,001 & 4,014 & & \\
\hline \multicolumn{7}{|c|}{ Only weak children die from pneumonia } \\
\hline Agree & 53.7 & 51.9 & 51.6 & 52.8 & 2,970 & 4.691 \\
\hline Disagree & 24.4 & 26.0 & 27.7 & 25.3 & 1,693 & 0.083 \\
\hline Don't know & 21.9 & 22.0 & 20.7 & 22.0 & 1,347 & \\
\hline Total & 100.0 & 100.0 & 100.0 & 100.0 & 6,010 & \\
\hline$N$ & 1,952 & 2,059 & 1,999 & 4,011 & & \\
\hline \multicolumn{7}{|c|}{$\begin{array}{l}\text { Most women go to drug shop for a child } \\
\text { with cough, rapid breaths }\end{array}$} \\
\hline Agree & 79.9 & 73.9 & 70.2 & 76.6 & 4,424 & 65.029 \\
\hline Disagree & 8.6 & 13.2 & 18.7 & 11.0 & 798 & 1.455 \\
\hline Don't know & 11.6 & 13.0 & 11.1 & 12.3 & 780 & \\
\hline Total & 100.0 & 100.0 & 100.0 & 100.0 & 6,002 & \\
\hline$N$ & 1,948 & 2,058 & 1,996 & 4,006 & & \\
\hline \multicolumn{7}{|c|}{$\begin{array}{l}\text { Antibiotics are the most effective treat- } \\
\text { ment for pneumonia }\end{array}$} \\
\hline Agree & 59.8 & 50.0 & 61.4 & 54.5 & 3,346 & 28.784 \\
\hline Disagree & 7.1 & 7.9 & 6.7 & 7.5 & 443 & 0.737 \\
\hline Don't know & 33.2 & 42.2 & 31.9 & 38.0 & 2,215 & \\
\hline Total & 100.0 & 100.0 & 100.0 & 100.0 & 6,004 & \\
\hline N & 1,951 & 2,055 & 1,998 & 4,006 & & \\
\hline \multicolumn{7}{|c|}{$\begin{array}{l}\text { Likelihood that child completes full } \\
\text { course of antibiotics }\end{array}$} \\
\hline Likely & 78.7 & 74.8 & 82.7 & 76.6 & 4,728 & 39.780 \\
\hline Unlikely & 10.5 & 10.3 & 6.5 & 10.4 & 529 & 0.934 \\
\hline Don't know & 10.8 & 14.9 & 10.8 & 13.0 & 754 & \\
\hline Total & 100.0 & 100.0 & 100.0 & 100.0 & 6,011 & \\
\hline$N$ & 1,953 & 2,056 & 2,002 & 4,009 & & \\
\hline \multicolumn{7}{|c|}{$\begin{array}{l}\text { Likelihood to seek care same/next day if } \\
\text { child develops cough, rapid breaths }\end{array}$} \\
\hline Likely & 85.0 & 79.6 & 89.1 & 82.1 & 5,070 & 64.159 \\
\hline Unlikely & 7.2 & 11.8 & 5.1 & 9.7 & 463 & 1.474 \\
\hline Don't know & 7.8 & 8.6 & 5.8 & 8.2 & 467 & \\
\hline Total & 100.0 & 100.0 & 100.0 & 100.0 & 6,000 & \\
\hline N & 1,950 & 2,055 & 1,995 & 4,005 & & \\
\hline \multicolumn{7}{|c|}{$\begin{array}{l}\text { Confidence to convince husband to seek } \\
\text { care for child with cough, rapid breaths }\end{array}$} \\
\hline Confident & 84.6 & 85.2 & 91.2 & 84.9 & 5,206 & 60.428 \\
\hline Uncertain & 8.9 & 8.5 & 4.5 & 8.7 & 456 & 1.403 \\
\hline Don't know & 6.5 & 6.3 & 4.3 & 6.4 & 344 & \\
\hline Total & 100.0 & 100.0 & 100.0 & 100.0 & 6,006 & \\
\hline $\mathrm{N}$ & 1,951 & 2,057 & 1,998 & 4,008 & & \\
\hline
\end{tabular}

Estimates based on low Ns (approx. $<30$ obs) have large relative standard errors and should be interpreted with caution. ${ }^{* * *}$ indicates $p<0.001,{ }^{* *}$ indicates $p<0.05$ and ${ }^{*}$ indicates $p<.0 .01$ for the chi ${ }^{2}$ statistic comparing integrated versus malaria-only study areas. 


\subsection{Diarrheal diseases}

\section{Key findings}

Diarrhea prevalence and care-seeking: Among last-born children in the past two years, less than one in five had diarrhea in the past two weeks. Among these children, 65 percent and 74 percent were taken for any care in Kebbi/ Sokoto and Zamfara, respectively. However, 34 percent and 56 percent who were taken for care were brought to a pharmacy in Kebbi/Sokoto and Zamfara, respectively, which often results in poor-quality care provision.

\section{Reasons for source of care or not seeking any care:}

The most common reasons for choosing the source of care across study areas were, trust in provider (39\%), nearby location (29\%), and effective treatment (25\%). This points to the importance of convenient, trusted, and high-quality care provision among respondents in these communities. The most important reasons for not seeking care included expense or cost (31\%), fatalism ("It's up to God") (29\%), and symptoms not perceived as severe or care provided at home (25\%).

Diarrhea treatment: Among children under 2 years with diarrhea, 36 percent and 56 percent were given ORS alone in Kebbi/Sokoto and Zamfara, with a statistically significant higher percentage in the latter area. Few children with diarrhea were given both ORS and zinc, although this proportion was again higher in Zamfara
(27\%) than in Kebbi/Sokoto (13\%). Similar to other priority behavioral outcomes, ORS and zinc treatment for children with diarrhea was far lower among those living in the poorest households or with less-educated mothers as compared with their wealthier and better-educated counterparts. Twenty percent of children with diarrhea still received traditional or homemade fluids across study areas, which merits further investigation if these fluids are used as alternatives to ORS and zinc. Antibiotics were also commonly given to treat diarrhea despite their lack of indication for most diarrhea-causing pathogens.

Diarrhea ideations: Among respondents who were currently pregnant or with a child under 2 years across study areas, 11 percent were unable to report any way to prevent pediatric diarrhea, while nearly half reported keeping surroundings clean (48\%), followed by not giving dirty water (39\%) or bad food (32\%) as prevention methods. While more than two-thirds of respondents recalled ORS as a diarrhea treatment (69\%), less than half could name zinc $(42 \%)$, and nearly one-third reported antibiotics as an effective diarrhea treatment despite its lack of indication for most diarrheal conditions. Nearly two-thirds of respondents (63\%) reported that ORS replaces lost water in a child with diarrhea, while 44 percent incorrectly noted that it stops diarrhea. One-quarter of respondents (24\%) had never heard of zinc, and approximately one-third of respondents reported that zinc makes diarrhea less severe and shortens its duration. Only 69 percent of respondents agreed that they knew how to prepare ORS themselves. 


\section{TABLE 7.3.A. ORS TREATMENT FOR PEDIATRIC DIARRHEA BY SOCIODEMOGRAPHIC CHARACTERISTICS}

Percentage of last-born children under 2 years with diarrhea in past two weeks given ORS by sociodemographic characteristics, September-October 2019

\begin{tabular}{|c|c|c|c|c|c|c|c|c|}
\hline & \multicolumn{2}{|c|}{ KEBBI } & \multicolumn{2}{|c|}{ sокото } & \multicolumn{2}{|c|}{$\begin{array}{l}\text { MALARIA-ONLY } \\
\text { (ZAMFARA) }\end{array}$} & \multicolumn{2}{|c|}{$\begin{array}{c}\text { INTEGRATED } \\
\text { (KEBBI/SOKOTO) }\end{array}$} \\
\hline & $\%$ & $\mathbf{N}$ & $\%$ & $\mathbf{N}$ & $\%$ & $\mathbf{N}$ & $\%$ & $\mathbf{N}$ \\
\hline Total & 51.0 & 153 & 26.5 & 210 & 56.0 & 171 & 35.9 & 364 \\
\hline \multicolumn{9}{|l|}{ Household wealth } \\
\hline Lowest & 42.3 & 40 & 29.6 & 67 & (..) & 13 & 33.7 & 107 \\
\hline Second & 62.9 & 32 & 22.5 & 43 & 38.0 & 31 & 37.4 & 75 \\
\hline Middle & 49.7 & 33 & 20.4 & 49 & 48.2 & 38 & 30.5 & 82 \\
\hline Fourth & (..) & 16 & 34.8 & 24 & 70.2 & 32 & 40.9 & 41 \\
\hline Highest & 54.3 & 32 & 33.7 & 27 & 64.5 & 57 & 45.4 & 59 \\
\hline \multicolumn{9}{|l|}{ Maternal age (in years) } \\
\hline $15-24$ years & 50.9 & 54 & 32.8 & 92 & 57.8 & 73 & 38.5 & 146 \\
\hline $25-34$ years & 52.6 & 74 & 22.3 & 98 & 55.6 & 72 & 34.8 & 172 \\
\hline $35-49$ years & 46.5 & 25 & (..) & 20 & 52.0 & 26 & 32.7 & 46 \\
\hline \multicolumn{9}{|c|}{$\begin{array}{l}\text { Maternal education } \\
\text { (highest level attended) }\end{array}$} \\
\hline None & 53.0 & 120 & 19.6 & 174 & 53.3 & 110 & 30.9 & 295 \\
\hline Primary & (..) & 6 & (..) & 11 & (..) & 11 & (..) & 17 \\
\hline Secondary or higher & (..) & 16 & (..) & 6 & 70.1 & 28 & 57.2 & 22 \\
\hline Islamic & (..) & 11 & (..) & 19 & 53.0 & 22 & 60.8 & 30 \\
\hline
\end{tabular}

(..) Value not shown due to low number of observations. Estimates based on low Ns (approx. $<30$ obs) have large relative standard errors and should be interpreted with caution. ${ }^{* *}$ indicates $p<0.001,{ }^{* *}$ indicates $p<0.05$ and ${ }^{*}$ indicates $p<.0 .01$ for the chi ${ }^{2}$ statistic comparing integrated versus malaria-only study areas.

\section{TABLE 7.3.B. ORS AND ZINC TREATMENT FOR PEDIATRIC DIARRHEA BY SOCIODEMOGRAPHIC CHARACTERISTICS}

Percentage of last-born children under 2 years with diarrhea in past two weeks given ORS and zinc by sociodemographic characteristics, September-October 2019

\begin{tabular}{|c|c|c|c|c|c|c|c|c|}
\hline & \multicolumn{2}{|c|}{ KEBBI } & \multicolumn{2}{|c|}{ SOKото } & \multicolumn{2}{|c|}{$\begin{array}{l}\text { MALARIA-ONLY } \\
\text { (ZAMFARA) }\end{array}$} & \multicolumn{2}{|c|}{$\begin{array}{c}\text { INTEGRATED } \\
\text { (KEBBI/SOKOTO) }\end{array}$} \\
\hline & $\%$ & $\mathbf{N}$ & $\%$ & $\mathbf{N}$ & $\%$ & $\mathbf{N}$ & $\%$ & $\mathbf{N}$ \\
\hline Total & 16.1 & 153 & 11.5 & 210 & 27.1 & 171 & 13.3 & 364 \\
\hline \multicolumn{9}{|l|}{ Household wealth } \\
\hline Lowest & 32.2 & 40 & 12.6 & 67 & (..) & 13 & 19.0 & 107 \\
\hline Second & 1.5 & 32 & 6.3 & 43 & 8.2 & 31 & 4.6 & 75 \\
\hline Middle & 16.2 & 33 & 11.4 & 49 & 30.3 & 38 & 13.1 & 82 \\
\hline Fourth & (..) & 16 & 14.3 & 24 & 45.2 & 32 & 10.4 & 41 \\
\hline Highest & 14.6 & 32 & 16.6 & 27 & 33.9 & 57 & 15.5 & 59 \\
\hline \multicolumn{9}{|l|}{ Maternal age (in years) } \\
\hline $15-24$ years & 23.8 & 54 & 11.2 & 92 & 19.2 & 73 & 15.2 & 146 \\
\hline 25-34 years & 14.4 & 74 & 10.6 & 98 & 37.2 & 72 & 12.2 & 172 \\
\hline $35-49$ years & 7.7 & 25 & (..) & 20 & 20.8 & 26 & 12.0 & 46 \\
\hline \multicolumn{9}{|c|}{$\begin{array}{l}\text { Maternal education } \\
\text { (highest level attended) }\end{array}$} \\
\hline None & 17.5 & 120 & (..) & 17 & 2.5 & 110 & 10.8 & 295 \\
\hline Primary & (..) & 6 & (..) & 11 & (..) & 11 & 12.6 & 17 \\
\hline Secondary or higher & (..) & 16 & (..) & 6 & 46.0 & 28 & 22.8 & 22 \\
\hline Islamic & (..) & 11 & (..) & 19 & 24.2 & 22 & 28.5 & 30 \\
\hline
\end{tabular}

(..) Value not shown due to low number of observations. Estimates based on low Ns (approx. <30 obs) have large relative standard errors and should be interpreted with caution. ${ }^{* * *}$ indicates $p<0.001,{ }^{* *}$ indicates $p<0.05$ and ${ }^{*}$ indicates $p<.0 .01$ for the chi ${ }^{2}$ statistic comparing integrated versus malaria-only study areas. 
TABLE 7.3.1. CARE-SEEKING FOR PEDIATRIC DIARRHEA

Percentage of last-born children under 2 years with diarrhea in the past two weeks who were taken for any advice or treatment by source of care, September-October 2019

\begin{tabular}{|c|c|c|c|c|c|c|}
\hline & KEBBI & sOKото & $\begin{array}{l}\text { MALARIA-ONLY } \\
\text { (ZAMFARA) }\end{array}$ & $\begin{array}{l}\text { INTEGRATED } \\
\text { (KEBBI/SOKOTO) }\end{array}$ & $\mathbf{N}$ & $\begin{array}{c}\mathrm{CHI}^{2} \\
\text { VALUE }\end{array}$ \\
\hline & $\%$ & $\%$ & $\%$ & $\%$ & & \\
\hline \multicolumn{7}{|c|}{$\begin{array}{l}\text { Has child had diarrhea at any time } \\
\text { in the last } 2 \text { weeks? }\end{array}$} \\
\hline No & 81.9 & 78.5 & 84.2 & 80.0 & 2,477 & 12.381 \\
\hline Yes & 17.2 & 20.6 & 15.6 & 19.2 & 534 & 1.486 \\
\hline Don't know & 0.9 & 0.9 & 0.3 & 0.9 & 28 & \\
\hline Total & 100.0 & 100.0 & 100.0 & 100.0 & 3,039 & \\
\hline$N$ & 892 & 1,078 & 1,069 & 1,970 & & \\
\hline \multicolumn{7}{|c|}{$\begin{array}{l}\text { Did you seek advice or treatment } \\
\text { from any source for the diarrhea? }\end{array}$} \\
\hline No & 26.8 & 39.8 & 25.8 & 34.8 & 148 & 5.131 \\
\hline Yes & 73.2 & 60.2 & 74.2 & 65.2 & 386 & 1.991 \\
\hline Total & 100.0 & 100.0 & 100.0 & 100.0 & 534 & \\
\hline$N$ & 153 & 210 & 171 & 363 & & \\
\hline \multicolumn{7}{|c|}{ Where did you seek advice or treatment? } \\
\hline \multicolumn{7}{|c|}{ Government hospital } \\
\hline No & 67.9 & 92.3 & 79.0 & 81.7 & 305 & 0.426 \\
\hline Yes & 32.1 & 7.7 & 21.0 & 18.3 & 81 & 0.086 \\
\hline Total & 100.0 & 100.0 & 100.0 & 100.0 & 386 & \\
\hline $\mathrm{N}$ & 119 & 135 & 132 & 254 & & \\
\hline \multicolumn{7}{|c|}{ Governmental PHC } \\
\hline No & 79.2 & 70.3 & 88.3 & 74.2 & 311 & 12.957 \\
\hline Yes & 20.8 & 29.7 & 11.7 & 25.8 & 75 & 3.706 \\
\hline Total & 100.0 & 100.0 & 100.0 & 100.0 & 386 & \\
\hline N & 119 & 135 & 132 & 254 & & \\
\hline \multicolumn{7}{|c|}{ Dispensary/health post } \\
\hline No & 98.4 & 88.8 & 96.9 & 93.0 & 362 & 3.217 \\
\hline Yes & 1.6 & 11.2 & 3.1 & 7.0 & 24 & 1.451 \\
\hline Total & 100.0 & 100.0 & 100.0 & 100.0 & 386 & \\
\hline N & 119 & 135 & 132 & 254 & & \\
\hline \multicolumn{7}{|c|}{ Community health outreach post } \\
\hline No & 94.9 & 96.4 & 98.6 & 95.7 & 375 & 2.980 \\
\hline Yes & 5.1 & 3.6 & 1.4 & 4.3 & 11 & 1.570 \\
\hline Total & 100.0 & 100.0 & 100.0 & 100.0 & 386 & \\
\hline N & 119 & 135 & 132 & 254 & & \\
\hline \multicolumn{7}{|c|}{ Private hospital/clinic } \\
\hline No & 97.4 & 94.1 & 96.7 & 95.5 & 374 & 0.378 \\
\hline Yes & 2.6 & 5.9 & 3.3 & 4.5 & 12 & 0.092 \\
\hline Total & 100.0 & 100.0 & 100.0 & 100.0 & 386 & \\
\hline N & 119 & 135 & 132 & 254 & & \\
\hline \multicolumn{7}{|c|}{ Pharmacy/chemist } \\
\hline No & 63.2 & 68.5 & 44.5 & 66.2 & 218 & 17.728 \\
\hline Yes & 36.9 & 31.5 & 55.5 & 33.8 & 168 & 9.273 \\
\hline Total & 100.0 & 100.0 & 100.0 & 100.0 & 386 & ** \\
\hline N & 119 & 135 & 132 & 254 & & \\
\hline
\end{tabular}

Estimates based on low Ns (approx. $<30$ obs) have large relative standard errors and should be interpreted with caution. ${ }^{* * *}$ indicates $p<0.001,{ }^{* *}$ indicates $p<0.05$ and * indicates $p<.0 .01$ for the chi ${ }^{2}$ statistic comparing integrated versus malaria-only study areas. 
TABLE 7.3.2. REASONS FOR CHOOSING SOURCE OF CARE FOR PEDIATRIC DIARRHEA

Percentage of last-born children under 2 years with diarrhea in the past two weeks taken to any source of care by reason for choosing treatment location, September-October 2019

\begin{tabular}{|c|c|c|c|c|c|c|}
\hline & $\begin{array}{c}\text { KEBBI } \\
\%\end{array}$ & $\begin{array}{c}\text { SOKOTO } \\
\%\end{array}$ & $\begin{array}{c}\text { MALARIA-ONLY } \\
\text { (ZAMFARA) } \\
\%\end{array}$ & $\begin{array}{c}\text { INTEGRATED } \\
\text { (KEBBI/SOKOTO) } \\
\%\end{array}$ & $\mathbf{N}$ & $\begin{array}{c}\mathrm{CHI}^{2} \\
\text { VALUE }\end{array}$ \\
\hline \multicolumn{7}{|c|}{ Free or inexpensive } \\
\hline No & 83.9 & 77.1 & 93.9 & 80.1 & 342 & 17.285 \\
\hline Yes & 16.1 & 22.9 & 6.1 & 19.9 & 44 & 6.652 \\
\hline Total & 100.0 & 100.0 & 100.0 & 100.0 & 386 & * \\
\hline N & 119 & 135 & 132 & 254 & & \\
\hline \multicolumn{7}{|c|}{ Nearby/transportation avail } \\
\hline No & 62.5 & 59.4 & 78.2 & 60.7 & 262 & 13.856 \\
\hline Yes & 37.5 & 40.6 & 21.8 & 39.3 & 124 & 4.114 \\
\hline Total & 100.0 & 100.0 & 100.0 & 100.0 & 386 & * \\
\hline $\mathrm{N}$ & 119 & 135 & 132 & 254 & & \\
\hline \multicolumn{7}{|c|}{ Medicines often in stock } \\
\hline No & 89.0 & 86.5 & 95.2 & 87.6 & 356 & 7.456 \\
\hline Yes & 11.0 & 13.5 & 4.8 & 12.4 & 30 & 3.173 \\
\hline Total & 100.0 & 100.0 & 100.0 & 100.0 & 386 & \\
\hline $\mathrm{N}$ & 119 & 135 & 132 & 254 & & \\
\hline \multicolumn{7}{|c|}{ Respectful care } \\
\hline No & 94.4 & 91.7 & 80.0 & 92.9 & 347 & 12.375 \\
\hline Yes & 5.6 & 8.3 & 20.0 & 7.1 & 39 & 6.581 \\
\hline Total & 100.0 & 100.0 & 100.0 & 100.0 & 386 & * \\
\hline $\mathrm{N}$ & 119 & 135 & 132 & 254 & & \\
\hline \multicolumn{7}{|c|}{ Trust to care for my child } \\
\hline No & 71.0 & 75.9 & 52.4 & 73.8 & 248 & 18.059 \\
\hline Yes & 29.0 & 24.1 & 47.6 & 26.2 & 138 & 6.516 \\
\hline Total & 100.0 & 100.0 & 100.0 & 100.0 & 386 & * \\
\hline$N$ & 119 & 135 & 132 & 254 & & \\
\hline \multicolumn{7}{|c|}{ Effective treatment } \\
\hline No & 88.9 & 79.7 & 69.8 & 83.7 & 304 & 9.812 \\
\hline Yes & 11.1 & 20.3 & 30.2 & 16.3 & 82 & 5.321 \\
\hline Total & 100.0 & 100.0 & 100.0 & 100.0 & 386 & * \\
\hline$N$ & 119 & 135 & 132 & 254 & & \\
\hline \multicolumn{7}{|c|}{ Short wait time } \\
\hline No & 95.2 & 85.5 & 90.7 & 89.7 & 344 & 0.099 \\
\hline Yes & 4.8 & 14.5 & 9.3 & 10.3 & 42 & 0.045 \\
\hline Total & 100.0 & 100.0 & 100.0 & 100.0 & 386 & \\
\hline $\mathrm{N}$ & 119 & 135 & 132 & 254 & & \\
\hline \multicolumn{7}{|l|}{ Privacy } \\
\hline No & 99.5 & 97.6 & 99.3 & 98.4 & 380 & 0.813 \\
\hline Yes & 0.5 & 2.4 & 0.7 & 1.6 & 6 & 0.592 \\
\hline Total & 100.0 & 100.0 & 100.0 & 100.0 & 386 & \\
\hline $\mathrm{N}$ & 119 & 135 & 132 & 254 & & \\
\hline \multicolumn{7}{|c|}{ Family/friends } \\
\hline No & 92.3 & 90.3 & 85.3 & 91.1 & 340 & 2.971 \\
\hline Yes & 7.8 & 9.7 & 14.71 & 8.9 & 46 & 1.443 \\
\hline Total & 100.0 & 100.0 & 100.0 & 100.0 & 386 & \\
\hline N & 119 & 135 & 132 & 254 & & \\
\hline \multicolumn{7}{|c|}{ Religious/community leaders } \\
\hline No & 91.4 & 100.0 & 100.0 & 96.3 & 374 & 8.535 \\
\hline Yes & 8.6 & 0.0 & 0.0 & 3.7 & 12 & 1.509 \\
\hline Total & 100.0 & 100.0 & 100.0 & 100.0 & 386 & \\
\hline N & 119 & 135 & 132 & 254 & & \\
\hline
\end{tabular}

Estimates based on low Ns (approx. $<30$ obs) have large relative standard errors and should be interpreted with caution. ${ }^{* \star *}$ indicates $p<0.001,{ }^{* *}$ indicates $p<0.05$ and $^{*}$ indicates $p<.0 .01$ for the chi ${ }^{2}$ statistic comparing integrated versus malaria-only study areas. 
TABLE 7.3.3. REASONS FOR NOT SEEKING CARE FOR PEDIATRIC DIARRHEA

Percentage of last-born children under 2 years with diarrhea in the past two weeks not taken to any source of care by reason for no care-seeking, September-October 2019

\begin{tabular}{|c|c|c|c|c|c|c|}
\hline & KEBBI & SOKOTO & $\begin{array}{l}\text { MALARIA-ONLY } \\
\text { (ZAMFARA) }\end{array}$ & $\begin{array}{c}\text { INTEGRATED } \\
\text { (KEBBI/SOKOTO) }\end{array}$ & $\mathbf{N}$ & $\begin{array}{c}\mathrm{CHI}^{2} \\
\text { VALUE }\end{array}$ \\
\hline & $\%$ & $\%$ & $\%$ & $\%$ & & \\
\hline \multicolumn{7}{|c|}{ Too expensive/could not find money } \\
\hline No & 61.9 & 77.8 & 63.6 & 73.0 & 102 & 1.531 \\
\hline Yes & 38.1 & 22.3 & 36.5 & 27.0 & 46 & 0.599 \\
\hline Total & 100.0 & 100.0 & 100.0 & 100.0 & 148 & \\
\hline $\mathrm{N}$ & 34 & 75 & 39 & 109 & & \\
\hline \multicolumn{7}{|c|}{ Facility closed/no one there } \\
\hline No & 91.1 & 100.0 & 96.8 & 97.3 & 143 & 0.034 \\
\hline Yes & 8.9 & 0.0 & 3.2 & 2.7 & 5 & 0.021 \\
\hline Total & 100.0 & 100.0 & 100.0 & 100.0 & 148 & \\
\hline N & 34 & 75 & 39 & 109 & & \\
\hline \multicolumn{7}{|c|}{ Distance/no transportation } \\
\hline No & 91.8 & 98.6 & 92.1 & 96.6 & 143 & 1.402 \\
\hline Yes & 8.2 & 1.4 & 7.9 & 3.4 & 5 & 0.879 \\
\hline Total & 100.0 & 100.0 & 100.0 & 100.0 & 148 & \\
\hline N & 34 & 75 & 39 & 109 & & \\
\hline \multicolumn{7}{|c|}{ No female provider at facility } \\
\hline No & 100.0 & 100.0 & 98.4 & 100.0 & 147 & 1.287 \\
\hline Yes & 0.0 & 0.0 & 1.7 & 0.0 & 1 & 0.922 \\
\hline Total & 100.0 & 100.0 & 100.0 & 100.0 & 148 & \\
\hline N & 34 & 75 & 39 & 109 & & \\
\hline \multicolumn{7}{|c|}{ Poor quality service } \\
\hline No & 100.0 & 97.9 & 100.0 & 98.5 & 146 & 1.077 \\
\hline Yes & 0.0 & 2.2 & 0.0 & 1.5 & 2 & 1.821 \\
\hline Total & 100.0 & 100.0 & 100.0 & 100.0 & 148 & \\
\hline$N$ & 34 & 75 & 39 & 109 & & \\
\hline \multicolumn{7}{|c|}{ Treatment not effective } \\
\hline No & 94.8 & 100.0 & 100.0 & 98.5 & 146 & 1.103 \\
\hline Yes & 5.2 & 0.0 & 0.0 & 1.5 & 2 & 1.573 \\
\hline Total & 100.0 & 100.0 & 100.0 & 100.0 & 148 & \\
\hline N & 34 & 75 & 39 & 109 & & \\
\hline \multicolumn{7}{|c|}{ Not severe/provided home care } \\
\hline No & 91.5 & 72.1 & 72.3 & 77.9 & 118 & 0.607 \\
\hline Yes & 8.6 & 27.9 & 27.7 & 22.1 & 30 & 0.160 \\
\hline Total & 100.0 & 100.0 & 100.0 & 100.0 & 148 & \\
\hline N & 34 & 75 & 39 & 109 & & \\
\hline \multicolumn{7}{|c|}{ Husband/partner oppose } \\
\hline No & 97.7 & 96.6 & 97.7 & 96.9 & 142 & 0.087 \\
\hline Yes & 2.3 & 3.4 & 2.3 & 3.1 & 6 & 0.072 \\
\hline Total & 100.0 & 100.0 & 100.0 & 100.0 & 148 & \\
\hline $\mathrm{N}$ & 34 & 75 & 39 & 109 & & \\
\hline \multicolumn{7}{|c|}{ Unable to leave work/home } \\
\hline No & 98.7 & 95.8 & 86.5 & 96.7 & 142 & 5.122 \\
\hline Yes & 1.3 & 4.2 & 13.5 & 3.3 & 6 & 2.614 \\
\hline Total & 100.0 & 100.0 & 100.0 & 100.0 & 148 & \\
\hline N & 34 & 75 & 39 & 109 & & \\
\hline \multicolumn{7}{|c|}{ Up to God } \\
\hline No & 84.6 & 62.9 & 73.0 & 69.4 & 96 & 0.243 \\
\hline Yes & 15.5 & 37.1 & 27.0 & 30.7 & 52 & 0.105 \\
\hline Total & 100.0 & 100.0 & 100.0 & 100.0 & 148 & \\
\hline $\mathrm{N}$ & 34 & 75 & 39 & 109 & & \\
\hline
\end{tabular}

Estimates based on low Ns (approx. $<30$ obs) have large relative standard errors and should be interpreted with caution. ${ }^{* \star *}$ indicates $p<0.001,{ }^{* *}$ indicates $p<0.05$ and * indicates $p<.0 .01$ for the chi ${ }^{2}$ statistic comparing integrated versus malaria-only study areas. 
TABLE 7.3.4. FLUIDS OR TREATMENTS GIVEN FOR PEDIATRIC DIARRHEA

Percentage of last-born children under 2 years with diarrhea in the past two weeks who were given treatments or fluids for the illness by type given, September-October 2019

\begin{tabular}{|c|c|c|c|c|c|c|}
\hline & $\begin{array}{l}\text { KEBBI } \\
\%\end{array}$ & $\begin{array}{l}\text { SOKOTO } \\
\%\end{array}$ & $\begin{array}{c}\text { MALARIA-ONLY } \\
\text { (ZAMFARA) } \\
\%\end{array}$ & $\begin{array}{c}\text { INTEGRATED } \\
\text { (KEBBI/SOKOTO) } \\
\%\end{array}$ & $\mathbf{N}$ & $\begin{array}{c}\mathrm{CHI}^{2} \\
\text { VALUE }\end{array}$ \\
\hline \multicolumn{7}{|c|}{$\begin{array}{l}\text { Has child had diarrhea at any time in the } \\
\text { last } 2 \text { weeks? }\end{array}$} \\
\hline No & 81.9 & 78.5 & 84.2 & 80.0 & 2,477 & 12.381 \\
\hline Yes & 17.2 & 20.6 & 15.6 & 19.2 & 534 & 1.486 \\
\hline Don't know & 0.9 & 0.9 & 0.3 & 0.9 & 28 & \\
\hline Total & 100.0 & 100.0 & 100.0 & 100.0 & 3,039 & \\
\hline N & 892 & 1,078 & 1,069 & 1,970 & & \\
\hline \multicolumn{7}{|c|}{ Was child given the following for diarrhea: } \\
\hline \multicolumn{7}{|l|}{ ORS and zinc } \\
\hline No & 82.4 & 88.2 & 72.8 & 86.0 & 409 & 13.019 \\
\hline Yes & 17.6 & 11.8 & 27.2 & 14.0 & 104 & 5.050 \\
\hline Total & 100.0 & 100.0 & 100.0 & 100.0 & 513 & * \\
\hline N & 137 & 206 & 170 & 343 & & \\
\hline \multicolumn{7}{|c|}{ ORS prepack fluid or mix } \\
\hline No & 48.2 & 72.8 & 44.0 & 63.3 & 276 & 19.325 \\
\hline Yes & 51.8 & 27.2 & 56.0 & 36.7 & 252 & 5.563 \\
\hline Total & 100.0 & 100.0 & 100.0 & 100.0 & 528 & * \\
\hline $\mathrm{N}$ & 150 & 207 & 171 & 357 & & \\
\hline \multicolumn{7}{|l|}{ Zinc } \\
\hline No & 62.0 & 78.7 & 63.1 & 72.2 & 358 & 23.574 \\
\hline Yes & 29.9 & 18.2 & 36.8 & 22.7 & 154 & 5.659 \\
\hline Don't know & 8.1 & 3.1 & 0.2 & 5.0 & 22 & * \\
\hline Total & 100.0 & 100.0 & 100.0 & 100.0 & 534 & \\
\hline N & 153 & 210 & 171 & 363 & & \\
\hline \multicolumn{7}{|c|}{ Traditional/homemade fluid } \\
\hline No & 72.3 & 79.1 & 77.8 & 76.5 & 407 & 0.989 \\
\hline Yes & 25.6 & 17.5 & 20.6 & 20.7 & 115 & 0.158 \\
\hline Don't know & 2.1 & 3.3 & 1.6 & 2.9 & 12 & \\
\hline Total & 100.0 & 100.0 & 100.0 & 100.0 & 534 & \\
\hline N & 153 & 210 & 171 & 363 & & \\
\hline \multicolumn{7}{|c|}{$\begin{array}{l}\text { How much was child given to drink during } \\
\text { the diarrhea including breastmilk }\end{array}$} \\
\hline Much less & 16.6 & 18.4 & 28.6 & 17.7 & 114 & 12.595 \\
\hline Somewhat less & 15.4 & 35.3 & 27.8 & 27.6 & 151 & 0.764 \\
\hline About the same & 35.7 & 27.6 & 21.6 & 30.7 & 141 & \\
\hline More to drink & 15.4 & 16.3 & 12.5 & 15.9 & 80 & \\
\hline Nothing to drink & 16.6 & 2.4 & 9.0 & 7.9 & 45 & \\
\hline Don't know & 0.4 & 0.1 & 0.5 & 0.2 & 3 & \\
\hline Total & 100.0 & 100.0 & 100.0 & 100.0 & 534 & \\
\hline $\mathrm{N}$ & 153 & 210 & 171 & 363 & & \\
\hline
\end{tabular}

Estimates based on low Ns (approx. $<30$ obs) have large relative standard errors and should be interpreted with caution. ${ }^{* * *}$ indicates $p<0.001,{ }^{* *}$ indicates $p<0.05$ and ${ }^{*}$ indicates $p<.0 .01$ for the chi ${ }^{2}$ statistic comparing integrated versus malaria-only study areas. 
TABLE 7.3.5. REPORTED WAYS TO PREVENT A CHILD FROM GETTING DIARRHEA

Percentage of women aged 15 to 49 years currently pregnant or with a child under 2 years reporting ways to prevent pediatric diarrhea, September-October 2019

\begin{tabular}{|c|c|c|c|c|c|c|}
\hline & KEBBI & SOKOTO & $\begin{array}{l}\text { MALARIA-ONLY } \\
\text { (ZAMFARA) }\end{array}$ & $\begin{array}{c}\text { INTEGRATED } \\
\text { (KEBBI/SOKOTO) }\end{array}$ & $\mathbf{N}$ & $\begin{array}{c}\mathrm{CHI}^{2} \\
\text { VALUE }\end{array}$ \\
\hline & $\%$ & $\%$ & $\%$ & $\%$ & & \\
\hline \multicolumn{7}{|c|}{ Ensure child is vaccinated } \\
\hline No & 75.9 & 79.7 & 68.9 & 78.0 & 4,502 & 60.227 \\
\hline Yes & 24.1 & 20.3 & 31.1 & 22.1 & 1,532 & 1.495 \\
\hline Total & 100.0 & 100.0 & 100.0 & 100.0 & 6,034 & \\
\hline $\mathrm{N}$ & 1,960 & 2,062 & 2,012 & 4,022 & & \\
\hline \multicolumn{7}{|c|}{ Use toilet/no open waste } \\
\hline No & 79.9 & 90.7 & 79.9 & 85.7 & 4,994 & 34.018 \\
\hline Yes & 20.1 & 9.3 & 20.1 & 14.3 & 1,040 & 1.354 \\
\hline Total & 100.0 & 100.0 & 100.0 & 100.0 & 6,034 & \\
\hline $\mathrm{N}$ & 1,960 & 2,062 & 2,012 & 4,022 & & \\
\hline \multicolumn{7}{|c|}{ Keep house/surroundings clean } \\
\hline No & 53.8 & 67.2 & 46.6 & 61.0 & 3,267 & 120.214 \\
\hline Yes & 46.3 & 32.8 & 53.4 & 39.0 & 2,767 & 3.887 \\
\hline Total & 100.0 & 100.0 & 100.0 & 100.0 & 6,034 & \\
\hline $\mathrm{N}$ & 1,960 & 2,062 & 2,012 & 4,022 & & \\
\hline \multicolumn{7}{|c|}{ Only breastmilk for first 6 months } \\
\hline No & 90.2 & 92.1 & 80.5 & 91.3 & 5,299 & 130.291 \\
\hline Yes & 9.8 & 7.9 & 19.5 & 8.8 & 735 & 4.507 \\
\hline Total & 100.0 & 100.0 & 100.0 & 100.0 & 6,034 & \\
\hline $\mathrm{N}$ & 1,960 & 2,062 & 2,012 & 4,022 & & \\
\hline \multicolumn{7}{|c|}{ Safe storage of drinking water } \\
\hline No & 72.9 & 85.0 & 68.9 & 79.4 & 4,543 & 81.764 \\
\hline Yes & 27.1 & 15.0 & 31.1 & 20.6 & 1,491 & 3.554 \\
\hline Total & 100.0 & 100.0 & 100.0 & 100.0 & 6,034 & \\
\hline $\mathrm{N}$ & 1,960 & 2,062 & 2,012 & 4,022 & & \\
\hline \multicolumn{7}{|c|}{ Don't give dirty water } \\
\hline No & 62.6 & 68.1 & 58.6 & 65.6 & 3,874 & 29.999 \\
\hline Yes & 37.4 & 31.9 & 41.4 & 34.4 & 2,160 & 1.227 \\
\hline Total & 100.0 & 100.0 & 100.0 & 100.0 & 6,034 & \\
\hline N & 1,960 & 2,062 & 2,012 & 4,022 & & \\
\hline \multicolumn{7}{|c|}{ Don't eat bad food } \\
\hline No & 73.2 & 66.1 & 66.4 & 69.4 & 4,141 & 6.217 \\
\hline Yes & 26.8 & 33.9 & 33.7 & 30.6 & 1,893 & 0.211 \\
\hline Total & 100.0 & 100.0 & 100.0 & 100.0 & 6,034 & \\
\hline $\mathrm{N}$ & 1,960 & 2,062 & 2,012 & 4,022 & & \\
\hline \multicolumn{7}{|c|}{ Good nutrition } \\
\hline No & 85.8 & 90.3 & 84.4 & 88.3 & 5,233 & 17.779 \\
\hline Yes & 14.2 & 9.7 & 15.6 & 11.8 & 801 & 0.888 \\
\hline Total & 100.0 & 100.0 & 100.0 & 100.0 & 6,034 & \\
\hline $\mathrm{N}$ & 1,960 & 2,062 & 2,012 & 4,022 & & \\
\hline \multicolumn{7}{|c|}{ Handwashing } \\
\hline No & 82.3 & 89.3 & 77.7 & 86.1 & 4,964 & 66.386 \\
\hline Yes & 17.7 & 10.7 & 22.3 & 13.9 & 1,070 & 2.886 \\
\hline Total & 100.0 & 100.0 & 100.0 & 100.0 & 6,034 & \\
\hline$N$ & 1,960 & 2,062 & 2,012 & 4,022 & & \\
\hline \multicolumn{7}{|c|}{ Proper disposal of infant stool } \\
\hline No & 88.4 & 93.8 & 88.3 & 91.3 & 5,424 & 13.791 \\
\hline Yes & 11.6 & 6.2 & 11.7 & 8.7 & 610 & 0.617 \\
\hline Total & 100.0 & 100.0 & 100.0 & 100.0 & 6,034 & \\
\hline N & 1,960 & 2,062 & 2,012 & 4,022 & & \\
\hline \multicolumn{7}{|l|}{ Other } \\
\hline No & 99.8 & 99.0 & 98.9 & 99.4 & 5,979 & 4.726 \\
\hline Yes & 0.2 & 1.0 & 1.1 & 0.6 & 55 & 1.430 \\
\hline Total & 100.0 & 100.0 & 100.0 & 100.0 & 6,034 & \\
\hline $\mathrm{N}$ & 1,960 & 2,062 & 2,012 & 4,022 & & \\
\hline \multicolumn{7}{|c|}{ Don't know } \\
\hline No & 83.7 & 78.8 & 94.2 & 81.0 & 5,167 & 257.108 \\
\hline Yes & 16.4 & 21.2 & 5.8 & 19.0 & 867 & 13.854 \\
\hline Total & 100.0 & 100.0 & 100.0 & 100.0 & 6,034 & *** \\
\hline $\mathrm{N}$ & 1,960 & 2,062 & 2,012 & 4,022 & & \\
\hline
\end{tabular}

Estimates based on low Ns (approx. $<30$ obs) have large relative standard errors and should be interpreted with caution. ${ }^{* \star *}$ indicates $p<0.001,{ }^{* *}$ indicates $p<0.05$ and ${ }^{*}$ indicates $p<.0 .01$ for the chi ${ }^{2}$ statistic comparing integrated versus malaria-only study areas. 
TABLE 7.3.6. REPORTED TREATMENTS PERCEIVED AS EFFECTIVE AGAINST PEDIATRIC DIARRHEA

Percentage of women aged 15 to 49 years currently pregnant or with a child under 2 years reporting effective treatments for pediatric diarrhea, September-October 2019

\begin{tabular}{|c|c|c|c|c|c|c|}
\hline & KEBBI & SOKOTO & $\begin{array}{l}\text { MALARIA-ONLY } \\
\text { (ZAMFARA) }\end{array}$ & $\begin{array}{c}\text { INTEGRATED } \\
\text { (KEBBI/SOKOTO) }\end{array}$ & $\mathbf{N}$ & $\begin{array}{c}\mathrm{CHI}^{2} \\
\text { VALUE }\end{array}$ \\
\hline & $\%$ & $\%$ & $\%$ & $\%$ & & \\
\hline \multicolumn{7}{|l|}{ ORS } \\
\hline No & 43.6 & 56.9 & 17.7 & 50.7 & 2,417 & 739.898 \\
\hline Yes & 56.4 & 43.2 & 82.3 & 49.3 & 3,617 & 21.421 \\
\hline Total & 100.0 & 100.0 & 100.0 & 100.0 & 6,034 & *** \\
\hline $\mathrm{N}$ & 1,960 & 2,062 & 2,012 & 4,022 & & \\
\hline \multicolumn{7}{|l|}{ Zinc } \\
\hline No & 62.5 & 77.5 & 50.2 & 70.5 & 3,838 & 247.764 \\
\hline Yes & 37.5 & 22.5 & 49.8 & 29.5 & 2,196 & 7.687 \\
\hline Total & 100.0 & 100.0 & 100.0 & 100.0 & 6,034 & ** \\
\hline $\mathrm{N}$ & 1,960 & 2,062 & 2,012 & 4,022 & & \\
\hline \multicolumn{7}{|c|}{ Breastmilk } \\
\hline No & 86.0 & 93.6 & 80.6 & 90.1 & 5,281 & 99.915 \\
\hline Yes & 14.0 & 6.4 & 19.4 & 9.9 & 753 & 3.330 \\
\hline Total & 100.0 & 100.0 & 100.0 & 100.0 & 6,034 & \\
\hline $\mathrm{N}$ & 1,960 & 2,062 & 2,012 & 4,022 & & \\
\hline \multicolumn{7}{|c|}{ Other fluids } \\
\hline No & 97.6 & 98.6 & 95.7 & 98.2 & 5,893 & 27.300 \\
\hline Yes & 2.4 & 1.4 & 4.3 & 1.9 & 141 & 1.023 \\
\hline Total & 100.0 & 100.0 & 100.0 & 100.0 & 6,034 & \\
\hline $\mathrm{N}$ & 1,960 & 2,062 & 2,012 & 4,022 & & \\
\hline \multicolumn{7}{|c|}{ Antibiotics } \\
\hline No & 75.4 & 67.2 & 68.2 & 71.0 & 4,249 & 5.060 \\
\hline Yes & 24.6 & 32.8 & 31.8 & 29.0 & 1,785 & 0.153 \\
\hline Total & 100.0 & 100.0 & 100.0 & 100.0 & 6,034 & \\
\hline $\mathrm{N}$ & 1,960 & 2,062 & 2,012 & 4,022 & & \\
\hline \multicolumn{7}{|l|}{ Other } \\
\hline No & 97.7 & 90.2 & 97.3 & 93.7 & 5,734 & 49.013 \\
\hline Yes & 2.3 & 9.8 & 2.7 & 6.3 & 300 & 5.675 \\
\hline Total & 100.0 & 100.0 & 100.0 & 100.0 & 6,034 & * \\
\hline N & 1,960 & 2,062 & 2,012 & 4,022 & & \\
\hline \multicolumn{7}{|c|}{ Don't know } \\
\hline No & 74.4 & 75.6 & 90.8 & 75.0 & 4,799 & 274.009 \\
\hline Yes & 25.6 & 24.5 & 9.2 & 25.0 & 1,235 & 8.328 \\
\hline Total & 100.0 & 100.0 & 100.0 & 100.0 & 6,034 & ** \\
\hline $\mathrm{N}$ & 1,960 & 2,062 & 2,012 & 4,022 & & \\
\hline
\end{tabular}

Estimates based on low Ns (approx. $<30$ obs) have large relative standard errors and should be interpreted with caution. ${ }^{* * *}$ indicates $p<0.001,{ }^{* *}$ indicates $p<0.05$ and ${ }^{*}$ indicates $p<.0 .01$ for the chi ${ }^{2}$ statistic comparing integrated versus malaria-only study areas. 
TABLE 7.3.7. REPORTED WAYS ORS MAY HELP A CHILD WITH DIARRHEA

Percentage of women aged 15 to 49 years currently pregnant or with a child under 2 years reporting ways ORS may help a child with diarrhea, September-October 2019

\begin{tabular}{|c|c|c|c|c|c|c|}
\hline & KEBBI & SOKOTO & $\begin{array}{l}\text { MALARIA-ONLY } \\
\text { (ZAMFARA) }\end{array}$ & $\begin{array}{c}\text { INTEGRATED } \\
\text { (KEBBI/SOKOTO) }\end{array}$ & $\mathbf{N}$ & $\begin{array}{c}\text { CHI }^{2} \\
\text { VALUE }\end{array}$ \\
\hline & $\%$ & $\%$ & $\%$ & $\%$ & & \\
\hline \multicolumn{7}{|c|}{ Never heard of it } \\
\hline No & 89.2 & 84.9 & 95.4 & 86.9 & 5,455 & 142.473 \\
\hline Yes & 10.8 & 15.2 & 4.6 & 13.2 & 579 & 3.784 \\
\hline Total & 100.0 & 100.0 & 100.0 & 100.0 & 6,034 & \\
\hline $\mathrm{N}$ & 1,960 & 2,062 & 2,012 & 4,022 & & \\
\hline \multicolumn{7}{|c|}{ Replaces water lost } \\
\hline No & 46.1 & 50.0 & 29.6 & 48.2 & 2,573 & 217.403 \\
\hline Yes & 53.9 & 50.0 & 70.4 & 51.8 & 3,461 & 7.588 \\
\hline Total & 100.0 & 100.0 & 100.0 & 100.0 & 6,034 & ** \\
\hline $\mathrm{N}$ & 1,960 & 2,062 & 2,012 & 4,022 & & \\
\hline \multicolumn{7}{|c|}{ Stops diarrhea } \\
\hline No & 61.1 & 73.5 & 48.1 & 67.8 & 3,685 & 227.488 \\
\hline Yes & 38.9 & 26.5 & 51.9 & 32.3 & 2,349 & 8.292 \\
\hline Total & 100.0 & 100.0 & 100.0 & 100.0 & 6,034 & ** \\
\hline $\mathrm{N}$ & 1,960 & 2,062 & 2,012 & 4,022 & & \\
\hline \multicolumn{7}{|c|}{ Prevents dehydration } \\
\hline No & 76.0 & 88.9 & 60.8 & 83.0 & 4,619 & 340.023 \\
\hline Yes & 24.0 & 11.1 & 39.2 & 17.0 & 1,415 & 14.453 \\
\hline Total & 100.0 & 100.0 & 100.0 & 100.0 & 6,034 & *** \\
\hline $\mathrm{N}$ & 1,960 & 2,062 & 2,012 & 4,022 & & \\
\hline \multicolumn{7}{|c|}{ Replaces salt/electrolyte } \\
\hline No & 88.5 & 92.8 & 73.3 & 90.8 & 5,136 & 282.720 \\
\hline Yes & 11.5 & 7.2 & 26.7 & 9.2 & 898 & 11.158 \\
\hline Total & 100.0 & 100.0 & 100.0 & 100.0 & 6,034 & *** \\
\hline N & 1,960 & 2,062 & 2,012 & 4,022 & & \\
\hline \multicolumn{7}{|c|}{ Restores energy } \\
\hline No & 73.6 & 80.9 & 60.1 & 77.5 & 4,375 & 199.122 \\
\hline Yes & 26.4 & 19.2 & 39.9 & 22.5 & 1,659 & 6.374 \\
\hline Total & 100.0 & 100.0 & 100.0 & 100.0 & 6,034 & * \\
\hline $\mathrm{N}$ & 1,960 & 2,062 & 2,012 & 4,022 & & \\
\hline \multicolumn{7}{|l|}{ Other } \\
\hline No & 100.0 & 99.7 & 99.7 & 99.8 & 6,021 & 1.352 \\
\hline Yes & 0.1 & 0.3 & 0.4 & 0.2 & 13 & 0.727 \\
\hline Total & 100.0 & 100.0 & 100.0 & 100.0 & 6,034 & \\
\hline $\mathrm{N}$ & 1,960 & 2,062 & 2,012 & 4,022 & & \\
\hline \multicolumn{7}{|c|}{ Don't know } \\
\hline No & 84.2 & 81.5 & 93.4 & 82.7 & 5,172 & 170.813 \\
\hline Yes & 15.8 & 18.5 & 6.6 & 17.3 & 862 & 8.227 \\
\hline Total & 100.0 & 100.0 & 100.0 & 100.0 & 6,034 & ** \\
\hline $\mathrm{N}$ & 1,960 & 2,062 & 2,012 & 4,022 & & \\
\hline
\end{tabular}

Estimates based on low Ns (approx. $<30$ obs) have large relative standard errors and should be interpreted with caution. ${ }^{* * *}$ indicates $p<0.001,{ }^{* *}$ indicates $p<0.05$ and ${ }^{*}$ indicates $p<.0 .01$ for the chi ${ }^{2}$ statistic comparing integrated versus malaria-only study areas. 
TABLE 7.3.8. REPORTED WAYS ZINC MAY HELP A CHILD WITH DIARRHEA

Percentage of women aged 15 to 49 years currently pregnant or with a child under 2 years reporting ways zinc may help a child with diarrhea, September-October 2019

\begin{tabular}{|c|c|c|c|c|c|c|}
\hline & KEBBI & SOKOTO & $\begin{array}{l}\text { MALARIA-ONLY } \\
\text { (ZAMFARA) }\end{array}$ & $\begin{array}{c}\text { INTEGRATED } \\
\text { (KEBBI/SOKOTO) }\end{array}$ & $\mathbf{N}$ & $\begin{array}{c}\mathrm{CHI}^{2} \\
\text { VALUE }\end{array}$ \\
\hline & $\%$ & $\%$ & $\%$ & $\%$ & & \\
\hline \multicolumn{7}{|c|}{ Never heard of it } \\
\hline No & 73.4 & 64.8 & 80.4 & 68.8 & 4,497 & 107.134 \\
\hline Yes & 26.6 & 35.3 & 19.6 & 31.2 & 1,537 & 3.499 \\
\hline Total & 100.0 & 100.0 & 100.0 & 100.0 & 6,034 & \\
\hline $\mathrm{N}$ & 1,960 & 2,062 & 2,012 & 4,022 & & \\
\hline \multicolumn{7}{|c|}{ Make less severe } \\
\hline No & 70.7 & 80.9 & 62.5 & 76.2 & 4,288 & 124.634 \\
\hline Yes & 29.3 & 19.1 & 37.5 & 23.8 & 1,746 & 5.076 \\
\hline Total & 100.0 & 100.0 & 100.0 & 100.0 & 6,034 & * \\
\hline $\mathrm{N}$ & 1,960 & 2,062 & 2,012 & 4,022 & & \\
\hline \multicolumn{7}{|c|}{ Shorter duration } \\
\hline No & 70.4 & 78.3 & 52.9 & 74.7 & 4,085 & 292.191 \\
\hline Yes & 29.6 & 21.7 & 47.1 & 25.3 & 1,949 & 11.623 \\
\hline Total & 100.0 & 100.0 & 100.0 & 100.0 & 6,034 & *** \\
\hline $\mathrm{N}$ & 1,960 & 2,062 & 2,012 & 4,022 & & \\
\hline \multicolumn{7}{|c|}{ Prevent dehydration } \\
\hline No & 83.0 & 89.2 & 67.0 & 86.3 & 4,824 & 290.22 \\
\hline Yes & 17.0 & 10.8 & 33.0 & 13.7 & 1,210 & 13.484 \\
\hline Total & 100.0 & 100.0 & 100.0 & 100.0 & 6,034 & *** \\
\hline $\mathrm{N}$ & 1,960 & 2,062 & 2,012 & 4,022 & & \\
\hline \multicolumn{7}{|c|}{ Less likely to reoccur } \\
\hline No & 95.0 & 96.1 & 82.7 & 95.6 & 5,524 & 228.305 \\
\hline Yes & 5.0 & 3.9 & 17.3 & 4.4 & 510 & 11.527 \\
\hline Total & 100.0 & 100.0 & 100.0 & 100.0 & 6,034 & *** \\
\hline N & 1,960 & 2,062 & 2,012 & 4,022 & & \\
\hline \multicolumn{7}{|c|}{ Restore energy } \\
\hline No & 80.5 & 87.1 & 72.6 & 84.0 & 4,800 & 108.909 \\
\hline Yes & 19.5 & 12.9 & 27.5 & 16.0 & 1,234 & 4.545 \\
\hline Total & 100.0 & 100.0 & 100.0 & 100.0 & 6,034 & * \\
\hline $\mathrm{N}$ & 1,960 & 2,062 & 2,012 & 4,022 & & \\
\hline \multicolumn{7}{|l|}{ Other } \\
\hline No & 99.8 & 100.0 & 99.8 & 99.9 & 6,027 & 0.345 \\
\hline Yes & 0.2 & 0.0 & 0.2 & 0.1 & 7 & 0.193 \\
\hline Total & 100.0 & 100.0 & 100.0 & 100.0 & 6,034 & \\
\hline$N$ & 1,960 & 2,062 & 2,012 & 4,022 & & \\
\hline \multicolumn{7}{|c|}{ Don't know } \\
\hline No & 78.2 & 76.4 & 84.4 & 77.2 & 4,724 & 48.944 \\
\hline Yes & 21.8 & 23.6 & 15.7 & 22.8 & 1,310 & 2.121 \\
\hline Total & 100.0 & 100.0 & 100.0 & 100.0 & 6,034 & \\
\hline $\mathrm{N}$ & 1,960 & 2,062 & 2,012 & 4,022 & & \\
\hline
\end{tabular}

Estimates based on low Ns (approx. $<30$ obs) have large relative standard errors and should be interpreted with caution. ${ }^{* * *}$ indicates $p<0.001,{ }^{* *}$ indicates $p<0.05$ and ${ }^{*}$ indicates $p<.0 .01$ for the chi ${ }^{2}$ statistic comparing integrated versus malaria-only study areas. 


\section{TABLE 7.3.9. DIARRHEA-RELATED BELIEFS, INTENTIONS, AND SELF-EFFICACY}

Percentage of women aged 15 to 49 years currently pregnant or with a child under 2 years reporting certain diarrhearelated beliefs, intentions, and self-efficacy, September-October 2019

\begin{tabular}{|c|c|c|c|c|c|c|}
\hline & $\begin{array}{c}\text { KEBBI } \\
\%\end{array}$ & $\begin{array}{c}\text { SOKOTO } \\
\%\end{array}$ & $\begin{array}{c}\text { MALARIA-ONLY } \\
\text { (ZAMFARA) } \\
\%\end{array}$ & $\begin{array}{c}\text { INTEGRATED } \\
\text { (KEBBI/SOKOTO) } \\
\%\end{array}$ & $\mathbf{N}$ & $\begin{array}{c}\mathrm{CHI}^{2} \\
\text { VALUE }\end{array}$ \\
\hline \multicolumn{7}{|c|}{$\begin{array}{l}\text { It is very common for children to get } \\
\text { diarrhea in my community }\end{array}$} \\
\hline Agree & 69.0 & 78.0 & 67.4 & 73.8 & 4,273 & 118.281 \\
\hline Disagree & 23.1 & 14.0 & 28.7 & 18.2 & 1,339 & 2.348 \\
\hline Don't know & 7.8 & 8.1 & 3.9 & 8.0 & 421 & \\
\hline Total & 100.0 & 100.0 & 100.0 & 100.0 & 6,033 & \\
\hline N & 1,960 & 2,062 & 2,011 & 4,022 & & \\
\hline \multicolumn{7}{|c|}{$\begin{array}{l}\text { In young children diarrhea can lead } \\
\text { to dehydration and even death }\end{array}$} \\
\hline Agree & 81.5 & 87.3 & 93.8 & 84.6 & 5,227 & 203.744 \\
\hline Disagree & 5.7 & 6.4 & 4.6 & 6.1 & 362 & 7.340 \\
\hline Don't know & 12.8 & 6.4 & 1.6 & 9.4 & 444 & ** \\
\hline Total & 100.0 & 100.0 & 100.0 & 100.0 & 6,033 & \\
\hline N & 1,960 & 2,062 & 2,011 & 4,022 & & \\
\hline \multicolumn{7}{|c|}{ I know how to prepare ORS } \\
\hline Agree & 50.5 & 53.1 & 80.8 & 51.9 & 3,674 & 568.968 \\
\hline Disagree & 24.3 & 23.0 & 8.8 & 23.6 & 1,145 & 10.686 \\
\hline Don't know & 25.2 & 23.9 & 10.4 & 24.5 & 1,214 & $* \star *$ \\
\hline Total & 100.0 & 100.0 & 100.0 & 100.0 & 6,033 & \\
\hline $\mathrm{N}$ & 1,960 & 2,062 & 2,011 & 4,022 & & \\
\hline \multicolumn{7}{|c|}{$\begin{array}{l}\text { Likelihood to continue breastfeeding } \\
\text { if child develops diarrhea }\end{array}$} \\
\hline Likely & 89.3 & 89.2 & 97.3 & 89.3 & 2,682 & 82.268 \\
\hline Unlikely & 6.0 & 4.9 & 1.7 & 5.4 & 129 & 6.385 \\
\hline Don't know & 4.7 & 5.8 & 1.0 & 5.3 & 102 & ** \\
\hline Total & 100.0 & 100.0 & 100.0 & 100.0 & 2,913 & \\
\hline$N$ & 864 & 1,045 & 1,004 & 1,909 & & \\
\hline \multicolumn{7}{|c|}{$\begin{array}{l}\text { Likelihood to continue ORS the next } \\
\text { time child develops diarrhea }\end{array}$} \\
\hline Likely & 85.0 & 73.7 & 92.8 & 78.9 & 5,042 & 254.019 \\
\hline Unlikely & 6.2 & 16.4 & 4.5 & 11.6 & 529 & 6.685 \\
\hline Don't know & 8.9 & 10.0 & 2.7 & 9.5 & 462 & ** \\
\hline Total & 100.0 & 100.0 & 100.0 & 100.0 & 6,033 & \\
\hline $\mathrm{N}$ & 1,960 & 2,062 & 2,011 & 4,022 & & \\
\hline \multicolumn{7}{|c|}{$\begin{array}{l}\text { Likelihood to give zinc the next time } \\
\text { child develops diarrhea }\end{array}$} \\
\hline Likely & 79.1 & 59.8 & 81.2 & 68.8 & 4,418 & 125.621 \\
\hline Unlikely & 8.5 & 23.1 & 10.3 & 16.3 & 786 & 2.095 \\
\hline Don't know & 12.4 & 17.1 & 8.5 & 14.9 & 829 & \\
\hline Total & 100.0 & 100.0 & 100.0 & 100.0 & 6,033 & \\
\hline $\mathrm{N}$ & 1,960 & 2,062 & 2,011 & 4,022 & & \\
\hline
\end{tabular}

Estimates based on low Ns (approx. $<30$ obs) have large relative standard errors and should be interpreted with caution. ${ }^{* * *}$ indicates $p<0.001,{ }^{* *}$ indicates $p<0.05$ and $^{*}$ indicates $p<.0 .01$ for the chi ${ }^{2}$ statistic comparing integrated versus malaria-only study areas. 


\subsection{Child health ideations}

\section{Key findings}

Among respondents, nearly all (90\%) agreed that a health provider was the best person to consult when a child is sick. Approximately 70 percent agreed that facilities in their community frequently have necessary treatment for sick children. Nearly all respondents (92\%) also felt confident that they could convince their partner to seek care or advice for a sick child. The most common influencers of decisions about seeking care for a sick child were spouses (81\%) followed by no one else $(16 \%)$ and mothers-in-law (10\%). Nevertheless, reported perceptions of quality care at health facilities do not appear to translate into correct behaviors; significant proportions of respondents choose pharmacies for care over health facilities, which merits further investigation.

\section{TABLE 7.4.1. PERCEPTIONS OF HEALTH SERVICES QUALITY FOR SICK CHILDREN}

Percentage of women aged 15 to 49 years currently pregnant or with a child under 2 years reporting perceptions of health services quality for sick children, September-October 2019

\begin{tabular}{|c|c|c|c|c|c|c|}
\hline & $\begin{array}{l}\text { KEBBI } \\
\%\end{array}$ & $\begin{array}{l}\text { SOKOTO } \\
\%\end{array}$ & $\begin{array}{c}\text { MALARIA-ONLY } \\
\text { (ZAMFARA) } \\
\%\end{array}$ & $\begin{array}{c}\text { INTEGRATED } \\
\text { (KEBBI/SOKOTO) } \\
\%\end{array}$ & $\mathbf{N}$ & $\begin{array}{c}\mathrm{CHI}^{2} \\
\text { VALUE }\end{array}$ \\
\hline \multicolumn{7}{|c|}{$\begin{array}{l}\text { Health provider is always the best } \\
\text { person to talk to when child is sick }\end{array}$} \\
\hline Agree & 88.0 & 84.0 & 92.2 & 85.8 & 5,335 & 91.895 \\
\hline Disagree & 9.0 & 12.2 & 7.2 & 10.7 & 544 & 2.398 \\
\hline Don't know & 3.0 & 3.8 & 0.7 & 3.4 & 154 & \\
\hline Total & 100.0 & 100.0 & 100.0 & 100.0 & 6,033 & \\
\hline $\mathrm{N}$ & 1,960 & 2,062 & 2,011 & 4,022 & & \\
\hline \multicolumn{7}{|c|}{$\begin{array}{l}\text { Facilities in my community often } \\
\text { have treatment needed for sick child }\end{array}$} \\
\hline Agree & 67.9 & 65.7 & 72.8 & 66.7 & 4,184 & 56.050 \\
\hline Disagree & 26.2 & 25.5 & 23.8 & 25.9 & 1,481 & 1.267 \\
\hline Don't know & 5.9 & 8.7 & 3.4 & 7.4 & 368 & \\
\hline Total & 100.0 & 100.0 & 100.0 & 100.0 & 6,033 & \\
\hline $\mathrm{N}$ & 1,960 & 2,062 & 2,011 & 4,022 & & \\
\hline
\end{tabular}

Estimates based on low Ns (approx. $<30$ obs) have large relative standard errors and should be interpreted with caution. ${ }^{* \star *}$ indicates $p<0.001,{ }^{* *}$ indicates $p<0.05$ and * indicates $p<.0 .01$ for the chi ${ }^{2}$ statistic comparing integrated versus malaria-only study areas.

\section{TABLE 7.4.2. SELF-EFFICACY IN CONVINCING PARTNER TO SEEK CARE FOR A SICK CHILD}

Percentage of women aged 15 to 49 years currently pregnant or with a child under 2 years reporting confidence to convince partner to seek care for a sick child, September-October 2019

\begin{tabular}{|c|c|c|c|c|c|c|}
\hline & KEBBI & sокото & $\begin{array}{l}\text { MALARIA-ONLY } \\
\text { (ZAMFARA) }\end{array}$ & $\begin{array}{l}\text { INTEGRATED } \\
\text { (KEBBI/SOKOTO) }\end{array}$ & $\mathbf{N}$ & $\begin{array}{l}\mathrm{CHI}^{2} \\
\text { VALUE }\end{array}$ \\
\hline & $\%$ & $\%$ & $\%$ & $\%$ & & \\
\hline \multicolumn{7}{|c|}{$\begin{array}{l}\text { Confidence to convince partner to } \\
\text { seek care for a sick child }\end{array}$} \\
\hline Confident & 87.7 & 88.2 & 95.0 & 88.0 & 5,467 & 114.650 \\
\hline Uncertain & 9.2 & 8.2 & 4.5 & 8.7 & 430 & 2.846 \\
\hline Don't know & 3.1 & 3.6 & 0.6 & 3.4 & 136 & \\
\hline Total & 100.0 & 100.0 & 100.0 & 100.0 & 6,033 & \\
\hline N & 1,960 & 2,062 & 2,011 & 4,022 & & \\
\hline
\end{tabular}

Estimates based on low Ns (approx. $<30$ obs) have large relative standard errors and should be interpreted with caution. ${ }^{* \star *}$ indicates $p<0.001,{ }^{* *}$ indicates $p<0.05$ and * indicates $p<.0 .01$ for the chi ${ }^{2}$ statistic comparing integrated versus malaria-only study areas. 
TABLE 7.4.3. WHO ELSE INFLUENCES DECISIONS ABOUT SEEKING CARE FOR A SICK CHILD?

Percentage of women aged 15 to 49 years currently pregnant or with a child under 2 years reporting who else influences a woman's decision or seek care for a sick child, September-October 2019

\begin{tabular}{|c|c|c|c|c|c|c|}
\hline & KEBBI & SOKOTO & $\begin{array}{l}\text { MALARIA-ONLY } \\
\text { (ZAMFARA) }\end{array}$ & $\begin{array}{c}\text { INTEGRATED } \\
\text { (KEBBI/SOKOTO) }\end{array}$ & $\mathbf{N}$ & $\begin{array}{c}\text { CHI }^{2} \\
\text { VALUE }\end{array}$ \\
\hline & $\%$ & $\%$ & $\%$ & $\%$ & & \\
\hline \multicolumn{7}{|c|}{ No one else } \\
\hline No & 82.1 & 86.8 & 83.0 & 84.7 & 5,032 & 3.047 \\
\hline Yes & 17.9 & 13.2 & 17.0 & 15.3 & 1,002 & 0.084 \\
\hline Total & 100.0 & 100.0 & 100.0 & 100.0 & 6,034 & \\
\hline $\mathrm{N}$ & 1,960 & 2,062 & 2,012 & 4,022 & & \\
\hline \multicolumn{7}{|c|}{ Husband/partner } \\
\hline No & 20.6 & 17.5 & 19.1 & 18.9 & 1,192 & 0.016 \\
\hline Yes & 79.4 & 82.5 & 81.0 & 81.1 & 4,842 & 0.000 \\
\hline Total & 100.0 & 100.0 & 100.0 & 100.0 & 6,034 & \\
\hline N & 1,960 & 2,062 & 2,012 & 4,022 & & \\
\hline \multicolumn{7}{|c|}{ Mother-in-law } \\
\hline No & 87.3 & 84.2 & 92.7 & 85.6 & 5,360 & 79.201 \\
\hline Yes & 12.8 & 15.8 & 7.3 & 14.4 & 674 & 5.187 \\
\hline Total & 100.0 & 100.0 & 100.0 & 100.0 & 6,034 & * \\
\hline N & 1,960 & 2,062 & 2,012 & 4,022 & & \\
\hline \multicolumn{7}{|l|}{ Mother } \\
\hline No & 89.6 & 93.7 & 93.0 & 91.8 & 5,535 & 2.806 \\
\hline Yes & 10.4 & 6.3 & 7.0 & 8.2 & 499 & 0.167 \\
\hline Total & 100.0 & 100.0 & 100.0 & 100.0 & 6,034 & \\
\hline $\mathrm{N}$ & 1,960 & 2,062 & 2,012 & 4,022 & & \\
\hline \multicolumn{7}{|c|}{ Friends } \\
\hline No & 99.0 & 98.4 & 95.8 & 98.7 & 5,885 & 42.471 \\
\hline Yes & 1.0 & 1.6 & 4.2 & 1.3 & 149 & 4.158 \\
\hline Total & 100.0 & 100.0 & 100.0 & 100.0 & 6,034 & * \\
\hline N & 1,960 & 2,062 & 2,012 & 4,022 & & \\
\hline \multicolumn{7}{|c|}{ Health provider } \\
\hline No & 98.4 & 97.0 & 95.8 & 97.6 & 5,859 & 15.493 \\
\hline Yes & 1.6 & 3.0 & 4.3 & 2.4 & 175 & 1.202 \\
\hline Total & 100.0 & 100.0 & 100.0 & 100.0 & 6,034 & \\
\hline N & 1,960 & 2,062 & 2,012 & 4,022 & & \\
\hline \multicolumn{7}{|c|}{ Religious/community leader } \\
\hline No & 99.5 & 99.9 & 99.6 & 99.8 & 6,017 & 0.865 \\
\hline Yes & 0.5 & 0.1 & 0.4 & 0.3 & 17 & 0.279 \\
\hline Total & 100.0 & 100.0 & 100.0 & 100.0 & 6,034 & \\
\hline $\mathrm{N}$ & 1,960 & 2,062 & 2,012 & 4,022 & & \\
\hline \multicolumn{7}{|c|}{ Partner's other family members } \\
\hline No & 99.9 & 99.2 & 99.2 & 99.5 & 6,002 & 2.505 \\
\hline Yes & 0.1 & 0.8 & 0.8 & 0.5 & 32 & 0.389 \\
\hline Total & 100.0 & 100.0 & 100.0 & 100.0 & 6,034 & \\
\hline $\mathrm{N}$ & 1,960 & 2,062 & 2,012 & 4,022 & & \\
\hline \multicolumn{7}{|c|}{ Own other family members } \\
\hline No & 99.6 & 99.4 & 99.8 & 99.5 & 6,007 & 3.024 \\
\hline Yes & 0.4 & 0.7 & 0.2 & 0.5 & 27 & 1.487 \\
\hline Total & 100.0 & 100.0 & 100.0 & 100.0 & 6,034 & \\
\hline$N$ & 1,960 & 2,062 & 2,012 & 4,022 & & \\
\hline \multicolumn{7}{|l|}{ Other } \\
\hline No & 99.8 & 99.7 & 99.9 & 99.8 & 6,017 & 1.264 \\
\hline Yes & 0.2 & 0.3 & 0.1 & 0.2 & 17 & 0.645 \\
\hline Total & 100.0 & 100.0 & 100.0 & 100.0 & 6,034 & \\
\hline $\mathrm{N}$ & 1,960 & 2,062 & 2,012 & 4,022 & & \\
\hline
\end{tabular}

Estimates based on low Ns (approx. $<30$ obs) have large relative standard errors and should be interpreted with caution. ${ }^{* \star *}$ indicates $p<0.001,{ }^{* \star}$ indicates $p<0.05$ and ${ }^{*}$ indicates $p<.0 .01$ for the chi ${ }^{2}$ statistic comparing integrated versus malaria-only study areas. 


\section{Key findings}

Community participation: Among respondents, most $(88 \%)$ reported regularly attending group or community meetings. Among those who reported regular meeting attendance, 44 percent participated in women's meetings, with slightly higher attendance in Zamfara (49\%) than in Kebbi/Sokoto (35\%). Attendance at other types of community meetings (e.g., agricultural, civic) was very low. This result could indicate potentially low participation of respondents in community meetings organized by Breakthrough ACTION/Nigeria to disseminate health messages, with possibly lower participation rates in Kebbi/Sokoto than in Zamfara. This should be considered during the planning of household visits and community events to ensure outreach targets women who may not be prone to community meeting participation, such as by engaging men and local leaders to support reticent women to attend these gatherings.

Household decision-making: Across study areas, many respondents reported that their spouse mainly makes decisions for the household, except in the case of how to use the respondents' own money, where half said they make that decision alone. Half (50\%) of respondents said that household decisions about major purchases, schooling, work outside the home, and how to use the partner's money were made exclusively by their partner. In these cases, approximately one-quarter of respondents reported joint decision-making between themselves and their spouse, with significantly higher joint decision-making in Zamfara than in Kebbi/Sokoto for nearly all of these decisions.

Gender roles: Across study areas, most respondents agreed that it is a woman's job to mainly take care of the home and cook for her family (88\%), that a good marriage is more important for a girl than a good education (79\%), and that it is more important for boys to get an education than it is for girls (71\%). Nevertheless, nearly all respondents (94\%) agreed that it is also important for couples to discuss and make decisions about child health together, and 85 percent agreed that a woman should play a role in making decisions about the household, even though reported joint decision-making was relatively low. 
TABLE 8.1.1. WOMEN'S COMMUNITY PARTICIPATION

Percentage of women aged 15 to 49 years currently pregnant or with a child under 2 years reporting regular attendance at group or community meetings by meeting type, September-October 2019

\begin{tabular}{|c|c|c|c|c|c|c|}
\hline & KEBBI & SOKOTO & $\begin{array}{l}\text { INTEGRATED } \\
\text { (KEBBI/SOKOTO) }\end{array}$ & $\begin{array}{l}\text { MALARIA-ONLY } \\
\text { (ZAMFARA) }\end{array}$ & $\mathbf{N}$ & $\begin{array}{c}\mathrm{CHI}^{2} \\
\text { VALUE }\end{array}$ \\
\hline & $\%$ & $\%$ & $\%$ & $\%$ & & \\
\hline \multicolumn{7}{|c|}{$\begin{array}{l}\text { Do you regularly attend any } \\
\text { meetings in your community? }\end{array}$} \\
\hline No & 10.8 & 6.6 & 8.6 & 10.5 & 579 & 6.343 \\
\hline Yes & 89.2 & 93.4 & 91.4 & 89.5 & 5,382 & 0.255 \\
\hline Total & 100.0 & 100.0 & 100.0 & 100.0 & 5,961 & \\
\hline $\mathrm{N}$ & 1,942 & 2,042 & 3,984 & 1,977 & & \\
\hline \multicolumn{7}{|c|}{$\begin{array}{l}\text { What types of community meetings } \\
\text { do you attend? }\end{array}$} \\
\hline \multicolumn{7}{|c|}{ Women's group } \\
\hline No & 97.5 & 96.6 & 97.0 & 94.8 & 5,741 & 17.211 \\
\hline Yes & 2.5 & 3.4 & 3.0 & 5.2 & 220 & 0.838 \\
\hline Total & 100.0 & 100.0 & 100.0 & 100.0 & 5,961 & \\
\hline $\mathrm{N}$ & 1,942 & 2,042 & 3,984 & 1,977 & & \\
\hline \multicolumn{7}{|c|}{ Civic group } \\
\hline No & 96.7 & 99.8 & 98.4 & 98.5 & 5,878 & 0.049 \\
\hline Yes & 3.3 & 0.2 & 1.6 & 1.5 & 83 & 0.001 \\
\hline Total & 100.0 & 100.0 & 100.0 & 100.0 & 5,961 & \\
\hline $\mathrm{N}$ & 1,942 & 2,042 & 3,984 & 1,977 & & \\
\hline \multicolumn{7}{|c|}{ Religious/church } \\
\hline No & 96.6 & 98.3 & 97.5 & 98.8 & 5,821 & 14.043 \\
\hline Yes & 3.4 & 1.7 & 2.5 & 1.2 & 140 & 0.873 \\
\hline Total & 100.0 & 100.0 & 100.0 & 100.0 & 5,961 & \\
\hline$N$ & 1,942 & 2,042 & 3,984 & 1,977 & & \\
\hline
\end{tabular}

Estimates based on low Ns (approx. $<30$ obs) have large relative standard errors and should be interpreted with caution. ${ }^{* * *}$ indicates $p<0.001,{ }^{* *}$ indicates $p<0.05$ and * indicates $p<.0 .01$ for the chi ${ }^{2}$ statistic comparing integrated versus malaria-only study areas. 
TABLE 8.1.2. WOMEN'S ROLE IN HOUSEHOLD DECISION-MAKING

Percentage of women aged 15 to 49 years currently pregnant or with a child under 2 years reporting household decision-making roles by decision type, September-October 2019

\begin{tabular}{|c|c|c|c|c|c|c|}
\hline & KEBBI & SOKOTO & $\begin{array}{l}\text { MALARIA-ONLY } \\
\text { (ZAMFARA) }\end{array}$ & $\begin{array}{c}\text { INTEGRATED } \\
\text { (KEBBI/SOKOTO) }\end{array}$ & $\mathbf{N}$ & $\begin{array}{c}\mathrm{CHI}^{2} \\
\text { VALUE }\end{array}$ \\
\hline & $\%$ & $\%$ & $\%$ & $\%$ & & \\
\hline \multicolumn{7}{|c|}{ How your money will be used } \\
\hline Respondent & 35.8 & 56.2 & 54.4 & 46.8 & 2,920 & 148.221 \\
\hline Spouse & 29.5 & 24.3 & 28.6 & 26.7 & 1,628 & 1.830 \\
\hline Both & 27.0 & 15.1 & 15.8 & 20.6 & 1,153 & \\
\hline Other & 0.9 & 0.3 & 0.4 & 0.6 & 39 & \\
\hline Don't know & 6.8 & 4.1 & 0.8 & 5.4 & 221 & \\
\hline Total & 100.0 & 100.0 & 100.0 & 100.0 & 5,961 & \\
\hline $\mathrm{N}$ & 1,942 & 2,042 & 1,977 & 3,984 & & \\
\hline \multicolumn{7}{|c|}{ How husband's money will be used } \\
\hline Respondent & 4.2 & 4.2 & 17.5 & 4.2 & 549 & 345.879 \\
\hline Spouse & 58.6 & 75.9 & 49.8 & 67.9 & 3,605 & 4.515 \\
\hline Both & 29.6 & 19.3 & 30.7 & 24.1 & 1,601 & ** \\
\hline Other & 1.1 & 0.4 & 0.9 & 0.7 & 53 & \\
\hline Don't know & 6.5 & 0.2 & 1.2 & 3.2 & 153 & \\
\hline Total & 100.0 & 100.0 & 100.0 & 100.0 & 5,961 & \\
\hline $\mathrm{N}$ & 1,942 & 2,042 & 1,977 & 3,984 & & \\
\hline \multicolumn{7}{|c|}{ Major household purchases } \\
\hline Respondent & 4.2 & 3.5 & 11.7 & 3.8 & 416 & 246.854 \\
\hline Spouse & 62.8 & 75.2 & 50.9 & 69.4 & 3,717 & 2.685 \\
\hline Both & 24.1 & 20.1 & 32.4 & 22.0 & 1,532 & * \\
\hline Other & 1.2 & 0.8 & 0.9 & 1.0 & 59 & \\
\hline Don't know & 7.7 & 0.4 & 4.2 & 3.8 & 237 & \\
\hline Total & 100.0 & 100.0 & 100.0 & 100.0 & 5,961 & \\
\hline N & 1,942 & 2,042 & 1,977 & 3,984 & & \\
\hline \multicolumn{7}{|l|}{ Schooling } \\
\hline Respondent & 3.5 & 2.7 & 7.9 & 3.1 & 306 & 347.146 \\
\hline Spouse & 51.1 & 50.9 & 43.1 & 51.0 & 2,966 & 3.382 \\
\hline Both & 17.4 & 9.3 & 29.2 & 13.1 & 1,072 & * \\
\hline Other & 1.4 & 0.7 & 1.1 & 1.0 & 66 & \\
\hline Don't know & 26.6 & 36.4 & 18.7 & 31.9 & 1,551 & \\
\hline Total & 100.0 & 100.0 & 100.0 & 100.0 & 5,961 & \\
\hline $\mathrm{N}$ & 1,942 & 2,042 & 1,977 & 3,984 & & \\
\hline \multicolumn{7}{|c|}{ Working outside home } \\
\hline Respondent & 5.5 & 2.9 & 19.9 & 4.1 & 561 & 427.573 \\
\hline Spouse & 59.2 & 70.7 & 48.9 & 65.4 & 3,544 & 3.863 \\
\hline Both & 16.4 & 9.8 & 16.0 & 12.9 & 851 & ** \\
\hline Other & 1.6 & 0.9 & 4.3 & 1.2 & 127 & \\
\hline Don't know & 17.4 & 15.7 & 10.9 & 16.5 & 878 & \\
\hline Total & 100.0 & 100.0 & 100.0 & 100.0 & 5,961 & \\
\hline $\mathrm{N}$ & 1,942 & 2,042 & 1,977 & 3,984 & & \\
\hline
\end{tabular}

Estimates based on low Ns (approx. <30 obs) have large relative standard errors and should be interpreted with caution. ${ }^{* \star *}$ indicates $p<0.001,{ }^{\star \star}$ indicates $p<0.05$ and ${ }^{*}$ indicates $p<.0 .01$ for the chi ${ }^{2}$ statistic comparing integrated versus malaria-only study areas. 
TABLE 8.1.3. GENDER-RELATED BELIEFS, ATTITUDES, AND PERCEPTIONS

Percentage of women aged 15 to 49 years currently pregnant or with a child under 2 years who agreed with certain gender-related beliefs, attitudes, or perceptions, September-October 2019

\begin{tabular}{|c|c|c|c|c|c|c|}
\hline & KEBBI & SOKOTO & $\begin{array}{l}\text { MALARIA-ONLY } \\
\text { (ZAMFARA) }\end{array}$ & $\begin{array}{l}\text { INTEGRATED } \\
\text { (KEBBI/SOKOTO) }\end{array}$ & $\mathbf{N}$ & $\begin{array}{l}\mathrm{CHI}^{2} \\
\text { VALUE }\end{array}$ \\
\hline & $\%$ & $\%$ & $\%$ & $\%$ & & \\
\hline \multicolumn{7}{|c|}{$\begin{array}{l}\text { Important for couples to discuss and } \\
\text { decide together about child health }\end{array}$} \\
\hline Agree & 93.8 & 92.9 & 96.6 & 93.3 & 5,653 & 55.394 \\
\hline Disagree & 4.3 & 3.4 & 2.8 & 3.8 & 205 & 1.930 \\
\hline Don't know & 1.9 & 3.7 & 0.6 & 2.9 & 103 & \\
\hline Total & 100.0 & 100.0 & 100.0 & 100.0 & 5,961 & \\
\hline $\mathrm{N}$ & 1,942 & 2,042 & 1,977 & 3,984 & & \\
\hline \multicolumn{7}{|c|}{$\begin{array}{l}\text { A woman should play a role in making } \\
\text { decisions about the household }\end{array}$} \\
\hline Agree & 84.7 & 84.3 & 87.1 & 84.5 & 5,135 & 42.484 \\
\hline Disagree & 13.9 & 12.2 & 12.4 & 13.0 & 732 & 0.848 \\
\hline Don't know & 1.4 & 3.5 & 0.6 & 2.5 & 94 & \\
\hline Total & 100.0 & 100.0 & 100.0 & 100.0 & 5,961 & \\
\hline$N$ & 1,942 & 2,042 & 1,977 & 3,984 & & \\
\hline \multicolumn{7}{|c|}{$\begin{array}{l}\text { It is more important for boys to get an } \\
\text { education than it is for girls }\end{array}$} \\
\hline Agree & 73.1 & 75.0 & 71.1 & 74.1 & 4,292 & 49.659 \\
\hline Disagree & 25.1 & 21.4 & 28.0 & 23.1 & 1,554 & 1.392 \\
\hline Don't know & 1.8 & 3.7 & 0.8 & 2.8 & 115 & \\
\hline Total & 100.0 & 100.0 & 100.0 & 100.0 & 5,961 & \\
\hline $\mathrm{N}$ & 1,942 & 2,042 & 1,977 & 3,984 & & \\
\hline \multicolumn{7}{|c|}{$\begin{array}{l}\text { A good marriage is more important for } \\
\text { a girl than a good education }\end{array}$} \\
\hline Agree & 78.3 & 86.7 & 77.9 & 82.9 & 4,841 & 62.878 \\
\hline Disagree & 19.9 & 10.9 & 21.5 & 15.1 & 1,038 & 1.695 \\
\hline Don't know & 1.8 & 2.3 & 0.6 & 2.1 & 82 & \\
\hline Total & 100.0 & 100.0 & 100.0 & 100.0 & 5,961 & \\
\hline$N$ & 1,942 & 2,042 & 1,977 & 3,984 & & \\
\hline \multicolumn{7}{|c|}{$\begin{array}{l}\text { It is a woman's job to mainly take care } \\
\text { of the home and cook for her family }\end{array}$} \\
\hline Agree & 92.9 & 92.2 & 86.8 & 92.5 & 5,405 & 146.210 \\
\hline Disagree & 5.5 & 5.3 & 13.0 & 5.4 & 486 & 6.481 \\
\hline Don't know & 1.6 & 2.5 & 0.2 & 2.1 & 70 & ** \\
\hline Total & 100.0 & 100.0 & 100.0 & 100.0 & 5,961 & \\
\hline $\mathrm{N}$ & 1,942 & 2,042 & 1,977 & 3,984 & & \\
\hline
\end{tabular}

Estimates based on low Ns (approx. $<30$ obs) have large relative standard errors and should be interpreted with caution. ${ }^{* \star *}$ indicates $p<0.001,{ }^{* *}$ indicates $p<0.05$ and * indicates $p<.0 .01$ for the chi ${ }^{2}$ statistic comparing integrated versus malaria-only study areas. 


\section{Media exposure}

\section{Key findings}

Media exposure: Radio listening is the most common form of media exposure, with about one-third of respondents reporting regular radio listening across study areas. Only a small percentage of respondents-less than 10 percent-report regularly viewing television. Beyond radio and television, exposure to other media sources is extremely low, with less than one percent of respondents reporting regular reading of newspapers or magazines or regular Internet use.

Radio health messages: Radio is, therefore, the most viable communication channel for widespread dissemination of health messages. Approximately one in three respondents -28 percent in Kebbi/Sokoto and 38 percent in Zamfara-report that they listen to the radio. As a result, a much higher proportion of respondents (28\%) across study areas report that they have heard any health messages or advertisements on the radio in the past 12 months compared with messages seen in other media forms. Among respondents who had heard any health messages, the most common topics were malaria (70\%), importance of ANC (61\%), and childhood immunizations (52\%). Unsurprisingly, reporting of malaria messages was significantly higher in Zamfara (75\%), where Breakthrough ACTION/Nigeria malaria programs have been ongoing, than in Kebbi/Sokoto (59\%). The least commonly noted messages related to maternal care after birth (7\%), newborn care (8\%), cough with rapid or difficult breathing (9\%), and maternal nutrition (9\%).

\section{Albishirin Ku! radio program exposure: Awareness of} Breakthrough ACTION/Nigeria's Albishirin Ku! campaign is already apparent, even as Breakthrough ACTION/Nigeria interventions are just beginning to roll out. In Kebbi/ Sokoto, nearly one-quarter of respondents have heard the Albishirin Ku! slogan on the radio, as have 13 percent of respondents in Zamfara. Furthermore, 15 percent of respondents in Kebbi/Sokoto have heard the radio program with Frank and Jamila, 26 percent have heard "iWannan Sakone Daga Ma'aikatar Lafiya Ta Kasa, Da Ta Jiha, Da Kuma," and 23 percent have heard the Albishirin $K u$ ! song. In Zamfara, more than one-quarter of respondents have heard the radio jingle about a mechanic and driver discussing malaria. A similar percentage have heard the radio jingle involving a football coach and his son discussing malaria.

Nevertheless, awareness of Breakthrough ACTION/ Nigeria's Albishirin Ku! campaign in Kebbi/Sokoto is lower among respondents living in the poorest households $(12 \%)$ than in the wealthiest households (42\%), and lower among respondents with no education (23\%) or Islamic education (20\%) compared with those having attended primary (37\%) or at least secondary (35\%) education. BSS baseline results point to the need for more focused Breakthrough ACTION/Nigeria outreach to these communities through other methods (e.g., household visits or community events) going forward since radio programming may not fully penetrate the poorest areas who also have worse health behaviors and outcomes.

\section{Breakthrough ACTION/Nigeria community volun- teer (CV) household visits or community events:}

Participation in a Breakthrough ACTION/Nigeria community volunteer (CV) community event or household visit was largely non-existent at the start of Breakthrough ACTION/Nigeria program implementation, as expected. Prior to Breakthrough ACTION/Nigeria program implementation, only 18 percent of respondents reported attending a community event in the past six months where a CV was present. These events included community dialogues, compound meetings, and naming ceremonies. Among all respondents, less than one percent reported participating in a community event in the past six months where a CV wearing either a Breakthrough ACTION/Nigeria, USAID, Albishirin Ku!, or Know Talk Go logo was present. Less than 2 percent reported participating in a household visit in the past six months with a CV wearing either a Breakthrough ACTION/Nigeria, USAID, Albishirin Ku!, or Know Talk Go logo. 


\section{TABLE 9.1. ALBISHIRIN KU! RADIO PROGRAM EXPOSURE BY SOCIODEMOGRAPHIC}

CHARACTERISTICS

Percentage of women aged 15 to 49 years currently pregnant or with a child under 2 years who reported hearing Albishirin Ku! radio programming by sociodemographic characteristics, September-October 2019

\begin{tabular}{|c|c|c|c|c|c|c|c|c|}
\hline & \multicolumn{2}{|c|}{ KEBBI } & \multicolumn{2}{|c|}{ SOKOTO } & \multicolumn{2}{|c|}{$\begin{array}{l}\text { MALARIA-ONLY } \\
\text { (ZAMFARA) }\end{array}$} & \multicolumn{2}{|c|}{$\begin{array}{c}\text { INTEGRATED } \\
\text { (KEBBI/SOKOTO) }\end{array}$} \\
\hline & $\%$ & $\mathbf{N}$ & $\%$ & $\mathbf{N}$ & $\%$ & $\mathbf{N}$ & $\%$ & $\mathbf{N}$ \\
\hline Total & 18.6 & 1,960 & 28.4 & 2,062 & 12.7 & 2,012 & 23.9 & 4,022 \\
\hline \multicolumn{9}{|l|}{ Household wealth } \\
\hline Lowest & 8.4 & 541 & 13.7 & 618 & 3.1 & 208 & 11.5 & 1,159 \\
\hline Second & 14.5 & 419 & 27.1 & 407 & 5.6 & 341 & 21.1 & 826 \\
\hline Middle & 23.9 & 63 & 36.1 & 408 & 9.9 & 407 & 30.7 & 771 \\
\hline Fourth & 15.8 & 276 & 40.4 & 286 & 17.5 & 488 & 28.3 & 563 \\
\hline Highest & 38.9 & 361 & 45.8 & 343 & 20.7 & 568 & 42.2 & 703 \\
\hline \multicolumn{9}{|l|}{ Maternal age (in years) } \\
\hline 15-24 years & 19.7 & 866 & 28.1 & 1,015 & 10.3 & 834 & 24.4 & 1,880 \\
\hline $25-34$ years & 17.5 & 850 & 27.4 & 837 & 13.7 & 888 & 22.7 & 1,687 \\
\hline $35-49$ years & 19.0 & 244 & 33.3 & 210 & 16.9 & 289 & 26.1 & 455 \\
\hline \multicolumn{9}{|c|}{$\begin{array}{l}\text { Maternal education } \\
\text { (highest level attended) }\end{array}$} \\
\hline None & 17.1 & 1,487 & 27.2 & 1,624 & 10.2 & 1,300 & 22.7 & 3111 \\
\hline Primary & 21.7 & 99 & 50.2 & 101 & 22.9 & 119 & 37.1 & 200 \\
\hline Secondary or higher & 34.7 & 210 & 35.0 & 141 & 16.0 & 341 & 34.8 & 351 \\
\hline Islamic & 13.9 & 164 & 25.0 & 196 & 19.4 & 251 & 19.8 & 360 \\
\hline
\end{tabular}

Estimates based on low Ns (approx. $<30$ obs) have large relative standard errors and should be interpreted with caution. ${ }^{* * *}$ indicates $p<0.001,{ }^{* *}$ indicates $p<0.05$ and * indicates $p<.0 .01$ for the chi ${ }^{2}$ statistic comparing integrated versus malaria-only study areas. 


\subsection{Radio}

\section{TABLE 9.1.1. RADIO EXPOSURE}

Percentage of women aged 15 to 49 years currently pregnant or with a child under 2 years who reported listening to the radio and hearing health messages on the radio, September-October 2019

\begin{tabular}{|c|c|c|c|c|c|c|}
\hline & KEBBI & SOKOTO & $\begin{array}{l}\text { MALARIA-ONLY } \\
\text { (ZAMFARA) }\end{array}$ & $\begin{array}{c}\text { INTEGRATED } \\
\text { (KEBBI/SOKOTO) }\end{array}$ & $\mathbf{N}$ & $\begin{array}{c}\mathrm{CHI}^{2} \\
\text { VALUE }\end{array}$ \\
\hline & $\%$ & $\%$ & $\%$ & $\%$ & & \\
\hline \multicolumn{7}{|c|}{ Do you ever listen to the radio? } \\
\hline No & 76.1 & 68.7 & 62.6 & 72.1 & 4,079 & 59.730 \\
\hline Yes & 23.9 & 31.3 & 37.5 & 27.9 & 1,954 & 2.134 \\
\hline Total & 100.0 & 100.0 & 100.0 & 100.0 & 6,033 & \\
\hline N & 1,960 & 2,062 & 2,011 & 4,022 & & \\
\hline \multicolumn{7}{|c|}{ How frequently do you listen to the radio? } \\
\hline Never & 76.1 & 68.7 & 62.6 & 72.1 & 4,080 & 75.721 \\
\hline$<1$ time a week & 6.4 & 7.4 & 9.1 & 6.9 & 461 & 1.347 \\
\hline Once a week & 2.9 & 6.8 & 4.5 & 5.0 & 297 & \\
\hline 2-3 times a week & 6.2 & 10.1 & 11.2 & 8.3 & 586 & \\
\hline 4+ times a week & 8.4 & 7.0 & 12.7 & 7.7 & 610 & \\
\hline Total & 100.0 & 100.0 & 100.0 & 100.0 & 6,034 & \\
\hline $\mathrm{N}$ & 1,960 & 2,062 & 2,012 & 4,022 & & \\
\hline \multicolumn{7}{|c|}{$\begin{array}{l}\text { In the last } 12 \text { months, did you hear any } \\
\text { health advertisements/messages on the } \\
\text { radio }\end{array}$} \\
\hline No & 82.0 & 73.4 & 68.8 & 77.4 & 4,421 & 53.284 \\
\hline Yes & 18.0 & 26.7 & 31.2 & 22.7 & 1,602 & 2.025 \\
\hline Total & 100.0 & 100.0 & 100.0 & 100.0 & 6,023 & \\
\hline $\mathrm{N}$ & 1,956 & 2,060 & 2,007 & 4,016 & & \\
\hline
\end{tabular}

Estimates based on low Ns (approx. $<30$ obs) have large relative standard errors and should be interpreted with caution. ${ }^{* * *}$ indicates $p<0.001,{ }^{* *}$ indicates $p<0.05$ and ${ }^{*}$ indicates $p<.0 .01$ for the chi ${ }^{2}$ statistic comparing integrated versus malaria-only study areas. 
TABLE 9.1.2. HEALTH MESSAGES HEARD ON THE RADIO

Percentage of women 15 to 49 years currently pregnant or with a child under 2 years having heard health messages on radio in past 12 months by type, September-October 2019

\begin{tabular}{|c|c|c|c|c|c|c|}
\hline & KEBBI & SOKOTO & $\begin{array}{l}\text { MALARIA-ONLY } \\
\text { (ZAMFARA) }\end{array}$ & $\begin{array}{l}\text { INTEGRATED } \\
\text { (KEBBI/SOKOTO) }\end{array}$ & $\mathbf{N}$ & $\begin{array}{c}\mathrm{CHI}^{2} \\
\text { VALUE }\end{array}$ \\
\hline & $\%$ & $\%$ & $\%$ & $\%$ & & \\
\hline \multicolumn{7}{|c|}{ Using contraception to space births } \\
\hline No & 52.6 & 69.8 & 69.8 & 63.5 & 1,043 & 6.451 \\
\hline Yes & 47.4 & 30.2 & 30.2 & 36.5 & 559 & 0.296 \\
\hline Total & 100.0 & 100.0 & 100.0 & 100.0 & 1,602 & \\
\hline $\mathrm{N}$ & 357 & 583 & 662 & 940 & & \\
\hline \multicolumn{7}{|c|}{ Using contraception for limiting births } \\
\hline No & 66.5 & 85.3 & 76.8 & 78.4 & 1,242 & 0.534 \\
\hline Yes & 33.5 & 14.7 & 23.2 & 21.6 & 360 & 0.019 \\
\hline Total & 100.0 & 100.0 & 100.0 & 100.0 & 1,602 & \\
\hline$N$ & 357 & 583 & 662 & 940 & & \\
\hline \multicolumn{7}{|c|}{$\begin{array}{l}\text { Giving birth in a health facility with } \\
\text { SBA }\end{array}$} \\
\hline No & 62.5 & 85.9 & 74.0 & 77.3 & 1,212 & 2.049 \\
\hline Yes & 37.5 & 14.2 & 26.0 & 22.7 & 390 & 0.089 \\
\hline Total & 100.0 & 100.0 & 100.0 & 100.0 & 1,602 & \\
\hline $\mathrm{N}$ & 357 & 583 & 662 & 940 & & \\
\hline \multicolumn{7}{|c|}{ Formulating a birth plan } \\
\hline No & 76.6 & 91.1 & 85.5 & 85.8 & 1,350 & 0.018 \\
\hline Yes & 23.4 & 8.9 & 14.5 & 14.2 & 252 & 0.001 \\
\hline Total & 100.0 & 100.0 & 100.0 & 100.0 & 1,602 & \\
\hline $\mathrm{N}$ & 357 & 583 & 662 & 940 & & \\
\hline \multicolumn{7}{|c|}{ Antenatal care } \\
\hline No & 46.7 & 37.6 & 37.7 & 40.9 & 640 & 1.575 \\
\hline Yes & 53.3 & 62.4 & 62.3 & 59.1 & 962 & 0.164 \\
\hline Total & 100.0 & 100.0 & 100.0 & 100.0 & 1,602 & \\
\hline$N$ & 357 & 583 & 662 & 940 & & \\
\hline \multicolumn{7}{|c|}{ Diarrhea } \\
\hline No & 66.8 & 81.9 & 76.6 & 76.4 & 1,239 & 0.014 \\
\hline Yes & 33.2 & 18.1 & 23.4 & 23.6 & 363 & 0.001 \\
\hline Total & 100.0 & 100.0 & 100.0 & 100.0 & 1,602 & \\
\hline$N$ & 357 & 583 & 662 & 940 & & \\
\hline \multicolumn{7}{|l|}{ Malaria } \\
\hline No & 47.2 & 37.3 & 25.4 & 40.9 & 583 & 40.578 \\
\hline Yes & 52.8 & 62.7 & 74.6 & 59.1 & 1,019 & 3.957 \\
\hline Total & 100.0 & 100.0 & 100.0 & 100.0 & 1,602 & * \\
\hline$N$ & 357 & 583 & 662 & 940 & & \\
\hline \multicolumn{7}{|c|}{ Cough with rapid/difficult breathing } \\
\hline No & 78.9 & 91.8 & 93.5 & 87.1 & 1,418 & 18.477 \\
\hline Yes & 21.1 & 8.2 & 6.5 & 12.9 & 184 & 0.680 \\
\hline Total & 100.0 & 100.0 & 100.0 & 100.0 & 1,602 & \\
\hline $\mathrm{N}$ & 357 & 583 & 662 & 940 & & \\
\hline \multicolumn{7}{|c|}{ Child nutrition } \\
\hline No & 79.2 & 89.9 & 86.9 & 86.0 & 1,378 & 0.256 \\
\hline Yes & 20.8 & 10.1 & 13.1 & 14.0 & 224 & 0.017 \\
\hline Total & 100.0 & 100.0 & 100.0 & 100.0 & 1,602 & \\
\hline N & 357 & 583 & 662 & 940 & & \\
\hline \multicolumn{7}{|c|}{ Immunizations } \\
\hline No & 59.8 & 60.2 & 42.1 & 60.1 & 850 & 45.780 \\
\hline Yes & 40.2 & 39.8 & 57.9 & 40.0 & 752 & 3.607 \\
\hline Total & 100.0 & 100.0 & 100.0 & 100.0 & 1,602 & \\
\hline
\end{tabular}




\begin{tabular}{|c|c|c|c|c|c|c|}
\hline $\mathrm{N}$ & 357 & 583 & 662 & 940 & & \\
\hline \multicolumn{7}{|c|}{ Breastfeeding } \\
\hline No & 74.8 & 83.8 & 68.7 & 80.5 & 1,233 & 24.695 \\
\hline Yes & 25.2 & 16.2 & 31.3 & 19.5 & 369 & 1.369 \\
\hline Total & 100.0 & 100.0 & 100.0 & 100.0 & 1,602 & \\
\hline $\mathrm{N}$ & 357 & 583 & 662 & 940 & & \\
\hline \multicolumn{7}{|c|}{ Maternal nutrition } \\
\hline No & 86.6 & 93.4 & 90.9 & 90.9 & 1,447 & 0.001 \\
\hline Yes & 13.4 & 6.6 & 9.1 & 9.1 & 155 & 0.000 \\
\hline Total & 100.0 & 100.0 & 100.0 & 100.0 & 1,602 & \\
\hline N & 357 & 583 & 662 & 940 & & \\
\hline \multicolumn{7}{|c|}{ Newborn care } \\
\hline No & 93.3 & 95.1 & 91.2 & 94.4 & 1,484 & 5.258 \\
\hline Yes & 6.7 & 4.9 & 8.9 & 5.6 & 118 & 0.458 \\
\hline Total & 100.0 & 100.0 & 100.0 & 100.0 & 1,602 & \\
\hline $\mathrm{N}$ & 357 & 583 & 662 & 940 & & \\
\hline \multicolumn{7}{|c|}{ Maternal care after giving birth } \\
\hline No & 98.0 & 96.2 & 91.3 & 96.8 & 1,500 & 16.902 \\
\hline Yes & 2.1 & 3.9 & 8.7 & 3.2 & 102 & 2.678 \\
\hline Total & 100.0 & 100.0 & 100.0 & 100.0 & 1,602 & \\
\hline N & 357 & 583 & 662 & 940 & & \\
\hline \multicolumn{7}{|c|}{ Water, sanitation and hygiene } \\
\hline No & 98.1 & 96.5 & 74.2 & 97.1 & 1,408 & 124.669 \\
\hline Yes & 1.9 & 3.5 & 25.8 & 2.9 & 194 & 22.959 \\
\hline Total & 100.0 & 100.0 & 100.0 & 100.0 & 1,602 & *** \\
\hline $\mathrm{N}$ & 357 & 583 & 662 & 940 & & \\
\hline
\end{tabular}

Estimates based on low Ns (approx. $<30$ obs) have large relative standard errors and should be interpreted with caution. ${ }^{* * *}$ indicates $p<0.001,{ }^{* *}$ indicates $p<0.05$ and ${ }^{*}$ indicates $p<.0 .01$ for the chi ${ }^{2}$ statistic comparing integrated versus malaria-only study areas. 


\subsection{Albishirin Ku!}

\section{TABLE 9.2.1. ALBISHIRIN KU! RADIO PROGRAM EXPOSURE}

Percentage of women aged 15 to 49 years currently pregnant or with a child under 2 years who reported hearing the Albishirin Ku! slogan on the radio, September-October 2019

\begin{tabular}{|c|c|c|c|c|c|c|}
\hline & KEBBI & SOKOTO & $\begin{array}{l}\text { MALARIA-ONLY } \\
\text { (ZAMFARA) }\end{array}$ & $\begin{array}{c}\text { INTEGRATED } \\
\text { (KEBBI/SOKOTO) }\end{array}$ & $\mathbf{N}$ & $\begin{array}{c}\mathrm{CHI}^{2} \\
\text { VALUE }\end{array}$ \\
\hline & $\%$ & $\%$ & $\%$ & $\%$ & & \\
\hline \multicolumn{7}{|c|}{$\begin{array}{l}\text { Have you heard the slogan Albishirin } \\
K u ! \text { on the radio? }\end{array}$} \\
\hline No & 75.7 & 65.9 & 83.7 & 70.4 & 4,447 & 152.812 \\
\hline Yes & 18.6 & 28.4 & 12.7 & 23.9 & 1,278 & 3.320 \\
\hline Don't know & 5.7 & 5.7 & 3.5 & 5.7 & 308 & * \\
\hline Total & 100.0 & 100.0 & 100.0 & 100.0 & 6,033 & \\
\hline $\mathrm{N}$ & 1,960 & 2,062 & 2,011 & 4,022 & & \\
\hline \multicolumn{7}{|c|}{$\begin{array}{l}\text { Heard radio program with couple } \\
\text { Faruk and Jamila }\end{array}$} \\
\hline No & 87.5 & 76.8 & 88.3 & 81.8 & 4,986 & 60.924 \\
\hline Yes & 9.2 & 19.1 & 8.2 & 14.5 & 822 & 1.312 \\
\hline Don't know & 3.3 & 4.1 & 3.5 & 3.7 & 225 & \\
\hline Total & 100.0 & 100.0 & 100.0 & 100.0 & 6,033 & \\
\hline $\mathrm{N}$ & 1,960 & 2,062 & 2,011 & 4,022 & & \\
\hline \multicolumn{7}{|c|}{$\begin{array}{l}\text { Heard iWannan Sakone Daga } \\
\text { Ma'aikatar Lafiya Ta Kasa, Da Ta Jiha, } \\
\text { Da Kuma }\end{array}$} \\
\hline No & 77.0 & 63.0 & 65.0 & 69.5 & 4,079 & 20.676 \\
\hline Yes & 18.7 & 33.0 & 31.7 & 26.4 & 1,733 & 0.353 \\
\hline Don't know & 4.3 & 4.0 & 3.4 & 4.1 & 221 & \\
\hline Total & 100.0 & 100.0 & 100.0 & 100.0 & 6,033 & \\
\hline$N$ & 1,960 & 2,062 & 2,011 & 4,022 & & \\
\hline \multicolumn{7}{|c|}{ Have you heard this song on the radio? } \\
\hline No & 79.7 & 68.0 & 80.3 & 73.4 & 4,509 & 43.130 \\
\hline Yes & 17.1 & 28.1 & 17.8 & 23.0 & 1,346 & 1.589 \\
\hline Don't know & 3.1 & 3.9 & 1.9 & 3.5 & 178 & \\
\hline Total & 100.0 & 100.0 & 100.0 & 100.0 & 6,033 & \\
\hline $\mathrm{N}$ & 1,960 & 2,062 & 2,011 & 4,022 & & \\
\hline
\end{tabular}

Estimates based on low Ns (approx. $<30$ obs) have large relative standard errors and should be interpreted with caution. ${ }^{* * *}$ indicates $p<0.001,{ }^{* *}$ indicates $p<0.05$ and ${ }^{*}$ indicates $p<.0 .01$ for the chi ${ }^{2}$ statistic comparing integrated versus malaria-only study areas. 
TABLE 9.2.2. ALBISHIRIN KU! HEALTH MESSAGES HEARD ON THE RADIO

Percentage of women 15 to 49 years currently pregnant or with a child under 2 years who had heard the Albishirin Ku! messages on the radio by type, September-October 2019

\begin{tabular}{|c|c|c|c|c|c|c|}
\hline & KEBBI & SOKOTO & $\begin{array}{l}\text { MALARIA-ONLY } \\
\text { (ZAMFARA) }\end{array}$ & $\begin{array}{l}\text { INTEGRATED } \\
\text { (KEBBI/SOKOTO) }\end{array}$ & $\mathbf{N}$ & $\begin{array}{c}\mathrm{CHI}^{2} \\
\text { VALUE }\end{array}$ \\
\hline & $\%$ & $\%$ & $\%$ & $\%$ & & \\
\hline \multicolumn{7}{|l|}{ Health } \\
\hline No & 45.3 & 79.3 & 82.2 & 67.6 & 934 & 38.555 \\
\hline Yes & 54.7 & 20.7 & 17.8 & 32.4 & 412 & 3.153 \\
\hline Total & 100.0 & 100.0 & 100.0 & 100.0 & 1,346 & \\
\hline $\mathrm{N}$ & 345 & 626 & 375 & 971 & & \\
\hline \multicolumn{7}{|c|}{ Albishirin Ku! } \\
\hline No & 63.2 & 57.1 & 43.2 & 59.2 & 745 & 34.514 \\
\hline Yes & 36.8 & 42.9 & 56.8 & 40.8 & 601 & 1.733 \\
\hline Total & 100.0 & 100.0 & 100.0 & 100.0 & 1,346 & \\
\hline $\mathrm{N}$ & 345 & 626 & 375 & 971 & & \\
\hline \multicolumn{7}{|c|}{$\begin{array}{l}\text { Pregnant women going to antenatal } \\
\text { care }\end{array}$} \\
\hline No & 48.6 & 75.8 & 70.7 & 66.4 & 877 & 2.798 \\
\hline Yes & 51.5 & 24.2 & 29.4 & 33.6 & 469 & 0.148 \\
\hline Total & 100.0 & 100.0 & 100.0 & 100.0 & 1,346 & \\
\hline $\mathrm{N}$ & 345 & 626 & 375 & 971 & & \\
\hline \multicolumn{7}{|c|}{ Childbirth spacing/family planning } \\
\hline No & 65.5 & 82.2 & 76.7 & 76.5 & 1,001 & 0.009 \\
\hline Yes & 34.5 & 17.8 & 23.3 & 23.5 & 345 & 0.000 \\
\hline Total & 100.0 & 100.0 & 100.0 & 100.0 & 1,346 & \\
\hline $\mathrm{N}$ & 345 & 626 & 375 & 971 & & \\
\hline \multicolumn{7}{|c|}{ Nutrition for children } \\
\hline No & 74.1 & 92.1 & 85.6 & 85.9 & 1,118 & 0.017 \\
\hline Yes & 26.0 & 8.0 & 14.4 & 14.1 & 228 & 0.001 \\
\hline Total & 100.0 & 100.0 & 100.0 & 100.0 & 1,346 & \\
\hline N & 345 & 626 & 375 & 971 & & \\
\hline \multicolumn{7}{|c|}{ Prompt care for malaria } \\
\hline No & 76.0 & 97.4 & 85.2 & 90.1 & 1,164 & 7.316 \\
\hline Yes & 24.0 & 2.6 & 14.9 & 10.0 & 182 & 0.282 \\
\hline Total & 100.0 & 100.0 & 100.0 & 100.0 & 1,346 & \\
\hline $\mathrm{N}$ & 345 & 626 & 375 & 971 & & \\
\hline \multicolumn{7}{|c|}{ Maternal nutrition during pregnancy } \\
\hline No & 80.7 & 90.4 & 86.0 & 87.1 & 1,137 & 0.363 \\
\hline Yes & 19.3 & 9.6 & 14.0 & 12.9 & 209 & 0.015 \\
\hline Total & 100.0 & 100.0 & 100.0 & 100.0 & 1,346 & \\
\hline $\mathrm{N}$ & 345 & 626 & 375 & 971 & & \\
\hline
\end{tabular}

Estimates based on low Ns (approx. $<30$ obs) have large relative standard errors and should be interpreted with caution. ${ }^{* * *}$ indicates $p<0.001,{ }^{* *}$ indicates $p<0.05$ and * indicates $p<.0 .01$ for the chi ${ }^{2}$ statistic comparing integrated versus malaria-only study areas. 
TABLE 9.2.3. ALBISHIRIN KU! JINGLES OR SHOWS HEARD ON THE RADIO

Percentage of women 15 to 49 years currently pregnant or with a child under 2 years who had heard the Albishirin Ku! radio jingles or shows by type, September-October 2019

\begin{tabular}{|c|c|c|c|c|c|c|}
\hline & KEBBI & SOKOTO & $\begin{array}{l}\text { MALARIA-ONLY } \\
\text { (ZAMFARA) }\end{array}$ & $\begin{array}{c}\text { INTEGRATED } \\
\text { (KEBBI/SOKOTO) }\end{array}$ & $\mathbf{N}$ & $\begin{array}{c}\mathrm{CHI}^{2} \\
\text { VALUE }\end{array}$ \\
\hline & $\%$ & $\%$ & $\%$ & $\%$ & & \\
\hline \multicolumn{7}{|c|}{ Have you heard a radio jingle featuring an auto } \\
\hline No & 86.4 & 76.4 & 71.6 & 81.0 & 4,650 & 124.067 \\
\hline Yes & 9.1 & 19.4 & 26.1 & 14.7 & 1,176 & 2.701 \\
\hline Don't know & 4.5 & 4.2 & 2.4 & 4.3 & 207 & \\
\hline Total & 100.0 & 100.0 & 100.0 & 100.0 & 6,033 & \\
\hline $\mathrm{N}$ & 1,960 & 2,062 & 2,011 & 4,022 & & \\
\hline \multicolumn{7}{|c|}{ Have you heard this radio jingle on the radio? } \\
\hline No & 86.1 & 71.4 & 69.5 & 78.2 & 4,520 & 141.258 \\
\hline Yes & 9.8 & 24.9 & 29.3 & 17.9 & 1,348 & 4.403 \\
\hline Don't know & 4.0 & 3.7 & 1.1 & 3.9 & 165 & * \\
\hline Total & 100.0 & 100.0 & 100.0 & 100.0 & 6,033 & \\
\hline $\mathrm{N}$ & 1,960 & 2,062 & 2,011 & 4,022 & & \\
\hline \multicolumn{7}{|c|}{$\begin{array}{l}\text { Heard radio jingle about football coach and } \\
\text { son discussing malaria? }\end{array}$} \\
\hline No & 87.7 & 78.4 & 71.6 & 82.7 & 4,725 & 157.389 \\
\hline Yes & 8.3 & 17.9 & 26.5 & 13.5 & 1,119 & 3.529 \\
\hline Don't know & 4.0 & 3.7 & 2.0 & 3.8 & 189 & * \\
\hline Total & 100.0 & 100.0 & 100.0 & 100.0 & 6,033 & \\
\hline N & 1,960 & 2,062 & 2,011 & 4,022 & & \\
\hline \multicolumn{7}{|c|}{ Have you heard this song on the radio? } \\
\hline No & 86.0 & 72.2 & 64.4 & 78.6 & 4,431 & 232.317 \\
\hline Yes & 10.2 & 24.4 & 34.5 & 17.8 & 1,446 & 6.617 \\
\hline Don't know & 3.8 & 3.4 & 1.0 & 3.6 & 156 & ** \\
\hline Total & 100.0 & 100.0 & 100.0 & 100.0 & 6,033 & \\
\hline N & 1,960 & 2,062 & 2,011 & 4,022 & & \\
\hline \multicolumn{7}{|c|}{ Have you heard of the following radio shows? } \\
\hline \multicolumn{7}{|c|}{ Taka Naka Rawan } \\
\hline No & 87.3 & 81.7 & 87.3 & 84.3 & 5,150 & 34.136 \\
\hline Yes & 7.5 & 12.9 & 10.2 & 10.4 & 631 & 0.866 \\
\hline Don't know & 5.2 & 5.4 & 2.5 & 5.3 & 252 & \\
\hline Total & 100.0 & 100.0 & 100.0 & 100.0 & 6,033 & \\
\hline N & 1,960 & 2,062 & 2,011 & 4,022 & & \\
\hline \multicolumn{7}{|c|}{ Don Tuwon Gobe } \\
\hline No & 87.0 & 81.5 & 86.0 & 84.0 & 5,095 & 28.607 \\
\hline Yes & 8.9 & 13.3 & 11.8 & 11.3 & 710 & 0.755 \\
\hline Don't know & 4.1 & 5.3 & 2.3 & 4.7 & 228 & \\
\hline Total & 100.0 & 100.0 & 100.0 & 100.0 & 6,033 & \\
\hline N & 1,960 & 2,062 & 2,011 & 4,022 & & \\
\hline \multicolumn{7}{|l|}{ Kai da lafiya } \\
\hline No & 83.2 & 77.2 & 83.4 & 80.0 & 4,862 & 44.832 \\
\hline Yes & 12.9 & 17.6 & 14.9 & 15.4 & 959 & 1.309 \\
\hline Don't know & 4.0 & 5.2 & 1.7 & 4.6 & 212 & \\
\hline Total & 100.0 & 100.0 & 100.0 & 100.0 & 6,033 & \\
\hline $\mathrm{N}$ & 1,960 & 2,062 & 2,011 & 4,022 & & \\
\hline \multicolumn{7}{|c|}{ Lafiyar mata da kananan yara } \\
\hline No & 79.7 & 69.0 & 68.6 & 74.0 & 4,323 & 117.426 \\
\hline Yes & 15.9 & 25.7 & 30.1 & 21.2 & 1,506 & 3.848 \\
\hline Don't know & 4.4 & 5.3 & 1.3 & 4.9 & 204 & \\
\hline Total & 100.0 & 100.0 & 100.0 & 100.0 & 6,033 & \\
\hline N & 1,960 & 2,062 & 2,011 & 4,022 & & \\
\hline \multicolumn{7}{|c|}{ Lafiyar Uwar Komai } \\
\hline No & 80.5 & 70.1 & 77.1 & 74.9 & 4,554 & 44.572 \\
\hline Yes & 15.7 & 24.3 & 21.1 & 20.3 & 1,259 & 1.324 \\
\hline Don't know & 3.8 & 5.7 & 1.8 & 4.8 & 220 & \\
\hline Total & 100.0 & 100.0 & 100.0 & 100.0 & 6,033 & \\
\hline $\mathrm{N}$ & 1,960 & 2,062 & 2,011 & 4,022 & & \\
\hline
\end{tabular}

Estimates based on low Ns (approx. $<30$ obs) have large relative standard errors and should be interpreted with caution. ${ }^{* \star *}$ indicates $p<0.001,{ }^{* *}$ indicates $p<0.05$ and ${ }^{*}$ indicates $p<.0 .01$ for the chi $^{2}$ statistic comparing integrated versus malaria-only study areas. 
TABLE 9.2.4. ALBISHIRIN KU! LOGO SEEN DURING HOUSEHOLD VISIT WITH COMMUNITY VOLUNTEER

Percentage of women 15 to 49 years currently pregnant or with a child under 2 years who had a household visit with a CV in the past six months and saw the Albishirin Ku!, Know Talk Go, USAID or Breakthrough ACTION/Nigeria logos on their clothing, Sep-Oct 2019

\begin{tabular}{|c|c|c|c|c|c|c|}
\hline & KEBBI & sокото & $\begin{array}{l}\text { MALARIA-ONLY } \\
\text { (ZAMFARA) }\end{array}$ & $\begin{array}{l}\text { INTEGRATED } \\
\text { (KEBBI/SOKOTO) }\end{array}$ & $\mathbf{N}$ & $\begin{array}{l}\mathrm{CHI}^{2} \\
\text { VALUE }\end{array}$ \\
\hline & $\%$ & $\%$ & $\%$ & $\%$ & & \\
\hline \multicolumn{7}{|l|}{ CV household visit in past 6 months } \\
\hline No & 84.2 & 85.5 & 89.1 & 84.9 & 5,245 & 33.963 \\
\hline Yes & 11.1 & 10.7 & 9.0 & 10.9 & 521 & 0.759 \\
\hline Don't know & 4.7 & 3.8 & 1.9 & 4.2 & 195 & \\
\hline Total & 100.0 & 100.0 & 100.0 & 100.0 & 5,961 & \\
\hline $\mathrm{N}$ & 1,942 & 2,042 & 1,977 & 3,984 & & \\
\hline \multicolumn{7}{|l|}{$\begin{array}{l}\text { Type of branding on CV clothing at } \\
\text { household visit }\end{array}$} \\
\hline No special branding & 44.4 & 12.4 & 19.8 & 26.3 & 117 & 195.934 \\
\hline Albishirin Ku! & 11.8 & 6.5 & 0.0 & 8.8 & 27 & 5.320 \\
\hline Know Talk Go & 0.0 & 0.0 & 0.4 & 0.0 & 1 & *** \\
\hline Nigerian or state government & 4.5 & 0.6 & 0.2 & 2.3 & 7 & \\
\hline USAID & 3.2 & 2.2 & 7.0 & 2.6 & 22 & \\
\hline Breakthrough ACTION & 11.8 & 2.1 & 0.4 & 6.3 & 22 & \\
\hline UNICEF & 0.5 & 11.8 & 8.9 & 6.9 & 34 & \\
\hline Plan International & 0.0 & 27.0 & 0.0 & 15.3 & 55 & \\
\hline Other & 0.7 & 2.5 & 49.5 & 1.7 & 61 & \\
\hline Don't know & 23.0 & 34.9 & 13.9 & 29.7 & 119 & \\
\hline Total & 100.0 & 100.0 & 100.0 & 100.0 & 465 & \\
\hline $\mathrm{N}$ & 140 & 208 & 117 & 348 & & \\
\hline \multicolumn{7}{|l|}{$\begin{array}{l}\text { Participation in household visit with } \\
\text { Breakthrough ACTION CV in past } 6 \\
\text { months }\end{array}$} \\
\hline No & 97.6 & 98.9 & 99.4 & 98.3 & 5,962 & 18.407 \\
\hline Yes & 2.4 & 1.1 & 0.6 & 1.7 & 72 & 2.407 \\
\hline Total & 100.0 & 100.0 & 100.0 & 100.0 & 6,034 & \\
\hline N & 1,960 & 2,062 & 2,012 & 4,022 & & \\
\hline \multicolumn{7}{|l|}{$\begin{array}{l}\text { Main messages discussed by Break- } \\
\text { through ACTION CV during household } \\
\text { visit }\end{array}$} \\
\hline Childbirth spacing/FP & 74.7 & 39.6 & 63.9 & 63.0 & 37 & 17.530 \\
\hline Antenatal care & 18.3 & 12.5 & 7.9 & 16.4 & 11 & 1.351 \\
\hline Pregnancy health/nutrition & 0.8 & 1.9 & 0.0 & 1.2 & 2 & \\
\hline Birth planning/delivery care & 4.9 & 0.0 & 0.0 & 3.3 & 2 & \\
\hline Newborn care & 0.0 & 4.5 & 0.0 & 1.5 & 2 & \\
\hline Breastfeeding & 1.2 & 0.0 & 0.0 & 0.8 & 1 & \\
\hline Immunizations & 0.0 & 35.3 & 0.0 & 11.7 & 7 & \\
\hline Malaria preventing/using mosquito nets & 0.0 & 1.9 & 28.2 & 0.6 & 6 & \\
\hline Malaria diagnosis and treatment & 0.0 & 2.6 & 0.0 & 0.9 & 1 & \\
\hline Malaria prevention during pregnancy/IPTp & 0.0 & 1.9 & 0.0 & 0.6 & 1 & \\
\hline Total & 100.0 & 100.0 & 100.0 & 100.0 & 70 & \\
\hline $\mathrm{N}$ & 30 & 28 & 12 & 58 & & \\
\hline
\end{tabular}

Estimates based on low Ns (approx. $<30$ obs) have large relative standard errors and should be interpreted with caution. ${ }^{* * *}$ indicates $p<0.001,{ }^{* *}$ indicates $p<0.05$ and ${ }^{*}$ indicates $p<.0 .01$ for the chi ${ }^{2}$ statistic comparing integrated versus malaria-only study areas. 
TABLE 9.2.5. ALBISHIRIN KU! LOGO SEEN DURING COMMUNITY EVENT WITH CV

Percentage of women 15 to 49 years currently pregnant or with a child under 2 years who attended a community event with a CV in the past six months and saw Albishirin Ku!, Know Talk Go, USAID or Breakthrough ACTION/Nigeria logos on their clothing, September-October 2019

\begin{tabular}{|c|c|c|c|c|c|c|}
\hline & $\begin{array}{l}\text { KEBBI } \\
\%\end{array}$ & $\begin{array}{l}\text { SOKOTO } \\
\%\end{array}$ & $\begin{array}{l}\text { MALARIA-ONLY } \\
\text { (ZAMFARA) } \\
\%\end{array}$ & $\begin{array}{c}\text { INTEGRATED } \\
\text { (KEBBI/SOKOTO) } \\
\%\end{array}$ & $\mathbf{N}$ & $\begin{array}{c}\mathrm{CHI}^{2} \\
\text { VALUE }\end{array}$ \\
\hline \multicolumn{7}{|c|}{$\begin{array}{l}\text { Participation in event with CV in the last } \\
6 \text { months }\end{array}$} \\
\hline No & 51.0 & 72.7 & 89.6 & 64.0 & 243 & 31.535 \\
\hline Yes & 44.8 & 25.7 & 10.2 & 33.4 & 75 & 3.925 \\
\hline Don't know & 4.2 & 1.5 & 0.3 & 2.6 & 9 & * \\
\hline Total & 100.0 & 100.0 & 100.0 & 100.0 & 327 & \\
\hline N & 94 & 119 & 114 & 213 & & \\
\hline \multicolumn{7}{|c|}{ Type of CV event in past 6 months } \\
\hline Compound meeting & 51.7 & 18.3 & 0.0 & 36.4 & 20 & 44.455 \\
\hline Community dialogue & 22.0 & 0.0 & 68.0 & 11.9 & 16 & 2.652 \\
\hline Marriage ceremony & 0.0 & 14.3 & 19.8 & 6.6 & 7 & \\
\hline Naming ceremony & 9.4 & 62.2 & 0.0 & 33.7 & 19 & \\
\hline Religious gathering & 10.6 & 0.0 & 0.0 & 5.7 & 5 & \\
\hline Household visit & 0.0 & 5.2 & 9.6 & 2.4 & 4 & \\
\hline Other event & 1.4 & 0.0 & 2.6 & 0.8 & 2 & \\
\hline Don't know & 4.9 & 0.0 & 0.0 & 2.6 & 2 & \\
\hline Total & 100.0 & 100.0 & 100.0 & 100.0 & 75 & \\
\hline $\mathrm{N}$ & 35 & 29 & 11 & 64 & & \\
\hline \multicolumn{7}{|c|}{ Type of branding on CV clothing at event } \\
\hline No special branding & 36.2 & 75.8 & 88.6 & 56.4 & 35 & 7.125 \\
\hline Albishirin Ku! & 22.5 & 11.9 & 0.0 & 17.1 & 6 & 0.677 \\
\hline Know Talk Go & 20.9 & 0.0 & 0.0 & 10.2 & 3 & \\
\hline Nigerian or state government & 2.7 & 0.0 & 0.0 & 1.3 & 1 & \\
\hline Don't know & 17.7 & 12.3 & 11.4 & 15.0 & 11 & \\
\hline Total & 100.0 & 100.0 & 100.0 & 100.0 & 56 & \\
\hline $\mathrm{N}$ & 24 & 25 & 7 & 49 & & \\
\hline \multicolumn{7}{|c|}{$\begin{array}{l}\text { Participation in community event with } \\
\text { Breakthrough ACTION CV in past } 6 \\
\text { months }\end{array}$} \\
\hline No & 99.5 & 99.9 & 100.0 & 99.7 & 6,025 & 11.404 \\
\hline Yes & 0.5 & 0.1 & 0.0 & 0.3 & 9 & 3.824 \\
\hline Total & 100.0 & 100.0 & 100.0 & 100.0 & 6,034 & \\
\hline $\mathrm{N}$ & 1,960 & 2,062 & 2,012 & 4,022 & & \\
\hline \multicolumn{7}{|c|}{$\begin{array}{l}\text { Main messages discussed by Break- } \\
\text { through ACTION CV at community event }\end{array}$} \\
\hline Childbirth spacing/FP & 100.0 & 0.0 & 77.8 & 77.8 & . & \\
\hline Postpartum care for mothers & 0.0 & 50.0 & 11.1 & 11.1 & . & \\
\hline Breastfeeding & 0.0 & 50.0 & 11.1 & 11.1 & . & \\
\hline Total & 100.0 & 100.0 & 100.0 & 100.0 & & \\
\hline $\mathrm{N}$ & 7 & 2 & 9 & 9 & & \\
\hline
\end{tabular}

Estimates based on low Ns (approx. $<30$ obs) have large relative standard errors and should be interpreted with caution. ${ }^{* * *}$ indicates $p<0.001,{ }^{* *}$ indicates $p<0.05$ and ${ }^{*}$ indicates $p<.0 .01$ for the chi ${ }^{2}$ statistic comparing integrated versus malaria-only study areas. 


\subsection{Television}

\section{TABLE 9.3.1. TELEVISION EXPOSURE}

Percentage of women aged 15 to 49 years currently pregnant or with a child under 2 years who reported watching TV and seeing health messages on TV, September-October 2019

\begin{tabular}{|c|c|c|c|c|c|c|}
\hline & KEBBI & SOKото & $\begin{array}{l}\text { MALARIA-ONLY } \\
\text { (ZAMFARA) }\end{array}$ & $\begin{array}{l}\text { INTEGRATED } \\
\text { (KEBBI/SOKOTO) }\end{array}$ & $\mathbf{N}$ & $\begin{array}{c}\mathrm{CHI}^{2} \\
\text { VALUE }\end{array}$ \\
\hline & $\%$ & $\%$ & $\%$ & $\%$ & & \\
\hline \multicolumn{7}{|c|}{ Do you currently watch TV? } \\
\hline No & 92.2 & 95.0 & 89.4 & 93.7 & 5,411 & 34.114 \\
\hline Yes & 7.8 & 5.0 & 10.6 & 6.3 & 622 & 2.026 \\
\hline Total & 100.0 & 100.0 & 100.0 & 100.0 & 6,033 & \\
\hline N & 1,960 & 2,062 & 2,011 & 4,022 & & \\
\hline \multicolumn{7}{|c|}{$\begin{array}{l}\text { About how many days in a normal week } \\
\text { do you watch TV? }\end{array}$} \\
\hline 0 times & 92.2 & 95.0 & 89.4 & 93.7 & 5,412 & 62.407 \\
\hline $1-2$ days & 1.2 & 1.7 & 1.2 & 1.5 & 108 & 2.744 \\
\hline $3-4$ days & 3.5 & 0.9 & 2.0 & 2.1 & 168 & 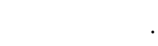 \\
\hline $5-7$ days & 3.1 & 2.4 & 7.5 & 2.7 & 346 & \\
\hline Total & 100.0 & 100.0 & 100.0 & 100.0 & 6,034 & \\
\hline N & 1,960 & 2,062 & 2,012 & 4,022 & & \\
\hline \multicolumn{7}{|c|}{$\begin{array}{l}\text { In past } 12 \text { months, have you seen any } \\
\text { health messages/ads on TV? }\end{array}$} \\
\hline No & 97.3 & 97.4 & 93.7 & 97.4 & 5,717 & 41.843 \\
\hline Yes & 2.7 & 2.6 & 6.3 & 2.7 & 317 & 4.005 \\
\hline Total & 100.0 & 100.0 & 100.0 & 100.0 & 6,034 & * \\
\hline $\mathrm{N}$ & 1,960 & 2,062 & 2,012 & 4,022 & & \\
\hline
\end{tabular}

Estimates based on low Ns (approx. $<30$ obs) have large relative standard errors and should be interpreted with caution. ${ }^{* * *}$ indicates $p<0.001,{ }^{* *}$ indicates $p<0.05$ and ${ }^{*}$ indicates $p<.0 .01$ for the chi ${ }^{2}$ statistic comparing integrated versus malaria-only study areas. 
TABLE 9.3.2. HEALTH MESSAGES SEEN ON TELEVISION

Percentage of women 15 to 49 years currently pregnant or with a child under 2 years who had seen health messages on TV in the past 12 months by type, September-October 2019

\begin{tabular}{|c|c|c|c|c|c|c|}
\hline & KEBBI & SOKOTO & $\begin{array}{l}\text { MALARIA-ONLY } \\
\text { (ZAMFARA) }\end{array}$ & $\begin{array}{c}\text { INTEGRATED } \\
\text { (KEBBI/SOKOTO) }\end{array}$ & $\mathbf{N}$ & $\begin{array}{c}\mathrm{CHI}^{2} \\
\text { VALUE }\end{array}$ \\
\hline & $\%$ & $\%$ & $\%$ & $\%$ & & \\
\hline \multicolumn{7}{|c|}{ Using contraception for birth spacing } \\
\hline No & 58.0 & 63.7 & 78.7 & 61.0 & 223 & 9.168 \\
\hline Yes & 42.0 & 36.3 & 21.3 & 39.0 & 94 & 2.966 \\
\hline Total & 100.0 & 100.0 & 100.0 & 100.0 & 317 & \\
\hline N & 70 & 79 & 168 & 149 & & \\
\hline \multicolumn{7}{|c|}{ Using contraception for limiting births } \\
\hline No & 69.1 & 79.3 & 87.4 & 74.4 & 256 & 7.080 \\
\hline Yes & 31.0 & 20.7 & 12.6 & 25.6 & 61 & 2.364 \\
\hline Total & 100.0 & 100.0 & 100.0 & 100.0 & 317 & \\
\hline N & 70 & 79 & 168 & 149 & & \\
\hline \multicolumn{7}{|c|}{$\begin{array}{l}\text { Giving birth in a health facility with } \\
\text { SBA }\end{array}$} \\
\hline No & 63.8 & 69.9 & 89.1 & 67.0 & 254 & 20.240 \\
\hline Yes & 36.2 & 30.1 & 10.9 & 33.0 & 63 & 10.788 \\
\hline Total & 100.0 & 100.0 & 100.0 & 100.0 & 317 & ** \\
\hline$N$ & 70 & 79 & 168 & 149 & & \\
\hline \multicolumn{7}{|c|}{ Birth plans } \\
\hline No & 81.5 & 81.2 & 85.1 & 81.3 & 273 & 0.591 \\
\hline Yes & 18.6 & 18.8 & 14.9 & 18.7 & 44 & 0.387 \\
\hline Total & 100.0 & 100.0 & 100.0 & 100.0 & 317 & \\
\hline N & 70 & 79 & 168 & 149 & & \\
\hline \multicolumn{7}{|c|}{ Antenatal care } \\
\hline No & 58.2 & 62.0 & 62.6 & 60.2 & 194 & 0.139 \\
\hline Yes & 41.8 & 38.0 & 37.4 & 39.8 & 123 & 0.071 \\
\hline Total & 100.0 & 100.0 & 100.0 & 100.0 & 317 & \\
\hline$N$ & 70 & 79 & 168 & 149 & & \\
\hline \multicolumn{7}{|c|}{ Diarrhea } \\
\hline No & 77.0 & 96.1 & 88.3 & 87.0 & 283 & 0.089 \\
\hline Yes & 23.0 & 3.9 & 11.7 & 13.0 & 34 & 0.044 \\
\hline Total & 100.0 & 100.0 & 100.0 & 100.0 & 317 & \\
\hline$N$ & 70 & 79 & 168 & 149 & & \\
\hline \multicolumn{7}{|l|}{ Malaria } \\
\hline No & 58.0 & 50.8 & 46.9 & 54.3 & 161 & 1.190 \\
\hline Yes & 42.0 & 49.2 & 53.1 & 45.8 & 156 & 0.332 \\
\hline Total & 100.0 & 100.0 & 100.0 & 100.0 & 317 & \\
\hline N & 70 & 79 & 168 & 149 & & \\
\hline \multicolumn{7}{|c|}{ Cough with rapid or difficult breathing } \\
\hline No & 98.1 & 81.0 & 93.8 & 89.2 & 295 & 1.748 \\
\hline Yes & 1.9 & 19.0 & 6.2 & 10.9 & 22 & 0.580 \\
\hline Total & 100.0 & 100.0 & 100.0 & 100.0 & 317 & \\
\hline N & 70 & 79 & 168 & 149 & & \\
\hline \multicolumn{7}{|c|}{ Child nutrition } \\
\hline No & 91.5 & 86.9 & 89.0 & 89.1 & 280 & 0.001 \\
\hline Yes & 8.5 & 13.1 & 11.1 & 10.9 & 37 & 0.001 \\
\hline Total & 100.0 & 100.0 & 100.0 & 100.0 & 317 & \\
\hline$N$ & 70 & 79 & 168 & 149 & & \\
\hline
\end{tabular}




\begin{tabular}{|c|c|c|c|c|c|c|}
\hline \multicolumn{7}{|c|}{ Immunizations } \\
\hline No & 75.7 & 70.2 & 66.5 & 72.8 & 214 & 0.985 \\
\hline Yes & 24.4 & 29.8 & 33.5 & 27.2 & 103 & 0.348 \\
\hline Total & 100.0 & 100.0 & 100.0 & 100.0 & 317 & \\
\hline $\mathrm{N}$ & 70 & 79 & 168 & 149 & & \\
\hline \multicolumn{7}{|c|}{ Breastfeeding } \\
\hline No & 90.9 & 85.2 & 81.6 & 87.9 & 266 & 1.527 \\
\hline Yes & 9.1 & 14.9 & 18.4 & 12.1 & 51 & 0.947 \\
\hline Total & 100.0 & 100.0 & 100.0 & 100.0 & 317 & \\
\hline$N$ & 70 & 79 & 168 & 149 & & \\
\hline \multicolumn{7}{|c|}{ Maternal nutrition } \\
\hline No & 97.6 & 90.3 & 91.9 & 93.8 & 289 & 0.292 \\
\hline Yes & 2.4 & 9.7 & 8.1 & 6.2 & 28 & 0.121 \\
\hline Total & 100.0 & 100.0 & 100.0 & 100.0 & 317 & \\
\hline $\mathrm{N}$ & 70 & 79 & 168 & 149 & & \\
\hline \multicolumn{7}{|c|}{ Newborn care } \\
\hline No & 99.2 & 97.3 & 96.7 & 98.2 & 305 & 0.442 \\
\hline Yes & 0.8 & 2.7 & 3.4 & 1.8 & 12 & 0.581 \\
\hline Total & 100.0 & 100.0 & 100.0 & 100.0 & 317 & \\
\hline $\mathrm{N}$ & 70 & 79 & 168 & 149 & & \\
\hline \multicolumn{7}{|c|}{ Maternal care after giving birth } \\
\hline No & 100.0 & 95.1 & 92.4 & 97.4 & 296 & 2.333 \\
\hline Yes & 0.0 & 4.9 & 7.6 & 2.6 & 21 & 1.286 \\
\hline Total & 100.0 & 100.0 & 100.0 & 100.0 & 317 & \\
\hline N & 70 & 79 & 168 & 149 & & \\
\hline \multicolumn{7}{|c|}{ Water, sanitation, and hygiene } \\
\hline No & 93.5 & 92.6 & 77.7 & 93.1 & 267 & 8.473 \\
\hline Yes & 6.5 & 7.4 & 22.3 & 7.0 & 50 & 4.159 \\
\hline Total & 100.0 & 100.0 & 100.0 & 100.0 & 317 & * \\
\hline $\mathrm{N}$ & 70 & 79 & 168 & 149 & & \\
\hline
\end{tabular}

Estimates based on low Ns (approx. $<30$ obs) have large relative standard errors and should be interpreted with caution. ${ }^{* * *}$ indicates $p<0.001,{ }^{* *}$ indicates $p<0.05$ and ${ }^{*}$ indicates $p<.0 .01$ for the chi ${ }^{2}$ statistic comparing integrated versus malaria-only study areas. 


\subsection{Newspaper or magazine}

\section{TABLE 9.4.1. NEWSPAPER OR MAGAZINE EXPOSURE}

Percentage of women 15 to 49 years currently pregnant or with a child under 2 years who had read newspapers or magazines and saw health messages, September-October 2019

\begin{tabular}{|c|c|c|c|c|c|c|}
\hline & $\begin{array}{c}\text { KEBBI } \\
\%\end{array}$ & $\begin{array}{l}\text { SOKOTO } \\
\%\end{array}$ & $\begin{array}{c}\text { MALARIA-ONLY } \\
\text { (ZAMFARA) } \\
\%\end{array}$ & $\begin{array}{c}\text { INTEGRATED } \\
\text { (KEBBI/SOKOTO) } \\
\%\end{array}$ & $\mathbf{N}$ & $\begin{array}{c}\mathrm{CHI}^{2} \\
\text { VALUE }\end{array}$ \\
\hline \multicolumn{7}{|l|}{$\begin{array}{l}\text { Do you ever read the newspaper or } \\
\text { magazines? }\end{array}$} \\
\hline No & 99.0 & 99.4 & 99.7 & 99.3 & 5,983 & 6.064 \\
\hline Yes & 1.0 & 0.6 & 0.3 & 0.8 & 50 & 2.159 \\
\hline Total & 100.0 & 100.0 & 100.0 & 100.0 & 6,033 & \\
\hline N & 1,960 & 2,062 & 2,011 & 4,022 & & \\
\hline \multicolumn{7}{|l|}{$\begin{array}{l}\text { How frequently do you read the news- } \\
\text { paper or magazines? }\end{array}$} \\
\hline 4 or more times a week & 32.0 & 20.7 & 52.0 & 27.5 & 10 & 8.097 \\
\hline 2-3 times a week & 15.1 & 3.4 & 20.6 & 10.4 & 6 & 0.819 \\
\hline Once a week & 4.4 & 26.3 & 0.0 & 13.1 & 7 & \\
\hline Less than once a week & 16.6 & 49.6 & 6.9 & 29.8 & 18 & \\
\hline Never reads newspapers/magazines & 32.0 & 0.0 & 20.5 & 19.2 & 9 & \\
\hline Total & 100.0 & 100.0 & 100.0 & 100.0 & 50 & \\
\hline$N$ & 22 & 21 & 7 & 43 & & \\
\hline \multicolumn{7}{|l|}{$\begin{array}{l}\text { In the last } 12 \text { months, did you read any } \\
\text { health advertisements or message }\end{array}$} \\
\hline No & 99.5 & 98.7 & 96.4 & 99.1 & 5,927 & 42.822 \\
\hline Yes & 0.5 & 1.3 & 3.6 & 0.9 & 106 & 2.474 \\
\hline Total & 100.0 & 100.0 & 100.0 & 100.0 & 6,033 & \\
\hline $\mathrm{N}$ & 1,960 & 2,062 & 2,011 & 4,022 & & \\
\hline
\end{tabular}

Estimates based on low Ns (approx. $<30$ obs) have large relative standard errors and should be interpreted with caution. ${ }^{* * *}$ indicates $p<0.001,{ }^{* *}$ indicates $p<0.05$ and * indicates $p<.0 .01$ for the chi $^{2}$ statistic comparing integrated versus malaria-only study areas. 
TABLE 9.4.2. HEALTH MESSAGES SEEN IN NEWSPAPERS OR MAGAZINES

Percentage of women 15 to 49 years currently pregnant or with a child under 2 years who had seen health messages in newspapers or magazines in the past 12 months by type, September-October 2019

\begin{tabular}{|c|c|c|c|c|c|c|}
\hline & KEBBI & SOKOTO & $\begin{array}{l}\text { MALARIA-ONLY } \\
\text { (ZAMFARA) }\end{array}$ & $\begin{array}{c}\text { INTEGRATED } \\
\text { (KEBBI/SOKOTO) }\end{array}$ & $\mathbf{N}$ & $\begin{array}{c}\mathrm{CHI}^{2} \\
\text { VALUE }\end{array}$ \\
\hline & $\%$ & $\%$ & $\%$ & $\%$ & & \\
\hline \multicolumn{7}{|c|}{ Using contraception to space births } \\
\hline No & 23.8 & 46.0 & 11.8 & 40.2 & 34 & 8.029 \\
\hline Yes & 76.2 & 54.0 & 88.2 & 59.8 & 72 & 2.359 \\
\hline Total & 100.0 & 100.0 & 100.0 & 100.0 & 106 & \\
\hline N & 14 & 33 & 59 & 47 & & \\
\hline \multicolumn{7}{|c|}{ Using contraception for limiting births } \\
\hline No & 65.0 & 77.9 & 12.7 & 74.5 & 49 & 29.862 \\
\hline Yes & 35.0 & 22.1 & 87.3 & 25.5 & 57 & 10.170 \\
\hline Total & 100.0 & 100.0 & 100.0 & 100.0 & 106 & ** \\
\hline N & 14 & 33 & 59 & 47 & & \\
\hline \multicolumn{7}{|c|}{$\begin{array}{l}\text { Giving birth in a health facility with } \\
\text { SBA }\end{array}$} \\
\hline No & 60.4 & 93.3 & 15.6 & 84.7 & 51 & 33.348 \\
\hline Yes & 39.6 & 6.7 & 84.4 & 15.3 & 55 & 27.795 \\
\hline Total & 100.0 & 100.0 & 100.0 & 100.0 & 106 & *** \\
\hline N & 14 & 33 & 59 & 47 & & \\
\hline \multicolumn{7}{|c|}{ Birth plans } \\
\hline No & 41.6 & 90.9 & 97.0 & 78.0 & 95 & 8.816 \\
\hline Yes & 58.4 & 9.1 & 3.0 & 22.0 & 11 & 3.339 \\
\hline Total & 100.0 & 100.0 & 100.0 & 100.0 & 106 & \\
\hline N & 14 & 33 & 59 & 47 & & \\
\hline \multicolumn{7}{|c|}{ Antenatal care } \\
\hline No & 33.0 & 43.6 & 16.4 & 40.8 & 37 & 5.006 \\
\hline Yes & 67.0 & 56.4 & 83.6 & 59.2 & 69 & 3.027 \\
\hline Total & 100.0 & 100.0 & 100.0 & 100.0 & 106 & \\
\hline N & 14 & 33 & 59 & 47 & & \\
\hline \multicolumn{7}{|c|}{ Diarrhea } \\
\hline No & 67.0 & 93.9 & 48.9 & 86.9 & 73 & 7.813 \\
\hline Yes & 33.0 & 6.1 & 51.1 & 13.1 & 33 & 12.704 \\
\hline Total & 100.0 & 100.0 & 100.0 & 100.0 & 106 & $\star \star \star *$ \\
\hline$N$ & 14 & 33 & 59 & 47 & & \\
\hline \multicolumn{7}{|l|}{ Malaria } \\
\hline No & 80.9 & 58.3 & 14.6 & 64.2 & 42 & 19.244 \\
\hline Yes & 19.1 & 41.7 & 85.4 & 35.8 & 64 & 18.341 \\
\hline Total & 100.0 & 100.0 & 100.0 & 100.0 & 106 & *** \\
\hline N & 14 & 33 & 59 & 47 & & \\
\hline \multicolumn{7}{|c|}{ Cough with rapid or difficult breathing } \\
\hline No & 89.7 & 89.3 & 98.1 & 89.4 & 100 & 3.200 \\
\hline Yes & 10.3 & 10.7 & 2.0 & 10.6 & 6 & 10.566 \\
\hline Total & 100.0 & 100.0 & 100.0 & 100.0 & 106 & ** \\
\hline N & 14 & 33 & 59 & 47 & & \\
\hline \multicolumn{7}{|c|}{ Child nutrition } \\
\hline No & 96.1 & 91.7 & 96.4 & 92.9 & 100 & 0.430 \\
\hline Yes & 4.0 & 8.3 & 3.6 & 7.2 & 6 & 1.473 \\
\hline Total & 100.0 & 100.0 & 100.0 & 100.0 & 106 & \\
\hline N & 14 & 33 & 59 & 47 & & \\
\hline
\end{tabular}




\begin{tabular}{|c|c|c|c|c|c|c|}
\hline \multicolumn{7}{|c|}{ Immunizations } \\
\hline No & 93.0 & 60.1 & 1.8 & 68.7 & 37 & 58.026 \\
\hline Yes & 7.0 & 39.9 & 98.2 & 31.3 & 69 & 37.493 \\
\hline Total & 100.0 & 100.0 & 100.0 & 100.0 & 106 & *** \\
\hline N & 14 & 33 & 59 & 47 & & \\
\hline \multicolumn{7}{|c|}{ Breastfeeding } \\
\hline No & 93.0 & 85.3 & 14.0 & 87.4 & 52 & 38.555 \\
\hline Yes & 7.0 & 14.7 & 86.0 & 12.7 & 54 & 33.744 \\
\hline Total & 100.0 & 100.0 & 100.0 & 100.0 & 106 & *** \\
\hline N & 14 & 33 & 59 & 47 & & \\
\hline \multicolumn{7}{|c|}{ Water, sanitation, and hygiene } \\
\hline No & 100.0 & 100.0 & 13.2 & 100.0 & 59 & 52.378 \\
\hline Yes & 0.0 & 0.0 & 86.8 & 0.0 & 47 & 63.498 \\
\hline Total & 100.0 & 100.0 & 100.0 & 100.0 & 106 & 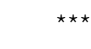 \\
\hline $\mathrm{N}$ & 14 & 33 & 59 & 47 & & \\
\hline
\end{tabular}

Estimates based on low Ns (approx. $<30$ obs) have large relative standard errors and should be interpreted with caution. ${ }^{* * *}$ indicates $p<0.001,{ }^{* *}$ indicates $p<0.05$ and ${ }^{*}$ indicates $p<.0 .01$ for the chi ${ }^{2}$ statistic comparing integrated versus malaria-only study areas. 


\subsection{Internet}

\section{TABLE 9.5.1. INTERNET EXPOSURE}

Percentage of women aged 15 to 49 years currently pregnant or with a child under 2 years who report using the internet and seeing health messages, September-October 2019

\begin{tabular}{|c|c|c|c|c|c|c|}
\hline & $\begin{array}{l}\text { KEBBI } \\
\%\end{array}$ & $\begin{array}{l}\text { SOKOTO } \\
\%\end{array}$ & $\begin{array}{c}\text { MALARIA-ONLY } \\
\text { (ZAMFARA) } \\
\%\end{array}$ & $\begin{array}{c}\text { INTEGRATED } \\
\text { (KEBBI/SOKOTO) } \\
\%\end{array}$ & $\mathbf{N}$ & $\begin{array}{c}\mathrm{CHI}^{2} \\
\text { VALUE }\end{array}$ \\
\hline \multicolumn{7}{|c|}{ Have you ever used the internet? } \\
\hline No & 99.3 & 98.5 & 99.4 & 98.9 & 5,949 & 4.232 \\
\hline Yes & 0.7 & 1.5 & 0.6 & 1.1 & 84 & 0.884 \\
\hline Total & 100.0 & 100.0 & 100.0 & 100.0 & 6,033 & \\
\hline N & 1,960 & 2,062 & 2,011 & 4,022 & & \\
\hline \multicolumn{7}{|c|}{$\begin{array}{l}\text { About how many days in a normal } \\
\text { week do you use the internet? }\end{array}$} \\
\hline 0 times & 99.3 & 98.5 & 99.4 & 98.9 & 5,950 & 9.342 \\
\hline 1-2 days & 0.3 & 0.2 & 0.0 & 0.2 & 12 & 1.274 \\
\hline $3-4$ days & 0.1 & 0.1 & 0.2 & 0.1 & 15 & \\
\hline $5-7$ days & 0.4 & 1.1 & 0.4 & 0.8 & 57 & \\
\hline Total & 100.0 & 100.0 & 100.0 & 100.0 & 6,034 & \\
\hline N & 1,960 & 2,062 & 2,012 & 4,022 & & \\
\hline \multicolumn{7}{|c|}{ How do you access the internet? } \\
\hline Public computer & 3.2 & 1.1 & 0.0 & 1.7 & 2 & 2.472 \\
\hline Own phone & 86.5 & 96.7 & 100.0 & 93.7 & 79 & 1.581 \\
\hline Someone else's phone & 10.3 & 2.2 & 0.0 & 4.6 & 3 & \\
\hline Total & 100.0 & 100.0 & 100.0 & 100.0 & 84 & \\
\hline $\mathrm{N}$ & 17 & 52 & 15 & 69 & & \\
\hline \multicolumn{7}{|c|}{$\begin{array}{l}\text { How easy for you to access internet to } \\
\text { look up health info }\end{array}$} \\
\hline Very easy & 42.9 & 72.9 & 55.9 & 64.2 & 57 & 10.952 \\
\hline Somewhat easy & 46.5 & 15.5 & 14.6 & 24.5 & 16 & 1.372 \\
\hline Somewhat difficult & 10.6 & 8.1 & 3.5 & 8.9 & 8 & \\
\hline Very difficult & 0.0 & 3.5 & 26.0 & 2.5 & 3 & \\
\hline Total & 100.0 & 100.0 & 100.0 & 100.0 & 84 & \\
\hline N & 17 & 52 & 15 & 69 & & \\
\hline \multicolumn{7}{|c|}{$\begin{array}{l}\text { In the last } 12 \text { months, did you see any } \\
\text { health messages on internet? }\end{array}$} \\
\hline No & 61.4 & 45.2 & 27.3 & 50.0 & 35 & 5.323 \\
\hline Yes & 38.6 & 52.6 & 72.7 & 48.5 & 47 & 1.868 \\
\hline Don't know & 0.0 & 2.2 & 0.0 & 1.6 & 2 & \\
\hline Total & 100.0 & 100.0 & 100.0 & 100.0 & 84 & \\
\hline $\mathrm{N}$ & 17 & 52 & 15 & 69 & & \\
\hline
\end{tabular}

Estimates based on low Ns (approx. $<30$ obs) have large relative standard errors and should be interpreted with caution. ${ }^{* * *}$ indicates $p<0.001,{ }^{* *}$ indicates $p<0.05$ and ${ }^{*}$ indicates $p<.0 .01$ for the chi ${ }^{2}$ statistic comparing integrated versus malaria-only study areas. 


\subsection{Other information sources}

\section{TABLE 9.6.1. HEALTH INFORMATION RECEIVED FROM OTHER SOURCES}

Percentage of women 15 to 49 years currently pregnant or with a child under 2 years who report receiving health messages from other sources by type, September-October 2019

\begin{tabular}{|c|c|c|c|c|c|c|}
\hline & $\begin{array}{c}\text { KEBBI } \\
\%\end{array}$ & $\begin{array}{l}\text { SOKOTO } \\
\%\end{array}$ & $\begin{array}{c}\text { MALARIA-ONLY } \\
\text { (ZAMFARA) } \\
\%\end{array}$ & $\begin{array}{c}\text { INTEGRATED } \\
\text { (KEBBI/SOKOTO) } \\
\%\end{array}$ & $\mathbf{N}$ & $\begin{array}{c}\mathrm{CHI}^{2} \\
\text { VALUE }\end{array}$ \\
\hline \multicolumn{7}{|c|}{$\begin{array}{l}\text { Last } 12 \text { months received/heard/seen } \\
\text { health information from other sources }\end{array}$} \\
\hline No & 85.0 & 87.3 & 92.0 & 86.2 & 5,266 & 52.302 \\
\hline Yes & 11.3 & 9.6 & 5.8 & 10.4 & 570 & 0.676 \\
\hline Don't know & 3.8 & 3.1 & 2.3 & 3.4 & 197 & \\
\hline Total & 100.0 & 100.0 & 100.0 & 100.0 & 6,033 & \\
\hline$N$ & 1,960 & 2,062 & 2,011 & 4,022 & & \\
\hline \multicolumn{7}{|c|}{$\begin{array}{l}\text { Health info source in the past } 3 \\
\text { months }\end{array}$} \\
\hline \multicolumn{7}{|l|}{ Billboards } \\
\hline No & 70.8 & 86.3 & 27.6 & 78.6 & 390 & 148.210 \\
\hline Yes & 29.2 & 13.7 & 72.4 & 21.4 & 180 & 5.756 \\
\hline Total & 100.0 & 100.0 & 100.0 & 100.0 & 570 & * \\
\hline N & 235 & 224 & 111 & 459 & & \\
\hline \multicolumn{7}{|c|}{ Mobile phone/SMS } \\
\hline No & 96.3 & 95.1 & 99.7 & 95.7 & 545 & 9.428 \\
\hline Yes & 3.8 & 4.9 & 0.3 & 4.3 & 25 & 8.415 \\
\hline Total & 100.0 & 100.0 & 100.0 & 100.0 & 570 & ** \\
\hline $\mathrm{N}$ & 235 & 224 & 111 & 459 & & \\
\hline \multicolumn{7}{|l|}{ Cinema } \\
\hline No & 99.3 & 100.0 & 100.0 & 99.7 & 569 & 0.850 \\
\hline Yes & 0.7 & 0.0 & 0.0 & 0.3 & 1 & 0.847 \\
\hline Total & 100.0 & 100.0 & 100.0 & 100.0 & 570 & \\
\hline N & 235 & 224 & 111 & 459 & & \\
\hline \multicolumn{7}{|c|}{ Live drama/puppet show } \\
\hline No & 98.9 & 98.9 & 99.1 & 98.9 & 565 & 0.029 \\
\hline Yes & 1.1 & 1.1 & 1.0 & 1.1 & 5 & 0.011 \\
\hline Total & 100.0 & 100.0 & 100.0 & 100.0 & 570 & \\
\hline N & 235 & 224 & 111 & 459 & & \\
\hline \multicolumn{7}{|c|}{ Leaflet/brochure } \\
\hline No & 98.3 & 98.8 & 98.3 & 98.5 & 562 & 0.042 \\
\hline Yes & 1.7 & 1.2 & 1.7 & 1.5 & 8 & 0.013 \\
\hline Total & 100.0 & 100.0 & 100.0 & 100.0 & 570 & \\
\hline $\mathrm{N}$ & 235 & 224 & 111 & 459 & & \\
\hline
\end{tabular}

Estimates based on low Ns (approx. $<30$ obs) have large relative standard errors and should be interpreted with caution. ${ }^{* \star *}$ indicates $p<0.001,{ }^{* *}$ indicates $p<0.05$ and * indicates $p<.0 .01$ for the chi ${ }^{2}$ statistic comparing integrated versus malaria-only study areas. 
TABLE 9.6.2. HEALTH INFORMATION RECEIVED FROM HEALTH SYSTEM SOURCES

Percentage of women 15 to 49 years currently pregnant or with a child under 2 years who received health messages from health system sources by type, September-October 2019

\begin{tabular}{|c|c|c|c|c|c|c|}
\hline & KEBBI & SOKоTO & $\begin{array}{l}\text { MALARIA-ONLY } \\
\text { (ZAMFARA) }\end{array}$ & $\begin{array}{l}\text { INTEGRATED } \\
\text { (KEBBI/SOKOTO) }\end{array}$ & $\mathbf{N}$ & $\begin{array}{c}\mathrm{CHI}^{2} \\
\text { VALUE }\end{array}$ \\
\hline & $\%$ & $\%$ & $\%$ & $\%$ & & \\
\hline \multicolumn{7}{|c|}{ Government hospital/health center } \\
\hline No & 57.3 & 68.6 & 16.8 & 63.0 & 298 & 123.298 \\
\hline Yes & 42.7 & 31.4 & 83.2 & 37.1 & 272 & 5.101 \\
\hline Total & 100.0 & 100.0 & 100.0 & 100.0 & 570 & * \\
\hline N & 235 & 224 & 111 & 459 & & \\
\hline \multicolumn{7}{|c|}{ Government post/dispensary } \\
\hline No & 96.7 & 99.2 & 33.3 & 97.9 & 497 & 275.033 \\
\hline Yes & 3.3 & 0.8 & 66.7 & 2.1 & 73 & 37.753 \\
\hline Total & 100.0 & 100.0 & 100.0 & 100.0 & 570 & $* * *$ \\
\hline $\mathrm{N}$ & 235 & 224 & 111 & 459 & & \\
\hline \multicolumn{7}{|c|}{ Women and children hospital } \\
\hline No & 98.3 & 98.8 & 68.7 & 98.5 & 533 & 99.190 \\
\hline Yes & 1.8 & 1.2 & 31.3 & 1.5 & 37 & 43.198 \\
\hline Total & 100.0 & 100.0 & 100.0 & 100.0 & 570 & 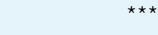 \\
\hline $\mathrm{N}$ & 235 & 224 & 111 & 459 & & \\
\hline \multicolumn{7}{|c|}{ Child welfare clinic } \\
\hline No & 99.6 & 99.5 & 100.0 & 99.5 & 567 & 1.179 \\
\hline Yes & 0.4 & 0.5 & 0.0 & 0.5 & 3 & 1.229 \\
\hline Total & 100.0 & 100.0 & 100.0 & 100.0 & 570 & \\
\hline $\mathrm{N}$ & 235 & 224 & 111 & 459 & & \\
\hline \multicolumn{7}{|c|}{ Church/mission/hospital } \\
\hline No & 100.0 & 99.8 & 100.0 & 99.9 & 569 & 0.257 \\
\hline Yes & 0.0 & 0.2 & 0.0 & 0.1 & 1 & 0.655 \\
\hline Total & 100.0 & 100.0 & 100.0 & 100.0 & 570 & \\
\hline $\mathrm{N}$ & 235 & 224 & 111 & 459 & & \\
\hline \multicolumn{7}{|c|}{ Private hospital/clinic } \\
\hline No & 100.0 & 97.4 & 100.0 & 98.7 & 565 & 3.388 \\
\hline Yes & 0.0 & 2.6 & 0.0 & 1.3 & 5 & 0.932 \\
\hline Total & 100.0 & 100.0 & 100.0 & 100.0 & 570 & \\
\hline$N$ & 235 & 224 & 111 & 459 & & \\
\hline \multicolumn{7}{|c|}{ Nursing/maternity home } \\
\hline No & 98.5 & 100.0 & 99.7 & 99.2 & 565 & 0.609 \\
\hline Yes & 1.5 & 0.0 & 0.3 & 0.8 & 5 & 0.469 \\
\hline Total & 100.0 & 100.0 & 100.0 & 100.0 & 570 & \\
\hline $\mathrm{N}$ & 235 & 224 & 111 & 459 & & \\
\hline \multicolumn{7}{|l|}{ Kiosk } \\
\hline No & 95.9 & 100.0 & 100.0 & 97.9 & 560 & 5.363 \\
\hline Yes & 4.1 & 0.0 & 0.0 & 2.1 & 10 & 1.165 \\
\hline Total & 100.0 & 100.0 & 100.0 & 100.0 & 570 & \\
\hline N & 235 & 224 & 111 & 459 & & \\
\hline \multicolumn{7}{|c|}{ Community health worker } \\
\hline No & 93.5 & 92.0 & 94.2 & 92.7 & 527 & 0.498 \\
\hline Yes & 6.5 & 8.1 & 5.8 & 7.3 & 43 & 0.088 \\
\hline Total & 100.0 & 100.0 & 100.0 & 100.0 & 570 & \\
\hline $\mathrm{N}$ & 235 & 224 & 111 & 459 & & \\
\hline \multicolumn{7}{|c|}{ Traditional birth attendant/healer } \\
\hline No & 95.7 & 96.6 & 96.4 & 96.2 & 551 & 0.018 \\
\hline Yes & 4.3 & 3.4 & 3.6 & 3.8 & 19 & 0.003 \\
\hline Total & 100.0 & 100.0 & 100.0 & 100.0 & 570 & \\
\hline $\mathrm{N}$ & 235 & 224 & 111 & 459 & & \\
\hline \multicolumn{7}{|c|}{ Mobile clinic } \\
\hline No & 100.0 & 99.8 & 100.0 & 99.9 & 569 & 0.231 \\
\hline Yes & 0.0 & 0.2 & 0.0 & 0.1 & 1 & 0.633 \\
\hline Total & 100.0 & 100.0 & 100.0 & 100.0 & 570 & \\
\hline $\mathrm{N}$ & 235 & 224 & 111 & 459 & & \\
\hline \multicolumn{7}{|c|}{ Pharmacy } \\
\hline No & 97.3 & 98.6 & 94.5 & 97.9 & 550 & 4.623 \\
\hline Yes & 2.7 & 1.5 & 5.5 & 2.1 & 20 & 0.600 \\
\hline Total & 100.0 & 100.0 & 100.0 & 100.0 & 570 & \\
\hline N & 235 & 224 & 111 & 459 & & \\
\hline
\end{tabular}

Estimates based on low Ns (approx. $<30$ obs) have large relative standard errors and should be interpreted with caution. ${ }^{* * *}$ indicates $p<0.001,{ }^{* *}$ indicates $p<0.05$ and * indicates $p<.0 .01$ for the chi ${ }^{2}$ statistic comparing integrated versus malaria-only study areas. 
TABLE 9.6.3. HEALTH INFORMATION RECEIVED FROM OTHER PERSONS OR SOURCES

Percentage of women 15 to 49 years currently pregnant or with a child under 2 years who received health messages from other persons or influencers, September-October 2019

\begin{tabular}{|c|c|c|c|c|c|c|}
\hline & KEBBI & SOKOTO & $\begin{array}{l}\text { MALARIA-ONLY } \\
\text { (ZAMFARA) }\end{array}$ & $\begin{array}{c}\text { INTEGRATED } \\
\text { (KEBBI/SOKOTO) }\end{array}$ & $\mathbf{N}$ & $\begin{array}{c}\mathrm{CHI}^{2} \\
\text { VALUE }\end{array}$ \\
\hline & $\%$ & $\%$ & $\%$ & $\%$ & & \\
\hline \multicolumn{7}{|c|}{ Parents } \\
\hline No & 85.4 & 85.2 & 99.5 & 85.3 & 501 & 37.653 \\
\hline Yes & 14.6 & 14.8 & 0.5 & 14.7 & 69 & 20.064 \\
\hline Total & 100.0 & 100.0 & 100.0 & 100.0 & 570 & *** \\
\hline $\mathrm{N}$ & 235 & 224 & 111 & 459 & & \\
\hline \multicolumn{7}{|c|}{ Sisters/brothers } \\
\hline No & 81.9 & 96.5 & 99.7 & 89.2 & 515 & 27.255 \\
\hline Yes & 18.1 & 3.6 & 0.3 & 10.8 & 55 & 19.596 \\
\hline Total & 100.0 & 100.0 & 100.0 & 100.0 & 570 & *** \\
\hline N & 235 & 224 & 111 & 459 & & \\
\hline \multicolumn{7}{|c|}{ Teacher/peer educator } \\
\hline No & 97.4 & 99.7 & 100.0 & 98.5 & 561 & 3.791 \\
\hline Yes & 2.6 & 0.4 & 0.0 & 1.5 & 9 & 1.473 \\
\hline Total & 100.0 & 100.0 & 100.0 & 100.0 & 570 & \\
\hline $\mathrm{N}$ & 235 & 224 & 111 & 459 & & \\
\hline \multicolumn{7}{|c|}{ Spouse/partner } \\
\hline No & 71.9 & 79.4 & 98.5 & 75.6 & 452 & 60.996 \\
\hline Yes & 28.1 & 20.6 & 1.5 & 24.4 & 118 & 14.373 \\
\hline Total & 100.0 & 100.0 & 100.0 & 100.0 & 570 & *** \\
\hline $\mathrm{N}$ & 235 & 224 & 111 & 459 & & \\
\hline \multicolumn{7}{|c|}{ Friends/neighbors } \\
\hline No & 58.1 & 56.8 & 96.2 & 57.5 & 380 & 112.824 \\
\hline Yes & 41.9 & 43.2 & 3.8 & 42.6 & 190 & 12.512 \\
\hline Total & 100.0 & 100.0 & 100.0 & 100.0 & 570 & 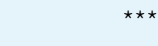 \\
\hline $\mathrm{N}$ & 235 & 224 & 111 & 459 & & \\
\hline
\end{tabular}

Estimates based on low Ns (approx. $<30$ obs) have large relative standard errors and should be interpreted with caution. ${ }^{* \star *}$ indicates $p<0.001,{ }^{* *}$ indicates $p<0.05$ and ${ }^{*}$ indicates $p<.0 .01$ for the chi ${ }^{2}$ statistic comparing integrated versus malaria-only study areas. 


\section{Discussion}

This technical report presents complete results of the baseline BSS survey undertaken between September and October 2019. Over the period from 2019 to 2021, baseline, midline, and endline waves of the BSS will be used to assess the effectiveness of the Breakthrough ACTION/Nigeria integrated SBC activities for malaria, family planning, and $\mathrm{MNCH}+\mathrm{N}$ in Kebbi and Sokoto relative to malaria-only SBC activities in Zamfara.

As part of this evaluation, the BSS not only measures changes in behavioral outcomes across family planning, malaria, and $\mathrm{MNCH}+\mathrm{N}$, but also measures whether certain behavioral drivers, or ideations, have been modified by SBC activities over the life of the Breakthrough ACTION/Nigeria project. Indeed, the BSS is unique in its focus on measuring these psychosocial influences - or emotional, social, and cognitive domains - that have been posited by various theories as intermediate determinants of behavioral outcomes. For some health areas, the BSS is pioneering in its collection of new metrics for behavioral drivers (e.g., pneumonia, breastfeeding, ANC, delivery care) while in other areas, the BSS has adapted published metrics for the Nigerian context (e.g., vaccination, diarrhea).

Based on this new data collection work, the BSS baseline report provides important program-relevant evidence to inform Breakthrough ACTION/Nigeria SBC adaption and scale-up during this early implementation period. In this report, we answer some key learning questions for Breakthrough ACTION/Nigeria programs, including: To what extent are respondents aware of health behaviors being promoted (e.g., how to prevent and treat certain diseases); To what extent are certain myths or beliefs held by respondents that could impede progress across health areas; How do respondents view health services in their communities, and what are the main reasons for choosing certain treatment locations or for not seeking care at all; Who mainly influences decisions and practices across health areas; What are key social norms around health behaviors; and Do respondents feel confident in their ability to take up promoted practices.

Key findings and recommendations for different health areas are outlined in the following sections. Going forward, in-depth analyses of the BSS baseline dataset will provide further evidence about the relative importance of different ideations on behavioral outcomes across health areas. We will also examine the differences among doers and non-doers of promoted behaviors, and take a deeper dive into the role of spousal communication, or other important topic areas, in the uptake of promoted behaviors by Breakthrough ACTION/Nigeria.

\section{Key Findings and Recommendations}

\section{Albishirin Ku!}

- Awareness of Breakthrough ACTION/Nigeria's Albishirin Ku! radio programming was already apparent even at this baseline period. In Kebbi/Sokoto, 24 percent of respondents had heard the Albishirin Ku! radio slogan as have 13 percent in Zamfara.

- Albishirin Ku! radio program awareness in Kebbi/Sokoto was significantly lower among respondents in the poorest households (12\%) than in wealthiest households (42\%) across study areas.

- This result suggests that Breakthrough ACTION/Nigeria radio programming may not penetrate to the poorest households that have demonstrably worse health outcomes according to BSS results (see below sections). Breakthrough ACTION/Nigeria may need to assure that additional outreach efforts through other channels or methods (e.g., household visits or community events) are targeted at the poorest areas, or consider ways to bring radios to communities (e.g., listening or discussion groups). 


\section{Family planning}

- Modern contraceptive use: There was low modern contraceptive use among non-pregnant respondents, with higher use in Zamfara (15\%) than in Kebbi/ Sokoto (11\%). Modern contraceptive use was lower among respondents in poorest households ( $6 \%$ and $7 \%$ ) than in wealthiest households (24\% and $34 \%$ ). Most respondents had heard of at least one modern method, with the most recognized methods being injectables, implants, and daily pills. Higher recall of methods was noted in Kebbi/Sokoto (30\%, 38\%, 33\%) than in Zamfara (16\%, 23\%, 19\%). Unsurprisingly, the most common methods used by non-pregnant respondents were injectables (48\%) and implants (25\%), with similar proportions across study areas.

\section{- Reasons for non-use, attitudes, and social norms:}

The most common reasons for not using contraception among current non-users were that "It's up to God" (25\%), currently breastfeeding (23\%), partner opposition (21\%), and respondent opposition (18\%). In line with this result, approximately half (52\%) of respondents agreed that they do not personally approve of using contraception for birth spacing, underscoring a critical hurdle to Breakthrough ACTION/Nigeria SBC messaging for changing family planning practices. In addition, one in five respondents believed that people in their community would call them bad names or avoid their company if others knew they were using contraception for birth spacing, which further highlights the importance of addressing social norms in family planning. About half of respondents agreed that religious leaders should speak publicly about using modern contraceptives, which is a pillar of Breakthrough ACTION/ Nigeria programming.

- Contraceptive myths: CCommon contraceptive myths also persist across study areas with firmer belief in these myths in Kebbi/Sokoto than in Zamfara. About one in three respondents in Kebbi/ Sokoto agreed that contraception could make a woman permanently infertile, harm a woman's womb, or lead to health problems, compared with about one-quarter of respondents in Zamfara. About one in five respondents in Kebbi/Sokoto agreed that contraceptives cause cancer, give you deformed babies, increase promiscuity, or reduce sexual urges of women and men. SBC programming needs to actively work to dispel these specific myths going forward to help promote behavior change.
- Spousal communication and decision-making: There were low rates of spousal communication about contraceptive use, although rates were higher in Zamfara (22\%) than in Kebbi/Sokoto (6\%). Unsurprisingly, respondents also noted that contraceptive decisions were mainly made by their partners, with higher reports in Kebbi/Sokoto (30\%) than in Zamfara (17\%). When respondents were asked who else influences a woman's decision about contraceptive use, the most commonly mentioned influencers were no one else (61\%) and partners/ spouses (30\%). Self-efficacy for negotiating contraceptive use with a partner was low. Only about half of respondents across study areas were confident in their ability to convince their partner to use modern contraception for spacing births. Finally, among non-users, only 15 percent intended to start contraceptive use in the next six months, representing another barrier to raising contraceptive use rates.

\section{Malaria}

- Malaria prevention: Overall, there was high long-lasting insecticide nets (LLIN) ownership. Approximately 75 percent and 69 percent of households in Zamfara and Kebbi/Sokoto, respectively, own at least one LLIN. However, fewer households had the recommended ratio of at least one LLIN for every two household members, with slightly higher proportions in Kebbi/Sokoto (25\%) than in Zamfara (18\%). In households where there were sufficient nets (at least one LLIN for every two household members), 81 percent and 88 percent of pregnant respondents slept under an LLIN the previous night in Kebbi/Sokoto and Zamfara, respectively, with little difference across wealth quintiles or maternal education. Similarly, in households with sufficient nets, over 90 percent of children under 2 years slept under an LLIN the previous night in integrated and malaria-only areas, with limited differences across sociodemographics. There was also extremely high overall awareness about the importance of LLINs in malaria prevention. Among respondents who were currently pregnant or with a child under 2 years, nearly all (97\%) agreed that malaria was caused by mosquito bites and 93 percent reported that sleeping under mosquito nets was an effective malaria prevention method. In addition, there was also strong intent to get all children under 5 sleeping under a mosquito net (93\%). The results suggest that resources building additional knowledge of the 
modes of transmission are not needed, but improving access, acceptability, and efficacy of LLIN may be a useful programmatic focus.

- Malaria during pregnancy: Malaria prevention efforts during pregnancy are substantially lacking in all three states. Less than one in five respondents in study areas took intermittent preventive treatment (three or more SP/Fansidar doses) during their last pregnancy, with lower rates among respondents in the poorest households (10\% and $12 \%$ ), as compared with the wealthiest (33\% and 34\%) in Kebbi/Sokoto and Zamfara, respectively. Lower percentages were also observed among the less educated (15\% and $14 \%)$ compared to respondents who attended at least some primary school (36\% and 25\%). The most common reasons for not taking SP/Fansidar during the last pregnancy were opposition by respondent $(34 \%)$ or spouse (33\%), which underscores a major barrier to increasing uptake. Among respondents attending ANC, few in Kebbi/Sokoto (11\%) and Zamfara (18\%) were given a mosquito net during the ANC visit. While 91 percent of respondents agreed that malaria in pregnancy can affect the fetus, far fewer could state the specific risks to the baby. Only about half across the study area reported fetal death $(45 \%)$ or miscarriage (44\%) as specific risks from malaria in pregnancy, while far fewer reported premature birth (26\%) and low birth weight (17\%) as specific risks. Building knowledge of malaria risks and specific health consequences for the fetus may be a driver toward greater adoption of prevention behaviors.

- Malaria treatment: While there were high rates of care-seeking for children under 2 years of age with fever, many respondents (33\% and $54 \%$ of those who sought care in Kebbi/Sokoto and Zamfara, respectively) went to a pharmacy where poor-quality care persists. The most important reasons for choosing the treatment location for pediatric fever care were trust (36\%), nearby location (33\%), and effective treatment (28\%). These findings underscore the importance to women of convenient and high-quality health services provision in their communities. Importantly, few febrile children were tested for malaria (19\% and $24 \%$ ), and few were given ACT treatment (22\% and $36 \%$ ) in Kebbi/Sokoto and Zamfara. The low prevalence of testing may be due in large part to care-seeking from pharmacies. Nearly all respondents reported intentions to take a child with fever to care the same/next day (92\%) and to make sure the child takes the entire treatment course (92\%), suggesting motivation is not a primary barrier to care-seeking. However, while 74 percent agreed that a blood test is the only way to know whether a person has malaria, most respondents (61\%) agreed that they still worry the illness could be malaria even if the test result is negative. This implies some lack of confidence in negative malaria test results, which points to an area for future SBC programming.

\section{$M N C H+N$}

- Antenatal care: There is low ANC attendance across the three states, with approximately one-third (32\% and $38 \%$ ) of respondents attending ANC one or more times and one-quarter (20\% and $26 \%$ ) attending ANC four or more times during the last pregnancy in Kebbi/Sokoto and Zamfara, respectively. The most common reasons for not attending ANC were lack of perceived need (42\%), spousal opposition (25\%), fatalism ("It's up to God") (20\%), and perceptions that ANC is not the norm (13\%). More than two-thirds (67\%) of respondents said their spouses influence their decision to go to ANC four or more times, making spousal support or opposition a critical facilitator or barrier to uptake. The strong influence of spouses on pregnancy and other health-related decisions underscores the need for continued and sustained engagment with men to improve the uptake of essential services for women and children in this area. There are also common ANC myths among respondents that persist. Overall, 48 percent and 35 percent of respondents agreed that pregnant women need ANC only when sick, and one-quarter of respondents agreed that only first-time mothers need ANC. A significant percentage of respondents -40 percent and 30 percent in Kebbi/Sokoto and Zamfara, respectively-noted that it is better to use a traditional provider than a health facility for ANC. Birth planning with a health worker during ANC does not appear to be standard practice, and only one in three respondents reported having discussed birth plans. These perceptions point to areas for SBC messaging to improve awareness of the benefits of ANC even for healthy pregnancies that could lead to additional ANC uptake going forward.

- Facility delivery: Facility delivery was an uncommon practice in Kebbi/Sokoto (14\%) and Zamfara (16\%) among respondents during their last pregnancy. More than 80 percent of respondents delivered at 
their own or another home, and births are commonly assisted by family members (36\%) or traditional birth attendants (29\%). The most common reasons for not delivering at a facility were that they did not feel it was necessary (75\% and 61\%) followed by spousal opposition (15\% and $36 \%$ ) in Kebbi/Sokoto and Zamfara, respectively. When respondents were asked who influences the decision to have a facility delivery, partner/spouse (57\%) was the most common response, making spousal opposition a critical barrier to service utilization. Only about half (54\%) of respondents agreed that the health facility is the best place to deliver a baby, and even fewer (44\%) felt confident that they could get to a health facility for delivery. Only about half (55\%) were confident that they could start a conversation with their husband about facility delivery, and a similar proportion (51\%) intended to deliver their next child at a health facility.

- Breastfeeding: Nearly all respondents (96\%) reported ever breastfeeding their last-born child under 2 years. Yet initiating breastfeeding within one hour of birth was low across study areas, although higher in Zamfara (46\%) than in Kebbi/Sokoto (36\%). Exclusive breastfeeding for the first six months of life was also low across study areas, with higher rates in Zamfara (46\%) than in Kebbi/Sokoto (26\%). This low coverage is in large part due to high rates of giving non-breastmilk liquids to a child in the first three days after birth, with much higher practice rates in Kebbi/Sokoto (73\%) than in Zamfara (50\%). In relation to this practice, 23 percent of respondents also agreed that mother's breastmilk after birth is bad, suggesting an area for future SBC messaging. Taken together, these responses suggest ways SBC messaging may help promote exclusive breastfeeding in this study area. In addition, while there was high awareness of breastfeeding and its benefits among respondents, only 55 percent of respondents felt confident to exclusively breastfeed their child for the first six months, with higher confidence in Zamfara (64\%) than Kebbi/Sokoto (42\%). Forty-one percent of respondents believed that most other respondents in their communities gave breastmilk exclusively to their infants, suggesting perceived social norms about breastfeeding. There was also relatively low intent to exclusively breastfeed their next child in Zamfara (59\%) and Kebbi/Sokoto (46\%).

- Vaccination: There was very low vaccination coverage in study areas, with 4 percent and 8 percent of children aged 12 to 23 months in Kebbi/Sokoto and Zamfara, respectively, fully vaccinated (BCG, measles, DPT3, and polio3) by the time of the survey interview. The most important reason for not vaccinating the child was spousal opposition (33\%) followed by distance to the health facility (14\%) and fear of needles (11\%). Across study areas, 73 percent of respondents reported that their spouses influence their decision about child vaccinations, making spousal opposition an important barrier to vaccine uptake. While respondents gave generally positive reports about their own vaccine knowledge and attitudes, more specific questions elicited a less rosy picture. Specifically, only about half (52\%) of respondents in Kebbi/Sokoto believed that health facilities frequently had vaccines available, as compared with 61 percent in Zamfara. Only 47 percent of respondents in Kebbi/Sokoto and 66 percent in Zamfara believed most respondents in their communities took children to facilities for routine vaccination. Approximately one in four respondents (26\%) agreed that vaccines contain dangerous chemicals that could harm a child's health. Few respondents knew when a child should first be vaccinated and the number of vaccinations children should receive by their first birthdays. Taken together, results suggest relatively low vaccine knowledge, common perceptions of frequent vaccine stockouts at health facilities, spousal opposition to vaccine uptake, and the persistence of vaccine myths across the study area.

- Acute respiratory infections: While there were high rates of care-seeking for under-twos with cough, rapid breathing, and a chest-related problem, only 49 percent and 33 percent of those who sought care were taken to a formal medical source in Kebbi/ Sokoto and Zamfara. Even fewer children with these respiratory symptoms were given antibiotics (41\% and 26\%). The most common reasons for choosing the source of care was nearby location (40\%), provider trust (36\%), family/friend recommendation (30\%), and effective treatment (27\%), which underscores the importance to women of convenient and high-quality health care provision in their communities. The most important reasons for not seeking care were expense/cost (33\%) and symptoms not perceived as severe or home care was provided (25\%). Pediatric pneumonia knowledge was low across study areas. Only 28 percent and 37 percent of respondents reported rapid or difficult breathing as a symptom of pediatric pneumonia, 45 percent and 31 percent could not report any way to 
help prevent a child from getting pneumonia, and 62 percent and 56 percent could not report any effective medicine to treat pneumonia in Kebbi/Sokoto and Zamfara, respectively. These results point to the need to shift care-seeking from pharmacies to formal medical sources and to improve overall pneumonia knowledge in study areas.

- Diarrhea: Few children with diarrhea were given both ORS and zinc, although this proportion was higher in Zamfara (27\%) than in Kebbi/Sokoto (13\%). Among children with diarrhea, 36 percent and 56 percent were given ORS alone in Kebbi/Sokoto and Zamfara, respectively. While there were high rates of care-seeking for children under 2 years with diarrhea, 34 percent and 56 percent of these children were taken to a pharmacy, where poor-quality care persists. The most important reasons for choosing the source of care were provider trust (39\%), nearby location (29\%), and effective treatment (24\%), which underscores the importance to women of convenient and high-quality health care provision in their communities. The most important reasons for not seeking care were expense/cost (32\%), fatalism ("It's up to God") (29\%), and symptoms not perceived as severe or care was provided at home (25\%). Approximately one in four respondents (24\%) had never heard of zinc across study areas. Across study areas, 63 percent of respondents reported that ORS replaces lost water in a child with diarrhea, while 44 percent incorrectly noted that it stops diarrhea. These results point to the need to shift care-seeking from pharmacies to formal medical sources and to improve overall diarrhea knowledge in study areas. 
1. USAID. Nigeria: Our Work. 2019 [accessed 2019 Jan 14]. https://www.usaid.gov/nigeria/our-work

2. Adebayo, S.B., E. Gayawan, C. Ujuju, and A. Ankomah. 2013. "Modelling geographical variations and determinants of use of modern family planning methods among women of reproductive age in Nigeria," Journal of Biosocial Science 45 (1): 57-77.

3. National Population Commission (NPC) [Nigeria] and ICF. 2019. Nigeria Demographic and Health Survey 2018 Key Indicators Report. Abuja, Nigeria, and Rockville, Maryland, USA.

4. World Bank. 2011. "Nigeria-Reproductive health at a glance." Washington, D.C. http://documents.worldbank. org/curated/en/446381468012689456/Nigeria-Reproductive-health-at-a-glance

5. Ankomah, A., J. Oladosu, and M. Anyanti. 2011. "Myths, misinformation, and communication about family planning and contraceptive use in Nigeria," Open Access Journal of Contraception 2: 95-105.

6. Measurement, Learning, and Evaluation (MLE) Project; National Population Council (NPC); Data, Research and Mapping Consult Ltd. 2015. "Measurement, learning \& evaluation of the Urban Reproductive Health Initiative: Nigeria 2014 endline survey." Chapel Hill, NC, USA: Measurement, Learning, and Evaluation (MLE) Project. Report No.: TWP 2-2015. https://www.nurhitoolkit.org/ sites/default/files/tracked_files/NURHI_Endline_Report. pdf

7. Countdown to 2030. Nigeria Profile. [accessed 2019 May 1]. http://countdown2030.org/wp-content/uploads/2018/01/Nigeria-CD2030.pdf

8. UN Inter-agency Group for Child Mortality Estimation. Child Mortality Estimates: Nigeria. [accessed 2019 Apr 15]. https://childmortality.org/data/Nigeria

9. National Malaria Elimination Program (Nigeria) and II. 2016. 2015 Nigeria Malaria Indicator Survey: Atlas of Key Indicators. Rockville, Maryland,USA. https://dhsprogram. com/pubs/pdf/ATR17/ATR17.pdf

10. President's Malaria Initiative. 2019. "President's Malaria Initiative Nigeria: Malaria Operational Plan FY 2019." Washington, D.C.. https://www.pmi.gov/docs/ default-source/default-document-library/malaria-operational-plans/fy19/fy-2019-nigeria-malaria-operational-plan.pdf?sfvrsn=3

11. Babalola, S., N. John, B. Ajao, and I. Speizer. 2015. "Ideation and intention to use contraceptives in Kenya and Nigeria." Demographic Research 33(8): 211-238.
12. Kincaid, D.L. 2000. "Mass media, ideation, and behavior: A longitudinal analysis of contraceptive change in the Philippines," Communication Research 27(6): 723-763.

13. FHI 360. 2014. "Integration of global health and other development stres: A review of the evidence." Washington, D.C. https://www.fhi360.org/sites/default/files/me$\mathrm{dia} /$ documents/sap-integration-ofglobal-health-full.pdf

14. Gulumbe, U., O. Alabi, O.A. Omisakin, and S. Omoleke. 2018. "Maternal mortality ratio in selected rural communities in Kebbi State, Northwest Nigeria," BMC Pregnancy and Childbirth 18(1): 503.

15. Audu, L.R., and Ekele, B.A. 2002. "A ten-year review of maternal mortality in Sokoto, northern Nigeria." West African Journal of Medicine 21(1):74-76. http://europepmc.org/abstract/MED/12081352

16. Maternal Newborn \& Child Health Programme (MNCH2). 2019. Where we work: Zamfara [accessed 2019 Oct 27]. https://www.mnch2.com/zamfara-state/.

17. Government of Sokoto State. 2018. Sokoto State: About Us [accessed 2019 Oct 27]. http://www.sokotostate.gov. ng/about-us

18. Larson, H.J., et al. 2015. "Measuring vaccine hesitancy: The development of a survey tool," Vaccine 33(34): 4165-4175.

19. National Bureau of Statistics (NBS) and United Nations Children's Fund (UNICEF). 2017. 2017 Multiple Indicator Cluster Survey 2016-17, Survey Findings Report. Abuja, Nigeria. https://www.unicef.org/nigeria/sites/unicef.org. nigeria/files/2018-09/Nigeria-MICS-2016-17.pdf

20. Kebbi State Government. 2018. About Kebbi State. http://www.kebbistate.gov.ng/about-kebbi-state

21. The World Bank. 2016. "Federal Republic of Nigeria Poverty Work Program: poverty reduction in Nigeria in the last decade." Washington, D.C. http://documents. worldbank.org/curated/en/103491483646246005/pdf/ ACS19141-REVISED-PUBLIC-Pov-assessment-final.pdf

22. Hornik, R.C., editor. 2002. "Public health communication: Evidence for behavior change." Public health communication: Evidence for behavior change, 435.

23. Hemming, K., and Marsh, J. 2013. "A menu-driven facility for sample-size calculations in cluster randomized controlled trials," Stata Journal 13(1): 114-135. 


\section{Breakthrough RESEARCH Nigeria}

Plot 839 Idris Ibrahim Crescent,

Jabi, Abuja NIGERIA

\section{Population Council}

4301 Connecticut Ave., NW | Suite 280

Washington, DC 20008 USA

+12022379400

breakthroughactionandresearch.org 